\title{
Arable plant diversity in conventionally managed farmland of Northwest Germany
}

Current state and a participative approach to increase farmland biodiversity

\author{
Dissertation \\ zur Erlangung des mathematisch-naturwissenschaftlichen Doktorgrades \\ "Doctor rerum naturalium" \\ der Georg-August-Universität Göttingen \\ im Promotionsprogramm Biologische Diversität und Ökologie \\ der Georg-August University School of Science (GAUSS)
}

vorgelegt von

ALEXANDER WIETZKE

aus Homberg/Efze

Göttingen, 2020 


\section{Betreuungsausschuss}

Prof. Dr. Christoph Leuschner

Prof. Dr. Erwin Bergmeier

Prof. Dr. Teja Tscharntke
Pflanzenökologie und Ökosystemforschung Georg-August-Universität Göttingen

Vegetationsanalyse und Phytodiversität

Georg-August-Universität Göttingen

Agrarökologie

Georg-August-Universität Göttingen

\section{$\underline{\text { Mitglieder der Prüfungskommission }}$}

Referent: Prof. Dr. Christoph Leuschner

Pflanzenökologie und Ökosystemforschung Georg-August-Universität Göttingen

Korreferent: Prof. Dr. Erwin Bergmeier Vegetationsanalyse \& Phytodiversität Georg-August-Universität Göttingen

2. Korreferent: Prof. Dr. Teja Tscharntke Agrarökologie Georg-August-Universität Göttingen

\section{Weitere Mitglieder der Prüfungskommission:}

Prof. Dr. Hermann Behling

Prof. Dr. Johannes Isselstein

Prof. Dr. Matthias Waltert
Palynologie und Klimadynamik

Georg-August-Universität Göttingen

Graslandwissenschaft

Georg-August-Universität Göttingen

Workgroup on Endangered Species

Georg-August-Universität Göttingen

Tag der mündlichen Prüfung: 12.08.2020 


\section{Table of contents}

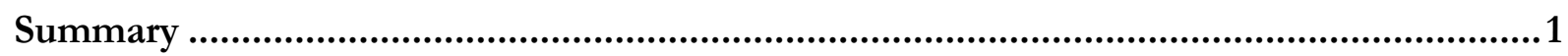

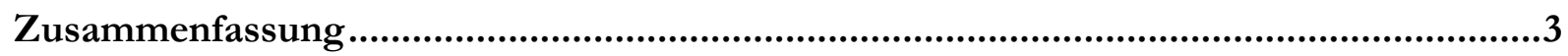

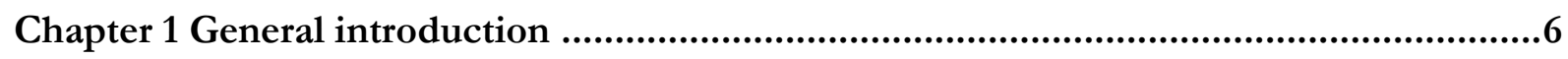

1.1 The importance of agricultural landscapes as habitat................................................................

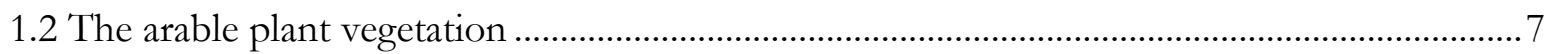

1.3 The impact of agricultural intensification on the arable flora .................................................. 8

1.4 Measures to stop biodiversity loss in agricultural landscapes...................................................... 9

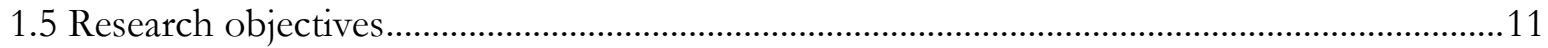

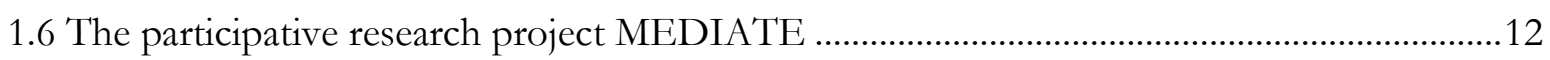

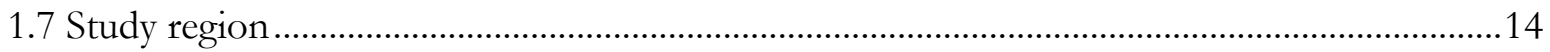

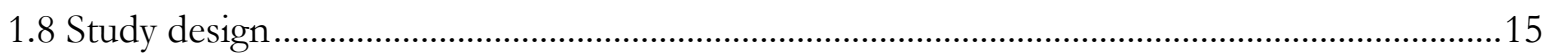

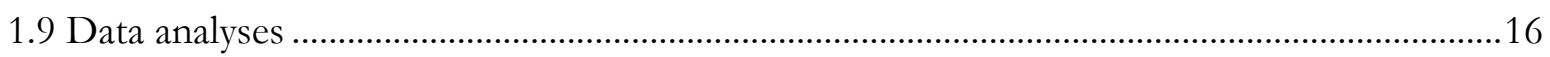

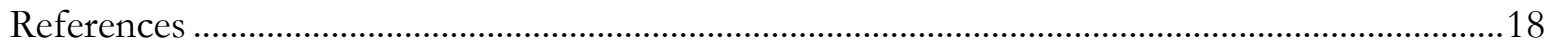

Chapter 2 Current state and drivers of arable plant diversity in conventionally managed

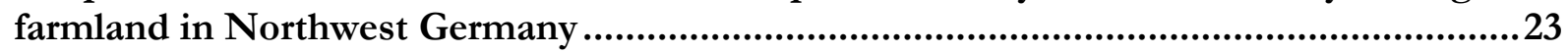

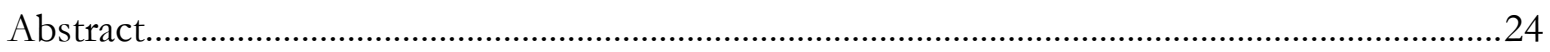

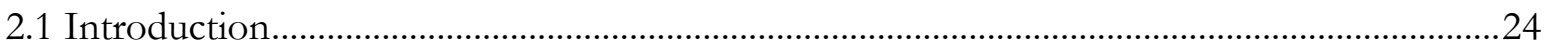

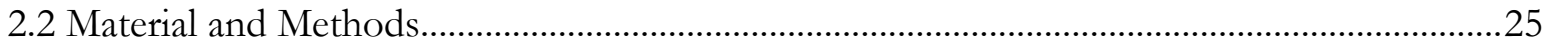

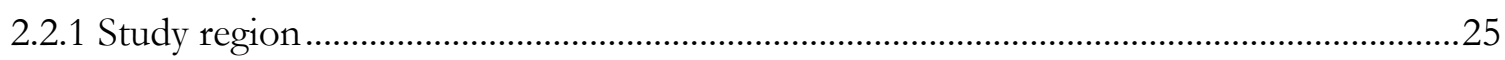

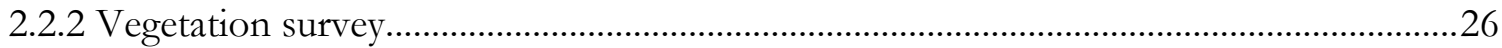

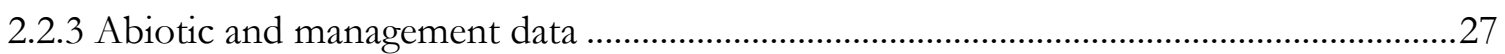

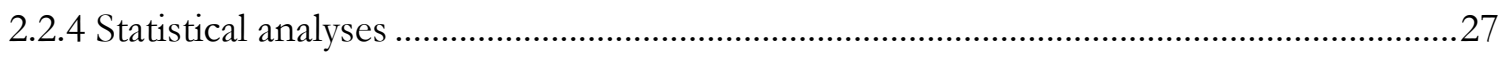

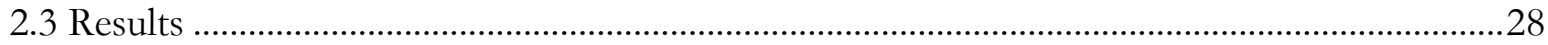

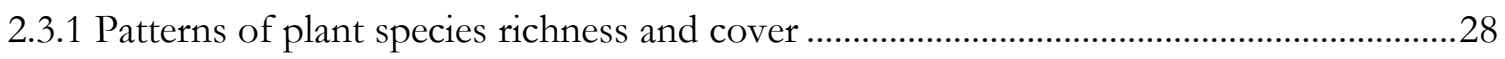

2.3.2 Variation in community composition................................................................................29

2.3.3 Factors determining arable species richness ........................................................................ 31

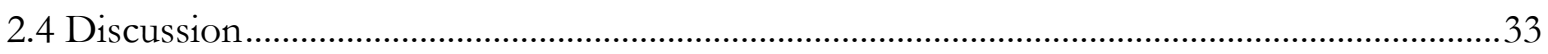

2.4.1 Quantifying phytodiversity loss in conventionally managed farmland ..............................33

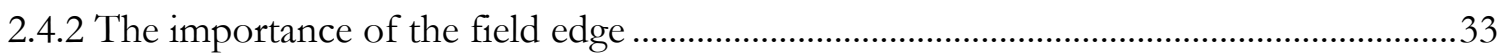

2.4.3 Composition of the recent arable vegetation and the role of field neighborhood..........34

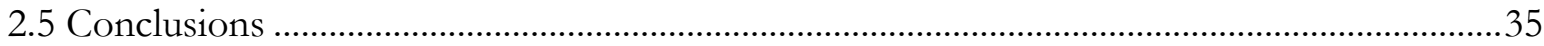




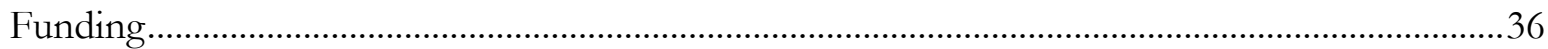

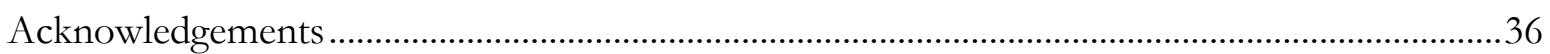

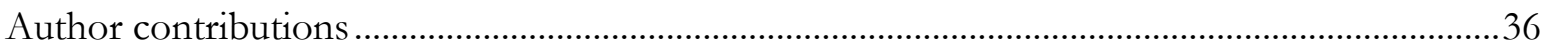

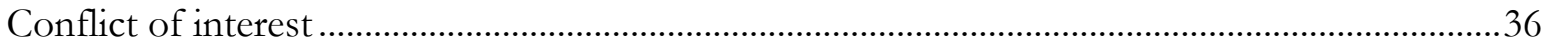

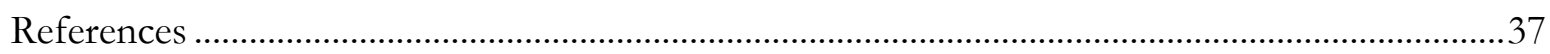

Electronic supplementary material ................................................................................................ 41

Chapter 3 Flower strips, conservation field margins and fallows promote the arable flora in intensively farmed landscapes: results of a 4 -year study ..............................................59

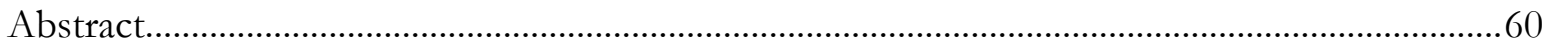

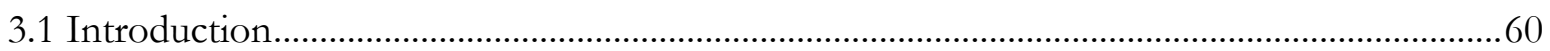

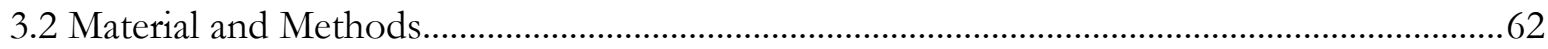

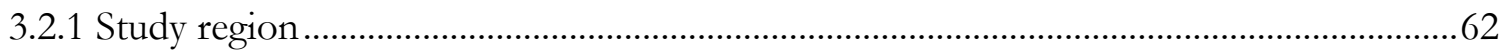

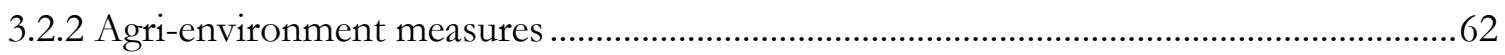

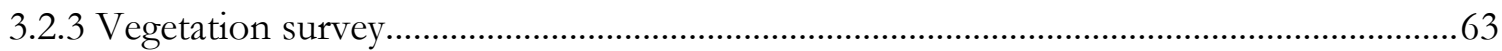

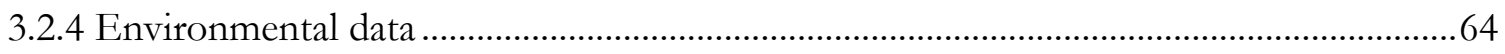

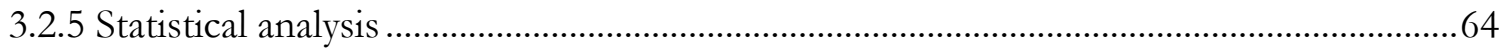

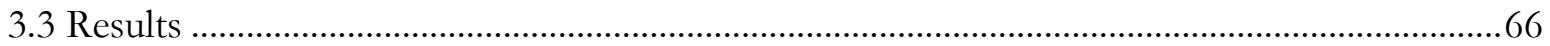

3.3.1 Effects of agri-environment measures on species richness and abundance .....................66

3.3.2 Effects of agri-environment measures on the species composition .................................67

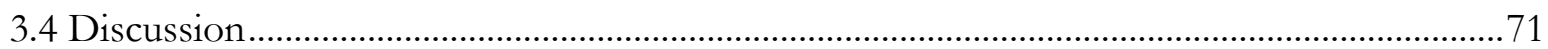

3.4.1 How were plant species richness and abundance affected by the measures? ..................71

3.4.2 How was plant species composition affected by the measures? .........................................72

3.4.3 Optimizing agri-environment measures targeted at arable plants ..................................... 74

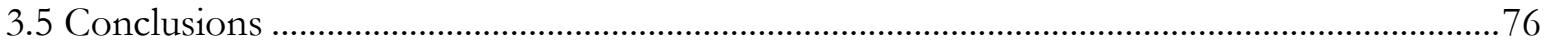

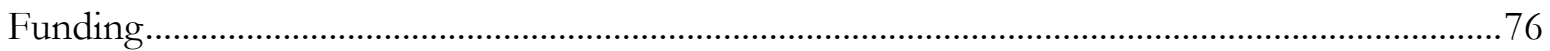

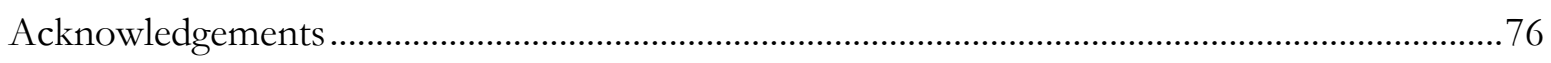

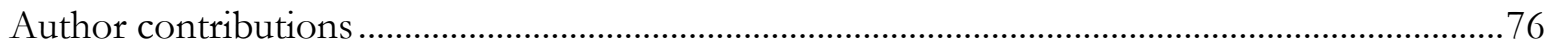

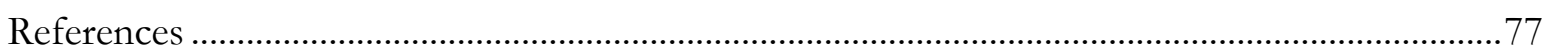

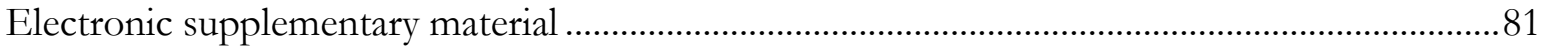

Chapter 4 Surveying the arable plant diversity of conventionally managed farmland: a

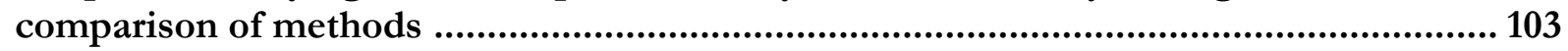

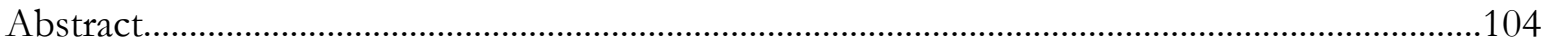




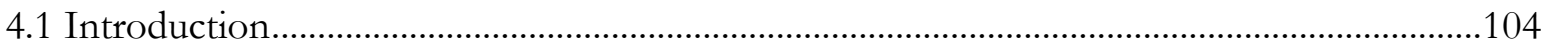

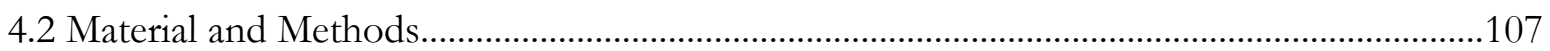

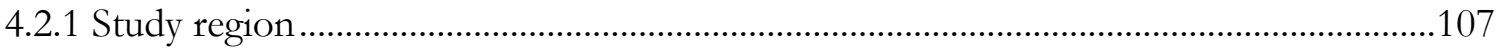

4.2.2 Vegetation survey..........................................................................................................108

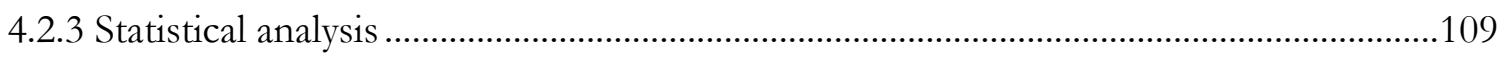

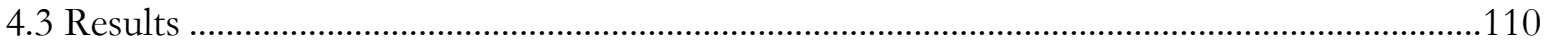

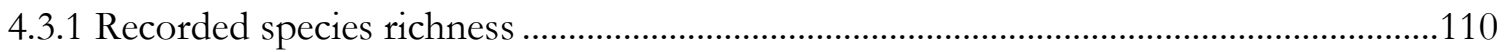

4.3.2 Expenditure of time ……………………...................................................................113

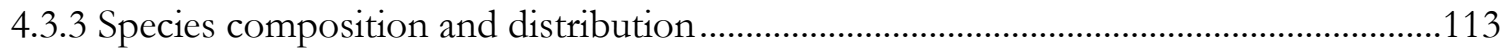

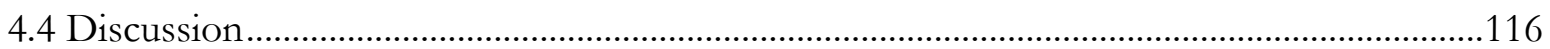

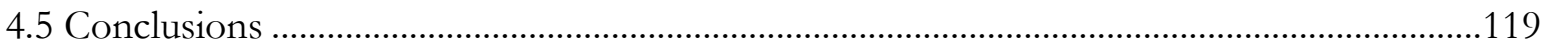

Acknowledgements .........................................................................................................................119

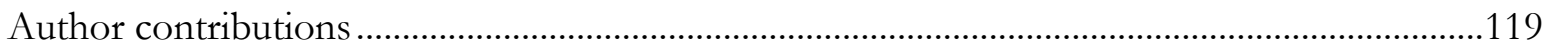

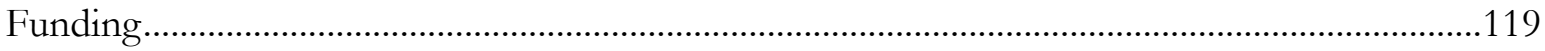

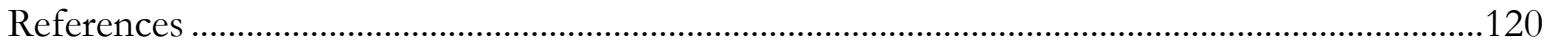

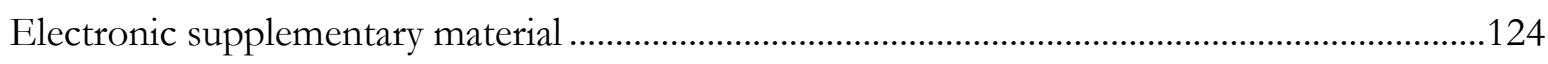

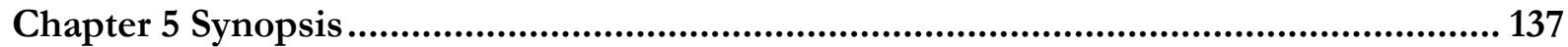

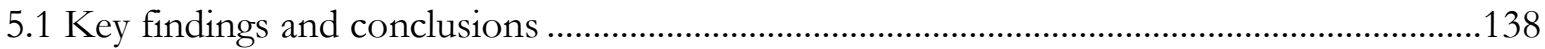

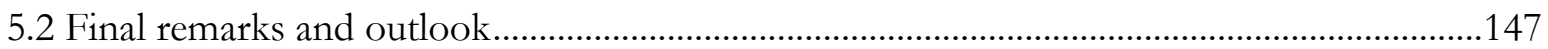

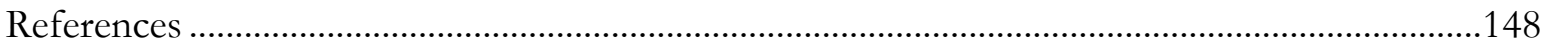

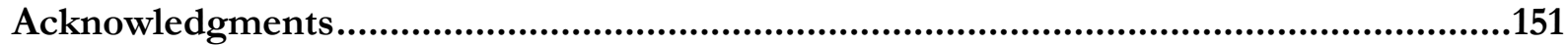

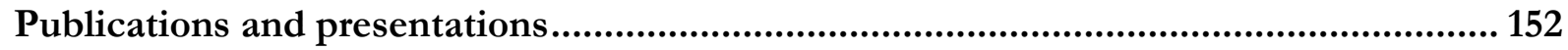

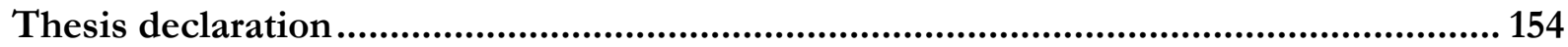




\section{Summary}

With the beginning of Central European agriculture in the Neolithic, man-made landscapes have been created, which are characterized by arable land cultivated with crops and grasslands needed for livestock farming. In these traditionally non-intensively farmed areas, a variety of animal and plant species have found a habitat. However, the industrialization of agriculture since the middle of the 19th century has been accompanied by an intensification of the management regime (in particular through the use of synthetic fertilizers and pesticides). This, in turn, has led to a strong decline in biodiversity in European agricultural landscapes within the last 50 to 60 years. The arable flora, which consists mainly of competitively weak plant species, has been particularly negatively affected by agricultural intensification. However, the maintenance of a rich arable flora is essential, as it is the basis of the food chain in agroecosystems (e.g. as a food source for many insects and farmland birds) and is therefore also crucial for the preservation of important ecosystem functions. Various conservation approaches, such as the introduction of agrienvironment measures by the European Union, have not yet been able to stop the ongoing loss of the arable flora in large parts of Europe's primarily conventionally managed farmland. Thus, further research is needed with respect to the current drivers of the plant diversity in conventionally managed fields (especially considering the effect of crop type and the landscape context) and which specific measures can effectively counteract the continuing impoverishment of the arable flora. In addition, currently conducted arable plant surveys strongly vary in their methodologies, making it difficult to reliably assess the conservation status of the arable flora and possible effects of conservation measures on a supra-regional scale. Here, the definition of a suitable standard arable plant monitoring approach is needed.

Based on the above-mentioned research needs, the present thesis is dedicated to the following research topics: Studying 200 conventionally managed arable fields, Chapter 2 shows the current state of the arable flora (species richness, abundance and composition) and investigates which factors (location in the field, cultivated crop, management regime, edaphic and landscape context) have a significant influence on the arable plant diversity. Chapter 3 compares four agrienvironment measures with regard to their effect on the arable plant vegetation within a two- to three-year study period. The measures were implemented in 67 arable fields and included i) conservation field margins, ii) annual fallows, iii) alternately managed biennial flower strips and iv) perennial flower strips. Before implementation, all measures were adapted with the participation of farmers and other relevant regional stakeholders (e.g. agricultural advisors, hunters, beekeepers, scientists, conservation authorities and organizations) to increase their effectiveness and acceptance. Based on 45 conventionally managed fields, Chapter 4 finally compares the efficiency (number of species recorded in a field and time required) of six established or newly developed survey methods for the assessment of the arable plant diversity. The research was conducted in the predominantly conventionally managed agricultural region of the districts of Nienburg and Diepholz (Lower Saxony, Northwest Germany) from 2016 to 2019.

This thesis shows that the current arable plant diversity of conventionally managed fields has reached a historical minimum. Today, the field interior is of almost no relevance as a habitat for arable plants (median species number and cover: 2 species and $0.5 \%$ ) and significant occurrences are only found in the field edge (median species number and cover: 11 species and $4 \%$; 
Chapter 2). The most important factors determining contemporary arable plant diversity in the field edge were the crop type and the presence of adjacent habitats (i.e. grass strips, hedges and ditch margins). Today's arable flora is severely impoverished and dominated by few widespread plant species, which are able to cope with the intensive management. Characteristic arable plant communities formerly associated with the specific management of crop types or soil factors are no longer detectable. The findings about the impoverishment of the arable flora presented here are transferable to a large part of Western and Central European farmland. Thus, there is an urgent need for action within the Common Agricultural Policy to counteract a further impoverishment of arable flora, related negative effects on dependent organism groups and the endangerment of important ecosystem services in agricultural landscapes. This requires the implementation of ecologically efficient measures on a large scale. The four agri-environment measures investigated in this thesis showed a similar positive effect on the arable plant diversity and abundance within the two- to three-year study period (median number of species and cover across all measures and years compared to conventionally managed field edges: 21 vs. 9 species and 68.5 vs. $9.5 \%$; Chapter 3). However, in order to preserve the arable plant diversity in the longer term, the establishment of conservation field margins and annual fallows are more suitable. Due to their adaptation for practicability on a modern conventional farm, all agrienvironmental measures tested here are expected to have a high acceptance among farmers. Thus, all measures tested are recommended for consideration in national or international agrienvironment schemes. Nevertheless, since many once widespread arable plant species are nowadays restricted to few arable fields, the introduction of such plant species via seed mixtures of local provenance should be considered in order to promote arable plant diversity at field level and to prevent the (local) extinction of rare species.

In addition, during the implementation of the agri-environment measures, it was found that different weather conditions in the study years can lead to significant changes in the observed arable plant species richness and cover. This supports the assumption that multi-year monitoring is absolutely necessary for a reliable evaluation of agri-environment measures. In this context, the choice of survey methodology can have a considerable influence on the monitoring results. This thesis demonstrated that the different currently applied arable plant survey methods lead to significantly different results regarding the recorded arable plant species pool of a conventionally managed field (Chapter 4). To analyze the future development of the arable flora and the effectiveness of implemented agri-environment measures in a larger spatial and temporal context, a standard national or international monitoring system is required, which should ideally be implemented within the framework of the Common Agricultural Policy. By capturing a large part of the arable plant species pool of a conventionally managed field (median: $75.6 \%$ ) in a reasonable time (median: 20 minutes), this thesis presented an elongated plot of $500 \times 1 \mathrm{~m}$ at the field edge as a promising survey method, which is recommended as future standard for arable plant monitoring. 


\section{Zusammenfassung}

Mit Beginn der mitteleuropäischen Landwirtschaft im Neolithikum entstanden durch den Menschen geformte Agrarlandschaften, welche bis heute durch den Anbau von Kulturfrüchten und Grünlandwirtschaft zur Viehhaltung geprägt sind. In diesen traditionell extensiv bewirtschafteten Nutzflächen hat eine Vielzahl von Tier- und Pflanzenarten einen Lebensraum gefunden. Mit der Industrialisierung der Landwirtschaft seit der Mitte des 19. Jahrhunderts ging jedoch eine intensivierte Nutzung von Agrarflächen einher (insbesondere durch den Einsatz von synthetischen Dünge- und Pflanzenschutzmitteln). Dies wiederum führte zu einem Rückgang der Artenvielfalt in europäischen Agrarlandschaften innerhalb der letzten 50 bis 60 Jahre. Die Ackerbegleitflora, welche überwiegend aus konkurrenzschwachen Pflanzenarten besteht, wurde besonders negativ von den intensivierten ackerbaulichen Nutzungspraktiken beeinflusst. Der Erhalt einer reichhaltigen Ackerbegleitflora ist jedoch essenziell, da diese ein wichtiges Fundament der Nahrungskette in Agrarökosystemen darstellt (u.a. als Nahrungsquelle für viele Insekten und Agrarvögel) und somit auch wichtig für den Erhalt bedeutender Ökosystemfunktionen ist. Unterschiedliche Schutzbestrebungen, wie etwa die Einführung von Agrarumweltmaßnahmen durch die Europäische Union, konnten den fortschreitenden Verlust der Ackerbegleitflora in weiten Teilen der hauptsächlich konventionell bewirtschafteten Agrarflächen Europas bislang nicht aufhalten. Es besteht daher noch weiterer Forschungsbedarf hinsichtlich der Faktoren, welche die Ackerwildkrautdiversität in konventionell bewirtschafteten Feldern derzeit bestimmen (insbesondere unter Berücksichtigung verschiedener Kulturfrüchte und des Landschaftskontextes) und der spezifischen Maßnahmen, die der fortschreitenden Verarmung der Ackerbegleitflora effektiv entgegenwirken können. Darüber hinaus unterscheiden sich derzeit durchgeführte Erhebungen der Ackervegetation zum Teil erheblich in der zugrundeliegenden Methodik, was eine verlässliche, überregionale Bewertung des Erhaltungszustandes der Ackerbegleitflora und möglicher Effekte von Schutzmaßnahmen schwierig macht. Hierfür ist die Definition eines geeigneten Standard-AckerwildkrautMonitoring-Ansatzes notwendig.

Basierend auf dem oben genannten Forschungsbedarf widmet sich die vorliegende Abschlussarbeit den nachfolgenden Forschungsthemen: Durch die Untersuchung von insgesamt 200 konventionell bewirtschafteten Ackerschlägen stellt Kapitel 2 den aktuellen Zustand der Ackerbegleitflora dar (Artenreichtum, Abundanz und Zusammensetzung) und überprüft, welche Faktoren (Position im Ackerschlag, angebaute Kulturfrucht, Bewirtschaftungsregime, edaphische und landschaftsbezogene Standortbedingungen) heutzutage einen wesentlichen Einfluss auf die Ackerwildkrautdiversität haben. Kapitel 3 vergleicht die Effekte von vier Agrarumweltmaßnahmen auf die Ackerbegleitflora innerhalb eines zwei- bis dreijährigen Untersuchungszeitraums. Die Maßnahmen wurden auf insgesamt 67 Ackerschlägen umgesetzt und umfassten i) extensiv bewirtschaftete Ackerflächen, ii) einjährige Bracheflächen, iii) alternierend bewirtschaftete, zweijährige Blühstreifen und iv) mehrjährige Blühstreifen. Vor der Umsetzung wurden alle Maßnahmen in Kooperation mit Landwirten und anderen relevanten regionalen Akteuren (z.B. Landwirtschaftsberatern, Jägern, Imkern, Wissenschaftlern, Naturschutzbehörden und -organisationen) angepasst, um ihre Wirksamkeit und Akzeptanz zu erhöhen. Basierend auf insgesamt 45 konventionell bewirtschafteten Ackerschlägen, werden in Kapitel 4 schließlich sechs etablierte oder neu entwickelte Erhebungsmethoden zur Erfassung der Ackerwildkrautdiversität hinsichtlich ihrer Effizienz (erfasste Artenzahl eines Ackerschlages 
und Zeitaufwand) miteinander verglichen. Die Forschungsarbeiten wurden in der überwiegend konventionell bewirtschafteten Agrarregion der Landkreise Nienburg und Diepholz (Niedersachsen, Nordwestdeutschland) von 2016 bis 2019 durchgeführt.

Diese Arbeit konnte zeigen, dass die heutige Ackerwildkrautdiversität konventionell bewirtschafteter Äcker ein historisches Minimum erreicht hat. Das Feldinnere hat dabei fast keine Relevanz mehr als Lebensraum für Ackerwildkräuter (Median-Artenzahl und -Deckung: 2 Arten und $0,5 \%$ ) und nennenswerte Vorkommen sind nur am Ackerrand vorzufinden (MedianArtenzahl und -Deckung: 11 Arten und $4 \%$; Kapitel 2). Die bedeutendsten Faktoren, welche die heutige pflanzliche Diversität im Ackerrandbereich bestimmen, waren die Art der angebauten Kulturfrucht und das Vorhandensein angrenzender Habitate (Feldraine, Gehölzstreifen und Grabenränder). Die heutige Ackerbegleitflora ist stark verarmt und wird von wenigen weit verbreiteten Ackerwildkräutern dominiert, welche fähig sind, das intensive Management zu überdauern. Charakteristische, ehemals mit dem typischen Management der Kulturpflanzenarten oder Bodenfaktoren verbundene Ackerwildkrautgesellschaften sind nicht mehr identifizierbar. Die hier dargestellten Erkenntnisse zur Verarmung der Ackerbegleitflora sind auf einen Großteil des West- und Zentraleuropäischen Ackerlandes übertragbar. Dies zeigt den dringenden Handlungsbedarf seitens der Gemeinsamen Europäischen Agrarpolitik auf, um einer weiteren Verarmung der Ackerbegleitflora, negative Auswirkungen auf abhängige Organismengruppen und der Gefährdung bedeutender Ökosystemleistungen in Agrarlandschaften entgegenzuwirken. Dazu bedarf es der großräumigen Umsetzung von ökologisch effizienten Maßnahmen. Alle vier der im Rahmen dieser Arbeit untersuchten Agrarumweltmaßnahmen zeigten eine vergleichbar positive Wirkung auf die Ackerwildkrautartenvielfalt und -abundanz innerhalb des zwei- bis dreijährigen Untersuchungszeitraumes (Vergleich der Median-Artenzahl und -Deckung aller Maßnahmenflächen mit konventionell bewirtschafteten Ackerrändern bezogen auf den gesamten Untersuchungszeitraum: 21 vs. 9 Arten und 68,5 vs. 9,5\%; Kapitel 3). Um jedoch die Ackerwildkrautdiversität auf längere Sicht zu erhalten, ist die Anlage von extensiv bewirtschafteten Ackerflächen und einjährigen Bracheflächen geeigneter. Alle hier untersuchten Maßnahmen dürften aufgrund ihrer Anpassung an die landwirtschaftliche Praxis in modernen konventionellen Betrieben eine hohe Akzeptanz unter Landwirten aufweisen. Daher werden alle in dieser Arbeit untersuchten Maßnahmen zur Berücksichtigung in nationalen oder internationalen Agrarumweltprogrammen empfohlen. Da heute das Vorkommen vieler typischer, einst weit verbreiteter Ackerwildkräuter auf wenige Ackerflächen beschränkt ist, sollte weiterhin die Einbringung solcher Pflanzenarten über Regio-Saatgut bedacht werden, um die Ackerwildkrautdiversität auf Feldebene zu fördern und das (lokales) Aussterben seltener Arten zu verhindern.

Zudem wurde während der Umsetzung der Agrarumweltmaßnahmen festgestellt, dass unterschiedliche Witterungsbedingungen in den Untersuchungsjahren zu signifikant veränderten Artenzahlen und -deckungen der Ackerbegleitflora führen können. Dies verdeutlicht, dass zur verlässlichen Evaluierung von Agrarumweltmaßnahmen ein mehrjähriges Monitoring zwingend notwendig ist. Die Wahl der angewandten Erhebungsmethodik kann hierbei einen entscheidenden Einfluss auf die Monitoring-Ergebnisse haben. So konnte diese Arbeit zeigen, dass die unterschiedlichen derzeit angewandten Erhebungsmethoden zur Erfassung der Ackerwildkrautdiversität zu signifikant unterschiedlichen Ergebnissen hinsichtlich des erfassten Ackerwildkraut-Artenpools in konventionell bewirtschafteten Äckern führen (Kapitel 4). Um die zukünftige Entwicklung der Ackerbegleitflora und die Effektivität von Agrarumweltmaßnahmen 
im größeren räumlichen und zeitlichen Kontext analysieren zu können, bedarf es daher eines einheitlichen nationalen bzw. internationalen Monitoringsystems, welches idealerweise im Rahmen der Gemeinsamen Europäischen Agrarpolitik implementiert werden sollte. Die vorliegende Arbeit konnte diesbezüglich zeigen, dass ein langgestreckter Plot von $500 \times 1 \mathrm{~m}$ am Ackerrand eine vielversprechende Methode darstellt, um einen Großteil des AckerwildkrautArtenpools in konventionell bewirtschafteten Äckern (Median: 75,6 \%) in einer vertretbaren Zeit erfassen zu können (Median: 20 Minuten). Diese Methode wird daher als zukünftiger Standard für das Ackerwildkraut-Monitoring auf konventionellen Ackerschlägen empfohlen. 


\section{Chapter 1}

\section{General Introduction}

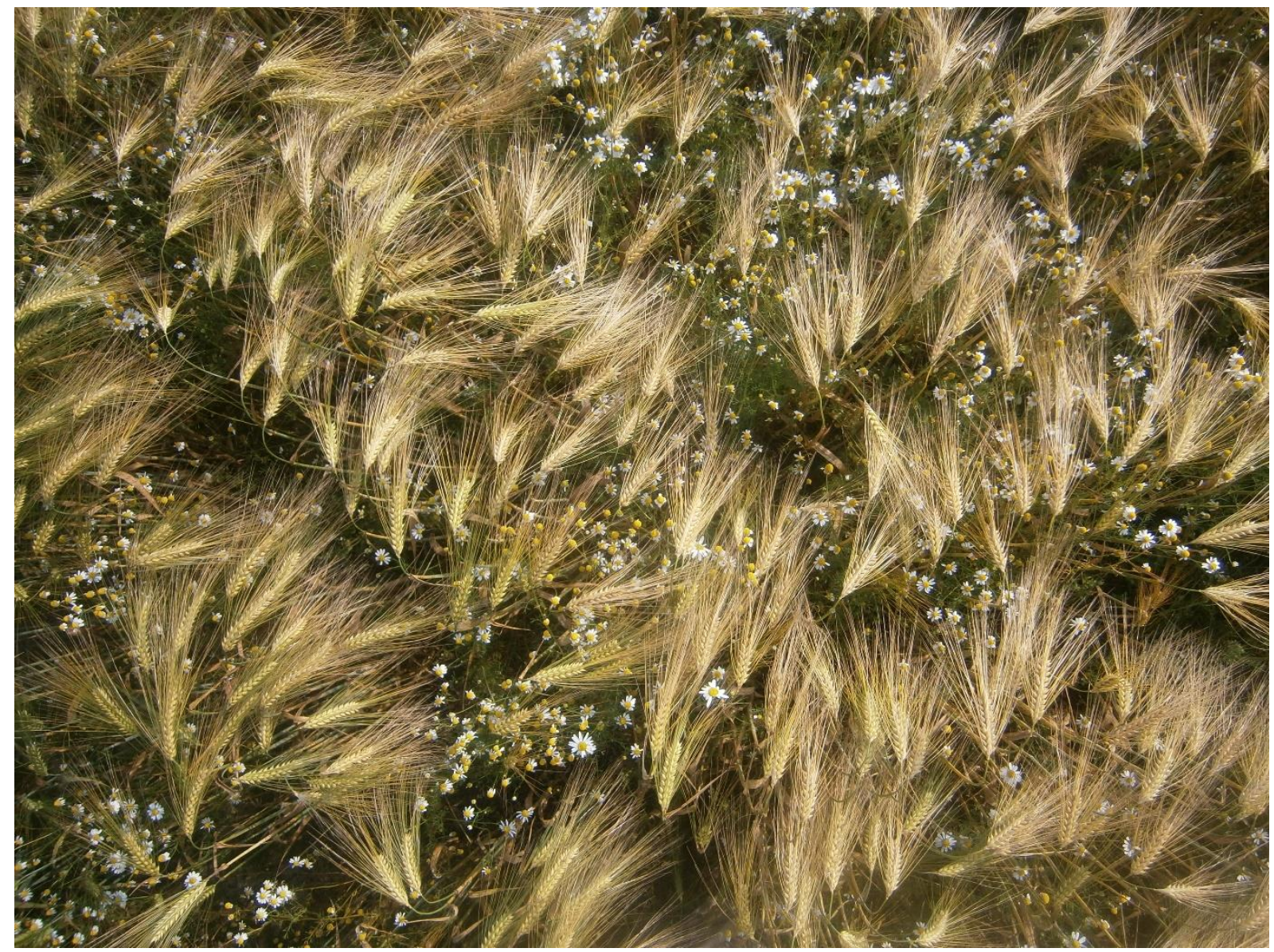




\subsection{The importance of agricultural landscapes as habitat}

Central European agriculture originated in the Neolithic (7500-6000 years before present) when humans began to settle, manage grassland for livestock farming and cultivate crops (Leuschner and Ellenberg, 2017a, 2017b). With the beginning of the agricultural practices, the historical landscape was likely a mixture of small-scale agricultural areas embedded in primeval habitat types such as marshes, bogs and forests (Hampicke, 2018). Today, nearly the half of the EU territory is under agricultural production with roughly two thirds of the area used as farmland cultivated with crops and one third as permanent grasslands (European Commission, 2015). The same ratio of farmland to grassland also applies to Germany (Bundesministerium für Ernährung und Landwirtschaft, 2017).

Non-intensively managed, small-scale and structurally diverse agricultural landscapes, as typical for historical traditional agriculture, created suitable habitat conditions for many species of different organism groups, e.g. farmland birds, arthropods, mammals or plants (Albrecht et al., 2016; Billeter et al., 2007; Hampicke, 2018; Poschlod and WallisDeVries, 2002; Šálek et al., 2018; Tscharntke et al., 2005; Zellweger-Fischer et al., 2018). For instance, Donald et al. (2006) classified 74 bird species as primary taxa of farm- and grassland in Europe. In addition, 1293 of 6185 vascular plant taxa known to be endemic to Europe occur in grassland habitats and 187 of in total 436 European butterfly species with known habitat preferences are considered as grassland specialists (Hobohm and Bruchmann, 2009; WallisDeVries and Van Swaay, 2009). Moreover, about 300-350 plant species are associated with arable habitats in Central Europe (Leuschner and Ellenberg, 2017a). Given the considerable proportion of agricultural area in the EU as well as in Germany and the large number of associated species puts the importance of agricultural landscapes for biodiversity beyond question. Moreover, maintaining this biodiversity is also important for agricultural production through providing ecosystem services, such as pollination or pest control (Dainese et al., 2019).

\subsection{The arable plant vegetation}

One species group that particularly depends on frequently disturbed man-made habitat conditions are arable plants (also called segetal plants). Since the beginning of arable land cultivation many plants have adapted to this special kind of anthropogenic habitat (see Chapter 1.1). For Central Europe, most of them are archeophytes originating from West Asia and Mediterranean countries (Albrecht et al., 2016; Meyer and Leuschner, 2015), i.e. species, which immigrated by human activities (e.g. transport via agricultural commodities such as cereal seeds) before the year 1492, but then could reproduce and establish themselves without further human intervention (Frey and Lösch, 2010). As the majority of arable plants are therophytes, their persistence relies on a continuous maintenance of the soil seed bank (Storkey et al., 2012), e.g. by natural spread or the use of regional crop seeds, which traditionally contained locally adapted weed seeds (Bergmeier and Strid, 2014). Driven by edaphic factors (acidic vs. base-rich), climatic conditions, crop type and related cultivation techniques (root crops or cereals; summer or winter crops), arable plant species formed different characteristic plant communities, which have been defined by the presence and frequency of specific plant taxa in a phytosociological hierarchical system (Hüppe and Hofmeister, 1990; Meyer et al., 2015). In addition, arable plant assemblages 
have also been classified into ecological groups related to specific site conditions (Hilbig et al., 1962; Hofmeister and Garve, 2006; Kaussmann and Kudocke, 1973). For Germany, Hofmeister and Garve (2006) list 287 species associated with typical arable plant communities with most of them being competitively weak and only few (about 30 species) may have negative effects on crop yield. However, European plant surveys of arable habitats normally consider all recorded herbaceous species as belonging to the arable plant vegetation, including plant species which mainly occur in ruderalized grasslands or hedges but occasionally spill over into arable fields. In this thesis, the analyses of species richness and cover in conventionally managed farmland are based on both all observed herbaceous species and typical arable plant taxa associated with specific arable plant communities according to Hofmeister and Garve (2006). If the latter group is meant, hereafter the terms 'arable plants sensu stricto' or 'typical arable plants' are used.

The arable plant vegetation is of great importance in agroecosystems not only for different species groups of the upper food web but also by contributing to ecosystem services. The plants provide food for rodents, the soil macrofauna (through the soil seed bank), seed-eating birds as well as phytophagous and pollinating insects (Fischer and Türke, 2016; Franke et al., 2009; Gabriel and Tscharntke, 2007; Heydemann and Meyer, 1983; Hyvönen and Huusela-Veistola, 2008; Marshall et al., 2003). Several insect species even rely on the occurrence of specific arable plant taxa in their life cycle (Marshall et al., 2003). Increased insect biomass through a species-rich and abundant arable plant vegetation, in turn, can serve as food source for insectivorous bird species (Marshall et al., 2003). In addition, a sufficiently dense arable plant vegetation can serve as erosion control and reduce nutrient leaching in periods when crop vegetation is missing (e.g. after harvest; Fagúndez, 2014; Gholamhoseini et al., 2013). By providing habitats and food sources for pollinating insects and natural enemies, the segetal flora can also contribute to pollination success and pest control in crops (Franke et al., 2009; Gabriel and Tscharntke, 2007; Hawes et al., 2003; Hyvönen and Huusela-Veistola, 2008; Médiène et al., 2011). Besides, arable plants have also an aesthetic value in cultural landscapes and contribute to people's well-being (Albrecht et al., 2016).

\subsection{The impact of agricultural intensification on the arable flora}

With the agricultural industrialization and related rapid advances in agricultural production techniques since the middle of the 20th century, crop yields in conventionally intensively used farmland have greatly increased, and today are on average three times higher than 50 years ago (Hampicke, 2018). On the one hand, raised yields made it possible to reduce food shortages with respect to the exponentially growing world population (Firbank, 2005). On the other hand, intensified agricultural land use has caused serious environmental problems, such as chemical pollution of soil and water (e.g. due to residues of pesticides and nitrogen leaching) and the strong decline in farmland biodiversity (Garibaldi et al., 2017; Sánchez-Bayo and Wyckhuys, 2019; Stoate et al., 2001).

One species group that has been particularly affected by the agricultural intensification is the arable plant vegetation, for which a sharp decline in species richness and abundance has been reported in Europe (Richner et al., 2015; Storkey et al., 2012). For instance, comparing the arable plant vegetation of the 1950s/60s with resurveyed plots in 2009 in Central Germany, Meyer et al. (2013b) found a decline in the plot-level species richness by about $70 \%$ and a reduction of the 
plant cover from 30 to $3 \%$. The plot-level decline of the arable plant species richness was accompanied by an overall impoverishment of the regional arable plant species pool by $23 \%$ (Meyer et al., 2013b). Several studies showed that the impoverishment is most pronounced in the field interior, whereas field edges exhibited slightly higher arable plant diversity and abundances, probably due to less intensive management and plant spillover from neighboring habitats (Aavik et al., 2008; Batáry et al., 2017; Fried et al., 2009; Meyer et al., 2013b; Nagy et al., 2018). Today, the arable plant vegetation belongs to one of the most threatened species groups in Central Europe with many red-listed species (Albrecht et al., 2016; Korneck et al., 1996; Leuschner and Ellenberg, 2017a; Richner et al., 2015; Still and Byfield, 2007; Storkey et al., 2012; Van Calster et al., 2008). A study of Meyer et al. (2015) in Central Germany revealed that floristic differences related to specific crop management and site conditions can hardly be detected today and typical arable plant communities are replaced by an impoverished and homogenized vegetation dominated by wide-spread, agro-tolerant species.

The reasons for the impoverishment of the arable flora are clearly related to changes introduced by intensified agriculture practices. Main changes include i) enhanced weed control (especially the use of highly efficient herbicides and seed cleaning techniques), ii) more competitive crop varieties and denser, strongly shading crop stands (enabled through increased sowing density and high fertilizer application), iii) a strongly impoverished diversity of cultivated crops (with especially strong reduction in summer crop cultivation), iv) deeper ploughing and early stubble breaking, v) drainage of arable habitats, and vi) increasing field sizes due to land consolidation and thus smaller less intensively managed field edge zones (Albrecht et al., 2016; Meyer et al., 2013b; Richner et al., 2015). These changes led to strong declines in the generally lightdemanding arable plant vegetation (Batlla and Benech-Arnold, 2014; Seifert et al., 2014) adapted to less-intensive management. For instance, deeper ploughing has created unsuitable habitat conditions for arable geophytes, such as Gagea villosa (Meyer et al., 2013a). The strong decline of arable land cultivated with summer crops and the immediate stubble breaking after harvest have increased the risk of incomplete reproduction of late germinating weeds, e.g. Stachys annua (Albrecht et al., 2016). Increasing drainage of arable fields has disadvantaged arable plant species adapted to temporarily waterlogged soils, e.g. Myosurus minimus (Albrecht et al., 2016). In addition, the strong reduction or abandonment of historically common cultivated crops, such as flax, has led to the strong decrease of specialized associated taxa, like Camelina alyssum (Meyer et al., 2013).

\subsection{Measures to stop biodiversity loss in agricultural landscapes}

In 1992, the Convention on Biological Diversity raised the conservation and sustainable use of biodiversity and related ecosystem functions to a global task (United Nations, 1992). As a consequence, during the last decades different international and national strategies were created in order to stop and reverse biodiversity loss, e.g. the United Nations' Strategic Plan for Biodiversity 2011-2020 (SCBD, 2010) or the EU biodiversity strategy to 2020 (EU, 2011). However, the increasing loss of biodiversity and related ecosystem functions is still one of the main challenges for humankind. One main driver of species loss is land use change, including significant changes in intensively used agricultural landscapes (Green, 2005; Pimm et al., 2014; Pimm and Raven, 2000; Sánchez-Bayo and Wyckhuys, 2019; Vié et al., 2009). Due to the high importance of agricultural landscapes for biodiversity and related ecosystem functioning, such as 
pest control or pollination (Dainese et al., 2019), the ongoing loss in species richness and abundance of various species groups has caused great political and public concern. In the European Union, environmental objectives were integrated into the Common Agricultural Policy, not only to counteract the biodiversity loss in agricultural landscapes, but also to address other environmental concerns such as water pollution, soil erosion or climate change (Matthews, 2013). Ecological focus areas and agri-environment and climate measures (hereafter both terms are combined under 'agri-environment measures') were introduced to increase the proportion of environmentally friendly managed agricultural land by providing financial support to farmers (European Union, 2013; Kleijn and Sutherland, 2003; Matthews, 2013).

Based on literature reviews and meta-analyses, a general positive effect of these measures on the overall farmland diversity could be demonstrated (Barral et al., 2015; Batáry et al., 2015, 2011). However, the effects of agri-environment measures on total farmland biodiversity have been shown to depend on the location, the overall landscape structure, the kind of implemented measure and the observed species group (Batáry et al., 2015, 2011; Gonthier et al., 2014; Scheper et al., 2013). In addition, measures implemented outside of managed areas (e.g. field margins, hedges or fallows) turned out to increase biodiversity of agricultural landscapes more than inproduction schemes (e.g. reduced herbicide and fertilizer input or organic farming; Batáry et al., 2015). With respect to arable plants, agri-environment measures also have been shown to generally increase species richness and abundance (Fischer and Milberg, 1997; Geiger et al., 2010; Kleijn et al., 2006; Walker et al., 2007). Such measures included organic farming, conservation fields (or field margins) and fallows (Albrecht et al., 2016; Fischer and Milberg, 1997).

However, from an agricultural point of view, there are still reservations about agri-environment measures funded through the Common Agricultural Policy. These reservations are based in particular on doubts about their effectiveness and on the inflexibility of the funding guidelines with regard to the implementation requirements (Stupak et al., 2019). In general, farmers prefer to implement conservation measures that are in accordance with their understanding of nature and 'good agricultural practice', thus, such measures are not necessarily congruent with those prioritized by conservationists (Stupak et al., 2019). As a result, farmers often implement measures that are not very effective from an ecological point of view (e.g. cover crops), but can be easily integrated into the agricultural practice and do not entail the risk of supporting 'weed infestation' of sites (Stupak et al., 2019; Zinngrebe et al., 2017). However, counteracting the ongoing loss of farmland biodiversity requires ecologically efficient measures that are widely implemented. It is therefore crucial to promote farmers' understanding of the ecological importance of such agri-environment measures and to take account of possible concerns from the agricultural practice when designing them (e.g. in terms of bureaucratic obstacles and implementation guidelines). 
In recent years, various initiatives and research projects have been launched to support the arable flora and other organism groups of agricultural landscapes, especially focusing on the improvement of the effectiveness of agri-environment measures and pointing out ways to increase their acceptance and thus the implementation area (e.g. by reducing bureaucratic obstacles). The present thesis is also based on such a participatory research project (see Chapter 1.6.1). Further examples for Germany include:

- the project '100 Äcker für die Vielfalt' (2008-2014; Meyer and Leuschner, 2015), which has created a long-term network of arable conservation fields in Germany in order to preserve threatened arable plant species;

- the project 'F.R.A.N.Z. - Für Ressourcen, Agrarwirtschaft \& Naturschutz mit Zukunft' (started in 2016 and still ongoing; https://www.franz-projekt.de/), which was launched to develop and test ecologically efficient and economically viable measures to support different organism groups in intensively used agricultural landscapes (e.g. amphibians, birds, hares, insects, and plants) based on the cooperation with ten demonstration farms distributed throughout Germany;

- or the initiative 'Landwirtschaft für Artenvielfalt' (started in 2012 and still ongoing; Gottwald and Stein-Bachinger, 2018), which also aims to counteract the overall biodiversity loss in agricultural landscapes with the focus on organic farming. Based on a whole-farm assessment, organic farms can receive credit and marketing advantages for supporting the biodiversity of agricultural landscapes through appropriate measures via the label 'Farming for Biodiversity'.

\subsection{Research objectives}

This thesis is dedicated to three overall research questions that were examined in an intensively managed farmland region in Northwest Germany (see Chapter 1.7 for more details about the study region). These research foci are briefly developed below and discussed in detail in the Chapters 2, 3 and 4. A synopsis of the key findings and the overall conclusions of this thesis is presented in Chapter 5.

Even though there are a number of studies observing the impact of intensified conventional agriculture on the arable plant vegetation in Europe (e.g. Fried et al. 2009; Gaba et al. 2010; Hanzlik and Gerowitt 2016; Albrecht et al. 2016; Hatcher and Froud-Williams 2017), most have addressed a single or very few crop species or carried out a non-differentiated analysis (all crops pooled). Thus, there is still missing information, how crop type and related conventional management practices, edaphic factors and the landscape context influence the arable plant vegetation. In addition, most studies have observed the impact of single agri-environment measures on the arable plant vegetation (Albrecht et al., 2016; Tscharntke et al., 2011; Wagner et al., 2017), but only very few compared the effects of different measure types with each other and observed their performance beyond one year (but see Eggenschwiler et al., 2009; Venclova et al., 2006; Walker et al., 2007). Especially the effect of flower strips on the arable plant vegetation has been rarely investigated (but see Rode et al., 2018), since this measure type was primarily introduced to support other organism groups, such as pollinating insects or farmland birds. Finally, many different methods are currently used for surveying the arable plant diversity, which 
differ considerably with respect to plot location in the field (edge and interior), surveyed area (ranging from $0.1 \mathrm{~m}^{2}$ up to $5,000 \mathrm{~m}^{2}$ ) and shape (e.g. rectangular or transects with dispersed plots) (Batáry et al., 2012; Chytrý and Otýpková, 2003; Fahrig et al., 2015; Hanzlik and Gerowitt, 2016; Meyer et al., 2015; Richner et al., 2015). The results of these survey methods are therefore difficult to compare. However, to evaluate the current state and development of the arable plant diversity in different regions and in course of time, standardized arable plant data are urgently needed. In order to recommend a suitable arable plant survey method and to establish a standardized monitoring approach - the basis for targeted nature conservation actions - a systematic comparison of the effectiveness of applied survey methods is required. However, such a comparative study is still missing.

Due to the knowledge gaps described above, the present thesis is devoted to the following three overarching questions:

i. Are there differences in the arable plant richness, cover and composition of seven conventionally intensively managed crop species (winter wheat, winter barley, winter rye, winter triticale, winter rapeseed, maize, and potato) and how is the arable plant richness influenced by crop cover, location in the field (field edge vs. field interior), management regime (herbicide and fertilizer application, tillage regime), edaphic conditions (soil pH and soil yield potential) and landscape context (type of adjacent habitat) (Chapter 2)?

ii. What is the impact of four agri-environment measures (annual fallow strips, conservation field margins, alternately managed biennial flower strips, and perennial flower strips) on the arable plant richness, cover and composition and are there spillover effects of the measures into the neighboring conventionally managed arable fields (Chapter 3)?

iii. How efficient are six traditional or novel arable plant survey methods, differing in plot size $\left(20\right.$ to $500 \mathrm{~m}^{2}$ ) and location in the field (interior vs. edge, corner vs. middle edge), with respect to recorded species diversity and time required in conventionally managed crops (winter rapeseed, winter wheat and maize) (Chapter 4)?

\subsection{The participative research project MEDIATE}

This thesis originated within the research project 'Development of targeted and efficient schemes to increase biodiversity in agricultural landscapes (MEDIATE)', which was conducted from 2016 to 2019 in the two districts Nienburg and Diepholz in Lower Saxony (Northwest Germany). MEDIATE was funded by the German Federal Environmental Foundation and involved the cooperation of the Lower Saxony chamber of agriculture, the Thünen Institute for biodiversity research, the Thünen Institute for farm economics and the Department of Plant Ecology and Ecosystems Research of the University of Göttingen. A key component of the project was a participative process, in which farmers, scientists and other relevant regional stakeholders (e.g. hunters, beekeepers, the chamber of agriculture, nature conservation authorities and organizations) were involved to develop and adapt agri-environment measures to be, on the one hand, ecologically and economically efficient and, on the other hand, also consider practical concerns of the farmers. 
The participative process included:

- Cooperation in the design of measures, e.g.:

O the selection of suitable seed mixtures for flower strips (from an agricultural and ecological perspective)

0 increasing the flexibility of measures: for instance, sowing of flower strips was feasible until the begin of May (latest 15.05.) to avoid possible damage by late frost events (agri-environment measure rules of the state allow this only until 15.04.)

- Meetings, workshops and field trips to promote the understanding of farmers and other relevant stakeholders with respect to the importance of farmland biodiversity in general, to demonstrate successful management of measures and to show the ecological effects of the implemented measures (monitoring results)

- Farmers had at any time the opportunity to clarify possible problems and questions about the implemented measures with the project team in personal contact

Due to the strong decline in the arable flora, the management of the chosen measure types was adapted to especially promote this species group. For instance, the management of three measure types included a regular soil disturbance to which the predominantly annual arable flora is adapted to. In addition, reduced sowing densities of cultivated crops and flower strips were considered to create less dense vegetation which allow the germination of arable plants still present in the soil seed bank. However, when planning and adapting the measures, creating suitable habitat conditions for other organism groups, such as farmland birds or insects, was also taken into account (e.g. through providing rich nectar and pollen sources by additionally sowing flowering plants). As a result of the participative process, the following four measure types were implemented in the study region from autumn 2016 or spring 2017 onwards (see also Fig. 1) and were financially compensated by project funds:

a) Conservation field margins ('CFM'): annual soil disturbance; $30 \%$ to $50 \%$ reduced sowing density of the cultivated crop (in autumn 2016/spring 2017; harvest possible

b) Annual fallows ('fallows'): annual soil disturbance; no seeding

c) Alternately managed biennial flower strips ('AFS'): sown with a seed mixture of crops, ornamental and native plant species (including typical arable plants; sowing density: $1 \mathrm{~g} \mathrm{~m}^{-2}$ ); alternate re-establishment of half of the flower strip area each year (including soil disturbance)

d) Perennial flower strips ('PFS'): sown with a seed mixture of perennial native plants (sowing density: $1 \mathrm{~g} \mathrm{~m}^{-2}$ ); annual mulching

Fertilizer and pesticide applications were excluded from all measure types (for more details on the measures see Chapter 3). From 2017 onwards, the arable flora of measure and control sites was surveyed each year. Beside the ecological focus, measures were also economically evaluated (not part of this thesis), since farmers have to consider the profitability of their farms. 


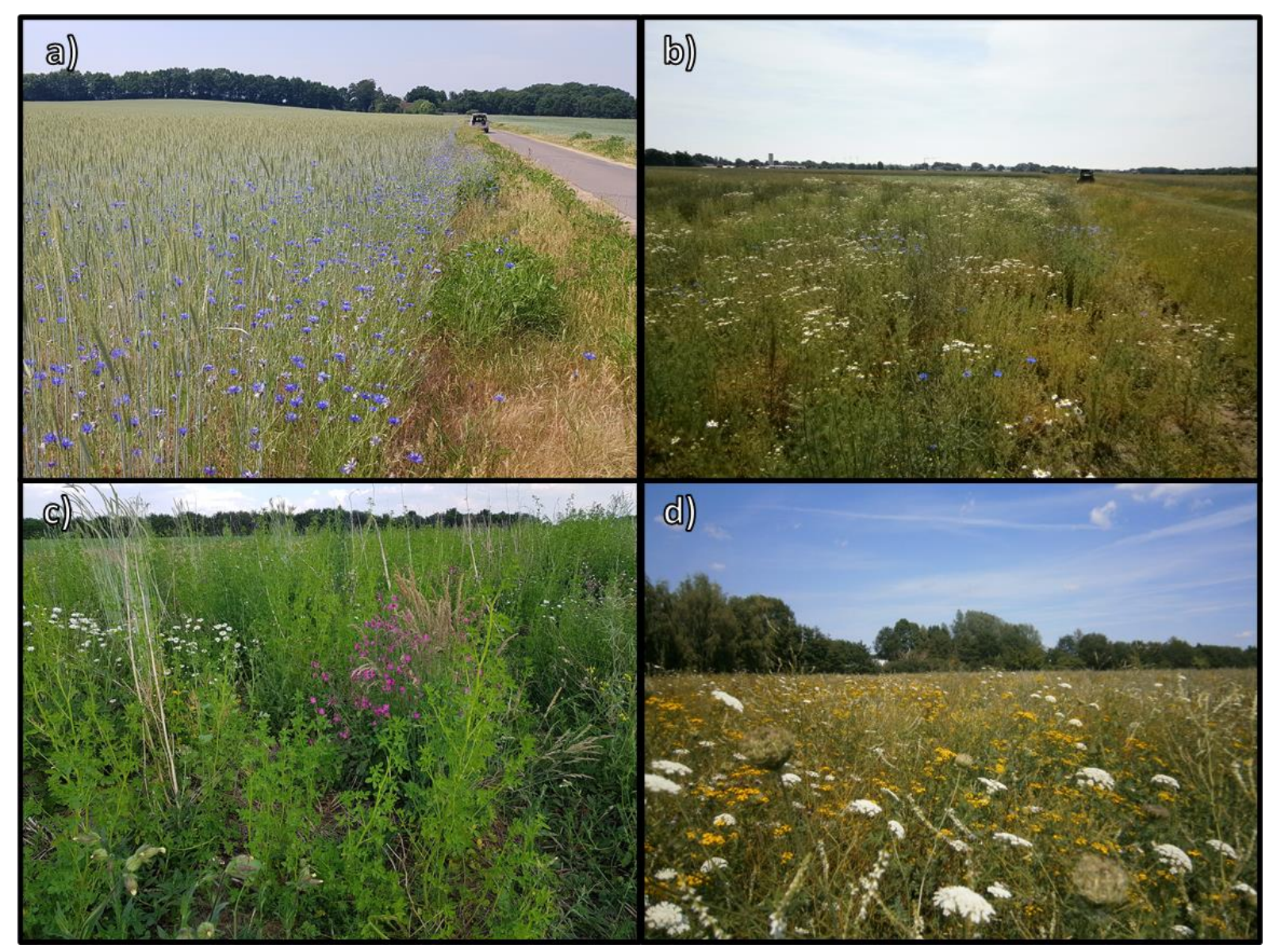

Fig. 1 Exemplary photographs of the four implemented measure types: a) conservation field margin, b) annual fallow, c) alternately managed biennial flower strip, and d) perennial flower strip.

\subsection{Study region}

The research project was conducted in the two neighboring districts Diepholz and Nienburg in Lower Saxony (Northwest Germany; see Fig. 2). The total district areas are 198,759 ha (Diepholz) and 139,893 ha (Nienburg; Niedersächsisches Landesamt für Statistik, 2007) with a high share of agriculturally used land: $65.2 \%$ (Diepholz) and $59.2 \%$ (Nienburg), thereof $80.8 \%$ (Diepholz) and $82.8 \%$ (Nienburg) for arable production (Landwirtschaftskammer Niedersachsen, 2018). The study region is characterized by intensive agriculture with $98 \%$ (Diepholz) and $97 \%$ (Nienburg) of the cropland managed conventionally in 2019 (Landwirtschaftskammer Niedersachsen, 2019). With respect to the cultivated area, the most important crop species are barley, rapeseed, maize, potato, rye, triticale and wheat (Landwirtschaftskammer Niedersachsen, 2018). The study area is part of the atlantic biogeographic region located in the North German Plain and includes two natural subregions: 'Ems-Hunte-Geest and Dümmer-Geestniederung' and 'Weser-Aller-Flachland' (von Drachenfels, 2010). The climate is cool-temperate and suboceanic with average annual precipitation and temperature (2016 to 2019 ) of $639.6 \mathrm{~mm} / 10.5{ }^{\circ} \mathrm{C}$ for Diepholz and $655.5 \mathrm{~mm} / 10.5{ }^{\circ} \mathrm{C}$ for Nienburg (due to missing measure values of the meteorological station in July and August 2018, the year 2018 was excluded from calculation for the district Nienburg; German Meteorological Service, 2019). The soils of the observed arable fields were sandy to loamy Cambisols, Podzols, 
Luvisols and Gleysols originating from Pleistocene and Holocene sediments (Federal Institute for Geosciences and Natural Resources, 2013).

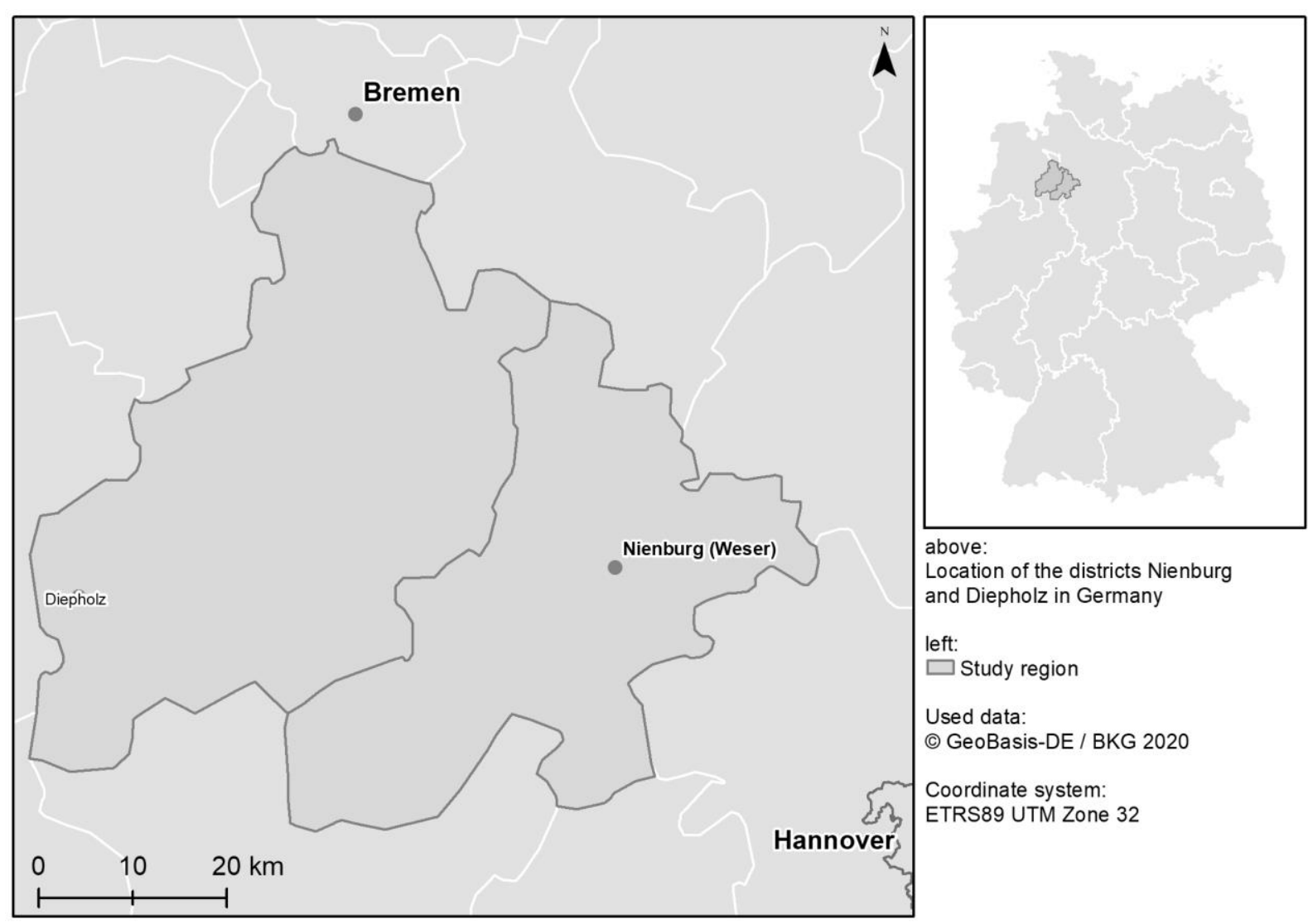

Fig. 2 Map of the study region with the districts of Nienburg and Diepholz in Lower Saxony (Northwest Germany).

\subsection{Study design}

In 2016, 200 conventionally managed arable fields cultivated with seven different crops were chosen to survey the current state of the arable vegetation in the study region (30 plots each in winter barley, maize, winter rapeseed, winter rye and winter wheat fields, 28 in potato and 22 in winter triticale; Chapter 2). Site selection was limited by the availability of fields of participating farmers (in total 15 farms) but aimed to ensure a wide dispersion of the chosen fields in the study region. Non-crop plant species richness and cover (Londo-scale; Londo, 1976) were recorded in plots (also called relevés) of $50 \times 2 \mathrm{~m}$ in the field edge (200 replicates) and field interior (at least $20 \mathrm{~m}$ apart from the field edge, 70 replicates) from end of May to the beginning of August. In addition, the total cover $(\%)$ of the cultivated crops, the non-crop vegetation and the tree and shrub layer were noted. Furthermore, the vegetation of 63 neighboring habitats to arable fields were surveyed: 22 hedges $(25 \times 2 \mathrm{~m}$ plots); 21 ditch margins $(8 \times 2 \mathrm{~m})$, and 20 grass strips $(16 \times$ $1 \mathrm{~m})$. Moreover, abiotic and management data of the observed arable fields were compiled to analyze their effects on the arable plant richness and composition. These data included fertilizer $(\mathrm{N}, \mathrm{P}, \mathrm{K})$ and herbicide input, soil cultivation techniques (tillage or no tillage), soil $\mathrm{pH}$ as well as soil type and quality. Information of the herbicide application (frequency and dose) was used to calculate the Standardized Treatment Index (STI after Roßberg et al., 2002; Sattler et al., 2007). 
From 2017 to 2019, the effects of agri-environment measures on the arable plant richness and abundance were observed (Chapter 3). For this, the implementation of four different agrienvironment measures was coordinated in the study region, namely conservation field margins (CFM), annual fallows (fallows), alternately managed biennial flower strips (AFS) and perennial flower strips (PFS). Measures were implemented in arable fields of 19 participating farms that were previously surveyed (to allow for the before-after-control-impact (BACI) approach). In total, 67 measure areas were implemented in arable fields, starting with 47 sites in autumn 2016 or spring 2017, supplemented by 20 measures in autumn 2017 or spring 2018. To evaluate the effects of the measures on the arable flora, the plant species richness and cover of measure areas and conventionally managed field edges (controls) and interiors were surveyed in $50 \times 2 \mathrm{~m}$ plots each year (except for 2017, when no interior plots could be observed due to time restriction). To examine possible effects of the implemented measures on the neighboring conventionally managed fields, spillover plots $(50 \times 2 \mathrm{~m})$ were implemented. The observed arable fields adjacent to the implemented measures were conventionally cultivated according to the crop rotation scheme of the participating farmer.

In addition, in 2017, six different relevé types for surveying the plant species richness in conventionally managed farmland were compared with respect to their detection success and time effort (Chapter 4). The different relevé types included field interior plots $(50 \times 2 \mathrm{~m})$ and five different types of field edge plots $(30 \times 2 \mathrm{~m}, 50 \times 2 \mathrm{~m}, 500 \times 1 \mathrm{~m}$, four dispersed subplots of $5 \times 1 \mathrm{~m}$, and corner plots of $50 \times 2 \mathrm{~m}$ ). The surveys were conducted in 45 arable fields $(15$ each of winter wheat, winter rapeseed and maize; 17 participating farms).

Beside the arable plant vegetation, also other organism groups were monitored within the research project (e.g. farmland birds and insects). For instance, the development of farmland bird populations was investigated in two 400 ha areas in the study region from 2016-2019 (including implemented measure areas). This thesis, however, will focus on the vegetation of arable fields.

For more detailed descriptions of the study design and the vegetation surveys see Chapters 2 to 4 .

\subsection{Data analyses}

In the following, a short overview of the used statistical approaches is presented. Detailed statistical descriptions can be found in the 'Material and Methods' sections of the Chapters 2 to 4 .

In general, statistical analyses were performed using the software R ( R Core Team, 2019). Depending on the specific research questions (Chapters 2 to 4), analyses were done for a) all observed herbaceous species (grasses included, but woody seedlings, crops and ornamental plants from seed mixtures excluded), b) arable plant species sensu stricto (according to Hofmeister and Garve 2006), c) indicators of High Nature Value arable land (as defined for Germany by Hünig and Benzler 2017), d) threatened arable plants (according to the Red List of Lower Saxony; Garve, 2004), e) proportions of forbs and graminoids, and f) proportions of annuals and perennials (according to Klotz et al. 2002). 
To characterize the observed study region, the proportions of the different landscape elements were analyzed (ArcGIS, ESRI, 2018) based on habitat mapping data (von Drachenfels, 2016; Chapter 2). Moreover, beta diversity was calculated (based on Sørensen and Jaccard's Index) to observe floristic similarity of the observed arable fields or plot types (Chapters 2 to 4). In addition, cluster analyses and non-metric multidimensional scaling (NMDS) were conducted to detect possible species formations with respect to the surveyed arable flora of seven crop species (Chapter 2) or to examine floristic differences between conventionally managed field edge plots and measure plots (Chapter 3). Furthermore, median numbers of diagnostic arable plant species for classes, orders, alliances or associations were calculated in order to verify if species assemblages found in the surveyed crops could be assigned to syntaxa in the phytosociological hierarchical system (Hüppe and Hofmeister, 1990; Preising et al., 1995, Chapter 2). In addition, Venn diagrams were used to display the number of species shared between a) field edge plots and neighboring habitats, b) different crop species, and c) measure sites and plots in conventionally managed fields, and to show how many species were found in only one of these habitats (Chapters 2 and 3). To account for weather effects on the total plant species number in a fouryear comparison (Chapter 3), interpolated high-resolution gridded climate data were used (German Meteorological Service, 2019). To evaluate the species detection success of six survey methods for arable plants, Spearman's rank correlation coefficients of observed species number per survey method and estimated total plant species richness per site were calculated (Chapter 4). In addition, species accumulation curves were used to show the effect of the survey method on the increase in species richness with increasing plot number (i.e. observed area).

In order to statistically examine the research questions of this thesis (see Chapter 1.5 and Chapters 2 to 4 ) linear or generalized linear mixed-effects models were used. To test for the significance of the response variables, likelihood-ratio tests (Type II sums of squares) and post hoc pairwise comparisons were performed. In some cases, e.g. when the replicate number was low, the Mann-Whitney-U test was applied to verify significant differences. 


\section{References}

Aavik, T., Augenstein, I., Bailey, D., Herzog, F., Zobel, M., Liira, J., 2008. What is the role of local landscape structure in the vegetation composition of field boundaries? Appl. Veg. Sci. 11, 375-386. https://doi.org/10.3170/2008-7-18486

Albrecht, H., Cambecèdes, J., Lang, M., Wagner, M., 2016. Management options for the conservation of rare arable plants in Europe. Bot. Lett. 163, 389-415. https://doi.org/10.1080/23818107.2016.1237886

Barral, M.P., Rey Benayas, J.M., Meli, P., Maceira, N.O., 2015. Quantifying the impacts of ecological restoration on biodiversity and ecosystem services in agroecosystems: A global meta-analysis. Agric. Ecosyst. Environ. 202, 223-231. https://doi.org/10.1016/j.agee.2015.01.009

Batáry, P., Baldi, A., Kleijn, D., Tscharntke, T., 2011. Landscape-moderated biodiversity effects of agri-environmental management: a meta-analysis. Proc. R. Soc. B Biol. Sci. 278, 1894-1902. https://doi.org/10.1098/rspb.2010.1923

Batáry, P., Dicks, L.V., Kleijn, D., Sutherland, W.J., 2015. The role of agri-environment schemes in conservation and environmental management: European Agri-Environment Schemes. Conserv. Biol. 29, 1006-1016. https://doi.org/10.1111/cobi.12536

Batáry, P., Gallé, R., Riesch, F., Fischer, C., Dormann, C.F., Mußhoff, O., Császár, P., Fusaro, S., Gayer, C., Happe, A.-K., Kurucz, K., Molnár, D., Rösch, V., Wietzke, A., Tscharntke, T., 2017. The former Iron Curtain still drives biodiversityprofit trade-offs in German agriculture. Nat. Ecol. Evol. 1, 1279-1284. https://doi.org/10.1038/s41559-017-0272-x

Batáry, P., Holzschuh, A., Orci, K.M., Samu, F., Tscharntke, T., 2012. Responses of plant, insect and spider biodiversity to local and landscape scale management intensity in cereal crops and grasslands. Agric. Ecosyst. Environ. 146, 130-136. https://doi.org/10.1016/j.agee.2011.10.018

Batlla, D., Benech-Arnold, R.L., 2014. Weed seed germination and the light environment: Implications for weed management: Light control of weed seed germination. Weed Biol. Manag. 14, 77-87. https://doi.org/10.1111/wbm.12039

Bergmeier, E., Strid, A., 2014. Regional diversity, population trends and threat assessment of the weeds of traditional agriculture in Greece: Weeds of Greek Traditional Agriculture. Bot. J. Linn. Soc. 175, 607-623. https://doi.org/10.1111/boj.12181

Billeter, R., Liira, J., Bailey, D., Bugter, R., Arens, P., Augenstein, I., Aviron, S., Baudry, J., Bukacek, R., Burel, F., Cerny, M., De Blust, G., De Cock, R., Diekötter, T., Dietz, H., Dirksen, J., Dormann, C., Durka, W., Frenzel, M., Hamersky, R., Hendrickx, F., Herzog, F., Klotz, S., Koolstra, B., Lausch, A., Le Coeur, D., Maelfait, J.P., Opdam, P., Roubalova, M., Schermann, A., Schermann, N., Schmidt, T., Schweiger, O., Smulders, M.J.M., Speelmans, M., Simova, P., Verboom, J., Van Wingerden, W.K.R.E., Zobel, M., Edwards, P.J., 2007. Indicators for biodiversity in agricultural landscapes: a panEuropean study: Biodiversity in European Agro-ecosystems. J. Appl. Ecol. 45, 141-150. https://doi.org/10.1111/j.1365-2664.2007.01393.x

Bundesministerium für Ernährung und Landwirtschaft (BMEL), 2017. Daten und Fakten: Land-, Forst- und Ernährungswirtschaft mit Fischerei und Wein- und Gartenbau [WWW Document]. URL https://www.bmel.de/SharedDocs/Downloads/Broschueren/DatenundFakten.pdf?_blob=publicationFile (accessed 3.2.20)

Chytrý, M., Otýpková, Z., 2003. Plot sizes used for phytosociological sampling of European vegetation. J. Veg. Sci. 14, 563-570. https://doi.org/10.1111/j.1654-1103.2003.tb02183.x

Dainese, M., Martin, E.A., Aizen, M.A., Albrecht, M., Bartomeus, I., Bommarco, R., Carvalheiro, L.G., Chaplin-Kramer, R., Gagic, V., Garibaldi, L.A., Ghazoul, J., Grab, H., Jonsson, M., Karp, D.S., Kennedy, C.M., Kleijn, D., Kremen, C., Landis, D.A., Letourneau, D.K., Marini, L., Poveda, K., Rader, R., Smith, H.G., Tscharntke, T., Andersson, G.K.S., Badenhausser, I., Baensch, S., Bezerra, A.D.M., Bianchi, F.J.J.A., Boreux, V., Bretagnolle, V., Caballero-Lopez, B., Cavigliasso, P., Ćetković, A., Chacoff, N.P., Classen, A., Cusser, S., da Silva e Silva, F.D., de Groot, G.A., Dudenhöffer, J.H., Ekroos, J., Fijen, T., Franck, P., Freitas, B.M., Garratt, M.P.D., Gratton, C., Hipólito, J., Holzschuh, A., Hunt, L., Iverson, A.L., Jha, S., Keasar, T., Kim, T.N., Kishinevsky, M., Klatt, B.K., Klein, A.-M., Krewenka, K.M., Krishnan, S., Larsen, A.E., Lavigne, C., Liere, H., Maas, B., Mallinger, R.E., Martinez Pachon, E., Martínez-Salinas, A., Meehan, T.D., Mitchell, M.G.E., Molina, G.A.R., Nesper, M., Nilsson, L., O’Rourke, M.E., Peters, M.K., Plećaš, M., Potts, S.G., Ramos, D. de L., Rosenheim, J.A., Rundlöf, M., Rusch, A., Sáez, A., Scheper, J., Schleuning, M., Schmack, J.M., Sciligo, A.R., Seymour, C., Stanley, D.A., Stewart, R., Stout, J.C., Sutter, L., Takada, M.B., Taki, H., Tamburini, G., Tschumi, M., Viana, B.F., Westphal, C., Willcox, B.K., Wratten, S.D., Yoshioka, A., Zaragoza-Trello, C., Zhang, W., Zou, Y., Steffan-Dewenter, I., 2019. A global synthesis reveals biodiversity-mediated benefits for crop production. Sci. Adv. 5, eaax0121. https://doi.org/10.1126/sciadv.aax0121

Donald, P.F., Sanderson, F.J., Burfield, I.J., van Bommel, F.P.J., 2006. Further evidence of continent-wide impacts of agricultural intensification on European farmland birds, 1990-2000. Agric. Ecosyst. Environ. 116, 189-196. https://doi.org/10.1016/j.agee.2006.02.007

Eggenschwiler, L., Jacot, K.A., Edwards, P.J., 2009. Vegetation development and nitrogen dynamics of sown and spontaneous set-aside on arable land. Ecol. Eng. 35, 890-897. https://doi.org/10.1016/j.ecoleng.2008.12.024

Environmental Systems Research Institute (ESRI), 2018. ArcGIS Desktop 10.6.1. [WWW Document]. URL https://www.esri.com/en-us/arcgis/products/arcgis-pro/overview (accessed 10.26.18) 
European Commission, 2015. Food \& Farming - Focus on Land. [WWW Document]. URL https://ec.europa.eu/agriculture/sites/agriculture/files/events/2015/outlook-conference/brochure-land_en.pdf (accessed 3.4.20)

European Union (EU), 2013. Overview of CAP reform 2014-2020 highlights improved framework for addressing 21st century challenges. [WWW Document]. URL https://ec.europa.eu/agriculture/sites/agriculture/files/policyperspectives/policy-briefs/05_en.pdf (accessed 3.14.19)

European Union (EU), 2011. EU biodiversity strategy to 2020 [WWW Document]. URL https://eur-lex.europa.eu/legalcontent/EN/TXT/PDF/?uri=CELEX:52011DC0244\&from=EN (accessed 1.23.19)

Fagúndez, J., 2014. The Paradox of Arable Weeds: Diversity, Conservation, and Ecosystem Services of the Unwanted, in: Benkeblia, N. (Ed.), Agroecology, Ecosystems, and Sustainability, Advances in Agroecology. CRC Press, pp. 139-150. https://doi.org/10.1201/b17775-8

Fahrig, L., Girard, J., Duro, D., Pasher, J., Smith, A., Javorek, S., King, D., Lindsay, K.F., Mitchell, S., Tischendorf, L., 2015. Farmlands with smaller crop fields have higher within-field biodiversity. Agric. Ecosyst. Environ. 200, $219-234$. https://doi.org/10.1016/j.agee.2014.11.018

Federal Institute for Geosciences and Natural Resources, 2013. Soil Map of the Federal Republic of Germany 1:1,000,000 (BÜK1000). [WWW https://www.bgr.bund.de/DE/Themen/Boden/Informationsgrundlagen/Bodenkundliche_Karten_Datenbanken/BU EK1000/buek1000_node.html (accessed 10.26.16)

Firbank, L.G., 2005. Striking a new balance between agricultural production and biodiversity. Ann. Appl. Biol. 146, $163-175$. https://doi.org/10.1111/j.1744-7348.2005.040078.x

Fischer, A., Milberg, P., 1997. Effects on the flora of extensified use of field margins. Swed. J. Agric. Res. Swed.

Fischer, C., Türke, M., 2016. Seed preferences by rodents in the agri-environment and implications for biological weed control. Ecol. Evol. 6, 5796-5807. https://doi.org/10.1002/ece3.2329

Franke, A.C., Lotz, L.A.P., Van Der Burg, W.J., Van Overbeek, L., 2009. The role of arable weed seeds for agroecosystem functioning: Arable weed seeds in agroecosystems. Weed Res. 49, 131-141. https://doi.org/10.1111/j.13653180.2009.00692.x

Frey, W., Lösch, R., 2010. Geobotanik. Spektrum Akademischer Verlag, Heidelberg. https://doi.org/10.1007/978-3-8274-2336-8

Fried, G., Petit, S., Dessaint, F., Reboud, X., 2009. Arable weed decline in Northern France: Crop edges as refugia for weed conservation? Biol. Conserv. 142, 238-243. https://doi.org/10.1016/j.biocon.2008.09.029

Gaba, S., Chauvel, B., Dessaint, F., Bretagnolle, V., Petit, S., 2010. Weed species richness in winter wheat increases with landscape heterogeneity. Agric. Ecosyst. Environ. 138, 318-323. https://doi.org/10.1016/j.agee.2010.06.005

Gabriel, D., Tscharntke, T., 2007. Insect pollinated plants benefit from organic farming. Agric. Ecosyst. Environ. 118, 43-48. https://doi.org/10.1016/j.agee.2006.04.005

Garibaldi, L.A., Gemmill-Herren, B., D’Annolfo, R., Graeub, B.E., Cunningham, S.A., Breeze, T.D., 2017. Farming Approaches for Greater Biodiversity, Livelihoods, and Food Security. Trends Ecol. Evol. 32, 68-80. https://doi.org/10.1016/j.tree.2016.10.001

Garve, E., 2004. Rote Liste und Florenliste der Farn- und Blütenpflanzen in Niedersachsen und Bremen, 5. Fassung vom 1.3.2004. Inform. d. Naturschutz Niedersachs. 24 (1) (1/04): 1-76, Hildesheim

Geiger, F., Bengtsson, J., Berendse, F., Weisser, W.W., Emmerson, M., Morales, M.B., Ceryngier, P., Liira, J., Tscharntke, T., Winqvist, C., Eggers, S., Bommarco, R., Pärt, T., Bretagnolle, V., Plantegenest, M., Clement, L.W., Dennis, C., Palmer, C., Oñate, J.J., Guerrero, I., Hawro, V., Aavik, T., Thies, C., Flohre, A., Hänke, S., Fischer, C., Goedhart, P.W., Inchausti, P., 2010. Persistent negative effects of pesticides on biodiversity and biological control potential on European farmland. Basic Appl. Ecol. 11, 97-105. https://doi.org/10.1016/j.baae.2009.12.001

German Meteorological Service, 2019. Climate Data Center (CDC) [WWW Document]. URL https://www.dwd.de/EN/climate_environment/cdc/cdc.html (accessed 11.21.19)

Gholamhoseini, M., AghaAlikhani, M., Mirlatifi, S.M., Sanavy, S.A.M.M., 2013. Weeds - Friend or foe? Increasing forage yield and decreasing nitrate leaching on a corn forage farm infested by redroot pigweed. Agric. Ecosyst. Environ. 179, 151162. https://doi.org/10.1016/j.agee.2013.08.016

Gonthier, D.J., Ennis, K.K., Farinas, S., Hsieh, H.-Y., Iverson, A.L., Batary, P., Rudolphi, J., Tscharntke, T., Cardinale, B.J., Perfecto, I., 2014. Biodiversity conservation in agriculture requires a multi-scale approach. Proc. R. Soc. B Biol. Sci. 281, 20141358-20141358. https://doi.org/10.1098/rspb.2014.1358

Gottwald, F., Stein-Bachinger, K., 2018. 'Farming for Biodiversity'-a new model for integrating nature conservation achievements on organic farms in north-eastern Germany. Org. Agric. 8, 79-86. https://doi.org/10.1007/s13165-0170198-2

Green, R.E., 2005. Farming and the Fate of Wild Nature. Science 307, 550-555. https://doi.org/10.1126/science.1106049

Hampicke, U., 2018. Kulturlandschaft: Äcker, Wiesen, Wälder und ihre Produkte: ein Lesebuch für Städter. Springer, Berlin, Germany 
Hanzlik, K., Gerowitt, B., 2016. Methods to conduct and analyse weed surveys in arable farming: a review. Agron. Sustain. Dev. 36. https://doi.org/10.1007/s13593-015-0345-7

Hatcher, P., Froud-Williams, R.J. (Eds.), 2017. Approaches and Objectives of Arable Weed Species Mapping: Where Next?, in: Weed Research: Expanding Horizons. John Wiley \& Sons Ltd, Hoboken, NJ, pp. 61-83

Hawes, C., Haughton, A.J., Osborne, J.L., Roy, D.B., Clark, S.J., Perry, J.N., Rothery, P., Bohan, D.A., Brooks, D.R., Champion, G.T., Dewar, A.M., Heard, M.S., Woiwod, I.P., Daniels, R.E., Young, M.W., Parish, A.M., Scott, R.J., Firbank, L.G., Squire, G.R., 2003. Responses of plants and invertebrate trophic groups to contrasting herbicide regimes in the Farm Scale Evaluations of genetically modified herbicide-tolerant crops. Philos. Trans. R. Soc. B Biol. Sci. 358, 1899-1913. https://doi.org/10.1098/rstb.2003.1406

Heydemann, B., Meyer, H., 1983. Auswirkungen der Intensivkultur auf die Fauna in den Agrarbiotopen. SchrR Dt Rat Für Landespfl 42, 174-191

Hilbig, W., Mahn, E.-G., Schubert, R. \& Wiedenroth, E. M. 1962. Die ökologisch-soziologischen Artengruppen der Ackerunkrautvegetation Mitteldeutschlands - Ein Beitrag zur Standortsgliederung im Bereich der mitteldeutschen Ackerlandschaften. Botanisches Jahrbuch 81 (4), 416-449

Hofmeister, H., Garve, E., 2006. Lebensraum Acker, Reprint der 2. neubearbeiteten Auflage. ed. Verlag Kessel, Remagen

Hobohm, C., Bruchmann, I., 2009. Endemische Gefäßpflanzen und ihre Habitate in Europa-Plädoyer für den Schutz der Grasland-Ökosysteme. Berichte Reinhold-Tüxen-Ges. 21, 142-161

Hünig, C., Benzler, A., 2017. Das Monitoring der Landwirtschaftsflächen mit hohem Naturwert in Deutschland, BfN-Skripten. Bundesamt für Naturschutz, Bonn-Bad Godesberg

Hüppe, J., Hofmeister, H., 1990. Syntaxonomische Fassung und Übersicht über die Ackerunkrautgesellschaften der Bundesrepublik Deutschland., - Ber. d. Reinh.-Tüxen-Ges. 2, S. 57-77, Hannover

Hyvönen, T., Huusela-Veistola, E., 2008. Arable weeds as indicators of agricultural intensity - A case study from Finland. Biol. Conserv. 141, 2857-2864. https://doi.org/10.1016/j.biocon.2008.08.022

Kaussmann, B., Kudocke, J., 1973. Die ökologisch-soziologischen Artengruppen der Ackerunkrautvegetation für den Norden der DDR. Feddes Repert. 84, 589-605. https://doi.org/10.1002/fedr.19730840707

Kleijn, D., Baquero, R.A., Clough, Y., Díaz, M., Esteban, J., Fernández, F., Gabriel, D., Herzog, F., Holzschuh, A., Jöhl, R., Knop, E., Kruess, A., Marshall, E.J.P., Steffan-Dewenter, I., Tscharntke, T., Verhulst, J., West, T.M., Yela, J.L., 2006. Mixed biodiversity benefits of agri-environment schemes in five European countries: Biodiversity effects of European agri-environment schemes. Ecol. Lett. 9, 243-254. https://doi.org/10.1111/j.1461-0248.2005.00869.x

Kleijn, D., Sutherland, W.J., 2003. How effective are European agri-environment schemes in conserving and promoting biodiversity? J. Appl. Ecol. 40, 947-969. https://doi.org/10.1111/j.1365-2664.2003.00868.x

Klotz, S., Kühn, I., Durka, W., Briemle, G., 2002. BIOLFLOR: Eine Datenbank mit biologisch-ökologischen Merkmalen zur Flora von Deutschland. Bundesamt für Naturschutz Bonn

Korneck, D., Schnittler, M., Vollmer, I., 1996. Rote Liste der Farn- und Blütenpflanzen (Pteridophyta et Spermatophyta) Deutschlands. Schriftenreihe für Vegetationskunde 28:21-187.

Landwirtschaftskammer Niedersachsen, 2019. Land use data of the districts Nienburg and Diepholz (Lower Saxony, Germany)

Landwirtschaftskammer Niedersachsen, 2018. Landwirtschaft in der Region Diepholz/Nienburg

Leuschner, C., Ellenberg, H., 2017a. Ecology of Central European Non-Forest Vegetation: Coastal to Alpine, Natural to ManMade Habitats: Vegetation Ecology of Central Europe, Volume II. Springer International Publishing, Cham. https://doi.org/10.1007/978-3-319-43048-5

Leuschner, C., Ellenberg, H., 2017b. Ecology of Central European Forests, Revised and extended version of the 6th German edition. ed, Vegetation ecology of central Europe. Springer International Publishing AG, Cham.

Londo, G., 1976. The decimal scale for releves of permanent quadrats. Vegetatio 33, 61-64. https://doi.org/10.1007/BF00055300

Marshall, E.J.P., Brown, V.K., Boatman, N.D., Lutman, P.J.W., Squire, G.R., Ward, L.K., 2003. The role of weeds in supporting biological diversity within crop fields. Weed Res. 43, 77-89. https://doi.org/10.1046/j.1365-3180.2003.00326.x

Matthews, A., 2013. Greening agricultural payments in the EU's Common Agricultural Policy. Bio-Based Appl. Econ. 2, 1-27

Médiène, S., Valantin-Morison, M., Sarthou, J.-P., de Tourdonnet, S., Gosme, M., Bertrand, M., Roger-Estrade, J., Aubertot, J.-N., Rusch, A., Motisi, N., Pelosi, C., Doré, T., 2011. Agroecosystem management and biotic interactions: a review. Agron. Sustain. Dev. 31, 491-514. https://doi.org/10.1007/s13593-011-0009-1

Meyer, S., Bergmeier, E., Becker, T., Wesche, K., Krause, B., Leuschner, C., 2015. Detecting long-term losses at the plant community level - arable fields in Germany revisited. Appl. Veg. Sci. 18, 432-442. https://doi.org/10.1111/avsc.12168

Meyer, S., Hilbig, W., Steffen, C., Schuch, S., 2013a. Ackerwildkrautschutz - eine Bibliographie: Ergebnisse aus dem F+EVorhaben (FKZ 351286 0300), BfN-Skripten. Bundesamt für Naturschutz, Bonn 
Meyer, S., Leuschner, C. (Eds.), 2015. 100 Äcker für die Vielfalt. Göttingen University Press, Göttingen. https://doi.org/10.17875/gup2015-815

Meyer, S., Wesche, K., Krause, B., Leuschner, C., 2013b. Dramatic losses of specialist arable plants in Central Germany since the 1950s/60s - a cross-regional analysis. Divers. Distrib. 19, 1175-1187. https://doi.org/10.1111/ddi.12102

Nagy, K., Lengyel, A., Kovács, A., Türei, D., Csergő, A.M., Pinke, G., 2018. Weed species composition of small-scale farmlands bears a strong crop-related and environmental signature. Weed Res. 58, 46-56. https://doi.org/10.1111/wre.12281

Niedersächsisches Landesamt für Statistik, 2007. Statistische Berichte Niedersachsen - Nutzungsarten der Bodenfläche.

Pimm, S.L., Jenkins, C.N., Abell, R., Brooks, T.M., Gittleman, J.L., Joppa, L.N., Raven, P.H., Roberts, C.M., Sexton, J.O., 2014. The biodiversity of species and their rates of extinction, distribution, and protection. Science 344, 1246752-1246752. https://doi.org/10.1126/science.1246752

Pimm, S.L., Raven, P., 2000. Extinction by numbers: Biodiversity. Nature 403, 843-845. https://doi.org/10.1038/35002708

Poschlod, P., WallisDeVries, M.F., 2002. The historical and socioeconomic perspective of calcareous grasslands-lessons from the distant and recent past. Biol. Conserv. 104, 361-376. https://doi.org/10.1016/S0006-3207(01)00201-4

Preising, E., Vahle, H.-C., Brandes, D., Hofmeister, H., Tüxen, J., Weber, H.E., 1995. Die Pflanzengesellschaften Niedersachsens - Bestandsentwicklung, Gefährdung und Schutzprobleme. Einjährige ruderale Pionier-, Tritt- und AckerwildkrautGesellschaften. Naturschutz und Landschaftspflege in Niedersachsen 20(6), 1-92

R Core Team, 2019. R: A Language and Environment for Statistical Computing. R Foundation for Statistical Computing, Vienna, Austria. [WWW Document]. URL https://www.R-project.org/ (accessed 11.2.19)

Richner, N., Holderegger, R., Linder, H.P., Walter, T., 2015. Reviewing change in the arable flora of Europe: a meta-analysis. Weed Res. 55, 1-13. https://doi.org/10.1111/wre.12123

Rode, M., Lischka, A., Schulz, G., 2018. Auswirkung von Blühstreifen auf die Biodiversität der Ackerbegleitflora in maisdominierten Agrarlandschaften. Umw. Raum 9, 81-114. https://doi.org/10.15488/3697

Roßberg, D., Gutsche, V., Enzian, S., Wick, M., 2002. NEPTUN 2000 - Erhebung von Daten zum tatsächlichen Einsatz chemischer Pflanzenschutzmittel im Ackerbau Deutschlands. Berichte aus der Biologischen Bundesanstalt für Landund Forstwirtschaft, Heft 98. SaphirVerlag, Ribbesbüttel

Šálek, M., Hula, V., Kipson, M., Daňková, R., Niedobová, J., Gamero, A., 2018. Bringing diversity back to agriculture: Smaller fields and non-crop elements enhance biodiversity in intensively managed arable farmlands. Ecol. Indic. 90, 65-73. https://doi.org/10.1016/j.ecolind.2018.03.001

Sánchez-Bayo, F., Wyckhuys, K.A.G., 2019. Worldwide decline of the entomofauna: A review of its drivers. Biol. Conserv. 232, 8-27. https://doi.org/10.1016/j.biocon.2019.01.020

Sattler, C., Kächele, H., Verch, G., 2007. Assessing the intensity of pesticide use in agriculture. Agric. Ecosyst. Environ. 119, 299304. https://doi.org/10.1016/j.agee.2006.07.017

Scheper, J., Holzschuh, A., Kuussaari, M., Potts, S.G., Rundlöf, M., Smith, H.G., Kleijn, D., 2013. Environmental factors driving the effectiveness of European agri-environmental measures in mitigating pollinator loss - a meta-analysis. Ecol. Lett. 16, 912-920. https://doi.org/10.1111/ele.12128

Secretariat of the Convention on Biological Diversity (SCBD), 2010. Strategic Plan for Biodiversity 2011-2020 and the Aichi Biodiversity Targets. Report of the tenth meeting of the Conference of the Parties to the Convention on Biological Diversity, Nagoya, Japan, 18-29 October 2010 (UNEP/CBD/COP/10/27), annex, decision X/2, annex. [WWW Document]. URL https://www.cbd.int/sp/ (accessed 1.23.19)

Seifert, C., Leuschner, C., Meyer, S., Culmsee, H., 2014. Inter-relationships between crop type, management intensity and light transmissivity in annual crop systems and their effect on farmland plant diversity. Agric. Ecosyst. Environ. 195, 173182. https://doi.org/10.1016/j.agee.2014.05.022

Still, K., Byfield, A., 2007. New priorities for arable plant conservation. Plantlife, Salisbury

Stoate, C., Boatman, N.D., Borralho, R.J., Carvalho, C.R., Snoo, G.R. de, Eden, P., 2001. Ecological impacts of arable intensification in Europe. J. Environ. Manage. 63, 337-365. https://doi.org/10.1006/jema.2001.0473

Storkey, J., Meyer, S., Still, K.S., Leuschner, C., 2012. The impact of agricultural intensification and land-use change on the European arable flora. Proc. R. Soc. B Biol. Sci. 279, 1421-1429. https://doi.org/10.1098/rspb.2011.1686

Stupak, N., Sanders, J., Heinrich, B., 2019. The Role of Farmers' Understanding of Nature in Shaping their Uptake of Nature Protection Measures. Ecol. Econ. 157, 301-311. https://doi.org/10.1016/j.ecolecon.2018.11.022

Tscharntke, T., Batáry, P., Dormann, C.F., 2011. Set-aside management: How do succession, sowing patterns and landscape context affect biodiversity? Agric. Ecosyst. Environ. 143, 37-44. https://doi.org/10.1016/j.agee.2010.11.025

Tscharntke, T., Klein, A.M., Kruess, A., Steffan-Dewenter, I., Thies, C., 2005. Landscape perspectives on agricultural intensification and biodiversity - ecosystem service management. Ecol. Lett. 8, 857-874. https://doi.org/10.1111/j.1461-0248.2005.00782.x

United Nations, 1992. Convention on biological diversity. Treaty Series, vol. 1760, No. 30619. [WWW Document]. URL https://www.cbd.int/convention/ (accessed 1.23.19) 
Van Calster, H., Vandenberghe, R., Ruysen, M., Verheyen, K., Hermy, M., Decocq, G., 2008. Unexpectedly high 20th century floristic losses in a rural landscape in northern France: Floristic changes in rural landscapes. J. Ecol. 96, 927-936. https://doi.org/10.1111/j.1365-2745.2008.01412.x

Venclova, V., Brant, V., Neckar, K., 2006. Weed succession during three years of set-aside experiment. Z. Für Pflanzenkrankh. Pflanzenschutz 611-618

Vié, J.-C., Hilton-Taylor, C., Stuart, S.N., IUCN--The World Conservation Union, IUCN Species Survival Commission (Eds.), 2009. Wildlife in a changing world: an analysis of the 2008 IUCN red list of threatened species. IUCN; Lynx Edicions, Gland, Switzerland: Barcelona, Spain

von Drachenfels, O., 2016. Kartierschlüssel für Biotoptypen in Niedersachsen unter besonderer Berücksichtigung der gesetzlich geschützten Biotope sowie der Lebensraumtypen von Anhang I der FFH-Richtlinie, Stand Juli 2016. - Naturschutz Landschaftspfl. Niedersachs. Heft A/4, 326

von Drachenfels, O., 2010. Überarbeitung der Naturräumlichen Regionen Niedersachsens. Inform.d. Naturschutz Niedersachsen 30, Nr. 4 (4/10): 249-252

Wagner, M., Bullock, J.M., Hulmes, L., Hulmes, S., Pywell, R.F., 2017. Cereal density and N-fertiliser effects on the flora and biodiversity value of arable headlands. Biodivers. Conserv. 26, 85-102. https://doi.org/10.1007/s10531-016-1225-4

Walker, K.J., Critchley, C.N.R., Sherwood, A.J., Large, R., Nuttall, P., Hulmes, S., Rose, R., Mountford, J.O., 2007. The conservation of arable plants on cereal field margins: An assessment of new agri-environment scheme options in England, UK. Biol. Conserv. 136, 260-270. https://doi.org/10.1016/j.biocon.2006.11.026

WallisDeVries, M.F., Van Swaay, C.A., 2009. Grasslands as habitats for butterflies in Europe, in: Grasslands in Europe. KNNV publishing, pp. 26-34

Zellweger-Fischer, J., Hoffmann, J., Korner-Nievergelt, P., Pfiffner, L., Stoeckli, S., Birrer, S., 2018. Identifying factors that influence bird richness and abundance on farms. Bird Study 1-13. https://doi.org/10.1080/00063657.2018.1446903

Zinngrebe, Y., Pe'er, G., Schueler, S., Schmitt, J., Schmidt, J., Lakner, S., 2017. The EU's ecological focus areas - How experts explain farmers' choices in Germany. Land Use Policy 65, 93-108. https://doi.org/10.1016/j.landusepol.2017.03.027 


\section{Chapter 2}

\section{Current state and drivers of arable plant diversity in conventionally managed farmland in Northwest Germany}

Alexander Wietzke, Clara-Sophie van Waveren, Erwin Bergmeier, Stefan Meyer, CHRISTOPH LEUSCHNER

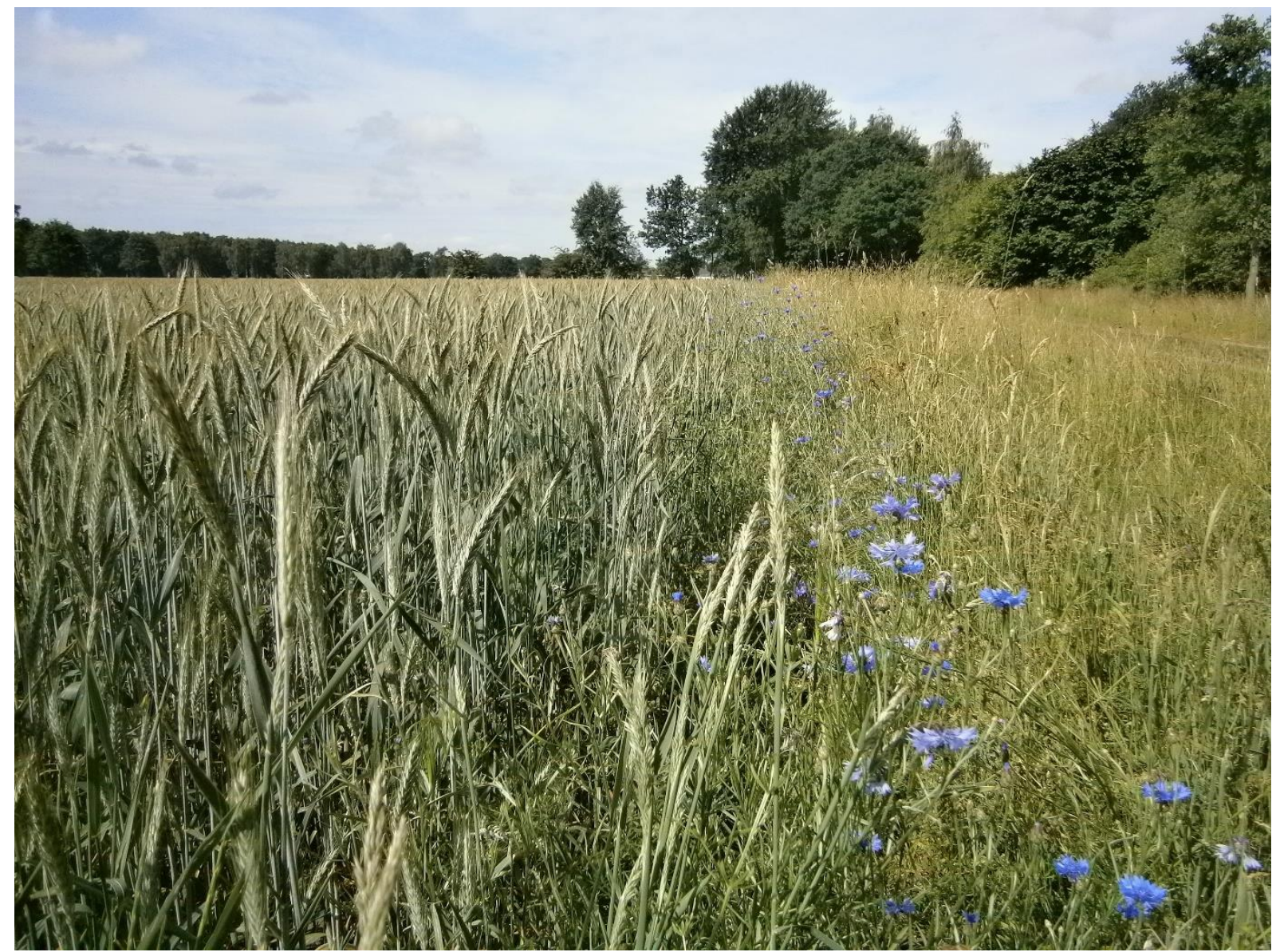

Submitted as

Alexander Wietzke, Clara-Sophie van Waveren, Erwin Bergmeier, Stefan Meyer and Christoph Leuschner. Current state and drivers of arable plant diversity in conventionally managed farmland in Northwest Germany. Diversity (MDPI).

Please note: During the review process of the submitted manuscript of Chapter 2 of the thesis, there were some marginal changes with regard to the text presented here (including some corrections in the raw data table and updated statistical analyses and figures). The revised article can be accessed at https://doi.org/10.3390/d12120469 (open access: Journal Diversity, MDPI). The corrected raw data Table S5 of Chapter 2 was uploaded to the eDiss-repository. In view of the changes that have become necessary during the review process (as compared to Chapter 2 and related statements in Chapter 5 "Synthesis" of the thesis), please use the above-mentioned revised article for citation purposes. 


\begin{abstract}
Agricultural intensification has led to dramatic diversity losses and homogenization of the arable vegetation in much of Europe. We analyzed the status of farmland phytodiversity and its determinants in 2016 in Northwest Germany by surveying 200 conventionally managed fields cultivated with seven crops (wheat, barley, rye, triticale, maize, rapeseed, potato). The study was combined with an analysis of edaphic (soil yield potential), agronomic (fertilizer and herbicide use) and landscape factors (adjacent habitats). In total, we recorded 150 non-crop plant species, many of them nitrophilous generalist species, while species of conservation value were almost completely absent. According to a mixed model, the cultivation of rapeseed and potato had a positive, that of maize a negative influence on non-crop plant richness as compared to cereals. The presence of grassy strips, ditch margins and hedges adjacent to fields increased field edge plant richness through putative spillover effects. Median values of non-crop plant richness and cover across all crops were only 2 species and $0.5 \%$ cover in the field interior, and 11 and $4 \%$ at the field edge. Agricultural intensification has wiped out non-crop plant life nearly completely from conventionally managed farmland, except for a narrow, floristically impoverished field edge strip.
\end{abstract}

Keywords: adjacent habitats; biodiversity loss; cereals; herbicides; maize; rapeseed.

\title{
2.1 Introduction
}

During the last decades, agricultural production has been greatly intensified in the industrialized countries with the consequence of dramatic biodiversity losses [1-5]. Increases in crop yield tend to correlate with decreases in farmland species richness, both in plants and various animal groups [1,6-8]. Plot-level plant species richness in arable fields has decreased by about $70 \%$ since the 1950s/60s in Central Germany with accompanying reductions in arable plant cover and regional species pool size [9]. A collapse of farmland phytodiversity has also been documented in many other Central and Western Europe regions [e.g., 10-13]. Since arable plants fulfil important functions such as nutrient retention and erosion reduction in fallow periods, the hosting of insect communities, and the provisioning of food for herbivorous animals, the drastic decline of plant cover and diversity in contemporary arable fields is alarming [14-18]. The large decrease in insect biomass [19] and farmland bird abundance that has been observed in the agricultural landscapes of Europe in recent time [6,20,21], is clearly related to reduced plant resources such as pollen, nectar and seed [17,22]. Continued systematic vegetation monitoring in the farmland is thus a prerequisite for measures to halt biodiversity erosion and to maintain at least basic ecosystem functions in intensively used agricultural landscapes for the benefit of farmers and the society as a whole [23-25].

Main determinants of arable plant diversity are environmental factors (precipitation, temperature, soil $\mathrm{pH}$, soil moisture and soil fertility), the type of cultivated crop (notably summer vs. winter crop) and crop rotation, and the associated type of management (application of herbicides and fertilizers and the tillage regime) [9,26-30]. Habitats neighboring the field can also influence farmland phytodiversity [31-33].

Today, the conventionally (intensively) managed field interior is colonized by only very few plant species of low cover, while slightly more plants typically persist at the field edges with somewhat 
lower management intensity [e.g., 12,29,34]. The pan-European reviews of Storkey et al. 2012 [4] and Richner et al. 2015 [2] revealed that the plant diversity losses concerned chiefly arable plant species and communities associated with traditional farming practices and from stony calcareous or acidic soils that are less suitable for intensive agriculture. In contrast, increases in absolute or relative terms have been observed in a few arable grasses, neophytes and plants with traits combining high nutrient demand, herbicide resistance and anemochory [2]. While some successful species are herbicide tolerant (e.g. Alopecurus myosuroides; [35]), others are able to escape herbicide treatment through a broad range of germination temperatures and high phenotypic plasticity $[30,36]$. As a consequence, the once existing variation in arable vegetation reflecting different crop types and along gradients in climate and soil fertility has largely disappeared [13,37]. Existing synopses of the arable vegetation of Central Europe consider farmland plant diversity up to the 1970/80s [e.g., 38-40]. This information may serve as a reference to analyze vegetation change since then. As about $95 \%$ of the arable land in Central Europe is managed conventionally [41], modern farmland vegetation surveys have to refer to all kinds of fields and especially the conventionally managed ones, if they are to represent the majority of the cropland. Several recent studies have investigated the vegetation of intensively managed fields [12,30,42-44], but mostly focused on a single crop or a few crops, or different crop species were pooled. Several studies addressed wheat fields in Central Europe, less often maize, triticale and/or rapeseed [34,43,45-47], or compared conventional and organic fields for a given crop, whereas studies on the species richness, composition and vegetation cover of a wider range of conventionally managed crop species in a landscape context are surprisingly scarce [but see 29,48]. Hence, the status quo of arable plant diversity in Central Europe in its dependence on agronomic and environmental factors is insufficiently known to date.

Here, we conduct a survey of arable plant diversity and species composition in fields of seven abundant cereal and root crop fields (wheat, barley, rye, triticale and rapeseed, maize, and potato) across an intensively managed farmland region in Northwest Germany and combine it with an analysis of edaphic (soil yield potential), agronomic (herbicide and fertilizer use) and landscape factors (type of adjacent habitat), which might influence species richness and composition. We tested the following hypotheses: (i) Decades of intensive agriculture have resulted in greatly impoverished and floristically homogenized arable plant communities that do not differ much across crop types and related management regimes (e.g., autumn-sown vs. spring-sown), (ii) the field edges are richer than the field interior, partly due to enrichment by plants from neighboring habitats, while the interior is nearly free of weeds, and (iii) the most important factors influencing the diversity and species composition of the arable vegetation are crop cover (plant density), herbicide treatment intensity, fertilizer amount, soil chemistry, and the type of adjacent habitat.

\subsection{Material and Methods}

\subsubsection{Study region}

The study was carried out in the north of the districts of Nienburg (centroid: N 52 36 '32.5334", E 9 $6^{\prime} 49.7118^{\prime \prime}$ ) and Diepholz (N 52 43'41.4940", E 842'4.1629"), Lower Saxony, Northwest Germany (Figure 1). The study region is part of the landscapes 'Ems-Hunte-Geest and DümmerGeestniederung' and 'Weser-Aller-Flachland' [49].

Situated in the Pleistocene lowlands (Saalian), the current land cover is predominantly arable land $(56.2 \%)$, followed by forest (14.5\%) and permanent agricultural grassland (12.4\%). About $97 \%$ of the arable land is managed conventionally. Habitats directly adjacent to arable fields include 
grass strips, ditches and hedges, which together cover about $2.4 \%$ of the area (Figure S1 and File S1). Annual average precipitation (2013-2017) ranges between $662 \mathrm{~mm}$ (Nienburg) and $684 \mathrm{~mm}$ (Diepholz) and the annual mean temperature (2013-2017) is $10.3^{\circ} \mathrm{C}$ (Nienburg) and $10.1^{\circ} \mathrm{C}$ (Diepholz) respectively [50]. The soils in the studied fields developed mostly from sandy to loamy Cambisols or Luvisols, in a few cases also from Podsols or Gleysols [51,52], depending on the sand content and groundwater level. Soil $\mathrm{pH}$ is acidic to neutral (4.1 to 6.7). Summer water deficits may develop more regularly in arable soils with higher sand content, while they are rare in the more loamy soils. According to the assessment by the Chamber of Agriculture, the soils in the study region mostly have a medium yield potential, a few sites are characterized by lower or higher potentials. The agricultural yield score (German Ackerwertzahl index) ranging from 1 (extremely poor) to 120 (extremely rich) varies for our fields by 20 to 69 .

Fifteen farmers from the two districts willing to grant access to their fields were included in the study. Thus, field selection was not fully random, but determined in the first instance by the location of the selected farms and the need to identify a sufficient number of replicate fields of a crop type. As far as possible, the fields were evenly distributed over the study region, which covered an area of c. $1300 \mathrm{~km}^{2}$ (Figure 1).

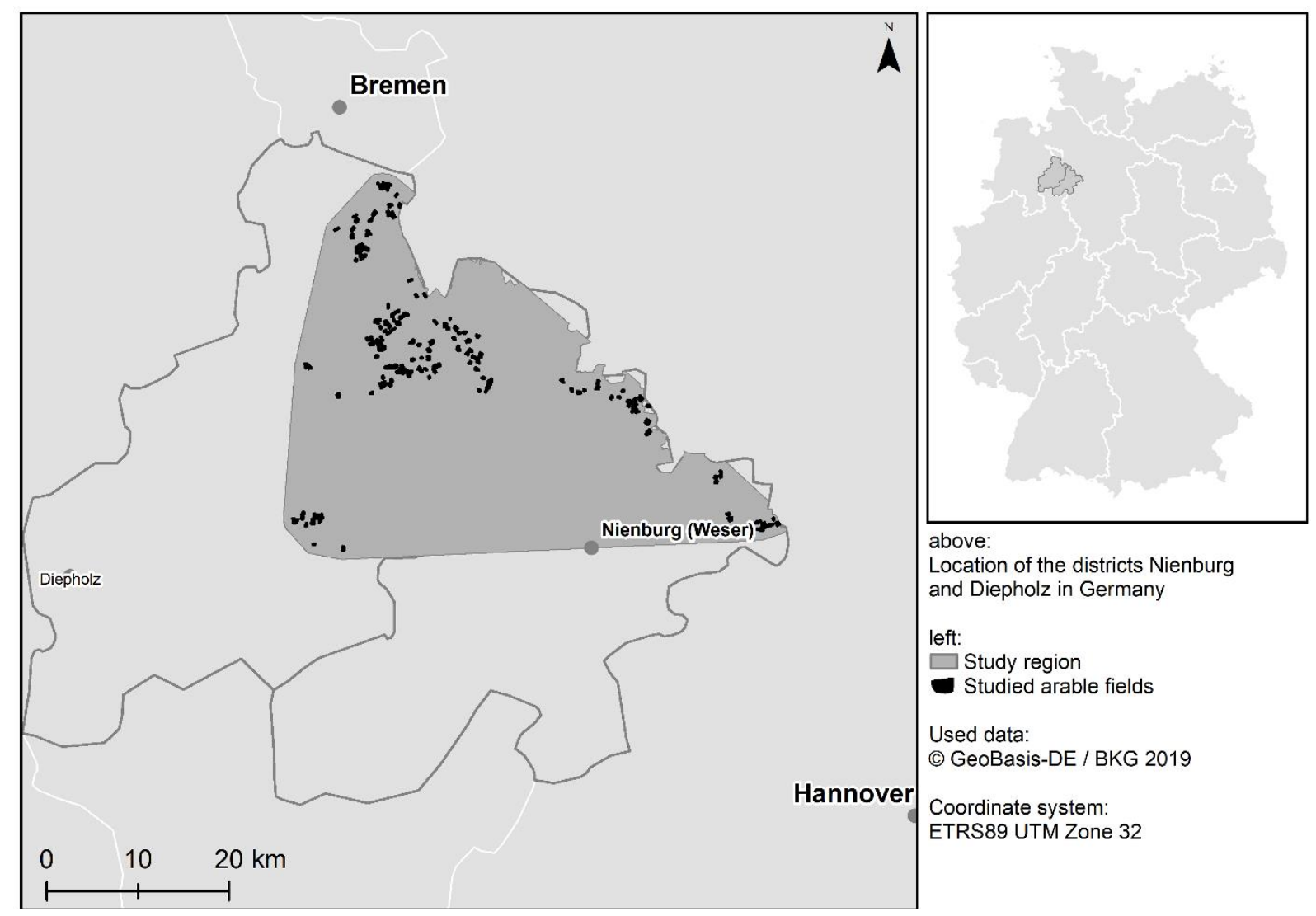

Fig. 1 Map of the study region with the districts of Nienburg and Diepholz in Lower Saxony, Northwest Germany. The location of the studied fields is indicated by black dots.

\subsubsection{Vegetation survey}

Herbaceous plant diversity of conventionally managed arable fields was studied in relevés of 100 $\mathrm{m}^{2}(50 \mathrm{~m} \times 2 \mathrm{~m})$ and vegetation cover estimated with the Londo scale [53] between end of May and August 2016. The relevés were placed either at the field edge (edge plots) or in the interior at 
least $20 \mathrm{~m}$ distant from the edge. Plot coordinates were determined by GPS (Garmin GPSMAP 64s). The type of habitat adjacent to edge plots was recorded in the categories hedge, grass strip (usually found along agricultural access tracks and between arable and non-arable habitats), ditch margin, or arable field (no other habitat between two fields), in order to analyze possible neighborhood effects on the field vegetation. We chose seven important crop species that together covered almost $90 \%$ of the arable land in the study region in 2016: winter wheat (24\% of 73,790 ha of arable land in the study region), maize (23\%), winter rapeseed (12\%), winter barley $(11 \%)$, winter rye $(9 \%)$, winter triticale (5\%), and potato (5\%; Figure S2). In total, 270 plots were investigated in the 200 studied fields: 200 edge plots (30 plots each in barley, maize, rapeseed, rye and wheat fields, 28 in potato and 22 in triticale fields), and 70 interior plots (10 per crop type). Pre-studies had shown that a smaller number of replicates of interior plots was sufficient to cover the greatly reduced number of occurring species. In addition, 63 plots in habitats directly adjacent to the fields were sampled to provide information on the local nonarable species pool, which would allow assessing spillover effects into the fields ( 22 hedges - plot size $2 \mathrm{~m} \times 25 \mathrm{~m}$; 21 ditch margins - plot size $2 \mathrm{~m} \times 8 \mathrm{~m} ; 20$ grass strips - plot size $1 \mathrm{~m} \times 16 \mathrm{~m}$ ). The studied hedge plots commonly included a narrow herbaceous fringe of about $0.5 \mathrm{~m}$ width. As the habitats border directly to the fields, some are irregularly disturbed by agricultural machinery or through tillage. Plant species names follow Buttler 2018 [54]. Raw vegetation data are presented in Table S5 in the Electronic Supplementary Materials (crop species, woody plant seedlings and a few plants not identified to the species level were excluded and omitted from the further analysis).

\subsubsection{Abiotic and management data}

The following management-related data were obtained through inquiry of 14 farmers (no data available of the 15th) for the agricultural business year 2015/16 (post-harvest 2015 - harvest 2016): cultivated crops, amount of fertilizers (N, P, K) and herbicides used and soil pH. Information on the soil yield potential (Ackerwertzahl) and soil type were retrieved by intersecting soil (scale 1:1,000,000) and agronomic maps (scale 1:5,000; [51,52]) using ArcMap [55]. Information on the frequency of application and dose of herbicides ('Herbicide Intensity Index') were used to calculate the Standardized Treatment Index (STI after [56,57]). STI values were calculated for every field in relative terms (herbicide amount applied relative to the amount permitted). The maximum permitted amount of herbicides was obtained from the Crop Protection Manager [58]. If the farmer used different herbicides per field within the agricultural business year 2015/16, the index was calculated by summing up the STI values of the different herbicides.

\subsubsection{Statistical analyses}

Statistical analyses and data visualization were conducted with $\mathrm{R}$ 3.5.1 Software [59] using the packages magrittr [60], tidyverse [61], goeveg [62], vegan [63] and reshape2 [64]. The Londo-scale cover values of plants were expressed as percentage values for the analysis [65]. Since the soil types of the studied arable fields were similar and pre-analysis revealed no significant soil effect on plant diversity, this factor was excluded from the analysis.

Plant species richness data were categorized into the groups (a) all herbaceous plants recorded (but without tree/shrub seedlings and crops), (b) plant species with close affinity to arable fields (according to Hofmeister and Garve 2006 [38]; hereafter termed 'arable plants sensu stricto' ), (c) 
arable plant species endangered in Lower Saxony [66], and (d) high-nature-value (HNV) species according to German Federal Agency for Nature Conservation 2018 [67] (Table S1). Arable plants sensu stricto [38] are taxa regularly found in arable fields and accompanying Central European agriculture. Part of these species are not restricted to arable habitats but may colonize other, e.g. ruderal, habitats too. HNV species are defined as taxa that are frequently associated with, and thus functioning as indicators of, habitats of increased plant diversity and nature value. For example, the presence of 4-5 HNV species stands for farmland of a moderate nature value, 6-7 species for high, and 8 or more species for very high-nature-value. Fields with three or fewer HNV species are not considered as high-nature-value farmland.

In an attempt to assign the species assemblages of the different studied crop types and field locations (edge vs. interior) to syntaxa in the phytosociological hierarchical system [39,68], we calculated the median numbers of diagnostic arable plant species for classes, orders, alliances or associations that were present in the assemblages. We expanded the vegetation analysis from the arable fields to the directly adjacent habitats (grass strips, ditch margins, hedges) and plotted Venn diagrams [69] to express the percent overlap in species pools. The floristic similarity between fields was determined with the Sørensen Dissimilarity Index (package vegan [63]) for edge and interior plots. Significant differences between field edge and interior plots for a given crop species were tested with the Mann-Whitney $U$ test for pairwise comparisons. In order to unravel biotic, environmental and management-related factors in their influence on plot-level species richness, we calculated a negative binomial generalized mixed effects model [70] with crop species (Crop), adjacent habitat (adj_habitat), soil yield potential (soil_y_p), Herbicide Intensity Index (STI), crop cover (cover), and total nitrogen input (N_tot) as fixed factors and farm as random factor. Due to multicollinearity, we introduced total nitrogen input instead of potassium and phosphorus input, and soil yield potential instead of soil $\mathrm{pH}$ in the analysis. The possible influence of shading through adjacent hedges or groves was introduced through the variable 'adjacent hedge'. Since the autumn-sown cereals wheat, barley, rye and triticale are managed in a similar way and have rather similar stand structures, these four winter cereals were pooled (variable 'cereals') in order to save degrees of freedom in the analysis. Nevertheless, due to the complexity of the analysis, no degrees of freedom were left for calculating factor interactions. The resulting mixed model had the form glmmTMB (species.number $\sim$ Crop+adj_habitat+soil_p_v + STI + cover + N_tot $+(1 /$ farm $)$, family $=$ nbinom1). Finally, we tested the significance of response variables by likelihood-ratio tests and a post hoc-pairwise comparison of categorial variables (Tukey [71]). Model structure and statistical results can be found in the Table S6 in the Electronic Supplementary Materials.

\subsection{Results}

\subsubsection{Patterns of plant species richness and cover}

In total, we observed 150 herbaceous non-crop plant species in the 270 plots examined in 200 arable fields. We take this number as a minimum estimate of the present species pool in the arable fields of the study region of c. $1300 \mathrm{~km}^{2}$. All 150 species were found at the field edges, while only 41 of them occurred also in the field interior (Table S2 and Table S3). Between-field variation, i.e. $\beta$ diversity, was very high (mean Sørensen Dissimilarity Index: 0.86 for interior plots [14 plots without species omitted]) and only slightly lower for edge plots (0.76). Fifty-nine of the 150 species $(39 \%)$ observed in the edge plots, and 25 of the 41 species $(61 \%)$ in the interior plots, were found in less than three of the 200 fields (Table S2). Median species number across all 
crop types in the edge plots was 11, and only 2 in the field interior plots. Among the seven crop types, the highest number of plant species was found in rapeseed field edge plots (median $=17$ ), followed by rye (13.5), whereas maize showed the lowest diversity (8; Figure 2). In field interior plots, median diversity varied between 0 and 3 in the seven crops. The same pattern across crop types was found for the diversity of arable plants sensu stricto (Figure 2). The number of HNV species of arable land was in general very low (median values between 0 and 3), but showed a tendency for higher values in the edge plots, especially in rapeseed and rye (Figure 2). We found only one plant species red-listed as Vulnerable in Lower Saxony (Odontites vernus) in a single edge plot (rye).

Crop cover was very high throughout the 270 plots (median of edge plots: $90 \%$; interior: $95 \%$ ), with lowest cover recorded for rye and triticale field edges (median: $85 \%$, Figure S4). Non-crop plant cover was in most fields $<1 \%$ in the interior (median: $0.5 \%$, all crops pooled; Figure 2 ) and reached at the field edge a median value of $4 \%$. An exception was rapeseed with medians of $11 \%$ (edge) and $10 \%$ (interior).
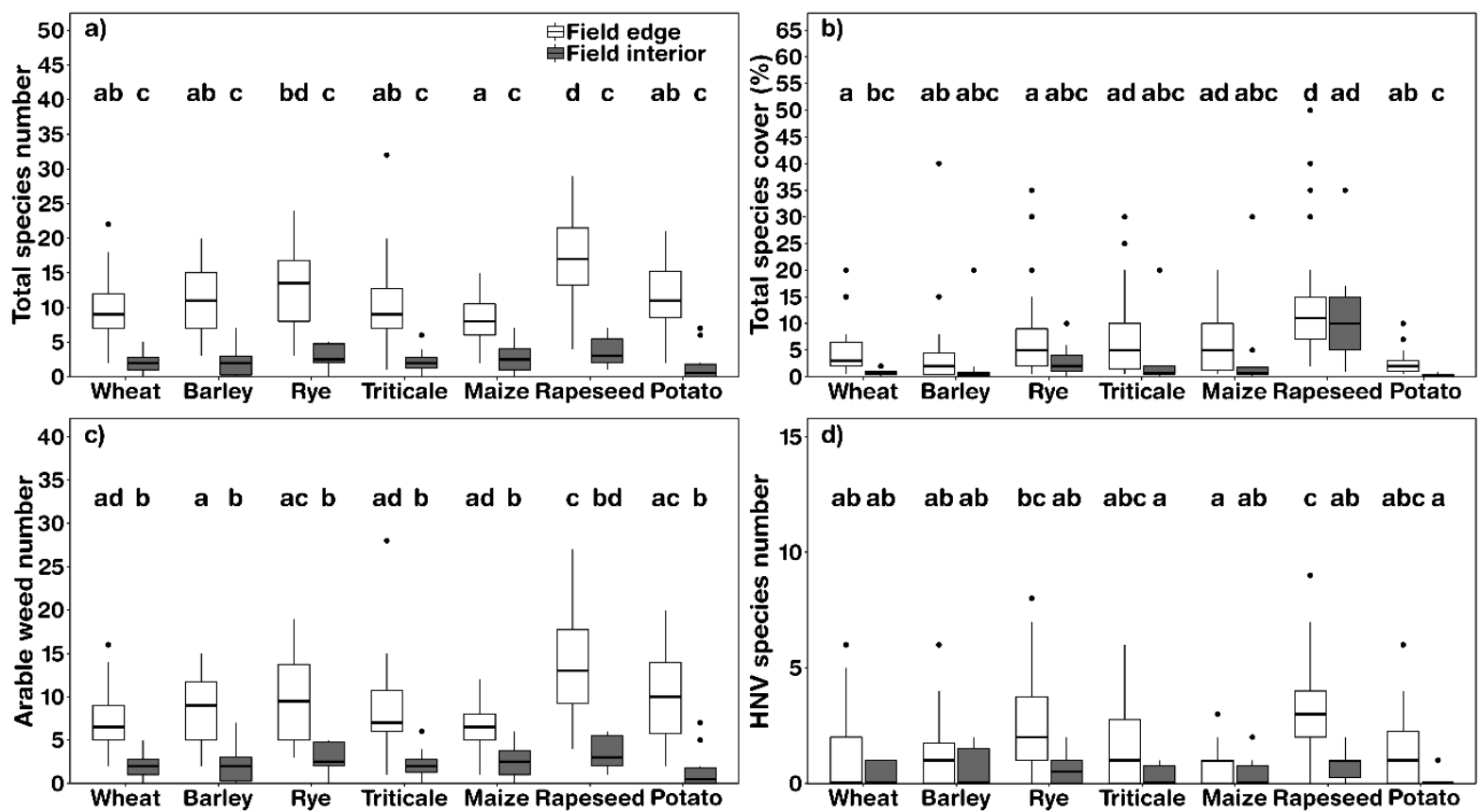

Fig. 2 Species richness (a, c, d) or plant cover (b) in $100 \mathrm{~m}^{2}$-plots in the field interior and at the edge in the seven crop types for all herbaceous species ( $a$ and $b$; total species number without woody plant seedlings and crop species), arable plant species sensu stricto (c; after Hofmeister and Garve [38]), and high-nature-value species of arable land (d; HNV species after German Federal Agency for Nature Conservation [67]). $\mathrm{n}=30$ fields for edge plots of wheat, barley, rye, maize, and rapeseed, $\mathrm{n}=28$ for potato, $\mathrm{n}$ $=22$ for triticale, $\mathrm{n}=10$ for interior plots. Significant differences between crop types and plot locations (field edge vs. interior) are indicated by different small letters (Mann-Whitney U-Test).

\subsubsection{Variation in community composition}

Despite a greatly impoverished arable species pool and low $\alpha$ diversity, species assemblages differed considerably between different crops. Only one species (Elymus repens) occurred in more than half of the plots ( $52 \%$; edge and interior plots pooled), another seven in more than 30 $\%$ (in decreasing order Galium aparine, Dactylis glomerata, Matricaria chamomilla, Fallopia convolvulus, Viola arvensis, Polygonum aviculare, Bromus sterilis; Table S3). While 34 herbaceous species were found in all seven crops, 23 were exclusively observed in winter cereals (wheat, barley, rye, triticale), and 16 only in rapeseed (Figure S3). A few species showed a preference for a certain crop, notably Echinochloa crus-galli for the interior of maize fields, and 
Viola arvensis for rapeseed fields (edge and interior), where they occurred with a particularly high frequency (Table S3). Yet, an assignment of the surveyed assemblages in the seven different crop types to defined plant associations was impossible due to the generally very low number of diagnostic species found ( 0 to 2; Table 1$)$; diagnostic species of alliances and orders were also commonly underrepresented.

Table 1 Number of arable plant species sensu stricto (defined by Hofmeister and Garve [38]) and number of species diagnostic for classes, orders, alliances or associations of arable plant communities (defined by Hüppe and Hofmeister, 1990). Given are median values; $\mathrm{n}=30$ for edge plots of barley, maize, rapeseed, rye and wheat, potato $=28$, triticale $=22 ; \mathrm{n}=10$ for interior plots per crop.

\begin{tabular}{llcccccc}
\hline Plot type & Crop & $\begin{array}{c}\text { Arable } \\
\text { plants }\end{array}$ & Class & Order & Alliance & Association & $\begin{array}{c}\text { Total number of diagnostic } \\
\text { species }\end{array}$ \\
\hline Field edge & Wheat & 6.5 & 0 & 0 & 0 & 1 & 1 \\
\hline Field interior & Wheat & 2 & 0 & 0 & 0 & 0 & 1 \\
\hline Field edge & Barley & 9 & 1 & 0 & 1 & 1 & 2.5 \\
\hline Field interior & Barley & 2 & 0 & 0 & 0 & 0 & 1 \\
\hline Field edge & Rye & 9.5 & 1 & 0 & 2 & 1 & 1 \\
\hline Field interior & Rye & 2.5 & 1 & 0 & 0 & 0 & 2 \\
\hline Field edge & Triticale & 7 & 0 & 0 & 0.5 & 1 & 0 \\
\hline Field interior & Triticale & 2 & 0 & 0 & 0 & 0 & 2 \\
\hline Field edge & Maize & 6.5 & 1 & 0 & 0 & 1 & 1.5 \\
\hline Field interior & Maize & 2.5 & 0 & 0 & 0 & 1 & 5 \\
\hline Field edge & Rapeseed & 13 & 2 & 0 & 1 & 2 & 2 \\
\hline Field interior & Rapeseed & 3 & 1 & 0 & 0 & 1 & 4 \\
\hline Field edge & Potato & 10 & 2 & 0 & 0.5 & 1 & 0 \\
\hline Field interior & Potato & 0.5 & 0 & 0 & 0 & 0 & 1 \\
\hline
\end{tabular}

Of the 150 herbaceous plant species found in the fields 112 (i.e. $74.7 \%$ ) occurred also in the directly adjacent habitats; 69 of these overlapping species (61.6\%) are arable plant species sensu stricto (Table S4). The largest fraction of overlapping species $(50.7 \%$ ) was shared with the grass strips bordering the fields, another $42.7 \%$ each with neighboring ditch margins and hedges (Figure 3). These three main neighboring habitats contained 51 species that were not found in the fields. Thus, the adjacent habitats were about as species-rich as the field edges (163 and 150 species, respectively), with roughly two thirds of the species overlapping. 
Field edges

Adjacent habitats

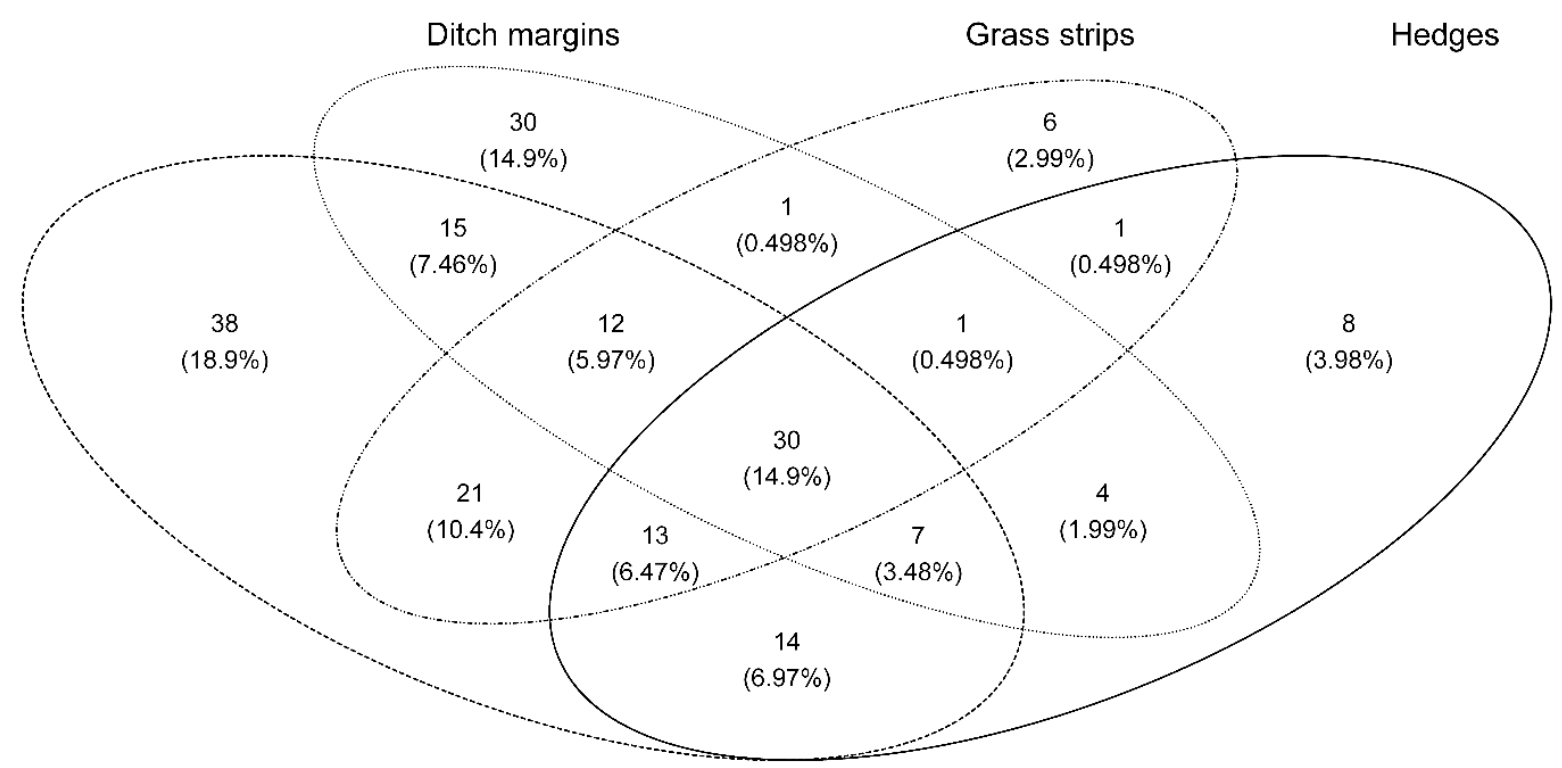

Fig. 3 Number of species exclusively occurring at field edges or in three types of adjacent habitats, or that are shared with other habitats in the study region. Indicated are the species number and the proportion of the total species pool (in \%) for herbaceous plant species richness (in total 201 species, woody plant seedlings and crops excluded); n: edge plots $=200$, ditch margins $=21$, grass strips $=20$, hedges $=22$.

\subsubsection{Factors determining arable species richness}

The generalized mixed effects model with crop type, plot location in the field, management regime and adjacent habitat as fixed effects and farm as random factor showed that plot location in the field (edge vs. interior) was the main factor determining plant diversity across all crop species (Figure 4; Table S6). Other significant, but somewhat smaller, effect sizes were found for the type of adjacent habitat, and crop type, while soil and management factors had only a small (in the case of Herbicide Intensity Index: insignificant) influence. Measured against winter cereals (wheat, barley, rye, triticale) field edge plots directly bordering other fields as a reference, species richness increased when grass strips, ditch margins and hedges were neighboring and rapeseed or potato were planted, while the cultivation of maize reduced diversity. Also, high management intensity as indicated by high soil yield potential, high total $\mathrm{N}$ input, greater herbicide use (Herbicide Intensity Index), and high crop cover decreased plot-level diversity at the field edge (Figure 5). The random factor farm (which introduces site and specific management effects) also had a significant effect on plot-level species richness (Table S6). 


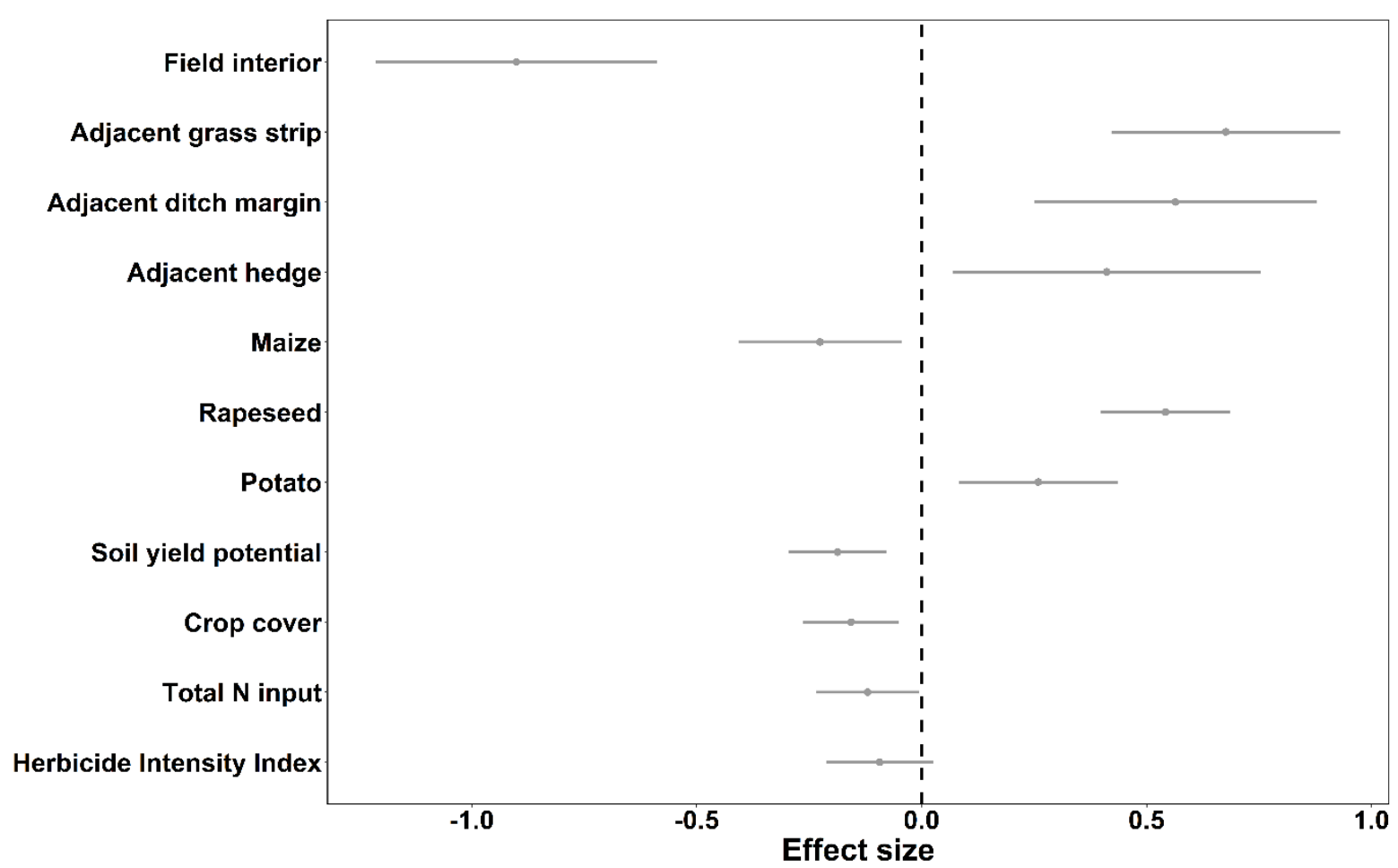

Fig. 4 Effect size (plotted estimates of the covariates and $95 \%$ confidence intervals) of the influence of plot location in the field (interior vs. edge), crop type (maize, potato, rapeseed), adjacent habitat type (grass strip, ditch margin, hedge), management-related factors (Herbicide Intensity Index, crop cover), and edaphic properties (soil yield potential, total nitrogen input) on total herbaceous plant species richness of the surveyed arable fields according to the generalized mixed effects model $(\mathrm{n}=255$ plots on 14 farms). The effects were calculated relative to species diversity in edge plots of cereal fields (wheat, barley, rye and triticale pooled) that bordered to another field, i.e. when no adjacent habitat (grass strip, ditch margin or hedge) between neighboring fields was present.

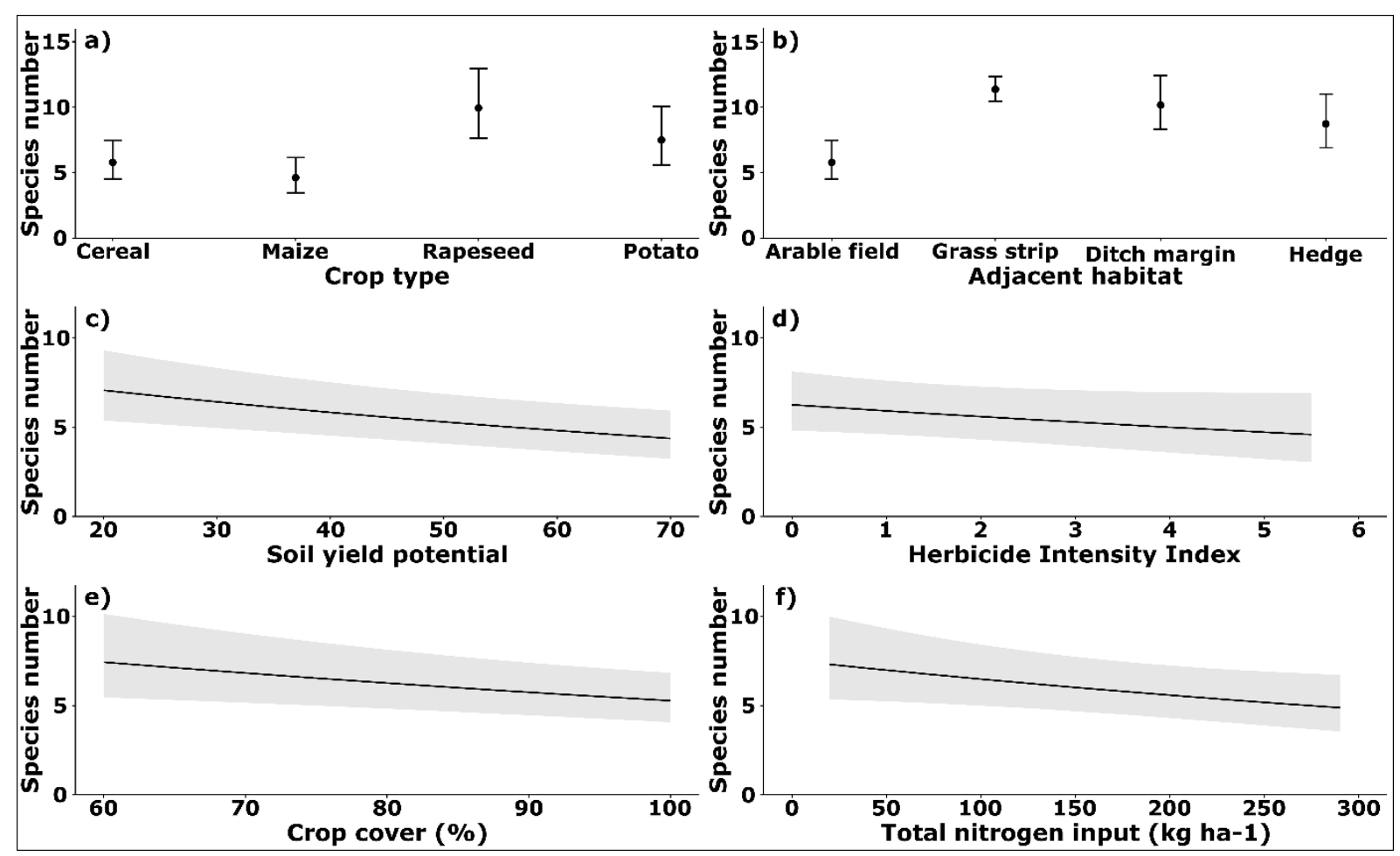

Fig. 5 Number of herbaceous plant species (without woody plant seedlings and crops) at the field level for different crop types (all four cereals pooled; field edge and interior pooled) (a), or for field edge plots bordering to different adjacent habitats (ditch margin, grass strip, hedge or no adjacent habitat: directly bordering to another arable field) (b), number of plant species (field edge and interior plots pooled) in relation to soil yield potential (Ackerwertzabb) (c), Herbicide Intensity Index (d), crop cover (e), or total nitrogen fertilizer added ( $\mathrm{f}$ ) according to the generalized mixed effects model ( $\mathrm{n}=255$ plots on 14 farms). Given are predicted means and $95 \%$ confidence intervals in (a) and (b), predicted values (black line) and $95 \%$ confidence intervals (grey, c to f). 


\subsection{Discussion}

\subsubsection{Quantifying phytodiversity loss in conventionally managed farmland}

With a median $\alpha$ diversity of 2 species per $100 \mathrm{~m}^{2}$ in the field interior, richness was even lower than in the extensive survey of Meyer et al. 2013 [9] conducted in 2009 in 392 arable fields in Central Germany (median: 7 species). Other studies in intensively managed arable land in Central and Western Europe used variable plot sizes but found mean richness at plot level in the field interior of 6 to 10 species [12,72-74]. The phytodiversity of field edges in our study area (median: 11 species per plot) is also lower than in the large-scale survey of Meyer et al. 2014 [75] in Central and North Germany (median: 18 species per plot). In fact, our richness values are among the lowest recorded so far for Central Europe. One likely reason is that the study region with its humid climate and relatively fertile soils enables high yields under intensive management. The once richer arable plant diversity in the region has vanished already 50 or more years ago due to the increasingly intensive management regime resulting in dense, shading crop stands [68,76]. In the 1950/60s, a median plot-level arable plant diversity of 24 species in the field interior was recorded in Central and North Germany [9], which underpins the strong decline in species diversity within the last 60-70 years. An increase in agricultural intensification and chemical weed control in the past years since the above-mentioned vegetation surveys in Central Germany and other regions may have caused further phytodiversity erosion.

With only 150 species found in the 200 fields in 2016 (Table S1), the regional species pool $(\gamma$ diversity) in the c. $1300 \mathrm{~km}^{2}$ large study region is certainly much impoverished compared to the situation decades ago, which supports our hypothesis (i). Hofmeister and Garve 2006 [38] list 287 species as typical associated with arable plant communities in Germany. Correspondingly, Meyer et al. 2013 [9] give a regional species pool of 301 taxa that were found in the 1950/60s in 392 fields in Central and North Germany; this number had decreased by $23 \%$ to 233 in 2009, which still exceeds by far the 150 species found in 2016 in our Northwest German study region. While low environmental variation in the study region explains part of the relatively small current species pool and our survey may have missed a few species persist in the diaspore bank of the arable fields, diversity losses in the recent past are indisputable, as is also indicated by the dominance of disturbance- and herbicide-tolerant generalist species in the current flora.

\subsubsection{The importance of the field edge}

In support of hypothesis (ii), we found that the location in the field, i.e. edge vs. interior, is more important for plant diversity than crop type. This reflects the steep diversity gradient across the first 1 or $2 \mathrm{~m}$ in the field margin, where herbicide and fertilizer amounts are often lower and crop densities reduced (Figure 4 and Figure S4). In our fields, the diversity decrease from the edge to the interior was even greater (from 11 to 2 species; median values) than in the study of Meyer et al. 2014 [75] in Central and North Germany (18 to 7 species). In a systematic comparison of field edge and interior plots, Wietzke \& Leuschner 2020 [77] could show that in conventionally managed fields of Northwest Germany, the entire remaining phytodiversity of arable plants is restricted to the small field edge strip, which occupies about $5 \%$ of the field area [78]. Thus, in terms of arable plant diversity and abundance, the field interior, i.e. $95 \%$ or more of the field, is of no relevance, a finding that is likely to be transferable to most of the conventionally managed arable land in Central Europe. Rapeseed fields differed from the other six crops in that the edgeinterior gradient existed for species richness but not for plant cover (Figure 2). This was caused by a remarkably high cover of Viola arvensis throughout the field edge and interior in rapeseed; 
this species seems to tolerate the shading of the closed stand and may profit from the less effective herbicide treatment against dicots in rapeseed.

\subsubsection{Composition of the recent arable vegetation and the role of field neighborhood}

Only 96 of the 150 species present in the studied arable fields can be considered as arable plants sensu stricto (according to [38]; Table S1) occurring mainly in arable land. A large fraction of these are widespread generalist arable plants, consisting of nitrophilous and herbicide-tolerant non-crop grasses and herbs. Similarly, the 54 additionally found plant species not characterized as arable plants sensu stricto include many nitrophilous and disturbance-tolerant taxa most frequently occurring in field-adjacent habitats in the farmland, notably eutrophic, irregularly mown grass strips and ditch margins, and fringes of hedges, from where they tend to spill over into the field edge. In general, arable plant assemblages of modern fields are not only much impoverished, but they contain a much larger proportion of ubiquitous grasses (such as Elymus repens, Bromus sterilis and Dactylis glomerata) and nitrophilous herbs (such as Galium aparine, Urtica dioica and Rumex obtusifolius) than in vegetation records of Northwest German arable fields decades ago $[68,79]$. As noted earlier, species with close dependence on edaphic factors (acidic vs. base-rich) and different types of field management (e.g. summer vs. winter crops, and root crops vs. cereals), and with them the characteristic habitat-specific arable plant communities have largely disappeared [2,30,37]. In correspondence, we found only a very small number of diagnostic species for arable plant associations (see Table 1), insufficient to identify the related communities, similarly as reported by Meyer et al. (2015) for Central and North Germany in 2009. The occasional occurrence of Matricaria chamomilla, Centaurea cyanus, Apera spica-venti and Vicia hirsuta in field edge plots reflects what is left of the Aphano-Matricarietum chamomillae, formerly the most common association in arable fields in the study area and wider northwest Germany [79].

Our mixed model analysis is among the few attempts to disentangle the influences of crop type, edaphic properties, agronomic and landscape factors on arable plant richness. In contradiction to our hypothesis (i), we found that crop is still a major determinant of plant diversity in modern arable fields. As compared to cereals, fields of rapeseed and potato positively influenced noncrop plant richness, whereas maize fields were less diverse (Figure 4 and 5). In a comparison of four crop species, Seifert et al. 2014 [29] also found a (non-significant) tendency toward higher species numbers in rapeseed. A likely explanation is that dicot weeds are more difficult to control in dicot crops (as rapeseed or potato) than in cereals. The lowest species numbers in maize may partly result from light deficiency which is more pronounced under the canopy of maize than under cereals (6 vs. 10-13\% relative light intensity; [29]), and perhaps from specific herbicide combinations.

Besides crop type and location in the field, the adjacent habitat was the third factor with a pronounced influence on plant diversity in the fields, which supports hypothesis (iii). Of the 150 species found in the fields, three quarters also occurred in the adjacent grassy habitats or hedges, and 69 of them were arable plants sensu stricto (Figure 3 and Table S4). In agreement with hypothesis (ii), this indicates that some arable taxa have refugia in adjacent habitats, and spill over from grass strips, ditch margins or hedges. This may buffer such species against population decreases or local extinctions in fields with adverse growing conditions. Correspondingly, six plant species listed as arable plants sensu stricto by Hofmeister and Garve 2006 [38] were solely found in adjacent habitats, including Vicia villosa, an obligatory arable plant. Population genetic studies will have to show whether adjacent habitats usually out of reach of herbicides harbor 
today source populations of certain arable plants decimated in the fields, or whether they profit from spillover from the field edge, but in turn do not benefit the field edge populations. The other 43 species found in both field edges and adjacent habitats are stress-tolerant and nitrophilous species with main occurrence in fertile and ruderalized grasslands and hedges. They occasionally spill over into the field edge, sometimes only shortly and then disappear again, but they increase the total number of field edge species [31-33]. They are of little conservation value with respect to the arable flora.

In many intensively used Central European farmland landscapes, the habitats between two neighboring fields occupy about $1-2(-5) \%$ of the area. If the grass strips and ditch margins not directly treated with fertilizers and pesticides are enlarged and mown once a year to reduce the nutrients stored in biomass and the competitiveness of some vigorous plants, and optionally supplied with seed mixtures of local origin arable and grassland plants, then these habitats may be revaluated to function as local refugium of a fraction of the farmland phytodiversity, benefitting at the same time farmland birds and insects [80,81]. Occasional tillage may increase the germination success. Such measures will be especially valuable when appropriate infield measures (such as ecological focus areas of non-intensively managed crop fields, arable fallow and indigenous flower strips) are not implemented. We hardly expect that the diversity of conventionally managed field edges, treated with fertilizers and herbicides, will profit much from revaluated grass strips and ditch margins, but landscape-level diversity will to some extent. To effectively increase both arable and non-arable diversity of farmland landscapes the improvement and enlargement of adjacent habitats should be combined with infield measures such as nonintensively managed cereal strips at the field edge.

\subsection{Conclusions}

This analysis of plant diversity in an intensively managed arable landscape, which is considered representative for much of Central and Western Europe's farmland, clearly demonstrates the dramatically low level of contemporary farmland phytodiversity. With a median non-crop plant cover of $0.5 \%$ in the field interior and $4 \%$ at its edge, weed control proves to be highly effective with arable plant cover reduced far below the level, where significant competition with the crop plants might occur. Overly weed control of such intensity leads to the collapse of arable plant diversity, and moreover has the potential to wreck important ecosystem functions provided by arable plants, notably the protection from soil erosion and the reduction of nitrate leaching during non-growth time of crops. Across all crops, species richness has reached historical minima with median values of eleven taxa at the edge and only two in the interior. Higher cover of arable non-crop species has only been found in fields of rapeseed, indicating possible gaps in chemical weed control rather than higher plant diversity associated with this crop species. The remaining arable plant species pool has lost nearly all taxa of conservation value. It nowadays consists mainly of widespread generalist arable plants, that are herbicide-tolerant or -avoiding, nitrophilous, non-crop grasses and herbs that are able to persist in the fields' seed bank or spill over from adjacent habitats. In arable landscapes, where infield measures to increase biodiversity (such as non-intensively managed field edge cereal strips) are not feasible under current agronomic conditions, we recommend to improve the habitat quality of field-adjacent grassy strips through mowing and, if helpful, tillage and sowing of local arable plant mixtures. Such management of these refugial habitats might result in an increase of the local farmland landscape species pool but will not compensate for the overall loss of farmland biodiversity. Agricultural policy at national and EU levels must take notice of the fact that agrochemical weed control has 
nearly wiped out non-crop plant life in at least $95 \%$ of the conventionally managed cropland of Central Europe, i.e. the field interior except for a narrow field edge strip, thereby jeopardizing important ecosystem services at the cost of future generations of farmers and the society as a whole.

\section{Funding}

The presented study is part of the research project 'Development of targeted and efficient schemes to increase biodiversity in agricultural landscapes (MEDIATE)' funded by the German Federal Environmental Foundation (DBU; 32873/01).

\section{Acknowledgements}

Many thanks to all farmers who granted us access to their fields. In addition, many thanks to Laura Sutcliffe, Stefan Mecke, Friedemann Goral, Jenny Schellenberg and Roman Link for helpful discussions regarding the study design and/or data analysis and to Christina Ewerhardy, Fionn Pape and Svenja Meyer for invaluable support during the field work.

\section{Author contributions}

Conceptualization, A.W. and C.L.; methodology, A.W., C.L., C.VW., E.B. and S.M.; formal analysis, A.W. and C.VW.; investigation, A.W.; data curation, A.W. and C.VW.; writing-original draft preparation, A.W. and C.VW; writing-review and editing, A.W., C.L., E.B., C.VW. and S.M.; project administration, A.W.; All authors have read and agreed to the published version of the manuscript; funding acquisition, C.L., J. Dauber (Thünen Institute of Biodiversity, Braunschweig, Germany), N. Kretzschmar and M. Polaschegg (Lower Saxony Chamber of Agriculture, Germany).

\section{Conflict of interest}

The authors declare that they have no conflict of interest. 


\section{References}

1. Geiger, F.; Bengtsson, J.; Berendse, F.; Weisser, W.W.; Emmerson, M.; Morales, M.B.; Ceryngier, P.; Liira, J.; Tscharntke, T.; Winqvist, C.; et al. Persistent negative effects of pesticides on biodiversity and biological control potential on European farmland. Basic Appl. Ecol. 2010, 11, 97-105, doi:10.1016/j.baae.2009.12.001.

2. Richner, N.; Holderegger, R.; Linder, H.P.; Walter, T. Reviewing change in the arable flora of Europe: a meta-analysis. Weed Res. 2015, 55, 1-13, doi:10.1111/wre.12123.

3. Stoate, C.; Boatman, N.D.; Borralho, R.J.; Carvalho, C.R.; Snoo, G.R. de; Eden, P. Ecological impacts of arable intensification in Europe. J. Environ. Manage. 2001, 63, 337-365, doi:10.1006/jema.2001.0473.

4. Storkey, J.; Meyer, S.; Still, K.S.; Leuschner, C. The impact of agricultural intensification and land-use change on the European arable flora. Proc. R. Soc. B Biol. Sci. 2012, 279, 1421-1429, doi:10.1098/rspb.2011.1686.

5. Habitats for birds in Europe: a conservation strategy for the wider environment; Tucker, G.M., Evans, M.I., BirdLife International, Eds.; BirdLife conservation series; BirdLife International: Cambridge, 1997; ISBN 978-0-946888-32-0.

6. Donald, P.F.; Sanderson, F.J.; Burfield, I.J.; van Bommel, F.P.J. Further evidence of continent-wide impacts of agricultural intensification on European farmland birds, 1990-2000. Agric. Ecosyst. Environ. 2006, 116, 189-196, doi:10.1016/j.agee.2006.02.007.

7. Gabriel, D.; Sait, S.M.; Kunin, W.E.; Benton, T.G. Food production vs. biodiversity: comparing organic and conventional agriculture. J. Appl. Ecol. 2013, 50, 355-364, doi:10.1111/1365-2664.12035.

8. Fokus Biodiversität: wie Biodiversität in der Kulturlandschaft erhalten und nachhaltig genutzt werden kann; Hotes, S., Wolters, V., Eds.; Oekom: München, 2010; ISBN 978-3-86581-172-1.

9. Meyer, S.; Wesche, K.; Krause, B.; Leuschner, C. Dramatic losses of specialist arable plants in Central Germany since the 1950s/60s - a cross-regional analysis. Divers. Distrib. 2013, 19, 1175-1187, doi:10.1111/ddi.12102.

10. Albrecht, H. Changes in the arable weed flora of Germany during the last five decades. Proc. 9th EWRS-Symp. Bp. 1995, 41-48.

11. Cirujeda, A.; Aibar, J.; Zaragoza, C. Remarkable changes of weed species in Spanish cereal fields from 1976 to 2007. Agron. Sustain. Dev. 2011, 31, 675-688, doi:10.1007/s13593-011-0030-4.

12. Fried, G.; Petit, S.; Dessaint, F.; Reboud, X. Arable weed decline in Northern France: Crop edges as refugia for weed conservation? Biol. Conserv. 2009, 142, 238-243, doi:10.1016/j.biocon.2008.09.029.

13. Leuschner, C.; Ellenberg, H. Ecology of Central European Non-Forest Vegetation: Coastal to Alpine, Natural to ManMade Habitats: Vegetation Ecology of Central Europe, Volume II; Springer International Publishing: Cham, 2017; ISBN 978-3-319-43046-1.

14. Gholamhoseini, M.; AghaAlikhani, M.; Mirlatifi, S.M.; Sanavy, S.A.M.M. Weeds - Friend or foe? Increasing forage yield and decreasing nitrate leaching on a corn forage farm infested by redroot pigweed. Agric. Ecosyst. Environ. 2013, 179, 151-162, doi:10.1016/j.agee.2013.08.016.

15. Hawes, C.; Haughton, A.J.; Osborne, J.L.; Roy, D.B.; Clark, S.J.; Perry, J.N.; Rothery, P.; Bohan, D.A.; Brooks, D.R.; Champion, G.T.; et al. Responses of plants and invertebrate trophic groups to contrasting herbicide regimes in the Farm Scale Evaluations of genetically modified herbicide-tolerant crops. Philos. Trans. R. Soc. B Biol. Sci. 2003, 358, 1899-1913, doi:10.1098/rstb.2003.1406.

16. Hyvönen, T.; Huusela-Veistola, E. Arable weeds as indicators of agricultural intensity - A case study from Finland. Biol. Conserv. 2008, 141, 2857-2864, doi:10.1016/j.biocon.2008.08.022.

17. Marshall, E.J.P.; Brown, V.K.; Boatman, N.D.; Lutman, P.J.W.; Squire, G.R.; Ward, L.K. The role of weeds in supporting biological diversity within crop fields. Weed Res. 2003, 43, 77-89, doi:10.1046/j.1365-3180.2003.00326.x.

18. Storkey, J.; Westbury, D.B. Managing arable weeds for biodiversity. Pest Manag. Sci. 2007, 63, 517-523, doi:10.1002/ps.1375.

19. Hallmann, C.A.; Sorg, M.; Jongejans, E.; Siepel, H.; Hofland, N.; Schwan, H.; Stenmans, W.; Müller, A.; Sumser, H.; Hörren, T.; et al. More than 75 percent decline over 27 years in total flying insect biomass in protected areas. PLOS ONE 2017, 12, e0185809, doi:10.1371/journal.pone.0185809.

20. Donald, P.F.; Green, R.E.; Heath, M.F. Agricultural intensification and the collapse of Europe's farmland bird populations. Proc. R. Soc. B Biol. Sci. 2001, 268, 25-29, doi:10.1098/rspb.2000.1325.

21. Vorríšek, P.; Jiguet, F.; van Strien, A.; Škorpilová, J.; Klvaňová, A.; Gregory, R. Trends in abundance and biomass of widespread European farmland birds: how much have we lost. BOU Proc. Lowl. Farml. Birds III 2010, 1-24.

22. Still, K.S. A future for rare arable plants. Aspects of Applied Biology, 81, 175-182. 2007.

23. Barral, M.P.; Rey Benayas, J.M.; Meli, P.; Maceira, N.O. Quantifying the impacts of ecological restoration on biodiversity and ecosystem services in agroecosystems: A global meta-analysis. Agric. Ecosyst. Environ. 2015, 202, 223 231, doi:10.1016/j.agee.2015.01.009. 
24. Cardinale, B.J.; Duffy, J.E.; Gonzalez, A.; Hooper, D.U.; Perrings, C.; Venail, P.; Narwani, A.; Mace, G.M.; Tilman, D.; Wardle, D.A.; et al. Biodiversity loss and its impact on humanity. Nature 2012, 486, 59-67, doi:10.1038/nature11148.

25. Millennium Ecosystem Assessment Ecosystems and Human Well-being: Biodiversity Synthesis. World Resources Institute, Washington, DC. 2005, 100.

26. Lososová, Z.; Chytrý, M.; Cimalová, S.; Kropáč, Z.; Otýpková, Z.; Pyšek, P.; Tichý, L. Weed vegetation of arable land in Central Europe: Gradients of diversity and species composition. J. Veg. Sci. 2004, 15, 415-422, doi:10.1111/j.16541103.2004.tb02279.x.

27. Fried, G.; Norton, L.R.; Reboud, X. Environmental and management factors determining weed species composition and diversity in France. Agric. Ecosyst. Environ. 2008, 128, 68-76, doi:10.1016/j.agee.2008.05.003.

Pinke, G.; Karácsony, P.; Czúcz, B.; Botta-Dukát, Z.; Lengyel, A. The influence of environment, management and site context on species composition of summer arable weed vegetation in Hungary. Appl. Veg. Sci. 2012, 15, 136-144, doi:10.1111/j.1654-109X.2011.01158.x.

Seifert, C.; Leuschner, C.; Meyer, S.; Culmsee, H. Inter-relationships between crop type, management intensity and light transmissivity in annual crop systems and their effect on farmland plant diversity. Agric. Ecosyst. Environ. 2014, 195, 173-182, doi:10.1016/j.agee.2014.05.022.

30. Albrecht, H.; Cambecèdes, J.; Lang, M.; Wagner, M. Management options for the conservation of rare arable plants in Europe. Bot. Lett. 2016, 163, 389-415, doi:10.1080/23818107.2016.1237886.

31. von Arx, G.; Biosshard, A.; Dietz, H. Land-use intensity and border structures as determinants of vegetation diversity in an agricultural area. Bull. Geobot. Inst. ETH 2002, 68, 3-15.

32. Aavik, T.; Augenstein, I.; Bailey, D.; Herzog, F.; Zobel, M.; Liira, J. What is the role of local landscape structure in the vegetation composition of field boundaries? Appl. Veg. Sci. 2008, 11, 375-386, doi:10.3170/2008-7-18486.

33. Nagy, K.; Lengyel, A.; Kovács, A.; Türei, D.; Csergő, A.M.; Pinke, G. Weed species composition of small-scale farmlands bears a strong crop-related and environmental signature. Weed Res. 2018, 58, 46-56, doi:10.1111/wre.12281.

34. Batáry, P.; Gallé, R.; Riesch, F.; Fischer, C.; Dormann, C.F.; Mußhoff, O.; Császár, P.; Fusaro, S.; Gayer, C.; Happe, A.K.; et al. The former Iron Curtain still drives biodiversity-profit trade-offs in German agriculture. Nat. Ecol. Evol. 2017, 1, 1279-1284, doi:10.1038/s41559-017-0272-x.

35. Maréchal, P.-Y.; Henriet, F.; Vancutsem, F.; Bodson, B. Ecological review of black-grass (Alopecurus myosuroides Huds.) propagation abilities in relationship with herbicide resistance. Biotechnol Agron Soc Env. 2012, 11.

36. Egan, J.F.; Graham, I.M.; Mortensen, D.A. A comparison of the herbicide tolerances of rare and common plants in an agricultural landscape: Herbicide tolerances of rare and common plants. Environ. Toxicol. Chem. 2014, 33, 696-702, doi:10.1002/etc.2491.

37. Meyer, S.; Bergmeier, E.; Becker, T.; Wesche, K.; Krause, B.; Leuschner, C. Detecting long-term losses at the plant community level - arable fields in Germany revisited. Appl. Veg. Sci. 2015, 18, 432-442, doi:10.1111/avsc.12168.

38. Hofmeister, H.; Garve, E. Lebensraum Acker; Reprint der 2. neubearbeiteten Auflage.; Verlag Kessel: Remagen, 2006; ISBN 978-3-935638-61-6.

39. Hüppe, J.; Hofmeister, H. Syntaxonomische Fassung und Übersicht über die Ackerunkrautgesellschaften der Bundesrepublik Deutschland., - Ber. d. Reinh.-Tüxen-Ges. 2, S. 57-77, Hannover. 1990.

40. Kaussmann, B.; Kudocke, J. Die ökologisch-soziologischen Artengruppen der Ackerunkrautvegetation für den Norden der DDR. Feddes Repert. 1973, 84, 589-605, doi:10.1002/fedr.19730840707.

41. Statistical Office of the European Union (EUROSTAT) Main farmland use by NUTS 2 regions \& organic crop area by agricultural production methods and crops. Available online: https://ec.europa.eu/eurostat/web/agriculture/data/database (accessed on Oct 24, 2018).

Gaba, S.; Chauvel, B.; Dessaint, F.; Bretagnolle, V.; Petit, S. Weed species richness in winter wheat increases with landscape heterogeneity. Agric. Ecosyst. Environ. 2010, 138, 318-323, doi:10.1016/j.agee.2010.06.005.

43. Hanzlik, K.; Gerowitt, B. Methods to conduct and analyse weed surveys in arable farming: a review. Agron. Sustain. Dev. 2016, 36, doi:10.1007/s13593-015-0345-7.

44. Approaches and Objectives of Arable Weed Species Mapping: Where Next? In Weed research: expanding horizons; Hatcher, P., Froud-Williams, R.J., Eds.; John Wiley \& Sons Ltd: Hoboken, NJ, 2017; pp. 61-83 ISBN 978-1-11996914-3.

45. Gabriel, D.; Roschewitz, I.; Tscharntke, T.; Thies, C. Beta diversity at different spatial scales: plant communities in organic and conventional agriculture. Ecol. Appl. 2006, 16, 2011-2021, doi:10.1890/10510761(2006)016[2011:BDADSS]2.0.CO;2.

46. Krauss, J.; Gallenberger, I.; Steffan-Dewenter, I. Decreased Functional Diversity and Biological Pest Control in Conventional Compared to Organic Crop Fields. PLoS ONE 2011, 6, e19502, doi:10.1371/journal.pone.0019502.

47. von Redwitz, C.; Gerowitt, B. Maize-dominated crop sequences in northern Germany: Reaction of the weed species communities. Appl. Veg. Sci. 2018, 21, 431-441, doi:10.1111/avsc.12384. 
48. Seifert, C.; Leuschner, C.; Culmsee, H. Arable plant diversity on conventional cropland-The role of crop species, management and environment. Agric. Ecosyst. Environ. 2015, 213, 151-163, doi:10.1016/j.agee.2015.07.017.

49. von Drachenfels, O. Überarbeitung der Naturräumlichen Regionen Niedersachsens. Inform.d. Naturschutz Niedersachsen 30, Nr. 4 (4/10): 249-252. 2010.

50. German Meteorological Service (DWD) Climate data (2013-2017) via the CDC FTP server. Available online: ftp://ftpcdc.dwd.de/pub/CDC/observations_germany/climate/annual/kl/recent/ \& https://www.dwd.de/DE/leistungen/klimadatendeutschland/statliste/statlex_html.html?view=nasPublication\&nn=16 102 (accessed on Oct 25, 2018).

51. Federal Institute for Geosciences and Natural Resources Soil Map of the Federal Republic of Germany 1:1,000,000 (BÜK1000). Available online: https://www.bgr.bund.de/DE/Themen/Boden/Informationsgrundlagen/Bodenkundliche_Karten_Datenbanken/BU EK1000/buek1000_node.html (accessed on Oct 26, 2016).

52. State Authority for Mining, Energy and Geology (LBEG) German soil assessment map 1:5,000 (BS5). Available online: https://www.lbeg.niedersachsen.de/karten_daten_publikationen/karten_daten/boden/bodenkarten/bodenschaetzung skarte_15000/bodenschaetzungskarte-von-niedersachsen-im-mastab-1-5-000-bs5-681.html (accessed on Oct 26, 2018).

53. Londo, G. The decimal scale for releves of permanent quadrats. Vegetatio 1976, 33, 61-64, doi:10.1007/BF00055300.

54. Buttler, K.P. Florenliste von Deutschland - Gefäßpflanzen. Available online: http://www.kp- buttler.de (accessed on Feb 13, 2019).

55. Environmental Systems Research Institute (ESRI) ArcGIS Desktop 10.6.1. Available online: https://www.esri.com/enus/arcgis/products/arcgis-pro/overview (accessed on Oct 26, 2018).

56. Roßberg, D.; Gutsche, V.; Enzian, S.; Wick, M. NEPTUN 2000 - Erhebung von Daten zum tatsächlichen Einsatz chemischer Pflanzenschutzmittel im Ackerbau Deutschlands. Berichte aus der Biologischen Bundesanstalt für Landund Forstwirtschaft, Heft 98. SaphirVerlag, Ribbesbüttel 2002.

57. Sattler, C.; Kächele, H.; Verch, G. Assessing the intensity of pesticide use in agriculture. Agric. Ecosyst. Environ. 2007, 119, 299-304, doi:10.1016/j.agee.2006.07.017.

58. Land24 GmbH Crop protection manger. Available online: http://www.raiffeisen.com/php/pflanzenschutzmittel/ (accessed on Oct 26, 2018).

59. R Core Team R: A language and environment for statistical computing. R Foundation for Statistical Computing, Vienna, Austria. Available online: https://www.R-project.org/ (accessed on Nov 6, 2018).

60. Bache, S.M.; Wickham, H. magrittr: A Forward-Pipe Operator for R. R package version 1.5. Available online: https://CRAN.R-project.org/package=magrittr (accessed on Nov 6, 2018).

61. Wickham, H. tidyverse: Easily Install and Load the "Tidyverse". R package version 1.2.1. Available online: https://CRAN.R-project.org/package=tidyverse (accessed on Nov 6, 2018).

62. Goral, F.; Schellenberg, J. goeveg: Functions for Community Data and Ordinations. R package version 0.3.3. Available online: https:/ /CRAN.R-project.org/package=goeveg (accessed on Nov 6, 2018).

63. Oksanen, J.; Blanchet, F.G.; Friendly, M.; Kindt, R.; Legendre, P.; McGlinn, D.; Minchin, P.R.; O’Hara, R.B.; Simpson, G.L.; Solymos, P.; et al. vegan: Community Ecology Package. R package version 2.5-2. Available online: https://CRAN.R-project.org/package $=$ vegan (accessed on Nov 6, 2018).

64. Wickham, H. Reshaping Data with the reshape Package. Journal of Statistical Software, 21(12), 1-20. Available online: http://www.jstatsoft.org/v21/i12/ (accessed on Nov 7, 2018).

65. Leyer, I.; Wesche, K. Multivariate Statistik in der Ökologie: eine Einführung; Springer-Lehrbuch; Korrigierter Nachdruck.; Springer: Berlin Heidelberg, 2008; ISBN 978-3-540-37706-1.

66. Garve, E. Rote Liste und Florenliste der Farn- und Blütenpflanzen in Niedersachsen und Bremen, 5. Fassung vom 1.3.2004. Inform. d. Naturschutz Niedersachs. 24 (1) (1/04): 1-76, Hildesheim. 2004.

67. German Federal Agency for Nature Conservation High-nature-value farmland, Germany. Available online: https://www.bfn.de/themen/monitoring/monitoring-von-landwirtschaftsflaechen-mit-hohem-naturwert.html (accessed on Oct 19, 2018).

68. Preising, E.; Vahle, H.-C.; Brandes, D.; Hofmeister, H.; Tüxen, J.; Weber, H.E. Die Pflanzengesellschaften Niedersachsens - Bestandsentwicklung, Gefährdung und Schutzprobleme. Einjährige ruderale Pionier-, Tritt- und Ackerwildkraut- Gesellschaften. 1995, 20(6), 1-92.

69. Chen, H. VennDiagram: Generate High-Resolution Venn and Euler Plots. R package version 1.6.20. Available online: https://CRAN.R-project.org/package=VennDiagram (accessed on Nov 7, 2018).

70. Brooks, M.E.; Kristensen, K.; Benthem, K.J. van; Magnusson, A.; Berg, C.W.; Nielsen, A.; Skaug, H.J.; Maechler, M.; Bolker, B.M. glmmTMB Balances Speed and Flexibility Among Packages for Zero-inflated Generalized Linear Mixed Modeling. R J. 2017, 9, 378-400. 
71. Hothorn, T.; Bretz, F.; Westfall, P. Simultaneous Inference in General Parametric Models. Biom. J. 2008, 50, 346-363, doi:10.1002/bimj.200810425.

72. Chamorro, L.; Masalles, R.M.; Sans, F.X. Arable weed decline in Northeast Spain: Does organic farming recover functional biodiversity? Agric. Ecosyst. Environ. 2016, 223, 1-9, doi:10.1016/j.agee.2015.11.027.

73. Kolářová, M.; Tyšer, L.; Soukup, J. Diversity of current weed vegetation on arable land in selected areas of the Czech Republic \&nbsp; Plant Soil Environ. 2013, 59, 208-213, doi:10.17221/783/2012-PSE.

74. Kovács-Hostyánszki, A.; Batáry, P.; Báldi, A.; Harnos, A. Interaction of local and landscape features in the conservation of Hungarian arable weed diversity: Weed richness in Hungarian cereal fields. Appl. Veg. Sci. 2011, 14, 40-48, doi:10.1111/j.1654-109X.2010.01098.x.

75. Meyer, S.; Wesche, K.; Krause, B.; Brütting, C.; Hensen, I.; Leuschner, C. Diversitätsverluste und floristischer Wandel im Ackerland seit 1950. Nat. Landsch. 2014, 89, 10.

76. Meisel, K.; von Hübschmann, A. Veränderungen der Acker- und Grünlandvegetation im nordwestdeutschen Flachland in jüngerer Zeit. Schriftenreihe Veg. 1976, 10, 109-124.

77. Wietzke, A.; Leuschner, C. Surveying the arable plant diversity of conventionally managed farmland: a comparison of methods. Environ. Monit. Assess. 2020, 192, 98, doi:10.1007/s10661-019-8042-7.

78. Leuschner, C.; Krause, B.; Meyer, S.; Bartels, M. Structural change in the arable land and grassland of Lower Saxony and Schleswig-Holstein since 1950. Nat. Landsch. 2014, 89, 386-391.

79. Meisel, K. Über die Artenverbindung des Aphanion arvensis J. et R. Tx. 1960 im west-und nordwestdeutschen Flachland. Schriftenreihe Veg. 1967, 2, 123-133 + table.

80.

Haaland, C.; Naisbit, R.E.; Bersier, L.-F. Sown wildflower strips for insect conservation: a review: Wildflower strips for insect conservation. Insect Conserv. Divers. 2011, 4, 60-80, doi:10.1111/j.1752-4598.2010.00098.x.

81. Vickery, J.A.; Feber, R.E.; Fuller, R.J. Arable field margins managed for biodiversity conservation: A review of food resource provision for farmland birds. Agric. Ecosyst. Environ. 2009, 133, 1-13, doi:10.1016/j.agee.2009.05.012. 


\title{
Electronic supplementary material
}

\author{
Supplementary methods:
}

File S1: Supplementary methods

\section{Landscape composition}

Proportions of landscape elements, crops and environmental measures were calculated with respect to the study region (see Figure 1 in the main text and Figures S1 and S2). The landscape analysis was based on habitat mapping data [1] of the two districts Nienburg and Diepholz [2,3] and with regard to crop composition on data of the Land Parcel Identification System (LPIS) within the Integrated Administration and Control System (IACS) which is supplied by the Ministry of Food, Agriculture and Consumer Protection, Lower Saxony [4-6]. Habitat mapping of the district Diepholz was based on aerial photograph interpretation from 2014 (ministerial map $1: 5,000$ with respect to legally protected habitats, ministerial map $1: 10,000$ for remaining habitats) and for the district of Nienburg regarding aerial photograph interpretation from 2011 with re-mapping of $20 \%$ of the area in 2013 (ministerial map $1: 10,000$ ) [2,3]. Habitat types were categorized into the following groups: 'arable land', 'settlements and infrastructure' (sealed area and related semi-natural habitats such as cemeteries, gardens or roadside greenery), 'grass strips' (usually found between arable and non-arable habitats, e.g. along agricultural access tracks), 'forb and ruderal stands', 'forests', 'marshlands (incl. degeneration stages)', 'permanent grasslands', 'standing waters', 'streams' and 'woody elements of the open landscape' mainly include hedges and scrub $(85 \%)$ and a small percentage of single trees, tree rows and orchards $(15 \%)$; not clearly assignable habitats were categorized as "others" (including headwater areas, dwarf shrub heathlands, inland dunes, bare soil areas). Since grass strips (usually found between arable and non-arable habitats, e.g. along agricultural access tracks) were not included in aerial habitat mapping, we extrapolated striped areas by buffering borderlines of "arable fields - adjacent habitat combinations" where usually grass strips occur (e.g. adjacent agricultural access tracks, meadows, heathlands, ruderal sites or forb stands) with $0.75 \mathrm{~m}$ (scaled by own experiences in the study region). Thus, grass strip areas have to be considered as an estimate. Buffered grass strip areas were intersected with the original habitat mapping data to calculate habitat proportions of the above-mentioned habitat types.

\section{References}

1. von Drachenfels, O. Kartierschlüssel für Biotoptypen in Niedersachsen unter besonderer Berücksichtigung der gesetzlich geschützten Biotope sowie der Lebensraumtypen von Anhang I der FFH-Richtlinie, Stand Juli 2016. Naturschutz Landschaftspfl. Niedersachs. Heft A/4, 3262016.

2. Landkreis Diepholz Biotope mapping data based on aerial photograph interpretation from 2014 (ministerial map 1 : 5,000 regarding legally protected biotopes, ministerial map 1: 10,000 for remaining biotopes). Available online: https://www.diepholz.de/ (accessed on Mar 15, 2019).

3. Landkreis Nienburg/Weser Biotope mapping data based on aerial photograph interpretation from 2011 with remapping of $20 \%$ of the area in 2013 (ministerial map 1: 10,000). Available online: https://www.lk-nienburg.de/ (accessed on Mar 15, 2019).

4. European Union (EU) Commission Regulation (EC) No 1122/2009 of 30 November 2009 laying down detailed rules for the implementation of Council Regulation (EC) No 73/2009 as regards cross-compliance, modulation and the integrated administration and control system, under the direct support schemes for farmers provided for that Regulation, as well as for the implementation of Council Regulation (EC) No 1234/2007 as regards cross-compliance 
under the support scheme provided for the wine sector Available online: https://eur-lex.europa.eu/legalcontent/EN/TXT/PDF/?uri=CELEX:32009R1122\&from=de (accessed on Nov 7, 2019).

5. European Union (EU) Council Regulation (EC) No 73/2009 of 19 January 2009 establishing common rules for direct support schemes for farmers under the common agricultural policy and establishing certain support schemes for farmers, amending Regulations (EC) No 1290/2005, (EC) No 247/2006, (EC) No 378/2007 and repealing Regulation (EC) No 1782/2003 Available online: https://eur-lex.europa.eu/legalcontent/EN/TXT/PDF/?uri=CELEX:32009R0073\&from=de (accessed on Nov 7, 2018).

6. Niedersächsischen Ministerium für Ernährung, Landwirtschaft und Verbraucherschutz (ML) Land use data from the Land Parcel Identification System (LPIS) as part of the Integrated Administration and Control System (IACS). Available online: https://www.ml.niedersachsen.de/startseite/ (accessed on Mar 15, 2019).

\section{Supplementary tables}

Table S1: Categorization of surveyed plant species in the 200 observed arable fields (crops or woody seedlings excluded): all herbaceous species, arable plants (sensu stricto; Hofmeister and Garve 2006), indicator species for High Nature Value arable land (HNV species; German Federal Agency for Nature Conservation, 2018) and red-listed arable plant species in Lower Saxony (Garve 2004); sorted by species names; full references see main manuscript.

\begin{tabular}{|c|c|c|c|c|}
\hline Species name & $\begin{array}{c}\text { All herbaceous } \\
\text { species (x) }\end{array}$ & $\begin{array}{c}\text { Arable plants } \\
\text { (A) }\end{array}$ & HNV & $\begin{array}{c}\text { Red-listed arable } \\
\text { plants }(x)\end{array}$ \\
\hline Achillea millefolium & $\mathrm{x}$ & $\mathrm{A}$ & & \\
\hline Aegopodium podagraria & $\mathrm{x}$ & & & \\
\hline Agrostis capillaris & $\mathrm{x}$ & A & & \\
\hline Agrostis stolonifera & $\mathrm{x}$ & A & & \\
\hline Alliaria petiolata & $\mathrm{x}$ & & & \\
\hline Allium vineale & $\mathrm{x}$ & A & & \\
\hline Alopecurus myosuroides & $\mathrm{x}$ & A & & \\
\hline Alopecurus pratense & $\mathrm{x}$ & & & \\
\hline Anchusa arvensis & $\mathrm{x}$ & A & & \\
\hline Anthoxanthum aristatum & $\mathrm{x}$ & A & & \\
\hline Anthriscus sylvestris & $\mathrm{x}$ & & & \\
\hline Apera spica-venti & $\mathrm{x}$ & A & & \\
\hline Aphanes arvensis & $\mathrm{x}$ & A & $\mathrm{HNV}$ & \\
\hline Arabidopsis thaliana & $\mathrm{x}$ & A & & \\
\hline Arrbenatherum elatius & $\mathrm{x}$ & & & \\
\hline Artemisia vulgaris & $\mathrm{x}$ & A & & \\
\hline Avena fatua & $\mathrm{x}$ & A & & \\
\hline Bromus commutatus subsp. decipiens & $\mathrm{x}$ & A & & \\
\hline Bromus bordeaceus & $\mathrm{x}$ & A & & \\
\hline Bromus inermis & $\mathrm{x}$ & & & \\
\hline Bromus sterilis & $\mathrm{x}$ & A & & \\
\hline Calystegia sepium & $\mathrm{x}$ & & & \\
\hline Capsella bursa-pastoris & $\mathrm{x}$ & A & & \\
\hline Carduus crispus & $\mathrm{x}$ & & & \\
\hline Carex acuta & $\mathrm{x}$ & & & \\
\hline Carex acutiformis & $\mathrm{x}$ & & & \\
\hline Carex birta & $\mathrm{x}$ & A & & \\
\hline Centaurea cyanus & $\mathrm{x}$ & A & $\mathrm{HNV}$ & \\
\hline Cerastium arvense & $\mathrm{x}$ & & & \\
\hline Cerastium glomeratum & $\mathrm{x}$ & A & & \\
\hline Cerastium holosteoides & $\mathrm{x}$ & A & & \\
\hline Ceratocapnos claviculata & $\mathrm{x}$ & & & \\
\hline Chaerophyllum temulum & $\mathrm{x}$ & & & \\
\hline Chenopodium album & $\mathrm{x}$ & A & & \\
\hline Chenopodium polyspermum & $\mathrm{x}$ & A & & \\
\hline Cirsium arvense & $\mathrm{x}$ & A & & \\
\hline Cirsium vulgare & $\mathrm{x}$ & & & \\
\hline Convolvulus arvensis & $\mathrm{x}$ & A & & \\
\hline Crepis biennis & $\mathrm{x}$ & & & \\
\hline Dactylis glomerata & $\mathrm{x}$ & & & \\
\hline Daucus carota & $\mathrm{x}$ & & & \\
\hline Deschampsia cespitosa & $\mathrm{x}$ & & & \\
\hline Descurainia sophia & $\mathrm{x}$ & A & & \\
\hline Digitaria ischaemum & $\mathrm{x}$ & A & & \\
\hline Echinochloa crus-galli & $\mathrm{x}$ & A & & \\
\hline Elymus repens & $\mathrm{x}$ & A & & \\
\hline
\end{tabular}




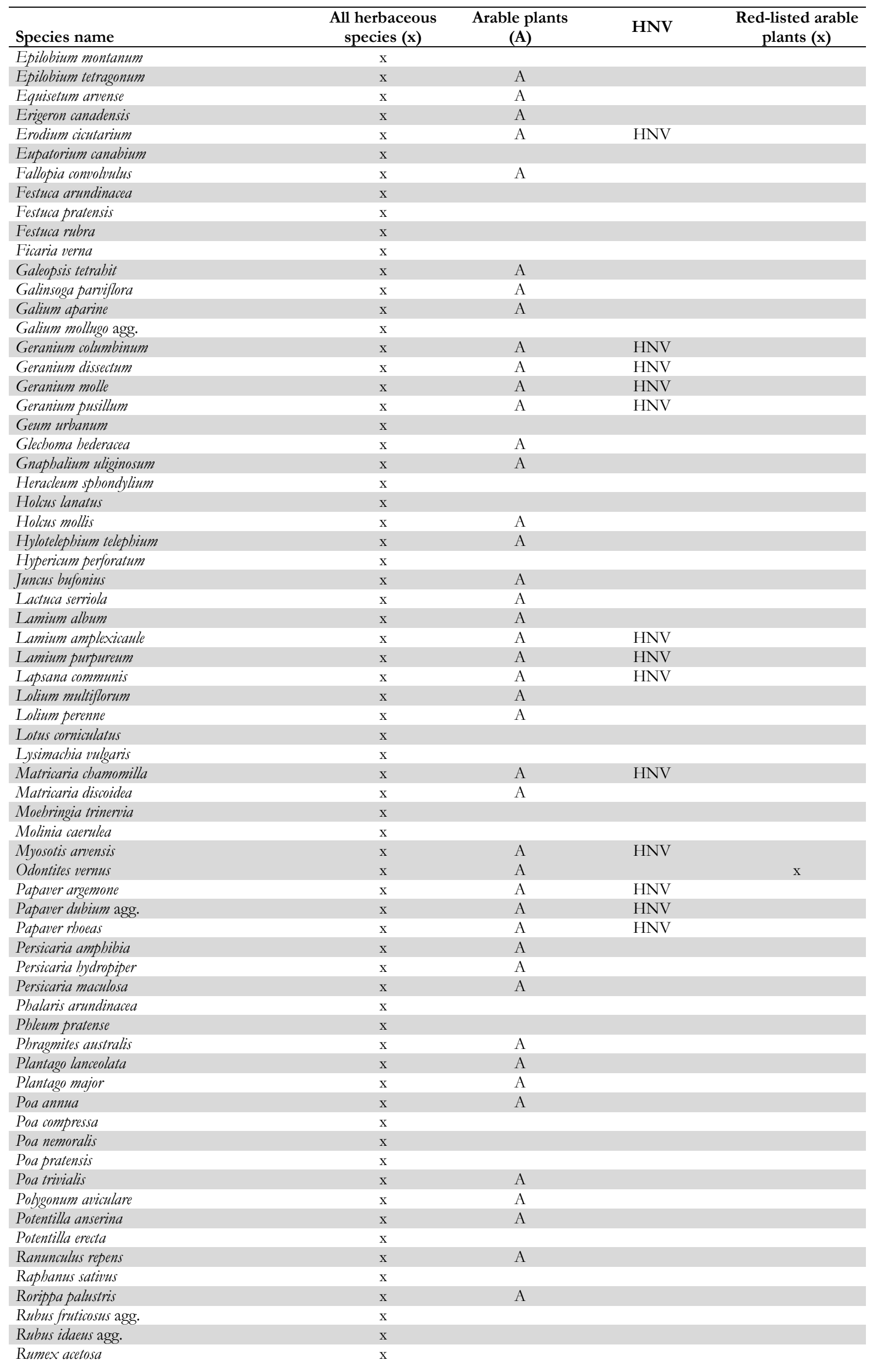




\begin{tabular}{|c|c|c|c|c|}
\hline Species name & $\begin{array}{l}\text { All herbaceous } \\
\text { species (x) }\end{array}$ & $\begin{array}{c}\text { Arable plants } \\
\text { (A) }\end{array}$ & HNV & $\begin{array}{c}\text { Red-listed arable } \\
\text { plants (x) }\end{array}$ \\
\hline Rumex acetosella & $\mathrm{x}$ & $\mathrm{A}$ & $\mathrm{HNV}$ & \\
\hline Rumex crispus & $\mathrm{x}$ & A & & \\
\hline Rumex bydrolapathum & $\mathrm{x}$ & & & \\
\hline Rumex obtusifolius & $\mathrm{x}$ & A & & \\
\hline Scleranthus annuus & $\mathrm{x}$ & A & & \\
\hline Senecio inaequidens & $\mathrm{x}$ & & & \\
\hline Senecio jacobaea & $\mathrm{x}$ & & & \\
\hline Senecio vulgaris & $\mathrm{x}$ & A & & \\
\hline Setaria viridis & $\mathrm{x}$ & A & & \\
\hline Silene latifolia & $\mathrm{x}$ & A & & \\
\hline Sisymbrium officinale & $\mathrm{x}$ & A & & \\
\hline Solanum nigrum & $\mathrm{x}$ & A & & \\
\hline Sonchus asper & $\mathrm{x}$ & A & & \\
\hline Sonchus oleraceus & $\mathrm{x}$ & A & & \\
\hline Stellaria graminea & $\mathrm{x}$ & A & & \\
\hline Stellaria media & $\mathrm{x}$ & A & & \\
\hline Tanacetum vulgare & $\mathrm{x}$ & & & \\
\hline Taraxacum sect. Ruderalia & $\mathrm{x}$ & A & & \\
\hline Thlaspi arvense & $\mathrm{x}$ & A & $\mathrm{HNV}$ & \\
\hline Trifolium dubium & $\mathrm{x}$ & & & \\
\hline Trifolium pratense & $\mathrm{x}$ & & & \\
\hline Trifolium repens & $\mathrm{x}$ & & & \\
\hline Tripleurospermum perforatum & $\mathrm{x}$ & A & & \\
\hline Urtica dioica & $\mathrm{x}$ & & & \\
\hline Urtica urens & $\mathrm{x}$ & A & & \\
\hline Veronica arvensis & $\mathrm{x}$ & A & & \\
\hline Veronica hederifolia & $\mathrm{x}$ & A & & \\
\hline Veronica persica & $\mathrm{x}$ & A & & \\
\hline Vicia angustifolia & $\mathrm{x}$ & A & & \\
\hline Vicia cracca & $\mathrm{x}$ & A & HNV & \\
\hline Vicia birsuta & $\mathrm{x}$ & A & $\mathrm{HNV}$ & \\
\hline Vicia sativa & $\mathrm{x}$ & & $\mathrm{HNV}$ & \\
\hline Vicia sepium & $\mathrm{x}$ & & $\mathrm{HNV}$ & \\
\hline Vicia tetrasperma & $\mathrm{x}$ & A & $\mathrm{HNV}$ & \\
\hline Viola arvensis & $\mathrm{x}$ & A & & \\
\hline Vulpia myuros & $\mathrm{x}$ & & & \\
\hline
\end{tabular}


Table S2: Total herbaceous species numbers per crop type in field edge and field interior plots and edge and interior pooled; only herbaceous non-crop species (woody seedlings excluded); infrequent species numbers (occurred in less than 3 plots, all crops pooled) are given in brackets; $n=30$ for edge plots of barley, maize, rapeseed, rye and wheat, potato $=28$, triticale $=22$; interior plots $=10$ per crop type.

\begin{tabular}{lccc}
\hline Crop type & Edge plots & Interior plots & Edge \& interior plots \\
\hline Barley & 79 & 17 & 80 \\
Maize & 56 & 15 & 58 \\
Potato & 67 & 14 & 67 \\
Rapeseed & 103 & 12 & 104 \\
Rye & 86 & 14 & 86 \\
Triticale & 66 & 14 & 69 \\
Wheat & 68 & 12 & 69 \\
\hline All crops (infrequent species) & $\mathbf{1 5 0}(\mathbf{5 9})$ & $\mathbf{4 1 ( 2 5 )}$ & $\mathbf{1 5 0}(\mathbf{5 6})$ \\
\hline
\end{tabular}

Table S3: Species occurrences in field edge and field interior plots (and edge and interior pooled) for observed crop types; sorted by species names; $\mathrm{n}=30$ for edge plots of barley, maize, rapeseed, rye and wheat, potato $=28$, triticale $=22$; interior plots $=10$ per crop.

\begin{tabular}{|c|c|c|c|c|c|c|c|c|c|c|c|c|c|c|c|c|c|}
\hline Species & 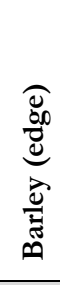 & 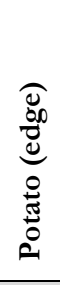 & 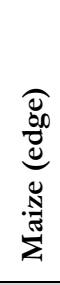 & 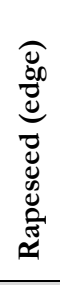 & 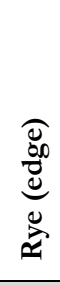 & 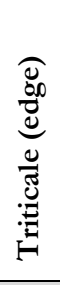 & 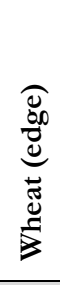 & 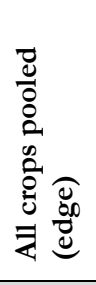 & 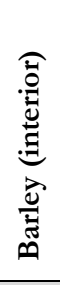 & 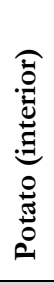 & 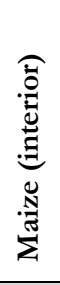 & 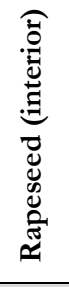 & 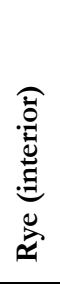 & 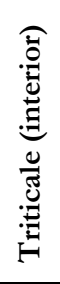 & 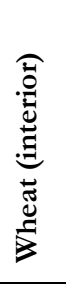 & 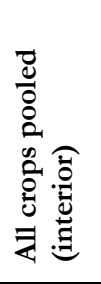 & 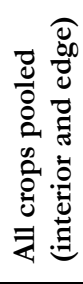 \\
\hline Achillea millefolium & 2 & 0 & 0 & 4 & 10 & 1 & 3 & 20 & 0 & 0 & 0 & 0 & 0 & 0 & 0 & 0 & 20 \\
\hline Aegopodium podagraria & 3 & 0 & 0 & 2 & 3 & 1 & 2 & 11 & 0 & 0 & 0 & 0 & 0 & 0 & 0 & 0 & 11 \\
\hline Agrostis capillaris & 1 & 4 & 0 & 0 & 1 & 3 & 3 & 12 & 0 & 0 & 0 & 0 & 0 & 0 & 0 & 0 & 12 \\
\hline Agrostis stolonifera & 0 & 7 & 1 & 1 & 5 & 0 & 0 & 14 & 0 & 0 & 0 & 0 & 0 & 0 & 1 & 1 & 15 \\
\hline Alliaria petiolata & 0 & 0 & 0 & 2 & 0 & 0 & 2 & 4 & 0 & 0 & 0 & 0 & 0 & 0 & 0 & 0 & 4 \\
\hline Allium vineale & 0 & 0 & 0 & 0 & 1 & 0 & 0 & 1 & 0 & 0 & 0 & 0 & 0 & 0 & 0 & 0 & 1 \\
\hline Alopecurus myosuroides & 4 & 2 & 1 & 6 & 2 & 0 & 11 & 26 & 1 & 0 & 0 & 0 & 0 & 0 & 0 & 1 & 27 \\
\hline Alopecurus pratensis & 7 & 1 & 1 & 10 & 3 & 2 & 3 & 27 & 0 & 0 & 0 & 0 & 0 & 0 & 0 & 0 & 27 \\
\hline Anchusa arvensis & 0 & 0 & 0 & 2 & 0 & 1 & 1 & 4 & 0 & 0 & 0 & 0 & 0 & 0 & 0 & 0 & 4 \\
\hline Anthoxanthum aristatum & 0 & 0 & 0 & 1 & 0 & 0 & 0 & 1 & 0 & 0 & 0 & 0 & 0 & 0 & 0 & 0 & 1 \\
\hline Anthriscus sylvestris & 3 & 4 & 0 & 7 & 6 & 2 & 5 & 27 & 0 & 0 & 0 & 0 & 0 & 0 & 0 & 0 & 27 \\
\hline Apera spica-venti & 8 & 5 & 0 & 0 & 6 & 7 & 8 & 34 & 0 & 0 & 0 & 0 & 0 & 0 & 2 & 2 & 36 \\
\hline Aphanes arvensis & 0 & 0 & 0 & 1 & 2 & 1 & 1 & 5 & 0 & 0 & 0 & 0 & 0 & 0 & 0 & 0 & 5 \\
\hline Arabidopsis thaliana & 0 & 1 & 0 & 2 & 2 & 2 & 0 & 7 & 0 & 0 & 0 & 1 & 0 & 0 & 0 & 1 & 8 \\
\hline Arrhenatherum elatius & 6 & 2 & 3 & 11 & 5 & 4 & 6 & 37 & 0 & 0 & 0 & 0 & 0 & 0 & 0 & 0 & 37 \\
\hline Artemisia vulgaris & 2 & 1 & 1 & 4 & 4 & 0 & 1 & 13 & 0 & 0 & 0 & 0 & 0 & 0 & 0 & 0 & 13 \\
\hline Avena fatua & 0 & 8 & 3 & 0 & 0 & 1 & 1 & 13 & 0 & 0 & 0 & 0 & 0 & 0 & 0 & 0 & 13 \\
\hline Bromus commutatus subsp. decipiens & 2 & 0 & 0 & 8 & 5 & 7 & 10 & 32 & 0 & 0 & 0 & 0 & 0 & 1 & 0 & 1 & 33 \\
\hline Bromus hordeaceus & 11 & 10 & 2 & 5 & 9 & 7 & 4 & 48 & 0 & 0 & 0 & 0 & 0 & 0 & 0 & 0 & 48 \\
\hline Bromus inermis & 1 & 0 & 0 & 0 & 0 & 0 & 1 & 2 & 0 & 0 & 0 & 0 & 0 & 0 & 0 & 0 & 2 \\
\hline Bromus sterilis & 16 & 7 & 2 & 15 & 16 & 11 & 20 & 87 & 0 & 0 & 0 & 0 & 0 & 0 & 0 & 0 & 87 \\
\hline Calystegia sepium & 0 & 0 & 2 & 0 & 1 & 0 & 0 & 3 & 1 & 0 & 1 & 1 & 0 & 0 & 0 & 3 & 6 \\
\hline Capsella bursa-pastoris & 4 & 4 & 0 & 17 & 2 & 2 & 2 & 31 & 0 & 1 & 0 & 3 & 0 & 0 & 1 & 5 & 36 \\
\hline Carduus crispus & 0 & 1 & 0 & 3 & 0 & 0 & 1 & 5 & 0 & 0 & 0 & 0 & 0 & 0 & 0 & 0 & 5 \\
\hline Carex acuta & 0 & 0 & 1 & 0 & 0 & 0 & 0 & 1 & 0 & 0 & 0 & 0 & 0 & 0 & 0 & 0 & 1 \\
\hline Carex acutiformis & 0 & 0 & 0 & 0 & 0 & 1 & 0 & 1 & 0 & 0 & 0 & 0 & 0 & 0 & 0 & 0 & 1 \\
\hline Carex birta & 0 & 0 & 0 & 0 & 1 & 0 & 0 & 1 & 0 & 0 & 0 & 0 & 0 & 0 & 0 & 0 & 1 \\
\hline Centaurea cyanus & 3 & 5 & 0 & 5 & 9 & 3 & 3 & 28 & 1 & 0 & 0 & 0 & 2 & 0 & 0 & 3 & 31 \\
\hline Cerastium arvense & 0 & 0 & 0 & 0 & 1 & 0 & 0 & 1 & 0 & 0 & 0 & 0 & 0 & 0 & 0 & 0 & 1 \\
\hline Cerastium glomeratum & 0 & 0 & 0 & 2 & 0 & 0 & 0 & 2 & 0 & 0 & 0 & 0 & 0 & 0 & 0 & 0 & 2 \\
\hline Cerastium holosteoides & 2 & 1 & 0 & 3 & 1 & 1 & 0 & 8 & 0 & 0 & 0 & 0 & 0 & 0 & 0 & 0 & 8 \\
\hline Ceratocapnos claviculata & 0 & 0 & 0 & 0 & 0 & 1 & 0 & 1 & 0 & 0 & 0 & 0 & 0 & 0 & 0 & 0 & 1 \\
\hline Chaerophyllum temulum & 2 & 0 & 0 & 1 & 1 & 0 & 1 & 5 & 0 & 0 & 0 & 0 & 0 & 0 & 0 & 0 & 5 \\
\hline Chenopodium album & 6 & 15 & 13 & 8 & 3 & 2 & 4 & 51 & 0 & 2 & 4 & 0 & 2 & 0 & 0 & 8 & 59 \\
\hline Chenopodium polyspermum & 0 & 0 & 2 & 1 & 0 & 0 & 0 & 3 & 0 & 0 & 0 & 0 & 0 & 0 & 0 & 0 & 3 \\
\hline Cirsium arvense & 8 & 8 & 2 & 10 & 3 & 4 & 7 & 42 & 0 & 0 & 0 & 0 & 1 & 0 & 0 & 1 & 43 \\
\hline Cirsium vulgare & 0 & 0 & 1 & 0 & 1 & 0 & 0 & 2 & 0 & 0 & 0 & 0 & 0 & 0 & 0 & 0 & 2 \\
\hline Convolvulus arvensis & 1 & 1 & 0 & 0 & 0 & 1 & 0 & 3 & 0 & 0 & 0 & 0 & 0 & 0 & 0 & 0 & 3 \\
\hline Erigeron canadensis & 0 & 1 & 0 & 1 & 1 & 0 & 0 & 3 & 0 & 0 & 0 & 0 & 0 & 0 & 0 & 0 & 3 \\
\hline Crepis biennis & 0 & 0 & 0 & 2 & 0 & 0 & 0 & 2 & 0 & 0 & 0 & 0 & 0 & 0 & 0 & 0 & 2 \\
\hline
\end{tabular}


Species

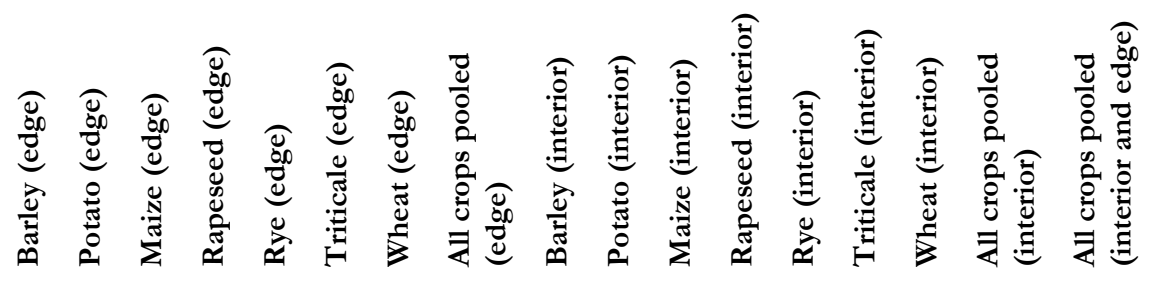

Dactylis glomerata

Daucus carota

$\begin{array}{lllllllll}13 & 16 & 11 & 19 & 15 & 13 & 16 & 103\end{array}$

Deschampsia cespitosa

Descurainia sophia

Digitaria ischaemum

$\begin{array}{llllllllllllllllll}\text { Echinocbloa crus-galli } & 0 & 9 & 12 & 0 & 1 & 0 & 1 & 23 & 0 & 2 & 6 & 0 & 0 & 1 & 0 & 9 & 32\end{array}$

$\begin{array}{llllllllllllllllll}\text { Elymus repens } & 23 & 26 & 22 & 20 & 15 & 13 & 10 & 129 & 3 & 1 & 3 & 0 & 1 & 2 & 1 & 11 & 140\end{array}$

Epilobium montanum

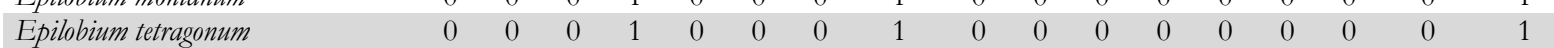

$\begin{array}{llllllllllllllllll}\text { Equisetum arvense } & 2 & 3 & 6 & 4 & 1 & 4 & 4 & 24 & 0 & 0 & 0 & 0 & 0 & 0 & 0 & 0 & 24\end{array}$

$\begin{array}{llllllllllllllllll}\text { Erodium cicutarium } & 1 & 1 & 2 & 1 & 2 & 2 & 0 & 9 & 0 & 0 & 0 & 0 & 0 & 0 & 0 & 0 & 9\end{array}$

Eupatorium cannabinum

$\begin{array}{lllllllllllllllllll}\text { Fallopia convolvulus } & 8 & 14 & 16 & 11 & 19 & 9 & 3 & 80 & 1 & 1 & 3 & 0 & 6 & 2 & 2 & 15 & 95\end{array}$

$\begin{array}{llllllllllllllllll}\text { Festuca arundinacea } & 1 & 0 & 0 & 2 & 0 & 0 & 1 & 4 & 0 & 0 & 0 & 0 & 0 & 0 & 0 & 0 & 4\end{array}$

$\begin{array}{llllllllllllllllll}\text { Festuca pratensis } & 5 & 0 & 0 & 4 & 2 & 2 & 0 & 13 & 0 & 0 & 0 & 0 & 0 & 0 & 0 & 0 & 13\end{array}$

$\begin{array}{llllllllllllllllll}\text { Festuca rubra } & 2 & 0 & 2 & 3 & 12 & 4 & 2 & 25 & 0 & 0 & 0 & 0 & 0 & 0 & 0 & 0 & 25\end{array}$

$\begin{array}{llllllllllllllllll}\text { Galeopsis tetrabit } & 2 & 0 & 2 & 6 & 4 & 2 & 2 & 18 & 1 & 0 & 0 & 0 & 0 & 0 & 0 & 1 & 19\end{array}$

Galinsoga parviflora

$\begin{array}{lllllllllllllllllll}\text { Galium aparine } & 20 & 11 & 7 & 18 & 19 & 15 & 19 & 109 & 2 & 1 & 0 & 1 & 2 & 4 & 1 & 11 & 120\end{array}$

Galium mollugo agg.

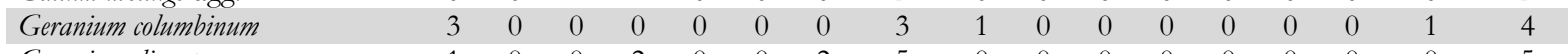

Geranium dissectum

$\begin{array}{llllllllllllllllll}\text { Geranium molle } & 0 & 1 & 0 & 0 & 0 & 0 & 0 & 1 & 0 & 0 & 0 & 0 & 0 & 0 & 0 & 0 & 1\end{array}$

Geranium pusillum

$\begin{array}{lllllllllllllllllll}\text { Geum urbanum } & 1 & 0 & 0 & 0 & 0 & 0 & 0 & 1 & 0 & 0 & 0 & 0 & 0 & 0 & 0 & 0 & 1\end{array}$

Glechoma hederacea

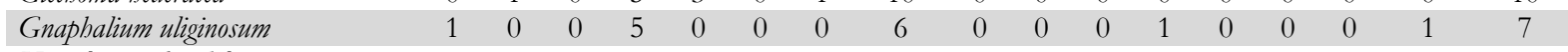

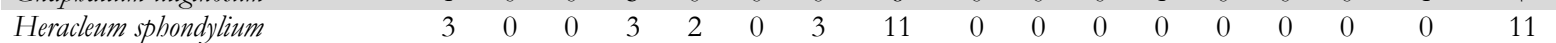

$\begin{array}{llllllllllllllllll}\text { Holcus lanatus } & 7 & 4 & 6 & 10 & 10 & 8 & 6 & 51 & 0 & 0 & 0 & 0 & 0 & 0 & 0 & 0 & 51\end{array}$

$\begin{array}{llllllllllllllllll}\text { Holcus mollis } & 13 & 7 & 0 & 11 & 12 & 11 & 5 & 59 & 2 & 0 & 0 & 0 & 0 & 0 & 0 & 2 & 61\end{array}$

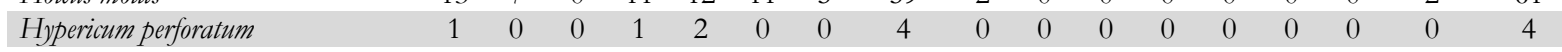

$\begin{array}{llllllllllllllllll}\text { Juncus bufonius } & 1 & 1 & 0 & 3 & 0 & 1 & 1 & 7 & 0 & 0 & 0 & 2 & 0 & 0 & 0 & 2 & 9 \\ \text { Lactuca serriola } & 0 & 1 & 0 & 1 & 0 & 0 & 0 & 2 & 0 & 0 & 0 & 0 & 0 & 0 & 0 & 0 & 2\end{array}$

$\begin{array}{llllllllllllllllll}\text { Lamium album } & 2 & 0 & 0 & 4 & 2 & 0 & 0 & 8 & 0 & 0 & 0 & 0 & 0 & 0 & 0 & 0 & 8\end{array}$

Lamium amplexicaule $\quad \begin{array}{llllllllllllllllll} & 0 & 0 & 0 & 1 & 1 & 0 & 0 & 2 & 0 & 0 & 0 & 0 & 0 & 0 & 0 & 0 & 2\end{array}$

$\begin{array}{llllllllllllllllll}\text { Lamium purpureum } & 4 & 1 & 0 & 4 & 2 & 1 & 2 & 14 & 0 & 0 & 0 & 0 & 0 & 0 & 0 & 0 & 14\end{array}$

$\begin{array}{llllllllllllllllll}\text { Lapsana communis } & 2 & 2 & 1 & 2 & 3 & 1 & 3 & 14 & 0 & 0 & 0 & 0 & 0 & 0 & 0 & 0 & 14\end{array}$

$\begin{array}{llllllllllllllllll}\text { Lolium multiflorum } & 2 & 2 & 2 & 2 & 4 & 2 & 4 & 18 & 0 & 0 & 0 & 0 & 0 & 0 & 0 & 0 & 18\end{array}$

$\begin{array}{llllllllllllllllll}\text { Lolium perenne } & 8 & 4 & 12 & 7 & 7 & 2 & 4 & 44 & 0 & 0 & 0 & 0 & 1 & 0 & 0 & 1 & 45\end{array}$

$\begin{array}{llllllllllllllllll}\text { Lotus corniculatus } & 0 & 0 & 1 & 0 & 0 & 0 & 0 & 1 & 0 & 0 & 0 & 0 & 0 & 0 & 0 & 0 & 1 \\ \text { L } & 0 & 1 & 0 & 0 & 1 & 1 & 0 & 3 & 0 & 0 & 0 & 0 & 0 & 0 & 0 & 0\end{array}$

$\begin{array}{lcccccccccccccccccc}\text { Lysimachia vulgaris } & 0 & 1 & 0 & 0 & 1 & 1 & 0 & 3 & 0 & 0 & 0 & 0 & 0 & 0 & 0 & 0 & 3\end{array}$

$\begin{array}{llllllllllllllllll}\text { Matricaria chamomilla } & 7 & 13 & 7 & 25 & 12 & 8 & 11 & 83 & 2 & 1 & 2 & 6 & 2 & 0 & 3 & 16 & 99\end{array}$

$\begin{array}{llllllllllllllllll}\text { Matricaria discoidea } & 0 & 1 & 1 & 0 & 0 & 0 & 0 & 2 & 0 & 0 & 0 & 0 & 0 & 0 & 0 & 0 & 2\end{array}$

$\begin{array}{llllllllllllllllll}\text { Moebringia trinervia } & 0 & 0 & 0 & 0 & 1 & 0 & 0 & 1 & 0 & 0 & 0 & 0 & 0 & 0 & 0 & 0 & 1\end{array}$

$\begin{array}{lllllllllllllllllc}\text { Molinia caerulea } & 0 & 0 & 0 & 0 & 0 & 1 & 0 & 1 & 0 & 0 & 0 & 0 & 0 & 0 & 0 & 0 & 1 \\ \text { Myosotis arvensis } & 3 & 8 & 3 & 15 & 9 & 3 & 4 & 45 & 1 & 0 & 0 & 2 & 1 & 2 & 1 & 7 & 52\end{array}$

$\begin{array}{llllllllllllllllll}\text { Odontites vernus } & 0 & 0 & 0 & 0 & 1 & 0 & 0 & 1 & 0 & 0 & 0 & 0 & 0 & 0 & 0 & 0 & 1 \\ \text { Papaver argemone } & 0 & 0 & 0 & 1 & 1 & 0 & 0 & 2 & 0 & 0 & 0 & 0 & 0 & 0 & 0 & 0 & 2\end{array}$

$\begin{array}{llllllllllllllllll}\text { Papaver argemone } & 0 & 0 & 0 & 1 & 1 & 0 & 0 & 2 & 0 & 0 & 0 & 0 & 0 & 0 & 0 & 0 & 2 \\ \text { Papaver dubium agg. } & 1 & 0 & 0 & 2 & 2 & 1 & 0 & 6 & 0 & 0 & 0 & 0 & 0 & 0 & 0 & 0 & 6\end{array}$

$\begin{array}{llllllllllllllllll}\text { Papaver rhoeas } & 0 & 0 & 1 & 2 & 2 & 0 & 0 & 5 & 0 & 0 & 0 & 0 & 0 & 0 & 0 & 0 & 5\end{array}$

$\begin{array}{llllllllllllllllll}\text { Persicaria amphibia } & 1 & 0 & 0 & 1 & 0 & 0 & 0 & 2 & 0 & 0 & 0 & 0 & 0 & 1 & 0 & 1 & 3\end{array}$

$\begin{array}{lllllllllllllllllll}\text { Persicaria maculosa } & 1 & 3 & 4 & 2 & 0 & 0 & 1 & 11 & 0 & 0 & 0 & 0 & 0 & 0 & 0 & 0 & 11\end{array}$

$\begin{array}{lllllllllllllllllll}\text { Phalaris arundinacea } & 0 & 0 & 0 & 1 & 0 & 0 & 0 & 1 & 0 & 0 & 0 & 0 & 0 & 0 & 0 & 0 & 1\end{array}$

$\begin{array}{llllllllllllllllll}\text { Phleum pratense } & 0 & 0 & 1 & 0 & 1 & 1 & 0 & 3 & 0 & 0 & 0 & 0 & 0 & 0 & 0 & 0 & 3 \\ \text { Phragmites australis } & 0 & 0 & 1 & 0 & 0 & 0 & 0 & 1 & 0 & 0 & 0 & 0 & 0 & 0 & 0 & 0 & 1\end{array}$

$\begin{array}{lllllllllllllllllll}\text { Plantago lanceolata } & 1 & 1 & 0 & 2 & 0 & 0 & 2 & 6 & 0 & 0 & 0 & 0 & 0 & 0 & 0 & 0 & 6\end{array}$

$\begin{array}{llllllllllllllllll}\text { Plantago major } & 1 & 1 & 0 & 1 & 0 & 1 & 0 & 4 & 0 & 0 & 0 & 0 & 0 & 0 & 0 & 0 & 4\end{array}$

$\begin{array}{lllllllllllllllllll}\text { Poa аппиа } & 7 & 6 & 1 & 3 & 4 & 1 & 5 & 27 & 0 & 1 & 1 & 1 & 1 & 1 & 1 & 6 & 33\end{array}$

$\begin{array}{llllllllllllllllll}\text { Poa compressa } & 0 & 0 & 0 & 0 & 1 & 0 & 0 & 1 & 0 & 0 & 0 & 0 & 0 & 0 & 0 & 0 & 1 \\ \text { Poa nemoralis } & 0 & 0 & 0 & 0 & 0 & 0 & 1 & 1 & 0 & 0 & 0 & 0 & 0 & 0 & 0 & 0 & 1\end{array}$

Poa pratensis

$\begin{array}{lllllllllllllllll}10 & 1 & 0 & 6 & 4 & 7 & 1 & 29 & 0 & 0 & 0 & 0 & 0 & 0 & 0 & 0 & 29\end{array}$ 
Species

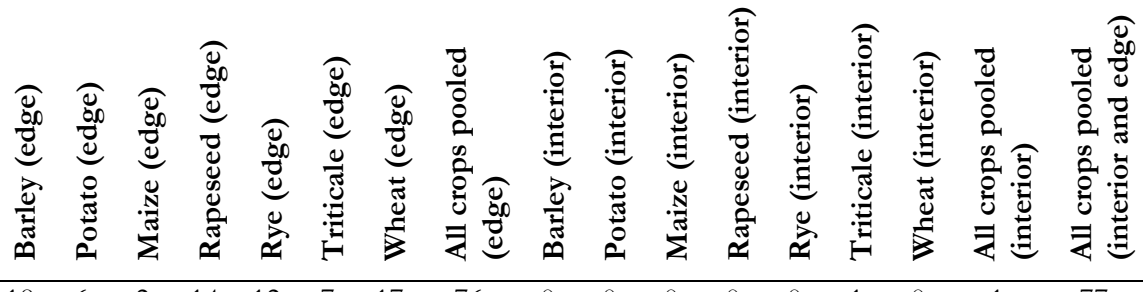

Poa trivialis

$\begin{array}{ccccccccccccccccc}18 & 6 & 2 & 14 & 12 & 7 & 17 & 76 & 0 & 0 & 0 & 0 & 0 & 1 & 0 & 1 & 77\end{array}$

$\begin{array}{llllllllllllllllll}\text { Polygonum aviculare } & 8 & 13 & 14 & 17 & 12 & 9 & 8 & 81 & 0 & 1 & 0 & 2 & 2 & 2 & 3 & 10 & 91\end{array}$

$\begin{array}{llllllllllllllllll}\text { Persicaria bydropiper } & 0 & 0 & 0 & 1 & 1 & 0 & 0 & 2 & 0 & 0 & 0 & 0 & 0 & 1 & 0 & 1 & 3\end{array}$

$\begin{array}{lllllllllllllllllll}\text { Potentilla anserina } & 0 & 1 & 0 & 0 & 0 & 0 & 0 & 1 & 0 & 0 & 0 & 0 & 0 & 0 & 0 & 0 & 1 \\ \text { Potentilla erecta } & 0 & 0 & 1 & 0 & 0 & 0 & 0 & 1 & 0 & 0 & 0 & 0 & 0 & 0 & 0 & 0 & 1 \\ \text { Pitaia }\end{array}$

$\begin{array}{llllllllllllllllll}\text { Ficaria verna } & 1 & 0 & 0 & 0 & 0 & 0 & 0 & 1 & 0 & 0 & 0 & 0 & 0 & 0 & 0 & 0 & 1 \\ \text { Ranunculus repens } & 1 & 0 & 0 & 2 & 0 & 0 & 2 & 5 & 0 & 0 & 0 & 0 & 0 & 0 & 0 & 0 & 5\end{array}$

$\begin{array}{llllllllllllllllll}\text { Raphanus sativus } & 0 & 0 & 0 & 1 & 0 & 0 & 0 & 1 & 0 & 0 & 0 & 1 & 0 & 0 & 0 & 1 & 2\end{array}$

Rorippa palustris

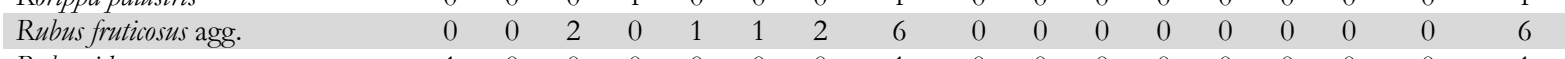

$\begin{array}{lllllllllllllllllll}\text { Rubus idaeus agg. } & 1 & 0 & 0 & 0 & 0 & 0 & 0 & 1 & 0 & 0 & 0 & 0 & 0 & 0 & 0 & 0 & 1\end{array}$

$\begin{array}{lllllllllllllllllll}\text { Rumex acetosa } & 3 & 0 & 1 & 3 & 2 & 0 & 2 & 11 & 0 & 0 & 0 & 0 & 0 & 0 & 0 & 0 & 11\end{array}$

$\begin{array}{lllllllllllllllllll}\text { Rumex acetosella } & 0 & 0 & 0 & 0 & 2 & 0 & 0 & 2 & 0 & 0 & 1 & 0 & 0 & 0 & 0 & 1 & 3\end{array}$

$\begin{array}{llllllllllllllllll}\text { Rumex crispus } & 0 & 0 & 0 & 0 & 2 & 3 & 3 & 8 & 0 & 0 & 0 & 0 & 0 & 0 & 0 & 0 & 8\end{array}$

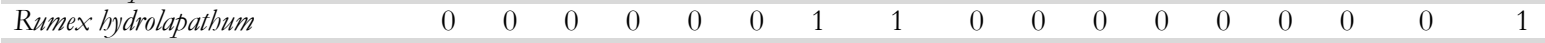

$\begin{array}{llllllllllllllllll}\text { Rumex obtusifolius } & 5 & 6 & 8 & 8 & 1 & 1 & 4 & 33 & 0 & 0 & 1 & 0 & 0 & 0 & 1 & 2 & 35\end{array}$

$\begin{array}{llllllllllllllllll}\text { Scleranthus annuus } & 0 & 0 & 0 & 1 & 1 & 0 & 0 & 2 & 0 & 0 & 0 & 0 & 0 & 0 & 0 & 0 & 2\end{array}$

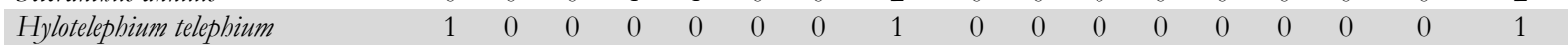

$\begin{array}{llllllllllllllllll}\text { Senecio inaequidens } & 0 & 0 & 0 & 1 & 0 & 0 & 0 & 1 & 0 & 0 & 0 & 0 & 0 & 0 & 0 & 0 & 1 \\ \text { Senecio jacobaea } & 0 & 0 & 0 & 1 & 0 & 0 & 0 & 1 & 0 & 0 & 0 & 0 & 0 & 0 & 0 & 0 & 1\end{array}$

$\begin{array}{llllllllllllllllll}\text { Senecio vulgaris } & 3 & 1 & 1 & 1 & 0 & 0 & 1 & 7 & 1 & 1 & 0 & 0 & 0 & 0 & 0 & 2 & 9\end{array}$

$\begin{array}{llllllllllllllllll}\text { Setaria viridis } & 0 & 0 & 1 & 0 & 0 & 0 & 0 & 1 & 0 & 0 & 0 & 0 & 0 & 0 & 0 & 0 & 1\end{array}$

$\begin{array}{llllllllllllllllll}\text { Silene latifolia } & 0 & 0 & 0 & 0 & 1 & 1 & 0 & 2 & 0 & 0 & 0 & 0 & 0 & 0 & 0 & 0 & 2\end{array}$

$\begin{array}{llllllllllllllllll}\text { Sisymbrium officinale } & 2 & 5 & 3 & 13 & 1 & 1 & 3 & 28 & 1 & 0 & 0 & 2 & 0 & 0 & 0 & 3 & 31\end{array}$

$\begin{array}{llllllllllllllllll}\text { Solanum nigrum } & 0 & 1 & 2 & 0 & 0 & 0 & 0 & 3 & 0 & 1 & 1 & 0 & 0 & 0 & 0 & 2 & 5\end{array}$

$\begin{array}{lllllllllllllllllll}\text { Sonchus asper } & 0 & 1 & 0 & 1 & 0 & 0 & 0 & 2 & 0 & 0 & 0 & 0 & 0 & 0 & 0 & 0 & 2\end{array}$

Sonchus oleraceus

$\begin{array}{lllllllllllllllllll}\text { Stellaria graminea } & 0 & 1 & 0 & 4 & 5 & 1 & 0 & 11 & 0 & 0 & 0 & 0 & 0 & 0 & 0 & 0 & 11\end{array}$

$\begin{array}{llllllllllllllllll}\text { Stellaria media } & 3 & 8 & 7 & 14 & 3 & 4 & 2 & 41 & 1 & 2 & 2 & 4 & 5 & 2 & 0 & 16 & 57\end{array}$

$\begin{array}{llllllllllllllllll}\text { Tanacetum vulgare } & 1 & 2 & 0 & 2 & 6 & 0 & 0 & 11 & 0 & 0 & 0 & 0 & 0 & 0 & 0 & 0 & 11\end{array}$

$\begin{array}{llllllllllllllllll}\text { Taraxacum sect. Ruderalia } & 1 & 1 & 0 & 2 & 3 & 3 & 5 & 15 & 0 & 0 & 0 & 0 & 0 & 0 & 0 & 0 & 15\end{array}$

$\begin{array}{llllllllllllllllll}\text { Thlaspi arvense } & 0 & 0 & 0 & 2 & 0 & 0 & 0 & 2 & 0 & 0 & 0 & 0 & 0 & 0 & 0 & 0 & 2\end{array}$

$\begin{array}{llllllllllllllllll}\text { Trifolium dubium } & 1 & 0 & 0 & 1 & 1 & 0 & 0 & 3 & 0 & 0 & 0 & 0 & 0 & 0 & 0 & 0 & 3\end{array}$

$\begin{array}{llllllllllllllllll}\text { Trifolium pratense } & 0 & 0 & 0 & 1 & 0 & 0 & 0 & 1 & 0 & 0 & 0 & 0 & 0 & 0 & 0 & 0 & 1 \\ \text { Trifolium repens } & 1 & 0 & 3 & 3 & 0 & 0 & 0 & 7 & 0 & 0 & 0 & 0 & 0 & 0 & 0 & 0 & 7\end{array}$

$\begin{array}{llllllllllllllllll}\text { Trifolium repens } & 1 & 0 & 3 & 3 & 0 & 0 & 0 & 7 & 0 & 0 & 0 & 0 & 0 & 0 & 0 & 0 & 7 \\ \text { Tripleurospermum perforatum } & 0 & 5 & 0 & 1 & 0 & 1 & 0 & 7 & 0 & 0 & 1 & 0 & 0 & 0 & 0 & 1 & 8\end{array}$

$\begin{array}{llllllllllllllllllll}\text { Urtica dioica } & 5 & 7 & 8 & 8 & 4 & 5 & 8 & 45 & 0 & 0 & 1 & 0 & 0 & 0 & 0 & 1 & 46\end{array}$

$\begin{array}{llllllllllllllllll}\text { Urtica urens } & 0 & 1 & 0 & 0 & 0 & 0 & 0 & 1 & 0 & 0 & 0 & 0 & 0 & 0 & 0 & 0 & 1\end{array}$

$\begin{array}{llllllllllllllllll}\text { Veronica arvensis } & 1 & 1 & 0 & 4 & 1 & 2 & 2 & 11 & 0 & 0 & 0 & 0 & 0 & 0 & 0 & 0 & 11\end{array}$

$\begin{array}{llllllllllllllllll}\text { Veronica hederifolia } & 9 & 0 & 0 & 5 & 4 & 3 & 2 & 23 & 1 & 0 & 0 & 0 & 0 & 0 & 0 & 1 & 24\end{array}$

$\begin{array}{llllllllllllllllll}\text { Veronica persica } & 0 & 1 & 0 & 0 & 0 & 0 & 0 & 1 & 0 & 0 & 0 & 0 & 0 & 0 & 0 & 0 & 1\end{array}$

$\begin{array}{llllllllllllllllll}\text { Vicia angustifolia } & 1 & 3 & 0 & 2 & 5 & 0 & 2 & 13 & 0 & 0 & 0 & 0 & 0 & 0 & 0 & 0 & 13\end{array}$

$\begin{array}{llllllllllllllllll}\text { Vicia cracca } & 0 & 0 & 0 & 1 & 0 & 0 & 0 & 1 & 0 & 0 & 0 & 0 & 0 & 0 & 0 & 0 & 1\end{array}$

$\begin{array}{llllllllllllllllll}\text { Vicia birsuta } & 5 & 6 & 3 & 13 & 11 & 5 & 5 & 48 & 1 & 0 & 0 & 0 & 2 & 1 & 0 & 4 & 52\end{array}$

$\begin{array}{lccccccccccccccccc}\text { Vicia sativa } & 2 & 0 & 1 & 7 & 12 & 4 & 0 & 26 & 0 & 0 & 0 & 0 & 0 & 0 & 0 & 0 & 26 \\ \text { Vicia sepium } & 0 & 0 & 0 & 1 & 0 & 0 & 0 & 1 & 0 & 0 & 0 & 0 & 0 & 0 & 0 & 0 & 1\end{array}$

$\begin{array}{llllllllllllllllll}\text { Vicia tetrasperma } & 2 & 0 & 0 & 2 & 0 & 1 & 0 & 5 & 0 & 0 & 0 & 0 & 0 & 0 & 0 & 0 & 5\end{array}$

$\begin{array}{llllllllllllllllll}\text { Viola arvensis } & 10 & 15 & 10 & 29 & 5 & 4 & 2 & 75 & 1 & 1 & 1 & 10 & 1 & 1 & 2 & 17 & 92\end{array}$

Vulpia myuros 
Table S4: Species occurrences in field edge $(n=200)$ and adjacent habitat plots (hedges $n=22$; ditch margins $n=21$; grass strips $\mathrm{n}=20$ ); sorted by species name; only herbaceous species (without crops and woody seedlings); $\mathrm{A}=$ arable plants (sensu stricto; Hofmeister and Garve, 2006), arable plants species occurring in both adjacent habitats and field edges (Shared arable plant species); full references see main manuscript.

\begin{tabular}{|c|c|c|c|c|c|c|}
\hline Species & Hedges & $\begin{array}{c}\text { Ditch } \\
\text { margins }\end{array}$ & $\begin{array}{l}\text { Grass } \\
\text { strips }\end{array}$ & $\begin{array}{l}\text { Field } \\
\text { edges }\end{array}$ & $\begin{array}{c}\text { Arable plants } \\
\text { (A) }\end{array}$ & $\begin{array}{c}\text { Shared arable } \\
\text { plant species (x) }\end{array}$ \\
\hline Achillea millefolium & 3 & 0 & 6 & 20 & $\mathrm{~A}$ & $\mathrm{x}$ \\
\hline Achillea ptarmica & 0 & 1 & 0 & 0 & & \\
\hline Aegopodium podagraria & 0 & 0 & 1 & 11 & & \\
\hline Agrostis capillaris & 2 & 7 & 9 & 12 & A & $\mathrm{x}$ \\
\hline Agrostis gigantea & 0 & 1 & 0 & 0 & A & \\
\hline Agrostis stolonifera & 0 & 2 & 2 & 14 & A & $\mathrm{x}$ \\
\hline Alliaria petiolata & 2 & 1 & 1 & 4 & & \\
\hline Allium vineale & 0 & 0 & 0 & 1 & A & \\
\hline Alopecurus myosuroides & 2 & 1 & 1 & 26 & A & $\mathrm{x}$ \\
\hline Alopecurus pratensis & 4 & 7 & 5 & 27 & & \\
\hline Anchusa arvensis & 0 & 0 & 0 & 4 & A & \\
\hline Angelica sylvestris & 0 & 1 & 0 & 0 & & \\
\hline Anthoxanthum aristatum & 0 & 0 & 0 & 1 & A & \\
\hline Anthoxanthum odoratum & 0 & 1 & 0 & 0 & & \\
\hline Anthriscus sylvestris & 9 & 1 & 5 & 27 & & \\
\hline Apera spica-venti & 2 & 1 & 4 & 34 & A & $\mathrm{x}$ \\
\hline Aphanes arvensis & 1 & 0 & 0 & 5 & A & $\mathrm{x}$ \\
\hline Arabidopsis thaliana & 0 & 1 & 1 & 7 & A & $\mathrm{x}$ \\
\hline Arenaria serpyllifolia & 0 & 0 & 1 & 0 & A & \\
\hline Arrbenatherum elatius & 5 & 7 & 8 & 37 & & \\
\hline Artemisia vulgaris & 3 & 0 & 0 & 13 & A & $\mathrm{x}$ \\
\hline Avena fatua & 1 & 0 & 0 & 13 & A & $\mathrm{x}$ \\
\hline Bromus commutatus subsp. decipiens & 0 & 3 & 5 & 32 & A & $\mathrm{x}$ \\
\hline Bromus hordeaceus & 3 & 0 & 11 & 48 & A & $\mathrm{x}$ \\
\hline Bromus inermis & 0 & 0 & 0 & 2 & & \\
\hline Bromus sterilis & 16 & 2 & 9 & 87 & A & $\mathrm{x}$ \\
\hline Calystegia sepium & 0 & 1 & 0 & 3 & & \\
\hline Capsella bursa-pastoris & 0 & 0 & 3 & 31 & A & $\mathrm{x}$ \\
\hline Carduus crispus & 0 & 0 & 0 & 5 & & \\
\hline Carex acuta & 0 & 1 & 0 & 1 & & \\
\hline Carex acutiformis & 0 & 3 & 0 & 1 & & \\
\hline Carex birta & 0 & 1 & 0 & 1 & A & $\mathrm{x}$ \\
\hline Centaurea cyanus & 0 & 0 & 5 & 28 & A & $\mathrm{x}$ \\
\hline Cerastium arvense & 0 & 0 & 0 & 1 & & \\
\hline Cerastium glomeratum & 0 & 0 & 1 & 2 & A & $\mathrm{x}$ \\
\hline Cerastium bolosteoides & 0 & 0 & 2 & 8 & A & $\mathrm{x}$ \\
\hline Ceratocapnos claviculata & 1 & 1 & 0 & 1 & & \\
\hline Chaerophyllum temulum & 2 & 0 & 0 & 5 & & \\
\hline Chenopodium album & 3 & 0 & 0 & 51 & A & $\mathrm{x}$ \\
\hline Chenopodium polyspermum & 0 & 0 & 1 & 3 & A & $\mathrm{x}$ \\
\hline Cirsium arvense & 4 & 3 & 2 & 42 & A & $\mathrm{x}$ \\
\hline Cirsium vulgare & 1 & 0 & 0 & 2 & & \\
\hline Convolvulus arvensis & 0 & 0 & 0 & 3 & A & \\
\hline Crepis biennis & 0 & 0 & 1 & 2 & & \\
\hline Dactylis glomerata & 16 & 13 & 20 & 103 & & \\
\hline Daucus carota & 0 & 0 & 0 & 1 & & \\
\hline Deschampsia cespitosa & 0 & 5 & 0 & 1 & & \\
\hline Descurainia sophia & 0 & 0 & 0 & 1 & A & \\
\hline Digitaria ischaemum & 0 & 0 & 0 & 1 & A & \\
\hline Dryopteris carthusiana & 1 & 0 & 0 & 0 & & \\
\hline Dryopteris dilatata & 1 & 3 & 0 & 0 & & \\
\hline Echinochloa crus-galli & 1 & 0 & 0 & 23 & A & $\mathrm{x}$ \\
\hline Elymus caninus & 1 & 1 & 0 & 0 & & \\
\hline Elymus repens & 15 & 10 & 14 & 129 & A & $\mathrm{x}$ \\
\hline Epilobium angustifolium & 0 & 1 & 0 & 0 & & \\
\hline Epilobium montanum & 0 & 3 & 0 & 1 & & \\
\hline Epilobium tetragonum & 0 & 3 & 1 & 1 & A & $\mathrm{x}$ \\
\hline Equisetum arvense & 0 & 3 & 2 & 24 & A & $\mathrm{x}$ \\
\hline Erigeron canadensis & 1 & 0 & 1 & 3 & A & $\mathrm{x}$ \\
\hline Erodium cicutarium & 0 & 0 & 0 & 9 & A & \\
\hline Eupatorium cannabinum & 0 & 0 & 0 & 1 & & \\
\hline Fallopia convolvulus & 4 & 2 & 3 & 80 & A & $\mathrm{x}$ \\
\hline Festuca arundinacea & 0 & 0 & 0 & 4 & & \\
\hline
\end{tabular}




\begin{tabular}{|c|c|c|c|c|c|c|}
\hline Species & Hedges & $\begin{array}{c}\text { Ditch } \\
\text { margins }\end{array}$ & $\begin{array}{l}\text { Grass } \\
\text { strips }\end{array}$ & $\begin{array}{l}\text { Field } \\
\text { edges }\end{array}$ & $\begin{array}{l}\text { Arable plants } \\
\text { (A) }\end{array}$ & $\begin{array}{c}\text { Shared arable } \\
\text { plant species (x) }\end{array}$ \\
\hline Festuca pratensis & 0 & 1 & 3 & 13 & & \\
\hline Festuca rubra & 4 & 6 & 8 & 25 & & \\
\hline Ficaria verna & 0 & 1 & 0 & 1 & & \\
\hline Filipendula ulmaria & 0 & 2 & 0 & 0 & & \\
\hline Galeobdolon luteum & 1 & 0 & 0 & 0 & & \\
\hline Galeopsis tetrabit & 4 & 2 & 0 & 18 & A & $\mathrm{x}$ \\
\hline Galinsoga parviflora & 0 & 0 & 0 & 1 & A & \\
\hline Galium aparine & 18 & 9 & 8 & 109 & A & $\mathrm{x}$ \\
\hline Galium mollugo agg. & 1 & 2 & 0 & 2 & & \\
\hline Galium palustre & 0 & 1 & 0 & 0 & & \\
\hline Galium saxatile & 0 & 2 & 0 & 0 & & \\
\hline Geranium columbinum & 0 & 0 & 0 & 3 & A & \\
\hline Geranium dissectum & 1 & 0 & 2 & 5 & A & $\mathrm{x}$ \\
\hline Geranium molle & 0 & 0 & 2 & 1 & A & $\mathrm{x}$ \\
\hline Geranium pusillum & 1 & 1 & 5 & 48 & A & $\mathrm{x}$ \\
\hline Geum urbanum & 0 & 1 & 0 & 1 & & \\
\hline Glechoma bederacea & 3 & 1 & 1 & 10 & A & $\mathrm{x}$ \\
\hline Glyceria fluitans & 0 & 2 & 0 & 0 & & \\
\hline Glyceria maxima & 0 & 2 & 0 & 0 & & \\
\hline Gnaphalium uliginosum & 0 & 0 & 0 & 6 & A & \\
\hline Hedera belix & 1 & 0 & 0 & 0 & & \\
\hline Heracleum sphondylium & 2 & 0 & 3 & 11 & & ( \\
\hline Holcus lanatus & 6 & 15 & 11 & 51 & & \\
\hline Holcus mollis & 5 & 7 & 5 & 59 & A & $\mathrm{x}$ \\
\hline Humulus lupulus & 1 & 0 & 0 & 0 & & \\
\hline Hylotelepbium telephium & 0 & 0 & 0 & 1 & A & \\
\hline Hypericum maculatum & 1 & 0 & 2 & 0 & & \\
\hline Hypericum perforatum & 2 & 0 & 0 & 4 & & \\
\hline Hypochaeris radicata & 0 & 0 & 2 & 0 & & \\
\hline Iris pseudacorus & 0 & 5 & 0 & 0 & & \\
\hline Juncus acutiflorus & 0 & 1 & 0 & 0 & & \\
\hline Juncus bufonius & 0 & 0 & 1 & 7 & A & $\mathrm{x}$ \\
\hline Juncus effusus & 1 & 14 & 0 & 0 & & \\
\hline Lactuca serriola & 0 & 0 & 0 & 2 & A & \\
\hline Lamium album & 0 & 0 & 3 & 8 & A & $\mathrm{x}$ \\
\hline Lamium amplexicaule & 0 & 0 & 0 & 2 & A & \\
\hline Lamium purpureum & 0 & 0 & 1 & 14 & A & $\mathrm{x}$ \\
\hline Lapsana communis & 1 & 1 & 2 & 14 & $\mathrm{~A}$ & $\mathrm{x}$ \\
\hline Lathyrus pratensis & 0 & 1 & 0 & 0 & & \\
\hline Lolium multiflorum & 1 & 0 & 1 & 18 & A & $\mathrm{x}$ \\
\hline Lolium perenne & 1 & 0 & 8 & 44 & $\mathrm{~A}$ & $\mathrm{x}$ \\
\hline Lotus corniculatus & 1 & 4 & 0 & 1 & & \\
\hline Luronium natans & 0 & 1 & 0 & 0 & & \\
\hline Luzula multiflora & 0 & 1 & 0 & 0 & & \\
\hline Lychnis flos-cuculi & 0 & 2 & 0 & 0 & & \\
\hline Lycopus europaeus & 0 & 1 & 0 & 0 & & \\
\hline Lysimachia nummularia & 0 & 1 & 0 & 0 & & \\
\hline Lysimachia vulgaris & 1 & 10 & 1 & 3 & & \\
\hline Lythrum salicaria & 0 & 1 & 0 & 0 & & \\
\hline Matricaria chamomilla & 1 & 0 & 4 & 83 & A & $\mathrm{x}$ \\
\hline Matricaria discoidea & 0 & 0 & 0 & 2 & A & \\
\hline Moebringia trinervia & 0 & 1 & 0 & 1 & & \\
\hline Molinia caerulea & 0 & 2 & 0 & 1 & & \\
\hline Myosotis arvensis & 5 & 2 & 4 & 45 & A & $\mathrm{x}$ \\
\hline Myosotis scorpioides & 0 & 1 & 0 & 0 & & \\
\hline Odontites vernus & 0 & 0 & 0 & 1 & A & \\
\hline Papaver argemone & 0 & 0 & 0 & 2 & A & \\
\hline Papaver dubium agg. & 0 & 0 & 1 & 6 & A & $\mathrm{x}$ \\
\hline Papaver rhoeas & 0 & 0 & 1 & 5 & A & $\mathrm{x}$ \\
\hline Persicaria amphibia & 0 & 1 & 0 & 2 & A & $\mathrm{x}$ \\
\hline Persicaria bydropiper & 0 & 0 & 0 & 2 & A & \\
\hline Persicaria maculosa & 1 & 0 & 0 & 11 & A & $\mathrm{x}$ \\
\hline Peucedanum palustre & 0 & 1 & 0 & 0 & & \\
\hline Phalaris arundinacea & 0 & 11 & 0 & 1 & & \\
\hline Phleum pratense & 1 & 2 & 0 & 3 & & \\
\hline Phragmites australis & 0 & 3 & 1 & 1 & A & $\mathrm{x}$ \\
\hline Plantago lanceolata & 1 & 1 & 8 & 6 & A & $\mathrm{x}$ \\
\hline Plantago major & 0 & 0 & 1 & 4 & A & $\mathrm{x}$ \\
\hline
\end{tabular}




\begin{tabular}{|c|c|c|c|c|c|c|}
\hline Species & Hedges & $\begin{array}{c}\text { Ditch } \\
\text { margins }\end{array}$ & $\begin{array}{l}\text { Grass } \\
\text { strips }\end{array}$ & $\begin{array}{l}\text { Field } \\
\text { edges }\end{array}$ & $\begin{array}{l}\text { Arable plants } \\
\text { (A) }\end{array}$ & $\begin{array}{c}\text { Shared arable } \\
\text { plant species (x) }\end{array}$ \\
\hline Poа аппиа & 2 & 0 & 0 & 27 & $\mathrm{~A}$ & $\mathrm{x}$ \\
\hline Poa compressa & 0 & 0 & 1 & 1 & & \\
\hline Poa nemoralis & 3 & 0 & 0 & 1 & & \\
\hline Poapratensis & 1 & 3 & 8 & 29 & & \\
\hline Poa trivialis & 6 & 5 & 10 & 76 & A & $\mathrm{x}$ \\
\hline Polygonum aviculare & 0 & 0 & 0 & 81 & A & \\
\hline Potentilla anserina & 1 & 0 & 0 & 1 & $\mathrm{~A}$ & $\mathrm{x}$ \\
\hline Potentilla erecta & 0 & 2 & 0 & 1 & & \\
\hline Potentilla reptans & 1 & 1 & 1 & 0 & A & \\
\hline Pteridium aquilinum & 1 & 0 & 0 & 0 & & \\
\hline Ranunculus acris & 0 & 1 & 0 & 0 & & \\
\hline Ranunculus repens & 0 & 0 & 2 & 5 & A & $\mathrm{x}$ \\
\hline Raphanus sativus & 0 & 0 & 0 & 1 & & \\
\hline Rorippa palustris & 0 & 0 & 0 & 1 & A & \\
\hline Rubus caesius & 0 & 1 & 0 & 0 & A & \\
\hline Rubus fruticosus agg. & 7 & 9 & 0 & 6 & & \\
\hline Rubus idaeus agg. & 1 & 1 & 0 & 1 & & \\
\hline Rumex acetosa & 0 & 4 & 4 & 11 & & \\
\hline Rumex acetosella & 0 & 0 & 2 & 2 & A & $\mathrm{x}$ \\
\hline Rumex crispus & 0 & 3 & 3 & 8 & A & $\mathrm{x}$ \\
\hline Rumex bydrolapatbum & 0 & 2 & 0 & 1 & & \\
\hline Rumex obtusifolius & 3 & 2 & 2 & 33 & A & $\mathrm{x}$ \\
\hline Scirpus sylvaticus & 0 & 1 & 0 & 0 & & \\
\hline Scleranthus annuиs & 0 & 0 & 0 & 2 & A & \\
\hline Scrophularia nodosa & 1 & 0 & 0 & 0 & & \\
\hline Scutellaria galericulata & 0 & 2 & 0 & 0 & & \\
\hline Senecio inaequidens & 0 & 0 & 0 & 1 & & \\
\hline Senecio jacobaea & 0 & 0 & 0 & 1 & & \\
\hline Senecio vulgaris & 1 & 0 & 0 & 7 & A & $\mathrm{x}$ \\
\hline Setaria viridis & 0 & 0 & 0 & 1 & A & \\
\hline Silene dioica & 1 & 1 & 0 & 0 & & \\
\hline Silene latifolia & 0 & 1 & 1 & 2 & A & $\mathrm{x}$ \\
\hline Sisymbrium officinale & 2 & 0 & 0 & 28 & A & $\mathrm{x}$ \\
\hline Solanum nigrum & 0 & 0 & 0 & 3 & A & \\
\hline Solidago canadensis & 1 & 0 & 0 & 0 & & \\
\hline Solidago gigantea & 1 & 0 & 0 & 0 & & \\
\hline Sonchus asper & 0 & 0 & 0 & 2 & A & \\
\hline Sonchus oleraceus & 0 & 0 & 1 & 2 & $\mathrm{~A}$ & $\mathrm{x}$ \\
\hline Sparganium erectum & 0 & 5 & 0 & 0 & & \\
\hline Stachys palustris & 0 & 1 & 0 & 0 & & \\
\hline Stellaria graminea & 0 & 1 & 5 & 11 & A & $\mathrm{x}$ \\
\hline Stellaria holostea & 0 & 0 & 1 & 0 & & \\
\hline Stellaria media & 1 & 0 & 2 & 41 & A & $\mathrm{x}$ \\
\hline Tanacetum vulgare & 3 & 0 & 5 & 11 & & \\
\hline Taraxacum sect. Ruderalia & 0 & 0 & 6 & 15 & A & $\mathrm{x}$ \\
\hline Thalictrum flavum & 0 & 1 & 0 & 0 & & \\
\hline Thlaspi arvense & 0 & 0 & 0 & 2 & A & \\
\hline Trifolium arvense & 0 & 0 & 1 & 0 & A & \\
\hline Trifolium campestre & 0 & 0 & 1 & 0 & & \\
\hline Trifolium dubium & 0 & 0 & 0 & 3 & & \\
\hline Trifolium pratense & 0 & 0 & 2 & 1 & & \\
\hline Trifolium repens & 1 & 0 & 2 & 7 & & \\
\hline Tripleurospermum perforatum & 0 & 0 & 0 & 7 & A & \\
\hline Typha latifolia & 0 & 2 & 0 & 0 & & \\
\hline Urtica dioica & 18 & 10 & 7 & 45 & & \\
\hline Urtica urens & 0 & 0 & 0 & 1 & A & \\
\hline Valeriana officinalis & 0 & 2 & 0 & 0 & & \\
\hline Veronica arvensis & 0 & 1 & 4 & 11 & A & $\mathrm{x}$ \\
\hline Veronica chamaedrys & 0 & 0 & 1 & 0 & & \\
\hline Veronica bederifolia & 1 & 0 & 2 & 23 & A & $\mathrm{x}$ \\
\hline Veronica persica & 0 & 0 & 0 & 1 & A & \\
\hline Vicia angustifolia & 1 & 1 & 5 & 13 & A & $\mathrm{x}$ \\
\hline Vicia cracca & 0 & 1 & 0 & 1 & A & $\mathrm{x}$ \\
\hline Vicia birsuta & 5 & 1 & 6 & 48 & A & $\mathrm{x}$ \\
\hline Vicia sativa & 3 & 1 & 4 & 26 & & \\
\hline Vicia sepium & 0 & 0 & 1 & 1 & & \\
\hline Vicia tetrasperma & 1 & 3 & 1 & 5 & A & $\mathrm{x}$ \\
\hline Vicia villosa & 0 & 1 & 1 & 0 & A & \\
\hline
\end{tabular}


CHAPTER 2

\begin{tabular}{lcccccc}
\hline Species & Hedges & $\begin{array}{c}\text { Ditch } \\
\text { margins }\end{array}$ & $\begin{array}{c}\text { Grass } \\
\text { strips }\end{array}$ & $\begin{array}{c}\text { Field } \\
\text { edges }\end{array}$ & $\begin{array}{c}\text { Arable plants } \\
\text { (A) }\end{array}$ & $\begin{array}{c}\text { Shared arable } \\
\text { plant species (x) }\end{array}$ \\
\hline Viola arvensis & 2 & 0 & 3 & 75 & A & $x$ \\
Vulpia myuros & 0 & 0 & 0 & 1 & & \\
\hline
\end{tabular}

Table S5: Raw data of the vegetation survey (2016) including management data (agricultural business year 2015/2016) and environmental data.

Can be downloaded from http://ediss.uni-goettingen.de/ (Niedersächsische Staats- und Universitätsbibliothek Göttingen). 


\section{CHAPTER 2}

Table S6: Summary of model structure and statistical results.

\begin{tabular}{|c|c|c|c|c|c|c|c|c|c|}
\hline Measured value & Model & $\mathrm{n}$ & $\begin{array}{c}\mathbf{R}^{2}{ }^{\prime} \mathbf{m} \\
\text { (fixed effects } \\
\text { only) }\end{array}$ & $\begin{array}{l}\mathbf{R}^{2} \mathbf{c} \\
\text { (fixed \& } \\
\text { random } \\
\text { effects) }\end{array}$ & $\begin{array}{l}\text { Explanatory } \\
\text { variables }\end{array}$ & $\begin{array}{c}\text { Z-value } \\
\text { (chi-square tests. } \\
\text { Type II Wald) }\end{array}$ & $\begin{array}{c}\text { Degrees of } \\
\text { freedom }\end{array}$ & $\begin{array}{c}\text { P-value } \\
(\operatorname{Pr}(\text { chi-square }))\end{array}$ & Significance \\
\hline \multirow{6}{*}{$\begin{array}{l}\text { Total } \\
\text { herbaceous } \\
\text { species number }\end{array}$} & \multirow{6}{*}{$\begin{array}{l}\text { glmmTMB(spec.num } \sim \text { poolCrop }+ \text { Adj_habitat }+ \\
\text { Soil_yield_potential }+ \text { Herbicide_Intensity }+ \\
\text { Cover_Crop }+ \text { N_tot }+(1 \mid \text { Hof }) . \text { family= } \\
\text { nbinom1. data }=\text { field })\end{array}$} & \multirow{6}{*}{$\begin{array}{c}255(14 \\
\text { farms })\end{array}$} & \multirow{6}{*}{0.94} & \multirow{6}{*}{0.94} & Pooled crop species & 76.9 & 3 & $<2.2 \mathrm{e}-16$ & *** \\
\hline & & & & & Adjacent habitat & 244.0 & 4 & $\begin{array}{l}<2.2 \mathrm{e}-16 \\
\end{array}$ & **** \\
\hline & & & & & Soil yield potential & 11.4 & 1 & 0,0007198 & $* * *$ \\
\hline & & & & & $\begin{array}{l}\text { Herbicide Intensity } \\
\text { Index }\end{array}$ & 2.3 & 1 & 0.1261987 & n.s. \\
\hline & & & & & Crop cover & 8.3 & 1 & 0.0039252 & ** \\
\hline & & & & & Total nitrogen input & 4.2 & 1 & 0.039758 & $*$ \\
\hline
\end{tabular}

\begin{tabular}{|c|c|c|c|c|c|c|c|}
\hline Measured value & Model & $\begin{array}{l}\text { Explanatory } \\
\text { variables }\end{array}$ & Estimate & $\begin{array}{c}\text { Standard } \\
\text { error }\end{array}$ & $\mathrm{z}$ value & $\operatorname{Pr}(>|z|)$ & Significance \\
\hline \multirow{12}{*}{ Total herbaceous species number } & \multirow{12}{*}{$\begin{array}{l}\text { glmmTMB(spec.num } \sim \text { poolCrop + Adj_habitat + Soil_yield_potential } \\
+ \text { Herbicide_Intensity }+ \text { Cover_Crop + N_tot }+(1 \mid \text { Hof). family }= \\
\text { nbinom1. data }=\text { field })\end{array}$} & Adjacent Field interior & -0.9006976 & 0.1593276 & -5.653 & $1.58 \mathrm{E}-08$ & $* * *$ \\
\hline & & Adjacent Grass strip & 0.6763108 & 0.1299435 & 5.205 & 0.000000194 & *** \\
\hline & & Adjacent Ditch margin & 0.5642157 & 0.1598911 & 3.529 & 0.000418 & $* * *$ \\
\hline & & Adjacent Hedge & 0.4111101 & 0.174575 & 2.355 & 0.018527 & * \\
\hline & & Maize & -0.2257731 & 0.0925184 & -2.44 & 0.014675 & * \\
\hline & & Rapeseed & 0.5421335 & 0.0738001 & 7.346 & $2.04 \mathrm{E}-13$ & $* * *$ \\
\hline & & $\begin{array}{l}\text { Potato } \\
\end{array}$ & 0.2592128 & 0.0898156 & 2.886 & 0.003901 & ** \\
\hline & & Soil yield potential & -0.0096231 & 0.0028455 & -3.382 & 0.00072 & $* * *$ \\
\hline & & Crop cover & -0.0086064 & 0.0029841 & -2.884 & 0.003925 & ** \\
\hline & & Total nitrogen input & -0.0014952 & 0.0007271 & -2.056 & 0.039758 & * \\
\hline & & Herbicide Intensity Index & -0.0564337 & 0.0369025 & -1.529 & 0.126199 & n.s. \\
\hline & & Random effect (farm) & 3.261846 & 0.3181355 & 10.253 & $<2 \mathrm{e}-16$ & $* * *$ \\
\hline
\end{tabular}


Multiple comparisons of means: Tukey Contrast

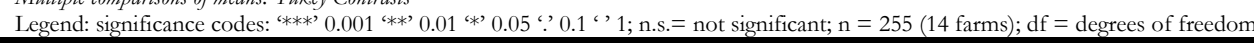

\begin{tabular}{|c|c|c|c|c|c|c|c|}
\hline \multirow{18}{*}{ Total herbaceous species number } & Crop species differences & Estimate & Std. Error & $\mathrm{z}$ value & $\operatorname{Pr}(>|z|)$ & Significance & df \\
\hline & Maize - Cereal & -0.226 & 0.093 & -2.440 & 0.068 & . & 241 \\
\hline & Potato - Cereal & 0.259 & 0.090 & 2.886 & 0.019 & * & 241 \\
\hline & Rapeseed - Cereal & 0.542 & 0.074 & 7.346 & $<0.001$ & $* * *$ & 241 \\
\hline & Potato - Maize & 0.485 & 0.113 & 4.274 & $<0.001$ & **** & 241 \\
\hline & Rapeseed - Maize & 0.768 & 0.102 & 7.536 & $<0.001$ & **** & 241 \\
\hline & Rapeseed - Potato & 0.283 & 0.098 & 2.892 & 0.020 & $*$ & 241 \\
\hline & Adjacent habitat differences & Estimate & Std. Error & $\mathrm{z}$ value & $\operatorname{Pr}(>|z|)$ & Significance & df \\
\hline & Adjacent Arable field - Adjacent Field interior & 0.901 & 0.159 & 5.653 & $<0.001$ & $* * *$ & 241 \\
\hline & Adjacent Grass strip - Adjacent Field interior & 1.577 & 0.104 & 15.107 & $<0.001$ & $* * *$ & 241 \\
\hline & Adjacent Ditch margin - Adjacent Field interior & 1.465 & 0.140 & 10.437 & $<0.001$ & $* * *$ & 241 \\
\hline & Adjacent Hedge - Adjacent Field interior & 1.312 & 0.155 & 8.471 & $<0.001$ & $* * *$ & 241 \\
\hline & Adjacent Grass strip - Adjacent Arable field & 0.676 & 0.130 & 5.205 & $<0.001$ & $* * *$ & 241 \\
\hline & Adjacent Ditch margin - Adjacent Arable field & 0.564 & 0.160 & 3.529 & 0.004 & $* *$ & 241 \\
\hline & Adjacent Hedge - Adjacent Arable field & 0.411 & 0.175 & 2.355 & 0.118 & n.s. & 241 \\
\hline & Adjacent Ditch margin - Adjacent Grass strip & -0.112 & 0.102 & -1.100 & 0.791 & n.s. & 241 \\
\hline & Adjacent Hedge - Adjacent Grass strip & -0.265 & 0.123 & -2.154 & 0.184 & n.s. & 241 \\
\hline & Adjacent Hedge - Adjacent Ditch margin & -0.153 & 0.154 & -0.994 & 0.846 & n.s. & 241 \\
\hline
\end{tabular}

\begin{tabular}{|c|c|c|c|c|c|c|}
\hline & & $\begin{array}{c}\text { Barley } \\
\text { (Field edge) }\end{array}$ & $\begin{array}{c}\text { Barley } \\
\text { (Field interior) }\end{array}$ & $\begin{array}{c}\text { Potato } \\
\text { (Field edge) }\end{array}$ & $\begin{array}{c}\text { Potato } \\
\text { (Field interior) }\end{array}$ & $\begin{array}{c}\text { Maize } \\
\text { (Field edge) }\end{array}$ \\
\hline & Barley (Field edge) & 1.00000 & 0.00098 & 1.00000 & 0.00112 & 0.27023 \\
\hline & Barley (Field interior) & 0.00098 & 1.00000 & 0.00141 & 1.00000 & 0.00218 \\
\hline & Potato (Field edge) & 1.00000 & 0.00141 & 1.00000 & 0.00128 & 0.11628 \\
\hline & Potato (Field interior) & 0.00112 & 1.00000 & 0.00128 & 1.00000 & 0.00294 \\
\hline & Maize (Field edge) & 0.27023 & 0.00218 & 0.11628 & 0.00294 & 1.00000 \\
\hline & Maize (Field interior) & 0.00181 & 1.00000 & 0.00246 & 1.00000 & 0.00601 \\
\hline & Rapeseed (Field edge) & 0.04239 & 0.00040 & 0.04466 & 0.00044 & 0.00002 \\
\hline & Rapeseed (Field interior) & 0.00393 & 1.00000 & 0.00481 & 0.81420 & 0.02276 \\
\hline & Rye (Field edge) & 1.00000 & 0.00065 & 1.00000 & 0.00072 & 0.01448 \\
\hline & Rye (Field interior) & 0.00110 & 1.00000 & 0.00178 & 1.00000 & 0.00262 \\
\hline & Triticale (Field edge) & 1.00000 & 0.00527 & 1.00000 & 0.00269 & 1.00000 \\
\hline & Triticale (Field interior) & 0.00065 & 1.00000 & 0.00113 & 1.00000 & 0.00142 \\
\hline & Wheat (Field edge) & 1.00000 & 0.00140 & 1.00000 & 0.00140 & 1.00000 \\
\hline & Wheat (Field interior) & 0.00044 & 1.00000 & 0.00085 & 1.00000 & 0.00081 \\
\hline & & $\begin{array}{l}\text { Maize (Field } \\
\text { interior) }\end{array}$ & $\begin{array}{c}\text { Rapeseed } \\
\text { (Field edge) }\end{array}$ & $\begin{array}{c}\text { Rapeseed } \\
\text { (Field } \\
\text { interior) }\end{array}$ & $\begin{array}{l}\text { Rye (Field } \\
\text { edge) }\end{array}$ & $\begin{array}{l}\text { Rye (Field } \\
\text { interior) }\end{array}$ \\
\hline & Barley (Field edge) & 0.00181 & 0.04239 & 0.00393 & 1.00000 & 0.00110 \\
\hline & Barley (Field interior) & 1.00000 & 0.00040 & 1.00000 & 0.00065 & 1.00000 \\
\hline & Potato (Field edge) & 0.00246 & 0.04466 & 0.00481 & 1.00000 & 0.00178 \\
\hline & Potato (Field interior) & 1.00000 & 0.00044 & 0.81420 & 0.00072 & 1.00000 \\
\hline & Maize (Field edge) & 0.00601 & 0.00002 & 0.02276 & 0.01448 & 0.00262 \\
\hline \multirow[t]{24}{*}{ Total herbaceous species number } & Maize (Field interior) & 1.00000 & 0.00051 & 1.00000 & 0.00110 & 1.00000 \\
\hline & Rapeseed (Field edge) & 0.00051 & 1.00000 & 0.00068 & 0.45046 & 0.00043 \\
\hline & Rapeseed (Field interior) & 1.00000 & 0.00068 & 1.00000 & 0.00164 & 1.00000 \\
\hline & Rye (Field edge) & 0.00110 & 0.45046 & 0.00164 & 1.00000 & 0.00086 \\
\hline & Rye (Field interior) & 1.00000 & 0.00043 & 1.00000 & 0.00086 & 1.00000 \\
\hline & Triticale (Field edge) & 0.00685 & 0.04531 & 0.01867 & 1.00000 & 0.00527 \\
\hline & Triticale (Field interior) & 1.00000 & 0.00035 & 1.00000 & 0.00054 & 1.00000 \\
\hline & Wheat (Field edge) & 0.00246 & 0.00107 & 0.00685 & 0.37228 & 0.00128 \\
\hline & Wheat (Field interior) & 1.00000 & 0.00030 & 1.00000 & 0.00043 & 1.00000 \\
\hline & & $\begin{array}{c}\text { Triticale } \\
\text { (Field edge) } \\
\end{array}$ & $\begin{array}{c}\text { Triticale } \\
\text { (Field interior) }\end{array}$ & $\begin{array}{c}\text { Wheat } \\
\text { (Field edge) }\end{array}$ & $\begin{array}{c}\text { Wheat } \\
\text { (Field interior) }\end{array}$ & \\
\hline & Barley (Field edge) & 1.00000 & 0.00065 & 1.00000 & 0.00044 & \\
\hline & Barley (Field interior) & 0.00527 & 1.00000 & 0.00140 & 1.00000 & \\
\hline & Potato (Field edge) & 1.00000 & 0.00113 & 1.00000 & 0.00085 & \\
\hline & Potato (Field interior) & 0.00269 & 1.00000 & 0.00140 & 1.00000 & \\
\hline & Maize (Field edge) & 1.00000 & 0.00142 & 1.00000 & 0.00081 & \\
\hline & Maize (Field interior) & 0.00685 & 1.00000 & 0.00246 & 1.00000 & \\
\hline & Rapeseed (Field edge) & 0.04531 & 0.00035 & 0.00107 & 0.00030 & \\
\hline & Rapeseed (Field interior) & 0.01867 & 1.00000 & 0.00685 & 1.00000 & \\
\hline & Rye (Field edge) & 1.00000 & 0.00054 & 0.37228 & 0.00043 & \\
\hline & Rye (Field interior) & 0.00527 & 1.00000 & 0.00128 & 1.00000 & \\
\hline & Triticale (Field edge) & 1.00000 & 0.00393 & 1.00000 & 0.00320 & \\
\hline & Triticale (Field interior) & 0.00393 & 1.00000 & 0.00083 & 1.00000 & \\
\hline & Wheat (Field edge) & 1.00000 & 0.00083 & 1.00000 & 0.00059 & \\
\hline & Wheat (Field interior) & 0.00320 & 1.00000 & 0.00059 & 1.00000 & \\
\hline
\end{tabular}




\begin{tabular}{|c|c|c|c|c|c|c|}
\hline & & $\begin{array}{c}\text { Barley } \\
\text { (Field edge) } \\
\end{array}$ & $\begin{array}{c}\text { Barley } \\
\text { (Field interior) } \\
\end{array}$ & $\begin{array}{c}\text { Potato } \\
\text { (Field edge) } \\
\end{array}$ & $\begin{array}{c}\text { Potato } \\
\text { (Field interior) }\end{array}$ & $\begin{array}{c}\text { Maize } \\
\text { (Field edge) } \\
\end{array}$ \\
\hline & Barley (Field edge) & 1.00000 & 0.00191 & 1.00000 & 0.00283 & 0.48640 \\
\hline & Barley (Field interior) & 0.00191 & 1.00000 & 0.00257 & 1.00000 & 0.01136 \\
\hline & Potato (Field edge) & 1.00000 & 0.00257 & 1.00000 & 0.00284 & 0.23745 \\
\hline & Potato (Field interior) & 0.00283 & 1.00000 & 0.00284 & 1.00000 & 0.00814 \\
\hline & Maize (Field edge) & 0.48640 & 0.01136 & 0.23745 & 0.00814 & 1.00000 \\
\hline & Maize (Field interior) & 0.00571 & 1.00000 & 0.00590 & 1.00000 & 0.03077 \\
\hline & Rapeseed (Field edge) & 0.02370 & 0.00043 & 0.69994 & 0.00049 & 0.00003 \\
\hline & Rapeseed (Field interior) & 0.01830 & 1.00000 & 0.01606 & 0.92889 & 0.15962 \\
\hline & Rye (Field edge) & 1.00000 & 0.00135 & 1.00000 & 0.00257 & 0.33494 \\
\hline & Rye (Field interior) & 0.00683 & 1.00000 & 0.00778 & 1.00000 & 0.03136 \\
\hline & Triticale (Field edge) & 1.00000 & 0.01785 & 1.00000 & 0.01092 & 1.00000 \\
\hline & Triticale (Field interior) & 0.00199 & 1.00000 & 0.00242 & 1.00000 & 0.01023 \\
\hline & Wheat (Field edge) & 1.00000 & 0.00479 & 1.00000 & 0.00571 & 1.00000 \\
\hline & Wheat (Field interior) & 0.00108 & 1.00000 & 0.00143 & 1.00000 & 0.00462 \\
\hline & & $\begin{array}{c}\text { Maize } \\
\text { (Field interior) } \\
\end{array}$ & $\begin{array}{c}\text { Rapeseed } \\
\text { (Field edge) } \\
\end{array}$ & $\begin{array}{c}\text { Rapeseed } \\
\text { (Field interior) }\end{array}$ & $\begin{array}{c}\text { Rye } \\
\text { (Field edge) } \\
\end{array}$ & $\begin{array}{c}\text { Rye } \\
\text { (Field interior) } \\
\end{array}$ \\
\hline & Barley (Field edge) & 0.00571 & 0.02370 & 0.01830 & 1.00000 & 0.00683 \\
\hline & Barley (Field interior) & 1.00000 & 0.00043 & 1.00000 & 0.00135 & 1.00000 \\
\hline & Potato (Field edge) & 0.00590 & 0.69994 & 0.01606 & 1.00000 & 0.00778 \\
\hline & Potato (Field interior) & 1.00000 & 0.00049 & 0.92889 & 0.00257 & 1.00000 \\
\hline & Maize (Field edge) & 0.03077 & 0.00003 & 0.15962 & 0.33494 & 0.03136 \\
\hline Arable plants sensu stricto & Maize (Field interior) & 1.00000 & 0.00049 & 1.00000 & 0.00444 & 1.00000 \\
\hline 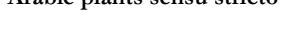 & Rapeseed (Field edge) & 0.00049 & 1.00000 & 0.00068 & 0.26542 & 0.00043 \\
\hline & Rapeseed (Field interior) & 1.00000 & 0.00068 & 1.00000 & 0.01355 & 1.00000 \\
\hline & Rye (Field edge) & 0.00444 & 0.26542 & 0.01355 & 1.00000 & 0.00571 \\
\hline & Rye (Field interior) & 1.00000 & 0.00043 & 1.00000 & 0.00571 & 1.00000 \\
\hline & Triticale (Field edge) & 0.02571 & 0.01519 & 0.08309 & 1.00000 & 0.02359 \\
\hline & Triticale (Field interior) & 1.00000 & 0.00038 & 1.00000 & 0.00141 & 1.00000 \\
\hline & Wheat (Field edge) & 0.01462 & 0.00135 & 0.06073 & 1.00000 & 0.01204 \\
\hline & Wheat (Field interior) & 1.00000 & 0.00031 & 1.00000 & 0.00078 & 1.00000 \\
\hline & & $\begin{array}{c}\text { Triticale } \\
\text { (Field edge) } \\
\end{array}$ & $\begin{array}{c}\text { Triticale } \\
\text { (Field interior) }\end{array}$ & $\begin{array}{c}\text { Wheat } \\
\text { (Field edge) }\end{array}$ & $\begin{array}{c}\text { Wheat } \\
\text { (Field interior) }\end{array}$ & \\
\hline & Barley (Field edge) & 1.00000 & 0.00199 & 1.00000 & 0.00108 & \\
\hline & Barley (Field interior) & 0.01785 & 1.00000 & 0.00479 & 1.00000 & \\
\hline & Potato (Field edge) & 1.00000 & 0.00242 & 1.00000 & 0.00143 & \\
\hline & Potato (Field interior) & 0.01092 & 1.00000 & 0.00571 & 1.00000 & \\
\hline & Maize (Field edge) & 1.00000 & 0.01023 & 1.00000 & 0.00462 & \\
\hline & Maize (Field interior) & 0.02571 & 1.00000 & 0.01462 & 1.00000 & \\
\hline & Rapeseed (Field edge) & 0.01519 & 0.00038 & 0.00135 & 0.00031 & \\
\hline & Rapeseed (Field interior) & 0.08309 & 1.00000 & 0.06073 & 1.00000 & \\
\hline & Rye (Field edge) & 1.00000 & 0.00141 & 1.00000 & 0.00078 & \\
\hline & Rye (Field interior) & 0.02359 & 1.00000 & 0.01204 & 1.00000 & \\
\hline & Triticale (Field edge) & 1.00000 & 0.01226 & 1.00000 & 0.00683 & \\
\hline & Triticale (Field interior) & 0.01226 & 1.00000 & 0.00380 & 1.00000 & \\
\hline & Wheat (Field edge) & 1.00000 & 0.00380 & 1.00000 & 0.00153 & \\
\hline & Wheat (Field interior) & 0.00683 & 1.00000 & 0.00153 & 1.00000 & \\
\hline
\end{tabular}




\begin{tabular}{|c|c|c|c|c|c|c|}
\hline & & $\begin{array}{c}\text { Barley } \\
\text { (Field edge) }\end{array}$ & $\begin{array}{c}\text { Barley } \\
\text { (Field interior) }\end{array}$ & $\begin{array}{c}\text { Potato } \\
\text { (Field edge) }\end{array}$ & $\begin{array}{c}\text { Potato } \\
\text { (Field interior) }\end{array}$ & $\begin{array}{c}\text { Maize } \\
\text { (Field edge) }\end{array}$ \\
\hline & Barley (Field edge) & 1.00000 & 1.00000 & 1.00000 & 0.20603 & 1.00000 \\
\hline & Barley (Field interior) & 1.00000 & 1.00000 & 1.00000 & 1.00000 & 1.00000 \\
\hline & Potato (Field edge) & 1.00000 & 1.00000 & 1.00000 & 0.10416 & 1.00000 \\
\hline & Potato (Field interior) & 0.20603 & 1.00000 & 0.10416 & 1.00000 & 0.20603 \\
\hline & Maize (Field edge) & 1.00000 & 1.00000 & 1.00000 & 0.20603 & 1.00000 \\
\hline & Maize (Field interior) & 1.00000 & 1.00000 & 1.00000 & 1.00000 & 1.00000 \\
\hline & Rapeseed (Field edge) & 0.00550 & 0.02939 & 0.13464 & 0.00232 & 0.00022 \\
\hline & Rapeseed (Field interior) & 1.00000 & 1.00000 & 1.00000 & 0.56160 & 1.00000 \\
\hline & Rye (Field edge) & 0.22509 & 0.22861 & 1.00000 & 0.00951 & 0.03221 \\
\hline & Rye (Field interior) & 1.00000 & 1.00000 & 1.00000 & 1.00000 & 1.00000 \\
\hline & Triticale (Field edge) & 1.00000 & 1.00000 & 1.00000 & 0.64701 & 1.00000 \\
\hline & Triticale (Field interior) & 1.00000 & 1.00000 & 0.64513 & 1.00000 & 1.00000 \\
\hline & Wheat (Field edge) & 1.00000 & 1.00000 & 1.00000 & 1.00000 & 1.00000 \\
\hline & Wheat (Field interior) & 1.00000 & 1.00000 & 1.00000 & 1.00000 & 1.00000 \\
\hline & & $\begin{array}{c}\text { Maize } \\
\text { (Field interior) }\end{array}$ & $\begin{array}{c}\text { Rapeseed } \\
\text { (Field edge) }\end{array}$ & $\begin{array}{c}\text { Rapeseed } \\
\text { (Field interior) }\end{array}$ & $\begin{array}{c}\text { Rye } \\
\text { (Field edge) } \\
\end{array}$ & $\begin{array}{c}\text { Rye } \\
\text { (Field interior) }\end{array}$ \\
\hline & Barley (Field edge) & 1.00000 & 0.00550 & 1.00000 & 0.22509 & 1.00000 \\
\hline & Barley (Field interior) & 1.00000 & 0.02939 & 1.00000 & 0.22861 & 1.00000 \\
\hline & Potato (Field edge) & 1.00000 & 0.13464 & 1.00000 & 1.00000 & 1.00000 \\
\hline & Potato (Field interior) & 1.00000 & 0.00232 & 0.56160 & 0.00951 & 1.00000 \\
\hline & Maize (Field edge) & 1.00000 & 0.00022 & 1.00000 & 0.03221 & 1.00000 \\
\hline & Maize (Field interior) & 1.00000 & 0.00911 & 1.00000 & 0.06369 & 1.00000 \\
\hline High nature value species & Rapeseed (Field edge) & 0.00911 & 1.00000 & 0.03152 & 1.00000 & 0.03137 \\
\hline & Rapeseed (Field interior) & 1.00000 & 0.03152 & 1.00000 & 0.38980 & 1.00000 \\
\hline & Rye (Field edge) & 0.06369 & 1.00000 & 0.38980 & 1.00000 & 0.30550 \\
\hline & Rye (Field interior) & 1.00000 & 0.03137 & 1.00000 & 0.30550 & 1.00000 \\
\hline & Triticale (Field edge) & 1.00000 & 0.28148 & 1.00000 & 1.00000 & 1.00000 \\
\hline & Triticale (Field interior) & 1.00000 & 0.00496 & 1.00000 & 0.03152 & 1.00000 \\
\hline & Wheat (Field edge) & 1.00000 & 0.00320 & 1.00000 & 0.08615 & 1.00000 \\
\hline & Wheat (Field interior) & 1.00000 & 0.00693 & 1.00000 & 0.05310 & 1.00000 \\
\hline & & $\begin{array}{c}\text { Triticale } \\
\text { (Field edge) }\end{array}$ & $\begin{array}{c}\text { Triticale } \\
\text { (Field interior) }\end{array}$ & $\begin{array}{c}\text { Wheat } \\
\text { (Field edge) }\end{array}$ & $\begin{array}{c}\text { Wheat } \\
\text { (Field interior) }\end{array}$ & \\
\hline & Barley (Field edge) & 1.00000 & 1.00000 & 1.00000 & 1.00000 & \\
\hline & Barley (Field interior) & 1.00000 & 1.00000 & 1.00000 & 1.00000 & \\
\hline & Potato (Field edge) & 1.00000 & 0.64513 & 1.00000 & 1.00000 & \\
\hline & Potato (Field interior) & 0.64701 & 1.00000 & 1.00000 & 1.00000 & \\
\hline & Maize (Field edge) & 1.00000 & 1.00000 & 1.00000 & 1.00000 & \\
\hline & Maize (Field interior) & 1.00000 & 1.00000 & 1.00000 & 1.00000 & \\
\hline & Rapeseed (Field edge) & 0.28148 & 0.00496 & 0.00320 & 0.00693 & \\
\hline & Rapeseed (Field interior) & 1.00000 & 1.00000 & 1.00000 & 1.00000 & \\
\hline & Rye (Field edge) & 1.00000 & 0.03152 & 0.08615 & 0.05310 & \\
\hline & Rye (Field interior) & 1.00000 & 1.00000 & 1.00000 & 1.00000 & \\
\hline & Triticale (Field edge) & 1.00000 & 1.00000 & 1.00000 & 1.00000 & \\
\hline & Triticale (Field interior) & 1.00000 & 1.00000 & 1.00000 & 1.00000 & \\
\hline & Wheat (Field edge) & 1.00000 & 1.00000 & 1.00000 & 1.00000 & \\
\hline & Wheat (Field interior) & 1.00000 & 1.00000 & 1.00000 & 1.00000 & \\
\hline
\end{tabular}




\begin{tabular}{|c|c|c|c|c|c|c|}
\hline \multirow{45}{*}{ Cover of herbaceous plants (\%) } & & $\begin{array}{c}\text { Barley } \\
\text { (Field edge) }\end{array}$ & $\begin{array}{c}\text { Barley } \\
\text { (Field interior) }\end{array}$ & $\begin{array}{c}\text { Potato } \\
\text { (Field edge) }\end{array}$ & $\begin{array}{c}\text { Potato } \\
\text { (Field interior) }\end{array}$ & $\begin{array}{c}\text { Maize } \\
\text { (Field edge) }\end{array}$ \\
\hline & Barley (Field edge) & 1.00000 & 0.75554 & 1.00000 & 0.00965 & 0.75554 \\
\hline & Barley (Field interior) & 0.75554 & 1.00000 & 0.29645 & 1.00000 & 0.17266 \\
\hline & Potato (Field edge) & 1.00000 & 0.29645 & 1.00000 & 0.00283 & 0.65606 \\
\hline & Potato (Field interior) & 0.00965 & 1.00000 & 0.00283 & 1.00000 & 0.00236 \\
\hline & Maize (Field edge) & 0.75554 & 0.17266 & 0.65606 & 0.00236 & 1.00000 \\
\hline & Maize (Field interior) & 1.00000 & 1.00000 & 1.00000 & 1.00000 & 0.68022 \\
\hline & Rapeseed (Field edge) & 0.00003 & 0.00662 & 0.00000 & 0.00024 & 0.06550 \\
\hline & Rapeseed (Field interior) & 0.18879 & 0.19913 & 0.10709 & 0.01332 & 1.00000 \\
\hline & Rye (Field edge) & 0.38980 & 0.05080 & 0.65606 & 0.00088 & 1.00000 \\
\hline & Rye (Field interior) & 1.00000 & 1.00000 & 1.00000 & 0.19644 & 1.00000 \\
\hline & Triticale (Field edge) & 0.74980 & 0.15348 & 0.65754 & 0.00391 & 1.00000 \\
\hline & Triticale (Field interior) & 1.00000 & 1.00000 & 1.00000 & 1.00000 & 0.42768 \\
\hline & Wheat (Field edge) & 1.00000 & 0.10725 & 1.00000 & 0.00102 & 1.00000 \\
\hline & Wheat (Field interior) & 0.75554 & 1.00000 & 0.11376 & 1.00000 & 0.04556 \\
\hline & & $\begin{array}{c}\text { Maize } \\
\text { (Field interior) }\end{array}$ & $\begin{array}{c}\text { Rapeseed } \\
\text { (Field edge) }\end{array}$ & $\begin{array}{c}\text { Rapeseed } \\
\text { (Field interior) }\end{array}$ & $\begin{array}{c}\text { Rye } \\
\text { (Field edge) } \\
\end{array}$ & $\begin{array}{c}\text { Rye } \\
\text { (Field interior) } \\
\end{array}$ \\
\hline & Barley (Field edge) & 1.00000 & 0.00003 & 0.18879 & 0.38980 & 1.00000 \\
\hline & Barley (Field interior) & 1.00000 & 0.00662 & 0.19913 & 0.05080 & 1.00000 \\
\hline & Potato (Field edge) & 1.00000 & 0.00000 & 0.10709 & 0.65606 & 1.00000 \\
\hline & Potato (Field interior) & 1.00000 & 0.00024 & 0.01332 & 0.00088 & 0.19644 \\
\hline & Maize (Field edge) & 0.68022 & 0.06550 & 1.00000 & 1.00000 & 1.00000 \\
\hline & Maize (Field interior) & 1.00000 & 0.01388 & 0.35419 & 0.35188 & 1.00000 \\
\hline & Rapeseed (Field edge) & 0.01388 & 1.00000 & 1.00000 & 0.04556 & 0.00780 \\
\hline & Rapeseed (Field interior) & 0.35419 & 1.00000 & 1.00000 & 1.00000 & 0.65606 \\
\hline & Rye (Field edge) & 0.35188 & 0.04556 & 1.00000 & 1.00000 & 1.00000 \\
\hline & Rye (Field interior) & 1.00000 & 0.00780 & 0.65606 & 1.00000 & 1.00000 \\
\hline & Triticale (Field edge) & 0.68022 & 0.29645 & 1.00000 & 1.00000 & 1.00000 \\
\hline & Triticale (Field interior) & 1.00000 & 0.00741 & 0.33759 & 0.15713 & 1.00000 \\
\hline & Wheat (Field edge) & 0.68022 & 0.00092 & 1.00000 & 1.00000 & 1.00000 \\
\hline & Wheat (Field interior) & 1.00000 & 0.00028 & 0.03824 & 0.00961 & 1.00000 \\
\hline & & $\begin{array}{c}\text { Triticale } \\
\text { (Field edge) }\end{array}$ & $\begin{array}{c}\text { Triticale } \\
\text { (Field interior) }\end{array}$ & $\begin{array}{c}\text { Wheat } \\
\text { (Field edge) }\end{array}$ & $\begin{array}{c}\text { Wheat } \\
\text { (Field interior) }\end{array}$ & \\
\hline & Barley (Field edge) & 0.74980 & 1.00000 & 1.00000 & 0.75554 & \\
\hline & Barley (Field interior) & 0.15348 & 1.00000 & 0.10725 & 1.00000 & \\
\hline & Potato (Field edge) & 0.65754 & 1.00000 & 1.00000 & 0.11376 & \\
\hline & Potato (Field interior) & 0.00391 & 1.00000 & 0.00102 & 1.00000 & \\
\hline & Maize (Field edge) & 1.00000 & 0.42768 & 1.00000 & 0.04556 & \\
\hline & Maize (Field interior) & 0.68022 & 1.00000 & 0.68022 & 1.00000 & \\
\hline & Rapeseed (Field edge) & 0.29645 & 0.00741 & 0.00092 & 0.00028 & \\
\hline & Rapeseed (Field interior) & 1.00000 & 0.33759 & 1.00000 & 0.03824 & \\
\hline & Rye (Field edge) & 1.00000 & 0.15713 & 1.00000 & 0.00961 & \\
\hline & Rye (Field interior) & 1.00000 & 1.00000 & 1.00000 & 1.00000 & \\
\hline & Triticale (Field edge) & 1.00000 & 0.35157 & 1.00000 & 0.04960 & \\
\hline & Triticale (Field interior) & 0.35157 & 1.00000 & 0.35652 & 1.00000 & \\
\hline & Wheat (Field edge) & 1.00000 & 0.35652 & 1.00000 & 0.01962 & \\
\hline & Wheat (Field interior) & 0.04960 & 1.00000 & 0.01962 & 1.00000 & \\
\hline
\end{tabular}

General linear bypotheses \& multiple comparisons for parametric models (results are given on the log, not the response scale; confidence level used: 0.95). Crop $=$ Cereal:

\begin{tabular}{|c|c|c|c|c|c|}
\hline Adj_habitat & 1smean & Std. Error & $\mathrm{df}$ & lower.CL & upper.CL \\
\hline Adjacent Field interior & 0.8536029 & 0.10180134 & 241 & 0.6530688 & 1.054137 \\
\hline Adjacent Arable field & 1.7543007 & 0.12933089 & 241 & 1.4995374 & 2.009064 \\
\hline Adjacent Grass strip & 2.4306114 & 0.04248508 & 241 & 2.3469219 & 2.514301 \\
\hline Adjacent Ditch margin & 2.3185162 & 0.10239739 & 241 & 2.116808 & 2.520224 \\
\hline Adjacent Hedge & 2.1654107 & 0.11872812 & 241 & 1.9315333 & 2.399288 \\
\hline \multicolumn{6}{|l|}{ poolCrop = Maize: } \\
\hline Adj_habitat & lsmean & Std. Error & df & lower.CL & upper.CL \\
\hline Adjacent Field interior & 0.6278297 & 0.12902022 & 241 & 0.3736785 & 0.881981 \\
\hline Adjacent Arable field & 1.5285276 & 0.14665129 & 241 & 1.2396456 & 1.81741 \\
\hline Adjacent Grass strip & 2.2048383 & 0.08390407 & 241 & 2.0395593 & 2.370117 \\
\hline Adjacent Ditch margin & 2.0927431 & 0.12108391 & 241 & 1.8542252 & 2.331261 \\
\hline Adjacent Hedge & 1.9396376 & 0.14837284 & 241 & 1.6473644 & 2.231911 \\
\hline \multicolumn{6}{|l|}{ poolCrop = Potato: } \\
\hline Adj_habitat & lsmean & Std. Error & $\mathrm{df}$ & lower.CL & upper.CL \\
\hline Adjacent Field interior & 1.1128158 & 0.12410358 & 241 & 0.8683496 & 1.357282 \\
\hline Adjacent Arable field & 2.0135137 & 0.14987294 & 241 & 1.7182855 & 2.308742 \\
\hline Adjacent Grass strip & 2.6898243 & 0.07982952 & 241 & 2.5325717 & 2.847077 \\
\hline Adjacent Ditch margin & 2.5777291 & 0.12215186 & 241 & 2.3371075 & 2.818351 \\
\hline Adjacent Hedge & 2.4246236 & 0.14183595 & 241 & 2.1452272 & 2.70402 \\
\hline \multicolumn{6}{|l|}{ poolCrop = Rapeseed: } \\
\hline Adj_habitat & lsmean & Std. Error & df & lower.CL & upper.CL \\
\hline Adjacent Field interior & 1.3957364 & 0.113675 & 241 & 1.171813 & 1.61966 \\
\hline Adjacent Arable field & 2.2964342 & 0.13448715 & 241 & 2.0315139 & 2.561355 \\
\hline Adjacent Grass strip & 2.9727449 & 0.06124566 & 241 & 2.8520997 & 3.09339 \\
\hline Adjacent Ditch margin & 2.8606497 & 0.11114617 & 241 & 2.6417077 & 3.079592 \\
\hline Adjacent Hedge & 2.7075442 & 0.13488665 & 241 & 2.4418369 & 2.973251 \\
\hline
\end{tabular}




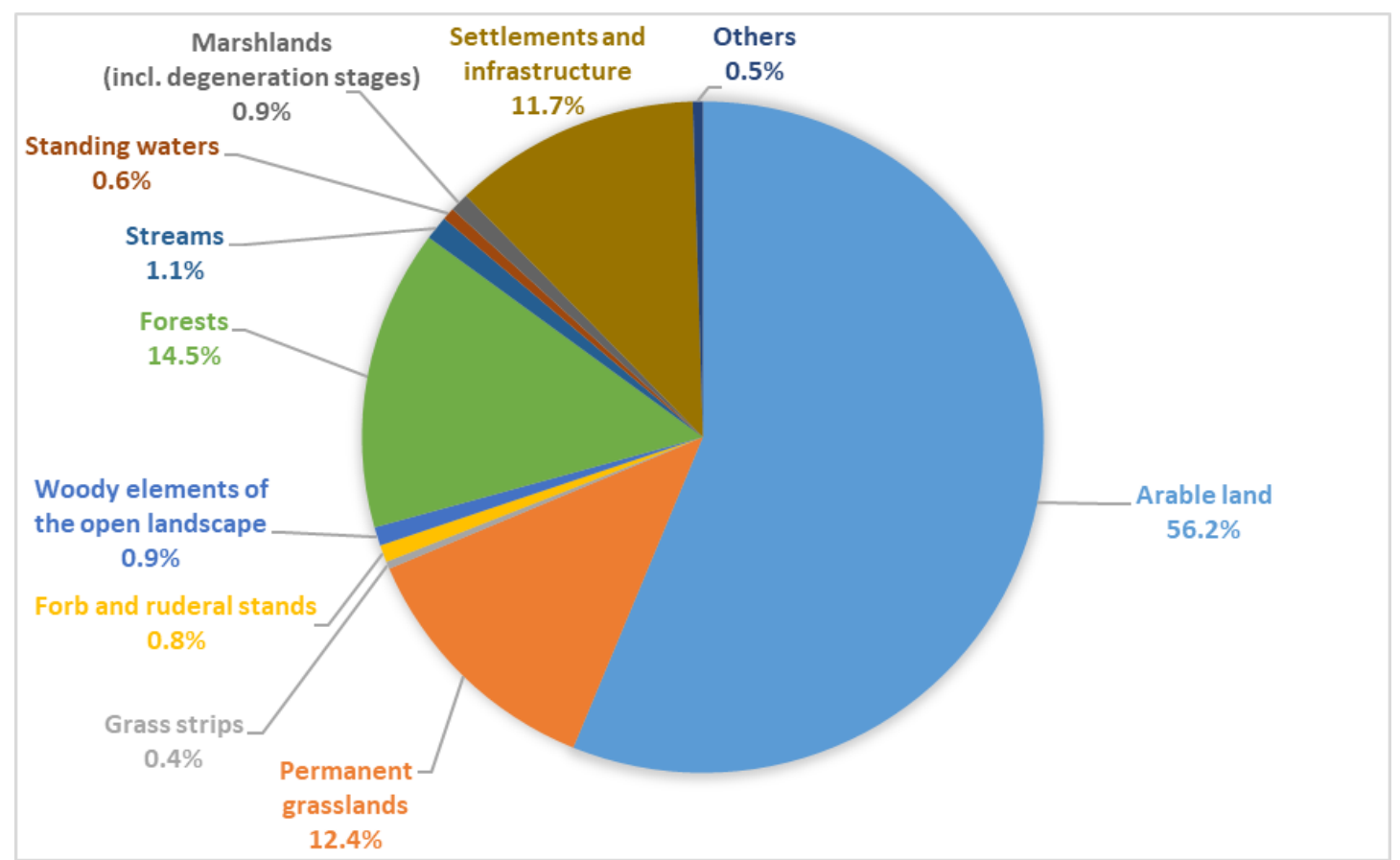

Figure S1: Relative frequency of habitat types in the study region in $2016(\%$ of total area $=132,275 \mathrm{ha})$, based on habitat mapping according to the key of Drachenfels (2016) using data of the two district administrations Nienburg (2016) and Diepholz (2016). Habitat types were categorized into the following groups: 'arable land', 'settlements and infrastructure' (sealed area and related semi-natural habitats such as cemeteries, gardens or roadside greenery), 'grass strips' (usually found between arable and non-arable habitats, e.g. along agricultural access tracks), 'forb and ruderal stands', 'forests', 'marshlands (incl. degeneration stages)', 'permanent grasslands', 'standing waters', 'streams' and 'woody elements of the open landscape' mainly include hedges and scrub $(85 \%)$ and a small percentage of single trees, tree rows and orchards (15\%); not clearly assignable habitats were categorized as 'others' (including headwater areas, dwarf shrub heathlands, inland dunes, bare soil areas); full references see main manuscript \& File S1 Supplementary methods.

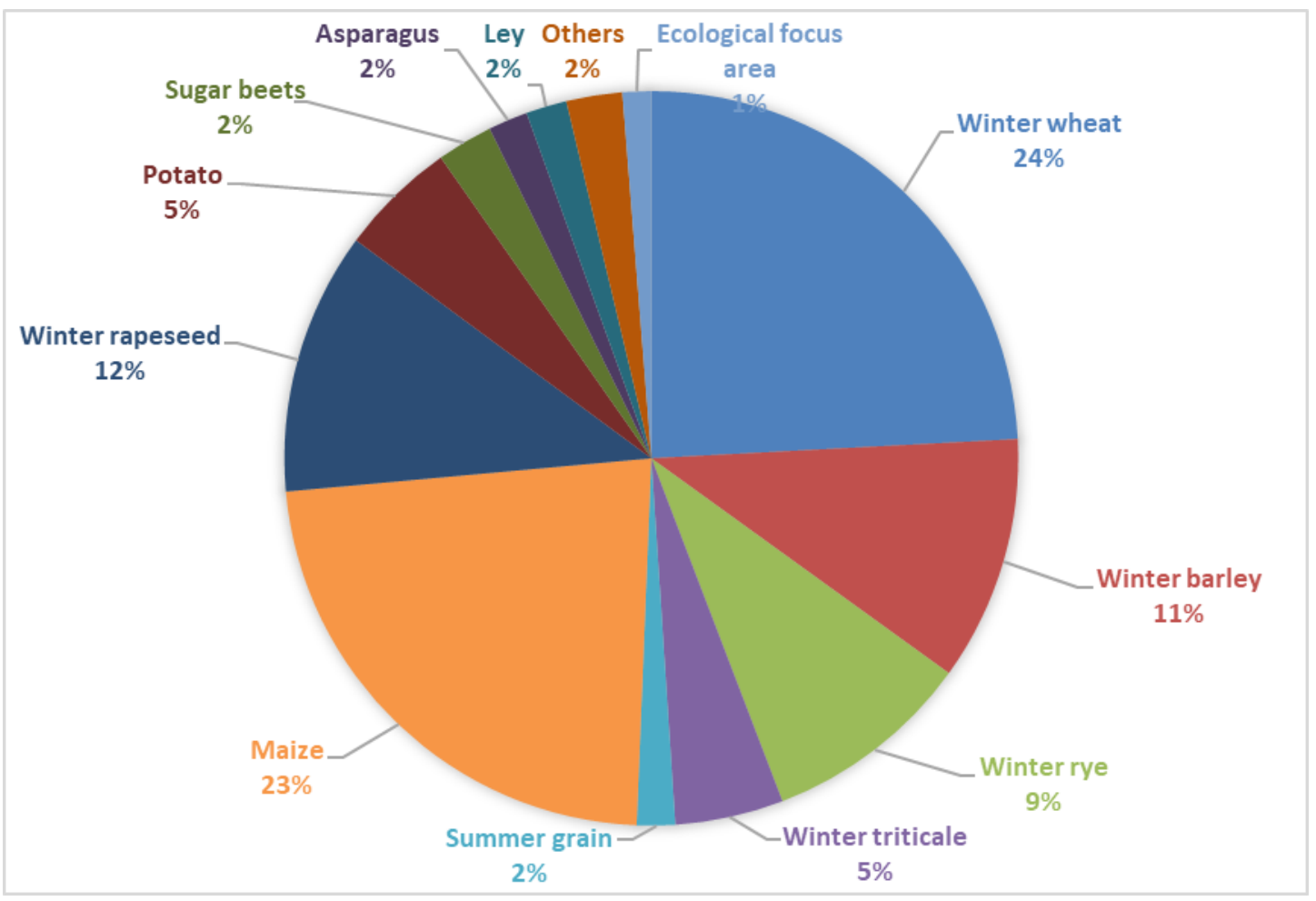

Figure S2: Proportion of crops in the farmland of the study region in 2016 [\% of total farmland area $=73,790$ ha]. Data from the Land Parcel Identification System (LPIS) as part of the Integrated Administration and Control System (IACS); provided by the Ministry of Food, Agriculture and Consumer Protection, Lower Saxony (EU, 2009a, 2009b; ML, 2016; full references see main manuscript \& File S1 Supplementary methods). 


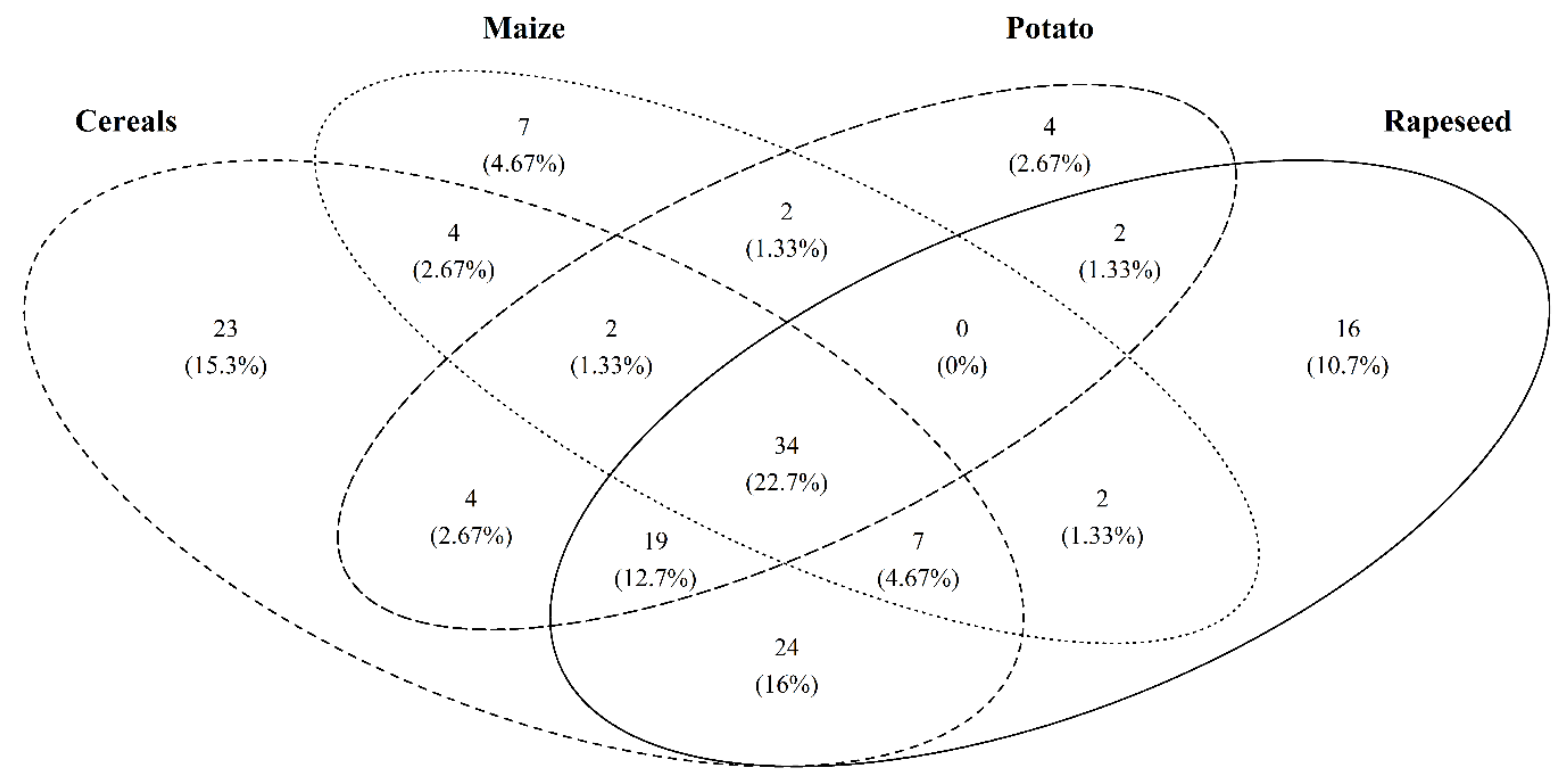

Figure S3: Plant species occurring only in one crop or with wider distribution across crop types (total species number: 150; crops and woody seedlings excluded); triticale, rye, wheat and barley were combined to the group (winter) cereals; $\mathrm{n}=30$ for edge plots of barley, maize, rapeseed, rye and wheat, potato $=28$, triticale $=22, \mathrm{n}$ for interior plots $=10$ per crop type.

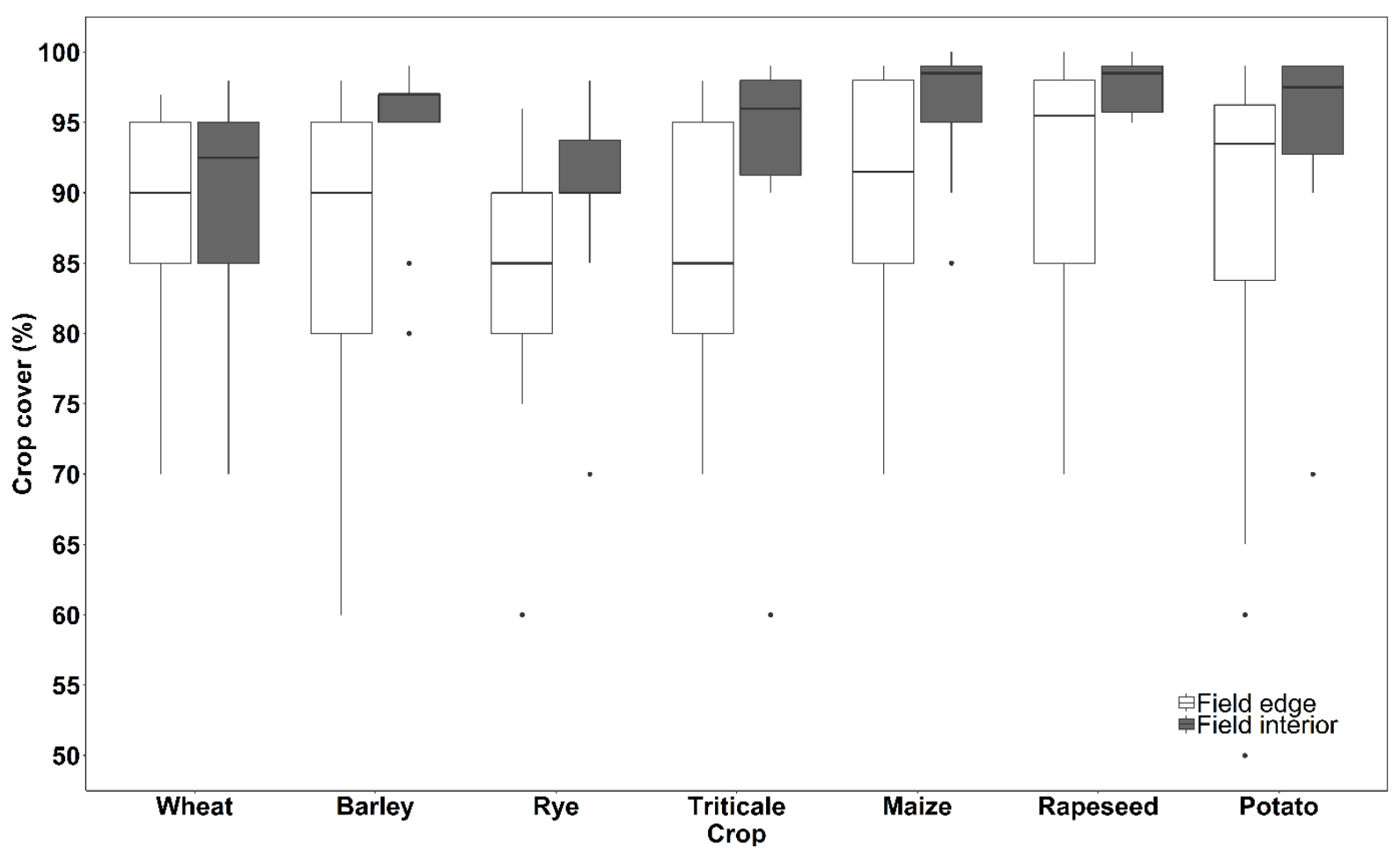

Figure S4: Crop cover (\%) in field edge and field interior plots; $\mathrm{n}=30$ for edge plots of barley, maize, rapeseed, rye and wheat, potato $=28$, triticale $=22$; interior plots $=10$ per crop. 


\section{Chapter 3}

\section{Flower strips, conservation field margins and fallows promote the arable flora in} intensively farmed landscapes: results of a 4-year study

Alexander Wietzke, Klara Albert, Erwin Bergmeier, Laura M. E. Sutcliffe, ClaraSOPHIE VAN WAVEREN, CHRISTOPH LEUSCHNER

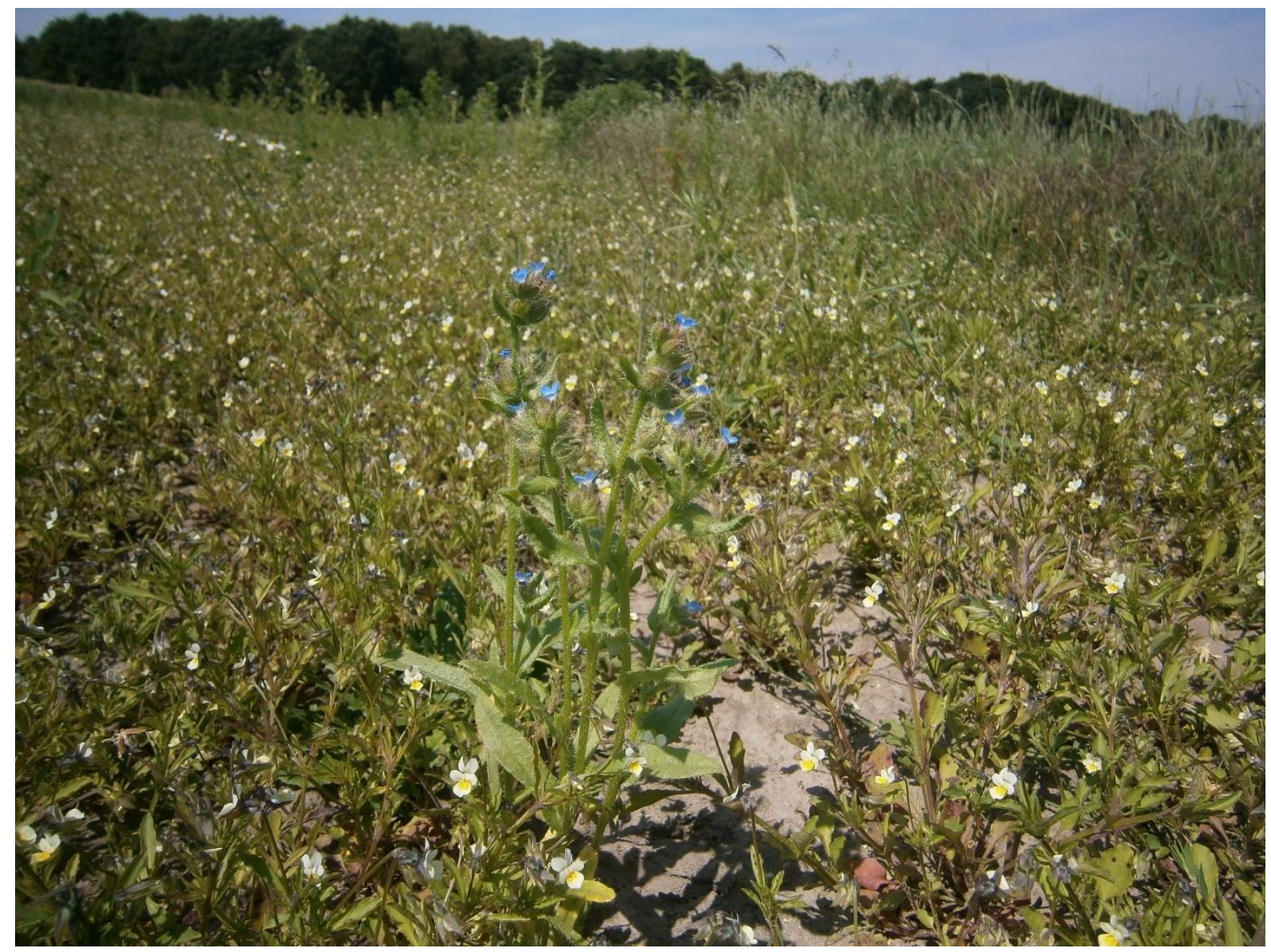

Published in

Agriculture, Ecosystems and Environment, Volume 304 (2020).

bttps:// doi.org/10.1016/j.agee.2020.107142

Please note: There have been marginal corrections to the published article (e.g. the correction of minor typing errors in the raw data table). A Corrigendum to the published article can be accessed at: https://doi.org/10.1016/j.agee.2021.107357. The corrected raw data Table A.6 of Chapter 3 has been uploaded to the eDiss repository. 


\begin{abstract}
Agri-environment measures to support biodiversity in intensively used agricultural landscapes have been implemented in many European countries, but the large-scale downward trend in many species groups continues. The arable flora is one of the species groups that suffered the steepest declines in European cultural landscapes. Despite their fundamental importance in agroecosystems as the basis of the food web, few studies have tested the effectiveness of different agri-environment measures on the arable flora in replicated field trials over several consecutive years. In a four-year participative project with the farmers, we recorded the effects of four agri-environment measures, namely conservation field margins (CFM), annual fallow strips (fallows), alternately managed biennial flower strips (AFS), and perennial flower strips (PFS), on the species richness, plant cover and composition of the non-crop vegetation in 67 intensively managed arable fields in Northwest Germany.

Compared to conventionally managed field edges, all measures led to a large increase in total plant cover (median values across measures and years: 68.5 vs. $9.5 \%$ ) and doubling of species richness (21 vs. 9 species per plot), promoting not only generalist species but also the typical arable flora (17 vs. 7 species, 56.5 vs. $8 \%$ cover). Of the 207 plant species recorded during sampling, 43 (including 16 typical arable species) were solely found in measures. All measures promoted forbs more than graminoids, while annual species benefited especially from CFM and fallows. The overall effects on diversity and cover remained stable over the 3 -yr implementation period but were significantly influenced by interannual weather fluctuation. Spillover effects of the measures into adjacent conventionally managed crops were not detectable.

We conclude that, if well-managed, all tested measures can have a positive effect on the native arable flora even in long-term intensively farmed landscapes. However, especially CFM and the annual fallows are particularly effective in naturally restoring and maintaining arable plant diversity. The limited occurrence of $1 / 4$ of typical arable plant species on less than four fields indicates severely impoverished soil seed banks in intensive farmland. Therefore, the reintroduction of native, rare arable plant species through seed mixtures should be considered in order to promote local diversity and save local populations of these species.
\end{abstract}

Keywords: Arable weeds; Common Agricultural Policy; Biodiversity conservation; Agrienvironment measure; Segetal flora

\title{
3.1 Introduction
}

The ongoing loss of global biodiversity is particularly acute in intensively used agricultural landscapes (Butchart et al., 2010; Flohre et al., 2011; Sánchez-Bayo and Wyckhuys, 2019; Storkey et al., 2012). The conventionally managed intensive farmland of Central and Western Europe has experienced a dramatic impoverishment of the arable flora and population collapses in many formerly common weed species. Most of the 287 plant species typically associated with arable communities in Germany are annuals, whose reproductive cycle is adapted to agricultural management, in particular regular soil disturbance (Hofmeister and Garve, 2006). Much of the 
typical arable flora of Germany consists of competitively weak species, and only about 30 species are able to compete more strongly with crops and may have harmful effects on crop yield (Hofmeister and Garve, 2006). However, in the last 60 to 70 years, a strong decline in the arable flora has made it one of the most endangered plant groups in Germany and elsewhere in Europe (Leuschner and Ellenberg, 2017; Richner et al., 2015; Storkey et al., 2012; Van Calster et al., 2008) with a high proportion of red-listed taxa (for Germany 35 \%; Korneck et al., 1996; Lang et al., 2016). This decline has strongly affected the field interior, whilst field edges (up to ca. $2 \mathrm{~m}$ into the crop) usually support somewhat higher arable plant diversity and abundance, reflecting less intensive management and possible spillover effects from adjacent habitats (Aavik et al., 2008; Batáry et al., 2017; Fried et al., 2009; Meyer et al., 2013; Nagy et al., 2018). Such strong losses in diversity and abundance of primary producers negatively impact the trophic web and ecosystem functioning of agricultural landscapes (Hyvönen and Huusela-Veistola, 2008; Marshall et al., 2003; Médiène et al., 2011). For instance, arable plants function as food sources for soil macrofauna, phytophagous insects (and, thus, insectivorous bird species), granivorous birds and pollinating insects (Hyvönen and Huusela-Veistola, 2008; Marshall et al., 2003, Franke et al., 2009). Thus, maintaining at least basic levels of non-crop vegetation richness and abundance in arable landscapes is crucial for both sustaining ecosystem functions and conserving overall farmland biodiversity.

To halt biodiversity loss in agricultural landscapes, the European Union introduced the requirement for Member States to implement agri-environment schemes in its Common Agricultural Policy (European Commission, 2013). Literature reviews and meta-analyses on the effectiveness of these measures revealed an overall positive influence on total farmland diversity and species abundance, although the effects varied considerably with landscape composition, management regime, location and organism group (Barral et al., 2015; Batáry et al., 2011, 2012, 2015; Gonthier et al., 2014). Analyses on the country level similarly demonstrated positive effects of agri-environment measures on arable plant diversity in Europe (e.g. Fischer and Milberg, 1997; Geiger et al., 2010; Kleijn et al., 2006; Walker et al., 2007). Fewer studies investigated the effects of agri-environment measures on plant species cover and abundance, but they demonstrate in general positive impacts (e.g. Fischer and Milberg, 1997; Walker et al., 2007).

Biodiversity conservation action in intensive farmland needs more clarity on which measure type is most effective in promoting arable plant diversity in a given landscape. Various studies have evaluated the effect of a particular measure type on the arable vegetation (e.g. Albrecht et al., 2016; Tscharntke et al., 2011; Wagner et al., 2017), or compared organic and conventional farming (e.g. Batáry et al., 2012; Gabriel et al., 2013; Romero et al., 2008), but investigations comparing the effects of different types of measures over several years are still scarce (but see Eggenschwiler et al., 2009; Venclova et al., 2006; Walker et al., 2007). In addition, the impact of flower strips on arable plant species richness and abundance has also rarely been examined (e.g. Rode et al., 2018), as this measure targets primarily insects and birds.

With this study in conventionally managed intensive farmland, we compare four different agrienvironment measures (annual fallow strips (hereafter termed fallows), conservation field margins (CFM), alternately managed biennial flower strips (AFS), and perennial flower strips (PFS)) with respect to their impact on the non-crop arable plant vegetation. These measures differ with respect to duration (annual vs. perennial), soil disturbance (with or without) and the mode of non-crop plant introduction (seeding vs. natural colonization). In contrast to agri-environment 
measures funded through the European Common Agricultural Policy, the measures investigated here were defined by the farmers implementing them in collaboration with ecologists and representatives of the local agricultural administration. The participating farmers were compensated for their yield loss and management costs.

We test the following hypotheses: i) The studied measures increase both total and arable plant species richness and abundance (cover) in comparison to conventionally farmed field edges; ii) the non-crop arable vegetation differs between the four measure types and from that of conventionally managed field edges in terms of species composition, plant life forms and trait spectra; and iii) species introduced through the agri-environment measures will not establish in the adjacent, conventionally managed crop field area, i.e. infield spillover is of low importance.

\subsection{Material and Methods}

\subsubsection{Study region}

This study was conducted in the two districts Nienburg (centroid: N 52³6'32.5334", E $\left.9^{\circ} 6^{\prime} 49.7118^{\prime \prime}\right)$ and Diepholz (N 52 43'41.4940", E 8 42'4.1629") in Lower Saxony, Northwest Germany (Appendix Fig. A.1). The study region is characterized by intensive conventional crop production on sandy to loamy Cambisols, Podzols, Luvisols and Gleysols (Federal Institute for Geosciences and Natural Resources, 2013). The climate is cool-temperate with suboceanic character with $662 \mathrm{~mm}$ mean annual precipitation and a mean annual temperature of $10.3{ }^{\circ} \mathrm{C}$ in Nienburg and $683.6 \mathrm{~mm} \mathrm{/} 10.1{ }^{\circ} \mathrm{C}$ in Diepholz (climate data from 2013-2017; German Meteorological Service, 2019). In consultation with 19 participating farmers, sixty-seven arable fields were chosen for the implementation of agri-environment measures, aiming to ensure wide dispersion within the study region.

\subsubsection{Agri-environment measures}

The selection of measures was decided together with the participating farmers in order to balance the benefit for biodiversity and the burden for the farmer in terms of labor effort and yield reduction (which were financially compensated: $700 € \mathrm{ha}^{-1}$ ). As far as possible, the measures were adapted to meet the farmers' preferences with respect to practicability. For instance, the sowing date of flower strips was postponed from April 15 (as it is required according to the agrienvironment measure rules of the state) to early May which is more practicable in the region to avoid late frost damage. Much effort was devoted to advice and information of the farmers in order to increase acceptance and improve the quality of the measures.

In general, measures were implemented in the form of strips at the edge of the field with the width varying from 5 to $18 \mathrm{~m}$ (in one case $3 \mathrm{~m}$ ). The strips were placed directly adjacent to the conventionally managed field, which was cultivated with winter crops (barley, rye, rapeseed, triticale or wheat) or summer crops (potato or maize) according to the normal crop rotation of the participating farmer (see Table A.1 in the Appendix for replicate numbers). In eight cases, measures were implemented on larger field sections or covered even the whole field and thus lacked a directly neighboring intensively cultivated field. In the latter case, we used a conventionally managed field close to the measure $($ c. $15 \mathrm{~m}$ ) as control, but then no spillover 
plots could be established. The separate control fields showed no conspicuous deviations in species diversity or cover, therefore we assumed they supported similar species pools. The area of the individual measures ranged between 400 and $6200 \mathrm{~m}^{2}$.

In autumn 2016 / spring 2017, 47 individual measures belonging to three measure types were implemented, namely annual fallow strips (fallows), conservation field margins (CFM) and alternately managed biennial flower strips (AFS). An additional 20 measure areas were established from autumn 2017 / spring 2018 onwards, including a fourth measure type, the perennial flower strips (PFS) (for replicate numbers in the different years see Table 1). In all measure types, the application of fertilizers or pesticides, mechanical weeding and access by machinery (except for harvest and mowing) was forbidden. Tillage was done with mouldboard plough (up to $30 \mathrm{~cm}$ ) and the direction of tillage in the adjacent field could be either parallel or perpendicular to the measure (depending on the field position of the measure).

Fallows were tilled once per year (autumn or spring, depending on the cultivation of summer or winter crops on the adjacent field). After tillage, the vegetation succession on the fallows proceeded without disturbance. Alternately managed biennial flower strips (AFS) were sown at the start of May using a seed mixture of 27 ornamental, crop and native plant species at a seed density of $1 \mathrm{~g} \mathrm{~m}^{-2}$ (12 annual and 15 perennial taxa, including five arable plants; see Table A.2 in the Appendix). From the second year onwards, half of the area of each strip was mown and the plant biomass left on the field, the area tilled and re-sown to generate structural diversity. In the following year, the other half of the strip was treated in the same manner. The regular soil disturbance on half of the area should support the establishment of arable plant species from the seed bank or from seed rain, which otherwise would have been suppressed by the dense cover of the sown flower strip. Perennial flower strips (PFS) were established by sowing a seed mixture of widespread perennial native herbaceous species (seed density $1 \mathrm{~g} \mathrm{~m}^{-2}$; in total 34 taxa, including nine native arable plants; Table A.3 in the Appendix). Sowing took place at the beginning of May 2018. The strips were mown annually between August and September and the plant biomass left on the field. Conservation field margins (CFM) were implemented by sowing the crop at lower seed density to reduce crop cover and improve the growth conditions for arable plant species. In 2016/2017, we applied $70 \%$ of the usual sowing density, and $50 \%$ from autumn 2017 onwards. This measure was applied in coordination with the regular crop rotation on the fields (see Table A.4 in the Appendix). Table A.5 in the Appendix summarizes the key management steps of the implemented measure types and show their average width, length and area.

\subsubsection{Vegetation survey}

Monitoring of arable vegetation and plants sown in flower strips took place from 2016 to 2019 (end of May to the beginning of August) in plots of $50 \times 2 \mathrm{~m}$ size $\left(100 \mathrm{~m}^{2}\right)$. We recorded the species richness and cover (Londo scale; Londo, 1976) of the chosen 67 conventionally managed arable fields (see Fig. A.1 in the Appendix; field size ranged between 0.4 and 20 ha) before the measures were implemented (pre-survey) and repeated the monitoring of the same plots after the implementation of the measures (before-after-control-impact approach). In addition, we recorded the arable vegetation of intensively managed fields in plots in the field interior (c. $20 \mathrm{~m}$ into the field), in so-called 'spillover plots' directly adjacent to the implemented measures in the field, and in field edge plots in the field opposite to the measure. The latter plots served as control to the 
measures in each implementation year. The field edge plots included the outermost furrow of the field and extended $2 \mathrm{~m}$ into the field. The monitoring design is shown in Fig. A.2 in the Appendix. Immature plants not identified to the species level were noted as genus (e.g. Setaria spec.). Nomenclature follows Buttler (2018). The raw vegetation data are compiled in Table A.6 in the Appendix. To account for the vegetation heterogeneity created by the alternate management of the AFS sites from the second year onwards, plots were located in equal shares in newly established and last-year measure strips, resulting in 7 and 6 plots in new and last-year measures, respectively, in the second implementation year, and 6 and 7 plots in the third year. In five cases, were the measure strip was relatively small, the plot was located in the strip center, covering both the newly established and last-year strip. Thus, the displayed results of plant species richness and cover from implementation year two onwards cover the structural heterogeneity in the AFS strips, which is created by the alternating management regime.

\subsubsection{Environmental data}

In order to analyze the influence of weather conditions on non-crop species richness and cover, we used interpolated high-resolution $\left(1 \times 1 \mathrm{~km}^{2}\right)$ gridded climate data (monthly precipitation totals and monthly averaged daily air temperatures) for the period from April to July in the four years (2016-2019, Climate Data Center, version v1.0 (German Meteorological Service, 2019); data download and interpolation with the $\mathrm{R}$ packages 'rdwd' (Boessenkool, 2019), 'raster' (Hijmans, 2019) and 'reshape' (Wickham, 2007)). The interval from April to July was chosen, as it covers the period of germination and development of the vegetation surveyed by us in summer. Climate diagrams for Nienburg and Diepholz are depicted in Fig. A.3 in the Appendix.

Soils in the study region developed from loamy to sandy glacial deposits with moderate to high fertility. Pre-analyses found no significant effect of soil type on plant diversity and species composition in the region (Wietzke et al., unpublished). Therefore, soil parameters were not included in the statistical analysis of the vegetation data of this study.

\subsubsection{Statistical analysis}

\subsubsection{Data preparation}

We used the statistical software R 3.5.3 (R Core Team, 2019) for data analysis. Species cover data (Londo scale) were transformed into percentage cover values (following Leyer and Wesche, 2008). In the analyses, species which are not part of the native herbaceous flora of the study region (seedlings of woody plants, crops and ornamental taxa) were excluded. Sown native plant species in the AFS and PFS measures were considered, as they cannot be distinguished with certainty from genuine plants originating from the diaspore bank or seed rain. Species numbers and total cover were calculated for a) all herbaceous species (grasses included), b) typical (noncrop) plant species of arable habitats according to the list of Hofmeister and Garve (2006; hereafter referred to as typical arable plants), c) indicators of High Nature Value arable land as defined for Germany by Hünig and Benzler (2017; hereafter referred to as HNV species), d) threatened arable plants according to the Red List of Lower Saxony (Garve, 2004), e) the proportion of forbs and graminoids (i.e. taxa of the families Poaceae, Juncaceae, Cyperaceae and Typhaceae), and $\mathrm{f}$ ) the proportion of annuals and perennials according to specifications made in 
Klotz et al. (2002). The assignment of the species to these plant groups is shown in Table A.7 in the Appendix. In eight cases, plant identification was only possible to the genus level. Total cover is the sum of the cover of all species and thus can exceed $100 \%$.

In the analyses, the surveyed plots were grouped according to the year of measure implementation (pre-survey, first, second and third year). The first year of implementation was 2017 for most measure areas but some were established later, with 2018 as first implementation year: see Table 1 for an overview of the replicates per year.

Table 1 Number of replicates per plot type in each calendar year and year of implementation. Plot types: alternately managed biennial flower strip (AFS), perennial flower strip (PFS), conservation field margin (CFM), annual fallow strip (Fallow), spillover plot next to the implemented measure, field edge (control) within the intensively managed field, field interior: plot in the intensively managed field interior; pre-survey plots in the implementation year ' 0 ' are summed within the field edge plots (in 2017 no field interior plots were surveyed).

\begin{tabular}{lcccc|cccc|c}
\hline Plot type & \multicolumn{4}{c}{ Calendar year } & \multicolumn{3}{c|}{ Implementation year } & \multicolumn{2}{c}{ Total plots } \\
& $\mathbf{2 0 1 6}$ & $\mathbf{2 0 1 7}$ & $\mathbf{2 0 1 8}$ & $\mathbf{2 0 1 9}$ & $\mathbf{0}$ & $\mathbf{1}$ & $\mathbf{2}$ & $\mathbf{3}$ & \\
\hline AFS & - & 18 & 19 & 17 & - & 19 & 18 & 17 & $\mathbf{5 4}$ \\
PFS & - & - & 14 & 13 & - & 14 & 13 & - & $\mathbf{2 7}$ \\
CFM & - & 14 & 17 & 16 & - & 18 & 16 & 13 & $\mathbf{4 7}$ \\
Fallow & - & 15 & 16 & 15 & - & 16 & 16 & 14 & $\mathbf{4 6}$ \\
Spillover & - & 47 & 61 & 58 & - & 63 & 59 & 44 & $\mathbf{1 6 6}$ \\
Field edge & 45 & 62 & 66 & 61 & 60 & 67 & 63 & 44 & 234 \\
Field interior & 20 & - & 66 & 61 & 20 & 20 & 63 & 44 & $\mathbf{1 4 7}$ \\
\hline Total plots & $\mathbf{6 5}$ & $\mathbf{1 5 6}$ & $\mathbf{2 5 9}$ & $\mathbf{2 4 1}$ & $\mathbf{8 0}$ & $\mathbf{2 1 7}$ & $\mathbf{2 4 8}$ & $\mathbf{1 7 6}$ & $\mathbf{7 2 1}$ \\
Total fields & $\mathbf{4 5}$ & $\mathbf{6 2}$ & $\mathbf{6 6}$ & $\mathbf{6 1}$ & $\mathbf{6 0}$ & $\mathbf{6 7}$ & $\mathbf{6 3}$ & $\mathbf{4 4}$ & \\
\hline
\end{tabular}

\subsubsection{Analysis of the effects of agri-environment measures and weather conditions on species richness and abundance}

To analyze the effect of the four measure types on non-crop plant diversity and cover, we performed negative binomial generalized mixed effects models ( $\mathrm{R}$ package 'glmmTMB'; Brooks et al., 2017) using the corresponding field edge as control. In addition, field interior plots were included in our study to serve as a reference level for the influence of the most intensive management on the non-crop arable vegetation. To investigate possible spillover effects from the measure site into the field, the spillover plots were compared with the field edge plots of the corresponding year. We introduced the different plot types as fixed factor (plots in the four measure types, and field edge, interior and spillover plots of the years 0 to 3). To account for possible effects of the farm with its specific management practices, farm location and previousyear effects in the fields, we introduced the farm and the studied arable field (field_ID) as random factors. Since 'farm' had only a marginal effect, this random factor was excluded in the final model. To test the significance of response variables, likelihood-ratio tests (Type II sums of squares; Fox et al., 2011) and post-hoc pairwise comparisons of estimated marginal means were conducted (Lenth, 2019). To test for the influence of precipitation and average temperature from April to July (regionalized grid data) in the different study years on herbaceous species richness and cover, we additionally performed negative binomial generalized mixed effects models with plot type and each one weather variable (temperature and precipitation were correlated to each other). 


\subsubsection{Analysis of the effect of agri-environment measures on species composition}

The floristic dissimilarity (beta diversity) of the plot types was calculated as distance to group spatial medians based on Jaccard's dissimilarity index (function 'betadisper' in the $\mathrm{R}$ package 'vegan'; Oksanen et al., 2019). Differences in beta diversity between the studied plot types were tested by fitting a linear mixed effects model (R package 'nlme'; Pinheiro et al., 2019) with the different fields (field_ID) as random factor. We performed non-metric multidimensional scaling (NMDS with two to three dimensions; Hellinger transformed abundance data; Legendre and Gallagher, 2001) for detecting differences in community composition between the measure types and controls. In this analysis, we only considered species which occurred more than two times in the dataset. The degree of overlap of species pools between the different plot types was analyzed with Venn diagrams (Chen, 2018).

Table A.8 in the Appendix presents the different models and statistical results.

\subsection{Results}

\subsubsection{Effects of agri-environment measures on species richness and abundance}

In total, we found 207 herbaceous plant species (crops, sown ornamentals, woody seedlings and eight species only determinable to genus level excluded) in the 67 studied arable fields over the 4yr study period (2016-2019, Table A.7 in the Appendix). 122 of these species are considered as typical arable plants in Germany (according to Hofmeister and Garve, 2006). The influence of plot type on species richness and cover was highly significant for all herbaceous species and the typical arable plants $(\mathrm{p}<0.0001$; Table A.8 in the Appendix). Compared to the field edge plots, the field interior showed significantly lower species numbers and, in most cases, cover values (Fig. 1 and Fig. A.4 in the Appendix). Even though interannual fluctuation was substantial, the richness and cover of all herbaceous species in the plots of the measures were higher (medians across all measures and years: 21 species and $68.5 \%$ cover) than in the control field edge plots (9 species and $9.5 \%$ cover; Fig. A.4 in the Appendix). However, the species richness of the CFM and AFS plots differed in the second year not significantly from the pre-survey plots (for CFM and AFS) and in the third year from the field edge plots (for CFM). We found no clear differences between the four measure types with respect to the increase in total plant species richness and cover compared to the control.

Similar patterns of species numbers and cover emerged when the typical arable plants are considered (medians across all years: 17 species and 56.5\% cover in the measures plots pooled over all four types vs. 7 species and $8 \%$ cover in the field edge plots; Fig. 1). In contrast to the full species count (all herbaceous species), however, the number of typical arable plant species did not increase in the PFS plots (Fig. 1 and Fig. A.4 in the Appendix).

Spillover effects were not significant in any of the three study years, i.e. a positive influence of the measures on the species richness and cover in the directly adjacent, conventionally managed arable field (spillover plots) was lacking. This is evident from the comparison of spillover plots to the corresponding field edge plots (Fig. 1 and Fig. A.4 in the Appendix). 
Comparison of the study years reveals a marked weather influence on the species richness and cover of all herbaceous plants and the typical arable plants ( $<<0.02$; Table A.8 in the Appendix). Higher precipitation from April to July had a positive effect, higher temperatures in this period a negative effect. Weather effects and species numbers and cover of observed plots per calendar year are shown in the Appendix (Fig. A.5 and Fig. A.6).
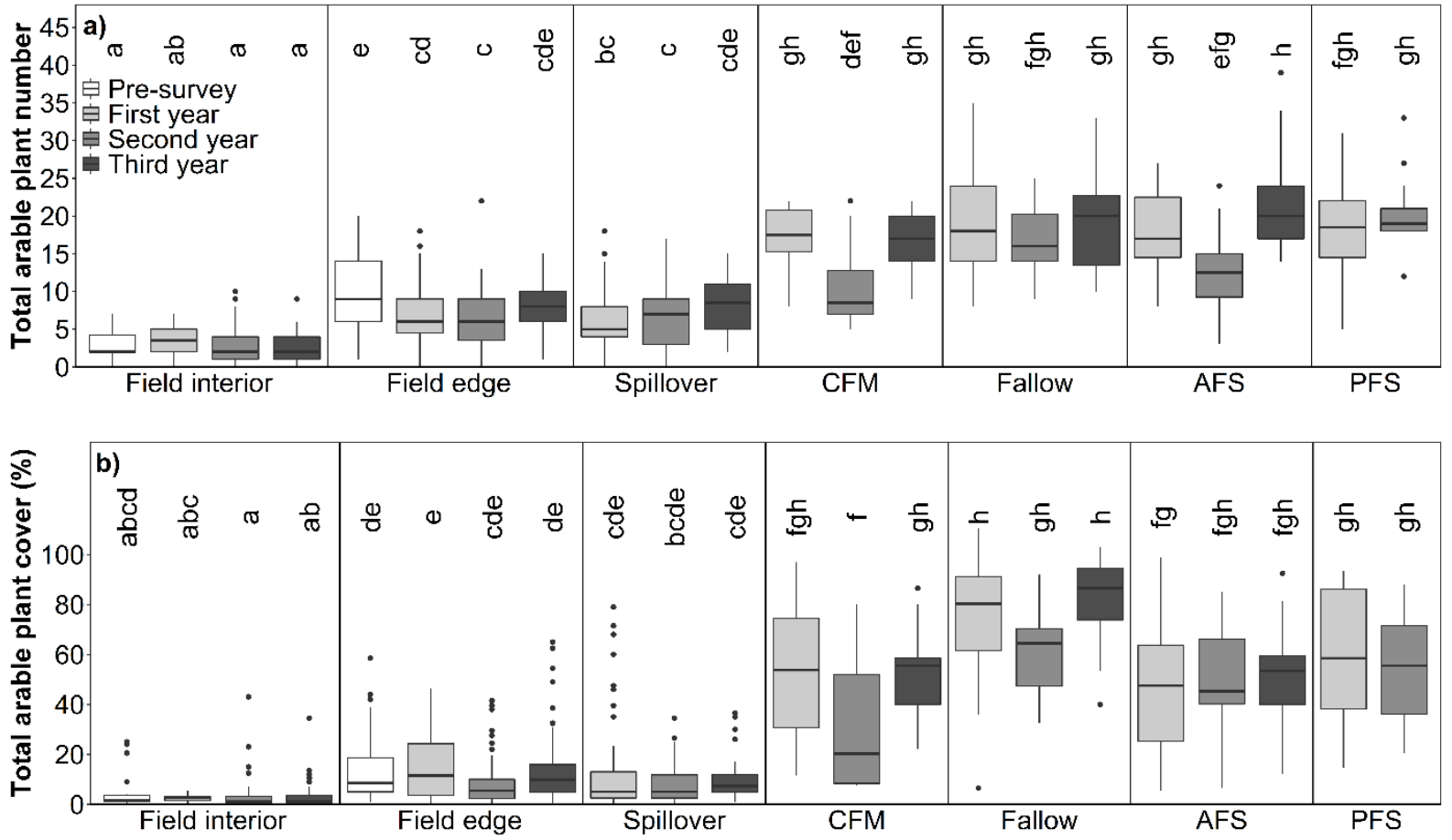

Fig. 1 Species richness (a) and cover (b) in $100 \mathrm{~m}^{2}$-plots of typical arable plants (according to the species list in Hofmeister and Garve, 2006) in the studied plot types (field interior, field edge as control, spillover, conservation field margin (CFM), fallow, alternately managed biennial flower strip (AFS) and perennial flower strip (PFS)) over the four years of the study (see legend). The total number of relevés was 721 (for details see Table 1). Significant differences between plot types and implementation years are indicated by different small letters (based on estimated marginal means and Tukey-adjusted comparisons); the models and statistical results are presented in Table A.8 in the Appendix.

\subsubsection{Effects of agri-environment measures on the species composition}

The total number of observed species (207) included 158 forbs and 49 graminoids, and 91 annuals and 116 perennials (Table A.7 in the Appendix). We found three regionally threatened species, namely Chenopodium bybridum, Galeopsis speciosa and Odontites vernus which occurred in only very few fields $(\leq 7)$ and with very low cover (Table A.6 and Table A.7 in the Appendix). Across all plot types and implementation years, the most frequent taxa (> 200 occurrences) were agrotolerant arable plants, notably Bromus sterilis, Chenopodium album, Elymus repens, Fallopia convolvulus, Galium aparine, Matricaria chamomilla, Polygonum aviculare and Viola arvensis (Table A.7 in the Appendix). In contrast, nearly a quarter of the typical arable plant species (27 of 122) were found in only three or even fewer fields, among them Erysimum cheiranthoides, Galeopsis speciosa, Scleranthus annuus and Urtica urens.

The separate analysis of forbs and graminoids shows that forbs profited more from the measures than graminoids. The species numbers and cover of forbs were consistently higher in measure plots than in field edge plots in all four measure types (Fig. A.7 in the Appendix). In contrast, graminoid species numbers increased only slightly above the field edge control, and this difference was not significant in several cases (an exception was the significantly higher graminoid species richness in the 2nd-year PFS plots; Fig. A.8 in the Appendix). Graminoid cover was 
significantly higher than in the control in most years for the fallow, AFS and PFS plots, while the CFM plots were similarly poor in graminoids as the field edge control in all study years.

Despite considerable variation between years, the species numbers and cover of annual plants were generally higher in the measure plots than in the field edge control plots, and this was valid for all four measure types (Fig. A.9 in the Appendix). While the cover of annual species remained at a constant level from the 1st to the 3rd year in the CFM and fallow plots, it tended to decrease in the AFS and PFS plots. The species number and cover of perennials were in general also higher in the measure plots than in the field edge control plots (Fig. A.10 in the Appendix), and this trend was more pronounced for the PFS plots than in the other three measure types. Over the three implementation years, the cover of perennials increased in the PFS, AFS and fallow plots, but not in the CFM plots. The annual:perennial ratio in terms of plant cover was rather large in fallow plots (median cover values across all years: $61.75: 11.75 \%$ ) and CFM plots $(37: 5$ $\%$ ), and smaller in AFS plots (31.75: $16.25 \%$ ), while it was close to 1 in PFS plots (27:26\%; Fig. A.9 and Fig. A.10 in the Appendix).

Of the 207 herbaceous non-crop species identified, 27 were indicators of High Nature Value (HNV) arable land. Although the plot-level richness of HNV species was generally quite low (ranging between 0 and 5 species in the four years), we found a positive effect of the measures on HNV species richness, as evidenced by the comparison with the field edge control plots (medians across all years: 3 species in the measures plots pooled over all four types vs. 1 species in the field edge plots; Fig. 2). Exceptions were the CFM and AFS plots in the second year of implementation and the PFS plots in the first year. In comparison to the median cover values of HNV species in the field edge plots $(0.5-1 \%)$, the measures CFM and fallow increased the HNV cover considerably (up to $23.25 \%$ in fallows of year three since establishment). In contrast, the measures AFS and PFS had only a very small effect on HNV species cover (median cover: $0.75-3.5 \%)$.
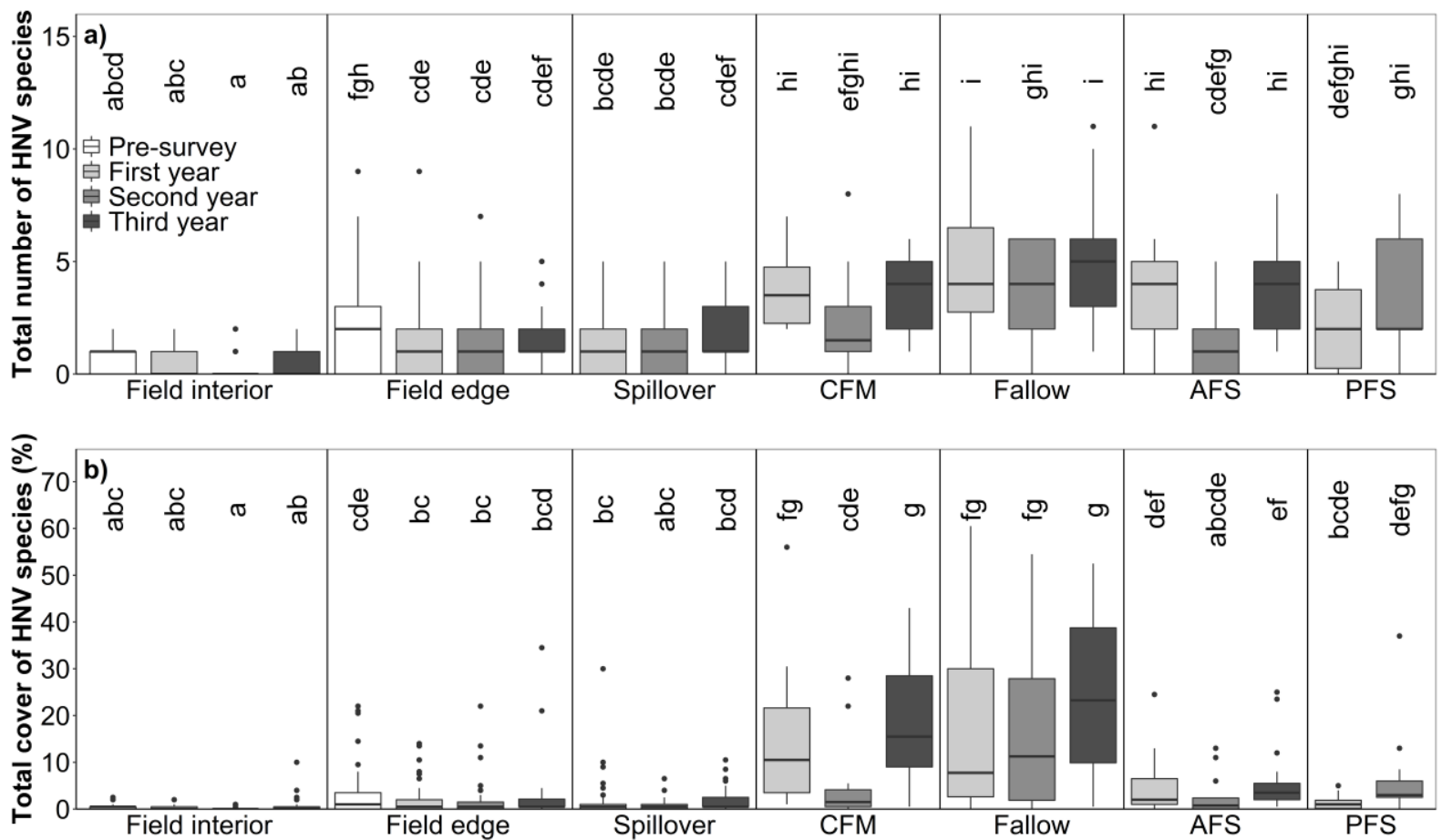

Fig. 2 Species richness (a) and plant cover (b) in $100 \mathrm{~m}^{2}$-plots of High Nature Value species of arable land (HNV species; according to Hünig and Benzler, 2017) in the studied plot types (field interior, field edge as control, spillover, CFM, fallow, AFS and PFS) over the four years of the study (see legend). The total number of relevés was 721 (for details see Table 1). Significant differences between plot types and implementation years are indicated by different small letters (based on estimated marginal means and Tukey-adjusted comparisons); the models and statistical results are presented in Table A.8 in the Appendix. 
Even though floristic dissimilarity tended to be higher in field interior, edge and spillover plots compared to the measure sites (distances to group spatial medians for a plot/year category ranging between 0.47 and 0.64; see Fig. A.11 in the Appendix), the pool of species overlapping between the seven observed plot types was large (Fig. 3). In accordance, non-metric multidimensional scaling showed no clearly distinguishable plant assemblages, when comparing the different measure types among each other and to the field edge plots (control; Fig. 4 and Fig. A.12 in the Appendix). Of the 207 recorded species, 130 (62.8\%) were found in both the measures and the intensively managed field (field edge plots), whereas 43 (20.8\%) of the species were only found in the measure plots (Fig. 3). Among the taxa exclusively found in the measures were 16 typical arable plants such as the native Aphanes arvensis, Spergula arvensis, Galeopsis speciosa and the neophyte Galinsoga quadriradiata (Fig. 3 and Table A.7 in the Appendix). Twenty-one species (including 12 typical arable plants) were only found in the conventionally managed fields (field edge, interior and spillover plots), but not in the measures (Table A.7 in the Appendix). Considering only the measure plots, $41.4 \%$ of the taxa occurring here (77 of 186 species; Fig. 5) were found in all four measure types. Among the typical arable plants, even 56.4\% (62 of 110 species) were present in all four measures, whereas only a small number of species were restricted to certain measures (Table A.7 in the Appendix). Nine species were exclusively found in CFM (including 3 typical arable plants such as Bromus secalinus and Torilis japonica), 11 in fallows (4 typical arable plants including Galeopsis speciosa and Urtica urens), 10 in AFS (2 typical arable plants: Setaria verticillata and Sonchus arvensis) and 15 in PFS (2 typical arable plants: Amaranthus retroflexus and Mentha arvensis).

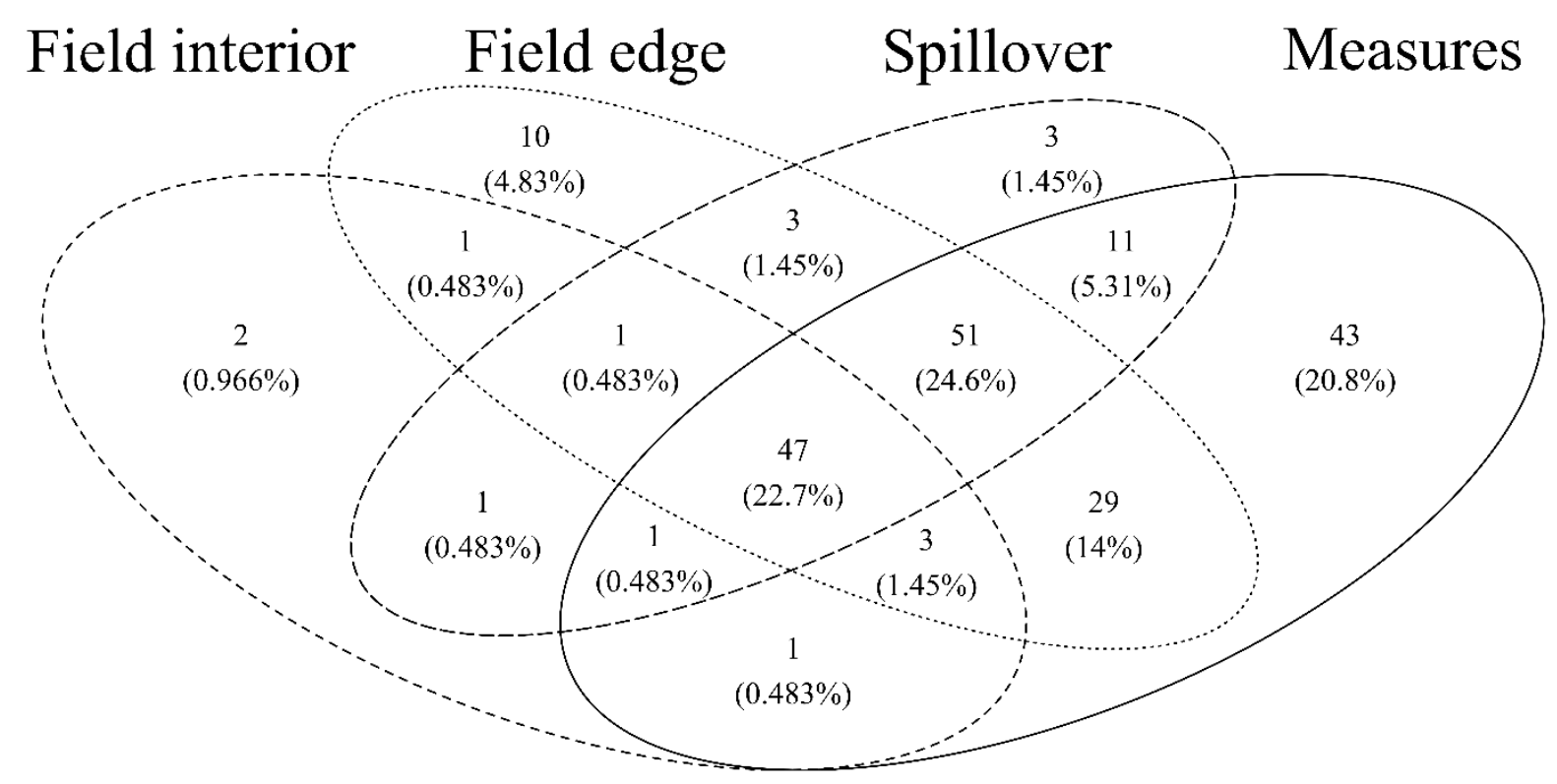

Fig. 3 Unique and shared species numbers (all herbaceous species considered) of the different studied plot types (field interior, field edge as control, spillover plots, and all four measure types pooled: CFM, fallow, AFS and PFS). The proportion of the total species pool (in \%) is given in brackets (in total 207 species; woody plant seedlings, ornamentals, crops and species only determinable to genus level excluded). The total number of relevés was 721 (for details see Table 1). 
a)

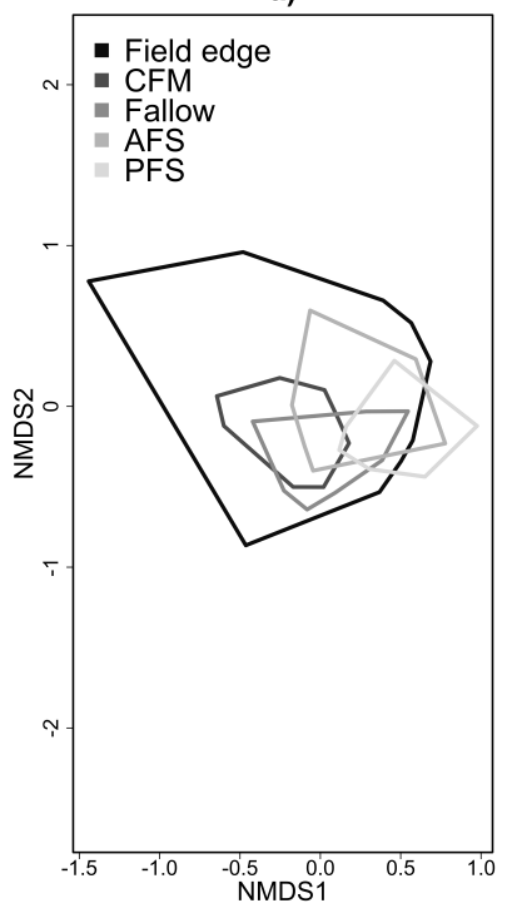

b)

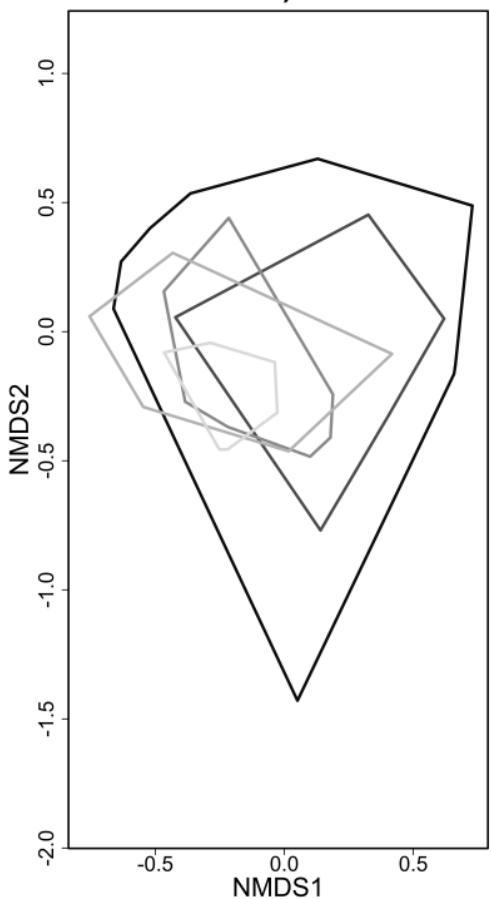

c)

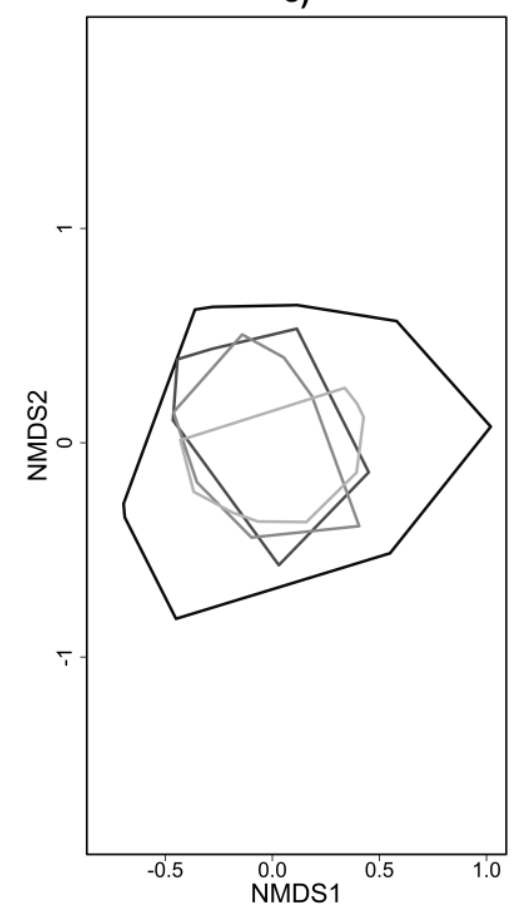

Fig. 4 Non-metric multidimensional scaling of the vegetation relevés conducted in the four studied measure types (CFM, fallow, AFS and PFS; see legend) in comparison to the field edge over the three implementation years: a) first implementation year ( $\mathrm{n}=$ $132, \mathrm{k}=3$, stress level $=0.201)$, b) second implementation year $(\mathrm{n}=125, \mathrm{k}=3$, stress level $=0.199)$ and $\mathrm{c})$ third implementation year $(\mathrm{n}=88, \mathrm{k}=3$, stress level $=0.216)$. NMDS Axis 1 and 2 are shown. PFS existed only for two implementation years.

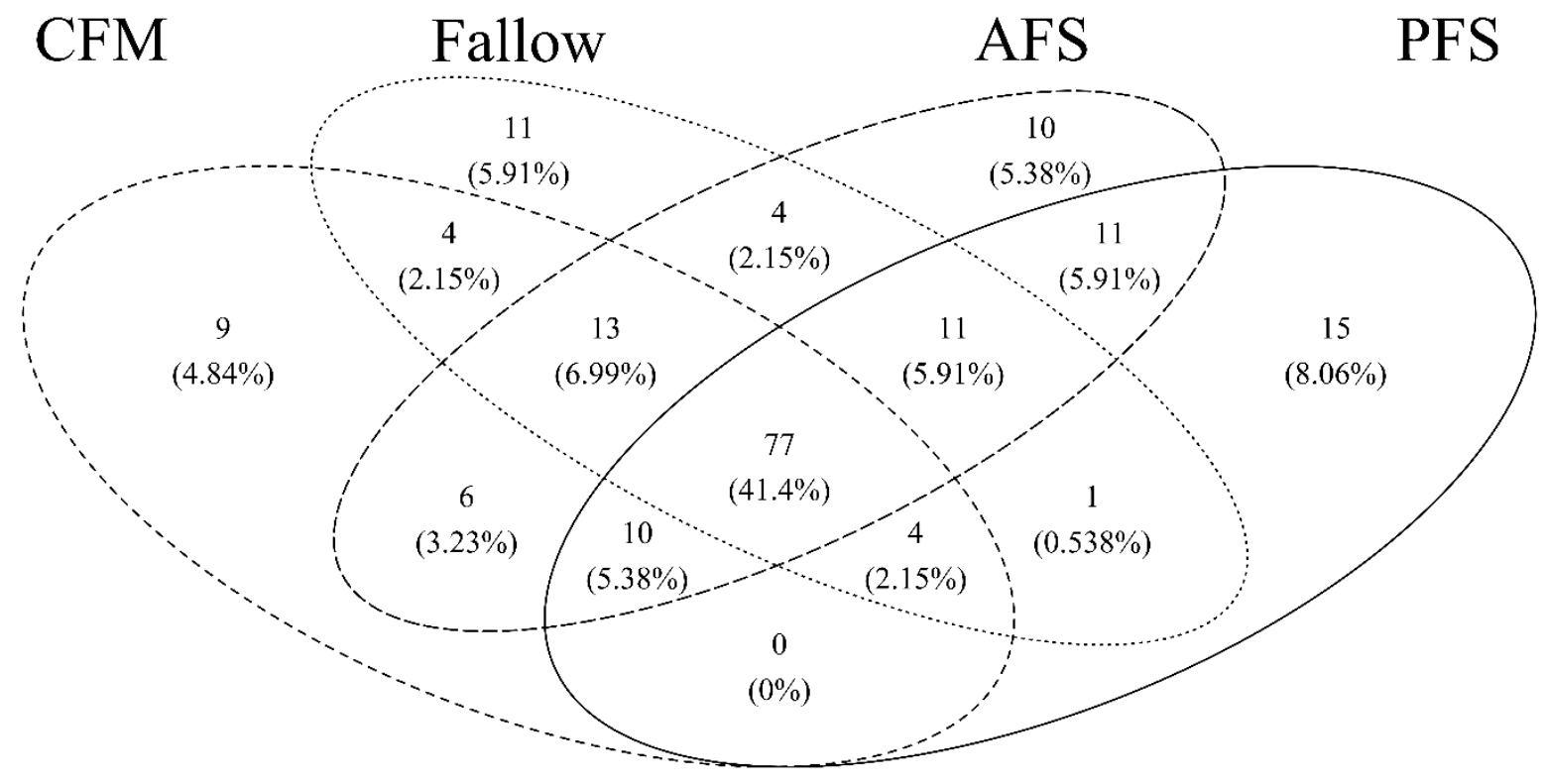

Fig. 5 Unique and shared species numbers (all herbaceous species considered) found in the four studied measure types (CFM, fallow, AFS and PFS). The proportion of the total species pool (in \%) is given in brackets (in total 186 species; woody plant seedlings, ornamentals, crops and species only determinable to order level excluded); $n=174$ relevés (for details see Table 1). 


\subsection{Discussion}

3.4.1 How were plant species richness and abundance affected by the measures?

While a number of studies have evaluated the effectiveness of agri-environment measures for farmland biodiversity in a more general way (e.g. Batáry et al., 2011; Geiger et al., 2010; Kleijn et al., 2006), very few studies directly compare the effectiveness of different measures on arable plant vegetation over a period of several years (but see e.g. Walker et al., 2007). In a participative procedure with farmers, we established a multi-annual field trial investigating the effect of four widely used types of agri-environment measures on the arable vegetation of conventional crop fields under real-world conditions. This approach has the additional strength that the measures have been adjusted for practicability on a modern conventional farm and are thus expected to have a high acceptance among farmers. In support of our hypothesis i), all four measures led in general to a significant increase in overall plant species richness and cover, and in the diversity and cover of typical arable plant species compared to the adjacent intensively managed field as control. The total plant cover increase (on average by $720 \%$ ) was in all measures greater than the diversity increase (on average by $230 \%$ ).

In general, these findings are in line with other studies which demonstrated overall positive effects of CFM, fallows and flower strips on plant species richness and abundance in arable habitats (Fischer and Milberg, 1997; Kovács-Hostyánszki et al., 2011; Rode et al., 2018; Van Buskirk and Willi, 2004). A comparison of the results of these studies reveals that soil type and fertility, landscape context, management history and seed bank composition have a large influence on the efficiency of agri-environment measures by affecting diaspore availability and the survival of arable plants (Aavik et al., 2008; Denys and Tscharntke, 2002; Richner et al., 2015; Tscharntke et al., 2011). As a major finding, our study demonstrated that the non-sown measure types fallows and CFM created suitable habitat conditions for the natural re-establishment of part of the native arable flora, even if there have been several decades of intensive management, as in our region. If only species numbers are considered, the plot-level diversity in our measures reached a similar level to that found in the 1950s/60s in the field interior of central and north German fields (medians: 21 species in fallows and 19 in CFMs vs. 24 in historical field interiors; Meyer et al., 2013). However, the species composition of historic surveys differs largely from that of recent intensive farmland in that the regional flora is greatly impoverished and homogenized, consisting predominantly of widely distributed agro-tolerant generalist species (Meyer et al., 2015). It is to be taken into account, too, that the plots in the implemented measures of this study were located at the field margin and thus possibly influenced by surrounding habitats, whereas the historical surveys in Meyer et al. (2013) refer to the field interior, i.e. the bulk of the arable land.

In support of hypothesis iii), we observed no spillover effects from the measure sites into the adjacent conventionally managed arable fields (Fig. 1 and Fig. A.4 in the Appendix). This finding can be explained by the intensive use of herbicides in the field which effectively excludes the invasion of non-herbicide-tolerant plants from outside the field and perhaps by low diaspore dispersal rates of many arable plants, found to be in the range of a few $\mathrm{m}$ per year (Albrecht et al., 1999; Bischoff, 1999). This finding is relevant for a better acceptance of agri-environment measures by farmers, who often are concerned about a possible weed infestation of their fields. Negligible spillover of arable plants contrasts with the well documented influence of neighboring 
natural habitats or agri-environment measures on the diversity and composition of various mobile animal groups in the farmland, e.g. Hymenoptera, Lepidoptera, Lycosidae, Coleoptera and Syrphidae (Blaauw and Isaacs, 2014; Holland et al., 2016; Loos et al., 2014; Tschumi et al., 2016). Spillover of plants may be slightly higher at the field edge, where the intensity of pesticide and fertilizer use and thus crop cover are somewhat lower than in the field interior, and some taxa of adjacent habitats may indeed colonize the field margin (Aavik et al., 2008; Cordeau et al., 2012; Nagy et al., 2018).

Considerable interannual variation in total plant species richness, especially prominent in the second year in CFM, fallow and AFS plots, and in the first year in PFS plots (Fig. A.4 in the Appendix), can most likely be explained by differences in weather conditions. As total species numbers and cover values increased with rising precipitation, and increased temperature led to reduced species richness and cover (Fig. A.5 in the Appendix), we assume that topsoil desiccation during the very dry and warm weather from April to July 2018 increased plant mortality and led to reduced species numbers. At this time, 46 of the 52 surveyed plots in 2018 of the measure types CFM, fallows and AFS were in the second year after establishment and all PFS plots were in their first year. Weather effects on arable plant community composition have been reported by e.g. Fried et al. (2008), Lososová and Cimalová (2009) and Pinke et al. (2012). This variation underpins the need of evaluating the effectiveness of agri-environment measures over several years to draw reliable conclusions, since weather may affect species richness and abundance, depending on the observed species group.

\subsubsection{How was plant species composition affected by the measures?}

In contradiction to hypothesis ii), non-metric multidimensional scaling did not reveal clear differences in the species composition of the communities found in the four measure types and at the intensively managed field edge (Fig. 4 and Fig. A.12 in the Appendix), even though diversity differed. Obviously, the species pools of implemented measures and managed fields have large overlaps and very few species occur exclusively in the managed field (edge or interior), while lacking in the measure sites. This is demonstrated by the species pool found in the four measures (186 species), which contained 77 species that occurred in all measure types (Fig. 5). In addition, measures and conventionally managed field edges shared 130 of in total 207 species (Fig. 3), most of which were widely distributed agro-tolerant taxa. This is in line with observations of Meyer et al. (2015) who found contemporary arable plant communities in Central Europe to be strongly impoverished and homogenized. These assemblages differ from the once characteristic, and now exceptionally rare, arable plant associations which contained a number of specialist taxa adapted to certain management and soil conditions (Albrecht et al., 2016; Meyer et al., 2015).

In support of hypothesis ii), the comparison of conventionally managed fields with the four measure types revealed differences in community structure, which relate to differences in vegetation continuity, fertilizer and pesticide use and soil disturbance regimes. Since three of the four measures were regularly ploughed (except for PFS), the median cover of annual taxa (pooled across all years) was much higher than that of perennials, as is characteristic for this kind of conservation management (Vickery et al., 2009; Westbury et al., 2008). However, after two or three years, the species richness and cover of perennials had significantly increased in the AFS 
and PFS plots, while annual species cover tended to decline (Fig. A.9 and Fig. A.10 in the Appendix). This indicates that continuous soil disturbance once a year, as conducted in our CFM and fallow sites, is most effective in conserving annual plants. As demonstrated in other studies, lacking soil disturbance (as in our PFS sites) leads to succession processes towards perennial vegetation with annual arable plants only dominating in the first one or two years (Boatman et al., 2011; Tscharntke et al., 2011). Leaving the vegetation without disturbance for two years, which was intended by the alternate management regime on half of the area in our AFS sites and which also happens in the second year in the PFS sites, appears to be a less effective measure of preserving annual plants over a longer time periods.

The dominance of graminoid species over forbs can threaten the success of perennial measures, as they reduce the diversity and thus the habitat value of the vegetation (Critchley et al., 2006; Critchley and Fowbert, 2000). Our results demonstrate that the measures investigated here generally promote the richness and cover of forbs more than that of graminoids, which likely is a consequence of the management (Fig. A.7 and Fig. A.8 in the Appendix). In a comparative review of conventional and organic farming, Hole et al. (2005) found that organic farming promotes the species richness and abundance of forbs more than that of graminoids, probably due to the absence of herbicides targeting dicots and less shading by the crop. That graminoid species richness and cover showed a stronger increase over the years only in PFS plots, but not in the plots of CFM and fallows with annual soil disturbance or AFS with regularly alternating management in half of the area, suggests that regular soil disturbance is generally better tolerated by forbs than graminoids in Central European arable fields.

The assessment of the four measures with respect to the goal of increasing plant diversity allows to conclude that the number of plant species indicating High Nature Value farmland tripled across all measures (median values across all years: 3 for measures and 1 for field edge plots; Fig. 2), which was linked to the general increase in the diversity of typical arable plants (Fig. 1). However, nearly a quarter (27 of 122) of the still occurring typical arable plant species were confined to fewer than four of the 67 studied fields (Table A.7 in the Appendix). Moreover, we found only three regionally threatened, red-listed arable species, which were present with very low cover values in few fields. This poses the question whether the population is still large enough to be viable in the long term. The measures did not have a positive effect on these rare and red-listed arable species over the 3-yr period, suggesting that despite their overall positive effect on biodiversity, the measures alone cannot restore the rarest species to conventional intensive farmland.

Kleijn et al. (2006) also found that agri-environment measures increased threatened red-listed species richness in only one of five studied countries. The potential effectiveness of biodiversitypromoting measures strongly depends on the local species pool in the soil seed bank (Denys and Tscharntke, 2002; Fischer and Milberg, 1997; Tscharntke et al., 2011), which likely applies in particular to rare species or threatened arable plants (Richner et al., 2015). We thus assume that exhausted seed banks, habitat isolation and intensive competition on fertile soils may have reduced the establishment success of threatened arable plants in our study (Albrecht et al., 2016; Denys and Tscharntke, 2002; Richner et al., 2015). The patchy distribution of the arable vegetation is mirrored in the observed relatively low floristic similarity of the plots in a given plot type (Fig. A.11 in the Appendix). Very rare and threatened arable plant species will need more targeted conservation actions, such as reintroduction with seed mixtures of local provenance and 
management regimes specifically adapted to the target species (Bischoff and Mahn, 2000; Kleijn et al., 2006; Lang et al., 2018, 2016). In this way, the (local) extinction of rare arable plant taxa could be prevented and the species pools at field level would be enriched. A network of conservation fields across the agricultural landscape could provide seeds of rare and threatened arable plants that might be added to the seed mix used in flower strips.

\subsubsection{Optimizing agri-environment measures targeted at arable plants}

Most agri-environment measures currently used in the EU to promote agrobiodiversity were designed primarily for birds and certain insect groups such as Hymenoptera and Lepidoptera, but less often targeted at arable plants. The recent awareness of the worldwide dramatic losses in insect abundance with intensive agriculture as a main driver (Sánchez-Bayo and Wyckhuys, 2019) has triggered growing interest in the implementation of flower strips in agricultural landscapes as food source and habitat primarily for insects. We have tested two types of flower strips (AFS and PFS) for their effect on arable plants. The comparison of the four measures tested in our study provides some general practical conclusions for the conservation of arable plants in intensive farmland: (i) the exclusion of fertilizers and herbicides strongly supports arable plant richness and abundance by particularly promoting non-graminoid species and reducing the nutrient-driven dominance of few competitive non-crop plants (Albrecht et al., 2016; Hole et al., 2005), (ii) continuous soil disturbance once a year benefits the predominantly annual arable flora and thus is preferable over measures which apply longer ploughing intervals, and (iii) crops or flower strips sown with reduced density are particularly effective, as dense vegetation is likely to suppress many light-demanding arable plants (Batlla and Benech-Arnold, 2014; Seifert et al., 2014). The exclusion of herbicides and fertilizer use in agri-environment measures is strongly recommended to support the arable flora. However, if CFM is widely implemented, the restricted application of fertilizers or selective herbicides (e.g. against graminoids) may also be considered, to support the acceptance of this measure by increasing the yield without strongly affecting arable plant species richness (Albrecht et al., 2016). By using a lower seed density $\left(1 \mathrm{~g} \mathrm{~m}^{-2}\right)$ than usually recommended for flower strips, we improved the establishment conditions for arable plants (Rode et al., 2018). In addition, PFS sites were mown annually to prevent the establishment of woody plants and dense stands of perennials and the succession toward communities dominated by ruderal and graminoid species (Hansson and Fogelfors, 1998; Hilbig, 1996). In our case, the cut plant mass was left on the field, but removing the cuttings is recommended in nutrient-rich sites to promote plant diversity by reducing the nutrient load (Kiehl et al., 2014). The patchy distribution of many arable plants in this study indicates strongly impoverished soil seed banks of the long-term intensively managed arable fields. In line with this, a reduction of the soil seed bank in arable habitats was also found in other European countries (Storkey et al., 2012). Thus, sowing arable plant species may represent the last option to promote arable plant diversity in impoverished intensively managed European farmland. For instance, flower strips could be adapted combining a mix of seeds of native arable plants of local origin with a small number of selected ornamental species known to be particularly attractive to insects (Albrecht et al., 2016). This approach was considered for our AFS and PFS sites and may be given preference over flower mixtures of mainly ornamental species such as phacelia and sunflower, as a large proportion of native arable plant species in the mixture helps to restore the once characteristic arable flora together with the species dependent on it (Albrecht et al., 2016). We suggest that future national and EU agri- 
environment schemes should adopt this more natural version of flower strips to halt plant diversity loss while also promoting insect and bird diversity.

Comparison of the four measures tested in our study indicates that they promoted arable plant diversity and cover to a similar degree. Due to their adaptation for practicability on a modern conventional farm, all measures are expected to have a high acceptance among farmers and can be recommended for consideration in national and EU agri-environment schemes, best in combination. Since the species pool overlap between the four measures was large and only few species were restricted to certain measures, one might conclude that the measures are equivalent in their effectiveness and thus should be given equal priority. However, a closer look at the change in species composition over the 3 -yr implementation period shows that CFM and annual fallow sites are better suited to promote the long-term establishment of more diverse arable plant communities than the two types of flower strips, as the cover of HNV species showed a much stronger increase and perennial plants did not expand as in AFS and especially PFS sites. As production-integrated measures, CFMs in cereal fields may offer the option to harvest the grain to use it as fodder, which might increase acceptance by farmers. As fallows and CFMs may bear a greater risk of the spread of harmful weeds on nutrient-rich sites (Denys and Tscharntke, 2002), these measures should preferentially be implemented on less fertile soil. AFS and PFS are an option on fertile soils as well, where no naturally occurring rare arable plants are suppressed. If well-managed, measures sown with seed mixtures can maintain plant diversity even over multiple years (Kiehl et al., 2014; Kirmer et al., 2018). When establishing such measures, the soil should be cultivated in a way that is appropriate for the germination of the sown species (seedbed preparation). To maintain plant diversity in perennial flower strips, a proper, site specific regular mowing regime should be considered, and biomass removal is particularly recommended on nutrient rich sites (Kiehl et al., 2014; Kirmer et al., 2018). In addition, creating more heterogeneous habitat conditions within a measure through ploughing and mowing only half the area at a time (as applied in AFS) can improve habitat conditions for different organism groups of agricultural landscapes, e.g. farmland birds (Gottschalk and Beeke, 2013).

In agreement with many other previous initiatives to promote agrobiodiversity, our practical experience from this trial has shown that the success of agri-environment schemes can be greatly improved when (i) the measures are planned in direct consultation with the farmers, (ii) the sites for measure implementation are chosen based on a biological pre-survey, (iii) the farmers receive advice also during the implementation phase, and (iv) a quality assessment is made in order to be able to optimize the scheme in the consecutive years. This requires skilled personnel with background in both agronomy and ecology, and a secure financial and legal framework the farmers can trust in and which enables compensation of the yield losses and additional management costs. Future agri-environment schemes also require the balancing of competing biodiversity goals in intensive farmland, as different organism groups may have different habitat and management requirements. It may be necessary to implement in every landscape a wellbalanced mix of flower strips, conservation field margins, annual fallows and further measures in order to generate benefit for all major organism groups. How the different components are weighted will depend on many factors, notably the landscape mosaic and the degree of biodiversity impoverishment, soil fertility, the agronomic structure and related cropping regimes, and the availability and suitability of agri-environment schemes. 


\subsection{Conclusions}

Halting and reversing biodiversity loss in European intensive farmland can only be successful when farmer acceptance of agri-environment measures increases and incentives are given for achieving greater farmer participation. This requires sufficient funding for compensation and the development of effective measures in collaboration with farmers that are flexible enough to be adapted to the local environmental and agronomic settings. The local or regional agencies that administrate agri-environment schemes must support the implementation process with continued advice through skilled personnel, which will be costly but worth the investment. The four tested measures of this trial have been found to be effective for arable plants in an intensively farmed region of Northwest Germany, adapted in cooperation with farmers. For targeting rare and threatened arable plants, the additional implementation of permanently established conservation fields is recommended that can also function as seed sources and for demonstration and education. Other organism groups should be addressed through the inclusion of further measures that proved to be particularly effective for farmland birds, hares, amphibians, and various groups of insects. Transferring this scheme to other farmland regions usually requires adaptation to local conditions. Monitoring concepts are an important tool for improving agri-environment schemes. The dry and warm summer 2018 has demonstrated that fluctuating weather can have a profound effect on the success of the measures, suggesting that surveys have to run over at least three, better five years to give reliable results.

\section{Funding}

This study was conducted within the research and demonstration project 'Development of targeted and efficient schemes to increase biodiversity in agricultural landscapes (MEDIATE)' which received funding from the German Federal Environmental Foundation (DBU; contract no. 32873/01).

\section{Acknowledgements}

We gratefully acknowledge the financial support granted by DBU. Many thanks go to all farmers for the implementation and management of the studied agri-environment measures and for granting access to their fields. We also thank Dr. Eckhard Gottschalk, Stefan Mecke, Dr. Stefan Meyer, Jenny Schellenberg and Dr. Robert Weigel for helpful discussions concerning the study design and/or data analysis, and Christina Ewerhardy, Fionn Pape, Svenja Meyer, Philine Zieschang, Marvin Thomas, Bettina Marth, Berit Weibel and Simone Blesinger for their support during the field work.

\section{Author contributions}

CL conceived the project together with J. Dauber (Thünen Institute of Biodiversity, Braunschweig, Germany), N. Kretzschmar and M. Polaschegg (Lower Saxony Chamber of Agriculture, Germany). AW, CL and LS developed the study design. AW and KA collected the 
data. AW, CVW and KA performed the data analysis. AW and CL wrote the manuscript with substantial input from all co-authors. All authors revised the manuscript critically.

\section{References}

Aavik, T., Augenstein, I., Bailey, D., Herzog, F., Zobel, M., Liira, J., 2008. What is the role of local landscape structure in the vegetation composition of field boundaries? Appl. Veg. Sci. 11, 375-386. https://doi.org/10.3170/2008-7-18486

Albrecht, H., Cambecèdes, J., Lang, M., Wagner, M., 2016. Management options for the conservation of rare arable plants in Europe. Bot. Lett. 163, 389-415. https://doi.org/10.1080/23818107.2016.1237886

Albrecht, H., Mayer, F., Mattheis, A., 1999. Veronica triphyllos L. in the Tertiärhügelland landscape in southern Bavaria - an example for habitat isolation of a stenoeceous plant species in agroecosystems. Z. Für Ökol. Naturschutz 8, 219-226.

Barral, M.P., Rey Benayas, J.M., Meli, P., Maceira, N.O., 2015. Quantifying the impacts of ecological restoration on biodiversity and ecosystem services in agroecosystems: A global meta-analysis. Agric. Ecosyst. Environ. 202, 223-231. https://doi.org/10.1016/j.agee.2015.01.009

Batáry, P., Baldi, A., Kleijn, D., Tscharntke, T., 2011. Landscape-moderated biodiversity effects of agri-environmental management: a meta-analysis. Proc. R. Soc. B Biol. Sci. 278, 1894-1902. https://doi.org/10.1098/rspb.2010.1923

Batáry, P., Dicks, L.V., Kleijn, D., Sutherland, W.J., 2015. The role of agri-environment schemes in conservation and environmental management: European Agri-Environment Schemes. Conserv. Biol. 29, 1006-1016. https://doi.org/10.1111/cobi.12536

Batáry, P., Gallé, R., Riesch, F., Fischer, C., Dormann, C.F., Mußhoff, O., Császár, P., Fusaro, S., Gayer, C., Happe, A.-K., Kurucz, K., Molnár, D., Rösch, V., Wietzke, A., Tscharntke, T., 2017. The former Iron Curtain still drives biodiversityprofit trade-offs in German agriculture. Nat. Ecol. Evol. 1, 1279-1284. https://doi.org/10.1038/s41559-017-0272-x

Batáry, P., Holzschuh, A., Orci, K.M., Samu, F., Tscharntke, T., 2012. Responses of plant, insect and spider biodiversity to local and landscape scale management intensity in cereal crops and grasslands. Agric. Ecosyst. Environ. 146, 130-136. https://doi.org/10.1016/j.agee.2011.10.018

Batlla, D., Benech-Arnold, R.L., 2014. Weed seed germination and the light environment: Implications for weed management: Light control of weed seed germination. Weed Biol. Manag. 14, 77-87. https://doi.org/10.1111/wbm.12039

Bischoff, A., 1999. Zeitliche und räumliche Dynamik segetaler Populationen von Lithospermum arvense L. und ihre Beeinflussung durch Bewirtschaftungsfaktoren. Flora 194, 127-136. https://doi.org/10.1016/S0367-2530(17)30889-7

Bischoff, A., Mahn, E.-G., 2000. The effects of nitrogen and diaspore availability on the regeneration of weed communities following extensification. Agric. Ecosyst. Environ. 77, 237-246. https://doi.org/10.1016/S0167-8809(99)00104-8

Blaauw, B.R., Isaacs, R., 2014. Larger patches of diverse floral resources increase insect pollinator density, diversity, and their pollination of native wildflowers. Basic Appl. Ecol. 15, 701-711. https://doi.org/10.1016/j.baae.2014.10.001

Boatman, N.D., Jones, N.E., Conyers, S.T., Pietravalle, S., 2011. Development of plant communities on set-aside in England. Agric. Ecosyst. Environ. 143, 8-19. https://doi.org/10.1016/j.agee.2011.05.003

Boessenkool, B., 2019. rdwd: Select and Download Climate Data from "DWD" (German Weather Service). R package version 1.2.0. [WWW Document]. URL https://CRAN.R-project.org/package $=$ rdwd (accessed 11.21.19).

Brooks, M.E., Kristensen, K., Benthem, K.J. van, Magnusson, A., Berg, C.W., Nielsen, A., Skaug, H.J., Maechler, M., Bolker, B.M., 2017. glmmTMB Balances Speed and Flexibility Among Packages for Zero-inflated Generalized Linear Mixed Modeling. R J. 9, 378-400.

Butchart, S.H.M., Walpole, M., Collen, B., van Strien, A., Scharlemann, J.P.W., Almond, R.E.A., Baillie, J.E.M., Bomhard, B., Brown, C., Bruno, J., Carpenter, K.E., Carr, G.M., Chanson, J., Chenery, A.M., Csirke, J., Davidson, N.C., Dentener, F., Foster, M., Galli, A., Galloway, J.N., Genovesi, P., Gregory, R.D., Hockings, M., Kapos, V., Lamarque, J.-F., Leverington, F., Loh, J., McGeoch, M.A., McRae, L., Minasyan, A., Morcillo, M.H., Oldfield, T.E.E., Pauly, D., Quader, S., Revenga, C., Sauer, J.R., Skolnik, B., Spear, D., Stanwell-Smith, D., Stuart, S.N., Symes, A., Tierney, M., Tyrrell, T.D., Vie, J.-C., Watson, R., 2010. Global Biodiversity: Indicators of Recent Declines. Science 328, 1164-1168. https://doi.org/10.1126/science.1187512

Buttler, K.P., 2018. Florenliste von Deutschland - Gefäßpflanzen. [WWW Document]. URL http://www.kp- buttler.de (accessed 2.13.19).

Chen, H., 2018. VennDiagram: Generate High-Resolution Venn and Euler Plots. R package version 1.6.20. [WWW Document]. URL https://CRAN.R-project.org/package=VennDiagram (accessed 11.7.18).

Cordeau, S., Petit, S., Reboud, X., Chauvel, B., 2012. The impact of sown grass strips on the spatial distribution of weed species in adjacent boundaries and arable fields. Agric. Ecosyst. Environ. 155, 35-40. https://doi.org/10.1016/j.agee.2012.03.022 
Critchley, C.N.R., Fowbert, J.A., 2000. Development of vegetation on set-aside land for up to nine years from a national perspective. Agric. Ecosyst. Environ. 79, 159-174. https://doi.org/10.1016/S0167-8809(99)00155-3

Critchley, C.N.R., Fowbert, J.A., Sherwood, A.J., 2006. The effects of annual cultivation on plant community composition of uncropped arable field boundary strips. Agric. Ecosyst. Environ. 113, 196-205. https://doi.org/10.1016/j.agee.2005.05.013

Denys, C., Tscharntke, T., 2002. Plant-insect communities and predator-prey ratios in field margin strips, adjacent crop fields, and fallows. Oecologia 130, 315-324. https://doi.org/10.1007/s004420100796

Eggenschwiler, L., Jacot, K.A., Edwards, P.J., 2009. Vegetation development and nitrogen dynamics of sown and spontaneous set-aside on arable land. Ecol. Eng. 35, 890-897. https://doi.org/10.1016/j.ecoleng.2008.12.024

European Commission, 2013. Overview of CAP reform 2014-2020 highlights improved framework for addressing 21st century challenges. [WWW Document]. URL https://ec.europa.eu/agriculture/sites/agriculture/files/policyperspectives/policy-briefs/05_en.pdf (accessed 3.14.19).

Federal Institute for Geosciences and Natural Resources, 2013. Soil Map of the Federal Republic of Germany 1:1,000,000 (BÜK1000). [WWWW Document]. URL https://www.bgr.bund.de/DE/Themen/Boden/Informationsgrundlagen/Bodenkundliche_Karten_Datenbanken/BU EK1000/buek1000_node.html (accessed 10.26.16).

Fischer, A., Milberg, P., 1997. Effects on the flora of extensified use of field margins. Swed. J. Agric. Res. Swed.

Flohre, A., Fischer, C., Aavik, T., Bengtsson, J., Berendse, F., Bommarco, R., Ceryngier, P., Clement, L.W., Dennis, C., Eggers, S., Emmerson, M., Geiger, F., Guerrero, I., Hawro, V., Inchausti, P., Liira, J., Morales, M.B., Oñate, J.J., Pärt, T., Weisser, W.W., Winqvist, C., Thies, C., Tscharntke, T., 2011. Agricultural intensification and biodiversity partitioning in European landscapes comparing plants, carabids, and birds. Ecol. Appl. 21, 1772-1781. https://doi.org/10.1890/100645.1

Fox, J., Weisberg, S., Fox, J., 2011. An R companion to applied regression, 2nd ed. ed. SAGE Publications, Thousand Oaks, Calif.

Fried, G., Norton, L.R., Reboud, X., 2008. Environmental and management factors determining weed species composition and diversity in France. Agric. Ecosyst. Environ. 128, 68-76. https://doi.org/10.1016/j.agee.2008.05.003

Fried, G., Petit, S., Dessaint, F., Reboud, X., 2009. Arable weed decline in Northern France: Crop edges as refugia for weed conservation? Biol. Conserv. 142, 238-243. https://doi.org/10.1016/j.biocon.2008.09.029

Gabriel, D., Sait, S.M., Kunin, W.E., Benton, T.G., 2013. Food production vs. biodiversity: comparing organic and conventional agriculture. J. Appl. Ecol. 50, 355-364. https://doi.org/10.1111/1365-2664.12035

Garve, E., 2004. Rote Liste und Florenliste der Farn- und Blütenpflanzen in Niedersachsen und Bremen, 5. Fassung vom 1.3.2004. Inform. d. Naturschutz Niedersachs. 24 (1) (1/04): 1-76, Hildesheim.

Geiger, F., Bengtsson, J., Berendse, F., Weisser, W.W., Emmerson, M., Morales, M.B., Ceryngier, P., Liira, J., Tscharntke, T., Winqvist, C., Eggers, S., Bommarco, R., Pärt, T., Bretagnolle, V., Plantegenest, M., Clement, L.W., Dennis, C., Palmer, C., Oñate, J.J., Guerrero, I., Hawro, V., Aavik, T., Thies, C., Flohre, A., Hänke, S., Fischer, C., Goedhart, P.W., Inchausti, P., 2010. Persistent negative effects of pesticides on biodiversity and biological control potential on European farmland. Basic Appl. Ecol. 11, 97-105. https://doi.org/10.1016/j.baae.2009.12.001

German Meteorological Service, 2019. Climate Data Center (CDC) [WWW Document]. URL https://www.dwd.de/EN/climate_environment/cdc/cdc.html (accessed 11.21.19).

Gonthier, D.J., Ennis, K.K., Farinas, S., Hsieh, H.-Y., Iverson, A.L., Batary, P., Rudolphi, J., Tscharntke, T., Cardinale, B.J., Perfecto, I., 2014. Biodiversity conservation in agriculture requires a multi-scale approach. Proc. R. Soc. B Biol. Sci. 281, 20141358-20141358. https://doi.org/10.1098/rspb.2014.1358

Gottschalk, E., Beeke, W., 2013. The partridge conservation project at the district of Göttingen-management of flower strips. Julius-Kühn-Arch. 104-111.

Hansson, M., Fogelfors, H., 1998. Management of permanent set-aside on arable land in Sweden. J. Appl. Ecol. 35, 758-771. https://doi.org/10.1046/j.1365-2664.1998.355350.x

Hijmans, R.J., 2019. raster: Geographic Data Analysis and Modeling. R package version 3.0-7. [WWW Document]. URL https://CRAN.R-project.org/package $=$ raster (accessed 11.21.19).

Hilbig, W., 1996. Die Vegetation mehrjähriger Ackerbrachen und ihre Veränderung im Verlaufe eines fünfjährigen Beobachtungszeitraumes. Angew. Bot. 70, 57-75.

Hofmeister, H., Garve, E., 2006. Lebensraum Acker, Reprint der 2. neubearbeiteten Auflage. ed. Verlag Kessel, Remagen.

Hole, D.G., Perkins, A.J., Wilson, J.D., Alexander, I.H., Grice, P.V., Evans, A.D., 2005. Does organic farming benefit biodiversity? Biol. Conserv. 122, 113-130. https://doi.org/10.1016/j.biocon.2004.07.018

Holland, J.M., Bianchi, F.J., Entling, M.H., Moonen, A.-C., Smith, B.M., Jeanneret, P., 2016. Structure, function and management of semi-natural habitats for conservation biological control: a review of European studies: Structure, function and management of semi-natural habitats for biological control. Pest Manag. Sci. 72, 1638-1651. https://doi.org/10.1002/ps.4318 
Hünig, C., Benzler, A., 2017. Das Monitoring der Landwirtschaftsflächen mit hohem Naturwert in Deutschland, BfN-Skripten. Bundesamt für Naturschutz, Bonn-Bad Godesberg.

Hyvönen, T., Huusela-Veistola, E., 2008. Arable weeds as indicators of agricultural intensity - A case study from Finland. Biol. Conserv. 141, 2857-2864. https://doi.org/10.1016/j.biocon.2008.08.022

Kiehl, K., Kirmer, A., Shaw, N., Tischew, S. (Eds.), 2014. Guidelines for native seed production and grassland restoration. Cambridge Scholars Publ, Newcastle upon Tyne, UK.

Kirmer, A., Rydgren, K., Tischew, S., 2018. Smart management is key for successful diversification of field margins in highly productive farmland. Agric. Ecosyst. Environ. 251, 88-98. https://doi.org/10.1016/j.agee.2017.09.028

Kleijn, D., Baquero, R.A., Clough, Y., Díaz, M., Esteban, J., Fernández, F., Gabriel, D., Herzog, F., Holzschuh, A., Jöhl, R., Knop, E., Kruess, A., Marshall, E.J.P., Steffan-Dewenter, I., Tscharntke, T., Verhulst, J., West, T.M., Yela, J.L., 2006. Mixed biodiversity benefits of agri-environment schemes in five European countries: Biodiversity effects of European agri-environment schemes. Ecol. Lett. 9, 243-254. https://doi.org/10.1111/j.1461-0248.2005.00869.x

Klotz, S., Kühn, I., Durka, W., Briemle, G., 2002. BIOLFLOR: Eine Datenbank mit biologisch-ökologischen Merkmalen zur Flora von Deutschland. Bundesamt für Naturschutz Bonn.

Korneck, D., Schnittler, M., Vollmer, I., 1996. Rote Liste der Farn- und Blütenpflanzen (Pteridophyta et Spermatophyta) Deutschlands. Schriftenreihe für Vegetationskunde 28:21-187.

Kovács-Hostyánszki, A., Kőrösi, Á., Orci, K.M., Batáry, P., Báldi, A., 2011. Set-aside promotes insect and plant diversity in a Central European country. Agric. Ecosyst. Environ. 141, 296-301. https://doi.org/10.1016/j.agee.2011.03.004

Lang, M., Prestele, J., Fischer, C., Kollmann, J., Albrecht, H., 2016. Reintroduction of rare arable plants by seed transfer. What are the optimal sowing rates? Ecol. Evol. 6, 5506-5516. https://doi.org/10.1002/ece3.2303

Lang, M., Prestele, J., Wiesinger, K., Kollmann, J., Albrecht, H., 2018. Reintroduction of rare arable plants: seed production, soil seed banks, and dispersal 3 years after sowing: Reintroduction of rare arable plants. Restor. Ecol. https://doi.org/10.1111/rec.12696

Legendre, P., Gallagher, E.D., 2001. Ecologically meaningful transformations for ordination of species data. Oecologia 129, 271280. https://doi.org/10.1007/s004420100716

Lenth, R., 2019. emmeans: Estimated Marginal Means, aka Least-Squares Means [WWW Document]. URL https://CRAN.Rproject.org/package $=$ emmeans (accessed 9.2.19).

Leuschner, C., Ellenberg, H., 2017. Ecology of Central European Non-Forest Vegetation: Coastal to Alpine, Natural to ManMade Habitats: Vegetation Ecology of Central Europe, Volume II. Springer International Publishing, Cham. https://doi.org/10.1007/978-3-319-43048-5

Leyer, I., Wesche, K., 2008. Multivariate Statistik in der Ökologie: eine Einführung, Korrigierter Nachdruck. ed, SpringerLehrbuch. Springer, Berlin Heidelberg.

Londo, G., 1976. The decimal scale for releves of permanent quadrats. Vegetatio 33, 61-64. https://doi.org/10.1007/BF00055300

Loos, J., Dorresteijn, I., Hanspach, J., Fust, P., Rakosy, L., Fischer, J., 2014. Low-Intensity Agricultural Landscapes in Transylvania Support High Butterfly Diversity: Implications for Conservation. PLoS ONE 9, e103256. https://doi.org/10.1371/journal.pone.0103256

Lososová, Z., Cimalová, Š., 2009. Effects of different cultivation types on native and alien weed species richness and diversity in Moravia (Czech Republic). Basic Appl. Ecol. 10, 456-465. https://doi.org/10.1016/j.baae.2008.11.001

Marshall, E.J.P., Brown, V.K., Boatman, N.D., Lutman, P.J.W., Squire, G.R., Ward, L.K., 2003. The role of weeds in supporting biological diversity within crop fields. Weed Res. 43, 77-89. https://doi.org/10.1046/j.1365-3180.2003.00326.x

Médiène, S., Valantin-Morison, M., Sarthou, J.-P., de Tourdonnet, S., Gosme, M., Bertrand, M., Roger-Estrade, J., Aubertot, J.-N., Rusch, A., Motisi, N., Pelosi, C., Doré, T., 2011. Agroecosystem management and biotic interactions: a review. Agron. Sustain. Dev. 31, 491-514. https://doi.org/10.1007/s13593-011-0009-1

Meyer, S., Bergmeier, E., Becker, T., Wesche, K., Krause, B., Leuschner, C., 2015. Detecting long-term losses at the plant community level - arable fields in Germany revisited. Appl. Veg. Sci. 18, 432-442. https://doi.org/10.1111/avsc.12168

Meyer, S., Wesche, K., Krause, B., Leuschner, C., 2013. Dramatic losses of specialist arable plants in Central Germany since the 1950s/60s - a cross-regional analysis. Divers. Distrib. 19, 1175-1187. https://doi.org/10.1111/ddi.12102

Nagy, K., Lengyel, A., Kovács, A., Türei, D., Csergő, A.M., Pinke, G., 2018. Weed species composition of small-scale farmlands bears a strong crop-related and environmental signature. Weed Res. 58, 46-56. https://doi.org/10.1111/wre.12281

Oksanen, J., Blanchet, F.G., Friendly, M., Kindt, R., Legendre, P., McGlinn, D., Minchin, P.R., O’Hara, R.B., Simpson, G.L., Solymos, P., Henry, M., Stevens, H., Szoecs, E., Wagner, H., 2019. vegan: Community Ecology Package. R package version 2.5-2. [WWW Document]. URL https://CRAN.R-project.org/package=vegan (accessed 11.6.18).

Pinheiro, J., Bates, D., DebRoy, S., Sarkar, D., R Core Team, 2019. nlme: Linear and Nonlinear Mixed Effects Models [WWW Document]. URL https://CRAN.R-project.org/package=nlme (accessed 11.18.19). 
Pinke, G., Karácsony, P., Czúcz, B., Botta-Dukát, Z., Lengyel, A., 2012. The influence of environment, management and site context on species composition of summer arable weed vegetation in Hungary. Appl. Veg. Sci. 15, 136-144. https://doi.org/10.1111/j.1654-109X.2011.01158.x

R Core Team, 2019. R: A Language and Environment for Statistical Computing. R Foundation for Statistical Computing, Vienna, Austria. [WWW Document]. URL https://www.R-project.org/ (accessed 11.2.19).

Richner, N., Holderegger, R., Linder, H.P., Walter, T., 2015. Reviewing change in the arable flora of Europe: a meta-analysis. Weed Res. 55, 1-13. https://doi.org/10.1111/wre.12123

Rode, M., Lischka, A., Schulz, G., 2018. Auswirkung von Blühstreifen auf die Biodiversität der Ackerbegleitflora in maisdominierten Agrarlandschaften. Umw. Raum 9, 81-114. https://doi.org/10.15488/3697

Romero, A., Chamorro, L., Sans, F.X., 2008. Weed diversity in crop edges and inner fields of organic and conventional dryland winter cereal crops in NE Spain. Agric. Ecosyst. Environ. 124, 97-104. https://doi.org/10.1016/j.agee.2007.08.002

Sánchez-Bayo, F., Wyckhuys, K.A.G., 2019. Worldwide decline of the entomofauna: A review of its drivers. Biol. Conserv. 232, 8-27. https://doi.org/10.1016/j.biocon.2019.01.020

Seifert, C., Leuschner, C., Meyer, S., Culmsee, H., 2014. Inter-relationships between crop type, management intensity and light transmissivity in annual crop systems and their effect on farmland plant diversity. Agric. Ecosyst. Environ. 195, 173182. https:// doi.org/10.1016/j.agee.2014.05.022

Storkey, J., Meyer, S., Still, K.S., Leuschner, C., 2012. The impact of agricultural intensification and land-use change on the European arable flora. Proc. R. Soc. B Biol. Sci. 279, 1421-1429. https://doi.org/10.1098/rspb.2011.1686

Tscharntke, T., Batáry, P., Dormann, C.F., 2011. Set-aside management: How do succession, sowing patterns and landscape context affect biodiversity? Agric. Ecosyst. Environ. 143, 37-44. https://doi.org/10.1016/j.agee.2010.11.025

Tschumi, M., Albrecht, M., Collatz, J., Dubsky, V., Entling, M.H., Najar-Rodriguez, A.J., Jacot, K., 2016. Tailored flower strips promote natural enemy biodiversity and pest control in potato crops. J. Appl. Ecol. 53, 1169-1176. https://doi.org/10.1111/1365-2664.12653

Van Buskirk, J., Willi, Y., 2004. Enhancement of farmland biodiversity within set-aside land. Conserv. Biol. 18, 987-994. https://doi.org/10.1111/j.1523-1739.2004.00359.x

Van Calster, H., Vandenberghe, R., Ruysen, M., Verheyen, K., Hermy, M., Decocq, G., 2008. Unexpectedly high 20th century floristic losses in a rural landscape in northern France: Floristic changes in rural landscapes. J. Ecol. 96, 927-936. https://doi.org/10.1111/j.1365-2745.2008.01412.x

Venclova, V., Brant, V., Neckar, K., 2006. Weed succession during three years of set-aside experiment. Z. Für Pflanzenkrankh. Pflanzenschutz 611-618.

Vickery, J.A., Feber, R.E., Fuller, R.J., 2009. Arable field margins managed for biodiversity conservation: A review of food resource provision for farmland birds. Agric. Ecosyst. Environ. 133, 1-13. https://doi.org/10.1016/j.agee.2009.05.012

Wagner, M., Bullock, J.M., Hulmes, L., Hulmes, S., Pywell, R.F., 2017. Cereal density and N-fertiliser effects on the flora and biodiversity value of arable headlands. Biodivers. Conserv. 26, 85-102. https://doi.org/10.1007/s10531-016-1225-4

Walker, K.J., Critchley, C.N.R., Sherwood, A.J., Large, R., Nuttall, P., Hulmes, S., Rose, R., Mountford, J.O., 2007. The conservation of arable plants on cereal field margins: An assessment of new agri-environment scheme options in England, UK. Biol. Conserv. 136, 260-270. https://doi.org/10.1016/j.biocon.2006.11.026

Westbury, D.B., Woodcock, B.A., Harris, S.J., Brown, V.K., Potts, S.G., 2008. The effects of seed mix and management on the abundance of desirable and pernicious unsown species in arable buffer strip communities. Weed Res. 48, 113-123. https://doi.org/10.1111/j.1365-3180.2007.00614.x

Wickham, H., 2007. Reshaping Data with the reshape Package. Journal of Statistical Software, 21(12), 1-20. [WWW Document]. URL http://www.jstatsoft.org/v21/i12/ (accessed 11.7.18). 


\section{Electronic supplementary material}

Table A.1 Number of replicates per crop in each calendar year and year of implementation.

\begin{tabular}{lcccc|cccc}
\hline Crop & \multicolumn{9}{c|}{ Implementation year } & \multicolumn{4}{c}{ Calendar Year } \\
& $\mathbf{0}$ & $\mathbf{1}$ & $\mathbf{2}$ & $\mathbf{3}$ & $\mathbf{2 0 1 6}$ & $\mathbf{2 0 1 7}$ & $\mathbf{2 0 1 8}$ & $\mathbf{2 0 1 9}$ \\
\hline Barley & 11 & 7 & 7 & 6 & 9 & 6 & 9 & 7 \\
Maize & 14 & 22 & 15 & 15 & 7 & 24 & 12 & 23 \\
Potato & 2 & 4 & 0 & 0 & 1 & 3 & 2 & 0 \\
Rapeseed & 11 & 11 & 8 & 2 & 8 & 14 & 7 & 3 \\
Rye & 7 & 2 & 10 & 5 & 7 & 2 & 7 & 8 \\
Triticale & 8 & 5 & 6 & 5 & 6 & 3 & 8 & 7 \\
Wheat & 7 & 16 & 17 & 11 & 7 & 10 & 21 & 13 \\
\hline
\end{tabular}

Table A.2 Seed mixture of the alternately managed biennial flower strips (AFS). Borago officinalis was removed from seed mixture from 2018 onwards due to feedback from regional beekeepers referring to potential contamination of produced honey with pyrrolizidine alkaloids; seed weight proportion of Borago officinalis was redistributed to seed weight proportion of the native plant species.

\begin{tabular}{lccc}
\hline Native plant species & Seed weight proportion 2016 and 2017 (\%) & Seed weight proportion 2018 (\%) \\
\hline Galium mollugo agg. & 0.44 & 0.67 \\
Hypericum perforatum & 0.44 & 0.67 \\
Leucanthemum vulgare agg. & 0.44 & 0.67 \\
Linaria vulgaris & 0.16 & 0.67 \\
Lotus corniculatus & 0.44 & 0.65 \\
Medicago lupulina & 0.44 & 0.65 \\
Melilotus alba & 2 & 2 \\
Melilotus officinalis & 2 & 2 \\
Papaver dubium & 0.44 & 0.67 \\
Prunella vulgaris & 0.44 & 0.67 \\
Silene dioica & 0.44 & 0.67 \\
Silene latifolia & 0.44 & 0.67 \\
Verbascum nigrum & 0.44 & 0.67 \\
Vicia cracca & 0.44 & 0.67 \\
& & \\
\hline Crop and ornamental species & & \\
\hline Anetbum graveolens & 3 & 3 \\
Avena sativa & 5 & 5 \\
Borago officinalis & 3 & $/$ \\
Calendula officinalis & 5 & 5 \\
Coriandrum sativum & 3 & 3 \\
\hline Fagopyrum esculentum & 14 & 14 \\
Heliantbus annuus & 15 & 15 \\
Linum usitatissimum & 17 & 17 \\
Malva sylvestris ssp. mauritiana & 5 & 5 \\
Medicago sativa & 7 & 7 \\
Ornithopus sativus & 4 & 4 \\
Raphanus sativus & 2 & 2 \\
Secale cereale 'multicaule' & 8 & 8 \\
\hline & & \\
\hline
\end{tabular}


Table A.3 Seed mixture of the perennial flower strips (PFS).

\begin{tabular}{|c|c|}
\hline Native plant species & Seed weight proportion ( $\%)$ \\
\hline Achillea millefolium & 8 \\
\hline Achillea ptarmica & 3 \\
\hline Agrostis capillaris & 1 \\
\hline Anthoxanthum odoratum & 2 \\
\hline Anthriscus sylvestris & 0.5 \\
\hline Arrbenatherum elatius & 0.5 \\
\hline Campanula rotundifolia & 0.1 \\
\hline Daucus carota & 8.4 \\
\hline Galium mollugo agg. & 7 \\
\hline Heracleum sphondylium & 0.5 \\
\hline Hypericum perforatum & 1.5 \\
\hline Hypochaeris radicata & 1 \\
\hline Lathyrus pratensis & 0.5 \\
\hline Scorzoneroides autumnalis & 4 \\
\hline Leucanthemum vulgare agg. & 9 \\
\hline Linaria vulgaris & 0.1 \\
\hline Lolium perenne & 2 \\
\hline Lotus corniculatus & 2.5 \\
\hline Lotus pedunculatus & 1.5 \\
\hline Lychnis-flos-cuculi & 3 \\
\hline Medicago lupulina & 1.5 \\
\hline Phleum pratense & 0.5 \\
\hline Plantago lanceolata & 9 \\
\hline Poapalustris & 1 \\
\hline Poapratensis & 3 \\
\hline Prunella vulgaris & 8.8 \\
\hline Rumex acetosa & 2.5 \\
\hline Scropbularia nodosa & 2.5 \\
\hline Silene latifolia ssp. alba & 9 \\
\hline Stellaria graminea & 0.5 \\
\hline Tanacetum vulgare & 1.5 \\
\hline Trifolium pratense & 3.5 \\
\hline Veronica chamaedrys & 0.1 \\
\hline Vicia cracca & 0.5 \\
\hline
\end{tabular}

Table A.4 Number of replicates of cultivated crops in each implementation year in conservation field margins.

\begin{tabular}{lccc}
\hline Crop & \multicolumn{3}{c}{ Implementation year } \\
& $\mathbf{1}$ & $\mathbf{2}$ & $\mathbf{3}$ \\
\hline Barley & 3 & 3 & 3 \\
Maize & 7 & 3 & 3 \\
Rapeseed & 3 & 1 & 1 \\
Rye & 0 & 1 & 0 \\
Triticale & 2 & 3 & 2 \\
Wheat & 3 & 5 & 4 \\
\hline
\end{tabular}


Table A.5 Key management steps and average width $(\mathrm{m})$, length $(\mathrm{m})$ and area $\left(\mathrm{m}^{2}\right)$ of the implemented measure types (in total 67 measure areas, for details see Table 1 in the main text): conservation field margin (CFM), annual fallow (fallow), alternately managed biennial flower strip (AFS) and perennial flower strip (PFS).

\begin{tabular}{|c|c|c|c|c|}
\hline Measure type & CFM & Fallow & AFS & PFS \\
\hline Average width (m) & 12,4 & 12,6 & 18,1 & 26,4 \\
\hline Average length (m) & 185,6 & 154,2 & 173,3 & 158,6 \\
\hline Average area $\left(\mathrm{m}^{2}\right)$ & 2159,8 & 1918,1 & 2683,8 & 2969,1 \\
\hline \multirow{3}{*}{ Management } & \multicolumn{4}{|c|}{$\begin{array}{l}\text { All measures: no application of fertilizers, pesticides, no mechanical weeding and no access by machinery } \\
\text { (except for harvest and mowing) }\end{array}$} \\
\hline & \multirow[t]{2}{*}{$\begin{array}{l}\text { Annual soil disturbance } \\
\text { (ploughing in autumn or } \\
\text { spring, depending on the } \\
\text { cultivation of summer or } \\
\text { winter crops) and cultivation } \\
\text { with crop; reduced sowing } \\
\text { rate ( } 70 \% \text { in autumn } \\
2016 / \text { spring } 2017 \text { and } 50 \% \\
\text { from autumn } 2017 \text { onwards) }\end{array}$} & \multirow[t]{2}{*}{$\begin{array}{l}\text { Annual soil } \\
\text { disturbance } \\
\text { (ploughing in autumn } \\
\text { or spring, depending } \\
\text { on the cultivation of } \\
\text { summer or winter } \\
\text { crops on the adjacent } \\
\text { field); no seeding }\end{array}$} & $\begin{array}{l}\text { After establishment, alternating re- } \\
\text { creation of half of the area from } \\
\text { the } 2 \text { nd year onwards (including } \\
\text { mowing with biomass left on the } \\
\text { field, ploughing and re-sowing); } \\
\text { seed mixture (see Table A.2): crop, } \\
\text { ornamental and native plant species } \\
\text { (annuals and perennials, including } \\
\text { typical arable plant species) }\end{array}$ & $\begin{array}{l}\text { Establishment by using a } \\
\text { seed mixture including } \\
\text { widespread perennial native } \\
\text { herbaceous species (see } \\
\text { Table A.3); annually } \\
\text { mowing between August } \\
\text { and September (plant } \\
\text { biomass left on the field) }\end{array}$ \\
\hline & & & \multicolumn{2}{|c|}{$\begin{array}{l}\text { Sowing at the begin of May; use of native plant seeds of local } \\
\text { provenance; reduced sowing rate }\left(1 \mathrm{~g} \mathrm{~m}^{-2}\right) \text { to promote naturally } \\
\text { present arable plant species in the soil seed bank (AFS and PFS) }\end{array}$} \\
\hline
\end{tabular}




\section{CHAPTER 3}

Table A.6 Raw data of the vegetation survey (2016-2019).

Can be downloaded from http://ediss.uni-goettingen.de/ (Niedersächsische Staats- und Universitätsbibliothek Göttingen).

Table A.7. Species occurrences per plot type and categorization of surveyed plant species (all herbaceous species, except crop species, woody seedlings and ornamental plants); sorted by species name; observed plot types: field interior (In), field edge (Ed), spillover plots (Sp) adjacent to implemented measures, conservation field margin (CFM), fallows ( $\mathrm{Fa}$ ), alternately managed biennial flower strip (AFS), perennial flower strip (PFS), species occurrences in plot types are given for each implementation year ( 0 (pre-survey), 1 (first year of measure implementation), 2 (second year of measure implementation)

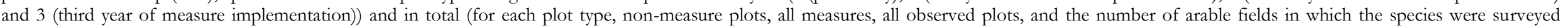
(occurrences in fields, 67 observed fields in total)). In addition, categorization of observed species are given: annuals or perennials $(\mathrm{A}=$ annual, $\mathrm{P}=$ perennial; Klotz et al., 2002), forbs or graminoids ( $\mathrm{F}=$ forb, $G$ = graminoid), typical arable plants (Arable plants, Hofmeister and Garve, 2006), indicator species for High Nature Value arable land (HNV species; Hünig und Benzler, 2017), threatened arable plant species (red-listed according to the Red List of Lower Saxony; Garve, 2004); species which were also sown in the flower strips are marked: AFS or/and PFS; $\mathrm{n}=721$ (for details see Table 1 in the main document; full references see main document).

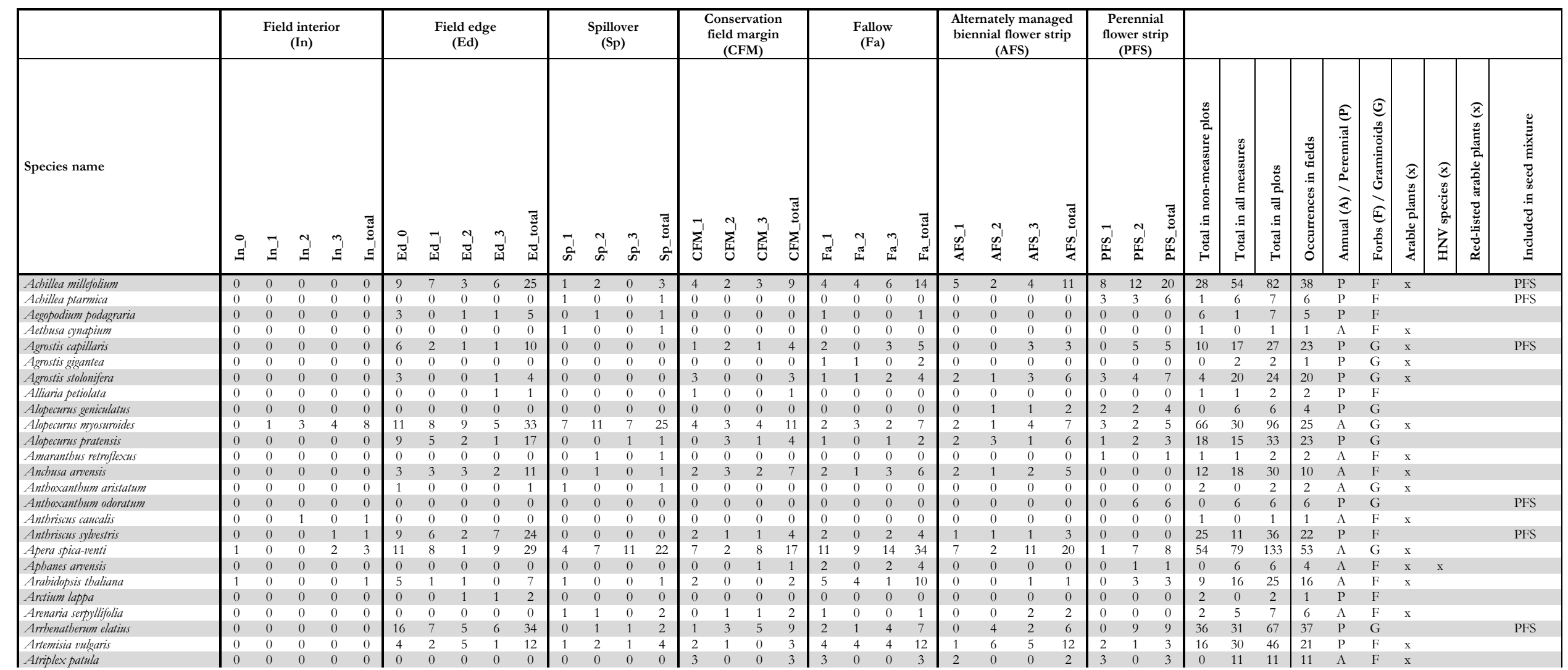




\section{CHAPTER 3}

\begin{tabular}{|c|c|c|c|c|c|c|c|c|c|c|c|c|c|c|c|c|c|c|c|c|c|c|c|c|c|c|c|c|c|c|c|c|c|c|c|c|c|c|c|}
\hline \multirow[b]{2}{*}{ Species name } & \multicolumn{5}{|c|}{$\begin{array}{l}\text { Field interior } \\
\quad \text { (In) }\end{array}$} & \multicolumn{5}{|c|}{$\begin{array}{c}\text { Field edge } \\
\text { (Ed) }\end{array}$} & \multicolumn{4}{|c|}{$\begin{array}{c}\text { Spillover } \\
(\mathrm{Sp})\end{array}$} & \multicolumn{4}{|c|}{$\begin{array}{l}\text { Conservation } \\
\text { field margin } \\
\text { (CFM) }\end{array}$} & & $\begin{array}{l}\text { Fall } \\
(\mathrm{F}\end{array}$ & & & & $(\mathrm{A}$ & $\frac{m}{\operatorname{man}}$ & & & $\begin{array}{l}\text { rennia } \\
\text { ver str } \\
\text { (PFS) }\end{array}$ & & & & & & & & & & & \\
\hline & $\begin{array}{l}0 \\
\Xi\end{array}$ & $\vec{\Xi}$ & $\begin{array}{l}N_{1} \\
\Xi\end{array}$ & $\stackrel{m}{\Xi}$ & 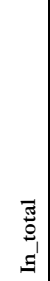 & 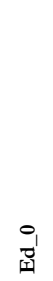 & 臭 & $\begin{array}{l}N_{1} \\
\vec{I}\end{array}$ & $\frac{m}{i}$ & 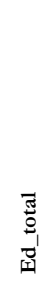 & $\begin{array}{l}\overrightarrow{1} \\
\text { के }\end{array}$ & $\begin{array}{l}N_{1} \\
\text { की }\end{array}$ & $\begin{array}{l}m_{1}^{1} \\
\hat{n}^{n}\end{array}$ & 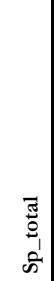 & 章 & 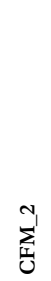 & $\sum_{\text {恶 }}^{m}$ & 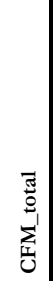 & F & $\begin{array}{l}N_{1} \\
\tilde{F}^{\prime}\end{array}$ & జ & 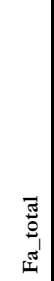 & $\begin{array}{l}\overrightarrow{1} \\
c^{\prime} \\
\text { 至 }\end{array}$ & 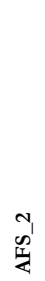 & 告 & 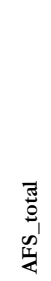 & 蒠 & $\begin{array}{l}1 \\
w_{1} \\
w^{\prime}\end{array}$ & 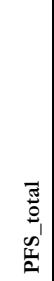 & 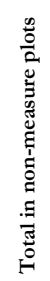 & 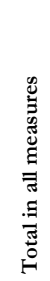 & 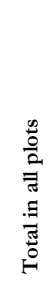 & 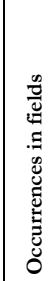 & 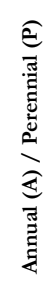 & 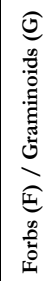 & 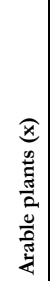 & 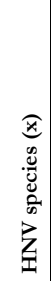 & 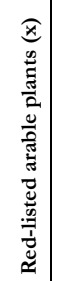 & 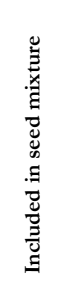 \\
\hline Avena fatua & 0 & 1 & 0 & 2 & 3 & 0 & 4 & 1 & 1 & 6 & 3 & 0 & 3 & 6 & 1 & 0 & 0 & 1 & 2 & 0 & 1 & 3 & 1 & 0 & 0 & 1 & 0 & 0 & 0 & 15 & 5 & 20 & 14 & $\bar{A}$ & $\bar{G}$ & $\mathrm{x}$ & & & \\
\hline Barbarea vulgaris & 0 & 0 & 0 & 0 & 0 & 1 & 1 & 1 & 0 & 3 & 0 & 1 & 0 & 1 & 0 & 1 & 0 & 1 & 0 & 0 & 0 & 0 & 1 & 1 & 1 & 3 & 1 & 2 & 3 & 4 & 7 & 11 & 7 & $\mathrm{P}$ & $\mathrm{F}$ & & & & \\
\hline Bellis perennis & 0 & 0 & 0 & 0 & 0 & 0 & 0 & 0 & 0 & 0 & 0 & 0 & 0 & 0 & 0 & 0 & 0 & 0 & 0 & 0 & 0 & 0 & 0 & 0 & 0 & 0 & 1 & 0 & 1 & 0 & 1 & 1 & 1 & $\mathrm{P}$ & $\mathrm{F}$ & & & & \\
\hline Berteroa incana & 0 & 0 & 0 & 0 & 0 & 0 & 0 & 0 & 0 & 0 & 0 & 0 & 0 & 0 & 0 & 0 & 0 & 0 & 0 & 0 & 0 & 0 & 0 & 0 & 0 & 0 & 0 & 1 & 1 & 0 & 1 & 1 & 1 & $\mathrm{P}$ & $\mathrm{F}$ & & & & \\
\hline Bromus commutatus subsp. decipiens & 1 & 0 & 2 & 1 & 4 & 7 & 7 & 8 & 4 & 26 & 4 & 3 & 7 & 14 & 2 & 2 & 3 & 7 & 1 & 2 & 4 & 7 & 3 & 1 & 2 & 6 & 0 & 1 & 1 & 44 & 21 & 65 & 24 & A & G & $\mathrm{x}$ & & & \\
\hline Bromus hordeacens & 0 & 0 & 1 & 0 & 1 & 15 & 5 & 8 & 10 & 38 & 0 & 3 & 2 & 5 & 3 & 1 & 1 & 5 & 6 & 3 & 5 & 14 & 1 & 3 & 8 & 12 & 2 & 1 & 3 & 44 & 34 & 78 & 40 & A & G & $\mathrm{x}$ & & & \\
\hline Bromus inermis & 0 & 0 & 0 & 0 & 0 & 0 & 0 & 0 & 1 & 1 & 0 & 0 & 0 & 0 & 0 & 0 & 0 & 0 & 0 & 0 & 0 & 0 & 0 & 0 & 0 & 0 & 0 & 0 & 0 & 1 & 0 & 1 & 1 & $\mathrm{P}$ & G & & & & \\
\hline Bromus secalinus & 0 & 0 & 0 & 0 & 0 & 1 & 2 & 0 & 0 & 3 & 0 & 1 & 1 & 2 & 1 & 0 & 0 & 1 & 0 & 0 & 0 & 0 & 0 & 0 & 0 & 0 & 0 & 0 & 0 & 5 & 1 & 6 & 6 & A & G & $\mathrm{x}$ & & & \\
\hline Bromus sterilis & 0 & 0 & 3 & 0 & 3 & 31 & 26 & 30 & 22 & 109 & 3 & 9 & 7 & 19 & 10 & 10 & 7 & 27 & 11 & 11 & 8 & 30 & 6 & 5 & 8 & 19 & 1 & 3 & 4 & 131 & 80 & 211 & 54 & A & G & $\mathrm{x}$ & & & \\
\hline Calystegia sepium & 1 & 0 & 0 & 0 & 1 & 0 & 0 & 0 & 0 & 0 & 0 & 0 & 1 & 1 & 0 & 0 & 0 & 0 & 0 & 0 & 0 & 0 & 1 & 1 & 1 & 3 & 0 & 0 & 0 & 2 & 3 & 5 & 2 & $\mathrm{P}$ & $\mathrm{F}$ & & & & \\
\hline Capsella burs. & 3 & 0 & 6 & 1 & 10 & 16 & 9 & 8 & 3 & 36 & 10 & 15 & 7 & 32 & 13 & 5 & 7 & 25 & 9 & 9 & 8 & 26 & 11 & 8 & 6 & 25 & 6 & 5 & 11 & 78 & 87 & 165 & 55 & A & $\mathrm{F}$ & $\mathrm{x}$ & & & \\
\hline Carduus crist & 0 & 0 & 0 & 0 & 0 & 0 & 1 & 0 & 1 & 2 & 0 & 0 & 1 & 1 & 1 & 0 & 2 & 3 & 0 & 0 & 0 & 0 & 0 & 0 & 1 & 1 & 0 & 0 & 0 & 3 & 4 & 7 & 3 & $\mathrm{P}$ & $\mathrm{F}$ & & & & \\
\hline Carduus nutans & 0 & 0 & 0 & 0 & 0 & 0 & 0 & 0 & 0 & 0 & 0 & 0 & 0 & 0 & 0 & 0 & 0 & 0 & 0 & 0 & 0 & 0 & 0 & 1 & 0 & 1 & 0 & 0 & 0 & 0 & 1 & 1 & 1 & $\mathrm{P}$ & F & & & & \\
\hline Carex act & 0 & 0 & 0 & 0 & 0 & 0 & 0 & 0 & 0 & 0 & 0 & 0 & 0 & 0 & 0 & 0 & 1 & 1 & 0 & 0 & 1 & 1 & 0 & 0 & 0 & 0 & 0 & 0 & 0 & 0 & 2 & 2 & 2 & $\mathrm{P}$ & G & & & & \\
\hline Carex acu & 0 & 0 & 0 & 0 & 0 & 1 & 0 & 0 & 1 & 2 & 0 & 0 & 0 & 0 & 0 & 0 & 0 & 0 & 0 & 0 & 0 & 0 & 0 & 0 & 0 & 0 & 0 & 0 & 0 & 2 & 0 & 2 & 2 & $\mathrm{P}$ & G & & & & \\
\hline Carex hirta & 0 & 0 & 0 & 0 & 0 & 2 & 0 & 0 & 0 & 2 & 0 & 0 & 0 & 0 & 0 & 0 & 0 & 0 & 1 & 0 & 0 & 1 & 0 & 0 & 0 & 0 & 1 & 1 & 2 & 2 & 3 & 5 & 3 & $\mathrm{P}$ & G & $\mathrm{x}$ & & & \\
\hline Carex spec. & 1 & 0 & 0 & 0 & 1 & 0 & 0 & 0 & 0 & 0 & 0 & 1 & 0 & 1 & 0 & 0 & 0 & 0 & 0 & 0 & 0 & 0 & 0 & 0 & 0 & 0 & 0 & 0 & 0 & 2 & 0 & 2 & 2 & $\mathrm{NA}$ & G & & & & \\
\hline Centaurea cyanus & 2 & 1 & 1 & 3 & 7 & 14 & 11 & 8 & 8 & 41 & 6 & 8 & 8 & 22 & 7 & 5 & 4 & 16 & 5 & 5 & 6 & 16 & 4 & 2 & 0 & 6 & 4 & 3 & 7 & 70 & 45 & 115 & 26 & $\mathrm{~A}$ & $\mathrm{~F}$ & $\mathrm{x}$ & & & \\
\hline Cerastium arvense & 0 & 0 & 0 & 0 & 0 & 1 & 0 & 0 & 0 & $\begin{array}{c}+1 \\
1\end{array}$ & 0 & 0 & 0 & 0 & 0 & 0 & 0 & $\begin{array}{c}10 \\
0\end{array}$ & 0 & 0 & 1 & 1 & 0 & 0 & 0 & 0 & 0 & 0 & 0 & 1 & 1 & 2 & 1 & P & F & & & & \\
\hline Cerastium glomereatum & 0 & 0 & 0 & 0 & 0 & 0 & 1 & 0 & 0 & 1 & 0 & 0 & 0 & 0 & 0 & 1 & 1 & 2 & 0 & 0 & 2 & 2 & 0 & 0 & 2 & 2 & 0 & 3 & 3 & 1 & 9 & 10 & 10 & A & $\mathrm{F}$ & $\mathrm{x}$ & & & \\
\hline Cerastium holosteoides & 0 & 0 & 0 & 0 & 0 & 4 & 0 & 0 & 0 & 4 & 0 & 1 & 0 & 1 & 3 & 0 & 0 & 3 & 3 & 2 & 1 & 6 & 3 & 0 & 1 & 4 & 2 & 1 & 3 & $\frac{1}{5}$ & 16 & 21 & 14 & $\begin{array}{l}\mathrm{A} \\
\mathrm{P}\end{array}$ & $\mathrm{F}$ & $x$ & & & \\
\hline Ceratocapnos clavicu & 0 & 0 & 0 & 0 & 0 & 1 & 0 & 0 & 0 & 1 & 0 & 0 & 0 & 0 & 1 & 0 & 1 & 2 & 0 & 1 & 0 & 1 & 0 & 0 & 0 & 0 & 0 & 0 & 0 & 1 & 3 & 4 & 2 & A & $\mathrm{F}$ & & & & \\
\hline Chaerophyllum bulbosum & 0 & 0 & 0 & 0 & 0 & 0 & 0 & 0 & 0 & 0 & 0 & 0 & 0 & 0 & 2 & 1 & 1 & 4 & 0 & 0 & 0 & 0 & 0 & 0 & 0 & 0 & 0 & 0 & 0 & 0 & 4 & 4 & 2 & P & F & & & & \\
\hline Chaerophyllum temulum & 0 & 0 & 0 & 0 & 0 & 2 & 0 & 0 & 0 & 2 & 0 & 0 & 0 & 0 & 0 & 0 & 0 & 0 & 1 & 0 & 0 & 1 & 0 & 0 & 0 & 0 & 0 & 0 & 0 & 2 & 1 & 3 & 3 & $\mathrm{P}$ & $\mathrm{F}$ & & & & \\
\hline Chenopodium album & 3 & 11 & 18 & 8 & 40 & 19 & 27 & 21 & 14 & 81 & 33 & 31 & 21 & 85 & 11 & 5 & 5 & 21 & 11 & 12 & 8 & 31 & 16 & 13 & 14 & 43 & 14 & 6 & 20 & 206 & 115 & 321 & 66 & A & $\mathrm{F}$ & $\mathrm{x}$ & & & \\
\hline Chenopodium hybridum & 0 & 1 & 1 & 0 & 2 & 0 & 0 & 1 & 0 & 1 & 1 & 1 & 0 & 2 & 0 & 1 & 0 & 1 & 0 & 0 & 0 & 0 & 2 & 0 & 0 & 2 & 1 & 0 & 1 & 5 & 4 & 9 & 7 & A & F & & & $\mathrm{x}$ & \\
\hline Chenopodium polyspermum & 0 & 0 & 2 & 0 & 2 & 2 & 1 & 2 & 1 & 6 & 3 & 3 & 4 & 10 & 5 & 1 & 1 & 7 & 5 & 2 & 0 & 7 & 6 & 1 & 3 & 10 & 3 & 1 & 4 & 18 & 28 & 46 & 24 & A & F & $\mathrm{x}$ & & & \\
\hline Cirsium arvense & 0 & 0 & 1 & 1 & 2 & 13 & 14 & 15 & 12 & 54 & 5 & 10 & 13 & 28 & 9 & 5 & 8 & 22 & 3 & 6 & 7 & 16 & 5 & 8 & 13 & 26 & 10 & 11 & 21 & 84 & 85 & 169 & 55 & $\mathrm{P}$ & $\mathrm{F}$ & $\mathrm{x}$ & & & \\
\hline Cirsium vulgare & 0 & 0 & 1 & 0 & 1 & 1 & 0 & 1 & 0 & 2 & 1 & 3 & 1 & 5 & 2 & 2 & 0 & 4 & 1 & 3 & 0 & 4 & 1 & 3 & 1 & 5 & 3 & 5 & 8 & 8 & 21 & 29 & 18 & $\mathrm{P}$ & F & & & & \\
\hline Convolvulus arvensis & 0 & 0 & 0 & 3 & 3 & 0 & 0 & 0 & 2 & 2 & 1 & 1 & 5 & 7 & 0 & 0 & 0 & 0 & 0 & 0 & 0 & 0 & 0 & 0 & 0 & 0 & 0 & 0 & 0 & 12 & 0 & 12 & $\begin{array}{l}10 \\
11\end{array}$ & $\mathrm{P}$ & $\mathrm{F}$ & $\mathrm{x}$ & & & \\
\hline Crepis biennis & 0 & 0 & 0 & 0 & 0 & 0 & 0 & 0 & 0 & 0 & 0 & 0 & 0 & 0 & 0 & 0 & 0 & 0 & 0 & 0 & 0 & 0 & 0 & 0 & 1 & 1 & 1 & 0 & 1 & 0 & 2 & 2 & 2 & $\mathrm{P}$ & $\mathrm{F}$ & & & & \\
\hline Dactylis glomerata & 0 & 0 & 0 & 0 & 0 & 27 & 14 & 17 & 11 & 69 & 3 & 3 & 2 & 8 & 5 & 6 & 4 & 15 & 7 & 4 & 7 & 18 & 3 & 7 & 7 & 17 & 4 & 9 & 13 & 77 & 63 & $\frac{2}{140}$ & 54 & $\mathrm{P}$ & G & & & & \\
\hline Daucus carota & 0 & 0 & 0 & 0 & 0 & 0 & 3 & 1 & 2 & 6 & 0 & 3 & 1 & 4 & 0 & 0 & 1 & 1 & 0 & 0 & 0 & 0 & 1 & 0 & 2 & 3 & 11 & 13 & 24 & 10 & 28 & 38 & 23 & $\mathrm{P}$ & $\mathrm{F}$ & & & & PFS \\
\hline Digitaria & 0 & 0 & 0 & 0 & 0 & 1 & 0 & 0 & 0 & 1 & 1 & 0 & 0 & 1 & 0 & 0 & 0 & 0 & 0 & 0 & 0 & 0 & 0 & 0 & 0 & 0 & 0 & 0 & 0 & 2 & 0 & 2 & 2 & A & G & $\mathrm{x}$ & & & \\
\hline Digitaria sangunanalis & 0 & 0 & 0 & 0 & 0 & 1 & 0 & 0 & 0 & 1 & 0 & 0 & 0 & 0 & 0 & 0 & 0 & 0 & 0 & 0 & 0 & 0 & 0 & 0 & 0 & 0 & 0 & 0 & 0 & 1 & 0 & 1 & 1 & A & G & $\mathrm{x}$ & & & \\
\hline Draba verna & 0 & 0 & 0 & 0 & 0 & 0 & 0 & 0 & 0 & 0 & 0 & 0 & 0 & 0 & 0 & 0 & 0 & 0 & 1 & 0 & 0 & 1 & 0 & 0 & 0 & 0 & 0 & 0 & 0 & 0 & 1 & 1 & 1 & A & $\mathrm{F}$ & $\mathrm{x}$ & & & \\
\hline Echinocbloa crus-galli & 2 & 7 & 9 & 7 & 25 & 7 & 12 & 5 & 5 & 29 & 18 & 11 & 14 & 43 & 4 & 1 & 3 & 8 & 8 & 5 & 0 & 13 & 10 & 7 & 3 & 20 & 12 & 3 & 15 & 97 & 56 & 153 & 51 & A & G & $\mathrm{x}$ & & & \\
\hline & 3 & 5 & 9 & 3 & 20 & 34 & 31 & 19 & 28 & 112 & 8 & 4 & 7 & 19 & 12 & 6 & 9 & 27 & 8 & 9 & 13 & 30 & 12 & 12 & 13 & 37 & 11 & 9 & 20 & 151 & 114 & 265 & 65 & $\mathrm{P}$ & G & $\mathrm{x}$ & & & \\
\hline & 0 & 1 & 0 & 0 & 1 & 0 & 0 & 0 & 0 & 0 & 0 & 0 & 0 & 0 & 1 & 0 & 0 & 1 & 0 & 0 & 0 & 0 & 0 & 0 & 0 & 0 & 0 & 0 & 0 & 1 & $\begin{array}{c}114 \\
1\end{array}$ & 2 & 2 & $\mathrm{P}$ & $\mathrm{F}$ & & & & \\
\hline & 0 & 0 & 0 & 0 & 0 & 0 & 0 & 1 & 0 & 1 & 0 & 1 & 0 & 1 & 0 & 0 & 0 & 0 & 1 & 1 & 1 & 3 & 0 & 0 & 1 & 1 & 0 & 2 & 2 & 2 & 6 & 8 & 7 & $\mathrm{P}$ & $\mathrm{F}$ & $\mathrm{x}$ & & & \\
\hline & 0 & 0 & 0 & 0 & 0 & 9 & 7 & 5 & 4 & 25 & 5 & 3 & 2 & 10 & 3 & 2 & 3 & 8 & 0 & 0 & 0 & 0 & 3 & 2 & 2 & 7 & 4 & 0 & 4 & 35 & 19 & 54 & 23 & $\mathrm{P}$ & $\mathrm{F}$ & $\mathrm{x}$ & & & \\
\hline & 0 & 0 & 0 & 0 & 0 & 0 & 1 & 0 & 0 & 1 & 1 & 1 & 0 & 2 & 0 & 0 & 0 & $\begin{array}{l}0 \\
0\end{array}$ & 0 & 0 & 0 & 0 & 0 & 1 & 0 & 1 & $\begin{array}{l}4 \\
0\end{array}$ & 1 & 1 & 3 & 2 & $\begin{array}{c}34 \\
5\end{array}$ & 23 & $P$ & F & & & & \\
\hline & 0 & 0 & 0 & 0 & 0 & 0 & 1 & 2 & 0 & 3 & 1 & 3 & 5 & 9 & 3 & 1 & 0 & & 5 & 0 & 0 & 14 & 1 & $\frac{1}{2}$ & 9 & 12 & 1 & 3 & & 12 & 35 & & 25 & & $\mathrm{~F}$ & $\mathrm{x}$ & & & \\
\hline & & 0 & 0 & 0 & 0 & 5 & 3 & 3 & 5 & 16 & 2 & 1 & 0 & 3 & 2 & 1 & & 4 & 2 & & 2 & 4 & 3 & & 4 & 9 & 0 & $i$ & & 19 & 17 & & 18 & & $\mathrm{~F}$ & $\mathrm{x}$ & & & \\
\hline & 0 & 0 & 0 & 0 & 0 & 0 & 0 & 0 & 0 & $\begin{array}{c}10 \\
0\end{array}$ & 0 & 0 & 0 & 0 & 0 & 0 & 0 & 0 & 0 & 0 & 0 & 0 & 0 & 0 & $\frac{4}{2}$ & 2 & & ( & & 0 & 3 & 3 & 3 & & F & $\mathrm{x}$ & & & \\
\hline lopia convolvulus & 5 & 6 & 15 & 9 & 35 & 21 & 30 & 26 & 23 & 100 & 35 & 25 & 20 & 80 & 13 & 6 & 7 & 26 & 12 & 10 & 9 & 31 & 16 & 9 & 14 & 39 & 9 & & & 215 & $\stackrel{3}{112}$ & 327 & 63 & $\mathrm{~A}$ & $\mathrm{~F}$ & x & & & \\
\hline
\end{tabular}




\section{CHAPTER 3}

\begin{tabular}{|c|c|c|c|c|c|c|c|c|c|c|c|c|c|c|c|c|c|c|c|c|c|c|c|c|c|c|c|c|c|c|c|c|c|c|c|c|c|c|c|}
\hline \multirow[b]{2}{*}{ Species name } & \multicolumn{5}{|c|}{$\begin{array}{l}\text { Field interior } \\
\text { (In) }\end{array}$} & \multicolumn{5}{|c|}{$\begin{array}{c}\begin{array}{c}\text { Field edge } \\
\text { (Ed) }\end{array} \\
\end{array}$} & \multicolumn{4}{|c|}{$\begin{array}{c}\text { Spillover } \\
\text { (Sp) }\end{array}$} & \multicolumn{4}{|c|}{$\begin{array}{l}\text { Conservation } \\
\text { field margin } \\
\text { (CFM) }\end{array}$} & & $\begin{array}{r}\text { Fallc } \\
(\mathrm{Fa}\end{array}$ & & & & (A) & & & & $\begin{array}{l}\text { ennia } \\
\text { er stri } \\
\text { PsF) }\end{array}$ & & & & & & & & & & & \\
\hline & $\begin{array}{l}\text { i } \\
\Xi\end{array}$ & $\vec{E}$ & $\begin{array}{l}\mathcal{I}_{1} \\
\Xi\end{array}$ & $\begin{array}{l}3 \\
\Xi\end{array}$ & 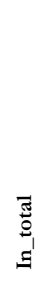 & 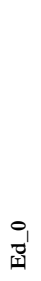 & ت्ञ & 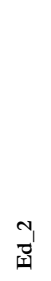 & $\vec{m}$ & 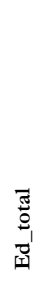 & $\frac{1}{2}$ & $\begin{array}{l}\tilde{1} \\
\hat{n}\end{array}$ & $\begin{array}{l}3 \\
\text { की }\end{array}$ & $\begin{array}{l}\text { 丞 } \\
\text { के }\end{array}$ & 至 & $\sum_{\text {岳 }}^{N}$ & $\sum_{\underbrace{m}_{U}}^{m}$ & 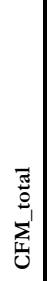 & F' & 胥 & ? & 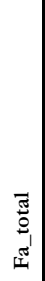 & 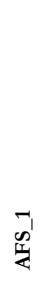 & 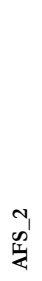 & 管 & 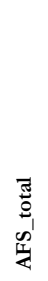 & $\approx$ & $\begin{array}{l}N_{1} \\
\text { 总 } \\
\text { 至 }\end{array}$ & 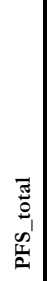 & 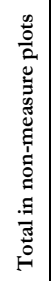 & 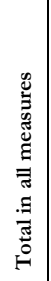 & 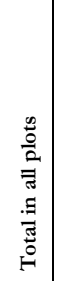 & 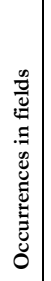 & 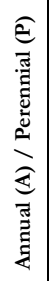 & 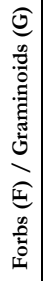 & $\begin{array}{l}\widehat{x} \\
0 \\
\frac{0}{2} \\
\frac{5}{2} \\
\frac{0}{0} \\
\frac{\pi}{4}\end{array}$ & $\begin{array}{l}\widehat{x} \\
\bar{g} \\
\bar{g} \\
\overline{0} \\
\overrightarrow{0} \\
\vec{z}\end{array}$ & 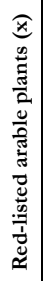 & 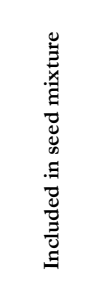 \\
\hline Festuca arundinacea & 0 & 0 & 0 & 0 & 0 & 0 & 0 & 0 & 0 & 0 & 0 & 0 & 0 & 0 & 0 & 0 & 0 & 0 & 0 & 0 & 0 & 0 & 0 & 0 & 0 & 0 & 0 & 1 & 1 & 0 & 1 & 1 & 1 & $\mathrm{P}$ & G & & & & \\
\hline Festuca pratensis & 0 & 0 & 0 & 0 & 0 & 5 & 2 & 1 & 2 & 10 & 0 & 0 & 0 & 0 & 0 & 1 & 0 & 1 & 0 & 0 & 0 & 0 & 1 & 1 & 0 & 2 & 0 & 0 & 0 & 10 & 3 & 13 & 12 & $\mathrm{P}$ & G & & & & \\
\hline Festuca rubra & 0 & 0 & 0 & 0 & 0 & 10 & 1 & 3 & 2 & 16 & 0 & 1 & 0 & 1 & 3 & 1 & 0 & 4 & 3 & 2 & 4 & 9 & 0 & 0 & 1 & 1 & 0 & 2 & 2 & 17 & 16 & 33 & 20 & P & G & & & & \\
\hline Fumaria officinalis & 0 & 0 & 0 & 0 & 0 & 0 & 0 & 0 & 0 & 0 & 0 & 0 & 0 & 0 & 0 & 0 & 1 & 1 & 1 & 0 & 0 & 1 & 0 & 0 & 0 & 0 & 1 & 0 & 1 & 0 & 3 & 3 & 3 & A & F & $\mathrm{x}$ & $\mathrm{x}$ & & \\
\hline Galeopsis speciosa & 0 & 0 & 0 & 0 & 0 & 0 & 0 & 0 & 0 & 0 & 0 & 0 & 0 & 0 & 0 & 0 & 0 & 0 & 1 & 0 & 1 & 2 & 0 & 0 & 0 & 0 & 0 & 0 & 0 & 0 & 2 & 2 & 1 & A & $\mathrm{F}$ & $\mathrm{x}$ & & $\mathrm{x}$ & \\
\hline Galeopsis tetrabit & 0 & 0 & 0 & 2 & 2 & 8 & 6 & 6 & 10 & 30 & 3 & 1 & 2 & 6 & 5 & 2 & 5 & 12 & 6 & 4 & 4 & 14 & 8 & 4 & 5 & 17 & 7 & 5 & 12 & 38 & 55 & 93 & 43 & A & $\mathrm{F}$ & $\mathrm{x}$ & & & \\
\hline Galinsogaga parviffora & 0 & 0 & 0 & 0 & 0 & 1 & 0 & 1 & 1 & 3 & 0 & 2 & 0 & 2 & 2 & 0 & 0 & 2 & 0 & 0 & 0 & 0 & 3 & 0 & 1 & 4 & 3 & 0 & 3 & 5 & 9 & 14 & 11 & A & F & $\mathrm{x}$ & & & \\
\hline Galinsoga quadriradiata & 0 & 0 & 0 & 0 & 0 & 0 & 0 & 0 & 0 & 0 & 0 & 0 & 0 & 0 & 1 & 0 & 0 & 1 & 0 & 0 & 0 & 0 & 0 & 1 & 0 & 1 & 0 & 0 & 0 & 0 & 2 & 2 & 2 & A & $\mathrm{F}$ & $\mathrm{x}$ & & & \\
\hline Galium aparine & 5 & 2 & 4 & 6 & 17 & 34 & 18 & 21 & 18 & 91 & 15 & 13 & 17 & 45 & 11 & 9 & 9 & 29 & 6 & 8 & 6 & 20 & 8 & 5 & 7 & 20 & 3 & 4 & 7 & 153 & 76 & 229 & 50 & A & $\mathrm{F}$ & $\mathrm{x}$ & & & \\
\hline Galium mollugo agg. & 0 & 0 & 0 & 0 & 0 & 1 & 1 & 0 & 1 & 3 & 0 & 3 & 2 & 5 & 0 & 0 & 0 & 0 & 1 & 0 & 2 & 3 & 1 & 8 & 4 & 13 & 1 & 12 & 13 & 8 & 29 & 37 & 27 & $\mathrm{P}$ & $\mathrm{F}$ & & & & AFS/PFS \\
\hline Galium uliginosum & 0 & 0 & 0 & 0 & 0 & 1 & 0 & 0 & 0 & 1 & 0 & 1 & 0 & 1 & 0 & 0 & 0 & 0 & 0 & 0 & 0 & 0 & 0 & 0 & 0 & 0 & 0 & 0 & 0 & 2 & 0 & 2 & 2 & $\mathrm{P}$ & F & & & & \\
\hline Galium verum & 0 & 0 & 0 & 0 & 0 & 0 & 0 & 0 & 0 & 0 & 0 & 0 & 0 & 0 & 0 & 0 & 0 & 0 & 0 & 0 & 0 & 0 & 0 & 0 & 0 & 0 & 0 & 1 & 1 & 0 & 1 & 1 & 1 & $\mathrm{P}$ & $\mathrm{F}$ & & & & \\
\hline Geranium dissectum & 0 & 0 & 0 & 0 & 0 & 1 & 1 & 1 & 3 & 6 & 1 & 0 & 2 & 3 & 3 & 1 & 3 & 7 & 0 & 0 & 1 & 1 & 1 & 0 & 2 & 3 & 1 & 2 & 3 & 9 & 14 & 23 & 12 & A & F & $\mathrm{x}$ & $\mathrm{x}$ & & \\
\hline Geranium molle & 0 & 0 & 0 & 1 & 1 & 1 & 1 & 0 & 1 & 3 & 0 & 0 & 0 & 0 & 0 & 0 & 0 & 0 & 0 & 0 & 0 & 0 & 0 & 0 & 0 & 0 & 0 & 0 & 0 & 4 & 0 & 4 & 4 & A & F & $\mathrm{x}$ & $\mathrm{x}$ & & \\
\hline Geranium pusillum & 0 & 2 & 1 & 5 & 8 & 16 & 17 & 14 & 14 & 61 & 12 & 8 & 13 & 33 & 7 & 4 & 7 & 18 & 7 & 7 & 10 & 24 & 9 & 2 & 6 & 17 & 5 & 8 & 13 & 102 & 72 & 174 & 53 & A & F & $\mathrm{x}$ & $\mathrm{x}$ & & \\
\hline Geranium robertianum & 0 & 0 & 0 & 0 & 0 & 0 & 0 & 0 & 0 & 0 & 0 & 0 & 0 & 0 & 0 & 0 & 0 & 0 & 1 & 0 & 0 & 1 & 0 & 0 & 0 & 0 & 0 & 0 & 0 & 0 & 1 & 1 & 1 & A & F & & $\mathrm{x}$ & & \\
\hline Geum urbanum & 0 & 0 & 0 & 0 & 0 & 0 & 0 & 0 & 0 & 0 & 1 & 0 & 0 & 1 & 1 & 0 & 0 & 1 & 0 & 0 & 0 & 0 & 0 & 0 & 0 & 0 & 0 & 0 & 0 & 1 & 1 & 2 & 1 & $\mathrm{P}$ & F & & & & \\
\hline Glecboma bederacea & 0 & 0 & 0 & 0 & 0 & 4 & 0 & 0 & 2 & 6 & 0 & 0 & 0 & 0 & 1 & 0 & 1 & 2 & 0 & 1 & 0 & 1 & 2 & 0 & 0 & 2 & 2 & 1 & 3 & 6 & 8 & 14 & 11 & $\mathrm{P}$ & F & $\mathrm{x}$ & & & \\
\hline Gyceria declinata & 0 & 0 & 0 & 0 & 0 & 0 & 0 & 0 & 0 & 0 & 0 & 0 & 0 & 0 & 0 & 0 & 0 & 0 & 0 & 0 & 0 & 0 & 0 & 0 & 0 & 0 & 1 & 0 & 1 & 0 & 1 & 1 & 1 & $\mathrm{P}$ & G & & & & \\
\hline Gnaphalium uliginosum & 0 & 0 & 1 & 0 & 1 & 0 & 1 & 1 & 1 & 3 & 2 & 3 & 0 & 5 & 0 & 0 & 0 & 0 & 1 & 2 & 0 & 3 & 1 & 1 & 1 & 3 & 1 & 0 & 1 & 9 & 7 & 16 & 12 & A & F & $\mathrm{x}$ & & & \\
\hline Heracleum sphondylium & 0 & 0 & 0 & 0 & 0 & 3 & 2 & 0 & 1 & 6 & 0 & 0 & 0 & 0 & 0 & 1 & 2 & 3 & 0 & 1 & 0 & 1 & 0 & 0 & 0 & 0 & 1 & 0 & 1 & 6 & 5 & 11 & 8 & $\mathrm{P}$ & $\mathrm{F}$ & & & & PFS \\
\hline Holcus lanatus & 0 & 0 & 0 & 0 & 0 & 17 & 4 & 12 & 12 & 45 & 1 & 1 & 2 & 4 & 4 & 2 & 4 & 10 & 3 & 8 & 8 & 19 & 4 & 8 & 11 & 23 & 3 & 10 & 13 & 49 & 65 & 114 & 53 & $\mathrm{P}$ & G & & & & \\
\hline Holcus mollis & 1 & 0 & 0 & 0 & 1 & 18 & 8 & 7 & 6 & 39 & 0 & 0 & 0 & 0 & 7 & 2 & 2 & 11 & 6 & 3 & 6 & 15 & 4 & 1 & 0 & 5 & 2 & 1 & 3 & 40 & 34 & 74 & 38 & $\mathrm{P}$ & G & $\mathrm{x}$ & & & \\
\hline Humulus lupulus & 0 & 0 & 0 & 0 & 0 & 0 & 0 & 0 & 1 & 1 & 0 & 1 & 0 & 1 & 0 & 0 & 0 & & 0 & 0 & 0 & 0 & 0 & 0 & 0 & 0 & 1 & 1 & 2 & 2 & 2 & 4 & 2 & $\mathrm{P}$ & $\mathrm{F}$ & & & & \\
\hline Hypericum maculatum & 0 & 0 & 0 & 0 & 0 & 0 & 0 & 0 & 0 & 0 & 0 & 0 & 0 & 0 & 0 & 0 & 0 & 0 & 1 & 1 & 1 & 3 & 0 & 0 & 0 & 0 & 0 & 0 & 0 & 0 & 3 & 3 & 2 & $\mathrm{P}$ & F & & & & \\
\hline Hypericum perforatum & 0 & 0 & 0 & 0 & 0 & 1 & 0 & 0 & 0 & 1 & 0 & 0 & 0 & 0 & 0 & 0 & 0 & & 0 & 0 & & 2 & 0 & 5 & 7 & 12 & 0 & 4 & 4 & 1 & 18 & 19 & 14 & P & F & & & & AFS/PFS \\
\hline Hypochaeris radicata & 0 & 0 & 0 & 0 & 0 & 0 & 0 & 1 & 0 & 1 & 0 & 0 & 0 & 0 & 0 & 0 & 0 & 0 & 0 & 0 & 0 & 0 & 0 & 1 & 1 & 2 & 1 & 0 & 1 & 1 & 3 & 4 & 4 & $\mathrm{P}$ & F & & & & PFS \\
\hline Iris psendacorus & 0 & 0 & 0 & 0 & 0 & 0 & 0 & 1 & 0 & 1 & 0 & 0 & 0 & 0 & 0 & 0 & 0 & 0 & 0 & 0 & 0 & 0 & 0 & 0 & 0 & 0 & 0 & 0 & 0 & 1 & & 1 & 1 & $\mathrm{P}$ & $\mathrm{F}$ & & & & \\
\hline Juncus bufonius & 0 & 0 & 0 & 0 & 0 & 1 & 0 & 0 & 0 & 1 & 0 & 0 & 0 & 0 & 1 & 0 & 0 & 1 & 2 & 1 & 0 & 3 & 1 & 0 & 1 & 2 & 0 & 0 & 0 & 1 & 6 & 7 & 7 & A & G & $\mathrm{x}$ & & & \\
\hline Juncus conglomeratus & 0 & 0 & 0 & 1 & 1 & 0 & 0 & 0 & 0 & 0 & 0 & 0 & 1 & 1 & 0 & 0 & 0 & 0 & 0 & 0 & 0 & 0 & 0 & 0 & 0 & 0 & 0 & 0 & 0 & 2 & 0 & 2 & 2 & $\mathrm{P}$ & G & & & & \\
\hline Lactuca serriola & 0 & 0 & 0 & 0 & 0 & 1 & 0 & 0 & 0 & 1 & 0 & 0 & 1 & 1 & 1 & 1 & 0 & 2 & 0 & 0 & 0 & 0 & 0 & 0 & 4 & 4 & 0 & 3 & 3 & 2 & 9 & 11 & 10 & A & $\mathrm{F}$ & $\mathrm{x}$ & & & \\
\hline Lamium alb. & 0 & 1 & 0 & 0 & 1 & 3 & 0 & 3 & 0 & 6 & 0 & 0 & 0 & 0 & 1 & 3 & 0 & 4 & 1 & 1 & 0 & 2 & 2 & 1 & 0 & 3 & 0 & 0 & 0 & 7 & 9 & 16 & 13 & $\mathrm{P}$ & $\mathrm{F}$ & $\mathrm{x}$ & $\mathrm{x}$ & & \\
\hline Lamin & 0 & 0 & 0 & 0 & 0 & 1 & 0 & 0 & 0 & 1 & 0 & 0 & 0 & 0 & 0 & 0 & 0 & 0 & 0 & 0 & 0 & 0 & 0 & 0 & 0 & 0 & 0 & 0 & 0 & 1 & 0 & 1 & 1 & A & F & $\mathrm{x}$ & $\mathrm{x}$ & & \\
\hline & 0 & 0 & 0 & 0 & 0 & 4 & 2 & 3 & 0 & 9 & 1 & 1 & 1 & 3 & 1 & 0 & 1 & 2 & 2 & 2 & 0 & 4 & 4 & 1 & 3 & 8 & 1 & 0 & 1 & 12 & 15 & 27 & 17 & A & $\mathrm{F}$ & $\mathrm{x}$ & $\mathrm{x}$ & & \\
\hline Lapsana communis & 0 & 0 & 0 & 0 & 0 & 3 & 0 & 2 & 2 & 7 & 0 & 0 & 2 & 2 & 2 & 1 & 1 & 4 & 2 & 1 & 1 & 4 & 4 & 0 & 3 & 7 & 1 & 1 & 2 & 9 & 17 & 26 & 13 & $\mathrm{P}$ & $\mathrm{F}$ & $\mathrm{x}$ & $\mathrm{x}$ & & \\
\hline Leucantbemum vulgare agg. & 0 & 0 & 0 & 0 & 0 & 0 & 0 & 0 & 0 & 0 & 0 & 1 & 1 & 2 & 0 & 0 & 0 & 0 & 0 & 0 & 0 & 0 & 1 & 7 & 9 & 17 & 5 & 12 & 17 & 2 & 34 & 36 & 25 & $\mathrm{P}$ & $\mathrm{F}$ & & & & AFS/PFS \\
\hline Linaria vulgaris & 0 & 0 & 0 & 0 & 0 & 0 & 0 & 0 & 0 & 0 & 0 & 0 & 0 & 0 & 0 & 0 & 1 & 1 & 0 & 0 & 1 & 1 & 1 & 3 & 7 & 11 & 1 & 4 & 5 & 0 & 18 & 18 & 14 & $\mathrm{P}$ & F & $\mathrm{x}$ & & & $\mathrm{AFS} / \mathrm{PFS}$ \\
\hline orrum & 0 & 0 & 1 & 3 & 4 & 4 & 3 & 5 & 3 & 15 & 1 & 3 & 3 & 7 & 3 & 1 & 1 & 5 & 1 & 2 & 0 & 3 & 1 & 1 & 2 & 4 & 0 & 1 & 1 & 26 & 13 & 39 & 21 & A & G & $\mathrm{x}$ & & & \\
\hline Lolium perenne & 1 & 0 & 0 & 1 & 2 & 12 & 7 & 5 & 6 & 30 & 5 & 6 & 5 & 16 & 1 & 3 & 3 & 7 & 3 & 3 & 3 & 9 & 6 & 2 & 5 & 13 & 3 & 4 & 7 & 48 & 36 & 84 & 35 & $P$ & G & $\mathrm{x}$ & & & PFS \\
\hline Lotus cormiculatus & 0 & 0 & 0 & 0 & 0 & 0 & 0 & 0 & 0 & 0 & 1 & 1 & 0 & 2 & 0 & 0 & 0 & 0 & 0 & 0 & 0 & 0 & 4 & 6 & 3 & 13 & 6 & 3 & 9 & 2 & 22 & 24 & 20 & $\mathrm{P}$ & $\mathrm{F}$ & & & & AFS/PFS \\
\hline Lychnis flos-cuculi & 0 & 0 & 0 & 0 & 0 & 0 & 0 & 0 & 0 & 0 & 0 & 0 & 0 & 0 & 0 & 0 & 0 & 0 & 0 & 0 & 0 & 0 & 0 & 0 & 0 & 0 & 1 & 0 & 1 & 0 & 1 & 1 & 1 & $P$ & $\mathrm{~F}$ & & & & PFS \\
\hline Lysimacbia nummularia & 0 & 0 & 0 & 0 & 0 & 0 & 0 & 1 & 0 & 1 & 0 & 0 & 0 & 0 & 0 & 0 & 0 & 0 & 0 & 0 & 0 & 0 & 0 & 0 & 0 & 0 & 0 & 0 & 0 & 1 & 0 & 1 & 1 & $\mathrm{P}$ & $\mathrm{F}$ & & & & \\
\hline Lysimacbia vulgaris & 0 & 0 & 0 & 0 & 0 & 1 & 0 & 1 & 0 & 2 & 0 & 0 & 0 & 0 & 0 & 1 & 1 & 2 & 0 & 0 & 0 & 0 & 0 & 0 & 0 & 0 & 0 & 0 & 0 & 2 & 2 & 4 & 4 & $\mathrm{P}$ & $\mathrm{F}$ & & & & \\
\hline Matricaria chamomilla & 7 & 2 & 10 & 8 & 27 & 29 & 19 & 19 & 15 & 82 & 22 & 29 & 21 & 72 & 12 & 7 & 11 & 30 & 10 & 11 & 12 & 33 & 13 & 6 & 12 & 31 & 2 & 7 & 9 & 181 & 103 & 284 & 57 & A & $\mathrm{F}$ & $\mathrm{x}$ & $\mathrm{x}$ & & \\
\hline Matricaria discoidea & 0 & 0 & 2 & 0 & 2 & 1 & 1 & 1 & 0 & 3 & 1 & 1 & 1 & 3 & 2 & 0 & 1 & 3 & 2 & 0 & 0 & 2 & 0 & 0 & 3 & 3 & 1 & 0 & 1 & 8 & 9 & 17 & 16 & A & $\mathrm{F}$ & $\mathrm{x}$ & & & \\
\hline Medicago lupulina & 0 & 0 & 0 & 0 & 0 & 0 & 0 & 1 & 1 & 2 & 0 & 2 & 1 & 3 & 0 & 0 & 1 & & 0 & 1 & 1 & 2 & 0 & 5 & 4 & 9 & & 2 & & 5 & 14 & 19 & 16 & $\mathrm{P}$ & F & $\mathrm{x}$ & & & AFS/PFS \\
\hline
\end{tabular}




\section{CHAPTER 3}

\begin{tabular}{|c|c|c|c|c|c|c|c|c|c|c|c|c|c|c|c|c|c|c|c|c|c|c|c|c|c|c|c|c|c|c|c|c|c|c|c|c|c|c|c|}
\hline \multirow[b]{2}{*}{ Species name } & \multicolumn{5}{|c|}{$\begin{array}{l}\text { Field interior } \\
\text { (In) }\end{array}$} & \multicolumn{5}{|c|}{$\begin{array}{c}\text { Field edge } \\
\text { (Ed) }\end{array}$} & \multicolumn{4}{|c|}{$\begin{array}{c}\text { Spillover } \\
(\mathrm{Sp})\end{array}$} & \multicolumn{4}{|c|}{$\begin{array}{l}\text { Conservation } \\
\text { field margin } \\
\text { (CFM) }\end{array}$} & & $\begin{array}{r}\text { Fall } \\
(\mathrm{F} .\end{array}$ & & & & & & & & $\begin{array}{l}\text { erenni- } \\
\text { wersts } \\
\text { (PFS) }\end{array}$ & & & & & & & & & & & \\
\hline & $\begin{array}{l}0 \\
\Xi\end{array}$ & $\vec{E}$ & $\begin{array}{l}N \\
\Xi\end{array}$ & $m_{\exists}$ & 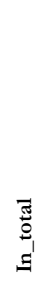 & 高 & 宔 & $\begin{array}{l}\text { Ni } \\
\text { İi }\end{array}$ & $\begin{array}{l}m \\
0 \\
0\end{array}$ & 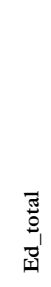 & $\begin{array}{l}1 \\
\text { के }\end{array}$ & $\begin{array}{l}\tilde{1} \\
\hat{n}\end{array}$ & $\begin{array}{l}3 \\
n^{2}\end{array}$ & 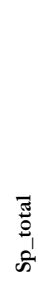 & 竞 & $\sum_{\text {戛 }}^{N}$ & 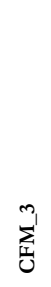 & 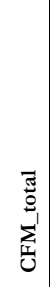 & का & 证 & ? & 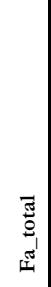 & 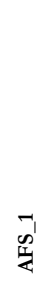 & 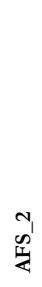 & 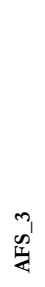 & 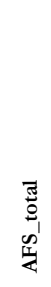 & $\begin{array}{c}\overrightarrow{1} \\
\text { 点 } \\
\text { 采 }\end{array}$ & 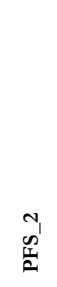 & 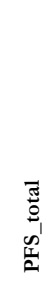 & 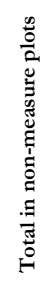 & 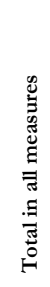 & 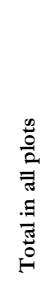 & 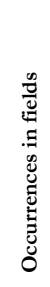 & 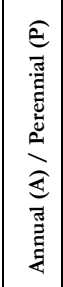 & 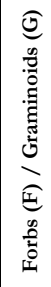 & 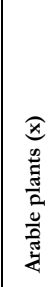 & 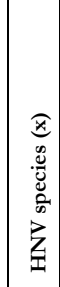 & 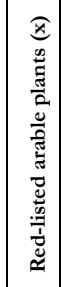 & 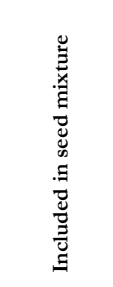 \\
\hline Melilotus alba & 0 & 0 & 0 & 0 & 0 & 0 & 0 & 0 & 0 & 0 & 0 & 0 & 0 & 0 & 0 & 0 & 0 & 0 & 0 & 0 & 0 & 0 & 2 & 1 & 4 & 7 & 0 & 1 & 1 & 0 & 8 & 8 & 6 & $P$ & F & & & & AFS \\
\hline Melilotus officinal & 0 & 0 & 0 & 0 & 0 & 0 & 0 & 0 & 0 & 0 & 0 & 1 & 0 & 1 & 0 & 0 & 0 & 0 & 0 & 0 & 0 & 0 & 2 & 6 & 5 & 13 & 0 & 1 & 1 & 1 & 14 & 15 & 12 & P & F & & & & \\
\hline Melilotu & 0 & 0 & 0 & 1 & 1 & 0 & 0 & 0 & 0 & 0 & 0 & 2 & 3 & 5 & 0 & 0 & 0 & 0 & 0 & 0 & 0 & 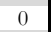 & 15 & 3 & 6 & 24 & 1 & 0 & 1 & 6 & 25 & 31 & 19 & NA & F & & & & AFS \\
\hline Mentha arvensis & 0 & 0 & 0 & 0 & 0 & 0 & 0 & 0 & 0 & 0 & 0 & 0 & 0 & 0 & 0 & 0 & 0 & 0 & 0 & 0 & 0 & 0 & 0 & 0 & 0 & 0 & 0 & 1 & 1 & 0 & 1 & 1 & 1 & $\mathrm{P}$ & $\mathrm{F}$ & $\mathrm{x}$ & & & \\
\hline Moebringia t & 0 & 0 & 0 & 0 & 0 & 1 & 0 & 0 & 0 & 1 & 0 & 0 & 0 & 0 & 0 & 0 & 0 & 0 & 0 & 0 & 0 & 0 & 0 & 0 & 0 & 0 & 0 & 0 & 0 & 1 & 0 & 1 & 1 & A & $\mathrm{F}$ & & & & \\
\hline Myosotis arvensis & 2 & 0 & 2 & 2 & 6 & 17 & 10 & 11 & 10 & 48 & 17 & 11 & 15 & 43 & 13 & 4 & 12 & 29 & 9 & 9 & 9 & 27 & 9 & 4 & 7 & 20 & 4 & 7 & 11 & 97 & 87 & 184 & 53 & A & $\mathrm{F}$ & $\mathrm{x}$ & $\mathrm{x}$ & & \\
\hline Odontites vernus & 0 & 0 & 0 & 0 & 0 & 2 & 1 & 1 & 0 & 4 & 0 & 1 & 2 & 3 & 0 & 1 & 0 & 1 & 1 & 1 & 0 & 2 & 0 & 0 & 1 & 1 & 0 & 0 & 0 & 7 & 4 & 11 & 6 & A & F & $\mathrm{x}$ & & $\mathrm{x}$ & \\
\hline Oxalis stricta & 0 & 0 & 0 & 0 & 0 & 0 & 0 & 0 & 0 & 0 & 0 & 0 & 0 & 0 & 0 & 1 & 0 & 1 & 0 & 0 & 0 & 0 & 0 & 0 & 0 & 0 & 0 & 0 & 0 & 0 & 1 & 1 & 1 & $\mathrm{P}$ & $\mathrm{F}$ & $\mathrm{x}$ & & & \\
\hline Panicum miliaceum & 0 & 0 & 0 & 0 & 0 & 0 & 0 & 0 & 0 & 0 & 0 & 0 & 0 & 0 & 0 & 0 & 0 & 0 & 0 & 0 & 0 & 0 & 0 & 1 & 0 & 1 & 0 & 0 & 0 & 0 & 1 & 1 & 1 & A & G & & & & \\
\hline Papaver argemone & 0 & 0 & 0 & 0 & 0 & 2 & 0 & 0 & 0 & 2 & 0 & 0 & 0 & 0 & 0 & 0 & 0 & 0 & 2 & 2 & 2 & 6 & 0 & 0 & 1 & 1 & 0 & 0 & 0 & 2 & 7 & 9 & 4 & A & F & $\mathrm{x}$ & $\mathrm{x}$ & & \\
\hline Papaver dubiumn agg. & 0 & 0 & 0 & 0 & 0 & 3 & 1 & 0 & 0 & 4 & 0 & 0 & 1 & 1 & 2 & 1 & 1 & 4 & 5 & 3 & 4 & 12 & 3 & 0 & 4 & 7 & 0 & 1 & 1 & 5 & 24 & 29 & 17 & A & F & $\mathrm{x}$ & $\mathrm{x}$ & & AFS \\
\hline Papaver rboeas & 0 & 0 & 0 & 0 & 0 & 3 & 2 & 1 & 0 & 6 & 2 & 3 & 2 & 7 & 0 & 0 & 0 & 0 & 3 & 1 & 3 & 7 & 2 & 2 & 10 & 14 & 0 & 2 & 2 & 13 & 23 & 36 & 21 & A & $\mathrm{F}$ & $\mathrm{x}$ & $\mathrm{x}$ & & \\
\hline Papaver spec. & 0 & 0 & 0 & 0 & 0 & 0 & 0 & 1 & 0 & 1 & 0 & 1 & 0 & 1 & 0 & 0 & 0 & 0 & 0 & 0 & 0 & 0 & 0 & 0 & 0 & 0 & 0 & 0 & 0 & 2 & 0 & 2 & 2 & NA & $\mathrm{F}$ & & $\mathrm{x}$ & & \\
\hline Persicaria amphibia & 1 & 0 & 0 & 0 & 1 & 2 & 0 & 2 & 1 & 5 & 2 & 4 & 0 & 6 & 1 & 0 & 1 & 2 & 1 & 1 & 0 & 2 & 0 & 0 & 1 & 1 & 4 & 3 & 7 & 12 & 12 & 24 & 16 & $\mathrm{P}$ & $\mathrm{F}$ & $\mathrm{x}$ & & & \\
\hline Persicaria hydropiper & 0 & 0 & 4 & 1 & 5 & 1 & 1 & 4 & 1 & 7 & 2 & 0 & 1 & 3 & 2 & 1 & 0 & 3 & 2 & 2 & 0 & 4 & 2 & 0 & 1 & 3 & 1 & 2 & 3 & 15 & 13 & 28 & 16 & A & F & $\mathrm{x}$ & & & \\
\hline Persicaria lapathijolia & 0 & 0 & 0 & 0 & 0 & 0 & 0 & 0 & 0 & 0 & 0 & 0 & 2 & 2 & 0 & 0 & 0 & 0 & 0 & 0 & 1 & 1 & 0 & 0 & 3 & 3 & 0 & 1 & 1 & 2 & 5 & 7 & 7 & A & F & $\mathrm{x}$ & & & \\
\hline Persicaria maculssa & 0 & 2 & 5 & 2 & 9 & 3 & 6 & 5 & 5 & 19 & 10 & 5 & 5 & 20 & 5 & 1 & 3 & 9 & 3 & 5 & 1 & 9 & 13 & 6 & 5 & 24 & 11 & 2 & 13 & 48 & 55 & 103 & 47 & A & F & $\mathrm{x}$ & & & \\
\hline Pbalaris arundinacea & 0 & 0 & 0 & 0 & 0 & 1 & 1 & 0 & 0 & 2 & 0 & 0 & 0 & 0 & 0 & 0 & 0 & 0 & 0 & 0 & 1 & 1 & 0 & 0 & 0 & 0 & 0 & 0 & 0 & 2 & 1 & 3 & 3 & $P$ & G & & & & \\
\hline Pbleum pratense & 0 & 0 & 0 & 0 & 0 & 0 & 1 & 0 & 0 & 1 & 1 & 1 & 0 & 2 & 0 & 0 & 0 & 0 & 0 & 0 & 0 & 0 & 2 & 3 & 2 & 7 & 0 & 6 & 6 & 3 & 13 & 16 & 13 & P & G & & & & PFS \\
\hline Phragnites australis & 0 & 0 & 0 & 0 & 0 & 1 & 0 & 2 & 2 & 5 & 0 & 0 & 1 & 1 & 2 & 1 & 0 & 3 & 0 & 0 & 0 & 0 & 0 & 1 & 1 & 2 & 0 & 1 & 1 & 6 & 6 & 12 & 5 & P & G & $\mathrm{x}$ & & & \\
\hline Plantago lanceolata & 0 & 0 & 0 & 0 & 0 & 1 & 3 & 1 & 2 & 7 & 1 & 1 & 1 & 3 & 2 & 2 & 0 & 4 & 1 & 2 & 2 & 5 & 3 & 2 & 2 & 7 & 10 & 13 & 23 & 10 & 39 & 49 & 27 & $\mathrm{P}$ & $\mathrm{F}$ & $\mathrm{x}$ & & & PFS \\
\hline Plantago major & 0 & 1 & 0 & 0 & 1 & 0 & 1 & 1 & 0 & 2 & 0 & 2 & 1 & 3 & 0 & 0 & 0 & 0 & 1 & 0 & 1 & 2 & 1 & 2 & 3 & 6 & 2 & 2 & 4 & 6 & 12 & 18 & 14 & $\mathrm{P}$ & $\mathrm{F}$ & $\mathrm{x}$ & & & \\
\hline Poa aпnиa & 2 & 6 & 6 & 2 & 16 & 4 & 5 & 6 & 2 & 17 & 13 & 8 & 4 & 25 & 1 & 2 & 0 & 3 & 3 & 6 & 2 & 11 & 2 & 3 & 3 & 8 & 2 & 2 & 4 & 58 & 26 & 84 & 45 & A & G & $\mathrm{x}$ & & & \\
\hline a comp & 0 & 0 & 0 & 0 & 0 & 0 & 0 & 0 & 0 & 0 & & 0 & 0 & 0 & 0 & 0 & 0 & 0 & 1 & 0 & 0 & 1 & 0 & 0 & 0 & 0 & 0 & 0 & 0 & 0 & 1 & 1 & 1 & P & G & & & & \\
\hline Poa palustris & 0 & 0 & 0 & 0 & 0 & 0 & 0 & 0 & 0 & 0 & 0 & 0 & 0 & 0 & 0 & 0 & 0 & 0 & 0 & 0 & 0 & 0 & 0 & 0 & 0 & 0 & 1 & 2 & 3 & 0 & 3 & 3 & 3 & P & G & & & & PFS \\
\hline Poa pratensis & 0 & 0 & 1 & 1 & 2 & 8 & 3 & 1 & 4 & 16 & 0 & 2 & 1 & 3 & 0 & 1 & 2 & 3 & 0 & 1 & 0 & 1 & 0 & 0 & 2 & 2 & 3 & 4 & 7 & 21 & 13 & 34 & 28 & P & G & & & & PFS \\
\hline Poa trivialis & 1 & 0 & 2 & 1 & 4 & 23 & 5 & 15 & 6 & 49 & 4 & 8 & 3 & 15 & 2 & 8 & 5 & 15 & 2 & 7 & 4 & 13 & 4 & 5 & 5 & 14 & 2 & 8 & 10 & 68 & 52 & 120 & 49 & P & G & $\mathrm{x}$ & & & \\
\hline Polygonum a & 3 & 4 & 18 & 6 & 31 & 27 & 28 & 26 & 22 & 103 & 26 & 26 & 26 & 78 & 15 & 7 & 8 & 30 & 14 & 10 & 10 & 34 & 15 & 7 & 10 & 32 & 10 & 4 & 14 & 212 & 110 & 322 & 64 & A & $\mathrm{F}$ & $\mathrm{x}$ & & & \\
\hline & 0 & 0 & 0 & 0 & 0 & 0 & 0 & 0 & 0 & 0 & 0 & 0 & 0 & 0 & 1 & 0 & 1 & 2 & 0 & 0 & 0 & 0 & 0 & 1 & 0 & 1 & 0 & 0 & 0 & 0 & 3 & 3 & 2 & P & F & $\mathrm{x}$ & & & \\
\hline Prunella & 0 & 0 & 0 & 0 & 0 & 0 & 0 & 0 & 0 & 0 & 0 & 0 & 0 & 0 & 0 & 0 & 0 & 0 & 0 & 0 & 1 & 1 & 0 & 1 & 1 & 2 & 0 & 2 & 2 & 0 & 5 & 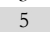 & 5 & P & $\mathrm{F}$ & & & & AFS/PFS \\
\hline & 0 & 0 & 0 & 0 & 0 & 0 & 1 & 0 & 0 & 1 & 0 & 0 & 0 & 0 & 0 & 1 & 0 & 1 & 1 & 1 & 1 & 3 & 0 & 1 & & 4 & 2 & 2 & 4 & 1 & 12 & 13 & 9 & P & $\mathrm{F}$ & $\mathrm{x}$ & & & \\
\hline$R$ & ( & 1 & 0 & 0 & 1 & 0 & 5 & 0 & 0 & 5 & 0 & 4 & 0 & 4 & 0 & 1 & 0 & 1 & 1 & 0 & 0 & 1 & 1 & 2 & 3 & 6 & 1 & 0 & 1 & 10 & 9 & 19 & 15 & A & $\mathrm{F}$ & $\mathrm{x}$ & & & \\
\hline & 0 & 0 & 0 & 0 & 0 & 3 & 1 & 2 & 3 & 9 & 1 & 0 & 0 & 1 & 1 & 1 & 1 & 3 & & 2 & 2 & 6 & 1 & 2 & 1 & 4 & 0 & 1 & 1 & 10 & 14 & 24 & 13 & P & $\mathrm{F}$ & & & & \\
\hline & 0 & 0 & 0 & 0 & 0 & 1 & 0 & 0 & 1 & 2 & 0 & 0 & 0 & 0 & 0 & 0 & 0 & 0 & 0 & 0 & 0 & 0 & 0 & 2 & 1 & 1 & 0 & 0 & 0 & 10 & $\begin{array}{l}14 \\
\end{array}$ & & 3 & 1 & $\mathrm{~F}$ & & & & \\
\hline & 0 & 0 & 0 & 0 & 0 & 2 & 0 & 1 & 0 & 3 & 1 & 0 & 0 & 1 & 0 & 1 & 0 & 1 & 0 & 2 & 0 & 2 & 0 & 2 & 1 & 3 & 2 & 2 & 4 & 4 & 10 & 14 & 11 & $P$ & F & & & & PFS \\
\hline & 0 & 0 & 0 & 0 & 0 & 4 & 1 & 1 & 0 & 6 & 0 & 0 & 0 & 0 & 1 & 2 & 0 & 3 & 4 & 4 & 4 & 12 & 1 & 1 & 1 & 3 & 0 & 0 & 0 & 6 & 18 & 24 & 13 & P & $\mathrm{F}$ & $\mathrm{x}$ & $x$ & & \\
\hline$R_{t}$ & 0 & 0 & 0 & 0 & 0 & 2 & 2 & 2 & 1 & 7 & 1 & 2 & 1 & 4 & 0 & 2 & 1 & 3 & 2 & 3 & 5 & 10 & 3 & 4 & 10 & 17 & 1 & 6 & 7 & 11 & 37 & 48 & 26 & $\mathrm{P}$ & F & $\mathrm{x}$ & & & \\
\hline & 1 & 0 & 1 & 0 & 2 & 7 & 3 & 4 & 1 & 15 & 4 & 6 & 3 & 13 & 5 & 3 & 4 & 12 & 5 & 4 & 3 & 12 & 6 & 6 & 2 & 14 & 4 & 4 & 8 & 30 & 46 & 76 & 30 & P & $\mathrm{F}$ & $\mathrm{x}$ & & & \\
\hline иtbus аппии & 0 & 0 & 0 & 0 & 0 & 1 & 0 & 0 & 0 & 1 & 0 & 0 & 0 & 0 & 1 & 0 & 1 & 2 & 1 & 1 & 2 & 4 & 0 & 0 & 0 & 0 & 0 & 0 & 0 & 1 & 6 & 7 & 3 & A & $\mathrm{F}$ & $\mathrm{x}$ & & & \\
\hline oneroides autumn & 0 & 0 & 0 & 0 & 0 & 1 & 0 & 0 & 0 & 1 & 0 & 0 & 0 & 0 & 1 & 0 & 0 & 1 & 1 & 0 & 0 & 1 & 1 & 1 & 0 & 2 & 3 & 6 & 9 & 1 & 13 & 14 & 13 & $\mathrm{P}$ & $\mathrm{F}$ & & & & PFS \\
\hline Scropbularia nodosa & 0 & 0 & 0 & 0 & 0 & 0 & 0 & 0 & 0 & 0 & 0 & 0 & 0 & 0 & 0 & 0 & 0 & 0 & 0 & 0 & 0 & 0 & 0 & 0 & 0 & 0 & 1 & 1 & 2 & 0 & 2 & 2 & 2 & $\mathrm{P}$ & F & & & & \\
\hline tellaria galericulata & 0 & 0 & 0 & 0 & 0 & 0 & 0 & 0 & 0 & 0 & 0 & 0 & 0 & 0 & 0 & 0 & 0 & 0 & 0 & 0 & 0 & 0 & 0 & 0 & 1 & 1 & 0 & 0 & 0 & 0 & 1 & 1 & 1 & $\mathrm{P}$ & $\mathrm{F}$ & & & & \\
\hline Senecio inaequidens & 0 & 0 & 0 & 0 & 0 & 0 & 0 & 0 & 0 & 0 & 0 & 0 & 0 & 0 & 0 & 0 & 0 & 0 & 1 & 0 & 0 & 1 & 0 & 0 & 2 & 2 & 0 & 0 & 0 & 0 & 3 & 3 & 3 & $\mathrm{P}$ & F & & & & \\
\hline Senecio jacobaea & 0 & 0 & 0 & 0 & 0 & 0 & 0 & 0 & 0 & 0 & 0 & 1 & 0 & 1 & 0 & 0 & 1 & 1 & 1 & 1 & 0 & 2 & 0 & 0 & 0 & 0 & 0 & 1 & 1 & 1 & 4 & 5 & 3 & $\mathrm{P}$ & $\mathrm{F}$ & & & & \\
\hline Senecio vulgaris & 2 & 0 & 1 & 0 & 3 & 2 & 1 & 0 & 0 & 3 & 3 & 5 & 1 & 9 & 1 & 0 & 0 & 1 & 3 & 2 & 1 & 6 & 2 & 0 & 3 & 5 & 0 & 0 & 0 & 15 & 12 & 27 & 15 & A & $\mathrm{F}$ & $\mathrm{x}$ & & & \\
\hline taria spec. & 0 & 0 & 0 & 1 & 1 & 0 & 0 & & 1 & 1 & 0 & 2 & 0 & & 0 & 0 & 1 & & 0 & 0 & 1 & & & & 0 & 0 & & 1 & & 4 & 3 & 7 & 6 & NA & G & & & & \\
\hline
\end{tabular}




\section{CHAPTER 3}

\begin{tabular}{|c|c|c|c|c|c|c|c|c|c|c|c|c|c|c|c|c|c|c|c|c|c|c|c|c|c|c|c|c|c|c|c|c|c|c|c|c|c|c|c|}
\hline \multirow[b]{2}{*}{ Species name } & \multicolumn{5}{|c|}{$\begin{array}{l}\text { Field interior } \\
\text { (In) }\end{array}$} & \multicolumn{5}{|c|}{$\begin{array}{l}\text { Field edge } \\
\text { (Ed) }\end{array}$} & \multicolumn{4}{|c|}{$\begin{array}{c}\text { Spillover } \\
\text { (Sp) }\end{array}$} & \multicolumn{4}{|c|}{$\begin{array}{l}\text { Conservation } \\
\text { field margin } \\
\text { (CFM) }\end{array}$} & & $\begin{array}{r}\text { Fallc } \\
(\mathrm{Fa}\end{array}$ & & & & (A) & & & & $\begin{array}{l}\text { erenniz } \\
\text { wer str } \\
\text { (PFS) }\end{array}$ & & & & & & & & & & & \\
\hline & $\begin{array}{l}\text { i } \\
\Xi\end{array}$ & $\vec{E}$ & $\begin{array}{l}N_{1} \\
\Xi\end{array}$ & $m_{\exists}$ & 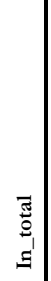 & 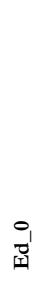 & i & $\begin{array}{l}N_{1} \\
\text { in }\end{array}$ & $\begin{array}{l}m \\
\text { mit }\end{array}$ & 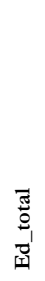 & की & $\begin{array}{l}\tilde{1} \\
\hat{n}\end{array}$ & $\begin{array}{l}3 \\
\text { की }\end{array}$ & $\begin{array}{l}\text { 丞 } \\
\text { के }\end{array}$ & 至 & $\sum_{\text {岳 }}^{N}$ & $\sum_{\underbrace{m}_{U}}^{m}$ & 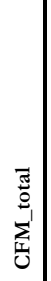 & F' & 胥 & ? & 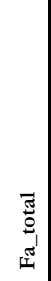 & 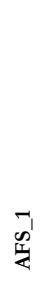 & 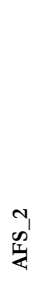 & 管 & 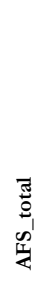 & 吾' & $\begin{array}{l}N^{\prime} \\
\text { 总 } \\
\text { 至 }\end{array}$ & 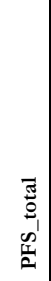 & 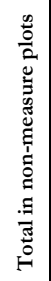 & 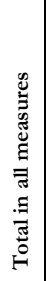 & 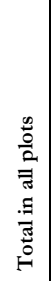 & 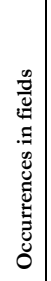 & 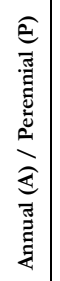 & 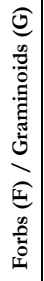 & $\begin{array}{l}\widehat{x} \\
0 \\
\frac{0}{2} \\
\frac{5}{2} \\
\frac{0}{0} \\
\frac{\pi}{4}\end{array}$ & $\begin{array}{l}\widehat{x} \\
\bar{g} \\
\bar{g} \\
\overline{0} \\
\overrightarrow{0} \\
\vec{z}\end{array}$ & 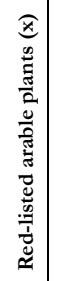 & 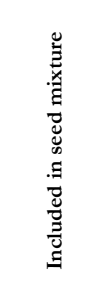 \\
\hline Setaria verticillata & 0 & 0 & 0 & 0 & 0 & 0 & 0 & 0 & 1 & 1 & 1 & 0 & 1 & 2 & 0 & 0 & 0 & 0 & 0 & 0 & 0 & 0 & 1 & 0 & 0 & 1 & 0 & 0 & 0 & 3 & 1 & 4 & 3 & A & $\overline{\mathrm{G}}$ & $\mathrm{x}$ & & & \\
\hline Setaria verticilliformis & 0 & 0 & 0 & 0 & 0 & 0 & 0 & 0 & 0 & 0 & 1 & 0 & 0 & 1 & 0 & 0 & 0 & 0 & 0 & 0 & 0 & 0 & 0 & 0 & 0 & 0 & 0 & 0 & 0 & 1 & 0 & 1 & 1 & A & G & $\mathrm{x}$ & & & \\
\hline Setaria viridis & 0 & 2 & 2 & 4 & 8 & 0 & 5 & 4 & 3 & 12 & 5 & 4 & 2 & 11 & 0 & 1 & 0 & 1 & 0 & 0 & 0 & 0 & 4 & 4 & 1 & 9 & 2 & 0 & 2 & 31 & 12 & 43 & 19 & A & G & $\mathrm{x}$ & & & \\
\hline Silene dioica & 0 & 0 & 0 & 0 & 0 & 0 & 0 & 0 & 2 & 2 & 0 & 1 & 1 & 2 & 1 & 0 & 0 & 1 & 1 & 0 & 0 & 1 & 2 & 8 & 5 & 15 & 1 & 3 & 4 & 4 & 21 & 25 & 18 & $\mathrm{P}$ & $\mathrm{F}$ & & & & $\mathrm{AFS}$ \\
\hline Silene latifolia & 0 & 0 & 0 & 0 & 0 & 1 & 3 & 2 & 1 & 7 & 1 & 6 & 4 & 11 & 1 & 0 & 0 & 1 & 1 & 1 & 0 & 2 & 6 & 12 & 14 & 32 & 11 & 12 & 23 & 18 & 58 & 76 & 35 & $\mathrm{P}$ & $\mathrm{F}$ & $\mathrm{x}$ & & & AFS/PFS \\
\hline Silene spec. & 0 & 0 & 0 & 0 & 0 & 0 & 0 & 0 & 0 & 0 & 0 & 2 & 0 & 2 & 0 & 0 & 0 & 0 & 0 & 0 & 0 & 0 & 0 & 0 & 0 & 0 & 0 & 0 & 0 & 2 & 0 & 2 & 2 & NA & $\mathrm{F}$ & & & & \\
\hline Silene vulgaris & 0 & 0 & 0 & 0 & 0 & 0 & 0 & 0 & 0 & 0 & 0 & 1 & 0 & 1 & 0 & 0 & 0 & 0 & 0 & 0 & 0 & 0 & 0 & 0 & 2 & 2 & 2 & 2 & 4 & 1 & 6 & 7 & 5 & $\mathrm{P}$ & F & & & & \\
\hline Sinapis arvensis & 0 & 0 & 0 & 0 & 0 & 0 & 0 & 0 & 1 & 1 & 2 & 1 & 0 & 3 & 1 & 0 & 0 & 1 & 1 & 0 & 0 & 1 & 4 & 3 & 1 & 8 & 2 & 0 & 2 & 4 & 12 & 16 & 13 & A & $\mathrm{F}$ & $\mathrm{x}$ & & & \\
\hline Sicymbrium officinale & 1 & 1 & 8 & 1 & 11 & 10 & 7 & 6 & 3 & 26 & 7 & 11 & 6 & 24 & 9 & 3 & 10 & 22 & 6 & 4 & 6 & 16 & 11 & 10 & 10 & 31 & 5 & 4 & 9 & 61 & 78 & 139 & 46 & A & $\mathrm{F}$ & $\mathrm{x}$ & & & \\
\hline Solanum nigrum & 0 & 2 & 4 & 1 & 7 & 1 & 3 & 4 & 4 & 12 & 6 & 5 & 4 & 15 & 1 & 1 & 2 & 4 & 4 & 0 & 1 & 5 & 8 & 4 & 4 & 16 & 4 & 0 & 4 & 34 & 29 & 63 & 29 & A & $\mathrm{F}$ & $\mathrm{x}$ & & & \\
\hline Solidago canadensis & 0 & 0 & 0 & 0 & 0 & 0 & 0 & 0 & 0 & 0 & 0 & 0 & 0 & 0 & 1 & 0 & 0 & 1 & 0 & 0 & 0 & 0 & 0 & 0 & 0 & 0 & 0 & 0 & 0 & 0 & 1 & 1 & 1 & P & F & & & & \\
\hline Solidago gigantea & 0 & 0 & 0 & 0 & 0 & 0 & 0 & 0 & 0 & 0 & 0 & 0 & 0 & 0 & 0 & 0 & 0 & 0 & 0 & 0 & 0 & 0 & 0 & 0 & 1 & 1 & 0 & 0 & 0 & 0 & 1 & 1 & 1 & P & F & & & & \\
\hline Sonchus arvensis & 0 & 0 & 0 & 0 & 0 & 0 & 0 & 0 & 1 & 1 & 0 & 0 & 0 & 0 & 0 & 0 & 0 & 0 & 0 & 0 & 0 & 0 & 1 & 1 & 0 & 2 & 0 & 0 & 0 & 1 & 2 & 3 & 2 & $\mathrm{P}$ & F & $\mathrm{x}$ & & & \\
\hline Sonchus asper & 0 & 0 & 0 & 0 & 0 & 0 & 1 & 1 & 1 & 3 & 1 & 2 & 5 & 8 & 7 & 0 & 4 & 11 & 3 & 1 & 1 & 5 & 4 & 1 & 5 & 10 & 6 & 2 & 8 & 11 & 34 & 45 & 33 & A & F & $\mathrm{x}$ & & & \\
\hline Soncbus oleraceus & 0 & 0 & 0 & 0 & 0 & 1 & 2 & 0 & 0 & 3 & 2 & 0 & 0 & 2 & 1 & 0 & 2 & 3 & 0 & 0 & 1 & 1 & 1 & 0 & 3 & 4 & 0 & 0 & 0 & 5 & 8 & 13 & 10 & A & F & $\mathrm{x}$ & & & \\
\hline Soncbus spec. & 0 & 0 & 0 & 0 & 0 & 0 & 0 & 1 & 3 & 4 & 0 & 0 & 3 & 3 & 0 & 0 & 0 & 0 & 0 & 0 & 0 & 0 & 0 & 0 & 1 & 1 & 0 & 0 & 0 & 7 & 1 & 8 & 7 & NA & F & & & & \\
\hline Spergula arvensis & 0 & 0 & 0 & 0 & 0 & 0 & 0 & 0 & 0 & 0 & 0 & 0 & 0 & 0 & 0 & 0 & 0 & 0 & 2 & 1 & 1 & 4 & 3 & 0 & 0 & 3 & 2 & 0 & 2 & 0 & 9 & 9 & 9 & A & F & $\mathrm{x}$ & $\mathrm{x}$ & & \\
\hline Stachys palusstris & 0 & 0 & 1 & 0 & 1 & 0 & 0 & 1 & 0 & 1 & 0 & 1 & 0 & 1 & 0 & 0 & 0 & 0 & 1 & 0 & 0 & 1 & 1 & 0 & 0 & 1 & 0 & 0 & 0 & 3 & 2 & 5 & 4 & $\mathrm{P}$ & F & $\mathrm{x}$ & & & \\
\hline Stellaria graminea & 0 & 0 & 0 & 0 & 0 & 5 & 0 & 1 & 0 & 6 & 0 & 0 & 0 & 0 & 0 & 1 & 0 & 1 & 3 & 2 & 1 & 6 & 0 & 1 & 1 & 2 & 0 & 2 & 2 & 6 & 11 & 17 & 11 & $\mathrm{P}$ & F & $\mathrm{x}$ & & & PFS \\
\hline Stellaria media & 6 & 3 & 6 & 4 & 19 & 13 & 19 & 8 & 7 & 47 & 18 & 19 & 12 & 49 & 8 & 4 & 7 & 19 & 6 & 13 & 9 & 28 & 9 & 3 & 7 & 19 & 6 & 4 & 10 & 115 & 76 & 191 & 60 & A & $\mathrm{F}$ & $\mathrm{x}$ & & & \\
\hline Symphytum officinale & 0 & 0 & 0 & 0 & 0 & 0 & 0 & 1 & 0 & 1 & 0 & 0 & 0 & 0 & 0 & 0 & 0 & 0 & 0 & 0 & 0 & 0 & 0 & 0 & 0 & 0 & 0 & 0 & 0 & 1 & 0 & 1 & 1 & $\mathrm{P}$ & $\mathrm{F}$ & $\mathrm{x}$ & & & \\
\hline Tanacetum vulgare & 0 & 0 & 0 & 0 & 0 & 5 & 4 & 2 & 2 & 13 & 0 & 3 & 0 & 3 & 3 & 3 & 2 & & 5 & 6 & 5 & 16 & 1 & 3 & 6 & 10 & 7 & 11 & 18 & 16 & 52 & 68 & 33 & $\mathrm{P}$ & $\mathrm{F}$ & & & & PFS \\
\hline Taraxacum sect. Ruderalia & 0 & 0 & 1 & 0 & 1 & 7 & 2 & 1 & 2 & 12 & 0 & 4 & 2 & 6 & 2 & 2 & 2 & 6 & 1 & 4 & 2 & 7 & 2 & 8 & 4 & 14 & 3 & 3 & 6 & 19 & 33 & 52 & 31 & $\mathrm{P}$ & F & $\mathrm{x}$ & & & \\
\hline Thlaspi arvense & 0 & 0 & 0 & 0 & 0 & 0 & 0 & 1 & 0 & 1 & 0 & 0 & 0 & 0 & 0 & 0 & 1 & & 0 & 0 & 0 & 0 & 1 & 0 & 0 & 1 & 1 & 0 & 1 & 1 & & 4 & 4 & A & $\mathrm{F}$ & $\mathrm{x}$ & $\mathrm{x}$ & & \\
\hline Torilis japonica & 0 & 0 & 0 & 0 & 0 & 0 & 0 & 0 & 0 & 0 & 0 & 0 & 0 & 0 & 0 & 1 & 0 & 1 & 0 & 0 & 0 & 0 & 0 & 0 & 0 & 0 & 0 & 0 & 0 & 0 & 1 & 1 & 1 & $\mathrm{P}$ & F & $\mathrm{x}$ & & & \\
\hline Trifolium arvense & 0 & 0 & 0 & 0 & 0 & 0 & 0 & 0 & 0 & 0 & 0 & 0 & 0 & 0 & 1 & 0 & 0 & 1 & 1 & 0 & 0 & 1 & 0 & 1 & 1 & 2 & 0 & 0 & 0 & 0 & 4 & 4 & 3 & A & F & $\mathrm{x}$ & $\mathrm{x}$ & & \\
\hline Trifolium dubium & 0 & 0 & 0 & 0 & 0 & 2 & 0 & 0 & 0 & 2 & 0 & 0 & 0 & 0 & 0 & 1 & 0 & 1 & 1 & 0 & 1 & 2 & 0 & 0 & 0 & 0 & 0 & 0 & 0 & 2 & 3 & 5 & 4 & A & F & & & & \\
\hline Trifolium pratense & 0 & 0 & 0 & 0 & 0 & 0 & 0 & 0 & 0 & 0 & 1 & 4 & 0 & 5 & 0 & 1 & 0 & 1 & 0 & 0 & 0 & 0 & 1 & 0 & 1 & 2 & 7 & 8 & 15 & 5 & 18 & 23 & 16 & $\mathrm{P}$ & $\mathrm{F}$ & & & & PFS \\
\hline Trifolium repens & 0 & 0 & 0 & 0 & 0 & 1 & 1 & 2 & 2 & 6 & 1 & 3 & 5 & 9 & 1 & 0 & 1 & 2 & 1 & 4 & 1 & 6 & 3 & 2 & 3 & 8 & 1 & 3 & 4 & 15 & 20 & 35 & 28 & P & $\mathrm{F}$ & & & & \\
\hline Trifolium resupinatum & 0 & 0 & 0 & 0 & 0 & 0 & 0 & 0 & 0 & 0 & 0 & 0 & 0 & 0 & 0 & 0 & 0 & 0 & 0 & 0 & 0 & 0 & 0 & 0 & 0 & 0 & 1 & 0 & 1 & 0 & 1 & 1 & 1 & A & F & & & & \\
\hline Trifolium spec. & 0 & 1 & 0 & 0 & 1 & 0 & 0 & 0 & 0 & 0 & 0 & 0 & 3 & 3 & 0 & 0 & 1 & 1 & 0 & 0 & 0 & 0 & 0 & 0 & 0 & 0 & 0 & 0 & 0 & 4 & 1 & 5 & 5 & NA & $\mathrm{F}$ & & & & \\
\hline Tripleuro. & 0 & 0 & 3 & 2 & 5 & 3 & 6 & 2 & 6 & 17 & 7 & 11 & 14 & 32 & 8 & 4 & 9 & 21 & 8 & 5 & 8 & 21 & 6 & 3 & 9 & 18 & 6 & 9 & 15 & 54 & 75 & 129 & 52 & A & $\mathrm{F}$ & $\mathrm{x}$ & & & \\
\hline & 0 & 0 & 0 & 0 & 0 & 0 & 0 & 0 & 0 & 0 & 1 & 0 & 0 & 1 & 0 & 0 & 0 & 0 & 0 & 0 & 0 & 0 & 0 & 0 & 0 & 0 & 0 & 0 & 0 & 1 & 0 & 1 & 1 & $\mathrm{P}$ & G & & & & \\
\hline & 0 & 0 & 0 & 0 & 0 & 0 & 1 & 0 & 0 & 1 & 0 & 0 & 0 & 0 & 1 & 0 & 0 & 1 & 0 & 0 & 0 & 0 & 1 & 0 & 0 & 1 & 0 & 0 & 0 & 1 & 2 & 3 & 3 & $\mathrm{P}$ & G & & & & \\
\hline Urtica dioica & 0 & 0 & 0 & 0 & 0 & 16 & 12 & 8 & 6 & 42 & 3 & 2 & 1 & 6 & 5 & 5 & 4 & 14 & 3 & 5 & 5 & 13 & 3 & 4 & 4 & 11 & 3 & 5 & 8 & 48 & 46 & 94 & 40 & $\mathrm{P}$ & $\mathrm{F}$ & & & & \\
\hline Urtica urens & 0 & 0 & 0 & 0 & 0 & 1 & 0 & 0 & 0 & 1 & 1 & 0 & 0 & 1 & 0 & 0 & 0 & 0 & 1 & 1 & 0 & 2 & 0 & 0 & 0 & 0 & 0 & 0 & 0 & 2 & 2 & 4 & 1 & A & F & $\mathrm{x}$ & & & \\
\hline Verbascum ychnitis & 0 & 0 & 0 & 0 & 0 & 0 & 0 & 0 & 0 & 0 & 0 & 0 & 0 & 0 & 0 & 0 & 0 & 0 & 0 & 0 & 0 & 0 & 0 & 0 & 1 & 1 & 0 & 0 & 0 & 0 & 1 & 1 & 1 & $P$ & $\mathrm{~F}$ & & & & \\
\hline ascum nigrt & 0 & 0 & 0 & 0 & 0 & 0 & 0 & 0 & 0 & 0 & 0 & 0 & 0 & 0 & 0 & 0 & 0 & 0 & 0 & 0 & 0 & 0 & 0 & 1 & 4 & 5 & 0 & 0 & 0 & 0 & 5 & 5 & 4 & $\mathrm{P}$ & $\mathrm{F}$ & & & & AFS \\
\hline Veronica arvensis & 0 & 0 & 0 & 0 & 0 & 6 & 2 & 0 & 1 & 9 & 0 & 1 & 0 & 1 & 2 & 1 & 1 & 4 & 5 & 1 & 4 & 10 & 2 & 1 & 1 & 4 & 1 & 2 & 3 & 10 & 21 & 31 & 20 & A & F & $\mathrm{x}$ & & & \\
\hline Veronica chamaedys & 0 & 0 & 0 & 0 & 0 & 0 & 0 & 1 & 0 & 1 & 0 & 1 & 0 & 1 & 0 & 0 & 0 & 0 & 0 & 0 & 0 & 0 & 0 & 0 & 0 & 0 & 0 & 2 & 2 & 2 & 2 & 4 & 4 & $\mathrm{P}$ & $\mathrm{F}$ & & & & PFS \\
\hline Veronica bederifolia & 0 & 0 & 1 & 0 & 1 & 7 & 2 & 3 & 2 & 14 & 0 & 1 & 0 & 1 & 1 & 1 & 2 & 4 & 0 & 0 & 0 & 0 & 0 & 0 & 1 & 1 & 0 & 0 & 0 & 16 & 5 & 21 & 17 & $\mathrm{~A}$ & $\mathrm{~F}$ & $\mathrm{x}$ & & & \\
\hline Veronica persica & 0 & 0 & 0 & 0 & 0 & 0 & 2 & 0 & 2 & 4 & 1 & 1 & 0 & 2 & 1 & 0 & 0 & 1 & 1 & 2 & 2 & 5 & 0 & 0 & 1 & 1 & 0 & 0 & 0 & 6 & 7 & 13 & 10 & A & F & $\mathrm{x}$ & & & \\
\hline Veronica polita & 0 & 0 & 0 & 0 & 0 & 0 & 1 & 0 & 1 & 2 & 0 & 0 & 0 & 0 & 0 & 0 & 0 & 0 & 0 & 0 & 0 & 0 & 0 & 0 & 0 & 0 & 0 & 0 & 0 & 2 & 0 & 2 & 2 & $\mathrm{~A}$ & $\mathrm{~F}$ & $\mathrm{x}$ & & & \\
\hline Vicia angustifolia & 0 & 0 & 0 & 0 & 0 & 4 & 1 & 0 & 0 & 5 & 1 & 0 & 0 & 1 & 3 & 2 & 1 & 6 & 3 & 5 & 2 & 10 & 2 & 1 & 3 & 6 & 1 & 1 & 2 & 6 & 24 & 30 & 17 & A & $\mathrm{F}$ & $\mathrm{x}$ & $\mathrm{x}$ & & \\
\hline Vicia cracca & 0 & 0 & 0 & 0 & 0 & 0 & 0 & 0 & 0 & 0 & 0 & 0 & 0 & 0 & 0 & 0 & 0 & 0 & 1 & 1 & 0 & 2 & 0 & 0 & 3 & 3 & 0 & 4 & 4 & 0 & 9 & 9 & 9 & $\mathrm{P}$ & F & $\mathrm{x}$ & $\mathrm{x}$ & & AFS/PFS \\
\hline Vicia birsuta & 2 & 0 & 3 & 0 & 5 & 18 & 8 & 6 & 6 & 38 & 2 & 3 & 4 & 9 & 8 & 5 & 3 & & 8 & 5 & 8 & 21 & 6 & 2 & 5 & 13 & 4 & 5 & 9 & 52 & 59 & & 40 & A & $\mathrm{F}$ & $\mathrm{x}$ & & & \\
\hline
\end{tabular}


CHAPTER 3

\begin{tabular}{|c|c|c|c|c|c|c|c|c|c|c|c|c|c|c|c|c|c|c|c|c|c|c|c|c|c|c|c|c|c|c|c|c|c|c|c|c|c|c|c|}
\hline \multirow[b]{2}{*}{ Species name } & \multicolumn{5}{|c|}{$\begin{array}{l}\text { Field interior } \\
\text { (In) }\end{array}$} & \multicolumn{5}{|c|}{$\begin{array}{c}\text { Field edge } \\
\text { (Ed) }\end{array}$} & \multicolumn{4}{|c|}{$\begin{array}{c}\text { Spillover } \\
\text { (Sp) }\end{array}$} & \multicolumn{4}{|c|}{$\begin{array}{l}\text { Conservation } \\
\text { field margin } \\
\text { (CFM) }\end{array}$} & \multicolumn{4}{|c|}{$\begin{array}{c}\begin{array}{c}\text { Fallow } \\
(\mathrm{Fa})\end{array} \\
\end{array}$} & \multicolumn{4}{|c|}{$\begin{array}{c}\text { Alternately managed } \\
\text { biennial flower strip } \\
\text { (AFS) }\end{array}$} & \multicolumn{3}{|c|}{$\begin{array}{c}\text { Perennial } \\
\text { flower strip } \\
\text { (PFS) } \\
\end{array}$} & \multirow[b]{2}{*}{ 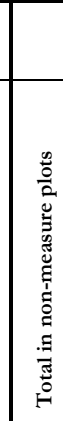 } & \multirow[b]{2}{*}{ 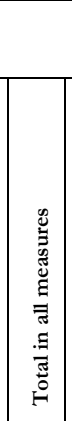 } & \multirow[b]{2}{*}{ 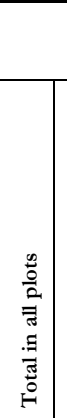 } & \multirow{2}{*}{\multicolumn{2}{|c|}{ 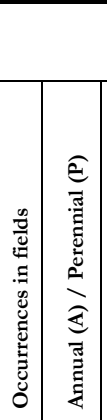 }} & \multirow[b]{2}{*}{ 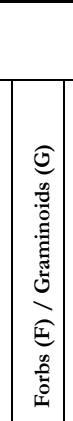 } & \multirow[b]{2}{*}{ 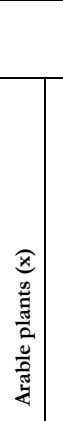 } & \multirow{2}{*}{\multicolumn{2}{|c|}{ 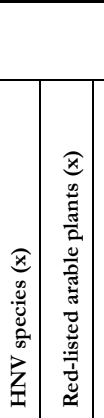 }} & \multirow[b]{2}{*}{ 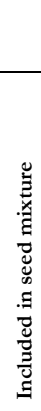 } \\
\hline & $\begin{array}{l}\hat{I} \\
\Xi\end{array}$ & $\exists$ & $\begin{array}{l}\tilde{I} \\
\Xi\end{array}$ & $\stackrel{m}{\Xi}$ & 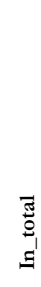 & i & 吾 & $\begin{array}{l}N_{1}^{\prime} \\
\text { I }\end{array}$ & i & 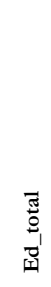 & $\frac{1}{\text { के }}$ & $\begin{array}{l}n \\
\text { के }\end{array}$ & $\begin{array}{l}3 \\
n_{1}\end{array}$ & $\begin{array}{l}\text { जٓ } \\
\stackrel{5}{\prime} \\
\text { की }\end{array}$ & $\sum_{\text {夏 }}^{-1}$ & $\sum_{\text {SI }}^{N}$ & $\sum_{0}^{m}$ & 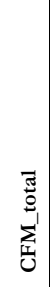 & का & $\tilde{F}^{1}$ & ? & 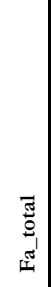 & 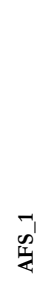 & 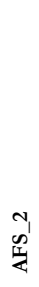 & $\begin{array}{l}m_{1}^{\prime} \\
0^{\prime}\end{array}$ & 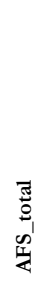 & 吾 & $\begin{array}{l}N_{1} \\
\text { 点 } \\
\text { 点 }\end{array}$ & 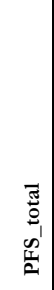 & & & & & & & & & & \\
\hline Vicia sativa & 0 & 0 & 0 & 0 & 0 & 7 & 2 & 3 & 6 & 18 & 1 & 1 & 2 & 4 & 3 & 1 & 2 & 6 & 3 & 2 & 3 & 8 & 1 & 0 & 1 & 2 & 0 & 2 & 2 & 22 & 18 & 40 & 20 & A & F & & $\mathrm{x}$ & & \\
\hline & 0 & 0 & 0 & 0 & 0 & 0 & 0 & 0 & 0 & 0 & 0 & 0 & 1 & 1 & 0 & 0 & 0 & 0 & 0 & 0 & 0 & 0 & 0 & 0 & 0 & 0 & 0 & 0 & 0 & 1 & 0 & 1 & 1 & NA & $\mathrm{F}$ & & $\mathrm{x}$ & & \\
\hline Vicia tetra. & 0 & 0 & 0 & 0 & 0 & 2 & 0 & 0 & 1 & 3 & 0 & 0 & 0 & 0 & 0 & 0 & 0 & 0 & 1 & 0 & 0 & 1 & 1 & 0 & 0 & 1 & 2 & 2 & 4 & 3 & 6 & 9 & 7 & A & $\mathrm{F}$ & x & $\mathrm{x}$ & & \\
\hline Vicia villo. & 0 & 1 & 0 & 0 & 1 & 0 & 0 & 0 & 0 & 0 & 0 & 0 & 0 & 0 & 0 & 0 & 0 & 0 & 0 & 0 & 0 & 0 & 0 & 0 & 0 & 0 & 0 & 0 & 0 & 1 & 0 & 1 & 1 & A & F & $\mathrm{x}$ & $\mathrm{x}$ & & \\
\hline Viola an & 3 & 6 & 22 & 17 & 48 & 23 & 25 & 22 & 19 & 89 & 33 & 20 & 30 & 83 & 15 & 11 & 10 & 36 & 13 & 8 & 12 & 33 & 12 & 3 & 7 & 22 & 5 & 6 & 11 & 220 & 102 & 322 & 58 & A & F & $\mathrm{x}$ & & & \\
\hline Vulpia myuros & 0 & 0 & 0 & 0 & 0 & 0 & 1 & 0 & 0 & 1 & 0 & 0 & 0 & 0 & 0 & 0 & 0 & 0 & 0 & 0 & 1 & 1 & 1 & 2 & 2 & 5 & 0 & 0 & 0 & 1 & 6 & & 4 & A & $\mathrm{G}$ & & & & \\
\hline
\end{tabular}




\section{CHAPTER 3}

Table A.8 Summary of model structures and statistical results.

Anova results (significance codes: '***' 0.001 '**' 0.01 '*' 0.05 '? 0.1 '” 1; n.s. = not significant)

\begin{tabular}{|c|c|c|c|c|c|c|c|c|c|}
\hline Measured value & Model & $\mathrm{n}$ & $\mathbf{R}^{2}$ conditional & $\mathbf{R}^{2}$ marginal & Explanatory variables & \begin{tabular}{|l|}
$\begin{array}{l}\text { Z-value } \\
\text { (chi-square tests. } \\
\text { Type II Wald) }\end{array}$ \\
\end{tabular} & $\begin{array}{l}\text { Degrees of } \\
\text { freedom }\end{array}$ & $\begin{array}{l}\text { P-value } \\
\text { (Pr(chi-square) })\end{array}$ & Significance \\
\hline $\begin{array}{l}\text { Total herbaceous species } \\
\text { number }\end{array}$ & 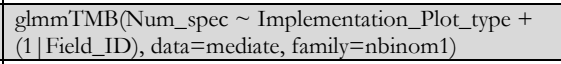 & \begin{tabular}{|l|}
721 plots \\
215 species \\
\end{tabular} & 0.726 & 0.693 & Implementation Plot type & 1330.500 & 21 & $<2.2 \mathrm{e}-16$ & *** \\
\hline Total herbaceous species cover & \begin{tabular}{|l|} 
glmmTMB(Cover $\sim$ Implementation_Plot_type + \\
$(1 \mid$ Field_ID), data =mediate, family $=$ nbinom1)
\end{tabular} & \begin{tabular}{|l|l|}
721 plots \\
215 species \\
\end{tabular} & 0.698 & 0.690 & Implementation Plot type & 1528.900 & 21 & $<2.2 \mathrm{e}-16$ & *** \\
\hline $\begin{array}{l}\text { Total species number of arable } \\
\text { plants }\end{array}$ & \begin{tabular}{|l} 
glmmTMB (Num_spec $\sim$ Implementation_Plot_type \\
$+(1 \mid$ Field_ID), data $=$ arable_plants, family=nbinom 1$)$ \\
\end{tabular} & \begin{tabular}{|l|l|}
721 plots \\
122 species \\
\end{tabular} & 0.689 & 0.649 & Implementation Plot type & 1120.300 & 21 & $<2.2 \mathrm{e}-16$ & *** \\
\hline Total cover of arable plants & \begin{tabular}{|l|} 
glmmTMB (Cover $\sim$ Implementation_Plot_type + \\
$(1 \mid$ Field_ID), data $=$ arable_plants, family $=$ nbinom1) \\
\end{tabular} & \begin{tabular}{|l|}
721 plots \\
122 species \\
\end{tabular} & 0.656 & 0.641 & Implementation Plot type & 1266.400 & 21 & $<2.2 \mathrm{e}-16$ & *** \\
\hline $\begin{array}{l}\text { Total species number of high } \\
\text { nature value species }\end{array}$ & $\begin{array}{l}\text { glmmTMB_Num_spec } \sim \text { Implementation_Plot_type + } \\
(1 \mid \text { Field_ID), data }=\text { HNV, family }=\text { nbinom1) } \\
\end{array}$ & \begin{tabular}{|l|l|}
721 plots \\
29 species \\
\end{tabular} & 0.630 & 0.442 & Implementation Plot type & 458.18 & 21 & $<2.2 \mathrm{e}-16$ & *** \\
\hline $\begin{array}{l}\text { Total species cover of high } \\
\text { nature value species }\end{array}$ & \begin{tabular}{|l|} 
glmmTMB(Cover $\sim$ Implementation_Plot_type + \\
$(1 \mid$ Field_ID), data $=$ HNV, family=nbinom1)
\end{tabular} & $\begin{array}{l}721 \text { plots } \\
29 \text { species }\end{array}$ & 0.549 & 0.404 & Implementation Plot type & 560.18 & 21 & $<2.2 \mathrm{e}-16$ & *** \\
\hline $\begin{array}{l}\text { Total species number of } \\
\text { annual plants in measure plots } \\
\text { and field edge }\end{array}$ & $\begin{array}{l}\text { glmmTMB(Num_spec } \sim \text { Implementation_Plot_type }+ \\
\text { (1|Field_ID), data }=\text { annuals, family=nbinom1) }\end{array}$ & $\begin{array}{l}408 \text { plots } \\
91 \text { species }\end{array}$ & 0.551 & 0.450 & Implementation Plot type & 390.25 & 14 & $<2.2 \mathrm{e}-16$ & *** \\
\hline $\begin{array}{l}\text { Total species cover of annual } \\
\text { plants in measure plots and } \\
\text { field edge }\end{array}$ & \begin{tabular}{|l} 
glmmTMB(Cover $\sim$ Implementation_Plot_type + \\
$(1 \mid$ Field_ID), data $=$ annuals, family $=$ nbinom1)
\end{tabular} & $\begin{array}{l}408 \text { plots } \\
91 \text { species }\end{array}$ & 0.533 & 0.519 & Implementation Plot type & 410.44 & 14 & $<2.2 \mathrm{e}-16$ & *** \\
\hline $\begin{array}{l}\text { Total species number of } \\
\text { perennial plants in measure } \\
\text { plots and field edge }\end{array}$ & $\begin{array}{l}\text { glmmTMB(Num_spec } \sim \text { Implementation_Plot_type }+ \\
(1 \mid \text { Field_ID), data }=\text { perennials, family }=\text { nbinom1) }\end{array}$ & $\begin{array}{l}408 \text { plots } \\
116 \text { species }\end{array}$ & 0.574 & 0.509 & Implementation Plot type & 455.75 & 14 & $<2.2 \mathrm{e}-16$ & *** \\
\hline $\begin{array}{l}\text { Total species cover of } \\
\text { perennial plants in measure } \\
\text { plots and field edge }\end{array}$ & $\begin{array}{l}\text { glmmTMB(Cover } \sim \text { Implementation_Plot_type }+ \\
(1 \mid \text { Field_ID), data }=\text { perennials, family=nbinom1) }\end{array}$ & $\begin{array}{l}408 \text { plots } \\
116 \text { species }\end{array}$ & 0,616 & 0,531 & Implementation Plot type & 368,19 & 14 & $<2.2 \mathrm{e}-16$ & *** \\
\hline $\begin{array}{l}\text { Total species number of } \\
\text { graminoids in measure plots } \\
\text { and field edge }\end{array}$ & $\begin{array}{l}\text { glmmTMB(Num_spec } \sim \text { Implementation_Plot_type }+ \\
\text { (1|Field_ID), data =graminoids, family=nbinom1) }\end{array}$ & $\begin{array}{l}408 \text { plots } \\
51 \text { species }\end{array}$ & 0.306 & 0.290 & Implementation Plot type & 152.03 & 14 & $<2.2 \mathrm{e}-16$ & *** \\
\hline $\begin{array}{l}\text { Total species cover of } \\
\text { graminoids in measure plots } \\
\text { and field edge }\end{array}$ & \begin{tabular}{|l} 
glmmTMB(Cover $\sim$ Implementation_Plot_type + \\
$(1 \mid$ Field_ID), data $=$ graminoids, family $=$ nbinom1)
\end{tabular} & $\begin{array}{l}408 \text { plots } \\
51 \text { species }\end{array}$ & 0,486 & 0,420 & Implementation Plot type & 207,76 & 14 & $<2.2 \mathrm{e}-16$ & *** \\
\hline $\begin{array}{l}\text { Total species number of forbs } \\
\text { in measure plots and field } \\
\text { edge }\end{array}$ & $\begin{array}{l}\text { glmmTMB(Num_spec } \sim \text { Implementation_Plot_type + } \\
\text { (1|Field_ID), data }=\text { forbs, family }=\text { nbinom1) }\end{array}$ & $\begin{array}{l}408 \text { plots } \\
164 \text { species }\end{array}$ & 0.618 & 0.552 & Implementation Plot type & 508.14 & 14 & $<2.2 \mathrm{e}-16$ & *** \\
\hline $\begin{array}{l}\text { Total species cover of forbs in } \\
\text { measure plots and field edge }\end{array}$ & $\begin{array}{l}\text { glmmTMB(Cover } \sim \text { Implementation_Plot_type }+ \\
(1 \mid \text { Field_ID), data=forbs, family=nbinom1) }\end{array}$ & \begin{tabular}{|l|}
408 plots \\
164 species \\
\end{tabular} & 0.605 & 0.584 & Implementation Plot type & 452.32 & 14 & $<2.2 \mathrm{e}-16$ & *** \\
\hline \multirow{2}{*}{$\begin{array}{l}\text { Total herbaceous species } \\
\text { number }\end{array}$} & \multirow{2}{*}{$\begin{array}{l}\text { glmmTMB(Num_spec } \sim \text { Plot_type + Precip_4_7 }+ \\
(1 \mid \text { Field_ID), data }=\text { mediate, family }=\text { nbinom1) }\end{array}$} & \multirow{2}{*}{\begin{tabular}{|l}
721 plots \\
215 species \\
\end{tabular}} & \multirow[t]{2}{*}{0.683} & \multirow[t]{2}{*}{0.654} & Plot_type & 1073.8395 & 6 & $<2 \mathrm{e}-16$ & $* * *$ \\
\hline & & & & & Precipitation_april_july & 6.1699 & 1 & 0.01 & * \\
\hline $\begin{array}{l}\text { Total herbaceous species } \\
\text { number }\end{array}$ & \begin{tabular}{|l|} 
glmmTMB(Num_spec $\sim$ Plot_type + Temp_4_7 + \\
$(1 \mid$ Field_ID), data $=$ mediate, family $=$ nbinom1) \\
\end{tabular} & \begin{tabular}{|l|}
721 plots \\
215 species \\
\end{tabular} & 0.704 & 0.673 & \begin{tabular}{|l|} 
Plot_type \\
Temperature_april_july \\
\end{tabular} & $\begin{array}{l}1138.486 \\
39.869 \\
\end{array}$ & $\begin{array}{l}6 \\
1 \\
\end{array}$ & \begin{tabular}{|l|}
$<2.2 \mathrm{e}-16$ \\
$2.72 \mathrm{E}-10$ \\
\end{tabular} & \begin{tabular}{|l|l}
$* * *$ \\
$* * *$ \\
\end{tabular} \\
\hline \multirow{2}{*}{ Total herbaceous species cover } & \multirow{2}{*}{$\begin{array}{l}\text { glmmTMB(Cover } \sim \text { Plot_type + Precip_4_7 + } \\
(1 \mid \text { Field_ID), data }=\text { mediate, family=nbinom1) }\end{array}$} & \multirow{2}{*}{\begin{tabular}{|l|}
721 plots \\
215 species \\
\end{tabular}} & \multirow{2}{*}{0.674} & \multirow{2}{*}{0.663} & Plot_type & \begin{tabular}{|l|l|}
1346.092 \\
\end{tabular} & 6 & $<2 \mathrm{e}-16$ & $* * *$ \\
\hline & & & & & Precipitation_april_july & 5.717 & 1 & 0.0168 & $*$ \\
\hline \multirow{2}{*}{ Total herbaceous species cover } & \multirow{2}{*}{\begin{tabular}{|l|}
$\begin{array}{l}\text { glmmTMB(Cover } \sim \text { Plot_type }+ \text { Temp_4_7 } \\
(1 \mid \text { Field_ID), data=mediate, family=nbinom1) }\end{array}$ \\
\end{tabular}} & \multirow{2}{*}{\begin{tabular}{|l|}
721 plots \\
215 species \\
\end{tabular}} & \multirow{2}{*}{0.685} & \multirow{2}{*}{0.675} & \begin{tabular}{|l|l|l|} 
Plot_type \\
\end{tabular} & 1405.614 & 6 & $<2.2 \mathrm{e}-16$ & $* * *$ \\
\hline & & & & & Temperature_april_july & \begin{tabular}{|l|}
27.094 \\
\end{tabular} & 1 & $1.94 \mathrm{E}-07$ & $* * *$ \\
\hline $\begin{array}{l}\text { Total herbaceous plant beta- } \\
\text { diversity (without genera) }\end{array}$ & $\begin{array}{l}\text { Ime(distances } \sim \text { Implementation_Plot_type, random }=\sim ~ \\
1 \mid \text { Field_ID, data }=\text { mediate_model) }\end{array}$ & \begin{tabular}{|l|}
693 plots \\
207 species \\
\end{tabular} & 0.381 & 0.302 & Implementation Plot type & 332.67 & 21 & $<2.2 \mathrm{e}-16$ & $* * *$ \\
\hline $\begin{array}{l}\text { Total herbaceous species } \\
\text { number per calendar year }\end{array}$ & $\begin{array}{l}\text { model_nbinom1_simpler_year <-glmmTMB(Num_spec } \\
\sim \text { Year_plot_type + (1|Field_ID), data=mediate, } \\
\text { family=nbinom1) }\end{array}$ & $\begin{array}{l}721 \text { plots } \\
215 \text { species }\end{array}$ & 0.734 & 0.700 & \begin{tabular}{|l|l} 
Year_plot_type \\
\end{tabular} & \begin{tabular}{|l|l}
1363.6 \\
\end{tabular} & 20 & $<2.2 \mathrm{e}-16$ & *** \\
\hline $\begin{array}{l}\text { Total herbaceous species cover } \\
\text { per calendar year }\end{array}$ & $\begin{array}{l}\text { model_nbinom1_simpler_year_cover <- } \\
\text { glmmTMB(Cover_all_plants } \sim \text { Year_plot_type + } \\
\text { (1|Field_ID), data=mediate, family=nbinom1) } \\
\end{array}$ & $\begin{array}{l}721 \text { plots } \\
215 \text { species }\end{array}$ & 0.722 & 0.714 & \begin{tabular}{|l|l} 
Year_plot_type \\
\end{tabular} & 1684.6 & 20 & $<2.2 \mathrm{e}-16$ & **** \\
\hline
\end{tabular}


Multiple comparisons of the different plot types per implementation year (results are given on the log scale); legend: significance codes: different letters indicate significance (< 0.05); Plot types: field edge and interior, spillover, conservation field margins (CFM), fallow, alternately managed biennial flower strip (AFS) and perennial flower strip (PFS)

\begin{tabular}{|c|c|c|c|c|c|c|c|}
\hline \multirow{23}{*}{$\begin{array}{l}\text { Total herbaceous } \\
\text { species number }\end{array}$} & Plot type differences & Emmean & Std. Error & Degrees of freedom & lower.CL & upper.CL & Significances \\
\hline & AFS_1 & 3.07 & 0.0788 & 697 & 2.834 & 3.32 & hij \\
\hline & AFS_2 & 2.87 & 0.0893 & 697 & 2.593 & 3.14 & fgh \\
\hline & AFS_3 & 3.35 & 0.074 & 697 & 3.119 & 3.57 & ij \\
\hline & Field_edge_0 & 2.52 & 0.0571 & 697 & 2.342 & 2.69 & ef \\
\hline & Field_edge_1 & 2.08 & 0.0654 & 697 & 1.883 & 2.28 & $\mathrm{~cd}$ \\
\hline & Field_edge_2 & 2.04 & 0.0689 & 697 & 1.826 & 2.25 & $\mathrm{~cd}$ \\
\hline & Field_edge_3 & 2.32 & 0.0718 & 697 & 2.105 & 2.54 & $\mathrm{de}$ \\
\hline & CFM_1 & 2.98 & 0.0834 & 697 & 2.725 & 3.23 & ghi \\
\hline & CFM_2 & 2.58 & 0.1052 & 697 & 2.256 & 2.9 & efg \\
\hline & CFM_3 & 3.02 & 0.0963 & 697 & 2.723 & 3.31 & ghij \\
\hline & Fallow_1 & 3.1 & 0.0837 & 697 & 2.841 & 3.35 & hij \\
\hline & Fallow_2 & 2.98 & 0.088 & 697 & 2.707 & 3.25 & ghi \\
\hline & Fallow_3 & 3.12 & 0.0876 & 697 & 2.857 & 3.39 & hij \\
\hline & Field_interior_0 & 1.16 & 0.1751 & 697 & 0.624 & 1.69 & $\mathrm{a}$ \\
\hline & Field_interior_1 & 1.29 & 0.168 & 697 & 0.772 & 1.8 & $\mathrm{ab}$ \\
\hline & Field_interior_2 & 1.01 & 0.1107 & 697 & 0.667 & 1.34 & $\mathrm{a}$ \\
\hline & Field_interior_3 & 1.01 & 0.1292 & 697 & 0.62 & 1.41 & $\mathrm{a}$ \\
\hline & PFS_1 & 3.13 & 0.0891 & 697 & 2.859 & 3.4 & hij \\
\hline & PFS_2 & 3.45 & 0.0809 & 697 & 3.203 & 3.7 & j \\
\hline & Spillover_1 & 1.87 & 0.0742 & 697 & 1.643 & 2.1 & $\mathrm{bc}$ \\
\hline & Spillover_2 & 2 & 0.0726 & 697 & 1.774 & 2.22 & $\mathrm{~cd}$ \\
\hline & Spillover_3 & 2.23 & 0.0747 & 697 & 2.003 & 2.46 & cde \\
\hline \multirow{23}{*}{$\begin{array}{l}\text { Total herbaceous } \\
\text { species cover }\end{array}$} & Plot type differences & Emmean & Std. Error & Degrees of freedom & lower.CL & upper.CL & Significances \\
\hline & AFS_1 & 3.800 & 0.108 & 697 & 3.466 & 4.130 & de \\
\hline & AFS_2 & 4.130 & 0.096 & 697 & 3.841 & 4.430 & efgh \\
\hline & AFS_3 & 4.230 & 0.094 & 697 & 3.944 & 4.520 & efgh \\
\hline & Field_edge_0 & 2.730 & 0.095 & 697 & 2.443 & 3.020 & $\mathrm{c}$ \\
\hline & Field_edge_1 & 2.700 & 0.092 & 697 & 2.418 & 2.980 & $\mathrm{c}$ \\
\hline & Field_edge_2 & 2.340 & 0.107 & 697 & 2.012 & 2.660 & bc \\
\hline & Field_edge_3 & 2.700 & 0.111 & 697 & 2.359 & 3.040 & $\mathrm{c}$ \\
\hline & CFM_1 & 3.960 & 0.103 & 697 & 3.646 & 4.280 & def \\
\hline & CFM_2 & 3.420 & 0.138 & 697 & 3.001 & 3.850 & $\mathrm{~d}$ \\
\hline & CFM_3 & 4.030 & 0.117 & 697 & 3.678 & 4.390 & defg \\
\hline & Fallow_1 & 4.320 & 0.093 & 697 & 4.037 & 4.600 & fgh \\
\hline & Fallow_2 & 4.240 & 0.096 & 697 & 3.946 & 4.530 & efgh \\
\hline & Fallow_3 & 4.480 & 0.093 & 697 & 4.201 & 4.770 & gh \\
\hline & Field_interior_0 & 1.760 & 0.220 & 697 & 1.088 & 2.430 & $\mathrm{ab}$ \\
\hline & Field_interior_1 & 1.620 & 0.227 & 697 & 0.927 & 2.320 & $\mathrm{ab}$ \\
\hline & Field_interior_2 & 1.400 & 0.143 & 697 & 0.958 & 1.830 & $\mathrm{a}$ \\
\hline & Field_interior_3 & 1.530 & \begin{tabular}{|l|l|}
0.163 \\
\end{tabular} & 697 & 1.030 & 2.020 & $\mathrm{a}$ \\
\hline & PFS_1 & 4.140 & 0.108 & 697 & 3.811 & 4.470 & efgh \\
\hline & PFS_2 & 4.580 & 0.092 & 697 & 4.297 & 4.860 & $\mathrm{~h}$ \\
\hline & Spillover_1 & 2.330 & 0.108 & 697 & 1.997 & 2.660 & $\mathrm{bc}$ \\
\hline & Spillover_2 & 2.240 & 0.114 & 697 & 1.889 & 2.590 & bc \\
\hline & Spillover_3 & 2.450 & 0.121 & 697 & 2.081 & 2.820 & $\mathrm{bc}$ \\
\hline \multirow{22}{*}{$\begin{array}{l}\text { Total arable plant } \\
\text { number }\end{array}$} & Plot type differences & Emmean & Std. Error & Degrees of freedom & lower.CL & upper.CL & Significances \\
\hline & AFS_1 & 2.920 & 0.078 & 697 & 2.681 & 3.160 & gh \\
\hline & AFS_2 & 2.531 & 0.095 & 697 & 2.240 & 2.820 & efg \\
\hline & AFS_3 & 3.087 & 0.077 & 697 & 2.851 & 3.320 & $\mathrm{~h}$ \\
\hline & Field_edge_0 & 2.289 & 0.059 & 697 & 2.109 & 2.470 & $\mathrm{e}$ \\
\hline & Field_edge_1 & 1.941 & 0.065 & 697 & 1.744 & 2.140 & $\mathrm{~cd}$ \\
\hline & Field_edge_2 & 1.874 & 0.069 & 697 & 1.664 & 2.080 & $\mathrm{c}$ \\
\hline & Field_edge_3 & 2.132 & 0.073 & 697 & 1.910 & 2.350 & cde \\
\hline & CFM_1 & 2.850 & 0.082 & 697 & 2.600 & 3.100 & gh \\
\hline & CFM_2 & 2.357 & 0.107 & 697 & 2.029 & 2.690 & def \\
\hline & CFM_3 & 2.852 & 0.096 & 697 & 2.558 & 3.150 & gh \\
\hline & Fallow_1 & 2.969 & 0.082 & 697 & 2.718 & 3.220 & $\mathrm{gh}$ \\
\hline & Fallow_2 & 2.833 & 0.087 & 697 & 2.567 & 3.100 & fgh \\
\hline & Fallow_3 & 2.952 & 0.088 & 697 & 2.684 & 3.220 & gh \\
\hline & Field_interior_0 & 1.103 & 0.169 & 697 & 0.587 & 1.620 & $\mathrm{a}$ \\
\hline & Field_interior_1 & 1.228 & 0.160 & 697 & 0.738 & 1.720 & $\mathrm{ab}$ \\
\hline & Field_interior_2 & 0.979 & 0.105 & 697 & 0.658 & 1.300 & $\mathrm{a}$ \\
\hline & Field_interior_3 & 0.949 & 0.125 & 697 & 0.567 & 1.330 & $\mathrm{a}$ \\
\hline & PFS_1 & 2.854 & 0.093 & 697 & 2.571 & 3.140 & fgh \\
\hline & PFS_2 & 2.950 & 0.092 & 697 & 2.668 & 3.230 & gh \\
\hline & Spillover_1 & 1.820 & 0.071 & 697 & 1.605 & 2.040 & bc \\
\hline & Spillover_2 & 1.890 & 0.071 & 697 & 1.675 & 2.110 & $\mathrm{c}$ \\
\hline
\end{tabular}


CHAPTER 3

\begin{tabular}{|c|c|c|c|c|c|c|c|}
\hline & Spillover_3 & 2.136 & 0.073 & 697 & 1.914 & 2.360 & cde \\
\hline \multirow{23}{*}{$\begin{array}{l}\text { Total arable plant } \\
\text { cover }\end{array}$} & Plot type differences & Emmean & Std. Error & Degrees of freedom & lower.CL & upper.CL & Significances \\
\hline & AFS_1 & 3.740 & 0.112 & 697 & 3.398 & 4.080 & fg \\
\hline & AFS_2 & 3.870 & 0.109 & 697 & 3.541 & 4.200 & fgh \\
\hline & AFS_3 & 3.950 & 0.107 & 697 & 3.625 & 4.280 & fgh \\
\hline & Field_edge_0 & 2.600 & 0.100 & 697 & 2.290 & 2.900 & de \\
\hline & Field_edge_1 & 2.610 & 0.095 & 697 & 2.322 & 2.900 & $\mathrm{e}$ \\
\hline & Field_edge_2 & 2.240 & 0.110 & 697 & 1.904 & 2.580 & cde \\
\hline & Field_edge_3 & 2.610 & 0.116 & 697 & 2.259 & 2.970 & $\mathrm{de}$ \\
\hline & CFM_1 & 3.910 & 0.107 & 697 & 3.578 & 4.230 & fgh \\
\hline & CFM_2 & 3.310 & 0.146 & 697 & 2.863 & 3.760 & $\mathrm{f}$ \\
\hline & CFM_3 & 3.990 & 0.121 & 697 & 3.618 & 4.360 & gh \\
\hline & Fallow_1 & 4.280 & 0.097 & 697 & 3.981 & 4.570 & $\mathrm{~h}$ \\
\hline & Fallow_2 & 4.140 & 0.102 & 697 & 3.829 & 4.450 & gh \\
\hline & Fallow_3 & 4.390 & 0.098 & 697 & 4.095 & 4.690 & $\mathrm{~h}$ \\
\hline & Field_interior_0 & 1.740 & 0.221 & 697 & 1.067 & 2.420 & abcd \\
\hline & Field_interior_1 & 1.610 & 0.228 & 697 & 0.910 & 2.310 & $\mathrm{abc}$ \\
\hline & Field_interior_2 & 1.390 & 0.144 & 697 & 0.947 & 1.830 & $\mathrm{a}$ \\
\hline & Field_interior_3 & 1.500 & 0.164 & 697 & 1.001 & 2.000 & $a b$ \\
\hline & PFS_1 & 4.060 & 0.115 & 697 & 3.707 & 4.410 & gh \\
\hline & PFS_2 & 4.030 & 0.120 & 697 & 3.658 & 4.390 & gh \\
\hline & Spillover_1 & 2.300 & 0.109 & 697 & 1.970 & 2.640 & cde \\
\hline & Spillover_2 & 2.200 & 0.116 & 697 & 1.843 & 2.550 & bcde \\
\hline & Spillover_3 & 2.400 & 0.124 & 697 & 2.018 & 2.770 & cde \\
\hline \multirow{23}{*}{$\begin{array}{l}\text { Total plant number } \\
\text { of high nature } \\
\text { value species }\end{array}$} & \begin{tabular}{|l|} 
Plot type differences \\
\end{tabular} & Emmean & \begin{tabular}{|l} 
Std. Error \\
\end{tabular} & \begin{tabular}{|l|} 
Degrees of freedom \\
\end{tabular} & \begin{tabular}{|l|} 
lower.CL \\
\end{tabular} & \begin{tabular}{|l|} 
upper.CL \\
\end{tabular} & Significances \\
\hline & AFS_1 & 1,235 & 0,150 & 697 & 0,776 & 1,693 & hi \\
\hline & AFS_2 & 0,228 & 0,225 & 697 & $-0,459$ & 0,916 & cdefg \\
\hline & AFS_3 & 1,283 & 0,154 & 697 & 0,814 & 1,753 & hi \\
\hline & Field_edge_0 & 0,699 & 0,111 & 697 & 0,361 & 1,036 & fgh \\
\hline & Field_edge_1 & 0,032 & 0,134 & 697 & $-0,378$ & 0,442 & cde \\
\hline & Field_edge_2 & 0,062 & 0,135 & 697 & \begin{tabular}{|l|}
$-0,350$ \\
\end{tabular} & 0,474 & cde \\
\hline & Field_edge_3 & 0,348 & 0,141 & 697 & \begin{tabular}{|l|}
$-0,083$ \\
\end{tabular} & 0,779 & cdef \\
\hline & CFM_1 & 1,198 & 0,153 & 697 & 0,730 & 1,667 & hi \\
\hline & CFM_2 & 0,643 & 0,195 & 697 & 0,046 & 1,240 & efghi \\
\hline & CFM_3 & 1,308 & 0,172 & 697 & 0,783 & 1,833 & hi \\
\hline & Fallow_1 & 1,299 & 0,149 & 697 & 0,844 & 1,753 & $\mathrm{i}$ \\
\hline & Fallow_2 & 1,078 & 0,160 & 697 & 0,588 & 1,567 & ghi \\
\hline & Fallow_3 & 1,342 & 0,153 & 697 & 0,874 & 1,810 & $\mathrm{i}$ \\
\hline & Field_interior_0 & $-0,625$ & 0,292 & 697 & $-1,518$ & 0,267 & abcd \\
\hline & Field_interior_1 & $-1,108$ & 0,393 & 697 & $-2,307$ & 0,092 & $\mathrm{abc}$ \\
\hline & Field_interior_2 & $-1,446$ & 0,257 & 697 & $-2,232$ & $-0,659$ & $\mathrm{a}$ \\
\hline & Field_interior_3 & $-0,955$ & 0,244 & 697 & $-1,700$ & $-0,211$ & $\mathrm{ab}$ \\
\hline & PFS_1 & 0,657 & 0,217 & 697 & $-0,007$ & 1,321 & defghi \\
\hline & PFS_2 & 1,218 & 0,184 & 697 & 0,656 & 1,779 & ghi \\
\hline & Spillover_1 & $-0,049$ & 0,141 & 697 & $-0,479$ & 0,381 & bcde \\
\hline & Spillover_2 & $-0,044$ & 0,143 & 697 & $-0,482$ & 0,394 & bcde \\
\hline & Spillover_3 & 0,365 & 0,140 & 697 & $-0,063$ & 0,794 & cdef \\
\hline \multirow{23}{*}{$\begin{array}{l}\text { Total plant cover of } \\
\text { high nature value } \\
\text { species }\end{array}$} & Plot type differences & Emmean & Std. Error & Degrees of freedom & \begin{tabular}{|l} 
lower.CL \\
\end{tabular} & upper.CL & Significances \\
\hline & AFS_1 & 1,538 & 0,224 & 697 & 0,855 & 2,222 & def \\
\hline & AFS_2 & 0,603 & 0,323 & 697 & $-0,384$ & 1,590 & abcde \\
\hline & AFS_3 & 1,721 & 0,221 & 697 & 1,045 & 2,397 & ef \\
\hline & Field_edge_0 & 0,985 & 0,158 & 697 & 0,501 & 1,469 & cde \\
\hline & Field_edge_1 & 0,487 & 0,179 & 697 & $-0,060$ & 1,034 & $\mathrm{bc}$ \\
\hline & Field_edge_2 & 0,432 & 0,182 & 697 & $-0,124$ & 0,989 & bc \\
\hline & Field_edge_3 & 0,701 & 0,189 & 697 & 0,123 & 1,279 & bcd \\
\hline & CFM_1 & 2,428 & 0,176 & 697 & 1,891 & 2,964 & fg \\
\hline & CFM_2 & 1,133 & 0,275 & 697 & 0,292 & 1,973 & cde \\
\hline & CFM_3 & 2,770 & 0,175 & 697 & 2,235 & 3,305 & $\mathrm{~g}$ \\
\hline & Fallow_1 & 2,474 & 0,173 & 697 & 1,947 & 3,001 & fg \\
\hline & Fallow_2 & 2,458 & 0,173 & 697 & 1,929 & 2,986 & $\mathrm{fg}$ \\
\hline & Fallow_3 & 2,754 & 0,166 & 697 & 2,245 & 3,263 & $\mathrm{~g}$ \\
\hline & Field_interior_0 & 0,096 & 0,325 & 697 & $-0,897$ & 1,089 & $a b c$ \\
\hline & Field_interior_1 & $-0,323$ & 0,428 & 697 & $-1,631$ & 0,986 & $a b c$ \\
\hline & Field_interior_2 & $-0,859$ & 0,287 & 697 & $-1,736$ & 0,017 & $\mathrm{a}$ \\
\hline & Field_interior_3 & $-0,274$ & 0,268 & 697 & $-1,091$ & 0,544 & $\mathrm{ab}$ \\
\hline & PFS_1 & 0,724 & 0,333 & 697 & $-0,294$ & 1,743 & bcde \\
\hline & PFS_2 & 1,773 & 0,259 & 697 & 0,982 & 2,563 & defg \\
\hline & Spillover_1 & 0,365 & 0,187 & 697 & $-0,205$ & 0,936 & bc \\
\hline & Spillover_2 & 0,277 & 0,189 & 697 & $-0,301$ & 0,856 & $a b c$ \\
\hline & Spillover_3 & 0,654 & 0,192 & 697 & 0,069 & 1,240 & bcd \\
\hline
\end{tabular}




\begin{tabular}{|c|c|c|c|c|c|c|c|}
\hline \multirow{16}{*}{$\begin{array}{l}\text { Total number of } \\
\text { annual plants }\end{array}$} & Plot type differences & Emmean & Std. Error & Degrees of freedom & lower.CL & upper.CL & Significances \\
\hline & AFS_1 & 2.66 & 0.0855 & 391 & 2.41 & 2.91 & $\mathrm{f}$ \\
\hline & AFS_2 & 2.05 & 0.1133 & 391 & 1.71 & 2.38 & bcde \\
\hline & AFS_3 & 2.71 & 0.0882 & 391 & 2.45 & 2.97 & $\mathrm{f}$ \\
\hline & Field_edge_0 & 1.95 & 0.0654 & 391 & 1.76 & 2.15 & $\mathrm{bc}$ \\
\hline & Field_edge_1 & 1.71 & 0.0687 & 391 & 1.51 & 1.91 & $\mathrm{ab}$ \\
\hline & Field_edge_2 & 1.59 & 0.0747 & 391 & 1.37 & 1.81 & $\mathrm{a}$ \\
\hline & Field_edge_3 & 1.86 & 0.0787 & 391 & 1.63 & 2.09 & $\mathrm{abc}$ \\
\hline & CFM_1 & 2.62 & 0.0886 & 391 & 2.36 & 2.88 & $\mathrm{f}$ \\
\hline & CFM_2 & 2.02 & 0.1196 & 391 & 1.67 & 2.37 & abcd \\
\hline & CFM_3 & 2.63 & 0.1036 & 391 & 2.32 & 2.93 & $\mathrm{f}$ \\
\hline & Fallow_1 & 2.75 & 0.0884 & 391 & 2.49 & 3.01 & $\mathrm{f}$ \\
\hline & Fallow_2 & 2.54 & 0.0959 & 391 & 2.26 & 2.83 & ef \\
\hline & Fallow_3 & 2.66 & 0.0971 & 391 & 2.37 & 2.94 & $\mathrm{f}$ \\
\hline & PFS_1 & 2.44 & 0.1084 & 391 & 2.12 & 2.76 & def \\
\hline & PFS_2 & 2.32 & 0.1181 & 391 & 1.97 & 2.66 & cdef \\
\hline \multirow{16}{*}{$\begin{array}{l}\text { Total cover of } \\
\text { annual plants }\end{array}$} & Plot type differences & Emmean & Std. Error & Degrees of freedom & \begin{tabular}{|l} 
lower.CL \\
\end{tabular} & upper.CL & Significances \\
\hline & AFS_1 & 3.62 & \begin{tabular}{|l|l|}
0.13 \\
\end{tabular} & 391 & 3.24 & 4.01 & def \\
\hline & AFS_2 & 3.46 & 0.142 & 391 & 3.04 & 3.87 & de \\
\hline & AFS_3 & 3.5 & 0.143 & 391 & 3.08 & 3.92 & de \\
\hline & Field_edge_0 & 2.39 & 0.118 & 391 & 2.04 & 2.74 & $\mathrm{ab}$ \\
\hline & Field_edge_1 & 2.5 & 0.109 & 391 & 2.18 & 2.82 & $a b c$ \\
\hline & Field_edge_2 & 2.15 & 0.125 & 391 & 1.78 & 2.52 & $\mathrm{a}$ \\
\hline & Field_edge_3 & 2.44 & 0.134 & 391 & 2.05 & 2.84 & $a b c$ \\
\hline & CFM_1 & 3.81 & 0.123 & 391 & 3.44 & 4.17 & ef \\
\hline & CFM_2 & 3.02 & 0.18 & 391 & 2.49 & 3.55 & bcd \\
\hline & CFM_3 & 3.91 & 0.137 & 391 & 3.51 & 4.32 & ef \\
\hline & Fallow_1 & 4.16 & 0.112 & 391 & 3.83 & 4.49 & $\mathrm{f}$ \\
\hline & Fallow_2 & 3.84 & 0.128 & 391 & 3.46 & 4.22 & ef \\
\hline & Fallow_3 & 4.17 & 0.119 & 391 & 3.82 & 4.52 & $\mathrm{f}$ \\
\hline & PFS_1 & 3.76 & 0.143 & 391 & 3.34 & 4.18 & def \\
\hline & PFS_2 & 3.21 & 0.186 & 391 & 2.66 & 3.75 & cde \\
\hline \multirow{16}{*}{$\begin{array}{l}\text { Total number of } \\
\text { perennial plants }\end{array}$} & Plot type differences & Emmean & \begin{tabular}{|l} 
Std. Error \\
\end{tabular} & Degrees of freedom & \begin{tabular}{|l|} 
lower.CL \\
\end{tabular} & upper.CL & Significances \\
\hline & AFS_1 & 1.828 & 0.1285 & 391 & 1.449 & 2.21 & $\mathrm{~cd}$ \\
\hline & AFS_2 & 2.252 & 0.1114 & 391 & 1.924 & 2.58 & def \\
\hline & AFS_3 & 2.513 & 0.1016 & 391 & 2.214 & 2.81 & $\mathrm{f}$ \\
\hline & Field_edge_0 & 1.656 & 0.0791 & 391 & 1.423 & 1.89 & $\mathrm{bc}$ \\
\hline & Field_edge_1 & 0.905 & 0.1048 & 391 & 0.596 & 1.21 & $\mathrm{a}$ \\
\hline & Field_edge_2 & 0.963 & 0.1049 & 391 & 0.654 & 1.27 & $\mathrm{a}$ \\
\hline & Field_edge_3 & 1.256 & 0.1096 & 391 & 0.933 & 1.58 & $\mathrm{ab}$ \\
\hline & CFM_1 & 1.747 & 0.1363 & 391 & 1.346 & 2.15 & bcd \\
\hline & CFM_2 & 1.706 & 0.1474 & 391 & 1.272 & 2.14 & bcd \\
\hline & CFM_3 & 1.855 & 0.1519 & 391 & 1.408 & 2.3 & bcde \\
\hline & Fallow_1 & 1.82 & 0.1395 & 391 & 1.408 & 2.23 & bcd \\
\hline & Fallow_2 & 1.87 & 0.137 & 391 & 1.467 & 2.27 & cde \\
\hline & Fallow_3 & 2.089 & 0.1324 & 391 & 1.698 & 2.48 & cdef \\
\hline & PFS_1 & 2.477 & 0.1159 & 391 & 2.135 & 2.82 & ef \\
\hline & PFS_2 & 3.072 & 0.0955 & 391 & 2.79 & 3.35 & $\mathrm{~g}$ \\
\hline \multirow{16}{*}{$\begin{array}{l}\text { Total cover of } \\
\text { perennial plants }\end{array}$} & Plot type differences & Emmean & Std. Error & Degrees of freedom & lower.CL & upper.CL & Significances \\
\hline & AFS_1 & 1,722 & 0,227 & 391 & 1,054 & 2,39 & $a b c$ \\
\hline & AFS_2 & 3,303 & 0,216 & 391 & 2,667 & 3,94 & efg \\
\hline & AFS_3 & 3,558 & 0,222 & 391 & 2,905 & 4,21 & $\mathrm{fg}$ \\
\hline & Field_edge_0 & 1,68 & 0,131 & 391 & 1,293 & 2,07 & $\mathrm{bc}$ \\
\hline & Field_edge_1 & 1,275 & 0,131 & 391 & 0,888 & 1,66 & $\mathrm{ab}$ \\
\hline & Field_edge_2 & 0,875 & 0,142 & 391 & 0,457 & 1,29 & $\mathrm{a}$ \\
\hline & Field_edge_3 & 1,342 & 0,161 & 391 & 0,867 & 1,82 & $a b c$ \\
\hline & CFM_1 & 2,229 & 0,228 & 391 & 1,557 & 2,9 & $\mathrm{~cd}$ \\
\hline & CFM_2 & 2,173 & 0,242 & 391 & 1,459 & 2,89 & bcd \\
\hline & CFM_3 & 1,93 & 0,269 & 391 & 1,139 & 2,72 & bcd \\
\hline & Fallow_1 & 2,261 & 0,24 & 391 & 1,555 & 2,97 & cde \\
\hline & Fallow_2 & 2,922 & 0,232 & 391 & 2,239 & 3,61 & def \\
\hline & Fallow_3 & 3,132 & 0,246 & 391 & 2,408 & 3,86 & defg \\
\hline & PFS_1 & 2,906 & 0,25 & 391 & 2,169 & 3,64 & def \\
\hline & PFS_2 & 4,24 & 0,249 & 391 & 3,507 & 4,97 & $\mathrm{~g}$ \\
\hline \multirow{6}{*}{$\begin{array}{l}\text { Total number of } \\
\text { graminoids }\end{array}$} & Plot type differences & Emmean & Std. Error & Degrees of freedom & \begin{tabular}{|l} 
lower.CL \\
\end{tabular} & upper.CL & Significances \\
\hline & AFS_1 & 1.45 & 0.1205 & 391 & 1.095 & 1.81 & $\mathrm{abc}$ \\
\hline & AFS_2 & 1.478 & \begin{tabular}{|l|}
0.1222 \\
\end{tabular} & 391 & 1.118 & 1.84 & $\mathrm{bc}$ \\
\hline & AFS_3 & 1.785 & \begin{tabular}{|l|l|l|l}
0.1084 \\
\end{tabular} & 391 & 1.465 & 2.1 & $\mathrm{~cd}$ \\
\hline & Field_edge_0 & 1.559 & 0.0646 & 391 & 1.369 & 1.75 & $\mathrm{bc}$ \\
\hline & Field_edge_1 & 0.971 & 0.0814 & 391 & 0.731 & 1.21 & $\mathrm{a}$ \\
\hline
\end{tabular}


CHAPTER 3

\begin{tabular}{|c|c|c|c|c|c|c|c|}
\hline & Field_edge_2 & 0.974 & 0.0837 & 391 & 0.727 & 1.22 & $\mathrm{a}$ \\
\hline & Field_edge_3 & 1.248 & 0.0881 & 391 & 0.989 & 1.51 & $\mathrm{ab}$ \\
\hline & CFM_1 & 1.48 & 0.1219 & 391 & 1.121 & 1.84 & $\mathrm{bc}$ \\
\hline & CFM_2 & 1.403 & 0.1342 & 391 & 1.008 & 1.8 & $\mathrm{abc}$ \\
\hline & CFM_3 & 1.61 & 0.1346 & 391 & 1.213 & 2.01 & bcd \\
\hline & Fallow_1 & 1.698 & 0.1167 & 391 & 1.354 & 2.04 & bcd \\
\hline & Fallow_2 & 1.632 & 0.1207 & 391 & 1.277 & 1.99 & $\mathrm{bcd}$ \\
\hline & Fallow_3 & 1.906 & 0.114 & 391 & 1.57 & 2.24 & $\mathrm{~cd}$ \\
\hline & PFS_1 & 1.463 & 0.1397 & 391 & 1.052 & 1.87 & $\mathrm{abc}$ \\
\hline & PFS_2 & 2.116 & 0.1057 & 391 & 1.805 & 2.43 & $\mathrm{~d}$ \\
\hline \multirow{16}{*}{$\begin{array}{l}\text { Total cover of } \\
\text { graminoids }\end{array}$} & Plot type differences & Emmean & Std. Error & Degrees of freedom & lower.CL & upper.CL & Significances \\
\hline & AFS_1 & 2,53 & 0,236 & 391 & 1,835 & 3,23 & bcd \\
\hline & AFS_2 & 2,96 & 0,238 & 391 & 2,259 & 3,66 & $\mathrm{~cd}$ \\
\hline & AFS_3 & 3,2 & 0,244 & 391 & 2,485 & 3,92 & $\mathrm{~cd}$ \\
\hline & Field_edge_0 & 1,77 & 0,139 & 391 & 1,365 & 2,18 & $\mathrm{~b}$ \\
\hline & Field_edge_1 & 1,85 & 0,132 & 391 & 1,463 & 2,24 & $\mathrm{~b}$ \\
\hline & Field_edge_2 & 1,05 & 0,145 & 391 & 0,626 & 1,48 & $\mathrm{a}$ \\
\hline & Field_edge_3 & 1,99 & 0,168 & 391 & 1,494 & 2,48 & $\mathrm{~b}$ \\
\hline & CFM_1 & 2,56 & 0,242 & 391 & 1,849 & 3,27 & bcd \\
\hline & CFM_2 & 2,12 & 0,26 & 391 & 1,355 & 2,89 & $\mathrm{bc}$ \\
\hline & CFM_3 & 2,53 & 0,29 & 391 & 1,68 & 3,39 & $\mathrm{bcd}$ \\
\hline & Fallow_1 & 3,14 & 0,249 & 391 & 2,408 & 3,88 & $\mathrm{~cd}$ \\
\hline & Fallow_2 & 3,1 & 0,25 & 391 & 2,36 & 3,83 & $\mathrm{~cd}$ \\
\hline & Fallow_3 & 3,46 & 0,265 & 391 & 2,677 & 4,24 & $\mathrm{~d}$ \\
\hline & PFS_1 & 2,81 & 0,273 & 391 & 2,004 & 3,61 & bcd \\
\hline & PFS_2 & 3,68 & 0,276 & 391 & 2,867 & 4,49 & $\mathrm{~d}$ \\
\hline \multirow{16}{*}{$\begin{array}{l}\text { Total number of } \\
\text { forbs }\end{array}$} & Plot type differences & Emmean & 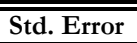 & Degrees of freedom & lower.CL & upper.CL & Significances \\
\hline & AFS_1 & 2.84 & 0.0911 & 391 & 2.57 & 3.1 & ef \\
\hline & AFS_2 & 2.58 & 0.1049 & 391 & 2.27 & 2.89 & $\mathrm{de}$ \\
\hline & AFS_3 & 3.09 & 0.0868 & 391 & 2.83 & 3.34 & $\mathrm{f}$ \\
\hline & Field_edge_0 & 2.02 & 0.0736 & 391 & 1.81 & 2.24 & bc \\
\hline & Field_edge_1 & 1.68 & 0.0809 & 391 & 1.44 & 1.92 & $\mathrm{ab}$ \\
\hline & Field_edge_2 & 1.59 & 0.0873 & 391 & 1.33 & 1.84 & $\mathrm{a}$ \\
\hline & Field_edge_3 & 1.9 & 0.0892 & 391 & 1.64 & 2.17 & $\mathrm{abc}$ \\
\hline & CFM_1 & 2.73 & 0.0975 & 391 & 2.45 & 3.02 & ef \\
\hline & CFM_2 & 2.21 & 0.1286 & 391 & 1.83 & 2.59 & $\mathrm{~cd}$ \\
\hline & CFM_3 & 2.74 & 0.1146 & 391 & 2.4 & 3.08 & def \\
\hline & Fallow_1 & 2.82 & 0.0993 & 391 & 2.52 & 3.11 & ef \\
\hline & Fallow_2 & 2.68 & 0.1048 & 391 & 2.37 & 2.99 & def \\
\hline & Fallow_3 & 2.78 & 0.1067 & 391 & 2.47 & 3.09 & ef \\
\hline & PFS_1 & 2.93 & 0.1028 & 391 & 2.63 & 3.24 & ef \\
\hline & PFS_2 & 3.16 & 0.097 & 391 & 2.88 & 3.45 & $\mathrm{f}$ \\
\hline \multirow[t]{16}{*}{ Total cover of forbs } & Plot type differences & $\overline{\text { Emmean }}$ & Std. Error & Degrees of freedom & lower.CL & upper.CL & Significances \\
\hline & AFS_1 & 3.49 & 0.133 & 391 & 3.1 & 3.88 & $\mathrm{bc}$ \\
\hline & AFS_2 & 3.72 & 0.124 & 391 & 3.35 & 4.09 & $\mathrm{bc}$ \\
\hline & AFS_3 & 3.77 & 0.125 & 391 & 3.4 & 4.14 & c \\
\hline & Field_edge_0 & 2.32 & 0.119 & 391 & 1.97 & 2.67 & $\mathrm{a}$ \\
\hline & Field_edge_1 & 2.23 & 0.117 & 391 & 1.89 & 2.58 & $\mathrm{a}$ \\
\hline & Field_edge_2 & 1.98 & 0.13 & 391 & 1.6 & 2.36 & $\mathrm{a}$ \\
\hline & Field_edge_3 & 2.23 & 0.14 & 391 & 1.82 & 2.64 & $\mathrm{a}$ \\
\hline & CFM_1 & 3.68 & 0.127 & 391 & 3.31 & 4.06 & $\mathrm{bc}$ \\
\hline & CFM_2 & 3.03 & 0.175 & 391 & 2.51 & 3.55 & $\mathrm{~b}$ \\
\hline & CFM_3 & 3.76 & 0.143 & 391 & 3.34 & 4.19 & $\mathrm{bc}$ \\
\hline & Fallow_1 & 3.87 & 0.124 & 391 & 3.51 & 4.24 & c \\
\hline & Fallow_2 & 3.77 & 0.129 & 391 & 3.39 & 4.15 & c \\
\hline & Fallow_3 & 3.92 & 0.129 & 391 & 3.53 & 4.3 & c \\
\hline & PFS_1 & 3.84 & 0.134 & 391 & 3.45 & 4.24 & c \\
\hline & PFS_2 & 4.07 & 0.126 & 391 & 3.7 & 4.44 & c \\
\hline \multirow{12}{*}{$\begin{array}{l}\text { Total herbaceous } \\
\text { plant beta-diversity }\end{array}$} & Plot type differences & Emmean & Std. Error & Degrees of freedom & lower.CL & upper.CL & Significances \\
\hline & AFS_1 & 0.533 & 0.0139 & 66 & 0.489 & 0.577 & abcd \\
\hline & AFS_2 & 0.571 & 0.01427 & 66 & 0.526 & 0.616 & bcdef \\
\hline & AFS_3 & 0.527 & 0.01467 & 66 & 0.481 & 0.574 & $a b c$ \\
\hline & Field_edge_0 & 0.602 & 0.00796 & 66 & 0.577 & 0.628 & efgh \\
\hline & Field_edge_1 & 0.616 & 0.00767 & 66 & 0.592 & 0.64 & fgh \\
\hline & Field_edge_2 & 0.626 & 0.00784 & 66 & 0.601 & 0.651 & fgh \\
\hline & Field_edge_3 & 0.603 & 0.00925 & 66 & 0.574 & 0.632 & efgh \\
\hline & CFM_1 & 0.534 & 0.01428 & 66 & 0.489 & 0.579 & abcd \\
\hline & CFM_2 & 0.599 & 0.01511 & 66 & 0.551 & 0.646 & cdefgh \\
\hline & CFM_3 & 0.512 & 0.01673 & 66 & 0.459 & 0.565 & $\mathrm{ab}$ \\
\hline & Fallow_1 & 0.546 & 0.01512 & 66 & 0.498 & 0.594 & abcde \\
\hline
\end{tabular}


CHAPTER 3

\begin{tabular}{|c|c|c|c|c|c|c|c|c|}
\hline & Fallow_2 & 0.543 & \multicolumn{2}{|l|}{0.01512} & 66 & 0.495 & 0.591 & abcde \\
\hline & Fallow_3 & 0.501 & \multicolumn{2}{|l|}{0.01614} & 66 & 0.45 & 0.552 & $\mathrm{ab}$ \\
\hline & Field_interior_0 & 0.638 & \multicolumn{2}{|l|}{0.01386} & 66 & 0.594 & 0.681 & \\
\hline & Field_interior_1 & 0.592 & 0.01432 & 66 & 0.5 & & 37 & efgh \\
\hline & Field_interior_2 & 0.632 & 0.0086 & 66 & 0.0 & & 59 & \\
\hline & Field_interior_3 & 0.645 & 0.00992 & 66 & 0.6 & & 76 & \\
\hline & PFS_1 & 0.511 & 0.01623 & 66 & 0.4 & & 62 & \\
\hline & PFS_2 & 0.473 & 0.01683 & 66 & 0.4 & & 26 & \\
\hline & Spillover_1 & 0.595 & 0.0079 & 66 & 0.5 & 0. & ef & \\
\hline & Spillover_2 & 0.619 & 0.00816 & 66 & 0.5 & & 45 & \\
\hline & Spillover_3 & 0.59 & 0.00925 & 66 & 0.5 & & 19 & $\mathrm{fg}$ \\
\hline Total herbaceous & Plot type differences & Emmear & & td. Error & Degrees of freedom & lower.CL & upper.CL & Significances \\
\hline species number per & AFS_2017 & 3.074 & & .0802 & 698 & 2.83 & 3.32 & ghi \\
\hline & AFS_2018 & 2.883 & & .0859 & 698 & 2.621 & 3.14 & efg \\
\hline & AFS_2019 & 3.349 & & .0735 & 698 & 3.125 & 3.57 & hi \\
\hline & Field_edge_2016 & 2.521 & & .0651 & 698 & 2.323 & 2.72 & $\mathrm{de}$ \\
\hline & Field_edge_2017 & 2.253 & & .0626 & 698 & 2.063 & 2.44 & $\mathrm{~cd}$ \\
\hline & Field_edge_2018 & 1.955 & & .0693 & 698 & 1.745 & 2.17 & bc \\
\hline & Field_edge_2019 & 2.275 & & .0621 & 698 & 2.086 & 2.46 & $\mathrm{~d}$ \\
\hline & CFM_2017 & 3.027 & & .0921 & 698 & 2.747 & 3.31 & fghi \\
\hline & CFM_2018 & 2.593 & & .1003 & 698 & 2.288 & 2.9 & def \\
\hline & CFM_2019 & 2.981 & & .0874 & 698 & 2.715 & 3.25 & fgh \\
\hline & Fallow_2017 & 3.112 & & .0847 & 698 & 2.854 & 3.37 & ghi \\
\hline & Fallow_2018 & 2.946 & & .0883 & 698 & 2.678 & 3.21 & fgh \\
\hline & Fallow_2019 & 3.137 & & .0839 & 698 & 2.882 & 3.39 & ghi \\
\hline & Field_interior_2016 & 1.155 & & .1741 & 698 & 0.626 & 1.68 & $\mathrm{a}$ \\
\hline & Field_interior_2018 & 1.189 & & .0985 & 698 & 0.89 & 1.49 & $\mathrm{a}$ \\
\hline & Field_interior_2019 & 0.901 & & .1146 & 698 & 0.552 & 1.25 & $\mathrm{a}$ \\
\hline & PFS_2018 & 3.129 & & .0883 & 698 & 2.86 & 3.4 & ghi \\
\hline & PFS_2019 & 3.448 & & .0803 & 698 & 3.204 & 3.69 & $\mathrm{i}$ \\
\hline & Spillover_2017 & 1.858 & & .0856 & 698 & 1.598 & 2.12 & $\mathrm{~b}$ \\
\hline & Spillover_2018 & 1.841 & & .0758 & 698 & 1.611 & 2.07 & $\mathrm{~b}$ \\
\hline & Spillover_2019 & 2.291 & & .063 & 698 & 2.1 & 2.48 & $\mathrm{~d}$ \\
\hline Total herbaceous & Plot type differences & Emmear & & td. Error & Degrees of freedom & lower.CL & upper.CL & Significances \\
\hline species cover per & AFS_2017 & 3.74 & & .1103 & 698 & 3.41 & 4.08 & $\mathrm{fg}$ \\
\hline calendar year & AFS_2018 & 4.16 & & .0891 & 698 & 3.89 & 4.43 & ghi \\
\hline & AFS_2019 & 4.23 & & .0909 & 698 & 3.95 & 4.5 & ghi \\
\hline & \begin{tabular}{|l|} 
Field_edge_2016 \\
\end{tabular} & 2.69 & & .1077 & 698 & 2.36 & 3.02 & cde \\
\hline & Field_edge_2017 & 2.88 & & .0865 & 698 & 2.62 & 3.14 & $\mathrm{de}$ \\
\hline & Field_edge_2018 & 2.26 & & .105 & 698 & 1.94 & 2.57 & $\mathrm{bc}$ \\
\hline & \begin{tabular}{|l|l|} 
Field_edge_2019 \\
\end{tabular} & 2.59 & & 0.0964 & 698 & 2.29 & 2.88 & cde \\
\hline & CFM_2017 & 4.19 & & .1016 & 698 & 3.89 & 4.5 & ghi \\
\hline & \begin{tabular}{|l} 
CFM_2018 \\
\end{tabular} & 3.19 & & .142 & 698 & 2.76 & 3.62 & ef \\
\hline & CFM_2019 & 4.08 & & .1001 & 698 & 3.77 & 4.38 & gh \\
\hline & Fallow_2017 & 4.31 & & .0935 & 698 & 4.02 & 4.59 & hi \\
\hline & Fallow_2018 & 4.24 & & .0929 & 698 & 3.96 & 4.52 & ghi \\
\hline & Fallow_2019 & 4.47 & & .087 & 698 & 4.21 & 4.73 & hi \\
\hline & Field_interior_2016 & 1.72 & & .2192 & 698 & 1.05 & 2.39 & $\mathrm{ab}$ \\
\hline & Field_interior_2018 & 1.49 & & .1342 & 698 & 1.08 & 1.9 & $\mathrm{a}$ \\
\hline & Field_interior_2019 & 1.37 & & .1425 & 698 & 0.94 & 1.81 & $\mathrm{a}$ \\
\hline & PFS_2018 & 4.14 & & .1051 & 698 & 3.82 & 4.46 & ghi \\
\hline & PFS_2019 & 4.57 & & .0895 & 698 & 4.3 & 4.85 & $\mathrm{i}$ \\
\hline & Spillover_2017 & 2.31 & & .1228 & 698 & 1.93 & 2.68 & $\mathrm{bc}$ \\
\hline & Spillover_2018 & 2.15 & & .1135 & 698 & 1.8 & 2.49 & bc \\
\hline & Spillover_2019 & 2.46 & & .1029 & 698 & 2.14 & 2.77 & bcd \\
\hline
\end{tabular}

Non-metric multidimensional scaling (NMDS) ordinations and their statistical results.

\begin{tabular}{|l|c|c|c|}
\hline & Dimensions & Stress & $\mathrm{n}$ \\
\hline Year 1 & 3 & 0.201 & 132 plots; 105 species \\
\hline Year 2 & Dimensions & Stress & $\mathrm{n}$ \\
\hline \hline & 3 & 0.199 & 125 plots; 105 species \\
\hline Year 3 & Dimensions & Stress & $\mathrm{n}$ \\
\hline \hline & 3 & 0.216 & 88 plots; 99 species \\
\hline Conservation field margin & Dimensions & Stress & $\mathrm{n}$ \\
\hline
\end{tabular}


CHAPTER 3

\begin{tabular}{|l|c|c|c|}
\hline & Dimensions & Stress & $\mathrm{n}$ \\
\hline Fallow & 3 & 0.195 & 46 plots; 79 species \\
\hline \hline & Dimensions & Stress & $\mathrm{n}$ \\
\hline Alternately managed flower strip & 3 & 0.180 & 54 plots; 96 species \\
\hline \hline & Dimensions & Stress & $\mathrm{n}$ \\
\hline Perennial flower strip & 2 & 0.163 & 27 plots; 82 species \\
\hline
\end{tabular}




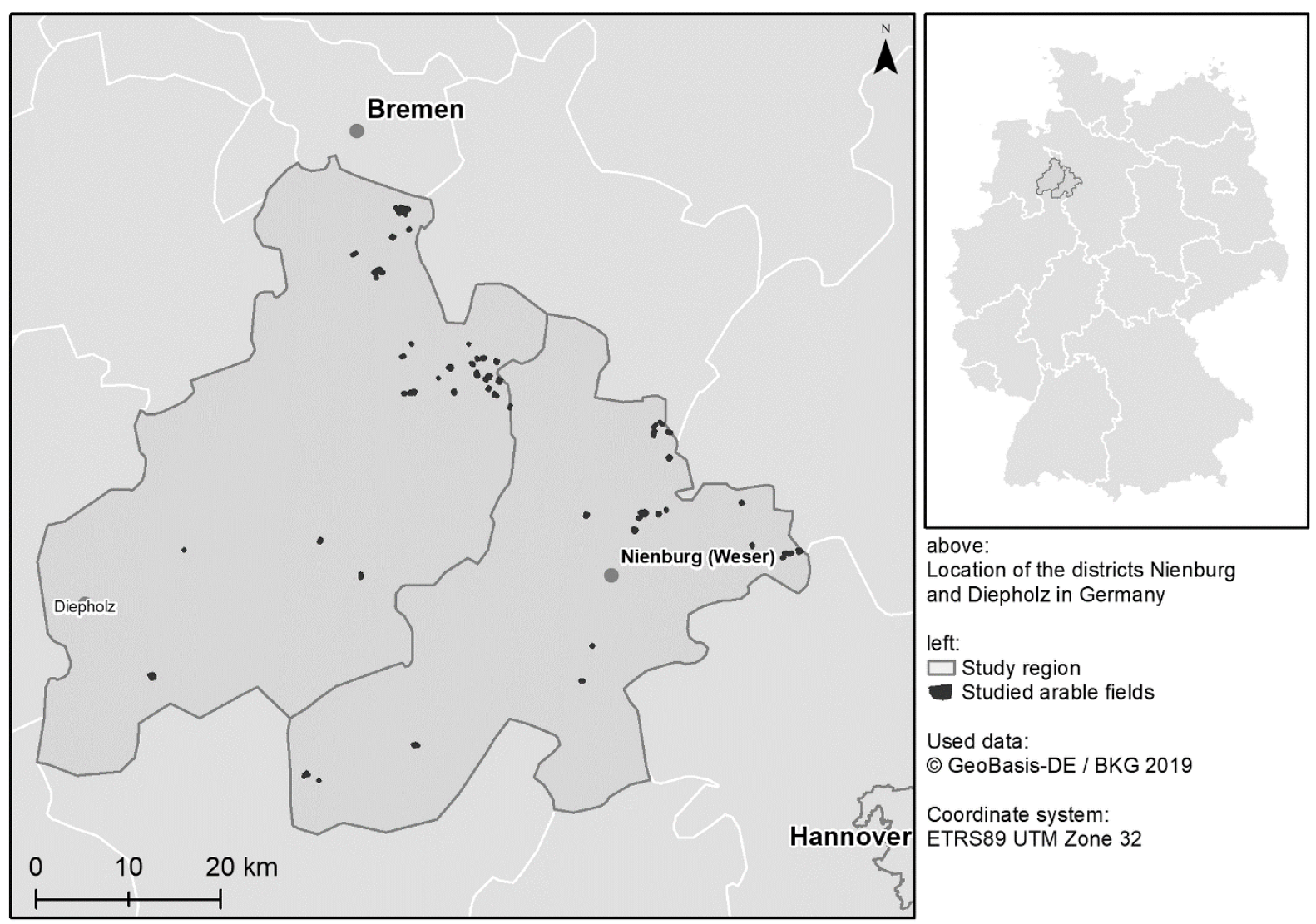

Figure A.1 Map of the study region in Lower Saxony, Northwest Germany with the districts of Nienburg and Diepholz. The location of the studied fields is indicated by black dots.

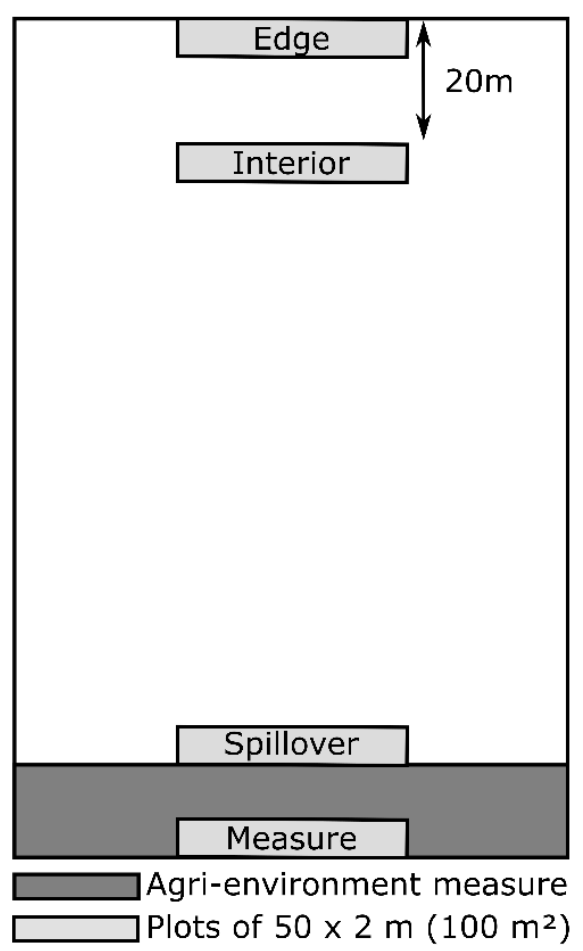

Figure A.2 Schematic map displaying the location of different types of relevé plots in the arable field. Each four plots of $50 \times 2 \mathrm{~m}\left(100 \mathrm{~m}^{2}\right)$ were placed in every field: one plot in the implemented measure and three plots in the intensively managed arable field directly adjacent to the measure (spillover plot), opposite to the measure at the field edge (field edge plot), and in the field interior $20 \mathrm{~m}$ distant to the edge (interior plot). The field edge plot served as direct control to the measure plot. 


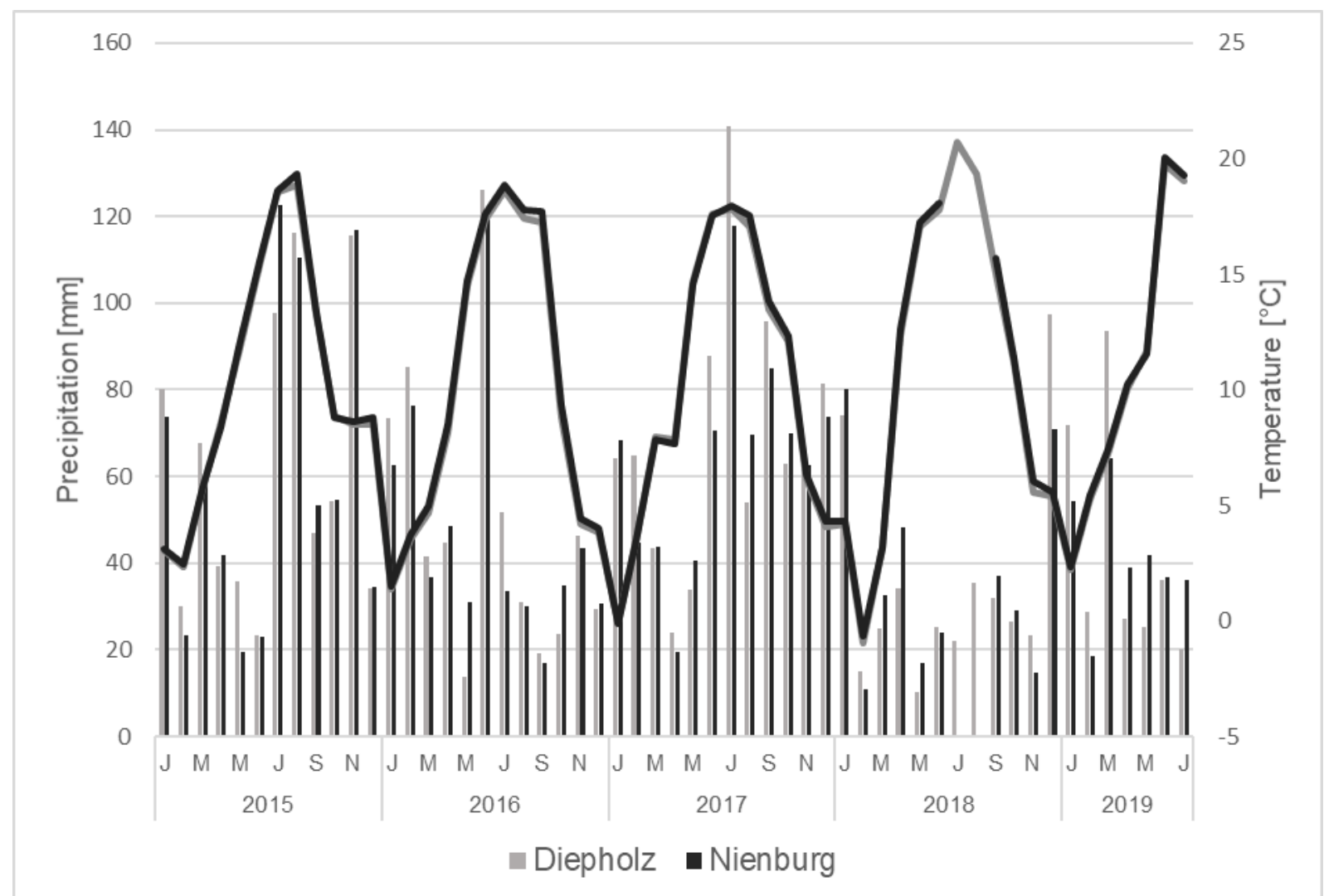

Figure A.3 Monthly precipitation totals (bars) and mean temperatures (line) during the study period (2016-2019) for the districts Nienburg and Diepholz (German Meteorological Service, 2019). No climate data are available for the district of Nienburg in July and August 2018 (full references see main text).
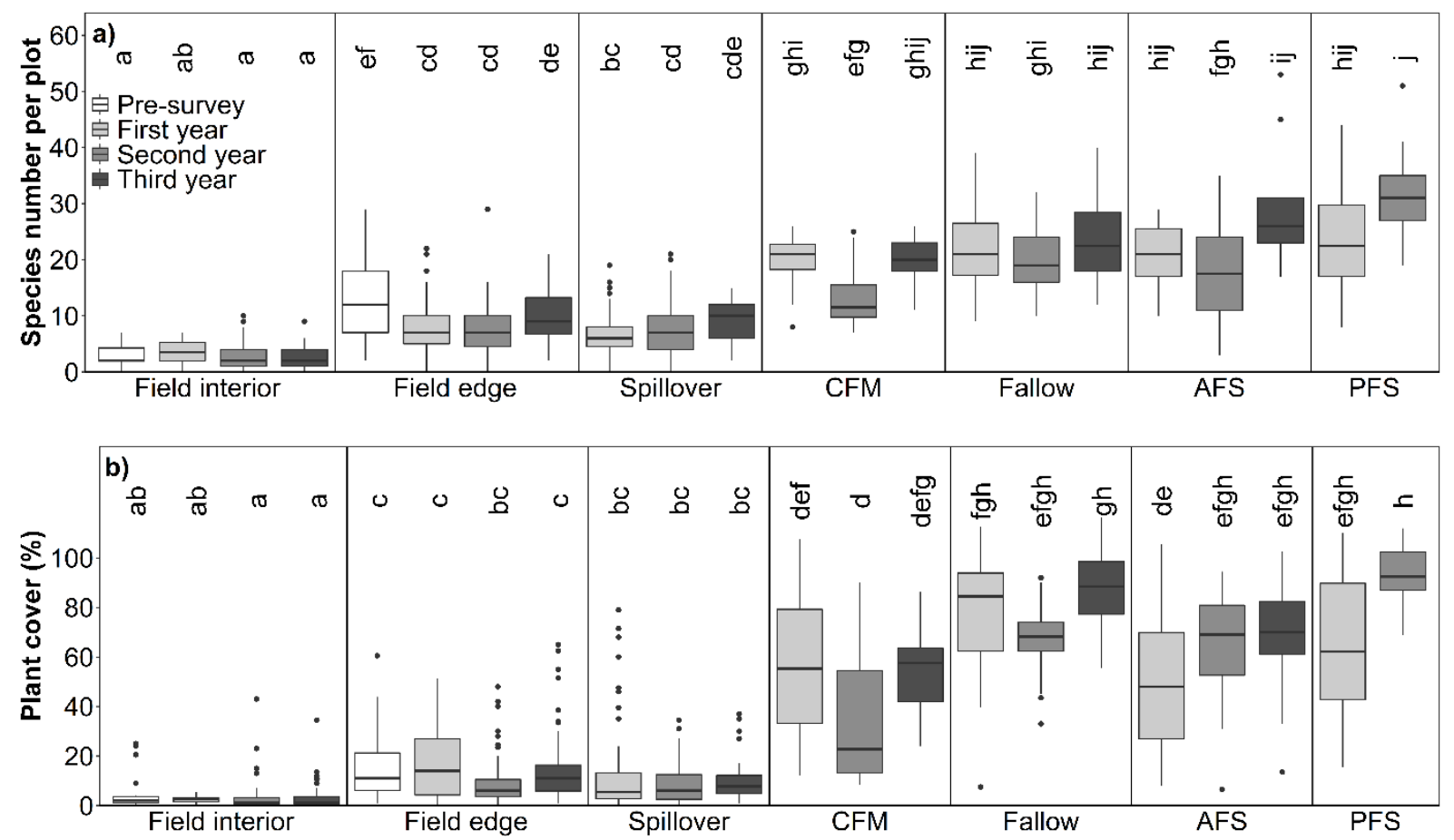

Figure A.4 Species richness (a) and plant cover (b) in $100 \mathrm{~m}^{2}$ plots of all herbaceous species (excluding woody plant seedlings, crop species and ornamentals) in the studied plot types (field edge, interior, spillover, conservation field margin (CFM), fallow, alternately managed biennial flower strip (AFS), perennial flower strip (PFS)) over the four years of the study (see legend). The total number of relevés was 721 (for details see Table 1 in the main document). Significant differences between plot types and implementation years are indicated by different small letters (based on estimated marginal means and Tukey-adjusted comparisons). The models and the model output are listed in Table A.8 in the Appendix. 

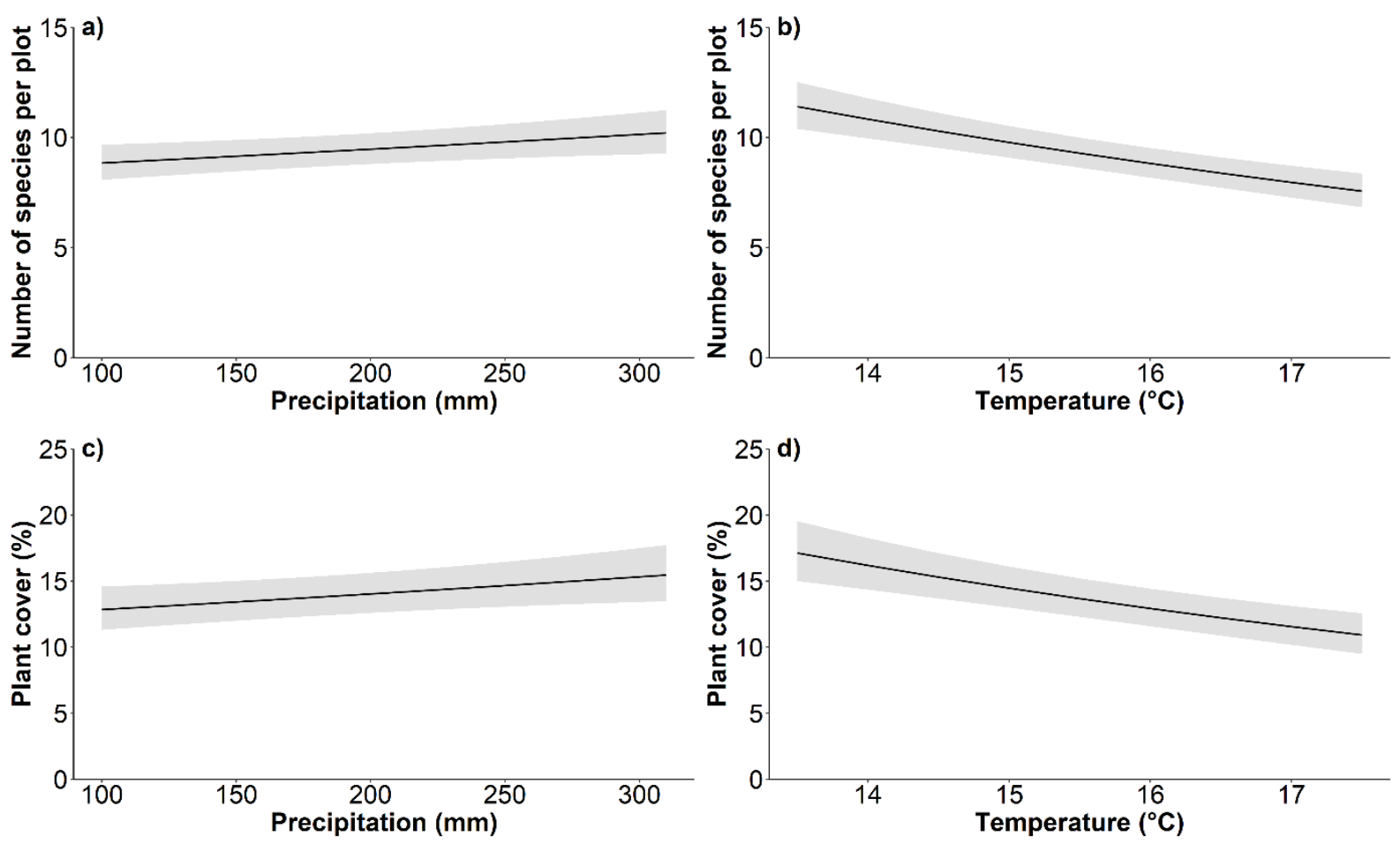

Figure A.5 Species richness ( $\mathrm{a}$ and b) and plant cover (c and d) in the study plots (all seven plot types pooled) in dependence on the sum of summer precipitation (April - July 2016-2019; a and c) and averaged summer temperature (b and d), considering all herbaceous species in the plots (excluding woody seedlings, crops and ornamentals). Predictions from negative binomial generalized mixed effects models ( $\mathrm{n}=721$ relevés on 19 farms). Given are predicted values (black line) and $95 \%$ confidence intervals (grey). The models and the model output are listed in Table A.8 in the Appendix (for more details about the data acquisition and analysis, see the main document).
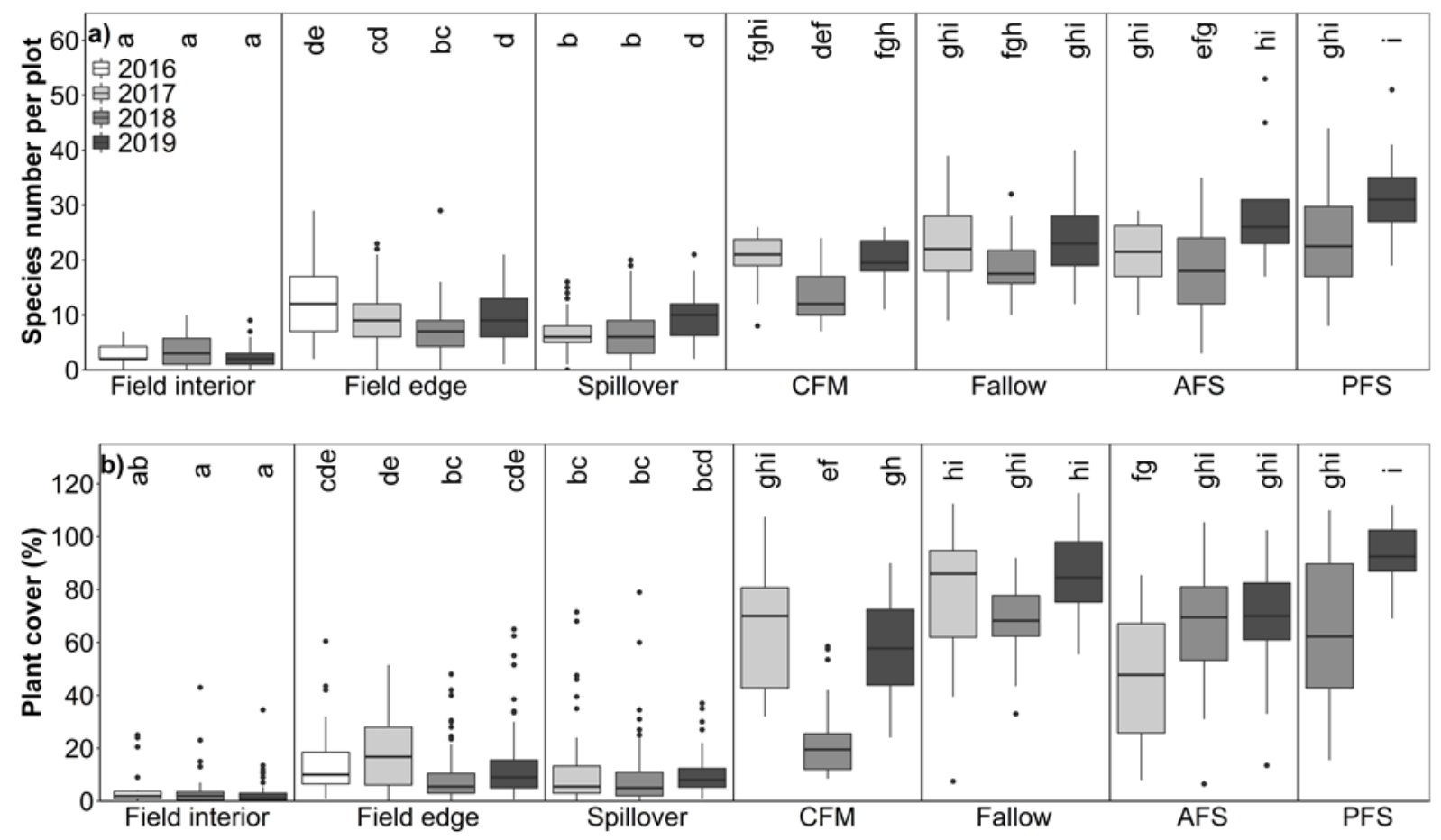

Figure A.6 Species richness (a) and plant cover (b) in $100 \mathrm{~m}^{2}$ plots of all herbaceous species (excluding woody plant seedlings, crop species and ornamentals) in the years 2016, 2017, 2018 and 2019 (see legend) in the seven plot types (field edge, interior, spillover, conservation field margin (CFM), fallow, alternately managed biennial flower strip (AFS), perennial flower strip (PFS)). PFS were only implemented in 2018 and no interior plots were surveyed in 2017. The total number of relevés was 721 (for details see Table 1 in the main document). Significant differences between plot types and years are indicated by different small letters (based on estimated marginal means and Tukey-adjusted comparisons). The models and the model output are listed in Table A.8 in the Appendix. 

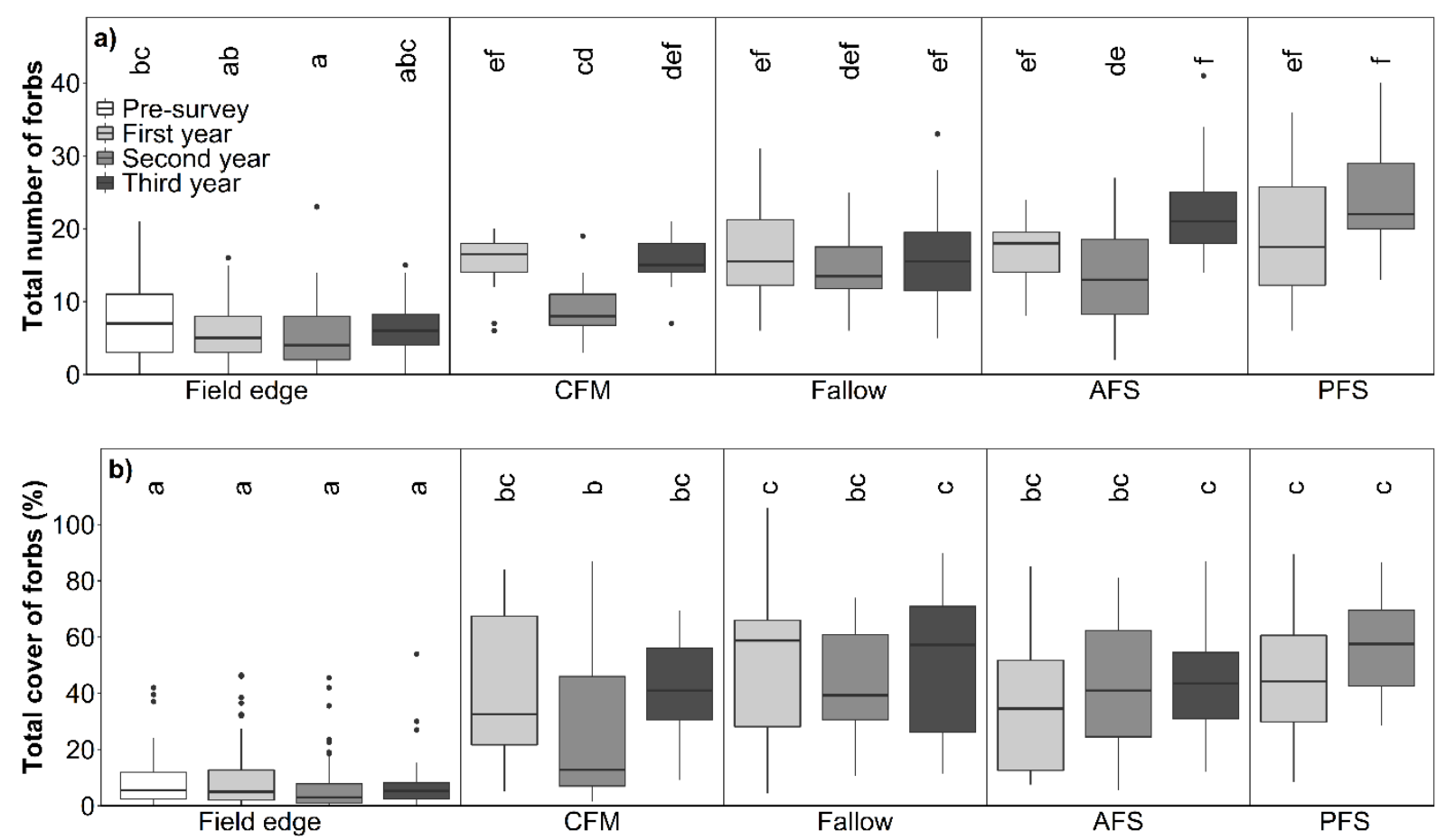

Figure A.7 Species richness (a) and plant cover (b) in $100 \mathrm{~m}^{2}$ plots of forbs (excluding crop species and ornamentals) in the studied plot types (field edge, interior, spillover, conservation field margin (CFM), fallow, alternately managed biennial flower strip (AFS), perennial flower strip (PFS)) over the four years of the study (see legend). The total number of relevés was 408 (for more details see Table 1 in the main document). Significant differences between plot types and implementation years are indicated by different small letters (based on estimated marginal means and Tukey-adjusted comparisons). The models and the model output are listed in Table A.8 in the Appendix.
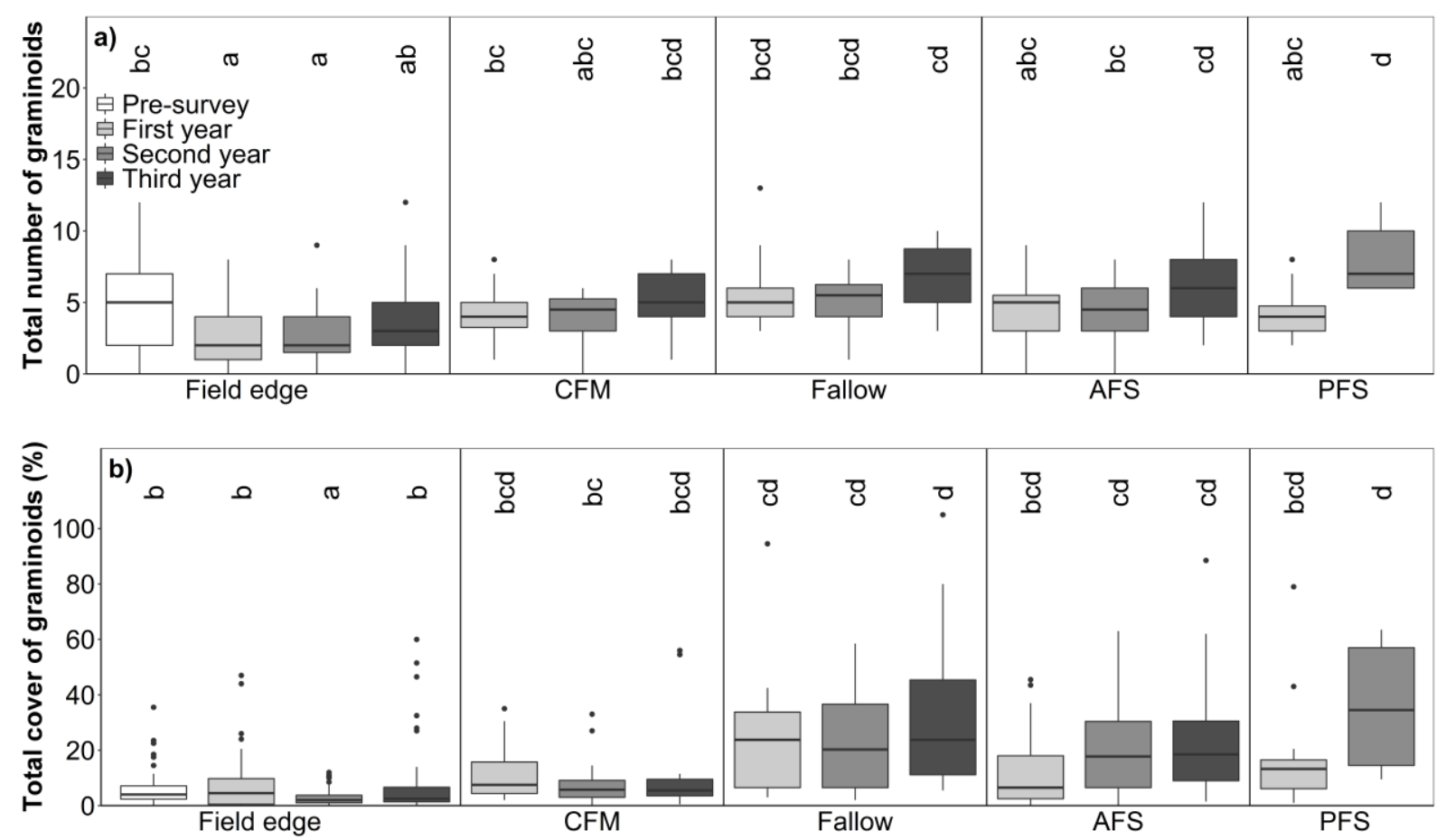

Fig. A.8 Species richness (a) and plant cover (b) in $100 \mathrm{~m}^{2}$ plots of graminoids (excluding crop species and ornamentals) in the studied plot types (field edge, interior, spillover, conservation field margin (CFM), fallow, alternately managed biennial flower strip (AFS), perennial flower strip (PFS)) over the four years of the study (see legend). The total number of relevés was 408 (for more details see Table 1 in the main document). Significant differences between plot types and implementation years are indicated by different small letters (based on estimated marginal means and Tukey-adjusted comparisons). The models and the model output are listed in Table A.8 in the Appendix. 

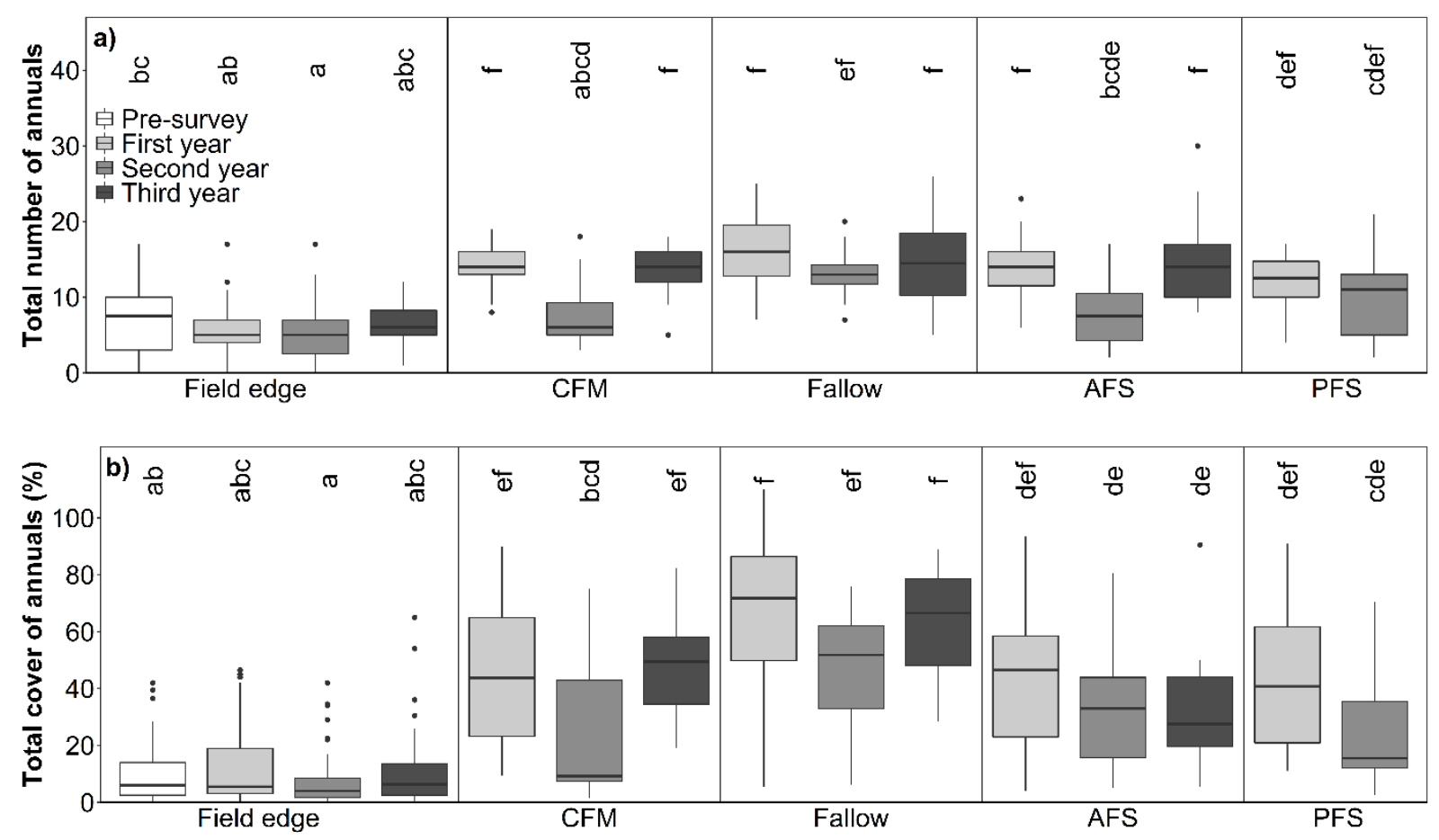

Figure. A.9 Species richness (a) and plant cover (b) in $100 \mathrm{~m}^{2}$ plots of annuals (excluding crop species and ornamentals) in the studied plot types (field edge, interior, spillover, conservation field margin (CFM), fallow, alternately managed biennial flower strip (AFS), perennial flower strip (PFS)) over the four years of the study (see legend). The total number of relevés was 408 (for more details see Table 1 in the main document). Significant differences between plot types and implementation years are indicated by different small letters (based on estimated marginal means and Tukey-adjusted comparisons). The models and the model output are listed in Table A.8 in the Appendix.
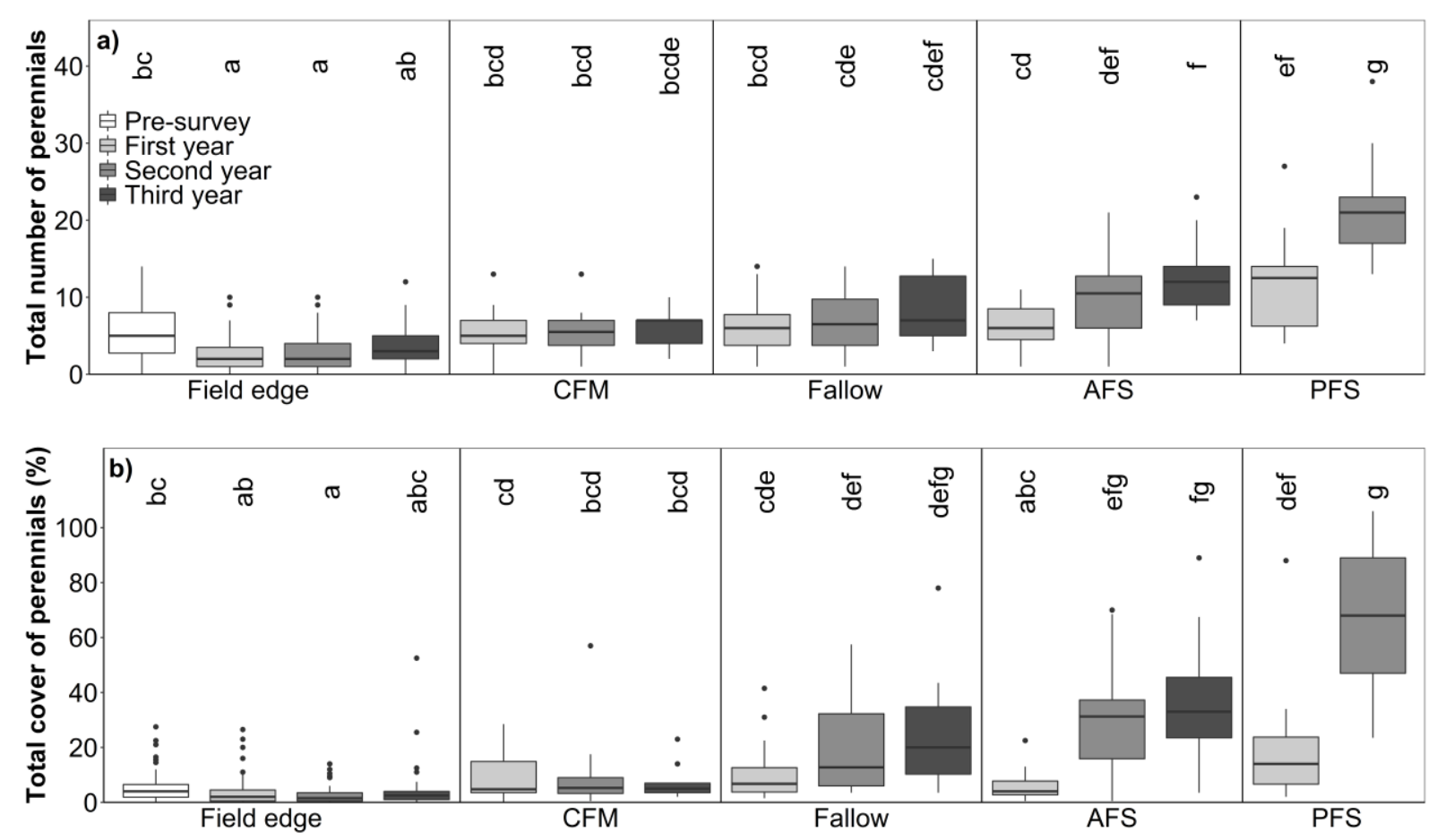

Figure A.10 Species richness (a) and plant cover (b) in $100 \mathrm{~m}^{2}$ plots of perennials (excluding crop species and ornamentals) in the studied plot types (field edge, interior, spillover, conservation field margin (CFM), fallow, alternately managed biennial flower strip (AFS), perennial flower strip (PFS)) over the four years of the study (see legend). The total number of relevés was 408 (for more details see Table 1 in the main document). Significant differences between plot types and implementation years are indicated by different small letters (based on estimated marginal means and Tukey-adjusted comparisons). The models and the model output are listed in Table A.8 in the Appendix. 


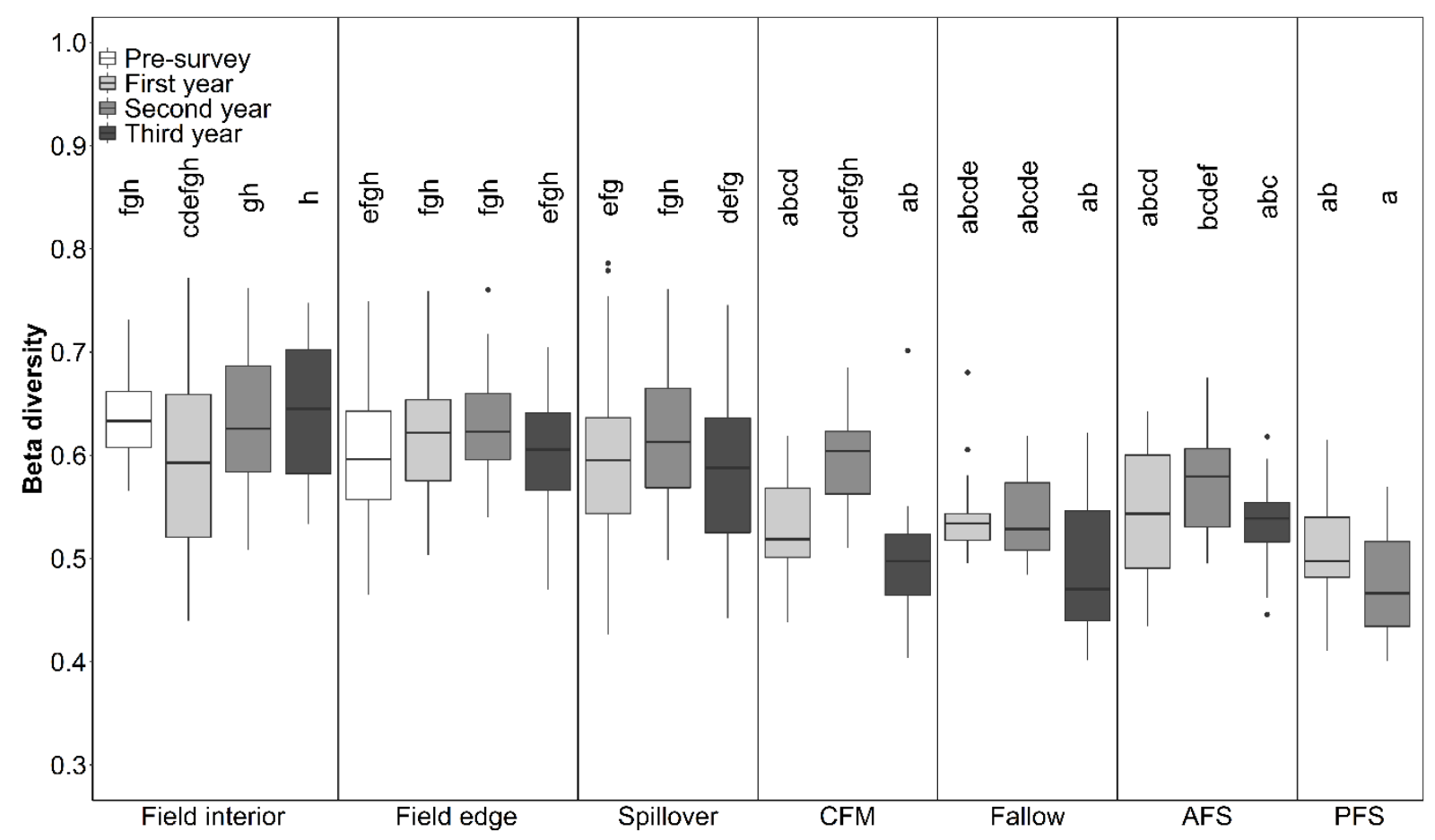

Figure A.11 Beta diversity, measured as distance to group spatial medians based on Jaccard dissimilarity index, for plots of the seven studied plot types (field edge, field interior, spillover, conservation field margin (CFM), fallow, alternately managed biennial flower strip (AFS), perennial flower strip (PFS); based on all herbaceous species excluding woody seedling, crops and species only determinable to the order level) of the 67 arable fields over the three years with measure implementation (see legend). The presurvey was conducted in the plots in the year before measure implementation. The lower the value, the more similar are the species compositions among the plots in a given plot type (range of values between 0 and 1 ). $n=693$ (28 plots with no observed species were excluded). Significant differences between plot types and implementation years are indicated by different small letters (based on estimated marginal means and Tukey-adjusted comparisons). The models and the model output are listed in Table A.8 in the Appendix.

a)

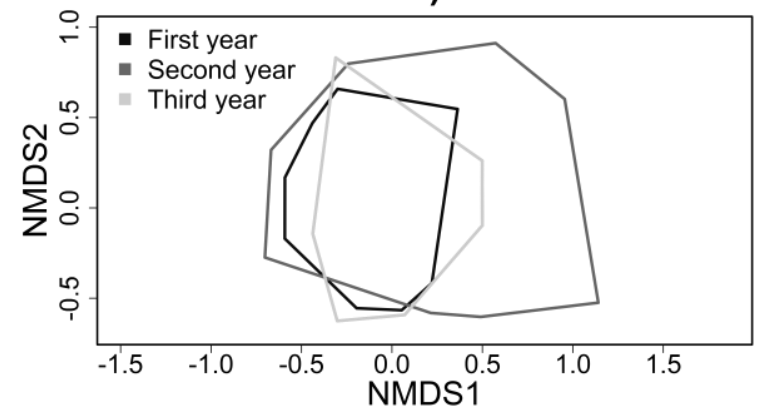

c)

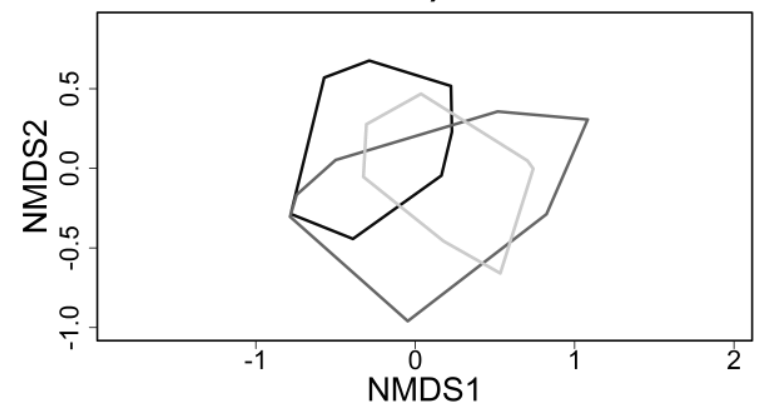

b)

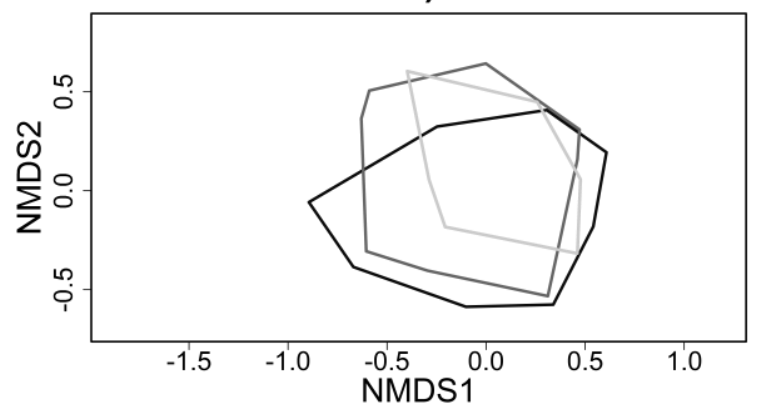

d)

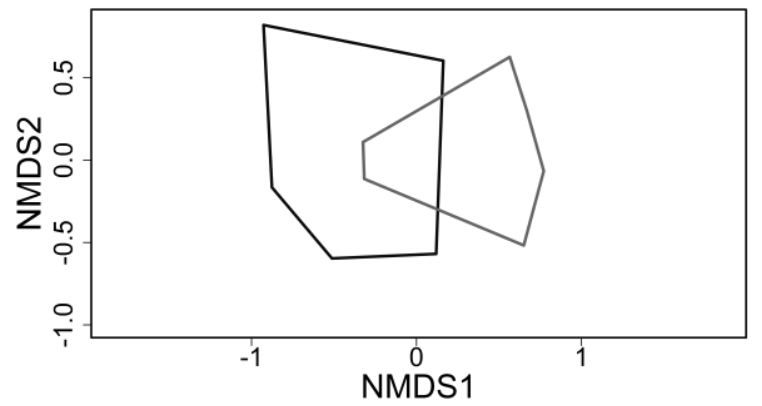

Figure A.12 Non-metric multidimensional scaling of the vegetation relevés conducted in the 1 st, 2 nd and 3 rd implementation year (black, dark grey and light grey lines) in the four different measure types: a) conservation field margin ( $\mathrm{n}=47, \mathrm{k}=3$, stress level $=0.161)$, b) fallow $(\mathrm{n}=46, \mathrm{k}=3$, stress level $=0.195), \mathrm{c})$ alternately managed biennial flower strip $(\mathrm{n}=54, \mathrm{k}=3$, stress level $=0.180)$ and $\mathrm{d})$ perennial flower strip $(\mathrm{n}=27, \mathrm{k}=2$, stress level $=0.163)$. Shown are the NMDS Axes 1 and 2. Perennial flower strips existed only for two implementation years. 


\section{Chapter 4}

\section{Surveying the arable plant diversity of conventionally managed farmland: a comparison of methods}

AlexAnder Wietzke, Christoph Leuschner

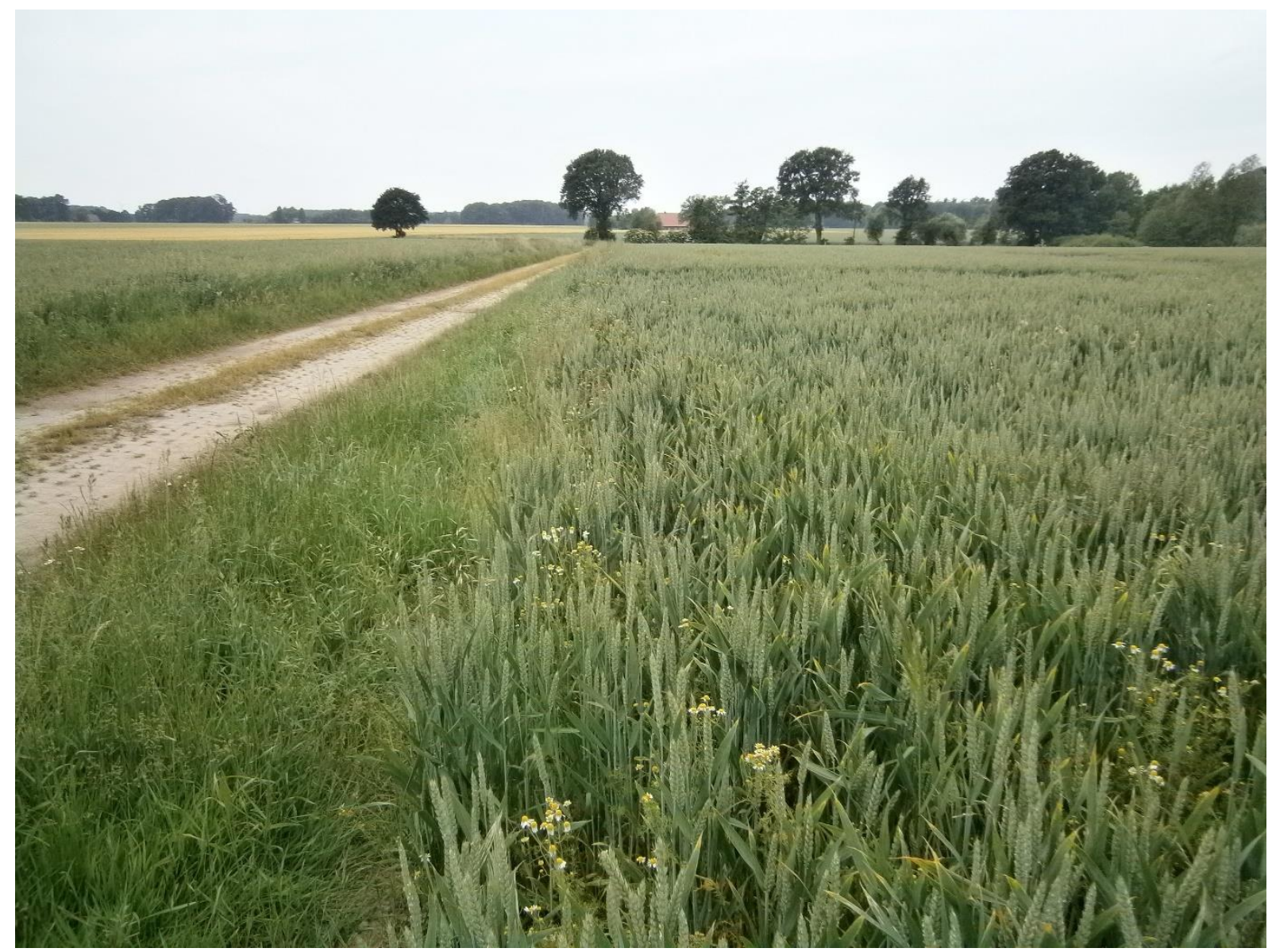

Published in

Environmental Monitoring and Assessment 192, 98 (2020).

bttps:// doi.org/10.1007/s10661-019-8042-7

Please note: There have been marginal corrections to the published article (e.g. the correction of minor typing errors in the raw data table). A Corrigendum to the published article can be accessed at: https://doi.org/10.1007/s10661-021-08940-3. The corrected raw data table (Online Resource 10) of Chapter 4 has been uploaded to the eDiss repository. 


\section{Abstract}

Agricultural intensification has led to drastic population declines in Europe's arable plant vegetation and continuous monitoring is a prerequisite for assessing measures to increase and conserve remnant populations of endangered arable plant species. Unfortunately, strong variation in plot sizes and in-field locations makes comparison of current arable plant monitoring approaches difficult. This study compares different relevé approaches in conventionally managed arable fields in Northwest German farmland with respect to plant species detection success and time expenditure.

We compared species detection rate and expenditure of time of six different relevé types in 45 conventionally managed arable fields (each 15 fields of wheat, maize and rapeseed): field 'Interior' plots $(50 \times 2 \mathrm{~m})$; field edge plots: 'Edge_30' $(30 \times 2 \mathrm{~m})$, 'Edge_50' $(50 \times 2 \mathrm{~m})$, 'Edge_500' (500 $\times 1 \mathrm{~m}$ ), 'Subplots' (four dispersed plots of $5 \times 1 \mathrm{~m})$ and 'Corner' plots $(50 \times 2 \mathrm{~m})$. To determine species detection rate, the species richness recorded with a survey method was related to the field's total plant species number as estimated from a survey of the entire field edge zone.

With a species detection rate of $8.3 \%$ (median), interior plots were inadequate for characterizing the field's arable plant vegetation. Edge_500 plots yielded the highest proportion of the field's arable plant species pool $(75.6 \%$, including taxa of conservation value), followed by 'Corner' plots (45.8 \%) and 'Sublots' (32.6\%). Edge_50 and Edge_30 plots detected less than $25 \%$ of the field's species pool. The average time needed for a relevé was 20 min in Edge_500 plots, and 5-11 min in the other plot types.

We suggest implementing Edge_500 plots as a standard monitoring approach in conventionally managed farmland due to its favorable ratio of detection success to expenditure of time. Our findings should be compared to methodological studies conducted in other regions, in different farmland management systems, and in landscapes of variable complexities.

Keywords: alpha diversity; arable weeds; field edge; field interior; plant detection rate; plot size.

\subsection{Introduction}

In former times, crop cultivation in the farmland was less intense and many plant species were able to coexist with the crop. In Central Europe, about 300-350 plant species have adapted to the frequently disturbed man-made habitat of arable fields. These weed species formed characteristic arable plant communities that have accompanied agriculture since centuries, if not millennia (Leuschner and Ellenberg 2017). Since the 1950s/1960s, advanced soil cultivation techniques, the widespread application of herbicides and increased fertilizer amounts that intensified competition with the crop have caused dramatic impoverishment of the arable plant vegetation in many regions of Central Europe and elsewhere, which manifested in large losses of arable plant cover and species richness and the collapse of once widespread arable plant (segetal) communities (Albrecht et al. 2016; Albrecht 1989; Májeková et al. 2010; Meisel and von Hübschmann 1976; Meyer et al. 2013). From a literature review, Leuschner \& Ellenberg (2017) concluded that, in comparison to other habitats in Central Europe, the species richness and population size of 
arable plants have experienced the most significant decrease within the past 50-60 years. For example, the study of Kläge (1999) in south-eastern Germany in the 1990s found that of 282 formerly recorded segetal species in the region, 90 taxa have disappeared and 72 showed a frequency decline of different extent. Albrecht (1989) observed a decline in the arable plant species pool of $20 \%$ between the intervals 1951-1968 and 1968-1988 in Bavaria, which was associated with a reduction in plot-level species richness from 23 to 16 species on average. In a large number of fields of Central and Northern Germany, the resampling study of Meyer et al. (2013) found a $23 \%$ reduction in the regional species pool and a decrease in median plot-level species richness from 24 to 7 species in the field interior compared to the 1950s/1960s. The species loss was associated with a large decline in arable plant coverage. Meyer et al. (2013) found that the median cover of arable plants declined from $30 \%$ in the 1959 s $/ 1960$ s to only $3 \%$ in 2009 in the interior of central and northern German fields.

Arable plants ('weeds') have always been a bane of the farmers due to reduced crop yield, but they are also fulfilling important ecosystem functions in the farmland, the benefits of which have only been fully recognized in the recent past. The root system of a sufficiently dense cover of arable plants can reduce soil erosion and the plants may catch nutrients (in particular mobile nitrate) during the summer fallow, thereby reducing leaching loss (Gholamhoseini et al. 2013). Equally important are positive effects on pollinator communities and related pollination success of crops and pest control through food webs supported by arable plants (Hawes et al. 2003; Hyvönen and Huusela-Veistola 2008; Médiène et al. 2011; Wietzke et al. 2018). Arable plants also support herbivorous insects which are eaten by insectivorous birds and they provide food for granivorous birds (Marshall et al. 2003). In Northern Germany, Heydemann \& Meyer (1983) counted 1200 insect species colonizing 102 arable plant species.

Thus, arable plants are of particular interest for biodiversity conservation in agricultural landscapes, and various agri-environment schemes such as conservation headlands have been introduced to halt plant biodiversity loss and reverse the trend in Europe (European Union 2013a, 2013b, 2014a, 2014b).

To assess the status quo of the segetal flora, monitor long-term trends and evaluate the effects of environment measures in the farmland, a consistent monitoring concept is needed. Vegetation surveys in the farmland have used a variety of plot sizes and plot geometries in the past, with plot size varying between 0.1 and $5000 \mathrm{~m}^{2}$ (Chytrý and Otýpková 2003; Hanzlik and Gerowitt 2016; Lososová et al. 2004; Meyer et al. 2013; Richner et al. 2015). Based on a comprehensive European dataset considering 2604 arable plant relevés between 1970 and 2000, Chytrý and Otýpková (2003) found a mean plot size of $74 \mathrm{~m}^{2}$, whereas the most frequently used plot size was $10 \mathrm{~m}^{2}$. For surveying arable plants, vegetation ecologists have traditionally made relevés in square or rectangular plots of 25 to $100 \mathrm{~m}^{2}$ size (Dierschke 1994; Hanzlik and Gerowitt 2016). For the analysis of the rich arable plant vegetation of the 1950s to $1970 \mathrm{~s}$, plots of $25 \mathrm{~m}^{2}$ were generally sufficiently large; this is certainly no longer possible in the nowadays intensively managed, species-poor farmland. In recent time, authors have investigated plots of largely different size, form and location in the field, when investigating different aspects of the arable plant vegetation. Examples of single-plot approaches are $100 \mathrm{~m}^{2}$ plots in the field interior (Fahrig et al. 2015; Lüscher et al. 2014), and $60 \mathrm{~m}^{2}$ plots at least $3 \mathrm{~m}$ distant to the field edge (German Federal Agency for Nature Conservation 2018). Split-plot designs include two paired $100 \mathrm{~m}^{2}$ plots at the field edge and in the interior (Meyer et al. 2015; Seifert et al. 2014, 2015); three $33 \mathrm{~m}^{2}$ - 
plots placed randomly in the interior (Gabriel et al. 2005), two field edge and two field interior plots of each $30 \mathrm{~m}^{2}$ (Roschewitz et al. 2005), three plots placed randomly in the interior and one edge plot of each $50 \mathrm{~m}^{2}$ (Pinke et al. 2012), two $2000 \mathrm{~m}^{2}$-plots in the field interior (Fried et al. 2008), and ten randomly placed $0.1 \mathrm{~m}^{2}$-plots (total area only $1 \mathrm{~m}^{2}$ ) (Hanzlik and Gerowitt 2011). Other authors used transects, e.g. one transect at the field edge and one in the interior consisting each of ten $5 \mathrm{~m}^{2}$-plots (Clough et al. 2007; Gabriel et al. 2006), one transect at the field edge and one in the interior consisting each of four or five $5 \mathrm{~m}^{2}$-plots (Batáry et al. 2012; Solé-Senan et al. 2014), one transect at the field edge and one in the interior consisting each of five $2 \mathrm{~m}^{2}$-plots (Krauss et al. 2011), three to ten $10 \mathrm{~m}^{2}$-plots in the field interior (depending on field size; Rotchés-Ribalta et al. 2015), and one transect in the field interior of ten $4 \mathrm{~m}^{2}$-plots (Petit et al. 2016). The above-mentioned studies used either square or rectangular plots, but oblong relevé plots placed at the field edge are increasingly plausible today, as the largest part of the remaining species pool is restricted to a narrow band along the field edge. In line with this, Bacaro et al. (2015) analyzed a large vegetation dataset consisting of 604 plots within different habitats (among others farmland, grassland or forests) and found elongated (rectangular) plots to record significantly more species than square plots. This can probably be traced back to an extended perimeter covered by oblong plots and, thus, the chance to include a wider range of environmental and habitat conditions with the associated plant species. The increasingly patchy distribution of arable plant vegetation in pesticide-treated, intensively managed fields may also suggest to use a larger number of small split plots to address vegetation heterogeneity. Such an approach may also account for the finding that the presence and type of adjacent habitats can influence field edge plant diversity by possible spillover effects (Aavik et al. 2008; Nagy et al. 2018; von Arx et al. 2002).

The large variation in available survey methods renders comparison of results difficult. As accurate data on the status and temporal change of the arable plant vegetation at regional, national and supra-national levels is urgently needed for agronomic and conservation purposes, this methodological diversity is highly unsatisfactory. Several authors have attempted to harmonize arable plant survey methods based on experiences gained in earlier studies. Examples are found in Hanzlik \& Gerowitt (2016) and Hatcher \& Froud-Williams (2017) who suggest to study several small square plots which can be placed randomly or within transects. It has also been proposed to place w-formed transects in the field interior. Species-area curve analysis may help to define a suitable plot size in the arable fields of interest (Pollnac et al. 2009). An example from an intensively farmed region is the study of Mulugeta et al. (2001) in corn and soybean fields of the US, which predicted that plot sizes between 32 and $185 \mathrm{~m}^{2}$, depending on the tillage regime, would be needed to find $75 \%$ of the field's arable plant species pool in the plot. To our knowledge, a systematic comparative study is missing which employs different arable plant survey methods in conventionally managed arable fields, and that could recommend methodological standardization and assess the effectivity of different methods in terms of time expenditure relative to plant detection success.

In this study, we compare six traditional or novel approaches to survey the arable plant vegetation of conventionally managed arable fields with the aim to identify methods that are efficient, but also time-economic. We recognize that most of farmland phytodiversity has disappeared from the field interior (Batáry et al. 2017; Clough et al. 2007; Gabriel et al. 2006; Seifert et al. 2014) and that survey methods today have to focus on the narrow field edge strip, which is often less than $2 \mathrm{~m}$ wide. We also accounted for the unwillingness of most farmers to 
allow vegetation relevés in the field interior, and thus focused on plots in the edge zone. We thus selected six different survey methods which use oblong plots of different size and placement in the field, (1) a $100 \mathrm{~m}^{2}$-plot $(50 \times 2 \mathrm{~m})$ in the field interior ('Interior'), which also was oblong and mostly served for comparison, (2) an equally-sized $100 \mathrm{~m}^{2}$-plot at the field edge ('Edge_50'), (3) a $60 \mathrm{~m}^{2}$-plot $\left(30 \times 2 \mathrm{~m}\right.$, 'Edge_30') at the field edge, (4) a $100 \mathrm{~m}^{2}$ oblong plot placed at the field corner $\left(50 \times 2 \mathrm{~m}\right.$, 'Corner'), (5) a plot area of $20 \mathrm{~m}^{2}$ split into four dispersed subplots of $5 \mathrm{~m}^{2}$ each ('Subplots'), and (6) an oblong $500 \mathrm{~m}^{2}$-plot $(500 \times 1 \mathrm{~m}$, 'Edge_500') along the field edge. The six approaches differ in plot size $\left(20\right.$ to $500 \mathrm{~m}^{2}$ ), plot location in the field (interior vs. edge, corner vs. middle edge), and amount of time needed for survey.

We tested the following hypotheses:

(i) Due to the patchy occurrence of the impoverished arable vegetation of today's intensively managed arable fields, the species detection rate will be highest in oblong $500 \mathrm{~m}^{2}$-plots at the field edge and lowest in $100 \mathrm{~m}^{2}$-plots in the field interior, where the most intensive management takes place,

(ii) field corner plots are species-richer than field edge plots of similar size,

(iii) beta diversity is lowest among Interior plots due to the universal occurrence of a small set of species well adapted to intensive field management,

(iv) additional species, i.e. taxa not detected with other survey methods, are only found in edge plots and not in the field interior, and

(v) splitting plots into subplots increases the number of species recorded per plot area, but also increases time expenditure.

\subsection{Material and Methods}

\subsubsection{Study region}

The study was conducted in an intensively managed agricultural landscape in the districts of Nienburg (centroid: N 52 36'32.5334”, E 96'49.7118”) and Diepholz (N 5243'41.4940”, E $\left.8^{\circ} 42^{\prime} 4.1629^{\prime \prime}\right)$ in the Pleistocene lowlands of western Lower Saxony (Northwest Germany; Online Resource 6). The districts are part of the natural regions 'Ems-Hunte-Geest and Dümmer-Geestniederung' and 'Weser-Aller-Flachland' south of the city of Bremen. Most of the farmland is conventionally managed arable land used to produce cereals, maize and rapeseed for the world market, or for use in local biogas plants. The climate of the two districts is temperateoceanic with mean annual precipitation (2013-2017) of $662.0 \mathrm{~mm}$ in Nienburg and $683.6 \mathrm{~mm}$ in Diepholz and mean annual temperatures of $10.3^{\circ} \mathrm{C}$ (Nienburg) and $10.1^{\circ} \mathrm{C}$ (Diepholz) (DWD 2018). The soils (mainly Cambisols, Podzols, Luvisols and Gleysols) are moderately fertile to fertile and developed from sandy to loamy deposits of the penultimate glaciation (Saalian) or Holocene loess deposits (BGR 2013; LBEG 2015). The farms included in the study were selected by the Chamber of Agriculture of Lower Saxony according to the criteria (i) conventional farming with a relatively high share of cereals, maize and rapeseed (ii) more or less even distribution of the farms in the two districts to avoid clumping and spatial autocorrelation, and (iii) willingness of the farmers to participate in the survey. 


\subsubsection{Vegetation survey}

The vegetation survey with determination of species identity and species richness was carried out from May to July 2017 in 45 fields (ranging in size from 3 to 11 ha) owned by 17 farmers. Fifteen fields had been planted with winter wheat, 15 with maize, and another 15 with winter rapeseed, which are the three dominant crops of the region and are of paramount economic importance for Central European agronomy. In the arable fields, we employed six different approaches to analyze the species richness of the arable plant vegetation at different spatial scales from the level of small plots $\left(5 \mathrm{~m}^{2}\right)$ to the field level (see schematic in Fig. 1). Some of the approaches have been used for a long time in vegetation surveys of farmland, others were adopted from more recent vegetation surveys or were introduced by us in response to floristic impoverishment. Since most of the floristic diversity in conventionally managed arable fields is found today in the narrow field edge, we used oblong plots of only 1 to $2 \mathrm{~m}$ width to account for the highly heterogeneous distribution of arable plants. We recorded all herbaceous plant species (grasses included, bryophytes excluded) except for juvenile woody plants and crop species. Species names follow Buttler (2018).

In all 45 fields, the following plot and transect types were studied: a) $100 \mathrm{~m}^{2}$-plots in the field interior ('Interior'; size $50 \times 2 \mathrm{~m}$, placed at least $20 \mathrm{~m}$ from the edge into the field), b) $60 \mathrm{~m}^{2}$-plots at the field edge ('Edge_30'; size $30 \times 2 \mathrm{~m}$, along the edge at least ten $\mathrm{m}$ from field corners), c) $100 \mathrm{~m}^{2}$-plots at the field edge ('Edge_50'; size $50 \times 2 \mathrm{~m}$, along the edge at least $10 \mathrm{~m}$ from field corners), d) $100 \mathrm{~m}^{2}$-plots at field corners ('Corner'; plots of $50 \times 2 \mathrm{~m}$ size, placed with two equally-sized legs in the field corner), e) four dispersed subplots of $20 \mathrm{~m}^{2}$ total size ('Subplots'; each $5 \times 1 \mathrm{~m}$, at the field edge at least $10 \mathrm{~m}$ distant to each other and to the field corner), and $\mathrm{f}$ ) linear edge plots of $500 \mathrm{~m}$ length and $1 \mathrm{~m}$ width $\left(500 \mathrm{~m}^{2}\right.$, 'Edge_500') along the field edge (consisting of twenty segments of $25 \times 1 \mathrm{~m}$ each, including at least one field corner) covering 30 $70 \%$ of the field's total perimeter. For obtaining an estimate of the field's overall species pool, we further inspected the 700 to $1500 \mathrm{~m}$-long total perimeter of the field (depending on field size) and counted all herbaceous plant species present along the $1 \mathrm{~m}$-wide margin (line plots of 700$1500 \mathrm{~m}^{2}$; 'total perimeter'). Based on our experience, we assumed that no additional species are occurring in the field Interior. This species count was used as a reference for the six approaches described above. However, if additional species were observed in a given field in other survey methods, they were added to the total species number.

We used a general plot size of $100 \mathrm{~m}^{2}$. In two cases, we also studied smaller plots: approach (b) uses $60 \mathrm{~m}^{2}$ in accordance with the plot size and design of the national farmland vegetation monitoring program of Germany (German Federal Agency for Nature Conservation 2018), while approach (e) studies four small plots of $5 \mathrm{~m}^{2}$ each, following the sampling scheme of Solé-Senan et al. (2014) and Batary et al. (2012). The latter approach with several small plots addresses the assumed high within-field variability in arable plant vegetation composition. Field edge plots were either 1 (approaches e and $\mathrm{f}$ ) or $2 \mathrm{~m}$ wide (approaches a to $\mathrm{d}$ ) and were aligned with the outermost furrow as a plot border. The location of the plots at the field edge and the starting points of the transects were selected by random (see Fig. 1). In each plot, all herbaceous species were listed, and the expenditure of time was noted (all approaches except for b, where the time was not recorded). In approach e), the total species number was calculated by summing over the four subplots. The location of all plots was determined with GPS. The raw data of the vegetation surveys are compiled in the Online Resource 10 (exclusive crop species, woody seedlings and few 
plant individuals which were non-determinable to species level; also excluded from further analysis).

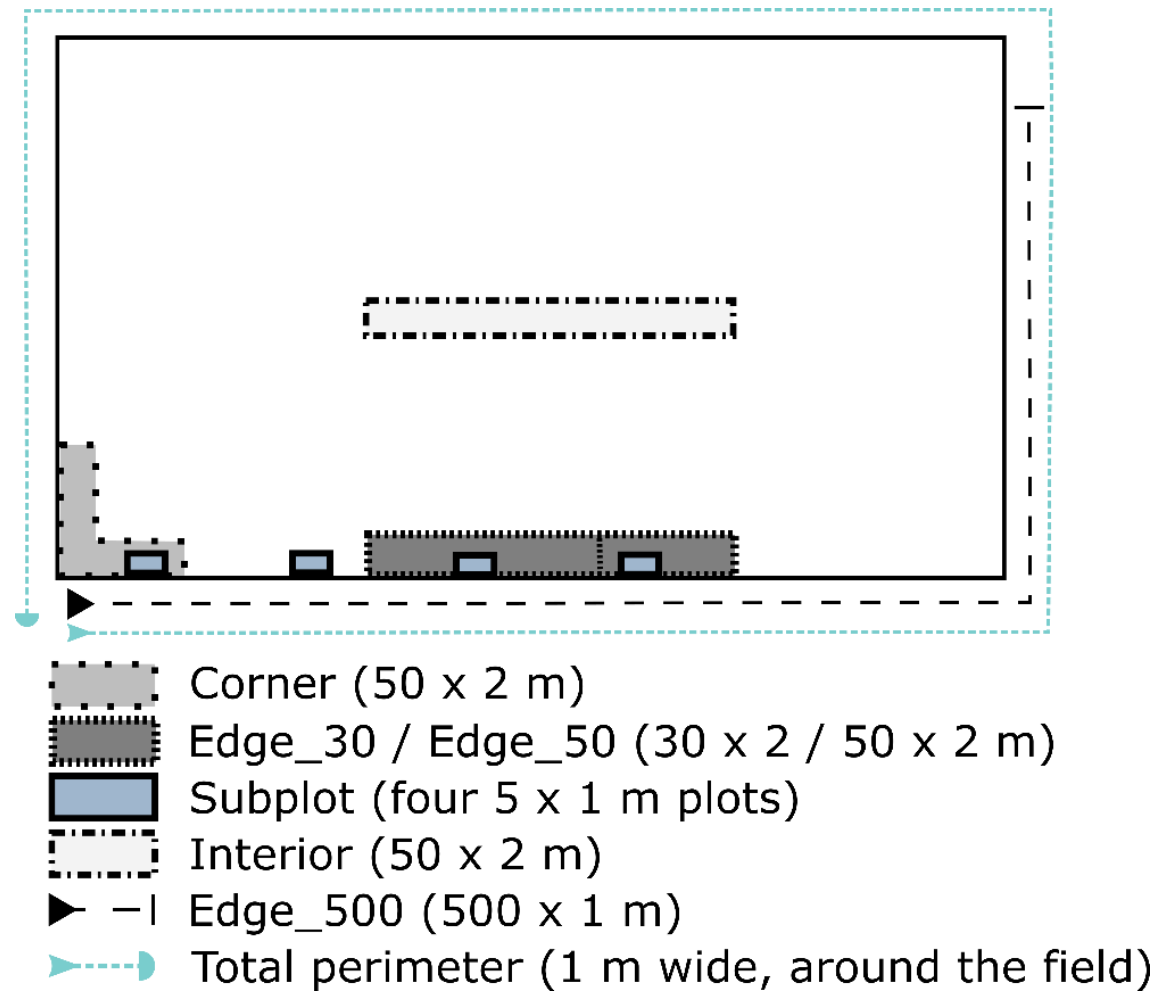

Fig. 1 Schematic map displaying the six survey methods that were tested in the study and their location in an arable field (for details see Material and Methods); survey methods: a) $100 \mathrm{~m}^{2}$-plots in the field interior ('Interior'; size $50 \times 2 \mathrm{~m}$, placed at least 20 $\mathrm{m}$ from the edge into the field), b) $60 \mathrm{~m}^{2}$-plots at the field edge ('Edge_30'; size $30 \times 2 \mathrm{~m}$, along the edge at least ten $\mathrm{m}$ from field corners), c) $100 \mathrm{~m}^{2}$-plots at the field edge ('Edge_50'; size $50 \times 2 \mathrm{~m}$, along the edge at least $10 \mathrm{~m}$ from field corners), d) $100 \mathrm{~m}^{2}$ plots at field corners ('Corner'; plots of $50 \times 2 \mathrm{~m}$ size, placed with two equally-sized legs in the field corner), e) four dispersed subplots of $20 \mathrm{~m}^{2}$ total size ('Subplots'; each $5 \times 1 \mathrm{~m}$, at the field edge at least $10 \mathrm{~m}$ distant to each other and to the field corner), and f) linear edge plots of $500 \mathrm{~m}$ length and $1 \mathrm{~m}$ width $\left(500 \mathrm{~m}^{2}\right.$, 'Edge_500').

\subsubsection{Statistical analysis}

Since most data sets were non-normally distributed, we present median values and use boxwhisker plots to visualize species richness data and the time consumed in the different survey methods. This was done for total species richness (all herbaceous non-crop species, including grasses, but without woody plant seedlings), the richness of arable plants sensu stricto according to the definition of Hofmeister \& Garve (2006), and the richness of high-nature-value species of arable land (according to the classification of the German Federal Agency for Nature Conservation 2018). High-nature-value species are defined as taxa which characterize through their presence farmland with high conservation value. The conservation value increases with the number of occurring high-nature-value species. We also compared the survey methods for the number of recorded red-listed arable plant species (Red List of Lower Saxony; Garve 2004).

All statistical analyses were conducted with R 3.5.2 software (R Core Team 2018) using the R packages 'magrittr' and 'tidyverse', among others (Bache and Wickham 2014; Wickham 2017). To test for spatial autocorrelation among the data from different fields, we calculated Moran's I (Paradis and Schliep 2018). Since no autocorrelation was detected, all 45 fields were treated as 
independent data points. To explore the effects of survey methods (explanatory variable) on species richness per plot (response variable), we employed generalized linear mixed-effects models (both with and without negative binomial family; R package 'Ime4'; Bates et al. 2015) using farmer and crop type as random factors to consider possible individual management effects and crop-dependent diversity patterns. To assess the goodness of the model in terms of the normality and heteroscedasticity of residuals, we inspected the residuals vs. the fitted values and QQ-plots, checked for over-dispersion (Bolker 2019) and calculated the variance explained $\left(\mathrm{R}^{2}\right)$ by fixed effects and by the entire model (Barton 2018; Fox et al. 2011). To test for significant effects of the explanatory variable (survey method) on species richness, we performed likelihoodratio tests using Type II sums of squares as criterion (R-package 'car'; Fox et al. 2011). Subsequently, a post-hoc two-sided Tukey test was applied ( $\mathrm{R}$ package 'multcomp'; Hothorn et al. 2008) to test for significant differences between survey methods. We also tested for differences between survey methods with respect to crop type effects. Due to the rather small sample size per crop ( $\mathrm{n}=15$ fields per crop type), we used the Mann-Whitney-U test for pairwise comparisons. Since crop type had a large effect on the time consumed in the different survey methods (as exemplified by hardly penetrable rapeseed vs. better accessible wheat fields), mixed effects models were not applied to explore the effect of survey method on the expenditure of time in the total data set (all crops pooled). As an alternative, we compared the expenditure of time in the different methods for the three crop types separately using the Mann-Whitney-U test. We also tested for differences between the survey methods in beta diversity among fields calculating the distances to group spatial medians based on Jaccard's dissimilarity index ( $R$ package 'vegan', function 'betadisper'; Oksanen et al. 2019). Significant differences in beta diversity between plots were also tested with the Mann-Whitney-U test. In addition, we plotted the species richness found by examining the six survey methods in the 45 fields against the estimated total species number in that field ('total perimeter') and calculated Spearman's rank correlation coefficient for the six relations to evaluate the accuracy of the different survey methods in terms of species detection. Finally, species accumulation curves were calculated with the R package 'vegan' (method = 'random'; permutations = 100; Oksanen et al. 2019) to analyze the influence of the survey method on the increase in species number with plot number or surveyed plot area. Information about model structures and test statistics is presented in Online Resource $9 \mathrm{a}$ and $9 \mathrm{~b}$.

\subsection{Results}

\subsubsection{Recorded species richness}

In the pooled sample of all 45 fields, we observed 197 herbaceous non-crop species (excluding seedlings of woody plants) and 125 arable plant species sensu stricto, when combining the results of all six survey methods (Online Resource 7; see Online Resource 8 for species richness per crop). The estimated total species pool of single wheat, maize and rapeseed fields in the study region consisted of 45, 40, and 52 herbaceous plant species, and 38, 32, and 40 arable plant species, respectively (median values), according to the total perimeter count. The highest total herbaceous species number in the plots was found in the $500 \mathrm{~m}^{2}$ Edge_500 plots (median of the three crops: 31 species), followed by the $100 \mathrm{~m}^{2}$-Corner plots (20) and the Subplots (14; Fig. 2). Edge_30 and Edge_50 plots (60 and $100 \mathrm{~m}^{2}$, respectively) reached very similar, but much lower, 
species richness (median $=10$ in both cases) than the aforementioned survey methods. The lowest median values were recorded in the $100 \mathrm{~m}^{2}$-plots in the field Interior (median $=4$ species). The same sequence in species richness was also found for the number of arable plant species (according to the classification of Hofmeister \& Garve 2006) and for the high-nature-value species of arable land (with exception of the Subplots; Fig. 2). A similar sequence of methods was also detected, when the three crop types are analyzed separately (Online Resource 1). With a few exceptions, the species richness differences between the six tested methods were significant at $\mathrm{p}$ $<0.05$.

In relation to the estimated total species pool of a field ('total perimeter'), the Edge_500 plots yielded the highest proportion of species recorded (median: $71.1 \%$ of all herbaceous plants, and $75.6 \%$ of all arable plant species; all crops pooled) followed by the Corner plots (median: 44.7 and $45.8 \%$ ) and the Subplots (31.3 and $32.6 \%$ ) (Fig. 2 and Online Resource 2). A much lower share of total species number was recorded in the Edge_50, Edge_30 and Interior plots (median: 24.3, 23.3 and $6.3 \%$ for all herbaceous plant species, and 25.0, $23.3 \%$ and $8.3 \%$ for the arable plant species). The proportion of high-nature-value species detected in the plots Edge_500, Corner, Subplots, Edge_50, Edge_30 and Interior was 75.0, 46.2, 30.8, 25.0, 22.2 and 6.7 \%, respectively, of the number found in the total perimeter count (Online Resource 3). The occurrence of red-listed arable plant species (with respect to Lower Saxony; Garve 2004) was very low. In total, we found nine occurrences of five red-listed species (total perimeter count) in eight of the 45 study fields (five rapeseed and three maize fields). In general, only one red-listed species was found per field (except for one field with two red-listed species). Corner und Edge_500 plots showed a slightly higher detection success compared to the other applied field edge survey methods (detection of four red-listed species vs. two or three occurrences), whereas no red-listed species were found in Interior plots. When analyzing the crop types separately, we found $70.5 \%$ of all herbaceous plants of the total perimeter count in the Edge_500 plots in wheat fields, $70.8 \%$ in maize, and $76.8 \%$ in rapeseed fields.

The relationship between recorded arable plant species number in a plot and estimated total arable plant species number in the field was strongest for the Edge_500 plots $(\mathrm{r}=0.83, \mathrm{p}<$ 0.0001), followed by the plot types Corner, Subplots, Edge_50 and Edge_30 (0.48 > r $>0.41$; $0.005>\mathrm{p}>0.001$, Fig. 3). No correlation was found between the arable plant species richness of Interior plots and total arable plant number per field $(\mathrm{r}=0.03)$. The same pattern was observed for all herbaceous plants instead of arable plant species (Online Resource 4). 

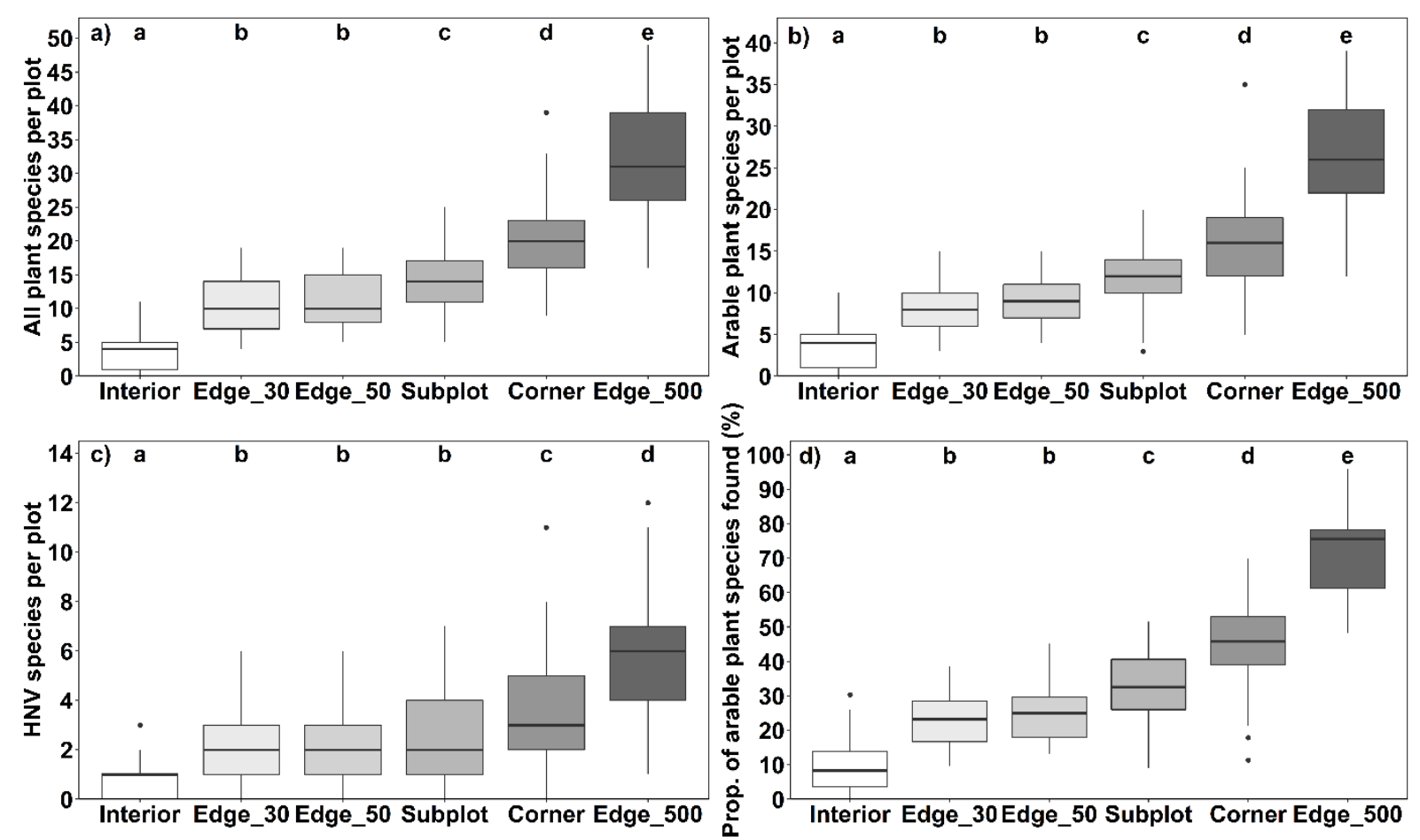

Fig. 2 Total number of herbaceous plant species (all plant species, woody seedlings and crops excluded) (a), number of arable plant species sensu stricto (as listed by Hofmeister and Garve 2006) (b), and number of high-nature-value species (HNV according to the German Federal Agency for Nature Conservation 2018) (c) in plots surveyed with six different methods. (d) Proportion (in \%) of the number of arable plant species sensu stricto present in the field that is found in plots of the six survey methods. All data are averages over wheat, maize and rapeseed fields. Black lines in boxplots represent medians; $n=45$ ( $3 \mathrm{crop}$ types $\times 15$ replicates per crop for each survey method), Tukey's test, $\alpha \leq 0.05$; different small letters indicate significant differences between survey methods. For model overview and statistical results see Online Resource 9.

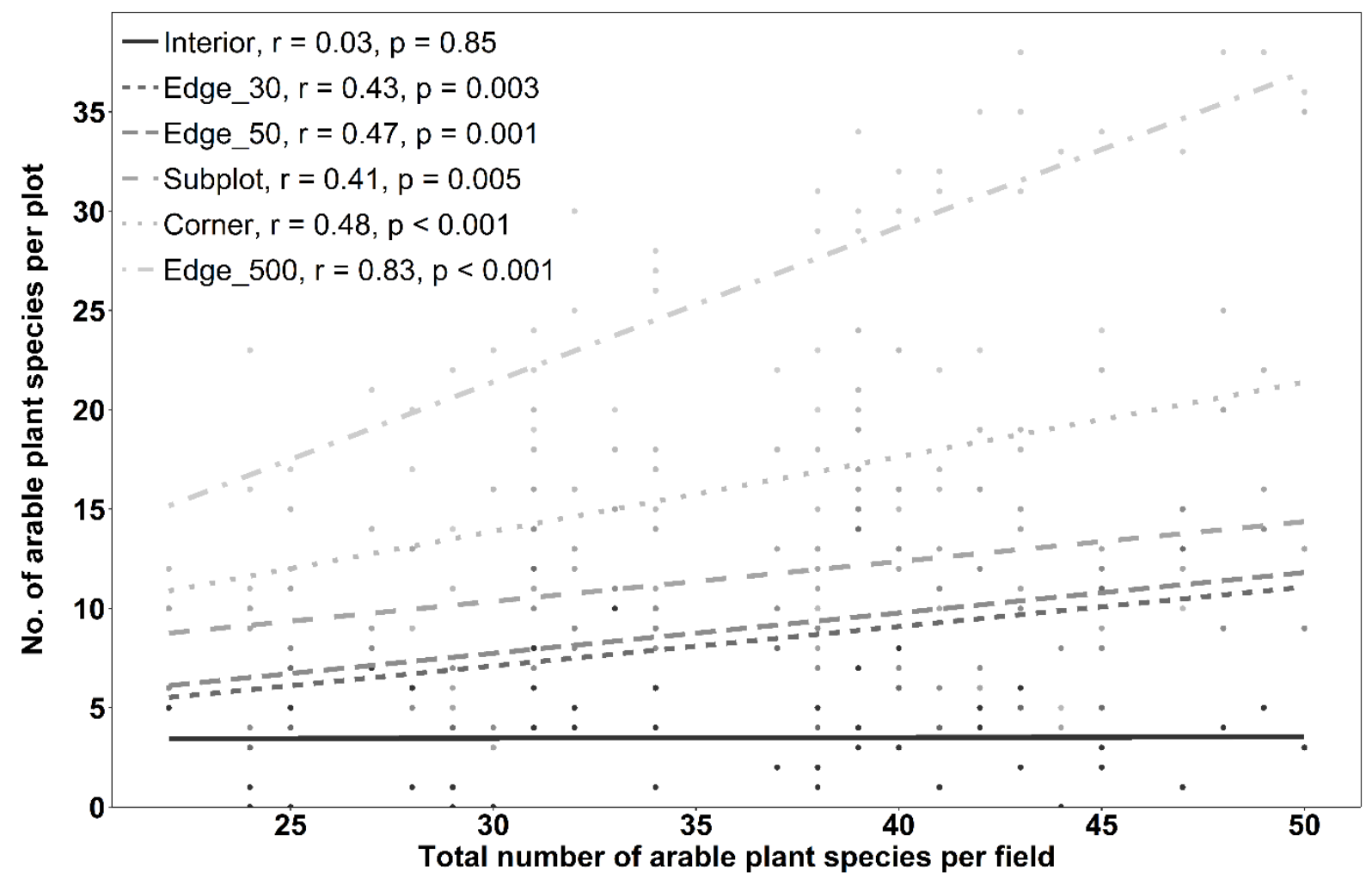

Fig. 3 Number of arable plant species found in plots of the six survey methods in relation to total arable plant species number in the respective field; $\mathrm{R}=$ Spearman's rank correlation coefficient with $\mathrm{p}$-values; $\mathrm{n}=45$ (per survey method). 


\subsubsection{Expenditure of time}

Due to differences in plot size and vegetation density, the six methods differed considerably in the time needed for the survey. Surveying the Edge_500 plots consumed the largest amount of time (median over all crops: 20 min, maize: 16, wheat: 20 min, rapeseed: 23 min; Fig. 4). In maize and wheat, Corner plots and Subplots required only half the time (median: 9-11 min, no significant difference between crop types), followed by Edge_50 plots with even less time (maize: $6.5 \mathrm{~min}$, wheat: $7 \mathrm{~min}$ ); the fastest surveys were possible in Interior plots (maize and wheat: 5 min; all figures without access to the plot). With 9.5-10 min, the Subplots required more time than the much larger contiguous Edge_30 and Edge_50 plots. Surveying rapeseed plots was in general more time-consuming due to the high crop density; a median value of 15 min was recorded for Interior plots and 23 min for Edge_500 plots.

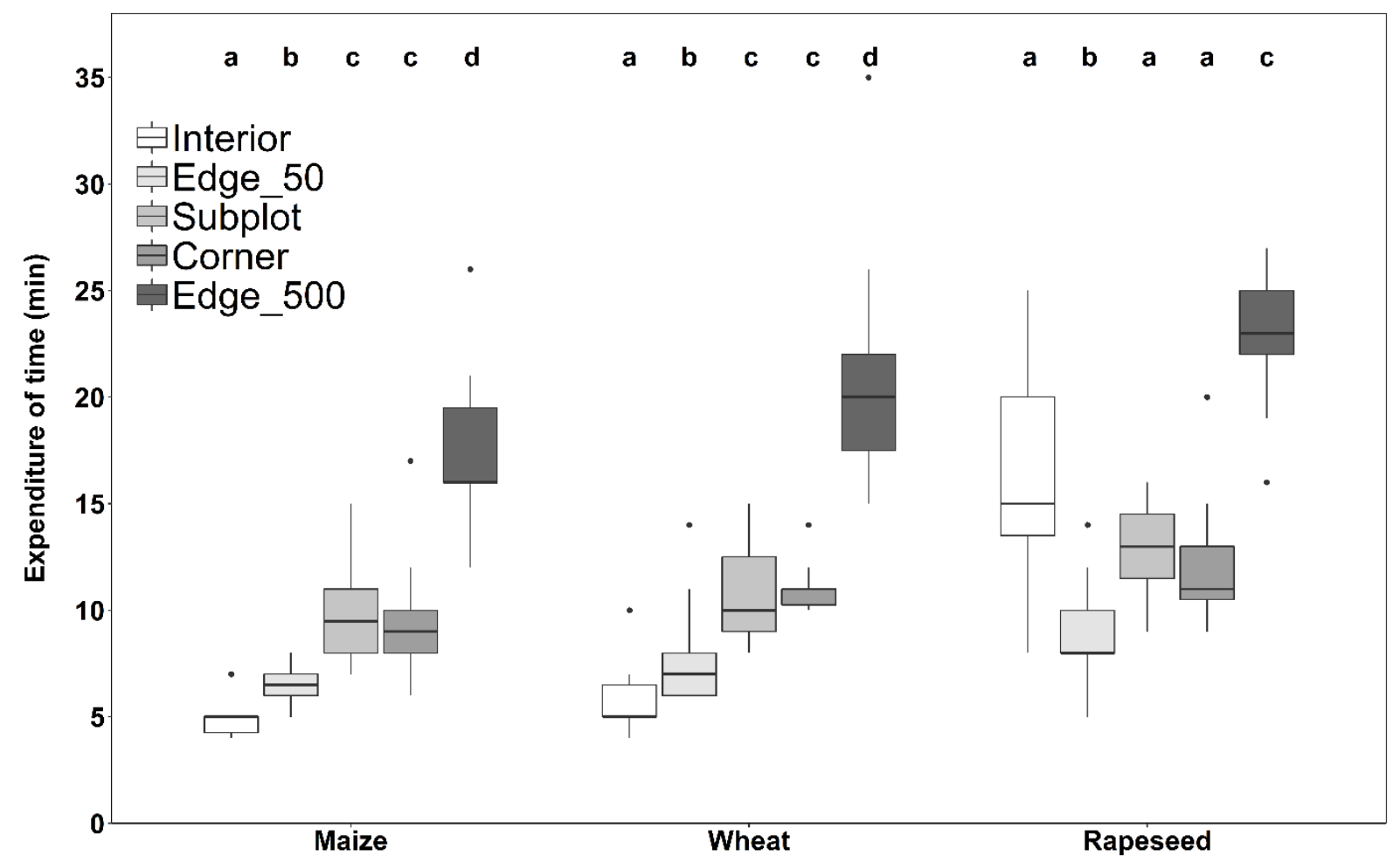

Fig. 4 Time required for surveying the plots of the six different survey methods. No expenditure of time measured for Edge_30 plots; black lines in boxplots represent medians; Mann-Whitney U-Test (pairwise comparisons within crop types using the Wilcoxon rank sum test, $\alpha \leq 0.05$ ); $\mathrm{n}=15$ per crop and survey method (except for each one missing value in a maize Interior plot, maize subplot, maize Edge_50 plot and wheat Corner plot).

\subsubsection{Species composition and distribution}

Across the whole field sample, the most frequent species were Elymus repens, Chenopodium album, Polygonum aviculare, Fallopia convolvulus, Galium aparine and Matricaria chamomilla (Online Resource 7). Due to the location of the plots in different parts of the field, the six survey methods recorded somewhat different subsets of the total species pool. The species list in Online Resource 7 shows that certain characteristic arable plant species, high-nature-value species, and red-listed species were only recorded with the more labor-intensive methods, notably the Edge_500 plots and the Corner plots. These methods recorded a number of taxa that were not found with the other methods: 34 species were only found in the Edge_500 plots, of which 13 were typical arable plants: Aethusa cynapium, Allium vineale, Anchusa arvensis, Anthoxanthum aristatum, Buglossoides arvensis, Cardamine hirsuta, Galinsoga quadriradiata, Glebionis segetum, Matricaria discoidea, Ornithopus perpusillus, 
Phragmites australis, Rorippa palustris, Senecio vulgaris. Four species occurred only in the Corner plots, of which two were typical arable plants (Anthemis arvensis and Anthriscus caucalis); one typical arable plant species (Urtica urens) was recorded only in the Subplots. There were no species which were solely found in Interior plots, Edge_30 and Edge_50 plots. There were also some herbaceous species exclusively appearing in a certain crop, 20 species in maize, 14 in wheat and 25 in rapeseed (for details see Online Resource 8). High-nature-value species occurred only very occasionally in Interior plots and always in very small numbers (Online Resource 7). Only five red-listed species were detected with all methods, namely Agrostemma githago, Anthemis arvensis, Buglossoides arvensis, Chenopodium bybridum, and Odontites vernus. No threatened species were found in wheat fields and in the interior of rapeseed and maize fields.

Beta diversity, related to the characteristic arable plant species, was higher for the Interior, Edge_30, and Edge_50 plot surveys (medians 0.57-0.58; Fig. 5) than for the Edge_500, Corner, and Subplots surveys $(0.48-0.54)$. Calculated distances to the group spatial medians indicate lower floristic similarity among fields from the data retrieved with the former three methods. Thus, Edge_500 and Corner plots showed highest floristic similarity across the fields. Species accumulation curves for the arable plant species showed a strong increase in species richness for the first 10 plots and the highest initial slope for the Edge_500 plots (Fig. 6). In contrast, the Interior plot curve showed a more continuous increase in species, but with very low slope. The other survey methods range between these two extremes. With respect to the twenty $25 \mathrm{~m}$-long subunits investigated in the Edge_500 plots, we found a relatively constant increase in arable plant species richness, until $500 \mathrm{~m}$ length was reached (Online Resource 5). Rapeseed showed the steepest, wheat an intermediate, and maize the lowest slope of the species-plot number curve. In none of the crop types, species richness saturated at $500 \mathrm{~m}$ plot length, as shown by comparison with the total perimeter count. 


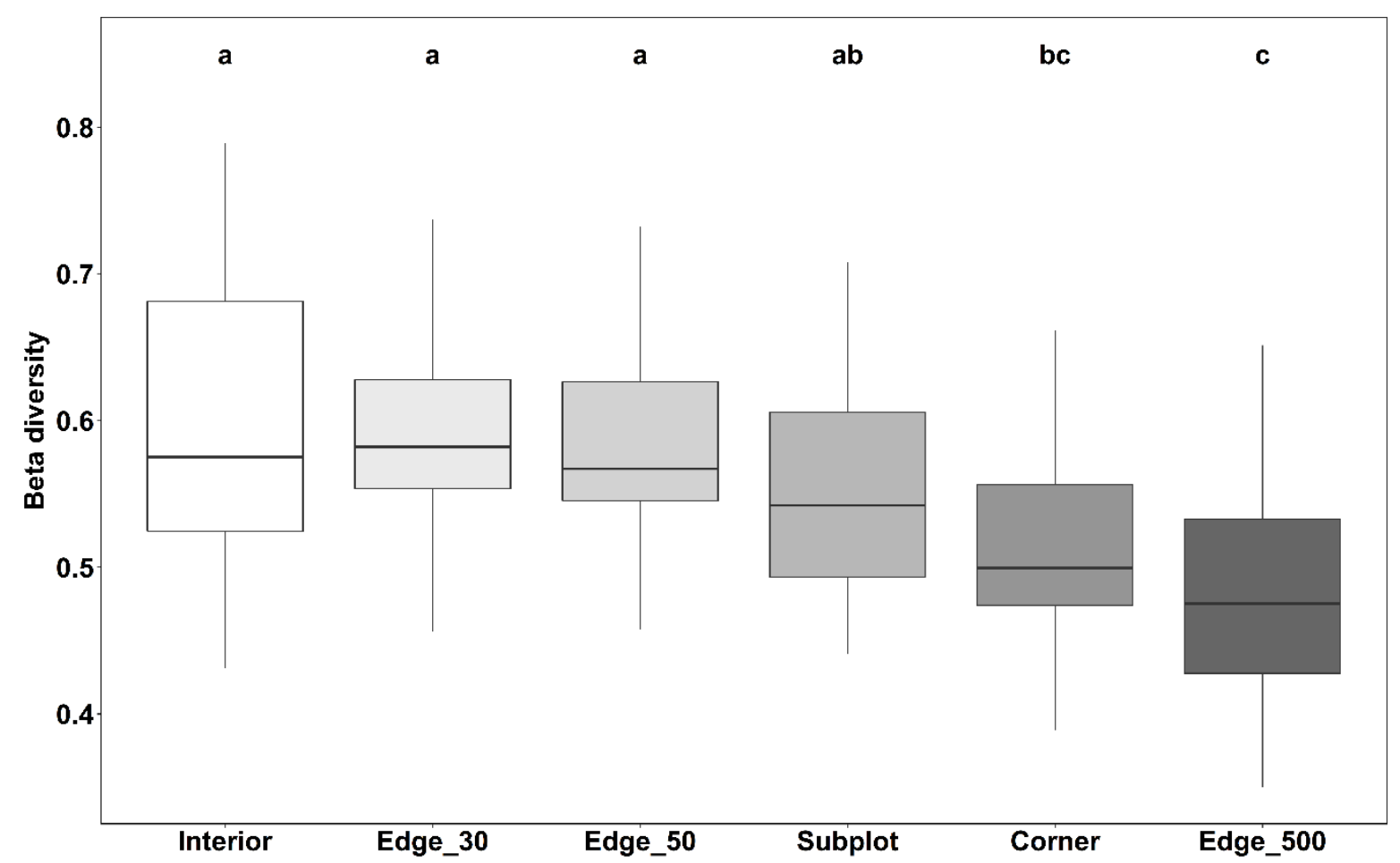

Fig. 5 Beta diversity, measured as distance to group spatial medians based on Jaccard dissimilarity index, for plots of the six survey methods in the 45 fields. Basis for the calculation were the number of arable plant species sensu stricto according to Hofmeister and Garve (2006). The lower the value, the more similar are species compositions for a given survey method (range of values between 0 and 1$) \cdot n=45$ for each survey method, except Interior $n=40$ (5 plots with no species were excluded); different small letters indicate significant differences between survey methods according to a Mann-Whitney U-Test $(\alpha \leq 0.05)$.

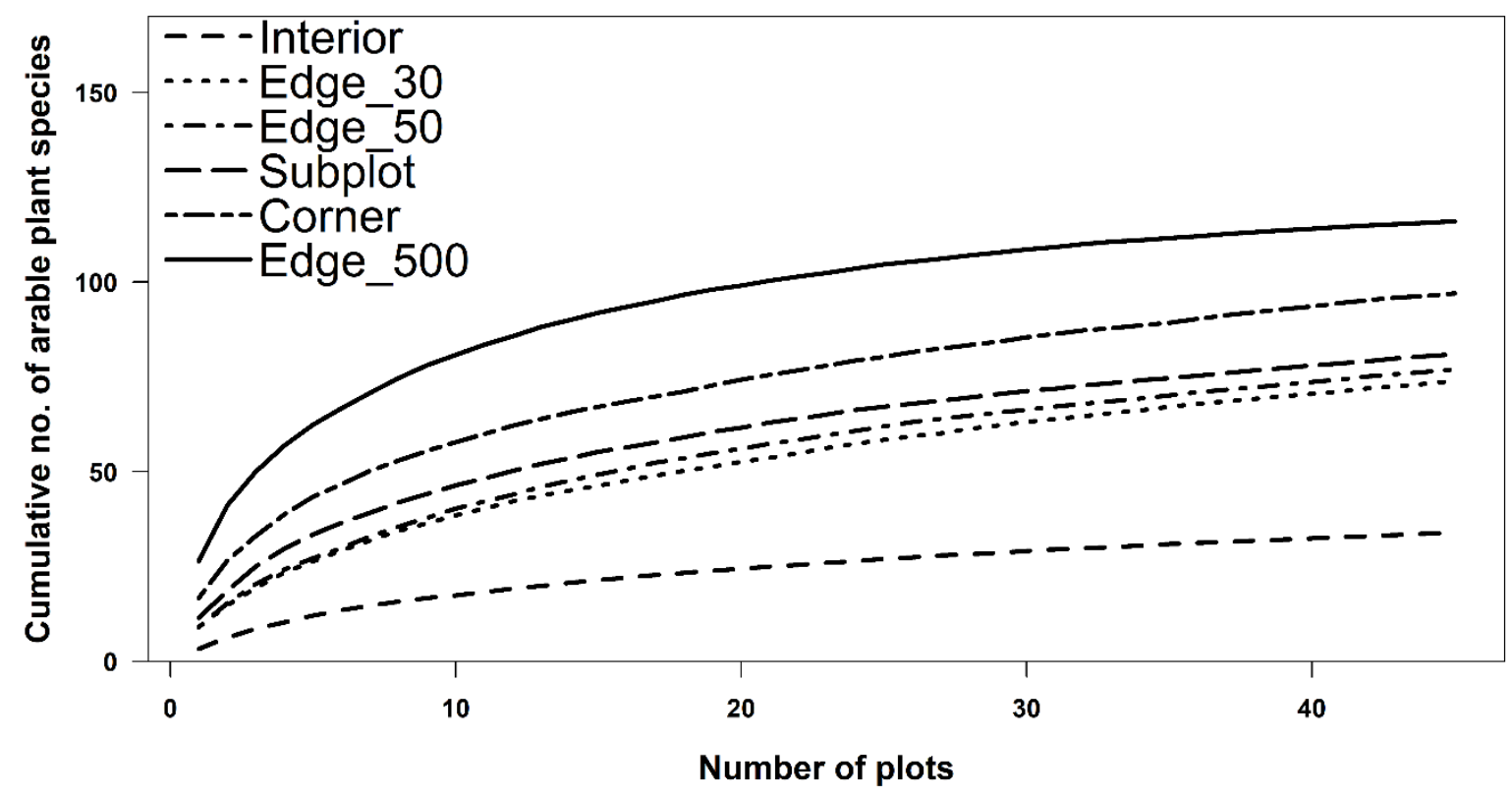

Fig. 6 Species accumulation curves for the number of arable plant species for the six survey methods with increasing plot number. Arable plant species sensu stricto according to Hofmeister \& Garve (2006); method = 'random', permutations $=100)$ for different survey methods (see legend); $\mathrm{n}=45$ for each survey method. 


\subsection{Discussion}

Our systematic comparison of six vegetation survey methods demonstrates high within-field heterogeneity in the arable plant vegetation of conventionally managed farmland. About $90 \%$ of arable plant species richness that has survived agricultural intensification was found in the $1 \mathrm{~m}$ wide marginal strip of the fields (Fig. 2d). This was similar in the three crop types despite contrasting stand structures, phenology and management regimes. This suggests that the main factors that drove the bulk of arable plant species out of the field interior is herbicide use and lower light penetration to the ground in the highly fertilized, dense crop stands, in conjunction with the tillage regime as the most influential environmental factors of conventional agriculture. This corroborates the findings of various studies that most of arable plant diversity today is restricted to the edge zone of arable fields (Albrecht et al. 2016; Batáry et al. 2017; Romero et al. 2008; Seifert et al. 2014, 2015). Thus, future arable plant monitoring should focus on field edge areas of 1 to $2 \mathrm{~m}$ width.

In line with hypothesis (iv), we did not find a single plant species in the Interior plots of the 45 fields that would have been missed, when exclusively investigating the edge zone. Only a few generalist arable plant species were found more frequently in Interior plots, mainly Viola arvensis (in 23 of 45 Interior plots), Polygonum aviculare (17), Chenopodium album (13), Matricaria chamomilla (13), Stellaria media (13) and Fallopia convolvulus (12; Online Resource 7). In addition, there was no correlation between observed arable plant richness in the Interior plots and total arable plant diversity in the field ( $r=0.03$; Fig. 3). Only 37 herbaceous plant species in total were recorded in the Interior of the 45 fields, 34 of which were arable plants sensu stricto. Decades of intense management have not only impoverished the actual arable plant vegetation in the field but also the seed bank and forced a large proportion of formerly widespread arable plant taxa to seek refuge in the edge zone or face extinction (Aavik et al. 2008; Andreasen et al. 2018; Meyer et al. 2013, 2015; von Arx et al. 2002). Directly neighboring, extensively managed habitats may nowadays function as source habitats for some plants, which spill over in the intensively managed field and increase plant diversity (Aavik et al. 2008; Nagy et al. 2018; von Arx et al. 2002). Before agricultural intensification, arable plant diversity was with 24 species per $100 \mathrm{~m}^{2}$ (median) six times higher in the field interior than it is today, explaining why earlier vegetation surveys focused on these areas (Meyer et al. 2013).

Floristic impoverishment justifies the use of strip-like plots of 2 or better $1 \mathrm{~m}$ width along the field edge without losing relevant information. Which of the five tested oblong plots at the field edge is preferable, depends on the purpose of study and available time. Rapid surveys in overregional or national farmland biodiversity monitoring schemes may rely on the 60- $\mathrm{m}^{2}$ Edge_30 plots, as used by the German Federal Agency for Nature Conservation (2018), which were surveyed by us in typically 6-7 min but we recorded with this survey method only $23.3 \%$ of the total species number and also of the arable plant species pool (median; Fig. 2 and Online Resource 2). The detection success is only insignificantly higher in Edge_50 plots $\left(100 \mathrm{~m}^{2}\right)$, while the time needed is probably only slightly higher (median Edge_50 plots across all crops: $7 \mathrm{~min}$ ). Therefore, it is mainly a question of standardization whether 60 or $100 \mathrm{~m}^{2}$ plots at the field edge are investigated. Given that further impoverishment in farmland biodiversity is likely to happen, we would recommend to prefer Edge_50 over Edge_30 plots. Our results further suggest that oblong plots located at field corners contain a larger fraction of the field's species pool than plots in the central part of the margin zone (Fig. 2), irrespective of crop species, which supports 
hypotheses (i) and (ii). This is certainly a consequence of typically less intensive herbicide use and fertilization at field corners, where tractors are turning, disturbance is higher and crop seed density is often reduced. Corner plots have the additional advantage that they may be more easily accessible than oblong plots along hedges or grass strips in the middle of the edge. The greatest advantage of oblong $100 \mathrm{~m}^{2}$-Corner plots is their reasonable detection success, which is nearly twice as high (45 vs. $25 \%$; Fig. 2) than that of similarly sized Edge_50 plots in the central part of the margin zone, while the expenditure of time is only ca. $64 \%$ higher (median 11 vs. 7 min across all crops). As postulated in hypothesis (v), an interesting alternative can be the Subplots approach, which has a similar time requirement as a single Corner plot (12 min), but has the potential to detect a relatively large number of species $(32.6 \%$ of the field's arable plant species pool) despite its small cumulated plot size $\left(20 \mathrm{~m}^{2}\right.$; Fig. 2 and Fig. 4). The effectiveness of this method is explained by the relatively low similarity in species composition in contemporary arable plant communities at the landscape scale, as is displayed by the quite high beta diversity and the relatively steep increase of the species-area curve at low plot numbers (Fig. 5 and Fig. 6). However, since the detection success is less than that of Corner plots, while the time needed is similar, the Corner plot approach seems to be more attractive. In addition, the Subplots method requires that the Subplots are placed either strictly by random or at fixed distances to guarantee comparability and to avoid cherry picking in terms of plant diversity along the field edge. If the Subplots approach is adopted, we would suggest placing at least two of the Subplots at the corner to increase the species richness recorded.

Clearly the highest detection success is achieved by the Edge_500 method, which investigates a five times larger plot area $\left(500 \mathrm{~m}^{2}\right)$ than all other methods. In smaller fields, this area accounts for more than $50 \%$ of the entire field edge area. With a median detection success of $75.6 \%$ (arable plant species; Fig. 2), the vegetation of the field is sufficiently well represented in this type of relevé to serve the goals of phytodiversity assessments and long-term monitoring schemes. Highnature-value species, if they occur, will mostly be detected, which is often not the case in the other approaches with less than 50 or even $25 \%$ detection success (Online Resource 3). For the Edge_500 approach, we found in the 45-field sample a highly significant correlation between the number of arable plant species detected in these plots and the total number of arable plant species present in the field $(\mathrm{r}=0.83, \mathrm{p}<0.0001$; Fig. 3). The method is clearly more timeconsuming than others $(\sim 20 \mathrm{~min}$ per plot across all crops) and perhaps not the choice for landscape-level surveys, where the focus is on a large number of plots. Yet, with only 9 min spent additionally (compared to Corner plots), about a quarter of the field's species pool is additionally recorded (Fig. 2). Moreover, the time needed to access fields and plots (which is not included in our figures) is often much more relevant than the time used for taking the releve itself. This may convince researchers to shift from smaller plots to the $500 \mathrm{~m}^{2}$-plot method, as the gain in additional information is considerable. For vegetation surveys in managed temperate grasslands, Ruff et al. (2013) similarly recommend large oblong plots as the most effective method. Bacaro et al. (2015) also found significantly higher species richness in rectangular (oblong) plots compared to square plots, but they state that plot alignment across possible environmental gradients may influence the species detection success. If the monitoring focus is on red-listed arable plant species, the total perimeter count should be considered. With respect to this species group, even the Edge_500 plots showed only a slightly higher detection success compared to the other field edge survey methods. 
By focusing in arable plant surveys on the edge zone, greater mixing of arable plants with taxa from adjacent habitats is to be expected and is frequently reflected in the relevés. This is a clear disadvantage of linear plot surveys along field edges compared to the historical approach of square plots in the field interior. We therefore recommend considering characteristic arable plant species (as listed by Hofmeister \& Garve 2006) in the relevé data in addition to the total herbaceous plant species list.

How much time is needed for a vegetation survey depends on crop density and thus season and crop species (Fig. 4). This is clearly demonstrated by rapeseed which generally requires more survey time due to its dense stand shortly before harvest. Surveys earlier in the year, when crop height is lower, may not be a good alternative, because many species are more difficult to identify, and some may even be overlooked.

Unexpected is our result that floristic similarity is higher at field edges than in the Interior across the sample of 45 fields (Fig. 5) which contradicts hypothesis (iii). Most vegetation surveys in intensively managed farmland noted a trend toward increasing homogenization of the arable plant vegetation in recent decades (Hanzlik and Gerowitt 2016; Meyer et al. 2013, 2015; Seifert et al. 2015), as phytodiversity has decreased greatly and many formerly rare, specialist taxa have disappeared entirely from the cultural landscape of large parts of Central Europe (Leuschner and Ellenberg 2017). Since the environment is most stressful to plants in the field interior, this suggests that a largely uniform basic set of highly stress- and herbicide-tolerant species should have survived, which is similar in all intensively used fields. We thus expected less between-field variation in community composition on a larger spatial scale (Hanzlik and Gerowitt 2016). In fact, there are very few widespread stress-tolerant species, such as Viola arvensis, Polygonum aviculare, Chenopodium album, Matricaria chamomilla, Stellaria media and Fallopia convolvulus, which were found in Interior plots more frequently. However, due to the impoverishment of the field interior community, the absence of two or three of the common species greatly increases floristic dissimilarity between fields. Thus, the lower beta diversity of the field edge communities as compared to the Interior plots can be explained by the much lower overall diversity of the latter. In addition, some of the common arable plant species such as Echinochloa crus-galli, Apera spicaventi, Myosotis arvensis or Sisymbrium officinale seem to have preferences in terms of crop species (Online Resource 8), edaphic conditions, field management (pest control, tillage and fertilization regime) and field neighborhood (Albrecht et al. 2016; Fried et al. 2008; Hanzlik and Gerowitt 2016; Lososová et al. 2004; Meyer et al. 2013; Pinke et al. 2012; Seifert et al. 2014).

Beta diversity, i.e. within- and between-field variation, is a major component of phytodiversity in the studied arable field complex. This is shown by the large interior-edge gradient in plot-level diversity and is also displayed by the species richness increase by $25 \%$ from the Edge_500 plot to the entire perimeter count (Fig. 2). Rising species numbers beyond a plot size of $500 \mathrm{~m}^{2}$ were also found by Mulugeta et al. (2001) in maize and soybean fields. Inspection of the species-area (plot number) curves further shows that regional variation is also playing a significant role for the landscape-level diversity (gamma diversity) of the region's arable plant vegetation (Fig. 6). All curves, in particular those of the field edge communities, tended to increase beyond 45 fields, probably reflecting differences in edaphic, climatic and agronomic conditions in the study region. The different components of phytodiversity need consideration in biodiversity assessment and monitoring schemes. 


\subsection{Conclusions}

This comparison of six approaches to survey arable plant vegetation showed large differences with respect to the proportion of detected species and the expenditure of time. The recent impoverishment of arable vegetation has the consequence that the former quadratic releve plots should be replaced by oblong strip-like plots at the field edge, preferably across a field corner. Except for studies where large plot numbers have to be surveyed in short time, we recommend $500 \mathrm{~m}$-long and $1 \mathrm{~m}$-wide linear plots at the edge, which include a field corner. An experienced botanist will need no more than about $20 \mathrm{~min}$ for the collection of presence/absence data in the $500 \mathrm{~m}^{2}$ plot with a high chance to record $75 \%$ or more of the field's overall arable plant diversity. When this is not possible due to time constraints, oblong $100 \mathrm{~m}^{2}$-plots stretching over a corner are also a promising option. Such plots should be introduced in addition to a subset of older plots that are surveyed for continuity of methods. Our conclusions about the efficiency of the examined vegetation survey methods should be tested in future studies for additional crop species, in other management systems (e.g. organic farming), and in additional regions such as the Mediterranean, where more species-rich farmland is still present. In addition, the influence of landscape heterogeneity and composition and the role of adjacent habitats on the arable plant diversity should be studied in more detail. Finally, more attention should be paid to between-field differences in segetal community composition and their causes. In the light of the large withinand between-field variation in species richness and the resulting poor comparability of relevé data, monitoring agencies should take initiatives to harmonize arable plant vegetation survey methods at national and international levels.

\section{Acknowledgements}

Many thanks go to all farmers who granted us access to their fields. We also thank Erwin Bergmeier, Jenny Schellenberg, Laura Sutcliffe, Stefan Meyer (all Göttingen) and Roman Link (Würzburg) for helpful discussions regarding study design and data analysis, and Christina Ewerhardy, Fionn Pape and Stefan Mecke for valuable support during the field work.

\section{Author contributions}

AW and CL set up the study design. AW collected the data and performed the data analysis. AW and CL wrote the manuscript.

\section{Funding}

The presented study is part of the research project 'Development of targeted and efficient schemes to increase biodiversity in agricultural landscapes (MEDIATE)' funded by the German Federal Environmental Foundation (DBU; 32873/01). 


\section{References}

Aavik, T., Augenstein, I., Bailey, D., Herzog, F., Zobel, M., \& Liira, J. (2008). What is the role of local landscape structure in the vegetation composition of field boundaries? Applied Vegetation Science, 11(3), 375-386. https://doi.org/10.3170/2008-7-18486

Albrecht, H. (1989). Untersuchungen zur Veränderung der Segetalflora an sieben bayerischen Ackerstandorten zwischen den Erhebungszeiträumen 1951/68 und 1986/88: mit 31 Tabellen im Text und im Anhang. Berlin Stuttgart: Cramer in d. Borntraeger-Verl.-Buchh.

Albrecht, H., Cambecèdes, J., Lang, M., \& Wagner, M. (2016). Management options for the conservation of rare arable plants in Europe. Botany Letters, 163(4), 389-415. https://doi.org/10.1080/23818107.2016.1237886

Andreasen, C., Jensen, H. A., \& Jensen, S. M. (2018). Decreasing diversity in the soil seed bank after 50 years in Danish arable fields. Agriculture, Ecosystems \& Environment, 259, 61-71. https://doi.org/10.1016/j.agee.2018.02.034

Bacaro, G., Rocchini, D., Diekmann, M., Gasparini, P., Gioria, M., Maccherini, S., et al. (2015). Shape matters in sampling plant diversity: Evidence from the field. Ecological Complexity, 24, 37-45. https://doi.org/10.1016/j.ecocom.2015.09.003

Bache, S. M., \& Wickham, H. (2014). magrittr: A Forward-Pipe Operator for R. R package version 1.5. https://CRAN.Rproject.org/package $=$ magrittr. Accessed 6 November 2018

Barton, K. (2018). MuMIn: Multi-Model Inference. R package version 1.42.1. https://CRAN.R-project.org/package=MuMIn. Accessed 18 February 2019

Batáry, P., Gallé, R., Riesch, F., Fischer, C., Dormann, C. F., Mußhoff, O., et al. (2017). The former Iron Curtain still drives biodiversity-profit trade-offs in German agriculture. Nature Ecology \& Evolution, 1(9), 1279-1284. https://doi.org/10.1038/s41559-017-0272-x

Batáry, P., Holzschuh, A., Orci, K. M., Samu, F., \& Tscharntke, T. (2012). Responses of plant, insect and spider biodiversity to local and landscape scale management intensity in cereal crops and grasslands. Agriculture, Ecosystems \& Environment, 146(1), 130-136. https://doi.org/10.1016/j.agee.2011.10.018

Bates, D., Mächler, M., Bolker, B., \& Walker, S. (2015). Fitting Linear Mixed-Effects Models using lme4. Journal of Statistical Software, 67(1). https://doi.org/10.18637/jss.v067.i01

Bolker, B. (2019). GLMM FAQ. http://bbolker.github.io/mixedmodels-misc/glmmFAQ.html\#count-data. Accessed 13 February 2019

Buttler, K. P. (2018). Florenliste von Deutschland - Gefäßpflanzen. http://www.kp- buttler.de. Accessed 13 February 2019

Chytrý, M., \& Otýpková, Z. (2003). Plot sizes used for phytosociological sampling of European vegetation. Journal of Vegetation Science, 14(4), 563-570. https://doi.org/10.1111/j.1654-1103.2003.tb02183.x

Clough, Y., Holzschuh, A., Gabriel, D., Purtauf, T., Kleijn, D., Kruess, A., et al. (2007). Alpha and beta diversity of arthropods and plants in organically and conventionally managed wheat fields: $\alpha$ - and $\beta$-diversity in wheat fields. Journal of Applied Ecology, 44(4), 804-812. https://doi.org/10.1111/j.1365-2664.2007.01294.x

Dierschke, H. (1994). Pflanzensoziologie: Grundlagen und Methoden; 55 Tabellen. Stuttgart: Ulmer

European Union (EU). (2013a). Regulation (EU) No 1305/2013 of the European Parliament and of the Council of 17 December 2013 on support for rural development by the European Agricultural Fund for Rural Development (EAFRD) and repealing Council Regulation (EC) No 1698/2005. https://eur-lex.europa.eu/legalcontent/EN/TXT/PDF/?uri=CELEX:32013R1305\&from=en. Accessed 4 February 2019

European Union (EU). (2013b). Regulation (EU) No 1307/2013 of the European Parliament and of the Council of 17 december 2013 establishing rules for direct payments to farmers under support schemes within the framework of the common agricultural policy and repealing Council Regulation (EC) No 637/2008 and Council Regulation (EC) No 73/2009. https://eur-lex.europa.eu/legal-

content/EN/TXT/PDF/?uri=CELEX:32013R1307\&qid=1436267634754\&from=EN. Accessed 4 February 2019

European Union (EU). (2014a). Commission Delegated Regulation (EU) No 639/2014 of 11 March 2014 supplementing Regulation (EU) No 1307/2013 of the European Parliament and of the Council establishing rules for direct payments to farmers under support schemes within the framework of the common agricultural policy and amending Annex X to that Regulation. https://eur-lex.europa.eu/legalcontent/EN/TXT/PDF/?uri=CELEX:32014R0639\&qid=1436267909117\&from=EN. Accessed 4 February 2019

European Union (EU). (2014b). Commission Implementing Regulation (EU) No 641/2014 of 16 June 2014 laying down rules for the application of Regulation (EU) No 1307/2013 of the European Parliament and of the Council establishing rules for direct payments to farmers under support schemes within the framework of the common agricultural policy. https://eur-lex.europa.eu/legalcontent/EN/TXT/PDF/?uri=CELEX:32014R0641\&qid=1436268294105\&from=EN. Accessed 4 February 2019

Fahrig, L., Girard, J., Duro, D., Pasher, J., Smith, A., Javorek, S., et al. (2015). Farmlands with smaller crop fields have higher within-field biodiversity. Agriculture, Ecosystems \& Environment, 200, 219-234. https://doi.org/10.1016/j.agee.2014.11.018 
Federal Institute for Geosciences and Natural Resources (BGR). (2013). Soil Map of the Federal Republic of Germany $1: 1,000,000$ https://www.bgr.bund.de/DE/Themen/Boden/Informationsgrundlagen/Bodenkundliche_Karten_Datenbanken/BU EK1000/buek1000_node.html. Accessed 26 October 2016

Fox, J., Weisberg, S., \& Fox, J. (2011). An R companion to applied regression (2nd ed.). Thousand Oaks, Calif: SAGE Publications.

Fried, G., Norton, L. R., \& Reboud, X. (2008). Environmental and management factors determining weed species composition and diversity in France. Agriculture, Ecosystems \& Environment, 128(1-2), 68-76. https://doi.org/10.1016/j.agee.2008.05.003

Gabriel, D., Roschewitz, I., Tscharntke, T., \& Thies, C. (2006). Beta diversity at different spatial scales: plant communities in organic and conventional agriculture. Ecological Applications, 16(5), 2011-2021. https://doi.org/10.1890/10510761(2006)016[2011:BDADSS]2.0.CO;2

Gabriel, D., Thies, C., \& Tscharntke, T. (2005). Local diversity of arable weeds increases with landscape complexity. Perspectives in Plant Ecology, Evolution and Systematics, 7(2), 85-93. https://doi.org/10.1016/j.ppees.2005.04.001

Garve, E. (2004). Rote Liste und Florenliste der Farn- und Blütenpflanzen in Niedersachsen und Bremen, 5. Fassung vom 1.3.2004. Inform. d. Naturschutz Niedersachs. 24 (1) (1/04): 1-76, Hildesheim

German Federal Agency for Nature Conservation. (2018). High-nature-value farmland, Germany. https://www.bfn.de/themen/monitoring/monitoring-von-landwirtschaftsflaechen-mit-hohem-naturwert.html. Accessed 19 October 2018

German Meteorological Service (DWD). (2018). Climate data (2013-2017) via the CDC FTP server. ftp://ftpcdc.dwd.de/pub/CDC/observations_germany/climate/annual/kl/recent/_ \& https://www.dwd.de/DE/leistungen/klimadatendeutschland/statliste/statlex_html.html?view=nasPublication\&nn=16 102. Accessed 25 October 2018

Gholamhoseini, M., AghaAlikhani, M., Mirlatifi, S. M., \& Sanavy, S. A. M. M. (2013). Weeds - Friend or foe? Increasing forage yield and decreasing nitrate leaching on a corn forage farm infested by redroot pigweed. Agriculture, Ecosystems \& Environment, 179, 151-162. https://doi.org/10.1016/j.agee.2013.08.016

Hanzlik, K., \& Gerowitt, B. (2011). The importance of climate, site and management on weed vegetation in oilseed rape in Germany. Agriculture, Ecosystems \& Environment, 141(3-4), 323-331. https://doi.org/10.1016/j.agee.2011.03.010

Hanzlik, K., \& Gerowitt, B. (2016). Methods to conduct and analyse weed surveys in arable farming: a review. Agronomy for Sustainable Development, 36(1). https://doi.org/10.1007/s13593-015-0345-7

Hatcher, P., \& Froud-Williams, R. J. (Eds.). (2017). Weed research: expanding horizons. Hoboken, NJ: John Wiley \& Sons Ltd.

Hawes, C., Haughton, A. J., Osborne, J. L., Roy, D. B., Clark, S. J., Perry, J. N., et al. (2003). Responses of plants and invertebrate trophic groups to contrasting herbicide regimes in the Farm Scale Evaluations of genetically modified herbicide-tolerant crops. Philosophical Transactions of the Royal Society B: Biological Sciences, 358(1439), 1899-1913. https://doi.org/10.1098/rstb.2003.1406

Heydemann, B., \& Meyer, H. (1983). Auswirkungen der Intensivkultur auf die Fauna in den Agrarbiotopen. SchrR. Dt. Rat für Landespfl., 42, 174-191

Hofmeister, H., \& Garve, E. (2006). Lebensraum Acker (Reprint der 2. neubearbeiteten Auflage.). Remagen: Verlag Kessel.

Hothorn, T., Bretz, F., \& Westfall, P. (2008). Simultaneous Inference in General Parametric Models. Biometrical Journal, 50(3), 346-363. https://doi.org/10.1002/bimj.200810425

Hyvönen, T., \& Huusela-Veistola, E. (2008). Arable weeds as indicators of agricultural intensity - A case study from Finland. Biological Conservation, 141(11), 2857-2864. https://doi.org/10.1016/j.biocon.2008.08.022

Kläge, H.-C. (1999). Segetalarten und -gesellschaften der nordwestlichen Niederlausitz und die Naturschutzstrategie zu ihrer Erhaltung: mit 31 Tabellen im Text sowie 12 Vegetationstabellen als Faltbeilagen. Berlin Stuttgart: J. Cramer in der Gebrüder Borntraeger Verlagsbuchhandlung

Krauss, J., Gallenberger, I., \& Steffan-Dewenter, I. (2011). Decreased Functional Diversity and Biological Pest Control in Conventional Compared to Organic Crop Fields. PLoS ONE, 6(5), e19502. https://doi.org/10.1371/journal.pone.0019502

Leuschner, C., \& Ellenberg, H. (2017). Ecology of Central European Non-Forest Vegetation: Coastal to Alpine, Natural to ManMade Habitats: Vegetation Ecology of Central Europe, Volume II. Cham: Springer International Publishing. https://doi.org/10.1007/978-3-319-43048-5

Lososová, Z., Chytrý, M., Cimalová, S., Kropáč, Z., Otýpková, Z., Pyšek, P., \& Tichý, L. (2004). Weed vegetation of arable land in Central Europe: Gradients of diversity and species composition. Journal of Vegetation Science, 15(3), 415-422. https://doi.org/10.1111/j.1654-1103.2004.tb02279.x

Lüscher, G., Jeanneret, P., Schneider, M. K., Turnbull, L. A., Arndorfer, M., Balázs, K., et al. (2014). Responses of plants, earthworms, spiders and bees to geographic location, agricultural management and surrounding landscape in European arable fields. Agriculture, Ecosystems \& Environment, 186, 124-134. https://doi.org/10.1016/j.agee.2014.01.020 
Májeková, J., Zaliberová, M., Šibík, J., \& Klimová, K. (2010). Changes in segetal vegetation in the Borská nížina Lowland (Slovakia) over 50 years. Biologia, 65(3). https://doi.org/10.2478/s11756-010-0035-5

Marshall, E. J. P., Brown, V. K., Boatman, N. D., Lutman, P. J. W., Squire, G. R., \& Ward, L. K. (2003). The role of weeds in supporting biological diversity within crop fields. Weed Research, 43(2), 77-89. https://doi.org/10.1046/j.13653180.2003.00326.x

Médiène, S., Valantin-Morison, M., Sarthou, J.-P., de Tourdonnet, S., Gosme, M., Bertrand, M., et al. (2011). Agroecosystem management and biotic interactions: a review. Agronomy for Sustainable Development, 31(3), 491-514. https://doi.org/10.1007/s13593-011-0009-1

Meisel, K., \& von Hübschmann, A. (1976). Veränderungen der Acker- und Grünlandvegetation im nordwestdeutschen Flachland in jüngerer Zeit. Schriftenreihe Vegetationskunde, 10, 109-124

Meyer, S., Bergmeier, E., Becker, T., Wesche, K., Krause, B., \& Leuschner, C. (2015). Detecting long-term losses at the plant community level - arable fields in Germany revisited. Applied Vegetation Science, 18(3), 432-442. https://doi.org/10.1111/avsc.12168

Meyer, S., Wesche, K., Krause, B., \& Leuschner, C. (2013). Dramatic losses of specialist arable plants in Central Germany since the 1950s/60s - a cross-regional analysis. Diversity and Distributions, 19(9), 1175-1187. https://doi.org/10.1111/ddi.12102

Mulugeta, D., Stoltenberg, D. E., \& Boerboom, C. M. (2001). Weed species-area relationships as influenced by tillage. Weed Science, 49(2), 217-223. https://doi.org/10.1614/0043-1745(2001)049[0217:WSARAT2.0.CO;2

Nagy, K., Lengyel, A., Kovács, A., Türei, D., Csergó, A. M., \& Pinke, G. (2018). Weed species composition of small-scale farmlands bears a strong crop-related and environmental signature. Weed Research, 58(1), 46-56. https://doi.org/10.1111/wre.12281

Oksanen, J., Blanchet, F. G., Friendly, M., Kindt, R., Legendre, P., McGlinn, D., et al. (2019). vegan: Community Ecology Package. R package version 2.5-2. https://CRAN.R-project.org/package= vegan. Accessed 6 November 2018

Paradis, E., \& Schliep, K. (2018). ape 5.0: an environment for modern phylogenetics and evolutionary analyses in R. Bioinformatics, 35(3), 526-528

Petit, S., Gaba, S., Grison, A.-L., Meiss, H., Simmoneau, B., Munier-Jolain, N., \& Bretagnolle, V. (2016). Landscape scale management affects weed richness but not weed abundance in winter wheat fields. Agriculture, Ecosystems \& Environment, 223, 41-47. https://doi.org/10.1016/j.agee.2016.02.031

Pinke, G., Karácsony, P., Czúcz, B., Botta-Dukát, Z., \& Lengyel, A. (2012). The influence of environment, management and site context on species composition of summer arable weed vegetation in Hungary. Applied Vegetation Science, 15(1), 136144. https://doi.org/10.1111/j.1654-109X.2011.01158.x

Pollnac, F. W., Maxwell, B. D., \& Menalled, F. D. (2009). Using Species-Area Curves to Examine Weed Communities in Organic and Conventional Spring Wheat Systems. Weed Science, 57(03), 241-247. https://doi.org/10.1614/WS-08-159.1

R Core Team. (2018). R: A language and environment for statistical computing. R Foundation for Statistical Computing, Vienna, Austria. https://www.R-project.org/. Accessed 6 November 2018

Richner, N., Holderegger, R., Linder, H. P., \& Walter, T. (2015). Reviewing change in the arable flora of Europe: a meta-analysis. Weed Research, 55(1), 1-13. https://doi.org/10.1111/wre.12123

Romero, A., Chamorro, L., \& Sans, F. X. (2008). Weed diversity in crop edges and inner fields of organic and conventional dryland winter cereal crops in NE Spain. Agriculture, Ecosystems \& Environment, 124(1-2), 97-104. https://doi.org/10.1016/j.agee.2007.08.002

Roschewitz, I., Gabriel, D., Tscharntke, T., \& Thies, C. (2005). The effects of landscape complexity on arable weed species diversity in organic and conventional farming: Landscape complexity and weed species diversity. Journal of Applied Ecology, 42(5), 873-882. https://doi.org/10.1111/j.1365-2664.2005.01072.x

Rotchés-Ribalta, R., Blanco-Moreno, J. M., Armengot, L., José-María, L., \& Sans, F. X. (2015). Which conditions determine the presence of rare weeds in arable fields? Agriculture, Ecosystems \& Environment, 203, 55-61. https://doi.org/10.1016/j.agee.2015.01.022

Ruff, M., Kuhn, G., Heinz, S., Kollmann, J., \& Albrecht, H. (2013). Evaluation of plant species diversity in grasslands for agrienvironmental schemes in small-structured landscapes - a methodological study. Naturschutz und Landschaftsplanung 45 (3), 76-82

Seifert, C., Leuschner, C., \& Culmsee, H. (2015). Arable plant diversity on conventional cropland-The role of crop species, management and environment. Agriculture, Ecosystems \& Environment, 213, 151-163. https://doi.org/10.1016/j.agee.2015.07.017

Seifert, C., Leuschner, C., Meyer, S., \& Culmsee, H. (2014). Inter-relationships between crop type, management intensity and light transmissivity in annual crop systems and their effect on farmland plant diversity. Agriculture, Ecosystems \& Environment, 195, 173-182. https://doi.org/10.1016/j.agee.2014.05.022

Solé-Senan, X. O., Juárez-Escario, A., Conesa, J. A., Torra, J., Royo-Esnal, A., \& Recasens, J. (2014). Plant diversity in Mediterranean cereal fields: Unraveling the effect of landscape complexity on rare arable plants. Agriculture, Ecosystems \& Environment, 185, 221-230. https://doi.org/10.1016/j.agee.2014.01.003 
State Authority for Mining, Energy and Geology (LBEG). (2015). German soil assessment map 1:5,000 (BS5). https://www.lbeg.niedersachsen.de/karten_daten_publikationen/karten_daten/boden/bodenkarten/bodenschaetzung skarte_15000/bodenschaetzungskarte-von-niedersachsen-im-mastab-1--5-000-bs5-681.html. Accessed 26 October 2018

von Arx, G., Biosshard, A., \& Dietz, H. (2002). Land-use intensity and border structures as determinants of vegetation diversity in an agricultural area. Bulletin of the Geobotanical Institute ETH, 68, 3-15

Wickham, H. (2017). tidyverse: Easily Install and Load the "Tidyverse". R package version 1.2.1. https://CRAN.Rproject.org/package=tidyverse. Accessed 6 November 2018

Wietzke, A., Westphal, C., Gras, P., Kraft, M., Pfohl, K., Karlovsky, P., et al. (2018). Insect pollination as a key factor for strawberry physiology and marketable fruit quality. Agriculture, Ecosystems \& Environment, 258, 197-204. https://doi.org/10.1016/j.agee.2018.01.036 


\section{Electronic supplementary material}

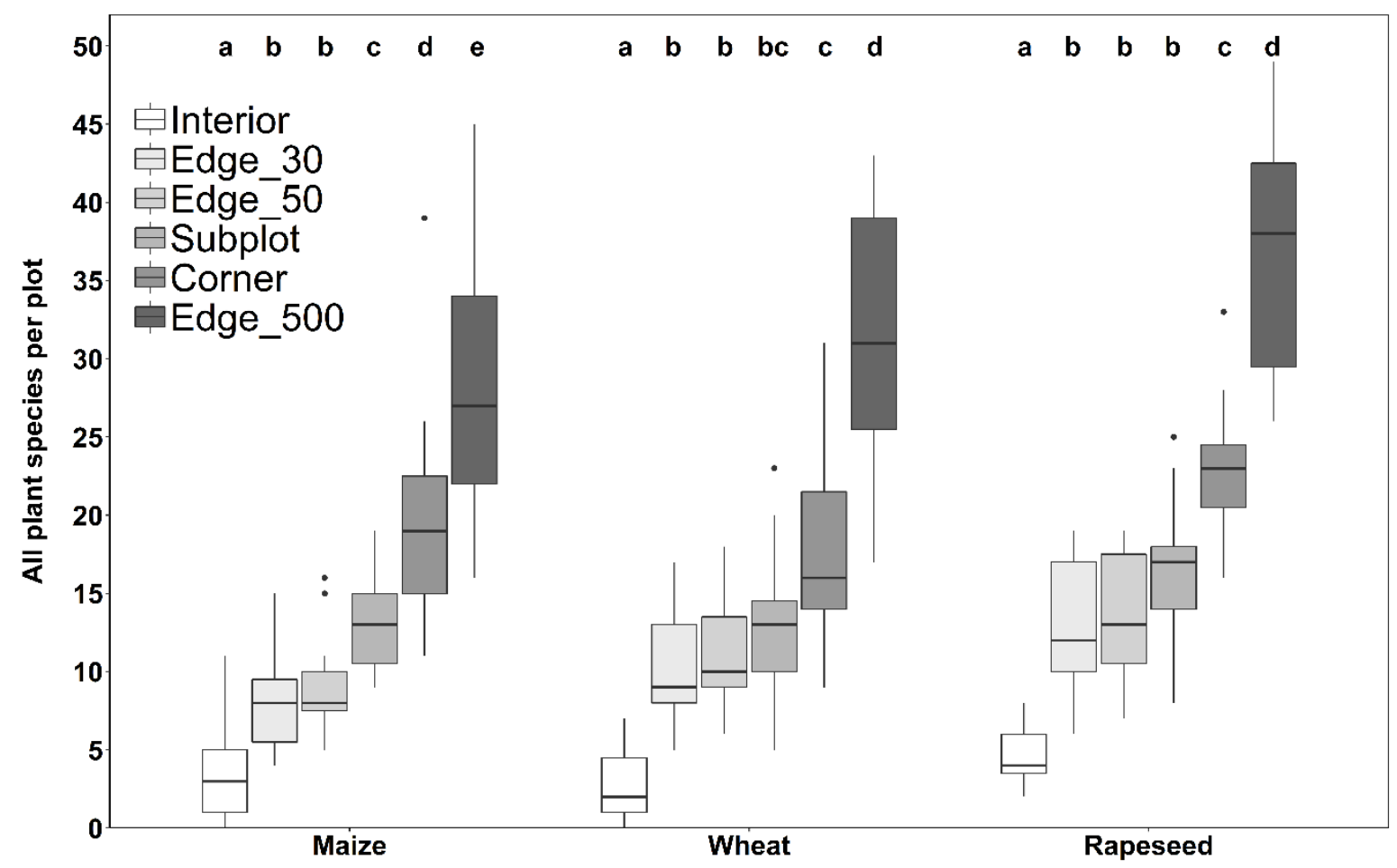

Online Resource 1 Total number of herbaceous species observed in the different crop types (winter wheat, winter rapeseed, maize); species number exclusive crop species and woody seedlings; survey methods in different colors (see legend): Interior $(50 \times 2 \mathrm{~m})$, Edge_30 $(30 \times 2 \mathrm{~m})$, Edge_50 $(50 \times 2 \mathrm{~m})$, Subplots (four plots of $5 \times 1 \mathrm{~m})$, Corner $(50 \times 2 \mathrm{~m})$ and Edge_500 $(500 \times \mathrm{x} 1 \mathrm{~m})$; black lines in boxplots represent medians; Mann-Whitney-U-Test (pairwise comparisons within crop types using Wilcoxon rank sum test, $\alpha \leq 0.05) ; \mathrm{n}=15$ per crop and survey method; different small letters indicate significant differences between survey methods within crop types; model overview and statistical results see Online Resource 9.

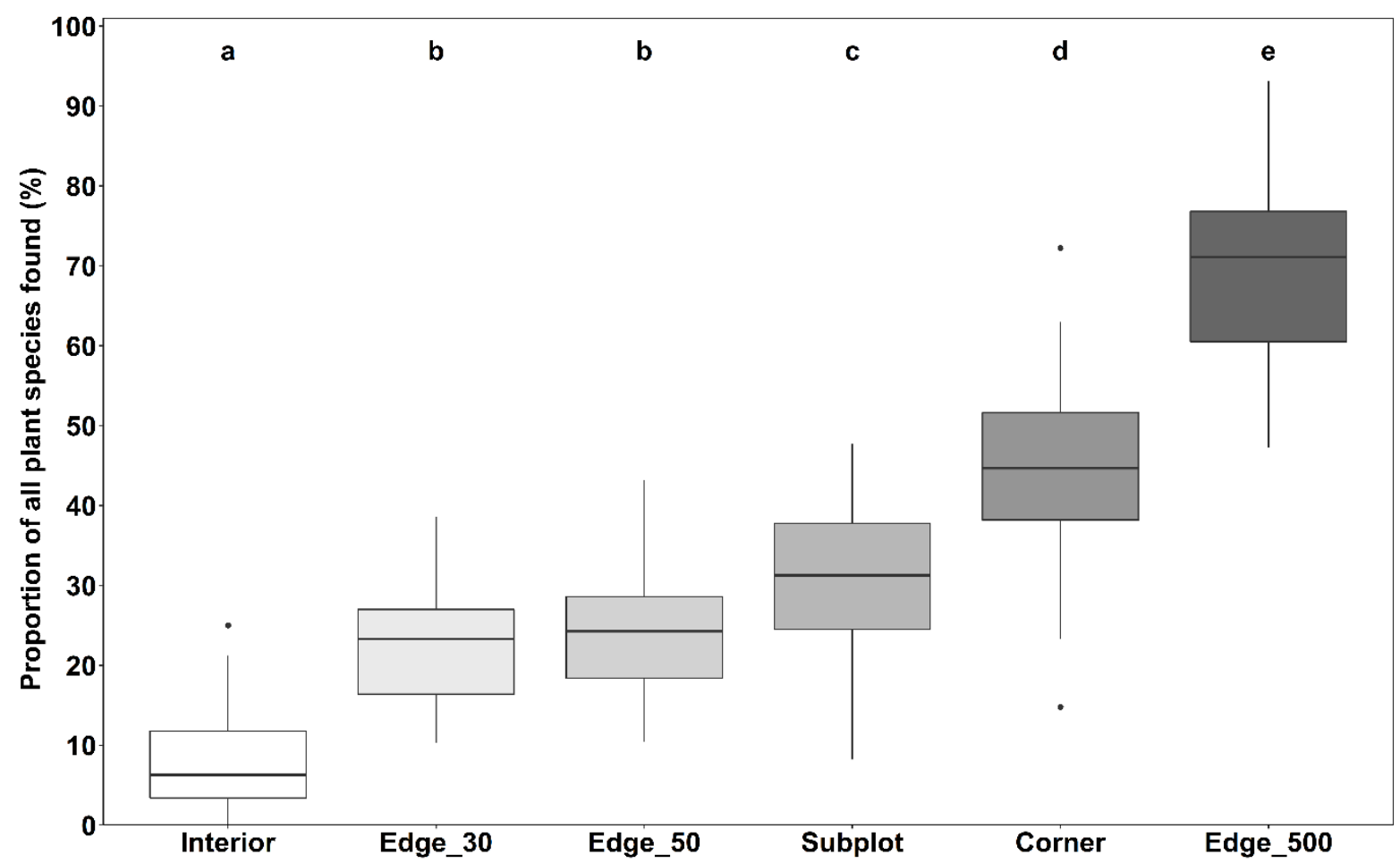

Online Resource 2 Proportion of the species number found in the plots (\%) of the different survey methods relative to the fir total species pool (total perimeter count; all herbaceous species considered, crops and woody seedlings excluded; winter wheat, winter rapeseed and maize were pooled); Interior $(50 \times 2 \mathrm{~m})$, Edge_30 $(30 \times 2 \mathrm{~m})$, Edge_50 $(50 \times 2 \mathrm{~m})$, Subplots (four plots o $\times 1 \mathrm{~m}$ ), Corner $(50 \times 2 \mathrm{~m})$ and Edge_500 $(500 \times 1 \mathrm{~m})$; black lines in boxplots represent medians; $\mathrm{n}=45$ (per survey method), Tukey's test $\alpha \leq 0.05$; different small letters indicate significant differences between survey methods; model overview and statis results see Online Resource 9. 


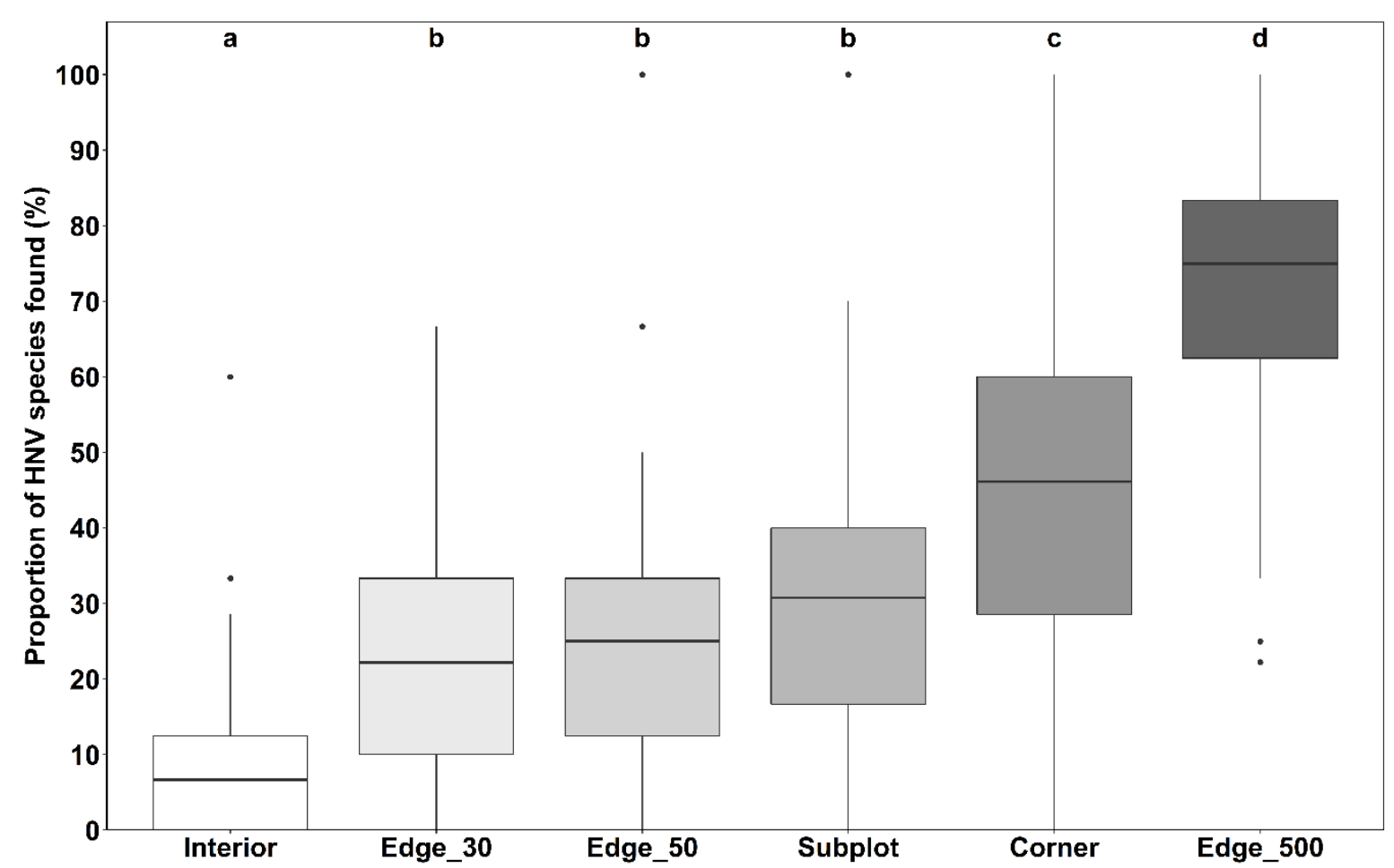

Online Resource 3 Proportion of the number of high-nature-value species (\%; HNV; according to German Federal Agency for Nature Conservation, 2018) found in the plots of the different survey methods relative to the field's total high-naturevalue (HNV) species pool (total perimeter count; all herbaceous species considered, crops and woody seedlings excluded; winter wheat, winter rapeseed and maize were pooled); Interior $(50 \times 2 \mathrm{~m})$, Edge_30 $(30 \times 2 \mathrm{~m})$, Edge_50 $(50 \times 2 \mathrm{~m})$, Subplots (four plots of $5 \times 1 \mathrm{~m})$, Corner $(50 \times 2 \mathrm{~m})$ and Edge_500 $(500 \times 1 \mathrm{~m})$; black lines in boxplots represent medians; $\mathrm{n}$ $=45$ (per survey method), Tukey's test $\alpha \leq 0.05$; different small letters indicate significant differences between survey methods; model overview and statistical results see Online Resource 9.

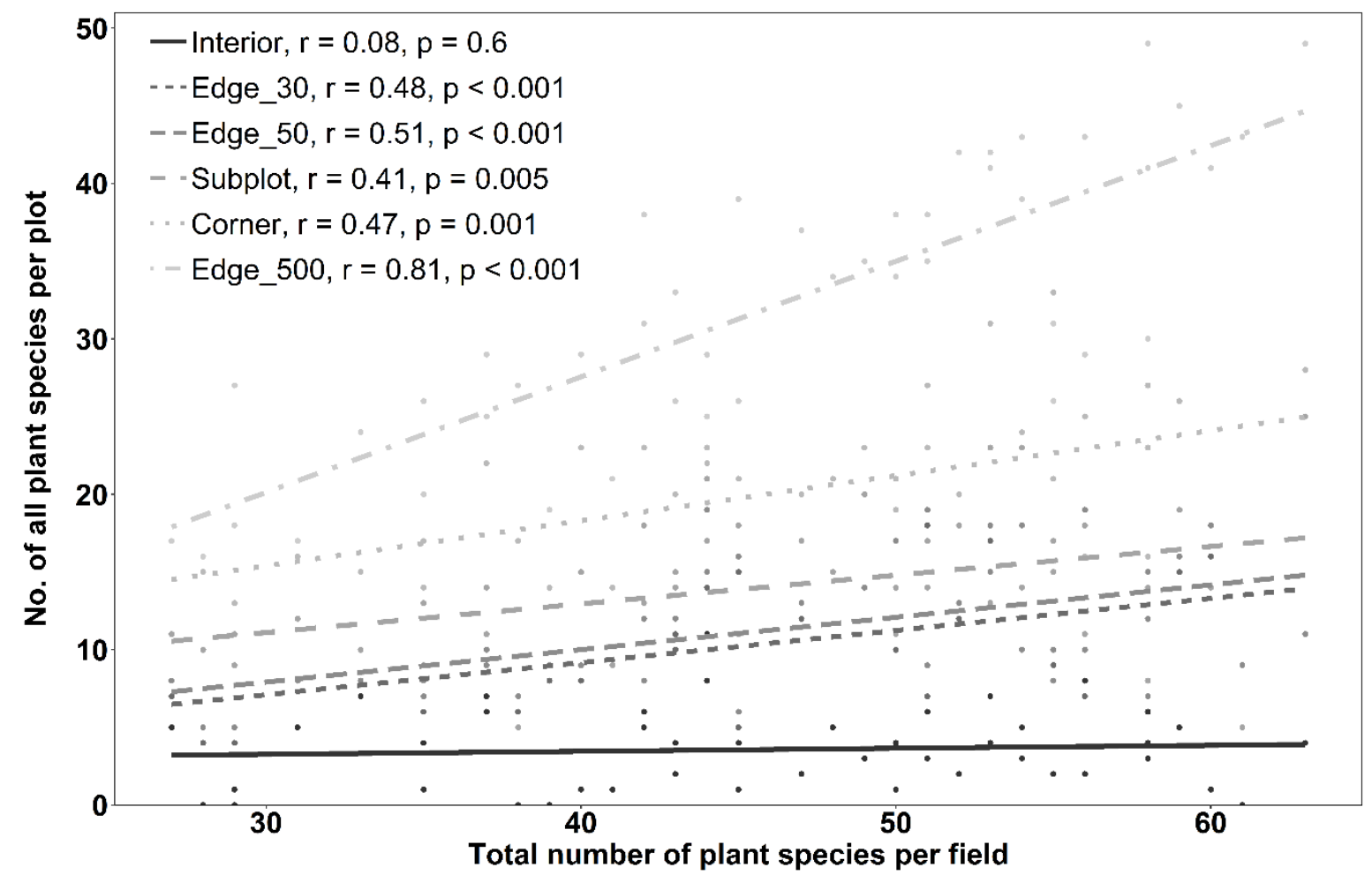

Online Resource 4 Number of all herbaceous plant species found in plots of the six survey methods in relation to the field's total herbaceous species number (crops and woody seedlings excluded); survey method (see legend): Interior $(50 \times 2 \mathrm{~m}$ ), Edge_30 $(30 \times 2 \mathrm{~m})$, Edge_50 $(50 \times 2 \mathrm{~m})$, Subplots (four plots of $5 \times 1 \mathrm{~m})$, Corner $(50 \times 2 \mathrm{~m})$ and Edge_500 $(500 \times 1 \mathrm{~m})$; R $=$ Spearman's rank correlation coefficient with $\mathrm{p}$-values; $\mathrm{n}=45$ (per survey method). 


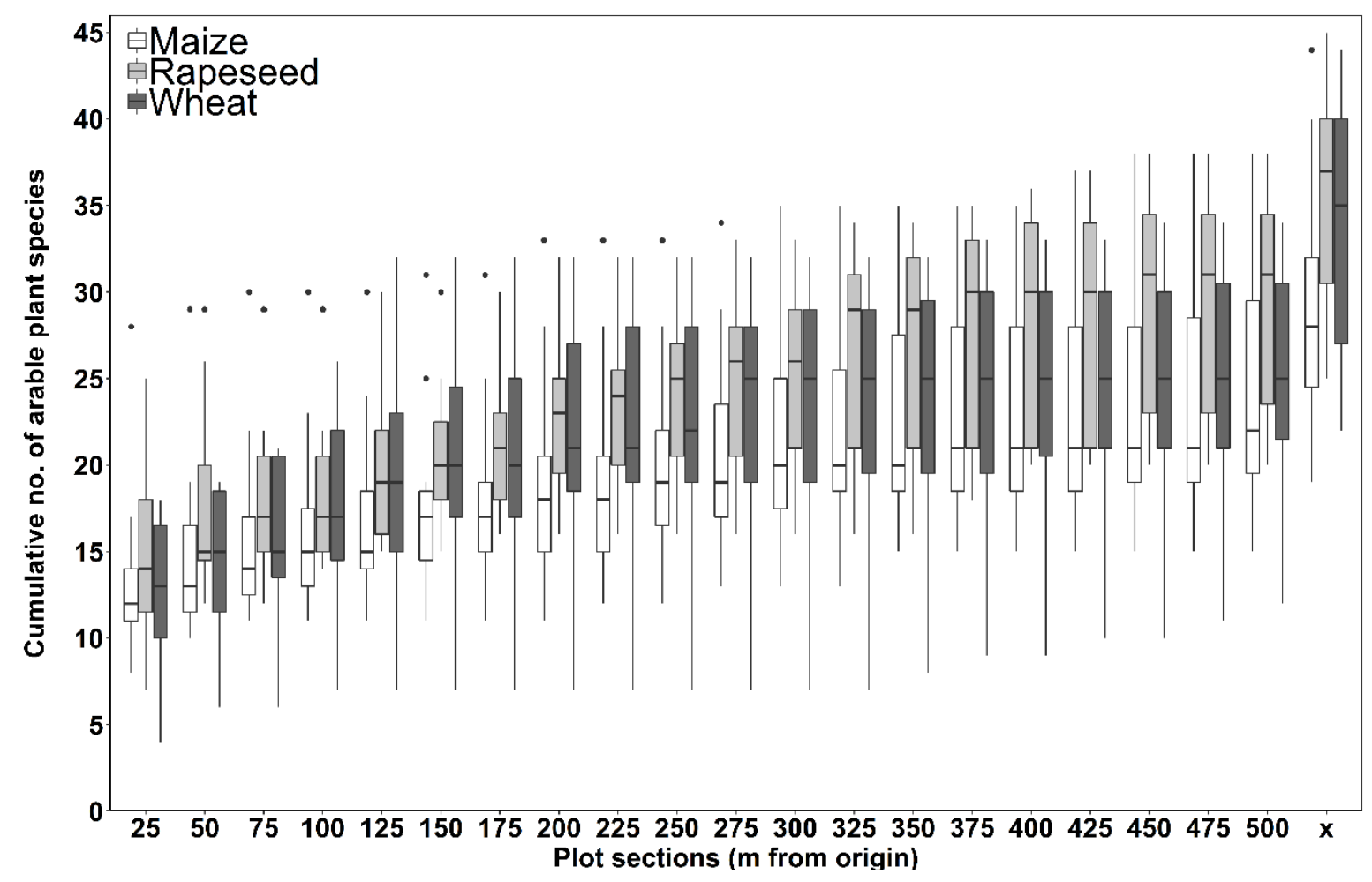

Online Resource 5 Cumulative number of arable plant species sensu stricto (Hofmeister \& Garve, 2006) in the 20 sections (each of $25 \mathrm{~m}$ length) of the Edge_500 plots (size: $500 \times 1 \mathrm{~m}$ ) in wheat, rapeseed and maize fields (see legend); ' $\mathrm{x}$ ' = additional species found subsequent to the $500 \mathrm{~m}$ plots (field perimeter ranged between 700 and $1500 \mathrm{~m}$ ); $\mathrm{n}=45$; black lines in boxplots represent medians.

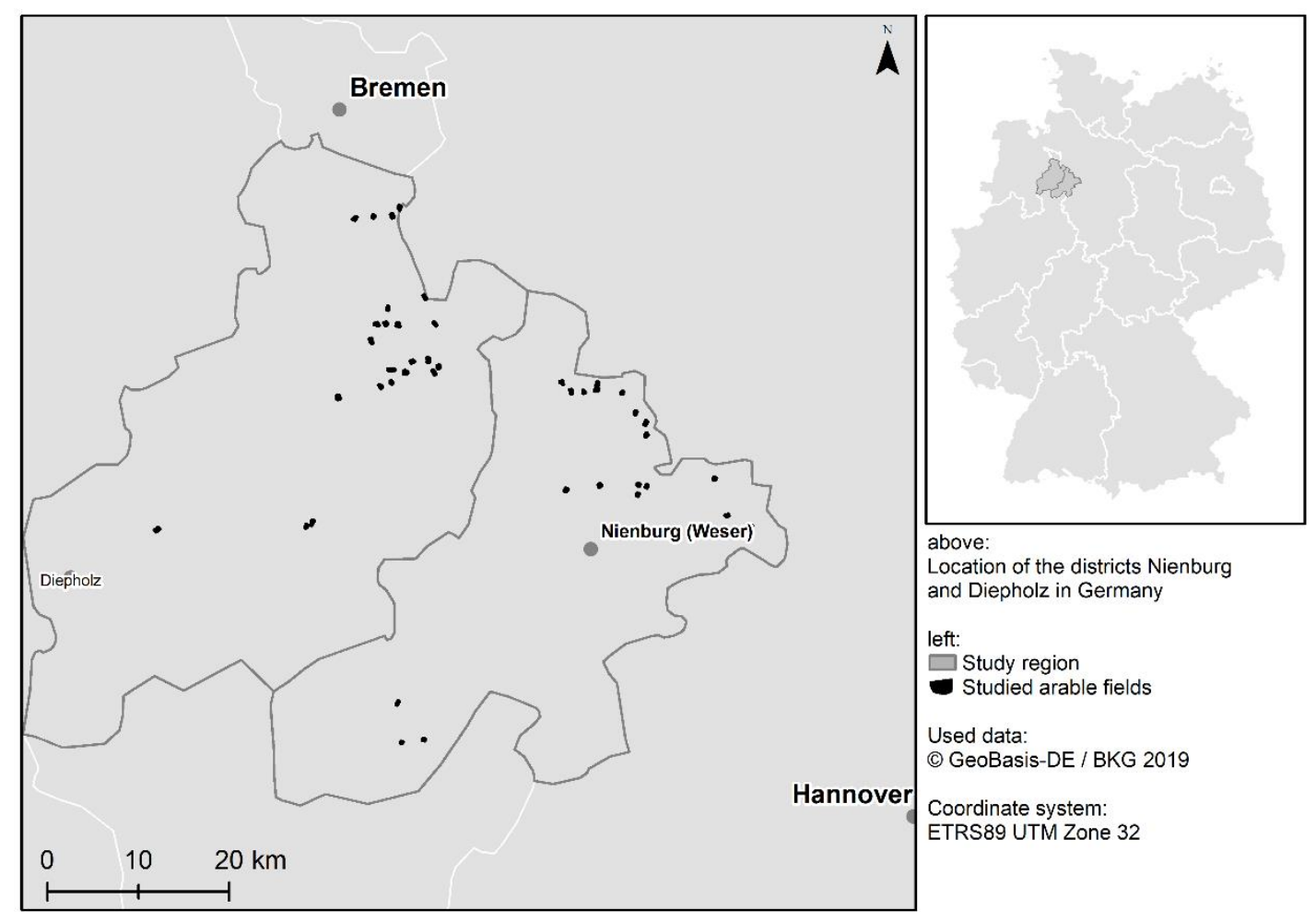

Online Resource 6 Map of the study region (the two districts of Nienburg and Diepholz in Lower Saxony, Northwest Germany). 
Online Resource 7 Frequency (absolute number) of all herbaceous species per survey method (crops and woody seedlings excluded; all crops pooled); Total $=$ species occurrences summed up for all survey methods and total field perimeter count); table is sorted by decreasing total species occurrences; survey methods: Interior $(50 \times 2 \mathrm{~m})$, Edge_30 $(30 \times 2 \mathrm{~m})$, Edge_50 $(50 \times 2 \mathrm{~m})$, Subplot (four plots of $5 \times 1 \mathrm{~m})$, Corner $(50 \times 2 \mathrm{~m})$ and Edge_500 $(500 \times 1 \mathrm{~m})$; arable plants (sensu stricto, Hofmeister and Garve, 2006), indicator species for High Nature Value arable land (HNV species; German Federal Agency for Nature Conservation, 2018) and red-listed species for Lower Saxony (lost, endangered and early warning stage; Garve, 2004) are marked with ' $\mathrm{x}$ ' in specific columns; $\mathrm{n}=45$ (observed fields); full references see main manuscript.

\begin{tabular}{|c|c|c|c|c|c|c|c|c|c|c|}
\hline Species & 节 & 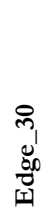 & $\begin{array}{l}8 \\
0 \\
5 \\
50 \\
5 \\
1\end{array}$ & 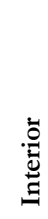 & $\begin{array}{l}\frac{0}{0} \\
\frac{0}{0} \\
\frac{0}{b}\end{array}$ & 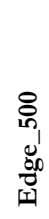 & & 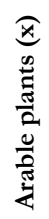 & $\underset{\text { 齐 }}{\overparen{Z}}$ & 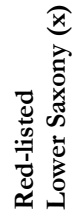 \\
\hline Elymus repens & 42 & 27 & 28 & 4 & 38 & 42 & 45 & $\mathrm{x}$ & & \\
\hline Chenopodium album & 24 & 17 & 18 & 13 & 21 & 35 & 44 & $\mathrm{x}$ & & \\
\hline Polygonum aviculare & 31 & 20 & 21 & 17 & 27 & 39 & 44 & $\mathrm{x}$ & & \\
\hline Fallopia convolvulus & 27 & 16 & 16 & 12 & 17 & 35 & 42 & $\mathrm{x}$ & & \\
\hline Galium aparine & 33 & 18 & 20 & 1 & 24 & 33 & 42 & $\mathrm{x}$ & & \\
\hline Matricaria chamomilla & 32 & 21 & 21 & 13 & 20 & 35 & 42 & $\mathrm{x}$ & $\mathrm{x}$ & \\
\hline Bromus sterilis & 33 & 25 & 27 & 0 & 29 & 39 & 41 & $\mathrm{x}$ & & \\
\hline Viola arvensis & 28 & 19 & 19 & 23 & 24 & 33 & 41 & $\mathrm{x}$ & & \\
\hline Cirsium arvense & 17 & 13 & 15 & 4 & 15 & 33 & 40 & $\mathrm{x}$ & & \\
\hline Dactylis glomerata & 29 & 14 & 15 & 0 & 15 & 29 & 40 & & & \\
\hline Geranium pusillum & 27 & 20 & 24 & 8 & 26 & 31 & 39 & $\mathrm{x}$ & $\mathrm{x}$ & \\
\hline Stellaria media & 16 & 10 & 11 & 13 & 14 & 34 & 38 & $\mathrm{x}$ & & \\
\hline Capsella bursa-pastoris & 12 & 5 & 5 & 4 & 4 & 31 & 37 & $\mathrm{x}$ & & \\
\hline Myosotis arvensis & 22 & 10 & 12 & 4 & 14 & 27 & 35 & $\mathrm{x}$ & $\mathrm{x}$ & \\
\hline Poa trivialis & 29 & 9 & 9 & 2 & 21 & 25 & 35 & $\mathrm{x}$ & & \\
\hline Urtica dioica & 24 & 13 & 15 & 2 & 16 & 27 & 35 & & & \\
\hline Lolium perenne & 26 & 4 & 4 & 1 & 11 & 22 & 33 & $\mathrm{x}$ & & \\
\hline Holcus mollis & 12 & 4 & 4 & 0 & 11 & 26 & 32 & $\mathrm{x}$ & & \\
\hline Vicia birsuta & 14 & 6 & 7 & 3 & 13 & 30 & 32 & $\mathrm{x}$ & $\mathrm{x}$ & \\
\hline Galeopsis tetrahit & 11 & 6 & 6 & 2 & 6 & 22 & 30 & $\mathrm{x}$ & & \\
\hline Sisymbrium officinale & 16 & 7 & 8 & 6 & 12 & 24 & 30 & $\mathrm{x}$ & & \\
\hline Bromus hordeaceus & 9 & 4 & 5 & 0 & 7 & 20 & 28 & $\mathrm{x}$ & & \\
\hline Роа аппиа & 13 & 3 & 3 & 3 & 5 & 18 & 28 & $\mathrm{x}$ & & \\
\hline Rumex obtusifolius & 11 & 3 & 4 & 1 & 7 & 23 & 28 & $\mathrm{x}$ & & \\
\hline Agrostis stolonifera & 11 & 0 & 0 & 0 & 4 & 20 & 27 & $\mathrm{x}$ & & \\
\hline Apera spica venti & 14 & 7 & 9 & 2 & 9 & 19 & 26 & $\mathrm{x}$ & & \\
\hline Ecbinocbloa crus-galli & 16 & 8 & 8 & 3 & 11 & 24 & 26 & $\mathrm{x}$ & & \\
\hline Holcus lanatus & 13 & 6 & 6 & 0 & 6 & 12 & 26 & & & \\
\hline Persicaria maculosa & 7 & 1 & 2 & 3 & 3 & 20 & 26 & $\mathrm{x}$ & & \\
\hline Vicia sativa & 9 & 3 & 4 & 0 & 4 & 20 & 26 & & $\mathrm{x}$ & \\
\hline Tripleurospermum perforatum & 8 & 0 & 0 & 0 & 6 & 17 & 24 & $\mathrm{x}$ & & \\
\hline Antbriscus syllestris & 12 & 8 & 8 & 0 & 10 & 16 & 23 & & & \\
\hline Bromus commutatus subsp. decipiens & 13 & 5 & 5 & 1 & 10 & 13 & 22 & $\mathrm{x}$ & & \\
\hline Equisetum arvense & 5 & 4 & 4 & 1 & 5 & 13 & 22 & $\mathrm{x}$ & & \\
\hline Festuca rubra & 7 & 2 & 2 & 0 & 10 & 10 & 22 & & & \\
\hline Lapsana communis & 8 & 0 & 1 & 0 & 3 & 13 & 21 & $\mathrm{x}$ & $\mathrm{x}$ & \\
\hline Arrbenatherum elatius & 13 & 10 & 10 & 0 & 9 & 8 & 20 & & & \\
\hline Avena fatua & 6 & 3 & 3 & 1 & 5 & 13 & 19 & $\mathrm{x}$ & & \\
\hline Sonchus asper & 6 & 1 & 1 & 0 & 1 & 9 & 19 & $\mathrm{x}$ & & \\
\hline Alopecurus pratensis & 8 & 6 & 6 & 0 & 4 & 7 & 18 & & & \\
\hline Lolium multiflorum & 8 & 7 & 7 & 1 & 4 & 12 & 18 & $\mathrm{x}$ & & \\
\hline Rumex crispus & 5 & 3 & 3 & 0 & 2 & 15 & 18 & $\mathrm{x}$ & & \\
\hline Papaver dubium agg. & 2 & 0 & 0 & 0 & 1 & 10 & 17 & $\mathrm{x}$ & $\mathrm{x}$ & \\
\hline Erigeron canadensis & 3 & 1 & 1 & 0 & 1 & 13 & 16 & $\mathrm{x}$ & & \\
\hline Glechoma bederacea & 3 & 4 & 5 & 1 & 5 & 8 & 16 & $\mathrm{x}$ & & \\
\hline Tanacetum vulgare & 7 & 4 & 4 & 0 & 4 & 10 & 16 & & & \\
\hline Achillea millefolium & 8 & 4 & 4 & 0 & 8 & 7 & 15 & $\mathrm{x}$ & & \\
\hline Centaurea cyanus & 7 & 4 & 4 & 1 & 1 & 10 & 15 & $\mathrm{x}$ & $\mathrm{x}$ & \\
\hline Solanum nigrum & 4 & 1 & 1 & 0 & 3 & 8 & 15 & $\mathrm{x}$ & & \\
\hline
\end{tabular}


Species

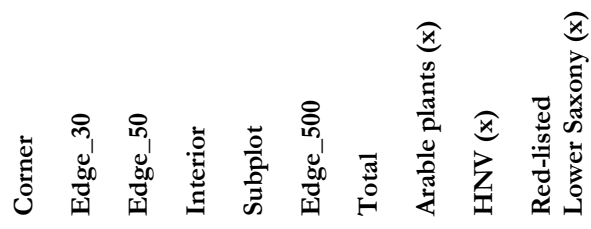

\begin{tabular}{lllllllllll}
\hline Taraxacum sect. Ruderalia & 7 & 1 & 1 & 0 & 2 & 7 & 15 & $\mathrm{x}$
\end{tabular}

$\begin{array}{lllllllll}\text { Artemisia vulgaris } & 3 & 1 & 1 & 0 & 2 & 9 & 14 & \mathrm{x} \\ \text { Chenopodium polyspermum } & 2 & 0 & 0 & 0 & 2 & 5 & 14 & \mathrm{x}\end{array}$

$\begin{array}{llllllll}\text { Chenopodium polyspermum } & 2 & 0 & 0 & 0 & 2 & 5 & 14 \\ \text { Cirsium vulgare } & 1 & 0 & 1 & 0 & 3 & 8 & 14\end{array}$

$\begin{array}{lllllllll}\text { Veronica arvensis } & 3 & 2 & 2 & 0 & 2 & 3 & 14 & \mathrm{x}\end{array}$

$\begin{array}{lllllllll}\text { Alopecurus myosuroides } & 6 & 3 & 3 & 1 & 3 & 12 & 13 & \mathrm{x}\end{array}$

$\begin{array}{lllllllll}\text { Gnaphalium uliginosum } & 2 & 0 & 0 & 0 & 1 & 8 & 13 & \mathrm{x}\end{array}$

$\begin{array}{llllllllll}\text { Lamium purpureum } & 1 & 2 & 2 & 0 & 1 & 8 & 13 & \mathrm{x} & \mathrm{x}\end{array}$

$\begin{array}{lllllllll}\text { Sonchus oleraceus } & 3 & 2 & 3 & 1 & 1 & 5 & 13 & \mathrm{x}\end{array}$

$\begin{array}{llllllll}\text { Trifolium repens } & 4 & 2 & 2 & 0 & 3 & 9 & 13\end{array}$

$\begin{array}{llllllll}\text { Agrostis capillaris } & 5 & 3 & 3 & 0 & 4 & 6 & 12\end{array}$

$\begin{array}{llllllll}\text { Heracleum sphondylium } & 4 & 5 & 6 & 0 & 6 & 4 & 12\end{array}$

$\begin{array}{lllllllll}\text { Persicaria amphibia } & 3 & 0 & 1 & 0 & 1 & 9 & 12 & \mathrm{x}\end{array}$

$\begin{array}{lllllllll}\text { Plantago major } & 2 & 0 & 0 & 0 & 2 & 10 & 12 & \mathrm{x}\end{array}$

$\begin{array}{llllllllll}\text { Cerastium bolosteoides } & 3 & 1 & 1 & 0 & 0 & 5 & 11 & \mathrm{x}\end{array}$

$\begin{array}{lllllllllll}\text { Vicia tetrasperma } & 2 & 1 & 1 & 0 & 1 & 9 & 11 & \mathrm{x} & \mathrm{x}\end{array}$

$\begin{array}{lllllllll}\text { Atriplex patula } & 2 & 1 & 1 & 2 & 2 & 8 & 10 & \mathrm{x}\end{array}$

$\begin{array}{lllllllll}\text { Juncus bufonius } & 1 & 1 & 1 & 1 & 0 & 5 & 10 & \mathrm{x}\end{array}$

$\begin{array}{llllllllll}\text { Plantago lanceolata } & 0 & 1 & 1 & 0 & 2 & 5 & 10 & \mathrm{x}\end{array}$

$\begin{array}{lllllllll}\text { Ranunculus repens } & 1 & 1 & 1 & 0 & 4 & 5 & 10 & \mathrm{x}\end{array}$

$\begin{array}{llllllll}\text { Rubus fruticosus agg. } & 4 & 1 & 1 & 0 & 3 & 3 & 10\end{array}$

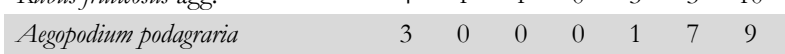

$\begin{array}{lllllllll}\text { Persicaria bydropiper } & 3 & 0 & 0 & 0 & 2 & 6 & 9 & \mathrm{x}\end{array}$

$\begin{array}{lllllllll}\text { Setaria viridis } & 4 & 1 & 1 & 3 & 3 & 7 & 9 & \mathrm{x}\end{array}$

$\begin{array}{lllllllll}\text { Veronica persica } & 3 & 0 & 0 & 0 & 5 & 5 & 9 & \mathrm{x}\end{array}$

$\begin{array}{llllllllllll}\text { Vicia angustifolia } & 3 & 0 & 0 & 0 & 0 & 2 & 9 & \mathrm{x} & \mathrm{x}\end{array}$

$\begin{array}{llllllll}\text { Carduus crispus } & 1 & 1 & 2 & 0 & 1 & 3 & 8\end{array}$

$\begin{array}{llllllll}\text { Daucus carota } & 2 & 3 & 3 & 0 & 0 & 4 & 8\end{array}$

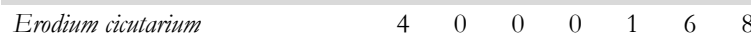

$\begin{array}{llllllll}\text { Poapratensis } & 2 & 1 & 1 & 0 & 2 & 4 & 8\end{array}$

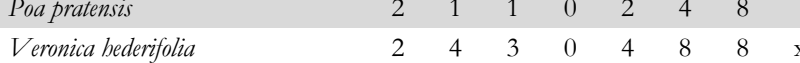

$\begin{array}{lllllllll}\text { Epilobium tetragonum } & 0 & 1 & 1 & 0 & 1 & 5 & 7 & \mathrm{x}\end{array}$

$\begin{array}{llllllllll}\text { Geranium dissectum } & 4 & 3 & 4 & 0 & 3 & 5 & 7 & \mathrm{x} & \mathrm{x}\end{array}$

$\begin{array}{lllllllllll}\text { Geranium molle } & 2 & 2 & 2 & 0 & 1 & 5 & 7 & \mathrm{x} & \mathrm{x}\end{array}$

$\begin{array}{llllllllll}\text { Papaver rhoeas } & 0 & 1 & 1 & 0 & 0 & 6 & 7 & \mathrm{x} & \mathrm{x}\end{array}$

\begin{tabular}{lllllllllll} 
Stellaria graminea & 1 & 2 & 2 & 1 & 1 & 3 & 7 & $\mathrm{x}$ & \\
Alliaria petiolata & 0 & 1 & 1 & 0 & 0 & 4 & 6 & & \\
Alopecurus geniculatus & 0 & 0 & 0 & 0 & 0 & 5 & 6 & & \\
Aphanes arvensis & 2 & 0 & 0 & 0 & 1 & 1 & 6 & $\mathrm{x}$ & $\mathrm{x}$ \\
\hline Arabidopsis thaliana & 1 & 0 & 0 & 0 & 0 & 2 & 6 & $\mathrm{x}$ & \\
Digitaria ischaemum & 4 & 2 & 2 & 0 & 3 & 4 & 6 & $\mathrm{x}$ & \\
Equisetum palustre & 2 & 2 & 2 & 0 & 2 & 4 & 6 & & \\
Hypericum perforatum & 2 & 0 & 0 & 0 & 2 & 5 & 6 & & \\
Lactuca serriola & 2 & 0 & 0 & 0 & 0 & 4 & 6 & $\mathrm{x}$ & \\
Phalaris arundinacea & 1 & 0 & 0 & 0 & 0 & 4 & 6 & & \\
Phragmites australis & 0 & 0 & 0 & 0 & 0 & 3 & 6 & $\mathrm{x}$ & \\
Rumex acetosa & 1 & 1 & 1 & 0 & 0 & 3 & 6 & & \\
Senecio vulgaris & 0 & 0 & 0 & 0 & 0 & 4 & 6 & $\mathrm{x}$ & \\
Sinapis arvensis & 1 & 0 & 0 & 0 & 0 & 4 & 6 & $\mathrm{x}$ & \\
Anchusa arvensis & 0 & 0 & 0 & 0 & 0 & 4 & 5 & $\mathrm{x}$ & \\
Bromus secalinus & 1 & 1 & 1 & 0 & 1 & 3 & 5 & $\mathrm{x}$ & \\
Cerastium glomeratum & 1 & 2 & 2 & 0 & 1 & 2 & 5 & $\mathrm{x}$ & \\
Chaerophyllum temulum & 2 & 0 & 0 & 0 & 0 & 4 & 5 & & \\
& & & & & & & \\
& & & & & & &
\end{tabular}


Species

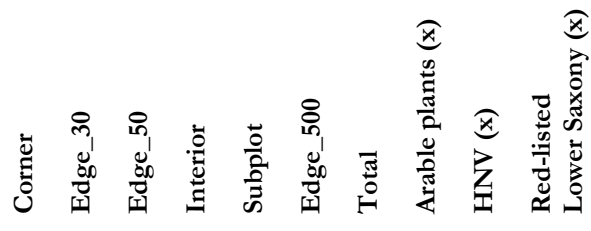

$\begin{array}{llllllllll}\text { Convolvulus arvensis } & 1 & 1 & 2 & 0 & 3 & 3 & 5 & \mathrm{x}\end{array}$

$\begin{array}{lllllllll}\text { Galinsoga parviflora } & 2 & 0 & 0 & 0 & 0 & 5 & 5 & \mathrm{x}\end{array}$

$\begin{array}{lllllllll}\text { Matricaria discoidea } & 0 & 0 & 0 & 0 & 0 & 4 & 5 & \mathrm{x}\end{array}$

$\begin{array}{llllllllllll}\text { Rumex acetosella } & 1 & 0 & 0 & 0 & 0 & 4 & 5 & \mathrm{x} & \mathrm{x} & \end{array}$

$\begin{array}{lllllllll}\text { Silene latifolia } & 0 & 0 & 2 & 0 & 0 & 2 & 5 & \mathrm{x}\end{array}$

$\begin{array}{lllllllllll}\text { Vicia villosa } & 1 & 1 & 1 & 0 & 0 & 4 & 5 & \mathrm{x} & \mathrm{x} & \end{array}$

Aethusa cynapium $\quad \begin{array}{lllllllll}0 & 0 & 0 & 0 & 0 & 1 & 4 & \mathrm{x}\end{array}$

$\begin{array}{llllllllll}\text { Carex birta } & 2 & 1 & 1 & 0 & 0 & 3 & 4 & \mathrm{x}\end{array}$

$\begin{array}{llllllllll}\text { Fumaria officinalis } & 0 & 3 & 3 & 0 & 3 & 3 & 4 & \mathrm{x} & \mathrm{x}\end{array}$

$\begin{array}{llllllll}\text { Geum urbanum } & 3 & 0 & 0 & 0 & 0 & 1 & 4\end{array}$

$\begin{array}{lllllllll}\text { Linaria vulgaris } & 0 & 1 & 1 & 0 & 0 & 3 & 4 & \mathrm{x}\end{array}$

Rorippapalustris $\quad 00 \begin{array}{llllllll} & 0 & 0 & 0 & 0 & 3 & 4 & \mathrm{x}\end{array}$

$\begin{array}{llllllllll}\text { Spergula arvensis } & 1 & 0 & 0 & 0 & 1 & 2 & 4 & \mathrm{x} & \mathrm{x}\end{array}$

$\begin{array}{llllllllll}\text { Stachys palustris } & 1 & 1 & 1 & 0 & 1 & 2 & 4 & \mathrm{x} & \end{array}$

$\begin{array}{llllllllll}\text { Vicia cracca } & 1 & 1 & 1 & 0 & 0 & 2 & 4 & \mathrm{x} & \mathrm{x}\end{array}$

$\begin{array}{llllllll}\text { Vulpia myuros } & 3 & 2 & 2 & 1 & 1 & 2 & 4\end{array}$

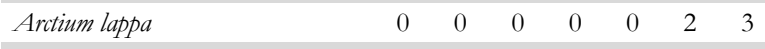

Chaerophyllum bulbosum $\quad \begin{array}{llllllll}0 & 0 & 0 & 0 & 0 & 2 & 3\end{array}$

$\begin{array}{llllllll}\text { Chelidonium majus } & 0 & 0 & 0 & 0 & 0 & 2 & 3\end{array}$

Galium mollugo agg. $\quad \begin{array}{lllllll}1 & 2 & 2 & 0 & 1 & 3 & 3\end{array}$

$\begin{array}{llllllll}\text { Lysimachia vulgaris } & 0 & 0 & 0 & 0 & 0 & 0 & 3\end{array}$

$\begin{array}{lllllllllll}\text { Odontites vernus } & 2 & 1 & 1 & 0 & 1 & 2 & 3 & \mathrm{x} & & \mathrm{x}\end{array}$

$\begin{array}{llllllllll}\text { Potentilla reptans } & 1 & 1 & 1 & 0 & 0 & 3 & 3 & \mathrm{x} & \end{array}$

Rubus idaeus agg. $\quad \begin{array}{lllllll}1 & 0 & 0 & 0 & 0 & 2 & 3\end{array}$

$\begin{array}{llllllll}\text { Stellaria bolostea } & 0 & 0 & 0 & 0 & 1 & 1 & 3\end{array}$

$\begin{array}{llllllllll}\text { Thlaspi arvense } & 0 & 0 & 1 & 0 & 1 & 2 & 3 & \mathrm{x} & \mathrm{x}\end{array}$

$\begin{array}{llllllll}\text { Trisetum flavescens } & 1 & 2 & 2 & 0 & 1 & 1 & 3\end{array}$

$\begin{array}{lllllllll}\text { Amaranthus retroflexus } & 1 & 0 & 0 & 0 & 0 & 1 & 2 & \mathrm{x}\end{array}$

$\begin{array}{llllllllll}\text { Arenaria serpyllifolia } & 0 & 1 & 1 & 0 & 0 & 2 & 2 & \mathrm{x}\end{array}$

$\begin{array}{llllllll}\text { Calystegia sepium } & 0 & 1 & 1 & 1 & 0 & 2 & 2\end{array}$

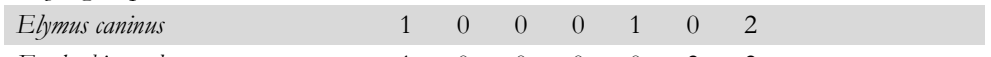

Euphorbia peplus $\quad \begin{array}{lllllllll}1 & 0 & 0 & 0 & 0 & 2 & 2 & \mathrm{x} & \mathrm{x}\end{array}$

$\begin{array}{llllllll}\text { Festuca pratensis } & 1 & 0 & 0 & 0 & 0 & 2 & 2\end{array}$

$\begin{array}{lllllllll}\text { Galinsoga quadriradiata } & 0 & 0 & 0 & 0 & 0 & 2 & 2 & \mathrm{x}\end{array}$

$\begin{array}{llllllll}\text { Humulus lupulus } & 1 & 0 & 0 & 0 & 2 & 0 & 2\end{array}$

$\begin{array}{llllllll}\text { Lotus corniculatus } & 0 & 0 & 0 & 0 & 0 & 0 & 2\end{array}$

$\begin{array}{lllllllllll}\text { Papaver argemone } & 1 & 0 & 0 & 0 & 0 & 1 & 2 & \mathrm{x} & \mathrm{x}\end{array}$

Poa compressa $\quad \begin{array}{lllllll}0 & 0 & 0 & 0 & 0 & 1 & 2\end{array}$

$\begin{array}{llllllllll}\text { Potentilla anserina } & 1 & 1 & 1 & 0 & 1 & 2 & 2 & \mathrm{x}\end{array}$

$\begin{array}{llllllll}\text { Pteridium aquilinum } & 0 & 0 & 0 & 0 & 0 & 2 & 2\end{array}$

$\begin{array}{lllllllll}\text { Scleranthus annuиs } & 1 & 0 & 0 & 0 & 0 & 2 & 2 & \mathrm{x}\end{array}$

$\begin{array}{llllllll}\text { Silene dioica } & 1 & 1 & 1 & 0 & 1 & 2 & 2\end{array}$

$\begin{array}{llllllll}\text { Solidago canadensis } & 0 & 0 & 0 & 0 & 0 & 1 & 2\end{array}$

$\begin{array}{lllllllll}\text { Symphytum officinale } & 1 & 0 & 0 & 0 & 1 & 1 & 2 & \mathrm{x}\end{array}$

$\begin{array}{llllllllllll}\text { Agrostemma githago } & 0 & 0 & 0 & 0 & 0 & 0 & 1 & \mathrm{x} & & \mathrm{x}\end{array}$

$\begin{array}{llllllllll}\text { Allium vineale } & 0 & 0 & 0 & 0 & 0 & 1 & 1 & \mathrm{x}\end{array}$

$\begin{array}{llllllllllll}\text { Anthemis arvensis } & 1 & 0 & 0 & 0 & 0 & 0 & 1 & \mathrm{x} & \mathrm{x} & & \mathrm{x}\end{array}$

$\begin{array}{llllllllll}\text { Anthoxanthum aristatum } & 0 & 0 & 0 & 0 & 0 & 1 & 1 & \mathrm{x}\end{array}$

$\begin{array}{lllllllll}\text { Anthriscus caucalis } & 1 & 0 & 0 & 0 & 0 & 0 & 1 & \mathrm{x}\end{array}$

Bromus inermis

$\begin{array}{llllllll}\text { Callitriche palustris agg. } & 0 & 0 & 0 & 0 & 0 & 0 & 1\end{array}$

$\begin{array}{llllllllll}\text { Cardamine hirsuta } & 0 & 0 & 0 & 0 & 0 & 1 & 1 & \mathrm{x}\end{array}$

$\begin{array}{llllllll}\text { Carduus nutans } & 0 & 0 & 0 & 0 & 0 & 1 & 1\end{array}$

$\begin{array}{llllllll}\text { Centaurea jacea } & 1 & 0 & 0 & 0 & 0 & 1 & 1\end{array}$

$\begin{array}{lllllllllll}\text { Glebionis segetum } & 0 & 0 & 0 & 0 & 0 & 1 & 1 & \mathrm{x} & \mathrm{x}\end{array}$ 


\begin{tabular}{|c|c|c|c|c|c|c|c|c|c|c|}
\hline Species & 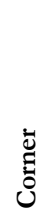 & 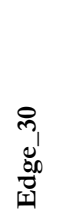 & 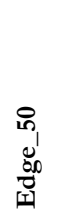 & 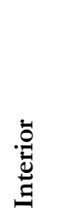 & $\begin{array}{l}\frac{\overrightarrow{0}}{0} \\
\frac{0}{0} \\
\vec{b}\end{array}$ & 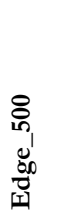 & تّٓँّ & $\begin{array}{l}\sqrt{x} \\
\frac{0}{5} \\
\frac{5}{2} \\
\frac{0}{0} \\
\frac{0}{0} \\
\frac{\pi}{4}\end{array}$ & 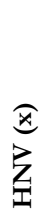 & 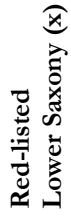 \\
\hline Digitaria sanguinalis & 1 & 1 & 1 & 0 & 1 & 1 & 1 & $\mathrm{x}$ & & \\
\hline Epilobium angustifolium & 0 & 0 & 0 & 0 & 0 & 1 & 1 & & & \\
\hline Epilobium birsutum & 0 & 0 & 0 & 0 & 0 & 1 & 1 & & & \\
\hline Epilobium montanum & 0 & 0 & 0 & 0 & 0 & 0 & 1 & & & \\
\hline Epilobium parviflorum & 0 & 0 & 0 & 0 & 0 & 1 & 1 & & & \\
\hline Equisetum sylvaticum & 0 & 0 & 0 & 0 & 0 & 1 & 1 & & & \\
\hline Erigeron annuus & 0 & 0 & 0 & 0 & 0 & 1 & 1 & & & \\
\hline Erysimum cheiranthoides & 1 & 0 & 0 & 0 & 0 & 1 & 1 & $\mathrm{x}$ & & \\
\hline Euphorbia esula & 0 & 0 & 0 & 0 & 0 & 1 & 1 & & $\mathrm{x}$ & \\
\hline Euphorbia belioscopia & 1 & 0 & 0 & 0 & 0 & 1 & 1 & $\mathrm{x}$ & $\mathrm{x}$ & \\
\hline Festuca arundinacea & 0 & 0 & 0 & 0 & 0 & 1 & 1 & & & \\
\hline Filago arvensis & 0 & 0 & 0 & 0 & 0 & 0 & 1 & $\mathrm{x}$ & & \\
\hline Geranium robertianum & 0 & 0 & 0 & 0 & 0 & 1 & 1 & & $\mathrm{x}$ & \\
\hline Geranium rotundifolium & 1 & 0 & 0 & 0 & 1 & 0 & 1 & $\mathrm{x}$ & $\mathrm{x}$ & \\
\hline Hypericum maculatum & 0 & 0 & 0 & 0 & 0 & 0 & 1 & & & \\
\hline Hypochaeris radicata & 0 & 0 & 0 & 0 & 0 & 1 & 1 & & & \\
\hline Impatiens glandulifera & 1 & 0 & 0 & 0 & 0 & 1 & 1 & & & \\
\hline Juncus effusus & 0 & 0 & 0 & 0 & 0 & 0 & 1 & & & \\
\hline Lamium amplexicaule & 1 & 0 & 0 & 0 & 0 & 1 & 1 & $\mathrm{x}$ & $\mathrm{x}$ & \\
\hline Scorzoneroides autumnalis & 0 & 0 & 0 & 0 & 0 & 1 & 1 & & & \\
\hline Buglossoides arvensis & 0 & 0 & 0 & 0 & 0 & 1 & 1 & $\mathrm{x}$ & $\mathrm{x}$ & $\mathrm{x}$ \\
\hline Lupinus polyphyllus & 0 & 0 & 0 & 0 & 0 & 0 & 1 & & & \\
\hline Lythrum salicaria & 0 & 0 & 0 & 0 & 0 & 1 & 1 & & $\mathrm{x}$ & \\
\hline Medicago lupulina & 0 & 0 & 0 & 0 & 0 & 0 & 1 & $\mathrm{x}$ & & \\
\hline Mentha arvensis & 0 & 0 & 0 & 0 & 0 & 0 & 1 & $\mathrm{x}$ & & \\
\hline Ornithopus perpusillus & 0 & 0 & 0 & 0 & 0 & 1 & 1 & $\mathrm{x}$ & $\mathrm{x}$ & \\
\hline Oxalis stricta & 0 & 0 & 0 & 0 & 0 & 1 & 1 & & & \\
\hline Persicaria mitis & 1 & 0 & 0 & 0 & 1 & 0 & 1 & & & \\
\hline Ranunculus acris & 0 & 0 & 0 & 0 & 0 & 1 & 1 & & & \\
\hline Rumex bydrolapatbum & 1 & 0 & 0 & 0 & 0 & 0 & 1 & & & \\
\hline Senecio vernalis & 0 & 1 & 0 & 0 & 0 & 1 & 1 & $\mathrm{x}$ & & \\
\hline Sonchus arvensis & 0 & 0 & 0 & 0 & 0 & 0 & 1 & $\mathrm{x}$ & & \\
\hline Trifolium bybridum & 0 & 0 & 0 & 0 & 0 & 0 & 1 & & & \\
\hline Trifolium pratense & 0 & 0 & 0 & 0 & 0 & 0 & 1 & & & \\
\hline Typha latifolia & 0 & 1 & 1 & 0 & 0 & 0 & 1 & & & \\
\hline Urtica urens & 0 & 0 & 0 & 0 & 1 & 0 & 1 & $\mathrm{x}$ & & \\
\hline
\end{tabular}


Online Resource 8 Frequency (absolute number) of herbaceous species per crop (crops and woody seedlings excluded; all crops pooled); Total $=$ species occurrences summed up for all survey methods and total perimeter count; table is sorted by decreasing total species occurrences; arable plants (sensu stricto, Hofmeister and Garve, 2006), indicator species for High Nature Value arable land (HNV species; German Federal Agency for Nature Conservation, 2018) and red-listed species for Lower Saxony (lost, endangered and early warning stage; Garve, 2004) are marked with ' $\mathrm{x}$ ' in specific columns; $n=45$ (observed fields); full references see main manuscript.

\begin{tabular}{|c|c|c|c|c|c|c|c|}
\hline Species & Maize & Rapeseed & Wheat & Total & $\begin{array}{l}\text { Arable plants } \\
\text { (x) }\end{array}$ & $\begin{array}{l}\text { HNV } \\
\text { (x) }\end{array}$ & $\begin{array}{c}\text { Red-listed } \\
\text { Lower Saxony } \\
(\mathbf{x}) \\
\end{array}$ \\
\hline Elymus repens & 15 & 15 & 15 & 45 & $\mathrm{x}$ & & \\
\hline Chenopodium album & 15 & 14 & 15 & 44 & $\mathrm{x}$ & & \\
\hline Polygonum aviculare & 14 & 15 & 15 & 44 & $\mathrm{x}$ & & \\
\hline Matricaria chamomilla & 12 & 15 & 15 & 42 & $\mathrm{x}$ & $\mathrm{x}$ & \\
\hline Fallopia convolvulus & 14 & 14 & 14 & 42 & $\mathrm{x}$ & & \\
\hline Galium aparine & 14 & 13 & 15 & 42 & $\mathrm{x}$ & & \\
\hline Bromus sterilis & 12 & 14 & 15 & 41 & $\mathrm{x}$ & & \\
\hline Viola arvensis & 12 & 15 & 14 & 41 & $\mathrm{x}$ & & \\
\hline Cirsium arvense & 11 & 14 & 15 & 40 & $\mathrm{x}$ & & \\
\hline Dactylis glomerata & 13 & 14 & 13 & 40 & & & \\
\hline Geranium pusillum & 15 & 15 & 9 & 39 & $\mathrm{x}$ & $\mathrm{x}$ & \\
\hline Stellaria media & 12 & 15 & 11 & 38 & $\mathrm{x}$ & & \\
\hline Capsella bursa-pastoris & 11 & 14 & 12 & 37 & $\mathrm{x}$ & & \\
\hline Myosotis arvensis & 6 & 15 & 14 & 35 & $\mathrm{x}$ & $\mathrm{x}$ & \\
\hline Poa trivialis & 10 & 10 & 15 & 35 & $\mathrm{x}$ & & \\
\hline Urtica dioica & 11 & 11 & 13 & 35 & & & \\
\hline Lolium perenne & 11 & 12 & 10 & 33 & $\mathrm{x}$ & & \\
\hline Vicia birsuta & 8 & 14 & 10 & 32 & $\mathrm{x}$ & $\mathrm{x}$ & \\
\hline Holcus mollis & 10 & 11 & 11 & 32 & $\mathrm{x}$ & & \\
\hline Galeopsis tetrabit & 10 & 10 & 10 & 30 & $\mathrm{x}$ & & \\
\hline Sisymbrium officinale & 6 & 14 & 10 & 30 & $\mathrm{x}$ & & \\
\hline Bromus hordeaceus & 7 & 9 & 12 & 28 & $\mathrm{x}$ & & \\
\hline Pоа аппиа & 10 & 7 & 11 & 28 & $\mathrm{x}$ & & \\
\hline Rumex obtusifolius & 7 & 11 & 10 & 28 & $\mathrm{x}$ & & \\
\hline Agrostis stolonifera & 8 & 9 & 10 & 27 & $\mathrm{x}$ & & \\
\hline Vicia sativa & 4 & 13 & 9 & 26 & & $\mathrm{x}$ & \\
\hline Apera spica venti & 2 & 13 & 11 & 26 & $\mathrm{x}$ & & \\
\hline Echinochloa crus-galli & 15 & 5 & 6 & 26 & $\mathrm{x}$ & & \\
\hline Holcus lanatus & 6 & 10 & 10 & 26 & & & \\
\hline Persicaria maculosa & 12 & 8 & 6 & 26 & $\mathrm{x}$ & & \\
\hline Tripleurospermum perforatum & 7 & 9 & 8 & 24 & $\mathrm{x}$ & & \\
\hline Anthriscus sylvestris & 3 & 9 & 11 & 23 & & & \\
\hline Bromus commutatus subsp. decipiens & 3 & 8 & 11 & 22 & $\mathrm{x}$ & & \\
\hline Equisetum arvense & 6 & 10 & 6 & 22 & $\mathrm{x}$ & & \\
\hline Festuca rubra & 6 & 7 & 9 & 22 & & & \\
\hline Lapsana communis & 4 & 8 & 9 & 21 & $\mathrm{x}$ & $\mathrm{x}$ & \\
\hline Arrhenatherum elatius & 5 & 8 & 7 & 20 & & & \\
\hline Avena fatua & 6 & 6 & 7 & 19 & $\mathrm{x}$ & & \\
\hline Sonchus asper & 6 & 8 & 5 & 19 & $\mathrm{x}$ & & \\
\hline Alopecurus pratensis & 4 & 8 & 6 & 18 & & & \\
\hline Lolium multiflorum & 5 & 8 & 5 & 18 & $\mathrm{x}$ & & \\
\hline Rumex crispus & 0 & 10 & 8 & 18 & $\mathrm{x}$ & & \\
\hline Papaver dubium agg. & 4 & 10 & 3 & 17 & $\mathrm{x}$ & $\mathrm{x}$ & \\
\hline Erigeron canadensis & 5 & 5 & 6 & 16 & $\mathrm{x}$ & & \\
\hline Glechoma hederacea & 5 & 6 & 5 & 16 & $\mathrm{x}$ & & \\
\hline Tanacetum vulgare & 4 & 6 & 6 & 16 & & & \\
\hline Centaurea cyanus & 2 & 8 & 5 & 15 & $\mathrm{x}$ & $\mathrm{x}$ & \\
\hline Achillea millefolium & 7 & 4 & 4 & 15 & $\mathrm{x}$ & & \\
\hline Solanum nigrum & 11 & 2 & 2 & 15 & $\mathrm{x}$ & & \\
\hline Taraxacum sect. Ruderalia & 6 & 2 & 7 & 15 & $\mathrm{x}$ & & \\
\hline Artemisia vulgaris & 7 & 4 & 3 & 14 & $\mathrm{x}$ & & \\
\hline Chenopodium polyspermum & 7 & 5 & 2 & 14 & $\mathrm{x}$ & & \\
\hline Cirsium vulgare & 0 & 7 & 7 & 14 & & & \\
\hline Veronica arvensis & 2 & 6 & 6 & 14 & $\mathrm{x}$ & & \\
\hline Lamium purpureum & 6 & 3 & 4 & 13 & $\mathrm{x}$ & $\mathrm{x}$ & \\
\hline Alopecurus myosuroides & 3 & 4 & 6 & 13 & $\mathrm{x}$ & & \\
\hline Gnaphalium uliginosum & 2 & 6 & 5 & 13 & $\mathrm{x}$ & & \\
\hline Sonchus oleraceus & 5 & 3 & 5 & 13 & $\mathrm{x}$ & & \\
\hline Trifolium repens & 5 & 5 & 3 & 13 & & & \\
\hline Agrostis capillaris & 3 & 4 & 5 & 12 & $\mathrm{x}$ & & \\
\hline Heracleum sphondylium & 2 & 4 & 6 & 12 & & & \\
\hline Persicaria amphibia & 2 & 5 & 5 & 12 & $\mathrm{x}$ & & \\
\hline Plantago major & 4 & 4 & 4 & 12 & $\mathrm{x}$ & & \\
\hline Vicia tetrasperma & 2 & 5 & 4 & 11 & $\mathrm{x}$ & $\mathrm{x}$ & \\
\hline Cerastium holosteoides & 2 & 4 & 5 & 11 & $\mathrm{x}$ & & \\
\hline Atriplex patula & 6 & 2 & 2 & 10 & $\mathrm{x}$ & & \\
\hline Juncus bufonius & 1 & 2 & 7 & 10 & $\mathrm{x}$ & & \\
\hline Plantago lanceolata & 3 & 3 & 4 & 10 & $\mathrm{x}$ & & \\
\hline
\end{tabular}




\begin{tabular}{|c|c|c|c|c|c|c|c|}
\hline Species & Maize & Rapeseed & Wheat & Total & $\begin{array}{l}\text { Arable plants } \\
\text { (x) }\end{array}$ & $\begin{array}{l}\text { HNV } \\
\text { (x) }\end{array}$ & $\begin{array}{c}\text { Red-listed } \\
\text { Lower Saxony } \\
\text { (x) }\end{array}$ \\
\hline Ranunculus repens & 1 & 6 & 3 & 10 & $\mathrm{x}$ & & \\
\hline Rubus fruticosus agg. & 3 & 3 & 4 & 10 & & & \\
\hline Vicia angustifolia & 1 & 6 & 2 & 9 & $\mathrm{x}$ & $\mathrm{x}$ & \\
\hline Aegopodium podagraria & 4 & 2 & 3 & 9 & & & \\
\hline Persicaria bydropiper & 2 & 3 & 4 & 9 & $\mathrm{x}$ & & \\
\hline Setaria viridis & 9 & 0 & 0 & 9 & $\mathrm{x}$ & & \\
\hline Veronica persica & 3 & 3 & 3 & 9 & $\mathrm{x}$ & & \\
\hline Erodium cicutarium & 4 & 2 & 2 & 8 & $\mathrm{x}$ & $\mathrm{x}$ & \\
\hline Lamium album & 1 & 5 & 2 & 8 & $\mathrm{x}$ & $\mathrm{x}$ & \\
\hline Carduus crispus & 1 & 4 & 3 & 8 & & & \\
\hline Daucus carota & 3 & 4 & 1 & 8 & & & \\
\hline Phleum pratense & 1 & 2 & 5 & 8 & & & \\
\hline Poa pratensis & 3 & 3 & 2 & 8 & & & \\
\hline Veronica bederifolia & 1 & 3 & 4 & 8 & $\mathrm{x}$ & & \\
\hline Geranium dissectum & 1 & 4 & 2 & 7 & $\mathrm{x}$ & $\mathrm{x}$ & \\
\hline Geranium molle & 4 & 3 & 0 & 7 & $\mathrm{x}$ & $\mathrm{x}$ & \\
\hline Papaver rhoeas & 2 & 2 & 3 & 7 & $\mathrm{x}$ & $\mathrm{x}$ & \\
\hline Epilobium tetragonum & 0 & 5 & 2 & 7 & $\mathrm{x}$ & & \\
\hline Raphanus sativus & 0 & 6 & 1 & 7 & & & \\
\hline Stellaria graminea & 1 & 3 & 3 & 7 & $\mathrm{x}$ & & \\
\hline Aphanes arvensis & 0 & 3 & 3 & 6 & $\mathrm{x}$ & $\mathrm{x}$ & \\
\hline Alliaria petiolata & 0 & 4 & 2 & 6 & & & \\
\hline Alopecurus geniculatus & 2 & 1 & 3 & 6 & & & \\
\hline Arabidopsis thaliana & 1 & 4 & 1 & 6 & $\mathrm{x}$ & & \\
\hline Digitaria ischaemum & 6 & 0 & 0 & 6 & $\mathrm{x}$ & & \\
\hline Equisetum palustre & 2 & 3 & 1 & 6 & & & \\
\hline Hypericum perforatum & 3 & 1 & 2 & 6 & & & \\
\hline Lactuca serriola & 0 & 4 & 2 & 6 & $\mathrm{x}$ & & \\
\hline Phalaris arundinacea & 0 & 4 & 2 & 6 & & & \\
\hline Phragmites australis & 3 & 0 & 3 & 6 & $\mathrm{x}$ & & \\
\hline Rumex acetosa & 1 & 3 & 2 & 6 & & & \\
\hline Senecio vulgaris & 2 & 3 & 1 & 6 & $\mathrm{x}$ & & \\
\hline Sinapis arvensis & 1 & 4 & 1 & 6 & $\mathrm{x}$ & & \\
\hline Rumex acetosella & 3 & 1 & 1 & 5 & $\mathrm{x}$ & $\mathrm{x}$ & \\
\hline Vicia villosa & 2 & 1 & 2 & 5 & $\mathrm{x}$ & $\mathrm{x}$ & \\
\hline Anchusa arvensis & 2 & 2 & 1 & 5 & $\mathrm{x}$ & & \\
\hline Bromus secalinus & 2 & 0 & 3 & 5 & $\mathrm{x}$ & & \\
\hline Cerastium glomeratum & 1 & 2 & 2 & 5 & $\mathrm{x}$ & & \\
\hline Chaerophyllum temulum & 0 & 3 & 2 & 5 & & & \\
\hline Convolvulus arvensis & 1 & 1 & 3 & 5 & $\mathrm{x}$ & & \\
\hline Galinsoga parviflora & 5 & 0 & 0 & 5 & $\mathrm{x}$ & & \\
\hline Matricaria discoidea & 1 & 4 & 0 & 5 & $\mathrm{x}$ & & \\
\hline Silene latifolia & 3 & 1 & 1 & 5 & $\mathrm{x}$ & & \\
\hline Fumaria officinalis & 2 & 1 & 1 & 4 & $\mathrm{x}$ & $\mathrm{x}$ & \\
\hline Spergula arvensis & 3 & 0 & 1 & 4 & $\mathrm{x}$ & $\mathrm{x}$ & \\
\hline Vicia cracca & 1 & 3 & 0 & 4 & $\mathrm{x}$ & $\mathrm{x}$ & \\
\hline Aetbusa cynapium & 0 & 2 & 2 & 4 & $\mathrm{x}$ & & \\
\hline Carex birta & 2 & 0 & 2 & 4 & $\mathrm{x}$ & & \\
\hline Geum urbanum & 2 & 2 & 0 & 4 & & & \\
\hline Linaria vulgaris & 1 & 2 & 1 & 4 & $\mathrm{x}$ & & \\
\hline Rorippa palustris & 1 & 3 & 0 & 4 & $\mathrm{x}$ & & \\
\hline Stachys palustris & 2 & 2 & 0 & 4 & $\mathrm{x}$ & & \\
\hline Vulpia myuros & 0 & 1 & 3 & 4 & & & \\
\hline Thlaspi arvense & 0 & 2 & 1 & 3 & $\mathrm{x}$ & $\mathrm{x}$ & \\
\hline Chenopodium bybridum & 3 & 0 & 0 & 3 & $\mathrm{x}$ & & $\mathrm{x}$ \\
\hline Odontites vernus & 0 & 3 & 0 & 3 & $\mathrm{x}$ & & $\mathrm{x}$ \\
\hline Arctium lappa & 1 & 0 & 2 & 3 & & & \\
\hline Chaerophyllum bulbosum & 0 & 2 & 1 & 3 & & & \\
\hline Chelidonium majus & 0 & 1 & 2 & 3 & & & \\
\hline Galium mollugo agg. & 0 & 3 & 0 & 3 & & & \\
\hline Lysimachia vulgaris & 3 & 0 & 0 & 3 & & & \\
\hline Potentilla reptans & 0 & 3 & 0 & 3 & $\mathrm{x}$ & & \\
\hline Rubus idaeus agg. & 2 & 0 & 1 & 3 & & & \\
\hline Stellaria bolostea & 0 & 2 & 1 & 3 & & & \\
\hline Trisetum flavescens & 0 & 0 & 3 & 3 & & & \\
\hline Euphorbia peplus & 0 & 1 & 1 & 2 & $\mathrm{x}$ & $\mathrm{x}$ & \\
\hline Papaver argemone & 0 & 0 & 2 & 2 & $\mathrm{x}$ & $\mathrm{x}$ & \\
\hline Amaranthus retroflexus & 1 & 0 & 1 & 2 & $\mathrm{x}$ & & \\
\hline Arenaria serpyllifolia & 1 & 1 & 0 & 2 & $\mathrm{x}$ & & \\
\hline Calystegia sepium & 2 & 0 & 0 & 2 & & & \\
\hline Elymus caninus & 1 & 0 & 1 & 2 & & & \\
\hline Festuca pratensis & 1 & 1 & 0 & 2 & & & \\
\hline Galinsoga quadriradiata & 2 & 0 & 0 & 2 & $\mathrm{x}$ & & \\
\hline Humulus lupulus & 0 & 1 & 1 & 2 & & & \\
\hline Lotus corniculatus & 0 & 1 & 1 & 2 & & & \\
\hline Poa compressa & 0 & 1 & 1 & 2 & & & \\
\hline
\end{tabular}


CHAPTER 4

\begin{tabular}{|c|c|c|c|c|c|c|c|}
\hline Species & Maize & Rapeseed & Wheat & Total & $\begin{array}{l}\text { Arable plants } \\
\text { (x) }\end{array}$ & $\begin{array}{l}\text { HNV } \\
\text { (x) }\end{array}$ & $\begin{array}{c}\text { Red-listed } \\
\text { Lower Saxony } \\
(\mathrm{x})\end{array}$ \\
\hline Potentilla anserina & 1 & 0 & 1 & 2 & $\mathrm{x}$ & & \\
\hline Pteridium aquilinum & 0 & 1 & 1 & 2 & & & \\
\hline Scleranthus annuиs & 1 & 1 & 0 & 2 & $\mathrm{x}$ & & \\
\hline Silene dioica & 1 & 1 & 0 & 2 & & & \\
\hline Solidago canadensis & 1 & 1 & 0 & 2 & & & \\
\hline Symphytum officinale & 0 & 1 & 1 & 2 & $\mathrm{x}$ & & \\
\hline Anthemis arvensis & 0 & 1 & 0 & 1 & $\mathrm{x}$ & $\mathrm{x}$ & $\mathrm{x}$ \\
\hline Buglossoides arvensis & 0 & 1 & 0 & 1 & $\mathrm{x}$ & $\mathrm{x}$ & $\mathrm{x}$ \\
\hline Glebionis segetum & 1 & 0 & 0 & 1 & $\mathrm{x}$ & $\mathrm{x}$ & \\
\hline Euphorbia esula & 1 & 0 & 0 & 1 & & $\mathrm{x}$ & \\
\hline Euphorbia helioscopia & 1 & 0 & 0 & 1 & $\mathrm{x}$ & $\mathrm{x}$ & \\
\hline Geranium robertianum & 0 & 1 & 0 & 1 & & $\mathrm{x}$ & \\
\hline Geranium rotundifolium & 0 & 1 & 0 & 1 & $\mathrm{x}$ & $\mathrm{x}$ & \\
\hline Lamium amplexicaule & 1 & 0 & 0 & 1 & $\mathrm{x}$ & $\mathrm{x}$ & \\
\hline Lythrum salicaria & 1 & 0 & 0 & 1 & & $\mathrm{x}$ & \\
\hline Ornithopus perpusillus & 1 & 0 & 0 & 1 & $\mathrm{x}$ & $\mathrm{x}$ & \\
\hline Agrostemma githago & 0 & 1 & 0 & 1 & $\mathrm{x}$ & & $\mathrm{x}$ \\
\hline Allium vineale & 1 & 0 & 0 & 1 & $\mathrm{x}$ & & \\
\hline Anthoxanthum aristatum & 0 & 0 & 1 & 1 & $\mathrm{x}$ & & \\
\hline Anthriscus caucalis & 0 & 1 & 0 & 1 & $\mathrm{x}$ & & \\
\hline Bromus inermis & 0 & 0 & 1 & 1 & & & \\
\hline Callitriche palustris agg. & 0 & 1 & 0 & 1 & & & \\
\hline Cardamine hirsuta & 0 & 1 & 0 & 1 & $\mathrm{x}$ & & \\
\hline Carduus nutans & 0 & 1 & 0 & 1 & & & \\
\hline Centaurea jacea & 0 & 1 & 0 & 1 & & & \\
\hline Digitaria sanguinalis & 1 & 0 & 0 & 1 & $\mathrm{x}$ & & \\
\hline Epilobium angustifolium & 0 & 1 & 0 & 1 & & & \\
\hline Epilobium hirsutum & 0 & 0 & 1 & 1 & & & \\
\hline Epilobium montanum & 0 & 1 & 0 & 1 & & & \\
\hline Epilobium parviflorum & 0 & 1 & 0 & 1 & & & \\
\hline Equisetum sylvaticum & 0 & 0 & 1 & 1 & & & \\
\hline Erigeron annuus & 0 & 1 & 0 & 1 & & & \\
\hline Erysimum cheiranthoides & 0 & 1 & 0 & 1 & $\mathrm{x}$ & & \\
\hline Festuca arundinacea & 0 & 0 & 1 & 1 & & & \\
\hline Filago arvensis & 1 & 0 & 0 & 1 & $\mathrm{x}$ & & \\
\hline Hypericum maculatum & 0 & 0 & 1 & 1 & & & \\
\hline Hypochaeris radicata & 0 & 1 & 0 & 1 & & & \\
\hline Impatiens glandulifera & 0 & 0 & 1 & 1 & & & \\
\hline Juncus effusus & 1 & 0 & 0 & 1 & & & \\
\hline Scorzoneroides autumnalis & 0 & 1 & 0 & 1 & & & \\
\hline Lupinus polyphyllus & 0 & 1 & 0 & 1 & & & \\
\hline Medicago lupulina & 1 & 0 & 0 & 1 & $\mathrm{x}$ & & \\
\hline Mentha arvensis & 1 & 0 & 0 & 1 & $\mathrm{x}$ & & \\
\hline Oxalis stricta & 0 & 1 & 0 & 1 & & & \\
\hline Persicaria mitis & 0 & 0 & 1 & 1 & & & \\
\hline Ranunculus acris & 0 & 0 & 1 & 1 & & & \\
\hline Rumex bydrolapatbum & 0 & 0 & 1 & 1 & & & \\
\hline Senecio vernalis & 0 & 1 & 0 & 1 & $\mathrm{x}$ & & \\
\hline Sonchus arvensis & 0 & 1 & 0 & 1 & $\mathrm{x}$ & & \\
\hline Trifolium bybridum & 0 & 1 & 0 & 1 & & & \\
\hline Trifolium pratense & 0 & 0 & 1 & 1 & & & \\
\hline Typha latifolia & 0 & 0 & 1 & 1 & & & \\
\hline Urtica urens & 1 & 0 & 0 & 1 & $\mathrm{x}$ & & \\
\hline
\end{tabular}




\section{CHAPTER 4}

Online resource 9a Overview of used models and statistical results.

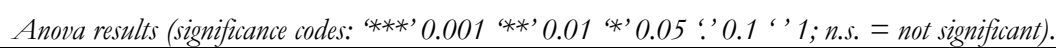

\begin{tabular}{|c|c|c|c|c|c|c|c|c|c|c|}
\hline Measured value & Model & $\mathrm{n}$ & $\begin{array}{c}\mathbf{R}^{2} \\
\text { (fixed } \\
\text { effects) }\end{array}$ & $\begin{array}{c}\mathbf{R}^{2} \\
\text { (fixed \& } \\
\text { random } \\
\text { effects) }\end{array}$ & $\begin{array}{l}\text { Explanatory } \\
\text { variables }\end{array}$ & $\begin{array}{c}\text { Z-value } \\
\text { (chi-square) }\end{array}$ & $\begin{array}{l}\text { Degrees of } \\
\text { freedom }\end{array}$ & $\begin{array}{c}\text { P-value } \\
\text { (Pr(chi-square)) }\end{array}$ & Significance & F-value \\
\hline Total herbaceous species number & $\begin{array}{l}\text { glmer.nb(Spec_No } \sim \text { Plot }+(1 \mid \text { Crop })+(1 \mid \text { Farmer }), \text { data }= \\
\text { kopfspec_without_total) }\end{array}$ & $\begin{array}{c}45 \\
\text { (per survey method) }\end{array}$ & 0.80 & 0.85 & Survey method & 905.3 & 5 & $<2.2 \mathrm{e}-16$ & *** & 180.9 \\
\hline Arable plant number sensu stricto & $\begin{array}{l}\text { glmer.nb(weed_no } \sim \text { Plot + (1 | Crop })+(1 \mid \text { Farmer }), \text { data }= \\
\text { kopfspec_without_total) }\end{array}$ & $\begin{array}{c}45 \\
\text { (per survey method) }\end{array}$ & 0.76 & 0.81 & Survey method & 798.05 & 5 & $<2.2 \mathrm{e}-16$ & $* * *$ & 159.5 \\
\hline High-nature-value species number & $\begin{array}{l}\text { glmer(Spec_No } \sim \text { Plot }+(1 \mid \text { Crop })+(1 \mid \text { Farmer }), \text { family=poisson, } \\
\text { data }=\text { kopfHNV })\end{array}$ & $\begin{array}{c}45 \\
\text { (per survey method) }\end{array}$ & 0.47 & 0.61 & Survey method & 222.72 & 5 & $<2.2 \mathrm{e}-16$ & *** & 44.114 \\
\hline $\begin{array}{l}\text { Detection rate of total species } \\
\text { number }\end{array}$ & $\begin{array}{l}\text { glmer(cbind(Spec_No, Total_spec_no) } \sim \text { Plot }+ \\
(1 \mid \text { Crop })+(1 \mid \text { Farmer), family=binomial, data = Prop_table })\end{array}$ & $\begin{array}{c}45 \\
\text { (per survey method) }\end{array}$ & 0.08 & 0.08 & Survey method & 904.26 & 5 & $<2.2 \mathrm{e}-16$ & *** & 180.89 \\
\hline $\begin{array}{l}\text { Detection rate of total arable plant } \\
\text { species number sensu stricto }\end{array}$ & $\begin{array}{l}\text { glmer(cbind(arten_ackerwp, total_arable_weeds_per_field }) ~ \\
\text { Plot }+(1 \mid \text { Crop })+(1 \mid \text { Farmer), family=binomial, } \\
\text { data = Prop_table_weeds) }\end{array}$ & $\begin{array}{l}45 \\
\text { (per survey method) }\end{array}$ & 0.07 & 0.07 & Survey method & 718.51 & 5 & $<2.2 \mathrm{e}-16$ & *** & 143.78 \\
\hline $\begin{array}{l}\text { Detection rate of total high-nature- } \\
\text { value species number }\end{array}$ & $\begin{array}{l}\text { glmer(cbind(HNV_No, total_HNV_per_field }) \sim \text { Plot }+ \\
(1 \mid \text { Crop })+(1 \mid \text { Farmer), family }=\text { binomial, data }=\text { Prop_HNV })\end{array}$ & $\begin{array}{c}45 \\
\text { (per survey method) }\end{array}$ & 0.12 & 0.12 & Survey method & 163.47 & 5 & $<2.2 \mathrm{e}-16$ & *** & 32.73 \\
\hline
\end{tabular}

Test for spatial autocorrelation: Moran's I (according to Paradis and Schliep 2018; full references see main text; significantp-values ( $<0.05)$ indicate spatial autocorrelation)

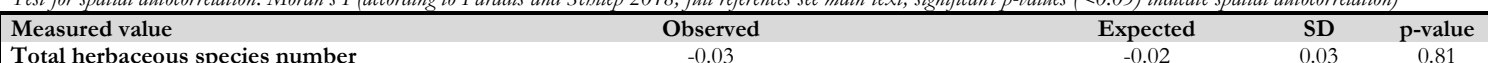

Total herbaceous species number

Observed

expected

0.03 
Online resource $9 \mathrm{~b}$ Statistical results.

Multiple Comparisons of Means: Tukey contrasts (based on model results: Online resource 9a)

Significance codes: '***' 0.001 '**' 0.01 '*' 0.05 ' 0.1 ' '1; $n=45$ (per survey method)

\begin{tabular}{|c|c|c|c|c|c|c|}
\hline Measured value & Compared survey method & Estimate & Std. Error & $\mathrm{z}$ value & $\operatorname{Pr}(>|z|)$ & Significance \\
\hline \multirow{15}{*}{$\begin{array}{l}\text { Total herbaceous } \\
\text { species number }\end{array}$} & \multirow{15}{*}{$\begin{array}{l}\text { Edge_30 - Corner } \\
\text { Edge_50 - Corner } \\
\text { Interior - Corner } \\
\text { Subplot - Corner } \\
\text { Edge_500 - Corner } \\
\text { Edge_50 - Edge_30 } \\
\text { Interior - Edge_30 } \\
\text { Subplot - Edge_30 } \\
\text { Edge_500 - Edge_30 } \\
\text { Interior - Edge_50 } \\
\text { Subplot - Edge_50 } \\
\text { Edge_500 - Edge_50 } \\
\text { Subplot - Interior } \\
\text { Edge_500 - Interior } \\
\text { Edge_500 - Subplot }\end{array}$} & -0.65662 & 0.06477 & -10.139 & $<0.001$ & $* * *$ \\
\hline & & -0.57842 & 0.06352 & -9.106 & $<0.001$ & $* * *$ \\
\hline & & -1.72798 & 0.09099 & -18.99 & $<0.001$ & $* * *$ \\
\hline & & -0.35595 & 0.06036 & -5.897 & $<0.001$ & $* * *$ \\
\hline & & 0.46908 & 0.05249 & 8.937 & $<0.001$ & $* * *$ \\
\hline & & 0.0782 & 0.07111 & 1.1 & 0.8766 & \\
\hline & & -1.07136 & 0.09644 & -11.109 & $<0.001$ & $* * *$ \\
\hline & & 0.30067 & 0.06831 & 4.402 & $<0.001$ & $* * *$ \\
\hline & & 1.1257 & 0.06147 & 18.313 & $<0.001$ & $* * *$ \\
\hline & & -1.14957 & 0.09562 & -12.023 & $<0.001$ & $* * *$ \\
\hline & & 0.22246 & 0.06713 & 3.314 & 0.0112 & $*$ \\
\hline & & 1.04749 & 0.06016 & 17.412 & $<0.001$ & $* * *$ \\
\hline & & 1.37203 & 0.09355 & 14.667 & $<0.001$ & $* * *$ \\
\hline & & 2.19706 & 0.08868 & 24.775 & $<0.001$ & $* * *$ \\
\hline & & 0.82503 & 0.05681 & 14.524 & $<0.001$ & $* * *$ \\
\hline \multirow{15}{*}{$\begin{array}{l}\text { Arable plant species } \\
\text { number sensu stricto }\end{array}$} & \multirow{15}{*}{$\begin{array}{l}\text { Edge_30 - Corner } \\
\text { Edge_50 - Corner } \\
\text { Interior - Corner } \\
\text { Subplot - Corner } \\
\text { Edge_500 - Corner } \\
\text { Edge_50 - Edge_30 } \\
\text { Interior - Edge_30 } \\
\text { Subplot - Edge_30 } \\
\text { Edge_500 - Edge_30 } \\
\text { Interior - Edge_50 } \\
\text { Subplot - Edge_50 } \\
\text { Edge_500 - Edge_50 } \\
\text { Subplot - Interior } \\
\text { Edge_500 - Interior } \\
\text { Edge_500 - Subplot }\end{array}$} & -0.66357 & 0.06879 & -9.646 & $<0.001$ & $* * *$ \\
\hline & & -0.58665 & 0.06736 & -8.71 & $<0.001$ & $* * *$ \\
\hline & & -1.54037 & 0.09192 & -16.757 & $<0.001$ & $* * *$ \\
\hline & & -0.33426 & 0.06315 & -5.293 & $<0.001$ & $* * *$ \\
\hline & & 0.48359 & 0.05406 & 8.946 & $<0.001$ & $* * *$ \\
\hline & & 0.07691 & 0.07631 & 1.008 & 0.91166 & \\
\hline & & -0.87681 & 0.09867 & -8.886 & $<0.001$ & $* * *$ \\
\hline & & 0.3293 & 0.07263 & 4.534 & $<0.001$ & $* * *$ \\
\hline & & 1.14716 & 0.06488 & 17.682 & $<0.001$ & $* * *$ \\
\hline & & -0.95372 & 0.09768 & -9.764 & $<0.001$ & $* * *$ \\
\hline & & 0.25239 & 0.07127 & 3.541 & 0.00507 & $* *$ \\
\hline & & 1.07025 & 0.06335 & 16.893 & $<0.001$ & $* * *$ \\
\hline & & 1.20611 & 0.09483 & 12.719 & $<0.001$ & $* * *$ \\
\hline & & 2.02397 & 0.08904 & 22.732 & $<0.001$ & $* * *$ \\
\hline & & 0.81786 & 0.05886 & 13.894 & $<0.001$ & $* * *$ \\
\hline \multirow{15}{*}{$\begin{array}{l}\text { High-nature-value } \\
\text { species number }\end{array}$} & \multirow{15}{*}{$\begin{array}{l}\text { Edge_30 - Corner } \\
\text { Edge_50 - Corner } \\
\text { Interior - Corner } \\
\text { Subplot - Corner } \\
\text { Edge_500 - Corner } \\
\text { Edge_50 - Edge_30 } \\
\text { Interior - Edge_30 } \\
\text { Subplot - Edge_30 } \\
\text { Edge_500 - Edge_30 } \\
\text { Interior - Edge_50 } \\
\text { Subplot - Edge_50 } \\
\text { Edge_500 - Edge_50 } \\
\text { Subplot - Interior } \\
\text { Edge_500 - Interior } \\
\text { Edge_500 - Subplot }\end{array}$} & -0.66905 & 0.13362 & -5.007 & $<0.001$ & $* * *$ \\
\hline & & -0.54598 & 0.1284 & -4.252 & $<0.001$ & $* * *$ \\
\hline & & -1.69867 & 0.19775 & -8.59 & $<0.001$ & $* * *$ \\
\hline & & -0.40851 & 0.12307 & -3.319 & 0.0107 & $*$ \\
\hline & & 0.47229 & 0.09909 & 4.766 & $<0.001$ & $* * *$ \\
\hline & & 0.12307 & 0.14915 & 0.825 & 0.9603 & \\
\hline & & -1.02962 & 0.21182 & -4.861 & $<0.001$ & $* * *$ \\
\hline & & 0.26054 & 0.14459 & 1.802 & 0.4479 & \\
\hline & & 1.14134 & 0.12481 & 9.145 & $<0.001$ & $* * *$ \\
\hline & & -1.15269 & 0.20856 & -5.527 & $<0.001$ & $* * *$ \\
\hline & & 0.13747 & 0.13978 & 0.984 & 0.9182 & \\
\hline & & 1.01828 & 0.11921 & 8.542 & $<0.001$ & $* * *$ \\
\hline & & 1.29016 & 0.20532 & 6.284 & $<0.001$ & $* * *$ \\
\hline & & 2.17096 & 0.19191 & 11.312 & $<0.001$ & $* * *$ \\
\hline & & 0.8808 & 0.11344 & 7.764 & $<0.001$ & $* * *$ \\
\hline \multirow{15}{*}{$\begin{array}{l}\text { Detection rate of } \\
\text { herbaceous total species } \\
\text { number }\end{array}$} & \multirow{15}{*}{$\begin{array}{l}\text { Edge_30 - Corner } \\
\text { Edge_50 - Corner } \\
\text { Interior - Corner } \\
\text { Subplot - Corner } \\
\text { Edge_500 - Corner } \\
\text { Edge_50 - Edge_30 } \\
\text { Interior - Edge_30 } \\
\text { Subplot - Edge_30 } \\
\text { Edge_500 - Edge_30 } \\
\text { Interior - Edge_50 } \\
\text { Subplot - Edge_50 } \\
\text { Edge_500 - Edge_50 } \\
\text { Subplot - Interior } \\
\text { Edge_500 - Interior } \\
\text { Edge_500 - Subplot } \\
\end{array}$} & -0.65457 & 0.06483 & -10.096 & $<0.001$ & $* * *$ \\
\hline & & -0.57682 & 0.06359 & -9.071 & $<0.001$ & $* * *$ \\
\hline & & -1.72725 & 0.09102 & -18.976 & $<0.001$ & $* * *$ \\
\hline & & -0.35572 & 0.06044 & -5.886 & $<0.001$ & $* * *$ \\
\hline & & 0.47165 & 0.05259 & 8.968 & $<0.001$ & $* * *$ \\
\hline & & 0.07775 & 0.07117 & 1.092 & 0.8796 & \\
\hline & & -1.07269 & 0.09647 & -11.12 & $<0.001$ & $* * *$ \\
\hline & & 0.29884 & 0.06837 & 4.371 & $<0.001$ & $* * *$ \\
\hline & & 1.12622 & 0.06155 & 18.298 & $<0.001$ & $* * *$ \\
\hline & & -1.15044 & 0.09564 & -12.029 & $<0.001$ & $* * *$ \\
\hline & & 0.2211 & 0.06719 & 3.291 & 0.0122 & $*$ \\
\hline & & 1.04847 & 0.06024 & 17.405 & $<0.001$ & $* * *$ \\
\hline & & 1.37153 & 0.09357 & 14.658 & $<0.001$ & $* * *$ \\
\hline & & 2.19891 & 0.08872 & 24.785 & $<0.001$ & $* * *$ \\
\hline & & 0.82738 & 0.0569 & 14.541 & $<0.001$ & $* * *$ \\
\hline \multirow{9}{*}{$\begin{array}{l}\text { Detection rate of total } \\
\text { arable plant species } \\
\text { number sensu stricto }\end{array}$} & \multirow{9}{*}{$\begin{array}{l}\text { Edge_30 - Corner } \\
\text { Edge_50 - Corner } \\
\text { Interior - Corner } \\
\text { Subplot - Corner } \\
\text { Edge_500 - Corner } \\
\text { Edge_50 - Edge_30 } \\
\text { Interior - Edge_30 } \\
\text { Subplot - Edge_30 } \\
\text { Edge_500 - Edge_30 }\end{array}$} & -0.66493 & 0.07254 & -9.166 & $<1 \mathrm{e}-04$ & $* * *$ \\
\hline & & -0.58787 & 0.07118 & -8.259 & $<1 \mathrm{e}-04$ & $* * *$ \\
\hline & & -1.54001 & 0.09472 & -16.259 & $<1 \mathrm{e}-04$ & $* * *$ \\
\hline & & -0.33511 & 0.06719 & -4.987 & $<1 \mathrm{e}-04$ & $* * *$ \\
\hline & & 0.48517 & 0.05872 & 8.263 & $<1 \mathrm{e}-04$ & $* * *$ \\
\hline & & 0.07706 & 0.07973 & 0.967 & 0.925701 & \\
\hline & & -0.87508 & 0.1013 & -8.638 & $<1 \mathrm{e}-04$ & $* * *$ \\
\hline & & 0.32982 & 0.0762 & 4.328 & 0.000195 & $* * *$ \\
\hline & & 1.15009 & 0.06885 & 16.704 & $<1 \mathrm{e}-04$ & $* * *$ \\
\hline
\end{tabular}


Detection rate of total high-nature-value species number
Interior - Edge 50

Subplot - Edge_50

Edge_500 - Edge_50

Subplot - Interior

Edge_500 - Interior

Edge_500 - Subplot

Edge_30 - Interior

Edge_50 - Interior

Subplot - Interior

Corner - Interior

Edge_500 - Interior

Edge_50 - Edge_30

Subplot - Edge_30

Corner - Edge_30

Edge_500 - Edge_30

Subplot - Edge_50

Corner - Edge_50

Edge_500 - Edge_50

Corner - Subplot

Edge_500 - Subplot

Edge_500 - Corner

\begin{tabular}{|l|l|l|l|l|}
\hline-0.95214 & 0.10033 & -9.49 & $<1 \mathrm{e}-04$ & $* * *$ \\
\hline 0.25275 & 0.0749 & 3.375 & 0.009159 & $* *$ \\
\hline 1.07303 & 0.06741 & 15.919 & $<1 \mathrm{e}-04$ & $* * *$ \\
\hline 1.2049 & 0.09754 & 12.352 & $<1 \mathrm{e}-04$ & $* * *$ \\
\hline 2.02518 & 0.09193 & 22.03 & $<1 \mathrm{e}-04$ & $* * *$ \\
\hline 0.82028 & 0.06319 & 12.982 & $<1 \mathrm{e}-04$ & $* * *$ \\
\hline 1.0295 & 0.2252 & 4.571 & $<0.001$ & $* * *$ \\
\hline 1.1526 & 0.2222 & 5.188 & $<0.001$ & $* * *$ \\
\hline 1.2905 & 0.2191 & 5.89 & $<0.001$ & $* * *$ \\
\hline 1.6991 & 0.212 & 8.016 & $<0.001$ & $* * *$ \\
\hline 2.1725 & 0.2065 & 10.52 & $<0.001$ & $* * *$ \\
\hline 0.1232 & 0.1671 & 0.737 & 0.97622 & \\
\hline 0.2611 & 0.163 & 1.601 & 0.58699 & \\
\hline 0.6696 & 0.1533 & 4.368 & $<0.001$ & $* * *$ \\
\hline 1.143 & 0.1457 & 7.846 & $<0.001$ & $* * *$ \\
\hline 0.1379 & 0.1587 & 0.869 & 0.95173 & \\
\hline 0.5465 & 0.1487 & 3.674 & 0.00297 & $* *$ \\
\hline 1.0199 & 0.1409 & 7.24 & $<0.001$ & $* * *$ \\
\hline 0.4086 & 0.1441 & 2.835 & 0.04963 & $*$ \\
\hline 0.882 & 0.1359 & 6.488 & $<0.001$ & $* * *$ \\
\hline 0.4734 & 0.1241 & 3.814 & 0.0018 & $* *$ \\
\hline
\end{tabular}

Pairwise comparisons using Wilcoxon rank sum test; pairwise p-value are given ( $p$ value adjustment method: bolm ); $n=15$ per crop and survey method (except required time: one missing value regarding maize interior plot, maize subplot, maize Edge_50 plot and wheat corner plot)

\begin{tabular}{|c|c|c|c|c|c|c|}
\hline \multirow{6}{*}{$\begin{array}{l}\text { Total herbaceous } \\
\text { species number } \\
\text { wheat }\end{array}$} & Survey method & Interior & Edge_30 & Edge_50 & Subplot & Corner \\
\hline & Edge_30 & 0.00011 & - & - & - & - \\
\hline & Edge_50 & $7.00 \mathrm{E}-05$ & 0.69555 & - & - & - \\
\hline & Subplot & 0.00011 & 0.5251 & 0.69555 & - & - \\
\hline & Corner & $4.90 \mathrm{E}-05$ & 0.00915 & 0.02137 & 0.16298 & - \\
\hline & Edge_500 & $4.90 \mathrm{E}-05$ & $4.90 \mathrm{E}-05$ & $5.80 \mathrm{E}-05$ & $9.70 \mathrm{E}-05$ & 0.00165 \\
\hline \multirow{5}{*}{$\begin{array}{l}\text { Total herbaceous } \\
\text { species number } \\
\text { maize }\end{array}$} & Edge_30 & 0.00452 & - & - & - & - \\
\hline & Edge_50 & 0.00116 & 0.31439 & - & - & - \\
\hline & Subplot & 0.0001 & 0.00205 & 0.00607 & - & - \\
\hline & Corner & $4.80 \mathrm{E}-05$ & 0.00012 & 0.00016 & 0.00614 & - \\
\hline & Edge_500 & $4.80 \mathrm{E}-05$ & $4.80 \mathrm{E}-05$ & $4.80 \mathrm{E}-05$ & 0.00012 & 0.01227 \\
\hline \multirow{5}{*}{$\begin{array}{l}\text { Total herbaceous } \\
\text { species number } \\
\text { rapeseed }\end{array}$} & Edge_30 & $7.30 \mathrm{E}-05$ & - & - & - & - \\
\hline & Edge_50 & $5.10 \mathrm{E}-05$ & 0.61722 & - & - & - \\
\hline & Subplot & 4.70E-05 & 0.18208 & 0.33909 & - & - \\
\hline & Corner & $4.70 \mathrm{E}-05$ & $9.00 \mathrm{E}-05$ & 0.00011 & 0.0032 & - \\
\hline & Edge_500 & 4.70E-05 & $4.70 \mathrm{E}-05$ & 4.70E-05 & $4.70 \mathrm{E}-05$ & 0.00011 \\
\hline \multirow{5}{*}{$\begin{array}{l}\text { Time expenditure } \\
\text { wheat }\end{array}$} & Survey method & Interior & $\overline{\text { Edge_50 }}$ & Subplot & Corner & \\
\hline & Edge_50 & 0.0036 & - & - & - & \\
\hline & Subplot & 4.70E-05 & 0.0018 & - & - & \\
\hline & Corner & $3.00 \mathrm{E}-05$ & 0.0011 & 0.4491 & - & \\
\hline & Edge_500 & $2.60 \mathrm{E}-05$ & $2.70 \mathrm{E}-05$ & $2.80 \mathrm{E}-05$ & $2.90 \mathrm{E}-05$ & \\
\hline \multirow{4}{*}{$\begin{array}{l}\text { Time expenditure } \\
\text { maize }\end{array}$} & Edge_50 & 0.00044 & - & - & - & \\
\hline & Subplot & $4.30 \mathrm{E}-05$ & 0.00022 & - & - & \\
\hline & Corner & $4.30 \mathrm{E}-05$ & 0.00188 & 0.35175 & - & \\
\hline & Edge_500 & $3.40 \mathrm{E}-05$ & $3.90 \mathrm{E}-05$ & $5.10 \mathrm{E}-05$ & $8.90 \mathrm{E}-05$ & \\
\hline \multirow{4}{*}{$\begin{array}{l}\text { Time expenditure } \\
\text { rapeseed }\end{array}$} & Edge_50 & 0.00059 & - & - & - & \\
\hline & Subplot & 0.10808 & 0.00107 & - & - & \\
\hline & Corner & 0.05375 & 0.00291 & 0.19469 & - & \\
\hline & Edge_500 & 0.00148 & $2.80 \mathrm{E}-05$ & $3.40 \mathrm{E}-05$ & $4.00 \mathrm{E}-05$ & \\
\hline
\end{tabular}

Pairwise comparisons using Wilcoxon rank sum test; pairwise p-value are given (p value adjustment method: holm); $n=45$ (per survey method), except Interior $n=$ 40 (5 plots with non-observed species were excluded).

\begin{tabular}{|l|l|l|l|l|l|l|}
\hline \multirow{4}{*}{$\begin{array}{l}\text { Beta diversity (based on } \\
\text { total arable weed } \\
\text { number sensu stricto) }\end{array}$} & Survey method & Interior & Edge_30 & Edge_50 & Subplot & Corner \\
\cline { 2 - 7 } & Edge_30 & 1 & - & - & - & - \\
\cline { 2 - 7 } & Edge_50 & 1 & 1 & - & - & - \\
\cline { 2 - 7 } & Subplot & 0.15723 & 0.07744 & 0.17369 & - & - \\
\cline { 2 - 7 } & Corner & 0.00076 & $2.00 \mathrm{E}-05$ & 0.00017 & 0.06623 & - \\
\cline { 2 - 6 } & Edge_500 & $1.80 \mathrm{E}-06$ & $1.20 \mathrm{E}-09$ & $1.40 \mathrm{E}-08$ & 0.00018 & 0.17649 \\
\hline
\end{tabular}

Online Resource 10 Raw data.

Can be downloaded from http://ediss.uni-goettingen.de/ (Niedersächsische Staats- und Universitätsbibliothek Göttingen). 


\section{Chapter 5}

\section{Synopsis}

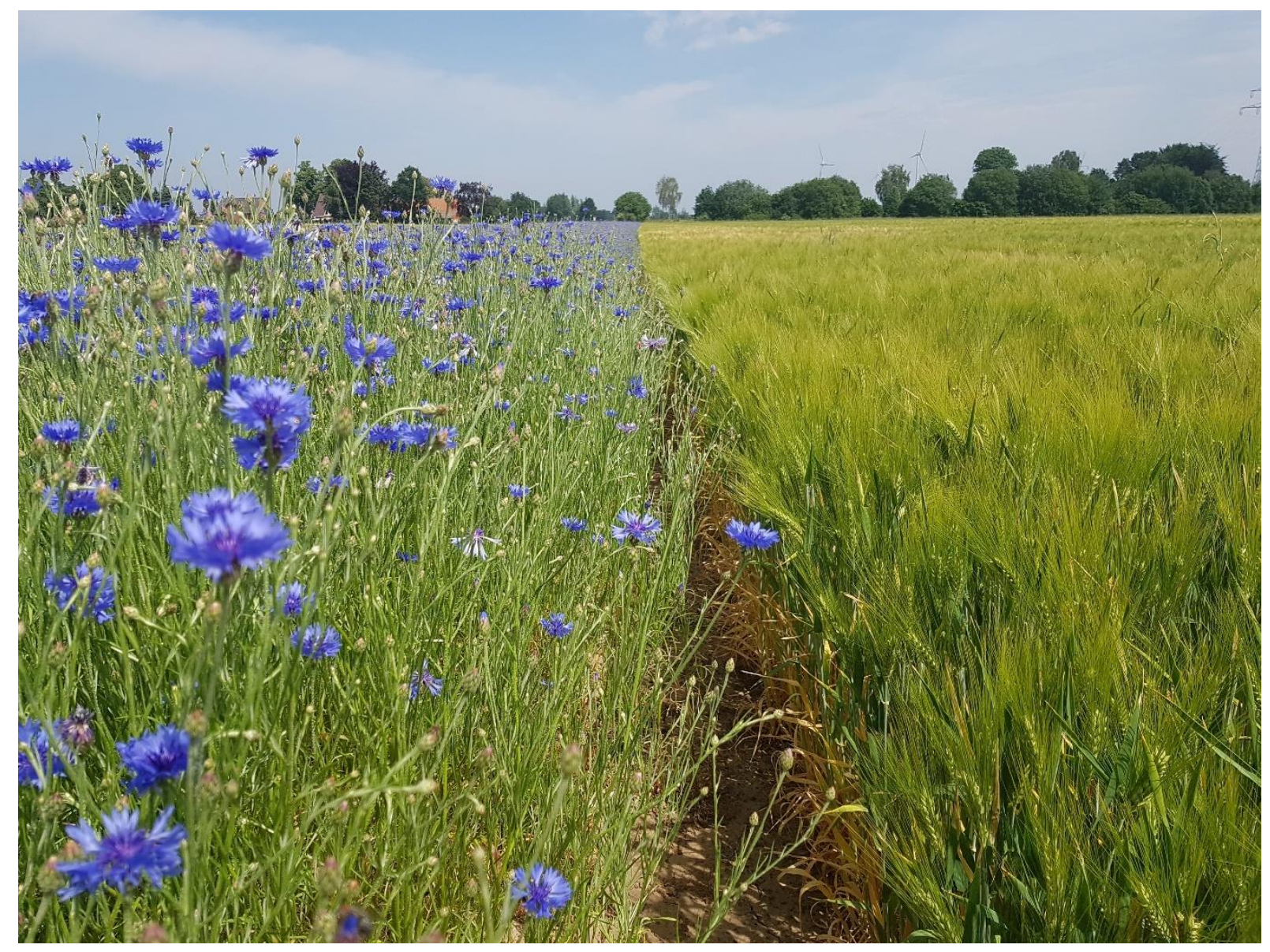

This thesis has advanced the scientific understanding of the arable flora of conventionally managed fields with respect to three major research questions that have insufficiently been investigated so far. First, it was shown how decades of intensive conventional management have affected the segetal vegetation of fields of seven important crop species in Northwest Germany and which factors significantly influence today's arable plant richness (Chapter 2). To stop the ongoing loss of arable plant diversity, four agri-environment measures have been implemented in a participative approach and have been evaluated over time, aiming to demonstrate their effectiveness in promoting the arable plant vegetation (Chapter 3). Finally, this thesis compared six arable plant survey methods in terms of their effectiveness (detection success and time effort) in conventionally managed farmland in order to recommend a future monitoring standard (Chapter 4).

In the following, four key findings related to the above-mentioned research priorities are presented and discussed, and conclusions are given about their significance for future nature conservation efforts, research and agricultural policy. 


\subsection{Key findings and conclusions}

i) Decades of intensive conventional management have led to a strongly impoverished farmland phytodiversity in the study region with the field interior today being of almost no relevance as habitat for arable plants. The observed non-crop plant species richness reached a historical minimum and notable occurrences of most arable plants were only found in the field edge. The cultivated crop species and adjacent habitats are the most important drivers of the field (edge) plant richness. As the finding of the strongly impoverished arable flora is likely to be transferrable to much of Western and Central European farmland, there is an urgent need for large-scale implementation of nature conservation measures, supported by European Common Agricultural Policy, to halt the ongoing loss of the arable plant diversity and associated ecosystem services.

Chapters 2 to 4 demonstrated the strongly impoverished arable plant vegetation of the conventionally managed arable fields in the study region, with nearly no threatened, red-listed arable plant species present anymore. This reflects the impact of the long-term intensified agricultural management since the middle of the $20^{\text {th }}$ century and underlines findings of other studies conducted in Western and Central Europe (e.g. Albrecht et al., 2016; Meyer et al., 2013; Richner et al., 2015; Storkey et al., 2012). The baseline survey of 200 conventionally managed arable fields in the study region in 2016 (Chapter 2) showed a very low plot-level median of the non-crop plant species richness across the observed seven crop species (11 and 2 species at the field edge and in the field interior, respectively). The corresponding plot-level species richness of typical arable plants (as listed by Hofmeister and Garve, 2006) was 8 species at the field edge and remained at 2 species in the field interior. Thus, the observed arable plant species richness has been among the lowest recorded so far for Central and Western Europe (Fried et al., 2009; Kolářová et al., 2013; Kovács-Hostyánszki et al., 2011a; Meyer et al., 2013). This may indicate an ongoing loss in the last years since the earlier studies have been conducted. Nowadays, the common arable plant taxa are agro-tolerant species, which are able to cope with intensive conventional management. Apart from some herbicide-resistant plant taxa (e.g. Alopecurus myosuroides; Maréchal et al., 2012), which can reach higher abundances and compete with the crops grown in some locations, the very low median cover value of the non-crop vegetation especially found in the field interior (median $0.5 \%$ across all crops) but also at the field edge (4 $\%)$ reflect the highly efficient weed control by the farmers. Today, notable occurrences of most arable plant species can only be found in the field edge, which can be explained by somewhat less intensive management and possible spillover from neighboring habitats (Chapters 2 and 4). In addition, there was a notable share of typical arable plant species whose occurrence was restricted to only few of the observed arable fields, which was also reflected by a relatively high beta diversity between the study fields (Chapters 2 to 4 ). This clearly demonstrates that many formerly typical arable plant species can hardly find a habitat in most modern conventionally intensively managed fields. Beside the location in the field, which influenced the observed noncrop plant species richness in the study region most strongly, also the cultivated crop species affected the observed species number (Chapter 2). Here, especially rapeseed, but also potato, positively influenced total arable plant species richness. This can likely be explained by the fact that rapeseed and potato are dicots and therefore it is more difficult to control dicot arable plants during their cultivation period, e.g. no application of highly effective broad-spectrum herbicides against dicotyls commonly used in cereals. Maize negatively affected the surveyed total plant species richness, which is likely to be driven by a combination of the strong shading effect of this crop (Seifert et al., 2014) and intensive herbicide application. Taking into account the crop- 
specific effects on arable plant species richness, which are at least partly due to specific management regimes, the use of selective rather than highly effective broad-spectrum herbicides can be a tool to promote arable plant diversity in conventional farming systems (Storkey and Westbury, 2007). Broad-spectrum herbicides should be the last resort and attempts should first be made to control problematic 'weed' species occurrences either mechanically (e.g. harrowing or tillage) or, if this is not effective, by using selective herbicides. As in particular grass species can become a problem in certain arable fields (Moray, 2005; Moss, 2017; Rolston et al., 2003), the diversification of crop rotation with the integration of dicot crops, such as rapeseed, could be one way to address this problem because this allows the efficient control of graminoids with selective herbicides. The selective control of dominant plant species that reduce yields spares the still existing typical arable plant species with conservation value, which are weak competitors anyway. In addition, including mass flowering dicot crops in crop rotation, such as rapeseed, can also support pollinating insects by providing nectar and pollen sources (Westphal et al., 2003).

However, even though cultivated crop species influenced total plant species richness, formerly found arable plant communities, typically associated with crop-specific cultivation (e.g. summer vs. winter crops), were no longer detectable. This demonstrates the through long-term intensive management nowadays strongly homogenized and impoverished arable plant vegetation also found by Meyer et al (2015) for Central and North Germany. Beside plot location and crop type, increasing soil yield potential, total $\mathrm{N}$ input, herbicide use and crop cover were found to negatively affect the total arable plant species richness (Chapter 2), but the influence of these factors was weaker. In contrast, the presence of adjacent habitats to arable fields (such as grass strips, ditch margins and hedges) was found to positively influence field edge plant diversity. These neighboring non-intensively managed habitats can be a plant refugium and spillover source also for plants typically associated with arable habitats. If grass strips adjacent to fields are revaluated through adequate management (i.e. mowing and, if appropriate, sowing of seed mixtures of local provenance including arable plants) they can contribute to increase field edge arable plant diversity and also benefit other farmland species, such as birds or insects (Haaland et al., 2011; Vickery et al., 2009). Taking this finding into account, increasing the ratio of adjacent habitat length to managed sites can be another possibility to promote farmland biodiversity (Batáry et al., 2017; von Arx et al., 2002), especially in highly productive and intensively used agricultural regions, where in-field measures are hardly to be implemented. However, such 'outof-field' measures cannot hold the overall decline of the arable plant diversity, which is mostly composed of competitively weak species dependent on less intensive arable habitat conditions.

In general, the strong loss of arable plant richness and abundance and its impact on associated organism groups of the upper food chain is alarming since it threatens important ecosystem functions, such as pollination as well as erosion and pest control (Fagúndez, 2014; Hawes et al., 2003; Hyvönen and Huusela-Veistola, 2008). The dramatic decline in insect biomass in the last decades in Europe and other parts of the world with agricultural intensification as one main driver (Sánchez-Bayo and Wyckhuys, 2019) can likely be related to the reduction of their food sources (i.e. pollen, nectar and seeds; Marshall et al., 2003; Still, 2007). In addition, both reduced insect biomass and seeds provided by arable plants can also be related to the overall decreasing populations of farmland birds (Donald et al., 2006, 2001; Marshall et al., 2003; Still, 2007; Vořríšek et al., 2010). 
National and European policies must take into account that the arable flora has almost completely vanished from a large part of conventionally intensive managed farmland, with the exception of a narrow field margin strip. The dramatic impoverishment of the arable plant vegetation and the related threat for important ecosystem functions highlights the urgent need for nature conservation action to be supported by agricultural policy. Sustainable forms of agricultural use are needed that ensure sufficient food production without depriving species living in agricultural landscapes of their habitat. One approach, which is already being implemented, is organic farming. Many studies demonstrated the positive effect of organic farming on different organism groups, such as insects, farmland birds and arable plants (Batáry et al., 2017; Rahmann, 2011; Tuck et al., 2014). However, as about $95 \%$ of the arable land in Central Europe is still managed conventionally (EUROSTAT 2018), it is necessary to implement ecologically effective measures on a large scale to counteract the dramatic loss of the arable flora and prevent the extinction of many formerly typical arable plants.

Well-managed conservation field margins (CFM), annual fallows (fallows), alternately managed biennial flower strips (AFS), and perennial flower strips (PFS) can be equally effective in promoting the arable plant vegetation in intensively farmed landscapes within a period of two to three years, but spillover effects into adjacent conventionally managed arable fields are not detectable. In a longer perspective, CFM and annual fallows are generally better suited to promote the establishment of more diverse arable plant communities. Despite the measures, the occurrence of many, formerly typical competitively weak arable plant species has been found to be restricted to very few arable fields. Thus, introducing native rare arable plant species through seed mixtures of local provenance should be considered to prevent their (local) extinction, the reby increasing overall farmland phytodiversity.

Although it has been shown so far that fallows, CFM and flower strips can positively affect the arable flora (Fischer and Milberg, 1997; Kovács-Hostyánszki et al., 2011b; Rode et al., 2018; Van Buskirk and Willi, 2004), there has been little research on which specific measures have the greatest effect in direct comparison over time. This information, however, is crucial for nature conservation efforts. This thesis has presented four ecologically efficient measures to halt the continuing loss of arable plant diversity (Chapter 3). Overall, CFM, fallows, AFS and PFS showed a similar effectiveness within the 3 years of implementation in a total of 67 arable fields. Compared to conventionally managed field edges, the median total and typical plant species number across all measures was doubled ( 9 and 7 vs. 21 and 17 species per plot) with a huge increase in the median plant cover ( 9.5 and $8 \%$ vs. 68.5 and $56.5 \%$ ). That graminoid species can become dominant over several years of implementation and thus threaten the success of agrienvironment measures is known from other studies (Critchley et al., 2006; Critchley and Fowbert, 2000). This risk was prevented by an adequate management of the measures tested in this study (i.e. regular soil disturbance in CFM, fallows and AFS as well as mowing in PFS; Hansson and Fogelfors, 1998; Hilbig, 1996; Rode et al., 2018). As a result, forbs were much stronger supported by all tested measures than graminoids. Sustaining a high coverage of forbs is not only important with respect to arable plant conservation (since about $90 \%$ of the typical arable plant species in Germany are forbs; Hofmeister and Garve, 2006), but can, in turn, also support the pollinating insect abundance by the provision of sufficient nectar and pollen sources (Hawes et al., 2003).

Although all measures have shown similar effects on total herbaceous and typical arable plant species richness and cover, differences in their performance were detectable over time. The cover 
of annual plants and indicator species of High Nature Value farmland was more strongly supported and preserved by CFM and fallows. In contrast, the annual plant cover tended to decline in AFS (with alternating management resulting in only biennial soil disturbance) and PFS (no soil disturbance), while perennial species richness and cover significantly increased over time in these two measures. This underlines findings of other studies that regular, yearly soil disturbance is important to sustainably promote the predominantly annual arable flora by preventing the vegetation succession with perennial plant taxa becoming dominant (Hansson and Fogelfors, 1998; Hilbig, 1996; Tscharntke et al., 2011; Vickery et al., 2009; Westbury et al., 2008). In addition, the cover of indicator species of High Nature Value farmland remained very low in AFS and PFS sites. Considering the above-mentioned, CFM and annual fallows should be given priority if arable plant conservation is the aim (especially if measures are to be implemented at the same location over a longer period of time). The success of those measures is based on the creation of historically typical non-intensive arable habitat conditions most arable plant species have been adapted to. In contrast, flower strips are known to be important for supporting other organism groups, such as insects and farmland birds (Gottschalk and Beeke, 2013; Haaland et al., 2011). However, considering a low sowing density of $1 \mathrm{~g} \mathrm{~m}^{-2}$ (as used in this study in AFS and PFS) also allows the germination of arable plants still present in the soil seed bank, which can additionally support native insect communities (Marshall et al., 2003). Nevertheless, many typical arable plant species were found to be rare and threatened, with red-listed taxa are hardly to be found any more, not even in measure areas (Chapters 2 to 4). This finding can likely be traced back to impoverished soil seed banks due to decades of intensive management (Albrecht et al., 2016; Denys and Tscharntke, 2002; Richner et al., 2015). Thus, including typical arable plants in seed mixtures can help to increase arable plant species richness at field level by refilling impoverished seed banks and preserve rare species from (local) extinction in conventionally intensively managed farmland. The effects of such adapted flower strips on the arable plant vegetation and the related soil seed bank should be further verified in future studies. However, the restricted occurrence of many typical arable plant species to few fields demonstrates the importance of more targeted conservation actions for rare arable plant species, such as the introduction through seed mixtures of local provenance and adapted management regimes (Albrecht et al., 2016; Lang et al., 2018). Seeds of local provenance could be provided by the establishment of conservation fields in the respective regions (Meyer and Leuschner, 2015). At the same time, such conservation fields can also be used for demonstration and education purposes.

As also shown in this thesis, continuous and species-group adapted management of agrienvironment measure sites is essential to ensure and maintain their desired effects (Batáry et al., 2011; Gonthier et al., 2014). In addition to the management adaptions already mentioned (regular soil disturbance and reduced sowing density of crops and flower strips), the exclusion of fertilizers and herbicides (as done for all tested measures in this thesis) is known to strongly positively affect the arable plant vegetation by particularly supporting non-graminoid species and reducing the risk of the nutrient-driven dominance of competitive non-crop plants (Albrecht et al., 2016; Hole et al., 2005). Furthermore, no or reduced nutrient addition likely improve habitat conditions for arable plants by creating less dense crop stands, which allow more light to pass through (Albrecht et al., 2016; Batlla and Benech-Arnold, 2014; Seifert et al., 2014). Both increased fertilizer and herbicide use are considered to have interactive effects, which cannot be completely disentangled from each other (Storkey et al., 2012). Herbicide use may have a more 
negative impact on total plant species richness (Hyvönen and Salonen, 2002), whereas higher amounts of fertilizer inputs can have a more adverse effect on threatened, low-competitive arable plant species (Storkey et al., 2012). To further reduce nitrogen concentration and, thus, supporting species richness especially of rare arable plants (Kleijn et al., 2009; Storkey et al., 2012), mowing and removing biomass should be considered in the management of agrienvironment measures in nutrient-rich sites. However, reductions of nutrient availability to levels before intensive fertilization can take decades, e.g. in the case of nitrogen and phosphorus (Horrocks et al., 2014).

In general, the landscape context should also be considered when aiming to increase farmland biodiversity (Batáry et al., 2011; Tscharntke et al., 2011). In this context, the measure types should be placed carefully with respect to landscape composition, local species pools and practical concerns of farmers. Here, pre-surveys are essential to enable a targeted implementation of the measures. In a given agricultural landscape, several measure types supporting different organism groups should be implemented in combination on a sufficient area. The necessary proportion of agri-environment measures in a given landscape depends on the target species, the quality of the implemented measures and the proportion of still existing (semi-)natural habitats suitable for the respective taxa (Meichtry-Stier et al., 2014; Tscharntke et al., 2011). Furthermore, measures should be linked to each other or/and to existing (semi-)natural habitats to serve as stepping stones for the spread of species and enable their genetic exchange (Saura et al., 2014). A diversity of well-linked measure types on a sufficient area can increase habitat heterogeneity, which is known to positively impact overall farmland biodiversity, especially in simple agricultural landscapes with only a small proportion of (semi-)natural habitats (Benton et al., 2003; KovácsHostyánszki et al., 2011a; Tscharntke et al., 2005). Habitat heterogeneity can also be increased within a given measure, e.g. by the regular renewal of part of the measure area. Such approaches can satisfy habitat requirements of different organism groups, e.g. annually managed areas for arable plants, perennial parts as overwintering possibility for insects (Haaland et al., 2011) and structurally diverse vegetation as breeding and food habitat for farmland birds (Gottschalk and Beeke, 2013). Besides, measures should be implemented for a sufficient time period, since the recovery of species populations may occur with a time lag (Watts et al., 2020). In addition, the revaluation of field-adjacent habitats should be considered, where necessary. For instance, the vegetation of grass strips next to arable fields in the study region was usually found to be species poor and dominated by few grass species, which is very likely the result of long-term inappropriate management practices (Chapter 2). With respect to such impoverished grass strips, perennial flower strips (such as the PFS presented in this thesis) are particularly suitable to improve habitat quality for farmland biodiversity (Vickery et al., 2009). In general, future research is still needed to evaluate the possible synergetic effects created by the linkage of different measure sites as well as of measures and (semi-)natural habitats.

Based on the results of this thesis and research findings of other studies, Table 1 gives an overview of the four tested agri-environment measures (i.e. the impact on the arable plant vegetation and expected influence on other organism groups as well as information about recommended management, site choice and possible agricultural usability). Through the participatory involvement of farmers in the design of the measures and their adaptation for practicability on a modern conventional farm, it is to be expected that these measures will be met with broad acceptance among farmers. Even though CFM and fallows turned out to be the best choice in aiming to promote arable plant diversity in the long-term, studies found that the 
implementation of all tested measure types is justified and important as they create diverse habitat conditions satisfying the requirements of different organism groups of agricultural landscapes (including farmland birds and insects; Gottschalk and Beeke, 2013; Haaland et al., 2011; Tscharntke et al., 2011; Vickery et al., 2009). Thus, all four measure types tested in this thesis can be recommended for consideration as part of national and European agri-environment schemes. However, future research should be carried out to evaluate more precisely the effectiveness of the four measures on different animal groups.

Table 1 Summary table of the four tested agri-environment measure types with respect to their impact on the arable plant vegetation and the expected influence on other organism groups as well as information about recommended management, site choice and possible agricultural usability. These information are based on the findings of this thesis and the following other studies: Albrecht et al., 2016; Becker et al., 2019; Denys and Tscharntke, 2002; Gottschalk and Beeke, 2013; Haaland et al., 2011; Hansson and Fogelfors, 1998; Hilbig, 1996; Hofmeister and Garve, 2006; Kiehl et al., 2014; Rode et al., 2018; Tscharntke et al., 2011; Vickery et al., 2009. Measure types: conservation field margin (CFM), annual fallow (fallow), alternately managed biennial flower strip (AFS) and perennial flower strip (PFS).

\begin{tabular}{|c|c|c|c|c|}
\hline Measure type & CFM & Fallow & AFS & PFS \\
\hline $\begin{array}{l}\text { Effects in the first } \\
\text { years }\end{array}$ & \multicolumn{4}{|c|}{ Very effective within the first two (PFS) to three years (CFM, fallow, AFS) } \\
\hline $\begin{array}{l}\text { Expected long- } \\
\text { term effects }\end{array}$ & \multicolumn{2}{|c|}{$\begin{array}{l}\text { Very effective } \\
\text { due to regular soil disturbance and related continuous } \\
\text { habitat conditions favored by arable plants }\end{array}$} & $\begin{array}{l}\text { Moderately effective } \\
\text { due to only biennial soil } \\
\text { disturbance }\end{array}$ & $\begin{array}{l}\text { Not effective } \\
\text { perennial plant species } \\
\text { will become dominant } \\
\text { due to missing soil } \\
\quad \text { disturbance }\end{array}$ \\
\hline & \multicolumn{4}{|c|}{ Pre-surveys are strongly recommended for site selection! } \\
\hline Site choice & \multicolumn{2}{|c|}{$\begin{array}{l}\text { Especially at sites with a still more diverse arable flora } \\
\text { (including rare arable plant species); less nutrient-rich sites } \\
\text { should be preferred to avoid the dominance of few } \\
\text { competitive strong arable plant species }\end{array}$} & $\begin{array}{l}\text { Species-poor and } \\
\text { nutritious sites, with no } \\
\text { rare arable plant species } \\
\text { present }\end{array}$ & $\begin{array}{l}\text { Species-poor and } \\
\text { nutritious sites; especially } \\
\text { suitable for the } \\
\text { restoration of } \\
\text { impoverished grass strips } \\
\text { next to arable fields }\end{array}$ \\
\hline \multirow{3}{*}{$\begin{array}{l}\text { Recommended } \\
\text { management }\end{array}$} & \multicolumn{4}{|c|}{$\begin{array}{l}\text { Continuous management to maintain measure effects is essential! } \\
\qquad \text { No application of fertilizers or pesticides }\end{array}$} \\
\hline & \multirow[t]{2}{*}{$\begin{array}{c}\text { Annual soil disturbance and } \\
\text { cultivation with crop; reduced } \\
\text { sowing density }(50 \%) \text { or double } \\
\text { space in rows }\end{array}$} & \multirow[t]{2}{*}{$\begin{array}{l}\text { Annual soil disturbance; } \\
\text { no seeding }\end{array}$} & $\begin{array}{l}\text { After establishment, } \\
\text { alternating re-creation } \\
\text { of half of the area from } \\
\text { the } 2 \text { nd year onwards } \\
\text { (including soil } \\
\text { disturbance); seed } \\
\text { mixture: ornamental } \\
\text { and native plant species } \\
\text { (annuals and perennials, } \\
\text { including typical arable } \\
\text { plants) }\end{array}$ & $\begin{array}{l}\text { Establishment by using a } \\
\text { seed mixture with native } \\
\text { perennial plants; cutting } \\
\text { once or twice per year } \\
\text { (depending on growth) to } \\
\text { maintain plant species } \\
\text { richness and prevent } \\
\text { predominance of } \\
\text { graminoids }\end{array}$ \\
\hline & & & \multicolumn{2}{|c|}{$\begin{array}{l}\text { Removal of cuttings from measure sites (if possible) } \\
\text { to reduce soil nutrient content; use of native plant } \\
\text { seeds of local provenance; reduced sowing density to } \\
\text { promote naturally present arable plant species }\end{array}$} \\
\hline $\begin{array}{l}\text { Agricultural } \\
\text { usability }\end{array}$ & $\begin{array}{l}\text { Harvesting in cereals is possible; } \\
\text { decreasing yield over time is } \\
\text { expected (due to missing } \\
\text { fertilizer application) }\end{array}$ & & $\begin{array}{l}\text { Possible use of cuttings } \\
\text { for hay production }\end{array}$ \\
\hline $\begin{array}{l}\text { Influence on other } \\
\text { organism groups }\end{array}$ & \multicolumn{4}{|c|}{$\begin{array}{c}\text { Based on other studies, all measure types are expected to create valuable habitats to support other organism groups of } \\
\text { agricultural landscapes, such as farmland birds and insects }\end{array}$} \\
\hline Remarks & \multicolumn{2}{|c|}{$\begin{array}{l}\text { Consideration of long-term implementation and seeding of } \\
\text { (rare) arable plant species of local provenance (recovery of } \\
\text { soil seed banks); alternately, biennial soil disturbance of half } \\
\text { of the area of fallows can improve the effects on other } \\
\text { organism groups (e.g. undisturbed areas for a certain time as } \\
\text { feeding/breeding/wintering habitat) }\end{array}$} & $\begin{array}{l}\text { Long-term effects on } \\
\text { the segetal flora can be } \\
\text { improved by a high } \\
\text { proportion of native } \\
\text { arable plant species in } \\
\text { the seed mixture }\end{array}$ & - \\
\hline
\end{tabular}


Beside the findings described above, spillover effects of measures into the adjacent conventionally managed fields were not detectable. This can be attributed almost certainly to the intensive management of the cultivated crops in the neighboring fields (in particular the use of highly effective herbicides). This is an important finding from an agricultural point of view, as farmers are often concerned that implemented agri-environment measures could also affect the adjacent cultivated crop stands by weed infestation. The present results serve well to weaken this concern.

iii) As weather conditions can strongly affect plant species richness and abundance in arable habitats, leading to significant variations between years, perennial field trials are essential to draw reliable conclusions about the effectiveness of agri-environment measures.

Chapter 3 demonstrated that interannual changes in precipitation and temperature regimes can significantly affect the plant species richness and abundance in arable habitats, including sites where agri-environment measures are implemented. The influence of weather conditions on the arable plant community composition was also shown in other studies (Fried et al., 2008; Lososová and Cimalová, 2009; Pinke et al., 2012). This highlights the necessity of evaluating the effects of agri-environment measures over several years. Short-term studies (e.g. commonly used one-year observations) run the risk of drawing wrong conclusions about the effectiveness of agrienvironment measures because, depending on the observed organism group, interannual variations in weather conditions may strongly influence the overall species richness and abundance. Thus, long-term trends in the community structure can remain hidden (e.g. changes in the proportions of specific plant life forms and trait spectra). Furthermore, the positive effects on farmland biodiversity can also occur with a time lag to the initial establishment of conservation measures (Watts et al., 2020). Beside long-term observations, reviews and metaanalyses are a more reliable assessment tool of the effectiveness of agri-environment measures since these include studies of various years and locations. But in order to be able to record timedependent effects of measures on farmland biodiversity, field studies over several years are essential.

In addition, the identified influence of weather conditions on the effectiveness of measures should also be considered in the context of climate change. Global climate change is accompanied by rising temperatures and changing precipitation regimes leading to the fragmentation and loss of habitats for various organism groups (Diffenbaugh and Field, 2013; Mantyka-Pringle et al., 2012). As the impact of climate change is considered to become more severe in the future (depending on the geographic region; Diffenbaugh and Field, 2013), it will not only impact future crop yields (Supit et al., 2012; Trnka et al., 2014) but also likely influence the performance of agri-environment measures. For example, warmer and drier weather conditions in spring and early summer may result in topsoil desiccation, which negatively affects the germination and establishment of sown plant species in flower strips, as it has been observed in many places of the study region in 2018 (with very warm and dry weather conditions from April to July; Chapter 3). Thus, future management changes may become necessary to preserve the effectiveness of those measures (e.g. appropriate site selection, the time of management (soil cultivation, sowing) or chosen species of seed mixtures). Details about how climate change can alter the effectiveness of measures and which adjustments in management can be useful have to be investigated in future studies. 
iv) The diversity of today's strongly impoverished arable flora can be examined most effectively by elongated field edge plots of $500 \times 1 \mathrm{~m}$. This arable plant survey method is recommended as standard monitoring approach in conventional farmland.

Chapter 4 described the considerable variation in current arable plant survey methods with respect to plots sizes (ranging from 0.1 to $5000 \mathrm{~m}^{2}$ ), shape (square or rectangular plots either coherent or splitted) and in-field location (edge or interior). By comparing six different survey methods in 45 conventionally managed arable fields (field 'Interior' plots $(50 \times 2 \mathrm{~m}$ ); field edge plots: 'Edge_30' $(30 \times 2 \mathrm{~m})$, 'Edge_50' $(50 \times 2 \mathrm{~m})$, 'Edge_500' $(500 \times 1 \mathrm{~m})$, 'Subplots' (four dispersed plots of $5 \times 1 \mathrm{~m})$ and 'Corner' plots $(50 \times 2 \mathrm{~m})$ ), it was found that they vary considerably in their species detection rate. Therefore, it is difficult to compare the results of studies based on different survey methods. Interior plots were shown to be least effective in characterizing typical arable plant diversity at field level (median species detection rate of solely $8.3 \%$ ). Edge_500 plots had by far the best detection rate of the field's typical arable plant species pool (median 75.6\%) with a justifiable expenditure of time (median 16-23 min, dependent on the observed crop type) followed by Corner plots (45.8\%, 9-11 min). Thus, to be able to reliably characterize todays strongly impoverished arable plant diversity of a field, elongated plots at the field edge should be surveyed because this is where locally typical arable plant species can still exist. Field corners support significantly more arable plant species than the middle of the field edge, likely driven by less intensive management in these field areas, which are more difficult to reach for weed control measures (e.g. herbicide applications). Thus, the use of elongated field edge plots of $500 \times 1 \mathrm{~m}$ including at least one field corner can be recommended as effective future standard survey method for arable plant monitoring in conventionally managed farmland. Corner plots of $50 \times 2 \mathrm{~m}$ can be recommended if time constraints rule out the Edge_500 plots. In addition, for the monitoring of threatened, red-listed arable plant species, total field edge perimeter counts should be used. With only a few occurrences and a very patchy distribution in conventional farmland, these species are otherwise hardly detectable. Nevertheless, besides answering specific research and conservation questions, defining a general standard survey method for arable plants, as the proposed Edge_500 plots, is important to ensure the spatial and temporal comparability of the collected data. However, the effectiveness of the tested arable plant survey methods in this thesis should be validated in future studies with respect to other regions (e.g. farmland harboring a higher floristic diversity such as the Mediterranean), additional crop species, further management systems (e.g. organic farming) and different landscape complexities (i.e. simple vs. complex).

In order to counteract the ongoing decline of farmland biodiversity and ecosystem services essential for agricultural production and human well-being (Macfadyen et al., 2012), a continuous and regular monitoring of species living in agricultural landscapes is crucial to document longterm changes and to assess the vulnerability status of threatened taxa and assemblages. Based on these data and the knowledge of important drivers of biodiversity loss appropriate conservation measures can be taken. Several monitoring schemes considering farmland biodiversity have been implemented at national level (e.g. in Canada, Germany, Norway, Sweden, Switzerland or United Kingdom; Herzog and Franklin, 2016). But many of these approaches focus on some indicator species, a single or only a few organism groups (e.g. farmland birds or plants), and/or are based on different methodologies (Herzog and Franklin, 2016). As farmland species groups are linked with each other through the food web, it is important to monitor the status of all relevant taxa in agroecosystems, so that timely and targeted actions can be taken before the trophic web and 
ecosystem functioning are negatively affected (Hyvönen and Huusela-Veistola, 2008; Marshall et al., 2003; Médiène et al., 2011). Thus, a harmonization of monitoring approaches should be aimed for in order to record the state and temporal changes of typical farmland species groups and the factors influencing them on a broader scale. This would allow the development of targeted, international nature conservation strategies. Some approaches at broader scale already exist, e.g. for Europe or North America, but they are also restricted to few species groups, i.e.: the Pan European Common Bird Monitoring Scheme (https://pecbms.info/), the European Butterfly Monitoring Scheme (https://butterfly-monitoring.net/) or the North American Breeding Bird Survey (https://www.usgs.gov/centers/pwrc/science/north-american-breedingbird-survey). In addition, these approaches rely on the input of volunteers, which may affect the quality and statistical representativeness of the individual records (Herzog and Franklin, 2016). Therefore, efforts should be made to further develop such programs, including the monitoring of other organism groups on the basis of appropriate standardized methods. Moreover, when introducing a new standardized national or international survey method, traditional sampling methods should be continued as a subset of the monitoring approach to maintain comparability with historical records. 


\subsection{Final remarks and outlook}

This thesis demonstrated that the arable flora of conventionally intensive farmland in Northwest Germany is strongly impoverished. This finding is reflected by other studies investigating Western and Central European conventional farmland. The loss not only concerns arable plants but also other organism groups of the agricultural landscape (e.g. farmland birds and insects). Therefore, national and European policies must take urgent action to counteract the loss of biodiversity in European agricultural landscapes. It needs ecologically effective agri-environment measures with sufficient funding for compensation that are widely accepted among land users and implemented on a large scale. Thus, cooperative processes are needed that define measures by the consideration of agronomic and ecological perspectives as well as their suitability in the agricultural practice (e.g. taking into account concerns of farmers with regard to the requirements of the funding policy guidelines). Such approaches should include all relevant stakeholders (e.g. farmers, landlords, hunters, beekeepers, scientists, nature conservation authorities or agricultural consultations) to consider all important aspects resulting in highly effective and accepted measures based on a broad consensus. In doing so, cooperative approaches can be a successful instrument to increase farmers' willingness to implement agri-environment measures by promoting their understanding of the significance of biodiversity conservation in agricultural landscapes and improving the practical feasibility of measures. Such a participative approach was successfully pursued in the research project MEDIATE underlying this thesis and enjoyed great popularity among the participants. In the implementation process of measures, regional nature conservation priorities and the suitable location of measures should be considered to make them effective at landscape level. Therefore, the measures should have flexible elements in terms of management so that they can be adapted to different regional circumstances and conservation focusses. Furthermore, the farmer should be accompanied by an expert advisor during the implementation process in order to clarify any problems that may arise and to preserve the effectiveness and acceptance of measures in the long term. These advisory services should be provided on a continuous basis, preferably integrated in agricultural authorities, and the advisors have to be trained in both agricultural and ecological matters and need advanced communication skills.

To reliably assess the conservation status of farmland biodiversity and the effectiveness of implemented measures, there is an urgent need for an ongoing, comprehensive and methodologically standardized (taxa-dependent) European monitoring system of farmland biodiversity, whose continued existence should be secured by long-term financing within the framework of the European Common Agricultural Policy. Based on such monitoring data, appropriate conservation strategies can be (further) developed to halt the continuing loss of biodiversity and associated ecosystem services in agricultural landscapes. 


\section{References}

Albrecht, H., Cambecèdes, J., Lang, M., Wagner, M., 2016. Management options for the conservation of rare arable plants in Europe. Bot. Lett. 163, 389-415. https://doi.org/10.1080/23818107.2016.1237886

Batáry, P., Baldi, A., Kleijn, D., Tscharntke, T., 2011. Landscape-moderated biodiversity effects of agri-environmental management: a meta-analysis. Proc. R. Soc. B Biol. Sci. 278, 1894-1902. https://doi.org/10.1098/rspb.2010.1923

Batáry, P., Gallé, R., Riesch, F., Fischer, C., Dormann, C.F., Mußhoff, O., Császár, P., Fusaro, S., Gayer, C., Happe, A.-K., Kurucz, K., Molnár, D., Rösch, V., Wietzke, A., Tscharntke, T., 2017. The former Iron Curtain still drives biodiversityprofit trade-offs in German agriculture. Nat. Ecol. Evol. 1, 1279-1284. https://doi.org/10.1038/s41559-017-0272-x

Batlla, D., Benech-Arnold, R.L., 2014. Weed seed germination and the light environment: Implications for weed management: Light control of weed seed germination. Weed Biol. Manag. 14, 77-87. https://doi.org/10.1111/wbm.12039

Becker, N., Muchow, T., Schmerlzer, M., 2019. AgrarNatur-Ratgeber - Arten erkennen - Maßnahmen umsetzen - Vielfalt bewahren. Hrsg. Stiftung Rheinische Kulturlandschaft, Bonn

Benton, T.G., Vickery, J.A., Wilson, J.D., 2003. Farmland biodiversity: is habitat heterogeneity the key? Trends Ecol. Evol. 18, 182-188. https://doi.org/10.1016/S0169-5347(03)00011-9

Critchley, C.N.R., Fowbert, J.A., 2000. Development of vegetation on set-aside land for up to nine years from a national perspective. Agric. Ecosyst. Environ. 79, 159-174. https://doi.org/10.1016/S0167-8809(99)00155-3

Critchley, C.N.R., Fowbert, J.A., Sherwood, A.J., 2006. The effects of annual cultivation on plant community composition of uncropped arable field boundary strips. Agric. Ecosyst. Environ. 113, 196-205. https://doi.org/10.1016/j.agee.2005.05.013

Denys, C., Tscharntke, T., 2002. Plant-insect communities and predator-prey ratios in field margin strips, adjacent crop fields, and fallows. Oecologia 130, 315-324. https://doi.org/10.1007/s004420100796

Diffenbaugh, N.S., Field, C.B., 2013. Changes in Ecologically Critical Terrestrial Climate Conditions. Science 341, 486-492. https://doi.org/10.1126/science.1237123

Donald, P.F., Green, R.E., Heath, M.F., 2001. Agricultural intensification and the collapse of Europe's farmland bird populations. Proc. R. Soc. B Biol. Sci. 268, 25-29. https://doi.org/10.1098/rspb.2000.1325

Donald, P.F., Sanderson, F.J., Burfield, I.J., van Bommel, F.P.J., 2006. Further evidence of continent-wide impacts of agricultural intensification on European farmland birds, 1990-2000. Agric. Ecosyst. Environ. 116, 189-196. https://doi.org/10.1016/j.agee.2006.02.007

Fagúndez, J., 2014. The Paradox of Arable Weeds: Diversity, Conservation, and Ecosystem Services of the Unwanted, in: Benkeblia, N. (Ed.), Agroecology, Ecosystems, and Sustainability, Advances in Agroecology. CRC Press, pp. 139-150. https://doi.org/10.1201/b17775-8

Fischer, A., Milberg, P., 1997. Effects on the flora of extensified use of field margins. Swed. J. Agric. Res. Swed.

Fried, G., Norton, L.R., Reboud, X., 2008. Environmental and management factors determining weed species composition and diversity in France. Agric. Ecosyst. Environ. 128, 68-76. https://doi.org/10.1016/j.agee.2008.05.003

Fried, G., Petit, S., Dessaint, F., Reboud, X., 2009. Arable weed decline in Northern France: Crop edges as refugia for weed conservation? Biol. Conserv. 142, 238-243. https://doi.org/10.1016/j.biocon.2008.09.029

Gonthier, D.J., Ennis, K.K., Farinas, S., Hsieh, H.-Y., Iverson, A.L., Batary, P., Rudolphi, J., Tscharntke, T., Cardinale, B.J., Perfecto, I., 2014. Biodiversity conservation in agriculture requires a multi-scale approach. Proc. R. Soc. B Biol. Sci. 281, 20141358-20141358. https://doi.org/10.1098/rspb.2014.1358

Gottschalk, E., Beeke, W., 2013. The partridge conservation project at the district of Göttingen-management of flower strips. Julius-Kühn-Arch. 104-111

Haaland, C., Naisbit, R.E., Bersier, L.-F., 2011. Sown wildflower strips for insect conservation: a review: Wildflower strips for insect conservation. Insect Conserv. Divers. 4, 60-80. https://doi.org/10.1111/j.1752-4598.2010.00098.x

Hansson, M., Fogelfors, H., 1998. Management of permanent set-aside on arable land in Sweden. J. Appl. Ecol. 35, 758-771. https://doi.org/10.1046/j.1365-2664.1998.355350.x

Hawes, C., Haughton, A.J., Osborne, J.L., Roy, D.B., Clark, S.J., Perry, J.N., Rothery, P., Bohan, D.A., Brooks, D.R., Champion, G.T., Dewar, A.M., Heard, M.S., Woiwod, I.P., Daniels, R.E., Young, M.W., Parish, A.M., Scott, R.J., Firbank, L.G., Squire, G.R., 2003. Responses of plants and invertebrate trophic groups to contrasting herbicide regimes in the Farm Scale Evaluations of genetically modified herbicide-tolerant crops. Philos. Trans. R. Soc. B Biol. Sci. 358, 1899-1913. https://doi.org/10.1098/rstb.2003.1406

Herzog, F., Franklin, J., 2016. State-of-the-art practices in farmland biodiversity monitoring for North America and Europe. Ambio 45, 857-871. https://doi.org/10.1007/s13280-016-0799-0

Hilbig, W., 1996. Die Vegetation mehrjähriger Ackerbrachen und ihre Veränderung im Verlaufe eines fünfjährigen Beobachtungszeitraumes. Angew. Bot. 70, 57-75

Hofmeister, H., Garve, E., 2006. Lebensraum Acker, Reprint der 2. neubearbeiteten Auflage. ed. Verlag Kessel, Remagen 
Hole, D.G., Perkins, A.J., Wilson, J.D., Alexander, I.H., Grice, P.V., Evans, A.D., 2005. Does organic farming benefit biodiversity? Biol. Conserv. 122, 113-130. https://doi.org/10.1016/j.biocon.2004.07.018

Horrocks, C.A., Dungait, J.A.J., Cardenas, L.M., Heal, K.V., 2014. Does extensification lead to enhanced provision of ecosystems services from soils in UK agriculture? Land Use Policy 38, 123-128. https://doi.org/10.1016/j.landusepol.2013.10.023

Hyvönen, T., Huusela-Veistola, E., 2008. Arable weeds as indicators of agricultural intensity - A case study from Finland. Biol. Conserv. 141, 2857-2864. https://doi.org/10.1016/j.biocon.2008.08.022

Hyvönen, T., Salonen, J., 2002. Weed species diversity and community composition in cropping practices at two intensity levels-a six-year experiment. Plant Ecol. 159, 73-81

Kiehl, K., Kirmer, A., Shaw, N., Tischew, S. (Eds.), 2014. Guidelines for native seed production and grassland restoration. Cambridge Scholars Publ, Newcastle upon Tyne, UK.

Kleijn, D., Kohler, F., Baldi, A., Batary, P., Concepcion, E.D., Clough, Y., Diaz, M., Gabriel, D., Holzschuh, A., Knop, E., Kovacs, A., Marshall, E.J.P., Tscharntke, T., Verhulst, J., 2009. On the relationship between farmland biodiversity and land-use intensity in Europe. Proc. R. Soc. B Biol. Sci. 276, 903-909. https://doi.org/10.1098/rspb.2008.1509

Kolářová, M., Tyšer, L., Soukup, J., 2013. Diversity of current weed vegetation on arable land in selected areas of the Czech Republic \&nbsp; Plant Soil Environ. 59, 208-213. https://doi.org/10.17221/783/2012-PSE

Kovács-Hostyánszki, A., Batáry, P., Báldi, A., Harnos, A., 2011a. Interaction of local and landscape features in the conservation of Hungarian arable weed diversity: Weed richness in Hungarian cereal fields. Appl. Veg. Sci. 14, 40-48. https://doi.org/10.1111/j.1654-109X.2010.01098.x

Kovács-Hostyánszki, A., Kőrösi, Á., Orci, K.M., Batáry, P., Báldi, A., 2011b. Set-aside promotes insect and plant diversity in a Central European country. Agric. Ecosyst. Environ. 141, 296-301. https://doi.org/10.1016/j.agee.2011.03.004

Lang, M., Prestele, J., Wiesinger, K., Kollmann, J., Albrecht, H., 2018. Reintroduction of rare arable plants: seed production, soil seed banks, and dispersal 3 years after sowing: Reintroduction of rare arable plants. Restor. Ecol. https://doi.org/10.1111/rec.12696

Lososová, Z., Cimalová, Š., 2009. Effects of different cultivation types on native and alien weed species richness and diversity in Moravia (Czech Republic). Basic Appl. Ecol. 10, 456-465. https://doi.org/10.1016/j.baae.2008.11.001

Macfadyen, S., Cunningham, S.A., Costamagna, A.C., Schellhorn, N.A., 2012. Managing ecosystem services and biodiversity conservation in agricultural landscapes: are the solutions the same?: Managing ecosystem services and biodiversity conservation. J. Appl. Ecol. 49, 690-694. https://doi.org/10.1111/j.1365-2664.2012.02132.x

Mantyka-Pringle, C.S., Martin, T.G., Rhodes, J.R., 2012. Interactions between climate and habitat loss effects on biodiversity: a systematic review and meta-analysis. Glob. Change Biol. 18, 1239-1252. https://doi.org/10.1111/j.13652486.2011.02593.x

Maréchal, P.-Y., Henriet, F., Vancutsem, F., Bodson, B., 2012. Ecological review of black-grass (Alopecurus myosuroides Huds.) propagation abilities in relationship with herbicide resistance. Biotechnol Agron Soc Env. 11

Marshall, E.J.P., Brown, V.K., Boatman, N.D., Lutman, P.J.W., Squire, G.R., Ward, L.K., 2003. The role of weeds in supporting biological diversity within crop fields. Weed Res. 43, 77-89. https://doi.org/10.1046/j.1365-3180.2003.00326.x

Médiène, S., Valantin-Morison, M., Sarthou, J.-P., de Tourdonnet, S., Gosme, M., Bertrand, M., Roger-Estrade, J., Aubertot, J.-N., Rusch, A., Motisi, N., Pelosi, C., Doré, T., 2011. Agroecosystem management and biotic interactions: a review. Agron. Sustain. Dev. 31, 491-514. https://doi.org/10.1007/s13593-011-0009-1

Meichtry-Stier, K.S., Jenny, M., Zellweger-Fischer, J., Birrer, S., 2014. Impact of landscape improvement by agri-environment scheme options on densities of characteristic farmland bird species and brown hare (Lepus europaeus). Agric. Ecosyst. Environ. 189, 101-109. https://doi.org/10.1016/j.agee.2014.02.038

Meyer, S., Bergmeier, E., Becker, T., Wesche, K., Krause, B., Leuschner, C., 2015. Detecting long-term losses at the plant community level - arable fields in Germany revisited. Appl. Veg. Sci. 18, 432-442. https://doi.org/10.1111/avsc.12168

Meyer, S., Leuschner, C. (Eds.), 2015. 100 Äcker für die Vielfalt. Göttingen University Press, Göttingen. https://doi.org/10.17875/gup2015-815

Meyer, S., Wesche, K., Krause, B., Leuschner, C., 2013. Dramatic losses of specialist arable plants in Central Germany since the 1950s/60s - a cross-regional analysis. Divers. Distrib. 19, 1175-1187. https://doi.org/10.1111/ddi.12102

Moray, R., 2005. Bromus-Arten in Winterweizen: Verbreitung, Bedeutung und Populationsdynamik. Hohenheim, Fakultät Agrarwissenschaften, Institut für Phytomedizin, Hohenheim

Moss, S., 2017. Black-grass (Alopecurus myosuroides): Why has this Weed become such a Problem in Western Europe and what are the Solutions? Outlooks Pest Manag. 28, 207-212. https://doi.org/10.1564/v28_oct_04

Pinke, G., Karácsony, P., Czúcz, B., Botta-Dukát, Z., Lengyel, A., 2012. The influence of environment, management and site context on species composition of summer arable weed vegetation in Hungary. Appl. Veg. Sci. 15, 136-144. https://doi.org/10.1111/j.1654-109X.2011.01158.x

Rahmann, G., 2011. Biodiversity and Organic farming: What do we know? VTI Agric. Forstery Res. 3, 189-208 
Richner, N., Holderegger, R., Linder, H.P., Walter, T., 2015. Reviewing change in the arable flora of Europe: a meta-analysis. Weed Res. 55, 1-13. https://doi.org/10.1111/wre.12123

Rode, M., Lischka, A., Schulz, G., 2018. Auswirkung von Blühstreifen auf die Biodiversität der Ackerbegleitflora in maisdominierten Agrarlandschaften. Umw. Raum 9, 81-114. https://doi.org/10.15488/3697

Rolston, M.P., Archie, W.J., Reddy, K., Dastgheib, F., 2003. Grass weed control and herbicide tolerance in cereals. N. Z. Plant Prot. 56, 220-226. https://doi.org/10.30843/nzpp.2003.56.6095

Sánchez-Bayo, F., Wyckhuys, K.A.G., 2019. Worldwide decline of the entomofauna: A review of its drivers. Biol. Conserv. 232, 8-27. https://doi.org/10.1016/j.biocon.2019.01.020

Saura, S., Bodin, Ö., Fortin, M.-J., 2014. Stepping stones are crucial for species’ long-distance dispersal and range expansion through habitat networks. J. Appl. Ecol. 51, 171-182. https://doi.org/10.1111/1365-2664.12179

Seifert, C., Leuschner, C., Meyer, S., Culmsee, H., 2014. Inter-relationships between crop type, management intensity and light transmissivity in annual crop systems and their effect on farmland plant diversity. Agric. Ecosyst. Environ. 195, 173182. https://doi.org/10.1016/j.agee.2014.05.022

Statistical Office of the European Union (EUROSTAT), 2018. Main farmland use by NUTS 2 regions \& organic crop area by agricultural production methods and crops. [WWW Document]. URL https://ec.europa.eu/eurostat/web/agriculture/data/database (accessed 10.24.18)

Still, K.S., 2007. A future for rare arable plants. Aspects of Applied Biology, 81, 175-182

Storkey, J., Meyer, S., Still, K.S., Leuschner, C., 2012. The impact of agricultural intensification and land-use change on the European arable flora. Proc. R. Soc. B Biol. Sci. 279, 1421-1429. https://doi.org/10.1098/rspb.2011.1686

Storkey, J., Westbury, D.B., 2007. Managing arable weeds for biodiversity. Pest Manag. Sci. 63, 517-523. https://doi.org/10.1002/ps.1375

Supit, I., van Diepen, C.A., de Wit, A.J.W., Wolf, J., Kabat, P., Baruth, B., Ludwig, F., 2012. Assessing climate change effects on European crop yields using the Crop Growth Monitoring System and a weather generator. Agric. For. Meteorol. 164, 96-111. https://doi.org/10.1016/j.agrformet.2012.05.005

Trnka, M., Rötter, R.P., Ruiz-Ramos, M., Kersebaum, K.C., Olesen, J.E., Žalud, Z., Semenov, M.A., 2014. Adverse weather conditions for European wheat production will become more frequent with climate change. Nat. Clim. Change 4, 637643. https://doi.org/10.1038/nclimate2242

Tscharntke, T., Batáry, P., Dormann, C.F., 2011. Set-aside management: How do succession, sowing patterns and landscape context affect biodiversity? Agric. Ecosyst. Environ. 143, 37-44. https://doi.org/10.1016/j.agee.2010.11.025

Tscharntke, T., Klein, A.M., Kruess, A., Steffan-Dewenter, I., Thies, C., 2005. Landscape perspectives on agricultural intensification and biodiversity - ecosystem service management. Ecol. Lett. 8, 857-874. https://doi.org/10.1111/j.1461-0248.2005.00782.x

Tuck, S.L., Winqvist, C., Mota, F., Ahnström, J., Turnbull, L.A., Bengtsson, J., 2014. Land-use intensity and the effects of organic farming on biodiversity: a hierarchical meta-analysis. J. Appl. Ecol. 51, 746-755. https://doi.org/10.1111/13652664.12219

Van Buskirk, J., Willi, Y., 2004. Enhancement of farmland biodiversity within set-aside land. Conserv. Biol. 18, 987-994. https://doi.org/10.1111/j.1523-1739.2004.00359.x

Vickery, J.A., Feber, R.E., Fuller, R.J., 2009. Arable field margins managed for biodiversity conservation: A review of food resource provision for farmland birds. Agric. Ecosyst. Environ. 133, 1-13. https://doi.org/10.1016/j.agee.2009.05.012

von Arx, G., Biosshard, A., Dietz, H., 2002. Land-use intensity and border structures as determinants of vegetation diversity in an agricultural area. Bull. Geobot. Inst. ETH 68, 3-15

Vořřšek, P., Jiguet, F., van Strien, A., Škorpilová, J., Klvaňová, A., Gregory, R., 2010. Trends in abundance and biomass of widespread European farmland birds: how much have we lost. BOU Proc. Lowl. Farml. Birds III 1-24

Watts, K., Whytock, R.C., Park, K.J., Fuentes-Montemayor, E., Macgregor, N.A., Duffield, S., McGowan, P.J.K., 2020. Ecological time lags and the journey towards conservation success. Nat. Ecol. Evol. 4, 304-311. https://doi.org/10.1038/s41559019-1087-8

Westbury, D.B., Woodcock, B.A., Harris, S.J., Brown, V.K., Potts, S.G., 2008. The effects of seed mix and management on the abundance of desirable and pernicious unsown species in arable buffer strip communities. Weed Res. 48, 113-123. https://doi.org/10.1111/j.1365-3180.2007.00614.x

Westphal, C., Steffan-Dewenter, I., Tscharntke, T., 2003. Mass flowering crops enhance pollinator densities at a landscape scale: Flowering crops enhance pollinator densities. Ecol. Lett. 6, 961-965. https://doi.org/10.1046/j.14610248.2003.00523.x 


\section{Acknowledgments}

The completion of this thesis would not have been possible without the support of many people.

First of all, I would like to express my sincere thanks to my first supervisor, Prof. Dr. Christoph Leuschner, who gave me the opportunity to do my $\mathrm{PhD}$ in this exciting topic and supported and guided me during this time. My special thanks also go to Prof. Dr. Erwin Bergmeier, my second supervisor, for his great helpfulness and support during my $\mathrm{PhD}$. I am also grateful to Prof. Dr. Teja Tscharntke for supportive discussions and his willingness to be available as the third member of my thesis committee.

Furthermore, I would like to thank my colleagues and friends for many inspiring talks and discussions during my PhD. I thank especially Roman Link, Stefan Meyer, Eva-Maria Rathke, Friederike Riesch, Jenny Schellenberg, Laura Sutcliffe, Clara-Sophie van Waveren and Annemarie Wurz.

I also thank all the people who supported me during the field work. Special thanks to Christina Ewerhardy, Stefan Mecke and Fionn Pape. Many thanks also to all farmers involved in the project for granting access to their fields, implementing and maintaining the agri-environment measures, and providing management data.

This thesis was embedded into the research project 'Development of targeted and efficient schemes to increase biodiversity in agricultural landscapes (MEDIATE)' which received funding from the German Federal Environmental Foundation (DBU). I am grateful for the financial support provided by the $\mathrm{DBU}$, which made the project and thus my $\mathrm{PhD}$ possible.

Finally, I would like to thank my husband very much, who always accompanied me with patience and helpful advice throughout my PhD.

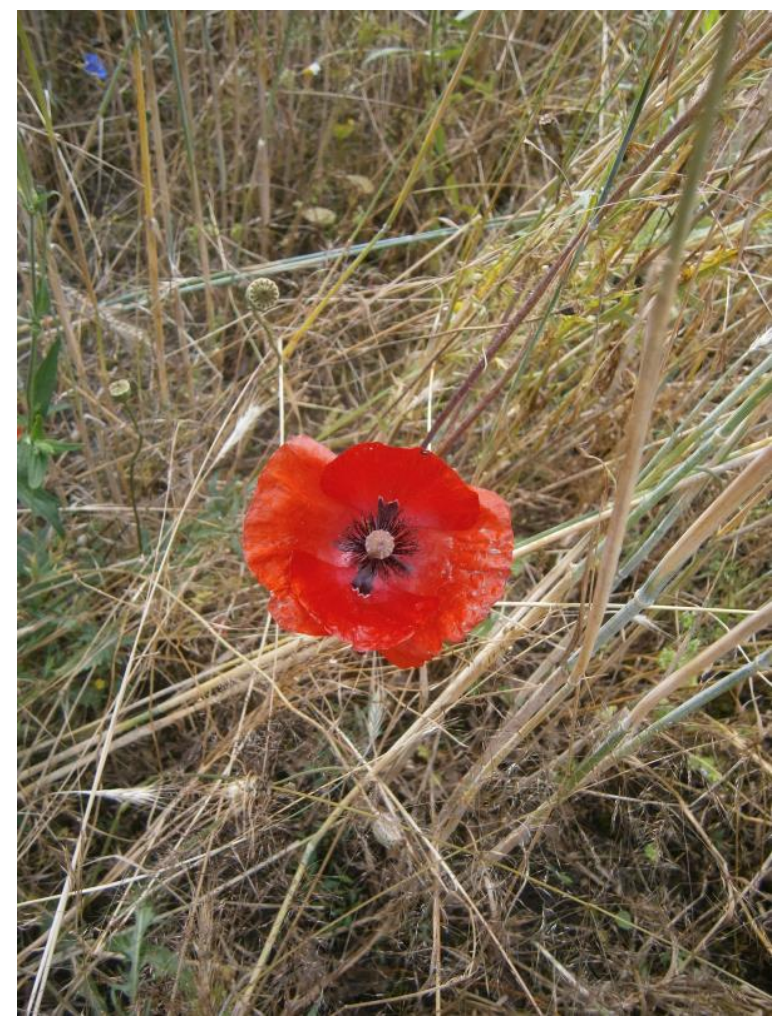




\section{Publications and presentations}

\section{Journal publications (published)}

- Wietzke, A., Albert, K., Bergmeier, E., Sutcliffe, L. M. E, van Waveren, C.-S., Leuschner, C. (2020): Flower strips, conservation field margins and fallows promote the arable flora in conventional intensive cropland: results of a 4-year study. Agriculture, Ecosystems and Environment, Volume 304. https://doi.org/10.1016/j.agee.2020.107142.

- WietzKe, A., Leuschner, C. (2020): Surveying the arable plant diversity of conventionally managed farmland: a comparison of methods. Environ. Monit. Assess. 192, 98. https://doi.org/10.1007/s10661019-8042-7.

- Wietzke, A., Bergmeier, E. (2019): Bedeutung von Parks und Friedhöfen für einheimische und verwilderte Frühjahrs-Geophyten. Erforsch. Erhalt. Phytodiversität Festschr. Zum 70. Geburtstag für Prof Dr Dietmar Brand. 13, 171-197. HTTPS://DOI.ORG/10.24355/DBBS.084-201902080856-0.

- Wietzke, A., Westphal, C., Gras, P., Kraft, M., Pfohl, K., Karlovsky, P., Pawelzik, E., TSCHARNTKE, T. \& SMIT, I. (2018): Insect pollination as a key factor for strawberry physiology and marketable fruit quality. Agriculture, Ecosystems and Environment 258: 197-204.

- Batáry, P., Gallé, R., Riesch, F., Fischer, C., Dormann, C.F., Mubhoff, O., Császár, P., Fusaro, S., Gayer, C., Happe, A.-K., Kurucz, K., Molnár, D., Rösch, V., Wietzke, A. \& Tscharntke, T. (2017): The former iron curtain still drives biodiversity-profit trade-offs in German agriculture. Nature Ecology \& Evolution 1: 1279-1284.

- Bergmeier, E.; Cloos, A.; Jonas, R.; Möhler, H.; Richter-Harder, N.; Schrader, J.; Teichmann, M. \& WOLTER F. (2014): Zeigerpflanzen historischer Weinberge und Mauern der Saale-Unstrut-Region. In: Siegesmund, S.; Hoppert, M. \& Epperlein, K. (Hg.) (2014): Natur Stein Kultur Wein. Zwischen Saale und Unstrut. 1. Aufl. Halle (Saale), Mitteldeutscher Verlag.

\section{Journal publications (submitted)}

- Hutchinson, L., Breeze, T., Oliver, T., Bailes, E., Bruenjes, L., Campbell, A., Erhardt, A., De Groot, G. A., Földesi, R., García, D., Goulson, D., Hainaut, H., Hambäck, P., Holzschuh, A., Jauker, F., KLatT, B., Kiein, A., Kleijn, D., Kovacs-Hostyanszki, A., Krimmer, E., McKerchar, M., Miñarro, M., Phillips, B., Potts, S., Pufal, G., RadzeviČiūtè, R., SAMnegard, U., Schulze, J., Shaw, R., TscharntKe, T., Vereecken, N., Westphal, C., Wietzke, A., Woodcock, B., Roberts, S., GARRATT, M.: Using field survey and ecological trait data to determine the wild bee pollinators of crops in Great Britain. Journal of Applied Ecology (submitted).

- Wietzke, A., VAn Waveren, C.-S., Bergmeier, E., Meyer, S., Leuschner, C.: Current state and drivers of arable plant diversity in conventionally managed farmland in Northwest Germany. Biodiversity and Conservation (submitted).

\section{Presentation at conferences}

- Heinrich, B., Wietzke, A., Polaschegg, M., van Waveren, C.-S. (2019): Entwicklung und Operationalisierung eines Zielkonzepts zur Erreichung gesellschaftlich erwünschter Biodiversitätsziele in der Agrarlandschaft. Poster presentation. 59th GEWISOLA Annual Conference 2019, Braunschweig. Conference topic: Agriculture and rural areas in the course of societal change.

- WietzKe, A., VAn Waveren, C.-S., Leuschner, C. (2018): Drivers of weed diversity and abundance in highly intensive agricultural landscapes of Northern Germany. Poster presentation. First international Biomove Symposium 2018, Integrating biodiversity research with movement ecology, Potsdam. Conference topic: Living in a landscape mosaic. 
- Wietzke, A., Dauber, J., Sanders, J., Polaschegg, M., Leuschner, C. (2018): Mediate Participative solutions to promote biodiversity in agricultural landscapes. Poster presentation. German Federal Environmental Foundation (DBU), Osnabrück. Conference topic: More biodiversity in agricultural landscapes - Is a trend reversal possible?

- Wietzke, A.; Westphal, C.; Kraft, M.; Gras, P.; TscharntKe, T.; Pawelzik, E. Smit, I. (2016): Pollination as a key factor for strawberry fruit physiology and quality. Poster presentation. Fiftieth session of the German Society for Quality (DGQ), Berlin. Conference topic: Plants for bioeconomy - Which challenges emanate to quality of renewable raw materials? 


\section{Thesis declaration}

I hereby affirm that I have written this thesis independently and without unauthorized assistance and that I have not made use of any services that contradict the purpose of the examination procedure. Furthermore, I hereby declare that I have not used this thesis or parts thereof for the application for a doctoral degree at any other university or institution.

Alexander Wietzke

Göttingen, 19.06.2020 